\title{
Environmental availability of micronutrients in tropical soils
}




\section{Thesis committee}

\section{Promotors}

Prof. Dr R.N.J. Comans

Professor of Soil Chemistry and Chemical Soil Quality

Wageningen University \& Research

\section{Co-promotor}

Prof. Dr E. Hoffland

Personal chair, Soil Biology Group

Wageningen University \& Research

\section{Other members}

Prof. Dr S.E.A.T.M. van der Zee, Wageningen University \& Research

Prof. Dr E. Smolders, KU Leuven, Belgium

Dr C. Tiberg, Swedish Geotechnical Institute, Sweden

Dr J. Dijkstra, TNO Geological Survey of the Netherlands, the Netherlands

This research was conducted under the auspices of the Graduate School for SocioEconomic and Natural Sciences of the Environment (SENSE) 


\title{
Environmental availability of micronutrients in tropical soils
}

\author{
Elise Van Eynde
}

Thesis

submitted in fulfilment of the requirements for the degree of doctor at Wageningen University

by the authority of the Rector Magnificus,

Prof. Dr A.P.J. Mol,

in the presence of the

Thesis Committee appointed by the Academic Board

to be defended in public

on Friday 27 August 2021

at 1.30 p.m. in the Aula. 
Elise Van Eynde

Environmental availability of micronutrients in tropical soils, 276 pages.

PhD thesis, Wageningen University, Wageningen, the Netherlands (2021) With references, with summary in English

ISBN 978-94-6395-880-6

DOI https://doi.org/10.18174/549565 


\section{Table of contents}

$\begin{array}{lll}\text { Chapter } 1 & \text { General Introduction } & 7\end{array}$

Chapter 2 Surface reactivity of the natural metal (hydr)oxides in 33 weathered tropical soils

Chapter 3 The role of metal (hydr)oxides in controlling organic carbon 61 storage of soils

Chapter 4 Boron Adsorption to Ferrihydrite with Implications for Surface 85 Speciation in Soils: Experiments and Modeling

Chapter 5 Interaction of $\mathrm{Zn}$ with ferrihydrite and its cooperative binding 119 in the presence of $\mathrm{PO}_{4}$

Chapter 6 Boron speciation and extractability in temperate and tropical 157 soils: a multi-surface modeling approach

Chapter 7 Solid-solution partitioning of micronutrients $\mathrm{Zn}, \mathrm{Cu}$ and B in 195 tropical soils: mechanistic and empirical models

$\begin{array}{lll}\text { Chapter } 8 \text { General Discussion } & 237\end{array}$

$\begin{array}{ll}\text { Summary } & 265\end{array}$

$\begin{array}{ll}\text { Acknowledgements } & 271\end{array}$

$\begin{array}{ll}\text { About the author } & 273\end{array}$

$\begin{array}{ll}\text { SENSE diploma } & 274\end{array}$ 

Chapter 1

General Introduction

Elise Van Eynde 


\section{Background}

The dominant presence of weathered soils with low inherent fertility has been formulated as one of the factors that hampers an African Green revolution to take place $^{1}$, and that explains why the average crop yield on the African continent has stagnated since the 1960s in contrast to other regions around the world ${ }^{2,3}$.

Sustainable agricultural intensification based on integrated soil fertility management, that includes the application of mineral fertilizers in combination with organic inputs and improved germplasm, has been formulated as a key strategy to increase yields in Sub-Saharan Africa (SSA) ${ }^{2,4-6}$. The current use of mineral fertilizers in SSA is on average $16 \mathrm{~kg} \mathrm{ha}^{-1}$, which is the lowest rate globally ${ }^{7}$. The challenge for sustainable mineral fertilizer management is to apply those elements that are actually growthlimiting. Next to oxygen, hydrogen and carbon, there are 13 elements essential for plant growth that are in general taken up from the soil. These elements are classified according to plant requirements: macronutrients (nitrogen, phosphorus, potassium), secondary macronutrients (calcium, magnesium and sulphur) and micronutrients (iron, zinc, copper, boron, manganese, molybdenum, chloride) ${ }^{8}$. During the last decades, soil fertility and crop nutrition research in Sub-Saharan Africa (SSA) has been mainly focused on the macronutrients, i.e. nitrogen $(\mathrm{N})$, phosphorus $(\mathrm{P})$ and potassium $(K)^{9-11}$, while research on micronutrient deficiencies was less frequent and more scattered ${ }^{12}$. However, global and regional studies have shown evidence that micronutrients can limit crop yields and crop growth responses to regular NPK fertilizers. Based on a meta-analysis that included more than 700 datapoints from various SSA countries, Kihara et al. ${ }^{10}$ concluded that there was an overall positive response in crop yield to the application of micronutrients, especially in regions with a low response to macronutrient application. In a different study, they identified low soil micronutrient concentrations typically for the poor-responsive soils to NPK fertilization ${ }^{13}$. Based on a global survey, Sillanpää ${ }^{14}$ identified micronutrient deficiencies for cereal growth in different SSA countries, and emphasized the need for supplying especially copper $(\mathrm{Cu})$, boron $(\mathrm{B})$ and zinc $(\mathrm{Zn})$ for correcting nutrient deficiencies. This is further illustrated by fertilizer trials in SSA countries that have shown a yield increase when micronutrients such as $\mathrm{Zn}, \mathrm{Cu}$ and $\mathrm{B}$, are applied ${ }^{9,15-}$ 17. The focus of this thesis will be on these three micronutrients.

The availability of $\mathrm{Zn}, \mathrm{Cu}$, and $\mathrm{B}$ in soils and the subsequent uptake by crops, not only has a potential effect on the attainable yields, but also affects the final concentration in the edible parts of the plant and the subsequent intake by humans. From the major micronutrients that are suspected to be problematic for crop growth in SSA, $\mathrm{Zn}$ and $\mathrm{Cu}$ are classified as essential trace elements for humans, while $\mathrm{B}$ is at this moment classified as probably essential ${ }^{18}$ with growing recognition that it should be considered essential ${ }^{19}$. Too low intake by humans of these micronutrients through their diet can result in severe health issues. Among all micronutrients, it is documented that $\mathrm{Zn}$ deficiency in humans is most widespread ${ }^{20}$. It is estimated that 
worldwide up to 2 billion people are prone to too low intake of vitamins and micronutrients, from which too low intake of $Z n$ affects 1.2 billion people ${ }^{21}$. In SSA, it is estimated that $50 \%$ of all children is at risk of $\mathrm{Zn}$ deficiency ${ }^{22}$. Micronutrient deficiencies among humans is widespread in regions where staple food is grown in soils with low micronutrient levels, as low levels in soils lead to low concentrations in the edible parts, and consequently to low intake by humans ${ }^{23,24}$. The latter has been confirmed by Berkhout et al.25, who found significant relations between the concentrations of micronutrients such as $\mathrm{Zn}$ and $\mathrm{Cu}$ in soils from SSA, and the prevalent rates of child mortality, stunting, wasting and underweight, which are typical health problems associated to micronutrient deficiencies.

Blanket fertilizer recommendations including only NPK are most common in SSA. They have been developed for large areas or agroecological zones based on general soil and climate information and do not take into account the spatial heterogeneity in soil and management factors ${ }^{26}$. Alternatively, science-based approaches have been developed for formulating site-specific fertilizer recommendations ${ }^{7}$. For example, the Quantitative Evaluation of Soil Fertility of Tropical soils (QUEFTS) model ${ }^{27}$ calculates the soil supply of NPK based on soil tests, and includes the interaction effects among these nutrients on the final yields. The use of QUEFTS results in fertilization recommendations that are superior to the blanket fertilizer schemes in terms of yield and agronomic use efficiencies ${ }^{7}$. Such science-based approaches could be extended to account for micronutrients, based on the knowledge about the soil chemical micronutrient status, and processes that affect their availability for plant uptake. Studying the soil micronutrient status, is therefore an important first step for the future development of fertilizer recommendation schemes that include micronutrients, and forms the starting point for this thesis research.

\section{Soil micronutrient status}

Soils contain often substantial total amounts of Zn $\left(\sim 50 \mathrm{mg} \mathrm{kg}^{-1}\right)^{28}$, Cu $(\sim 20 \mathrm{mg} \mathrm{kg}$ $\left.{ }^{1}\right)^{29}$ and $\mathrm{B}\left(\sim 40 \mathrm{mg} \mathrm{kg}^{-1}\right)^{29}$. A large part of this total amount is inert, because it is typically occluded in the crystal matrix of soil constituents such as metal (hydr)oxides and clay minerals ${ }^{30,31}$. As a result, the total micronutrient content in soils is mainly related to the parent material, next to other soil-forming processes ${ }^{32}$. Part of this total content is assumed to be released from the soil matrix by very slow processes only, like mineral weathering, and is therefore not readily available for plant uptake. There is a general agreement that the total element content in soils, measured by for example X-ray fluorescence spectroscopy ${ }^{33}$ or soil sample destruction using Aqua Regia or $\mathrm{HF}^{34}$, does not provide information on the possible toxicity or deficiency for biota ${ }^{35-37}$ and consequently should not be used for fertilizer recommendations or risk assessments.

More relevant for plant uptake are the reactive and soluble pools. Part of the total content interacts with the soil solution through sorption/desorption and (rapid) 
precipitation/dissolution processes (Figure 1). This nutrient pool is often referred to as the reactive pool ${ }^{31,38}$, the labile pool ${ }^{39,40}$, the nutrient buffer capacity or quantity $(\mathrm{Q})^{39,41}$ or potential availability ${ }^{37}$. The soluble pool consists of the dissolved species in the soil solution that are buffered by the adsorbed and/or precipitated species in the solid phase. This pool is also referred to as the intensity (I),dissolved concentration $(\mathrm{C})^{39,41}$ or actual availability ${ }^{37}$. Together, the soluble and potential available pool represent the soil environmental available nutrient concentration ${ }^{42}$.

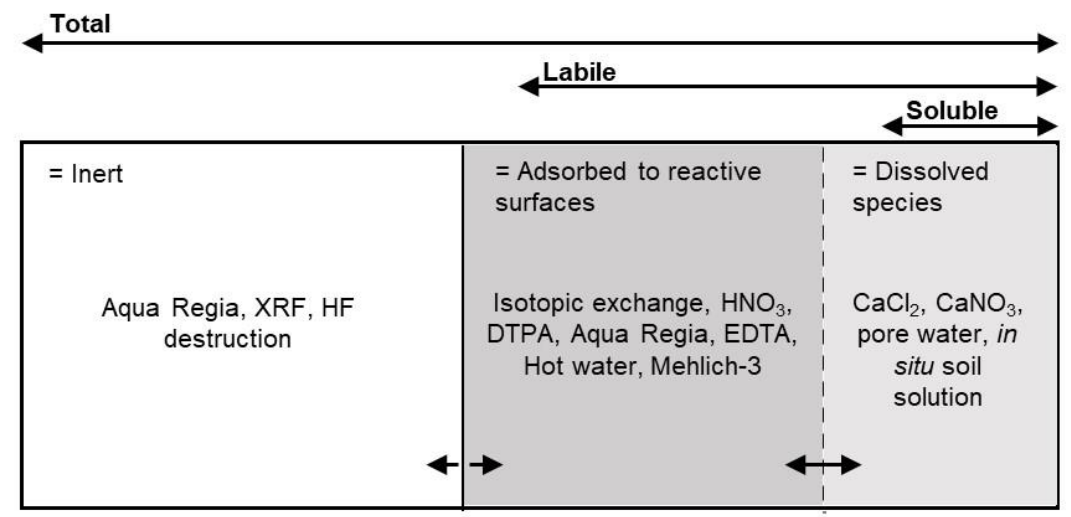

Figure 1: Different nutrient pools in soils, adapted from Römkens et al ${ }^{43}$ Part of the total nutrient content is inert. The release from the inert fraction that is part of the crystal mineral matrix into the labile pool (dotted arrow) is assumed to be slow. As a result, this pool is not relevant for plant availability. The labile pool consists mainly of adsorbed species in the solid phase, and of the dissolved species in solution. The last two pools are in equilibrium via adsorption/desorption and precipitation/dissolution processes. In each box, different methods are given which have been used to estimate the total, labile and soluble micronutrient (i.e., $\mathrm{Zn}, \mathrm{Cu}$ and $\mathrm{B}$ ) concentrations in soils. The scale of the figure is not absolute, since the contribution of the different pools to the total amount may differ among elements and soils.

The reactive pool can be estimated by isotopic dilution: stable or radio-isotopes are added to a soil sample, and due to mixture with the particular element naturally present in the soil, the isotope that has been added is diluted. The extent of dilution is related to the pool that is exchangeable with the solution phase, which is by definition the reactive pool ${ }^{40,44,45}$. Conceptually, isotopic dilution is the most solid approach to quantify the reactive nutrient concentration ${ }^{46}$. However, the use of soil extractions rather than isotopic dilution is often more convenient, since this requires less-specialized laboratories and is associated with lower costs, especially when multi-element concentrations can be analyzed simultaneously. One such commonly used method is the $0.43 \mathrm{M} \mathrm{HNO}_{3}$ extraction. ${ }^{31}$ Marzouk et al. ${ }^{44}$ showed that there was an agreement between the $0.43 \mathrm{M} \mathrm{HNO}_{3}$-extractable concentration and the isotopically exchangeable $\mathrm{Zn}$ for acidic soils. Based on geochemical modelling, the efficiency of the $\mathrm{HNO}_{3}$ extraction for measuring the reactive concentration has been evaluated for a wide range of elements ${ }^{31}$. These results showed a reasonable agreement between $\mathrm{HNO}_{3}$-extractable $\mathrm{Zn}$ and $\mathrm{Cu}$ and the reactive concentration, except for $\mathrm{Zn}$ in high $\mathrm{pH}$ soils and soils with low $\mathrm{Cu}$ loadings ${ }^{31}$. The $0.43 \mathrm{M} \mathrm{HNO}_{3}$ 
extraction has been recently adopted as the international standard for measuring the reactive concentration of trace elements in soils ${ }^{38}$.

In the context of soil fertility research, extraction methods have been developed not with the aim of targeting a specific soil nutrient pool as shown by Figure 1, but more as diagnostic soil tests for assessing nutrient bioavailability, based on the premise that the extractable amount is related to the plant nutrient uptake. For $\mathrm{Cu}$ and $\mathrm{Zn}$, chelating agents such as diethylenetriaminepentaacetic acid (DTPA) ${ }^{47}$ and ethylenediaminetetraacetic acid (EDTA $)^{48}$ have been used in extraction solutions for the identification of soils with insufficient levels for crop growth ${ }^{44}$. The DTPA extraction, according to Lindsay and Norvell ${ }^{47}$, is a very common soil test for identifying low Zn soils. However, previous studies have shown that the DTPAextractable $\mathrm{Zn}$ and $\mathrm{Cu}$ are not good estimations of the reactive pool, since they are consistently lower than what is measured based on isotopic dilution methods ${ }^{44,49}$. According to the conceptual framework depicted in Figure 1, a DTPA extraction can therefore be considered as an intermediate between I and $Q$ soil tests.

For B, the above mentioned extraction methods are less common. Specifically for B, the use of a hot water extraction is often used for estimating $B$ availability in soils ${ }^{50}$. In addition, solutions with competing anions such as phosphate ${ }^{51}$, or sugars or polyols that are known to have a strong affinity for complexing $B$ at neutral to alkaline $\mathrm{pH}$ values ${ }^{52,53}$ have been used for measuring the exchangeable soil $\mathrm{B}$, but the extent to which these extraction methods quantify the reactive pool has not been tested with isotopic dilution or geochemical modelling, as it has been done for $\mathrm{Zn}$ and $\mathrm{Cu}^{31,44}$.

Multi-element extractions in which macro-and micronutrients can be measured simultaneously are often used for the assessment of the soil nutrient status, due to their convenience and associated lower cost. One frequently used example is the Mehlich-3 extraction ${ }^{54}$, an acidic extraction solution that contains a mixture of chemical compounds $\left(\mathrm{CH}_{3} \mathrm{COOH}, \mathrm{NH}_{4} \mathrm{NO}_{3}, \mathrm{NH}_{4} \mathrm{~F}, \mathrm{HNO}_{3} \text { and EDTA }\right)^{54}$. It has been used for assessing the availability of macronutrients such as phosphorus, but also for $\mathrm{Zn}, \mathrm{Cu}$ and $\mathrm{B}^{55,56}$ and it is a commonly used method in African laboratories for measuring the nutrient availability in agricultural soils ${ }^{57,58}$. Previous studies have shown that micronutrient concentrations in a Mehlich-3 extraction of for example $\mathrm{Zn}$, are higher than in DTPA extractions ${ }^{59}$, but the relations between the two extraction methods are not the same among different soils with different physical-chemical properties. To the best of our knowledge, a Mehlich-3 extraction has not yet been compared with reactive concentrations based on isotopic dilution methods, nor has its efficiency for measuring the reactive concentration been evaluated based on geochemical modelling as it has been done for example for $0.43 \mathrm{M} \mathrm{HNO}_{3}{ }^{31}$. Especially the short equilibration time that is part of the extraction protocol ${ }^{60}$, may possibly limit the applicability for quantifying the reactive nutrient pool. 
The soil solution provides the immediate source of nutrients for plants since it is in direct contact with the plant roots. Ideally, the soluble nutrient concentration should be measured directly in the soil solution. However, the soil solution composition is variable in time ${ }^{61}$ and it is often more convenient to apply soil extractions in the laboratory that aim to mimic the soil solution. Often, calcium (Ca) is used as a cation in these extractions ${ }^{62}$, as this is expected to be the dominant ion in the actual soil solution ${ }^{63,64}$. In general, soil extractions are always associated with a certain conditionality (i.e. composition of extraction solution, solution-to-solid ratio and equilibration time) that needs to be taken into account when interpreting concentrations in term of nutrient pools ${ }^{65}$.

According to the conceptual framework in Figure 1, both the reactive and soluble nutrient pools are important in terms of availability for crop uptake: a plant takes up nutrients from the solution, which are replenished by the adsorbed or precipitated forms from the reactive pool. Which of the two pools is most relevant for predicting micronutrient deficiencies or toxicities is a complex issue. It has resulted in numerous studies that try to relate measured concentrations in various soil extractions to crop uptake, tissue concentrations or yields. It is often concluded that the extraction method for which the strongest relation between extractable concentrations and plant parameters is found, is considered to be the best method ${ }^{66}$. Unfortunately, very few studies include both reactive and soluble fractions for analyzing the influence of the soil micronutrient status on plant parameters ${ }^{67,68}$. In some cases, the combination of both soil nutrient fractions has led to better relations with plant parameters, than using only one single fraction ${ }^{41,67,68}$. In addition, such analyses could help to improve the understanding of how certain factors influence the relative importance of each nutrient pool for plant uptake. For instance, the choice of extraction method might depend on the nutrient of interest: for contaminated soils, it has been suggested that measuring the soluble pool might be a better soil diagnostic test for the risk assessment of relatively mobile and easily desorbed elements, such as $\mathrm{B}$ and $\mathrm{Zn}$, compared to $\mathrm{Cu}$ which is more strongly adsorbed and for which an acid extraction might be more appropriate ${ }^{69,70}$. When the transport of elements towards the plant roots exceeds the uptake rate, the concentrations near the plant roots are higher than the physiological need of the plants. As such, measuring the soluble concentrations may relate better to plant uptake ${ }^{71}$. In uncontaminated soils, the transport to the soil roots by diffusion and mass flow may become more important, thereby increasing the importance of the soil buffering capacity (i.e. reactive concentrations). The adsorption affinity of a soil, which is related to the distribution coefficient (i.e., $\mathrm{Q} / \mathrm{C}$ or $\mathrm{Q} / \mathrm{I}$ ), can determine which extractions are more appropriate. For soils with high adsorption affinity, soil tests that approximate the soluble pool may be more related to plant uptake, while for soils with limited adsorption affinity, the reactive concentrations may be a better indicator for nutrient availability ${ }^{41}$.

To summarize, for assessing the micronutrient status, ideally both the reactive and soluble concentrations are known, since both pools are important in terms of the 
availability for plant uptake, being direct or indirect, and the ratio of both fractions can be informative for defining the main limitation for plant uptake. In general, for soils with low levels, the soil tests that are used for measuring the intensity or the soluble pool, are less common than soil tests that approximate the reactive content due to the low concentrations in the soluble pool and the associated analytical challenges. As already mentioned, a Mehlich-3 extraction is most often used by African laboratories for measuring the micronutrient nutrient levels in soils ${ }^{57,58}$. This is also reflected by the results from the African Soil Information Service Project (AfSIS). The AfSIS project was created to produce spatial agronomic and soil data in SSA. This has resulted in a large set of point data which contains total ${ }^{32}$ and reactive concentrations based on Mehlich-3 extractions ${ }^{72,73}$ of macro-and micronutrients in soils across the region, next to other general soil properties such as organic carbon, $\mathrm{pH}$, cation exchange capacity and texture ${ }^{73,74}$. In addition, these data have been used to produce soil maps that predict general soil properties, and labile macro-and micronutrient concentrations for the whole of SSA ${ }^{58,73,74}$. No such information exists for the soluble concentrations of micronutrients in SSA so far, but could be derived from the reactive concentrations, if the solid-solution partitioning is known.

The solid-solution partitioning, i.e. the distribution of the reactive content over the solid and solution phase, is controlled by adsorption/desorption processes and precipitation/dissolution processes (Figure 1). These processes are governed by the amount and reactivity of the different mineral and organic surfaces for adsorption, the soil $\mathrm{pH}$, the ionic strength and co-occurring ions. The solid-solution partitioning of trace elements has been studied extensively in, often contaminated, soils from temperate regions in the context of risk assessment, but rarely for tropical soils with inherently low levels ${ }^{75}$. However, a good understanding of the soil chemical processes that control the solid-solution partitioning of $\mathrm{Zn}, \mathrm{Cu}$ and $\mathrm{B}$ in SSA soils can enhance the development of more accessible prediction tools of the soluble pool based on existing data and routine measurements. Improving the understanding of soil chemical processes that control the micronutrient solid-solution partitioning, and translating this knowledge into accessible prediction tools, will be the main aim of this thesis. To do so, mechanistic and empirical geochemical models will be used.

\section{Modeling solid-solution partitioning}

A basic model for the solid-solution partitioning of elements in soils is shown by the distribution coefficient $\left(K_{d}\right)$ in equation 1 , in which $Q$ is the adsorbed concentration $\left(\mathrm{mol} \mathrm{kg}^{-1}\right)$ and $C$ the concentration in solution $\left(\mathrm{mol} \mathrm{L}^{-1}\right)$.

$$
K_{d}=\frac{Q}{C}
$$

The distribution coefficient as shown in Equation 1 assumes a constant $K_{d}$ value independent of other soil properties and the concentration. But linear adsorption is only true at the lower concentration ranges, since the binding affinity generally 
decreases with increasing concentrations ${ }^{76}$. Otherwise a Freundlich model (Equation 2) is more appropriate in which the increase in $Q$ decreases with increasing concentrations, following from an $n$ value smaller than 1 .

$$
K_{d}=\frac{Q}{c^{n}}
$$

The values of $K_{d}$ can vary greatly among soils ${ }^{64}$, since the adsorption is governed by soil-specific properties such as $\mathrm{pH}$ and the amount and type of reactive surfaces. Therefore, the model from equation 2 can be extended with soil properties in the form of a partition relation:

$$
\log K_{d}=\log \left(\frac{Q}{C^{n}}\right)=a_{0}+\sum a_{i} \log X_{i}
$$

in which $X_{i}$ represents a set of important soil properties such as $\mathrm{pH}$, while $a_{0}$ and $a_{i}$ are the regression coefficients. Based on equation 3, empirical prediction models have been developed to model the solid-solution partitioning in various soil samples, from which some examples are shown in Table 1. Most existing models have been calibrated on temperate soils, often from contaminated sites, to calculate the solidsolution partitioning of metals such as $\mathrm{Zn}$ and $\mathrm{Cu}$ for risk assessment ${ }^{77}$. These models have rarely been developed and used in the context of plant nutrition ${ }^{78}$, and they do not exist for tropical soils from SSA nor for trace elements such as B. In contrast to mechanistic models, which are introduced in the next paragraph, the applicability of empirical partition relations is restricted to the range of soil properties that were used for model derivation ${ }^{79}$, which could imply differences in model equations for tropical and temperate soils. 
Table 1: Examples of empirical prediction models or partition relations from literature to calculate the solid-solution partitioning of $\mathrm{Zn}$ and $\mathrm{Cu}$ in soils. The prediction model is given, together with how the element concentration in the solid (Q) and solution (C) phase was measured (see section 2), and the type of soils that were used to calibrate the prediction model with the corresponding reference. Other soil properties in the regression models are the soil organic matter (SOM), Clay, the metal (hydr)oxides (Al, $\mathrm{Fe}$ ), $\mathrm{pH}$, dissolved organic carbon (DOC), soil solution ratio (SSR) and the cation exchange capacity (CEC).

\begin{tabular}{|c|c|c|c|}
\hline Prediction model & Solid phase & Solution phase & Soils \\
\hline $\begin{array}{l}\text { Log } C_{Z n}=0.93+0.99 \log Q- \\
0.43 \log S O M-0.22 \log C l a y- \\
0.14 \log A I F e+0.12 \log D O C- \\
0.46 p H\end{array}$ & $\begin{array}{l}0.43 \mathrm{M} \mathrm{HNO}_{3} \\
\text { (Q) }\end{array}$ & $\begin{array}{l}0.01 / 0.002 \mathrm{M} \\
\mathrm{CaCl}_{2} / \mathrm{Ca}\left(\mathrm{NO}_{3}\right)_{2}(\mathrm{C})\end{array}$ & $\begin{array}{l}118 \text { Dutch soil } \\
\text { samples, } \\
\text { contaminated and } \\
\text { uncontaminated }^{80}\end{array}$ \\
\hline \multicolumn{4}{|l|}{$\begin{array}{l}\log C_{C u}=-3.74+0.60 \log Q- \\
0.28 \operatorname{logSOM}-0.79 \operatorname{logAIFe}+ \\
0.79 \mathrm{DOC}\end{array}$} \\
\hline $\begin{array}{l}\log \mathrm{C}_{\mathrm{Zn}}=-0.66 \mathrm{pH}+1.04 \mathrm{SOM}- \\
5.92\end{array}$ & $\begin{array}{l}\mathrm{HNO}_{3}-\mathrm{HClO}_{4} \\
\text { digest }\end{array}$ & $\begin{array}{l}\text { Free } Z n \text { activity in } \\
\text { EDTA }(C)\end{array}$ & $\begin{array}{l}18 \text { neutral to alkaline } \\
\mathrm{pH} \text { agricultural soils } \\
\text { from Colorado }^{78}\end{array}$ \\
\hline $\begin{array}{l}\log Q_{z n}=-1.07+0.697 \log C+0.675 \\
\log C E C+0.282 p H \\
\log Q_{C u}=-0.775+0.567 \log C+ \\
0.445 \log C E C+0.225 p H- \\
0.625 \log S S R\end{array}$ & $\begin{array}{l}\text { Adsorbed } \\
\text { amount from } \\
\text { batch } \\
\text { experiments } \\
\text { (Q) }\end{array}$ & $\begin{array}{l}\text { Total concentrations } \\
\text { in different } \\
\text { background } \\
\text { electrolytes }(\mathrm{C})\end{array}$ & $\begin{array}{l}478 / 408 \text { datapoints } \\
\text { from literature } 81\end{array}$ \\
\hline $\begin{array}{l}\log C_{Z n}=3.68-0.55 p H+0.94 \\
\log Q-0.34 \log S O M \\
\log C_{C u}=1.37-0.21 p H+0.93 \\
\log -0.21 \log S O M\end{array}$ & $\begin{array}{l}\text { Total content } \\
\text { via different } \\
\text { acid digestions } \\
\text { (Q) }\end{array}$ & $\begin{array}{l}\text { Total concentrations } \\
\text { in water } \\
\text { displacement, } \\
\text { lysimeter, water or } \\
\text { neutral salt } \\
\text { extractions (C) }\end{array}$ & $\begin{array}{l}212 / 353 \text { datapoints } \\
\text { from literature }\end{array}$ \\
\hline
\end{tabular}

Next to empirical prediction models, mechanistic models have been developed to describe ion adsorption to the soil reactive surfaces with permanent and $\mathrm{pH}$ dependent surface charge. These surface-specific models are combined with thermodynamic constants for inorganic speciation and mineral equilibria, in a multisurface geochemical model (Figure 2) ${ }^{83}$. In fact, the empirical partition relations are models in which the complex processes described in a multi-surface model, are lumped into one regression equation. ${ }^{79}$ Since a multi-surface model calculates the chemical speciation of elements in soils, it cannot only be used to predict the solidsolution partitioning of elements, but also provides information on which processes are most important for controlling the solid-solution partitioning, that in turn helps to interpret and develop partition relations in the form of statistical regression models. 


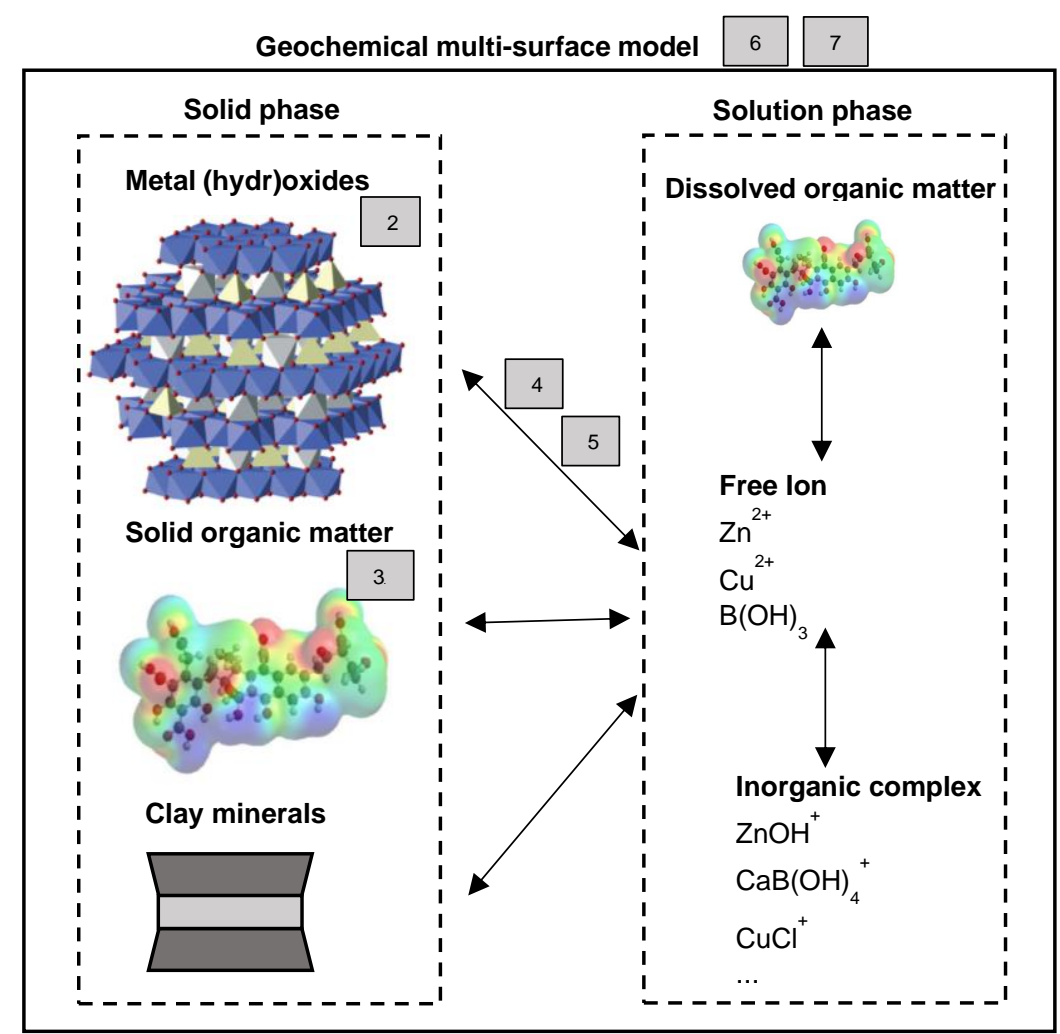

Figure 2: Schematic picture of a multi-surface model for calculating the speciation of $\mathrm{Zn}, \mathrm{Cu}$ and $\mathrm{B}$ in soils. In the solid phase, the nutrients can be adsorbed to metal (hydr)oxides (represented by a ferrihydrite particle ${ }^{85}$ ), solid soil organic matter and clay minerals. For each compartment in the solid phase, a surfacespecific ion adsorption model is used. In the solution phase, the nutrients are present as free ions, as inorganic complexes, or complexes with dissolved organic matter. The numbers refer to the different chapters of this thesis: (2) The reactivity of the natural (hydr)oxide fraction in tropical soils, (3) The role of metal (hydr)oxides in the capacity of soils to bind store soil organic carbon, (4) (5) The adsorption of $\mathrm{Zn}$ and B to ferrihydrite nanoparticles, (6) (7) Application of a multi-surface model in soils from Sub-Saharan Africa.

Ion adsorption models balance between a mechanistic description of the adsorption process based on thermodynamic principles, and a simplification of the complexity of adsorption processes in order to be convenient for applications ${ }^{84}$. In general, ion adsorption models differentiate between electrostatic and ion-specific binding, and account for competitive and cooperative effects among ions for binding to the surfaces $^{83}$. In contrast to empirical prediction models (Table 1, Equations 1-3), their generic nature makes them widely applicable, and not limited to specific environmental conditions ${ }^{77}$. The derivation of generic model parameters is done independently based on laboratory adsorption experiments.

Multi-surface models have been applied for studying the solid-solution partitioning in $\mathrm{Zn}$ and $\mathrm{Cu}$ in soils $\mathrm{s}^{75,80,86-93}$, waste materials ${ }^{94}$, and composts ${ }^{95}$. To do so, the solution phase has often been approximated by a soil extraction with low salt levels, to mimic 16 
the actual soil solution. In addition, such models have been applied for example to study the effect of root exudates on nutrient availability ${ }^{96,97}$, the efficiency of a chelate micronutrient fertilizer ${ }^{98}$, or the effect of management practices such as liming and fertilization on the micronutrient speciation in soils ${ }^{75}$. Similarly to the empirical prediction models, these models have rarely been used in soils from the tropics with low levels of $\mathrm{Zn}$ and $\mathrm{Cu}^{75}$. In addition, previous multi-surface modelling calculations of $\mathrm{Zn}$ and $\mathrm{Cu}$ have shown that for $\mathrm{Cu}$, the model performs relatively good and the important processes that control $\mathrm{Cu}$ solid-solution partitioning, which are dominated by the adsorption to particulate and dissolved organic matter, are generally well understood ${ }^{31,95}$. This is different for $\mathrm{Zn}^{88,95}$, for which modelling calculations have shown systematic deviations from measured concentrations, suggesting that the chemical processes that control solid-solution partitioning of $\mathrm{Zn}$ are generally less understood and that more research is needed.

Goldberg and colleagues have done most work regarding modelling the solidsolution partitioning of $B$ in soils ${ }^{99-101}$. They did not use a multi-surface model as shown by Figure 2, but instead they used a generalized composite approach, in which the binding to the different reactive surfaces in soils is approximated by one single composite surface plane ${ }^{83}$. The binding constants were fitted to $\mathrm{pH}$ dependent $B$ adsorption data for the soil-specific assemblage, and subsequently related to soil properties ${ }^{101}$. As a result, the adsorption parameters are not generically transferable to other soils, and little information is gained about the individual soil chemical processes that affect the distribution of natural reactive $B$ over the solid and solution phase.

Although clay minerals are often included, previous multi-surface modelling calculations have shown that the soil organic matter followed by the metal (hydr)oxides are the most important reactive surfaces for $\mathrm{Cu}$ and $\mathrm{Zn}$ adsorption ${ }^{62,87,90,93}$. Next to $\mathrm{pH}$, the amounts of both reactive surfaces have been identified as the most important soil properties for explaining the solid-solution partitioning of these two trace elements in soils ${ }^{80}$. In addition, spectroscopic analyses of soil samples have also confirmed the importance of soil organic matter and metal (hydr)oxides for the adsorption of $\mathrm{Zn}$ and $\mathrm{Cu}^{90,102-104}$. It has also been shown that these surfaces adsorb $B^{105-107}$, and it has been found that the content of soil organic matter and metal (hydr)oxides is important for explaining the binding constants of $B$ to soils when using a generalized composite modelling approach ${ }^{101}$.

Understanding and modelling the solid-solution partitioning of $\mathrm{Zn}, \mathrm{Cu}$ and $\mathrm{B}$ therefore requires knowledge of the soil organic matter and metal (hydr)oxide content, their corresponding reactivity and the adsorption mechanisms. These properties are discussed in the following sections. 


\section{Soil metal (hydr)oxides}

The core mineral structure of metal (hydr)oxides consists of metals, such as Fe and $\mathrm{Al}$, surrounded by oxygen atoms (see an example in Figure 1). At the surface, the charge of the oxygens is undersaturated which is compensated by proton adsorption. As a result, a pH dependent surface charge is developed that leads to ion adsorption.

The overall energy related to the ion adsorption to the charged metal (hydr)oxide surface consists of a chemical component (i.e., intrinsic binding affinity, $K^{\text {int }}$ ), and an electrostatic component. Due to the charge at the surface, ions of opposite charge in the aqueous phase accumulate near the surface, and their concentration decreases towards the solution. The opposite is true for ions with similar charge as the surface. This phenomenon is described by the diffuse double layer (DDL) as shown by Figure $3^{108}$.
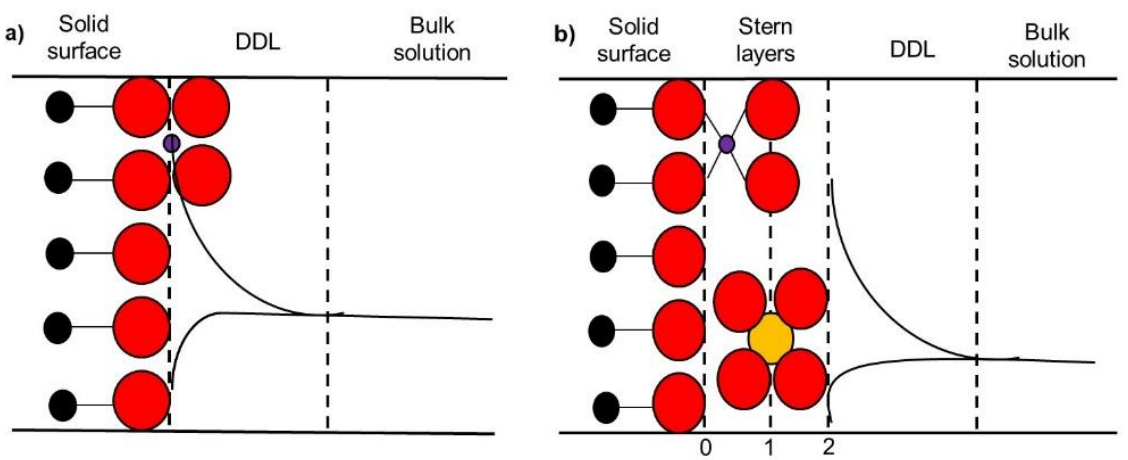

Figure 3: Schematic representation of a metal (hydr)oxide and solution interface following a) the diffuse double layer model that is part of the generalized two layer ion adsorption model (GTLM) ${ }^{108}$ and b) the extended Stern layer model that is part of the charge distribution (CD) ion adsorption model ${ }^{112}$. The solid particle consists of metal ions (black spheres) that are coordinated with oxygen atoms (red spheres) which at the surface interact with ions that form inner-sphere complexes (purple sphere) or outer-sphere complexes in which the ion does not lose its hydration sphere (yellow sphere). Protons are not shown for simplicity. Due to surface charge, ions of opposite charge accumulate near the surface, and their concentration decreases towards the solution while the opposite is true for ions with equal charge as the surface (black lines). a) In the GTLM, the adsorbed surface species are considered as point charges, and this charge is completely attributed to the surface plane that is simultaneously the head-end of the diffuse double layer. Outer-sphere surface species are not considered in this ion adsorption model. b) The extended Stern layer model defines an inner and outer Stern layer. The adsorbed surface species are not treated as point charges, but instead the total charge of the adsorption complex is distributed over the different planes.

Most multi-surface modelling studies have applied the Generalized Two Layer Model $(G T L M)^{108}$ for describing trace element adsorption to the metal (hydr)oxides ${ }^{31,75,87,93,95}$. In the GTLM, the adsorbed surface species are treated as point charges, and the total charge of the adsorbed complex is attributed to the surface plane (Figure 3a). Based on the surface charge, the surface potential is 18 
calculated, which affects the intrinsic binding affinity of the surface species due to electrostatic effects (i.e. $K^{a p p}=K^{\text {int }} \exp \left(-\Delta z_{0} \psi / R T\right.$ ) with $\Delta z_{0}$ the change in surface charge due to the adsorbed species, $\psi$ the surface potential, $\mathrm{R}$ the gas constant and $\mathrm{T}$ the temperature ${ }^{83,108}$ ). A more realistic physical representation of the interface is that the DDL is separated from the surface by a Stern layer ${ }^{109}$. In the basic Stern model, the surface is separated from the DDL by a layer that is related to the minimum distance at which hydrated counter-ions can approach the mineral surface ${ }^{109}$. In the Extended Stern model, an additional charge layer is introduced in order to separate the minimum distance of approach from the head end of the DDL (Figure $3 b)^{110}$.

This representation of the solid-solution interphase of the metal (hydr)oxides is used in the most recent version of the charge distribution (CD) model to describe ion adsorption to metal (hydr)oxides ${ }^{111}$. In addition, in the CD model the adsorbed surface species are not treated as point charges, but instead, the total charge of the adsorbed complex is distributed over the different planes that separate the surface and the two Stern layers (Figure 3b). The electrostatic component of the adsorption energy is consequently related to the change in charge and corresponding potentials of the different planes (i.e. $\left.K^{\text {app }}=K^{\text {int }} \exp \left(-\Delta z_{0} \psi_{0} / R T\right) \exp \left(-\Delta z_{1} \psi_{1} / R T\right)^{109}\right)$. The change in charge upon adsorption at the 0 - and 1-plane is given by the charge distribution values, i.e., $\Delta \mathrm{z}_{0}$ and $\Delta \mathrm{z}_{1}$, which represent the fraction of charge of the adsorbed surface species that is attributed to the corresponding plane. Since the location of the ion charge in the solid-solution interface strongly affects the electrostatic interactions, the CD modelling approach results in a better description of ion adsorption processes to metal (hydr)oxides compared to other models such as the GTLM $^{109}$.

The GTLM has been used for modelling ion adsorption to iron $(\mathrm{Fe})$ oxides such as microcrystalline ferrihydrite $(\mathrm{Fh})$ nanoparticles ${ }^{108}$ and crystalline minerals such as goethite ${ }^{113}$, and adsorption to aluminum (Al) oxides such as gibbsite ${ }^{114}$ and for manganese oxides ${ }^{115}$. In the GTLM, the site densities (sites $\mathrm{nm}^{-2}$ ) of hypothetical reactive surface groups have been fitted based on different series of ion adsorption data for each type of metal (hydr)oxide ${ }^{108}$. With these fixed values, the adsorption data of protons and various cations and anions were analyzed with the GTLM for developing a consistent database with ion adsorption constants for different elements $^{108}$. This modelling approach with hypothetical surface groups with fitted site densities differs from the Multi Site Ion complexation (MUSIC) model in which knowledge of the surface structure of the metal (hydr)oxide particle is used to define the different reactive sites and corresponding site densities ${ }^{116}$. The CD model in combination with a mineral-specific MUSIC model, has been used extensively for modelling anion and cation adsorption to goethite, including $\mathrm{Zn}^{93,117}, \mathrm{Cu}^{118}$ and $\mathrm{B}^{107}$. Thanks to recent improved insights into the mineral structure of Fh nanoparticles ${ }^{119-}$ ${ }^{121}$, a MUSIC model has been developed for $\mathrm{Fh}^{85}$. Only recently, the CD model in 
combination with the MUSIC model for $\mathrm{Fh}$, has been applied for modeling adsorption of $\mathrm{PO}_{4}{ }^{85}, \mathrm{CO}_{3}{ }^{122}, \mathrm{Ca}^{123,124}, \mathrm{Mg}^{123,124}, \mathrm{H}_{4} \mathrm{SiO}_{4}{ }^{125} \mathrm{As}(\mathrm{OH})_{3}{ }^{85}$ to $\mathrm{Fh}$ nanoparticles in a consistent manner. Such parameters do not yet exist for micronutrients such as $\mathrm{Zn}$, $\mathrm{Cu}$ and $\mathrm{B}$. In chapter 3 and 4 of this thesis, the interaction of respectively $\mathrm{Zn}$ and $\mathrm{B}$ will be interpreted with the CD-MUSIC model, based on experimental adsorption data in combination with molecular orbital calculations ${ }^{126}$ of the surface species that have been previously identified with spectroscopy.

The parameters for ion adsorption to metal (hydr)oxides are based on laboratory experiments with synthetic preparations of the metal (hydr)oxide of interest. When moving from synthetic laboratory experiments to natural systems such as soils, a choice needs to be made which model oxide is representative for the natural metal (hydr)oxide. The fraction of metal (hydr)oxide in soils consists of a wide variety of minerals with variable chemical composition, mineral structure and crystallinity. Laboratory adsorption experiments have shown that these mineral features clearly imply different adsorption interactions with ions ${ }^{123}$. The dominant mass fraction of the metal (hydr)oxide often varies across different environments ${ }^{127-129}$. Although the soils from the tropics are as heterogeneous as soils from temperate regions ${ }^{130}$, soils from the humid tropics are often relatively old and have experienced a prolonged and intensive weathering regime. In possible combination with low organic matter content and low phosphate levels, these intensively weathered soils are typically characterized by a high content of $\mathrm{Fe}$ and $\mathrm{Al}$ (hydr)oxides that are mainly wellcrystallized minerals such as goethite and gibbsite ${ }^{131,132}$. In general, these wellcrystallized metal (hydr)oxides have a lower reactivity, illustrated by a lower specific surface area (SSA in $\mathrm{m}^{2} \mathrm{~g}^{-1}$ oxide), compared to micro-crystalline oxide nanoparticles such as ferrihydrite (Fh).

Recently, it has been shown that synthetic Fh nanoparticles are a better proxy than goethite for describing the reactivity of the natural metal (hydr)oxides for a series of Dutch topsoil samples ${ }^{133}$. The question that arises is whether this is also the case for soils from tropical regions where crystalline materials contribute most to the overall mass fraction of metal (hydr)oxide. In chapter 2 of this thesis (Figure 2), an attempt is made to answer this question with a novel method that uses phosphate as probe-ion for assessing the reactive surface area of soils ${ }^{134}$.

\section{Soil organic matter}

Soil organic matter (SOM) consists of a mixture of diverse organic compounds. Understanding and modelling ion adsorption to soil organic matter is complex. It requires a proper description of the electrostatic component of the adsorption processes in combination with a formulation of the intrinsic binding reaction, similarly as discussed for ion adsorption to metal (hydr)oxides. In addition, the diversity in chemical structure of the soil organic matter leads to a large variety in affinity for ion adsorption. This chemical heterogeneity needs to be included in ion adsorption 
models and this is obviously less straightforward than for minerals ${ }^{135}$. The Non Ideal Competitive Adsorption (NICA) model takes chemical heterogeneity into account, using a continuous affinity distribution that is ion- and substrate specific ${ }^{136}$ :

$$
\boldsymbol{\theta}_{i}=\frac{\boldsymbol{n}_{i}}{n_{H}} \boldsymbol{\theta}_{i} \boldsymbol{Q}_{H, \text { max }}=\frac{n_{i}}{n_{H}} \boldsymbol{Q}_{H, \max } \frac{\left(\widetilde{K_{l}} a_{i}\right)^{n_{i}}}{\sum_{i}\left(\widetilde{K_{l}} a_{i}\right)^{n_{i}}} \frac{\left(\sum_{i}\left(\left(\widetilde{K_{l}} a_{i}\right)^{n_{i}}\right)^{p}\right.}{1+\left(\sum_{i}\left(\widetilde{K_{l}} a_{i}\right)^{n_{i}}\right)^{p}}
$$

with:
$\theta_{i}$ the fraction of reactive sites that is occupied with ion $i$
$\mathrm{QH}_{\mathrm{H}, \text { max }}$ the maximum adsorption sites for protons
$\mathrm{n}_{\mathrm{i}}$ the non-ideality parameter for ion $\mathrm{i}$
$\mathrm{n}_{\mathrm{H}}$ the non-ideality parameter for proton adsorption
$\mathrm{K}_{i}$ is the median value of the affinity distribution for ion $\mathrm{i}$
$a_{i}$ is the activity of ion $i$
$p$ is the heterogeneity parameter for the adsorption surface

The ion-specific non-ideality parameter in equation 4 can be interpreted as a reaction stoichiometry. For example, if $n_{i}$ is half of $n_{H}$, the maximal adsorption of ion $i$ is only half of the maximal proton adsorption. Since the non-ideality parameter is also part of the exponent $m_{i}$ of $K_{i}\left(i . e . m_{i}=n_{i} \times p\right)$, it also defines the width of the affinity distribution and the extent to which the affinity distributions of different ions are correlated ${ }^{137}$. To account for electrostatic effects on ion binding to organic matter, the ion activities of the NICA isotherm shown by equation 4 are translated to the activities near the surface based on the surface potential. In the Donnan model, the structure of the organic matter substances for ion binding is interpreted as a hydrated gel in which the surface charge $(\sigma)$ is completely neutralized by counter-ions present in the Donnan volume $\left(V_{D}\right)^{138}$ :

$$
\sigma=V_{D} \sum_{i} z_{i} C_{D, i}=V_{D} \sum_{i} z_{i} C_{s, i} e^{-z_{i} F \psi_{D} / R T}
$$

in which $z_{i}$ is the ion valence, $C_{D, i}$ is the concentration in the Donnan phase, $C_{s, i}$ is the concentration in the bulk solution, $\psi_{D}$ is the electrostatic potential in the Donnan volume, and $F, R$ and $T$ respectively the Faraday constant, the gas constant and the temperature. Based on the analysis of $\mathrm{pH}$-dependent surface charge of a variety of organic matter substances, an empirical relationship was established between the ionic strength and the Donnan volume ${ }^{138}$. If the Donnan volume is calculated accordingly, the electrostatic potential and subsequently the ion concentrations in the Donnan volume can be calculated based on the surface charge. The NICAdonnan model as shown by equations 4 and 5 has been parameterized for the adsorption of protons and a large series of trace elements, including $\mathrm{Zn}, \mathrm{Cu}$ and $\mathrm{B}^{105,139,140}$. The major assumption on which these models are based, is that humic and fulvic acids represent the reactive fraction of soil organic matter for ion adsorption ${ }^{141}$.

For modelling and understanding $\mathrm{Zn}, \mathrm{Cu}$ and $\mathrm{B}$ speciation and availability, the soil organic matter content is a key soil property as it is an important reactive surface for 
adsorption $80,93,106$. Next to compound retention and nutrient cycling, soil organic matter provides a wide range of soil functions. Currently the focus of soil organic matter research is mainly on carbon sequestration in the context of climate change mitigation ${ }^{141}$. However, the total soil organic matter content that is controlled by carbon stabilization has also its impact on micronutrient availability. This is illustrated by Figure 4 that shows the relation between soil organic carbon content and the concentrations of $\mathrm{Zn}, \mathrm{Cu}$ and $\mathrm{B}$ measured by a Mehlich-3 extraction in Rwanda. The soil organic matter stabilization in mineral soils is mainly through interactions with the soil mineral matrix ${ }^{142}$ from which the Fe- and Al-(hydr)oxides are being increasingly recognized as the most important reactive surfaces for stabilizing soil organic matter, via the formation of organo-mineral associations ${ }^{143}$. Especially the metal (hydr)oxide nanoparticles like ferrihydrite are expected to play a major role for carbon stabilization, due to their high affinity for adsorbing soil organic matter, ${ }^{144}$ and due to their large contribution to the overall reactivity of soils ${ }^{145}$. Recently, it has been shown that not only the reactivity, but also the particle size are key properties of the soil metal (hydr)oxides that control soil organic matter content ${ }^{133}$. In this thesis, the question whether this is a universal feature and thus also applies to soils from SSA with a very different composition of the metal (hydr)oxide fraction, will be studied in Chapter 3 (Figure 2). 

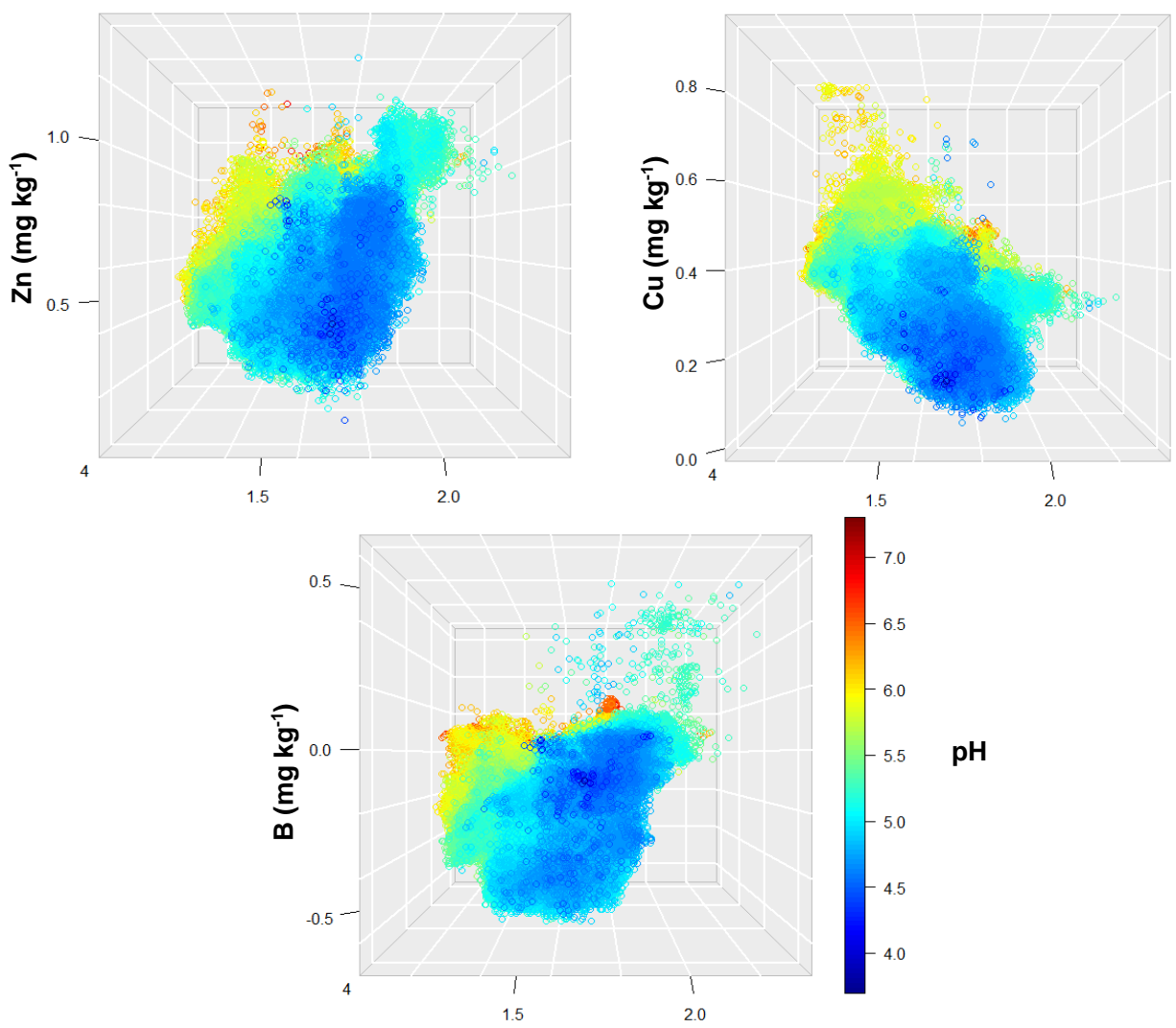

Soil organic carbon $\left(\mathrm{g} \mathrm{kg}^{-1}\right)$

Figure 4: The $\mathrm{Zn}, \mathrm{Cu}$ and $\mathrm{B}$ concentrations in Mehlich-3 (vertical z-axis) are plotted against the soil organic carbon (horizontal $x$-axis) and the soil $\mathrm{pH}\left(\mathrm{H}_{2} \mathrm{O}\right)$ ( $y$-axis and colour scale of the points) for Rwanda. Note that the nutrient concentrations and soil organic carbon content are plotted on a log10 scale. The nutrient concentrations were extracted from the nutrient maps created by Hengl et al. ${ }^{58}$, the pH $(5-15 \mathrm{~cm}$ depth) and soil organic carbon content $(5-15 \mathrm{~cm}$ depth) were extracted from the global maps from Soilgrids ${ }^{72}$. The concentrations of $\mathrm{Zn}$ and $\mathrm{B}$ measured in a Mehlich-3 extraction increase with increasing soil organic carbon content, (pearson correlation coefficient of 0.33 for $\mathrm{Zn}$ and 0.35 for $\mathrm{B}$ ) and this effect is enhanced by an increase in $\mathrm{pH}$. For $\mathrm{Cu}$, the opposite is true, and the $\mathrm{Cu}$ concentrations decrease with increasing soil organic carbon content (a negative pearson correlation coefficient of -0.67). All three figures illustrate the importance of soil organic carbon content for micronutrient concentrations in soils. In addition, it gives already some possible insights into the extraction efficiency of Mehlich-3: the positive relation between $\mathrm{Zn}$ and $\mathrm{B}$ and soil organic carbon suggests that Mehlich-3 may approximate the reactive concentration, while the results for $\mathrm{Cu}$ suggests that the adsorption capacity negatively affects the $\mathrm{Cu}$ concentration measured in Mehlich-3. A comparison between micronutrient concentrations in Mehlich-3 and $0.43 \mathrm{M} \mathrm{HNO}_{3}$ will be made in the final chapter of this thesis.

\section{Objectives and outline of this thesis}

In this thesis, the general objective is to study the soil chemical processes that control the solid-solution partitioning of $\mathrm{Zn}, \mathrm{Cu}$, and $\mathrm{B}$, particularly in soils from tropical areas in SSA. As already stated in the first section of this introduction, these 
micronutrients have been identified as possible candidates to explain the incidence of micronutrient deficiencies for crop production, and in the case of $\mathrm{Zn}$, for human health problems. Studying the solid-solution partitioning of $\mathrm{Zn}, \mathrm{Cu}$ and $\mathrm{B}$ for soils from SSA with a potentially high risk of deficiencies in crops and humans will enhance the knowledge on the soil micronutrient availability. This knowledge can then be translated into prediction models for the soluble micronutrient pool which can increase the possible applications of currently available data.

In addition, $\mathrm{Zn}, \mathrm{Cu}$, and $\mathrm{B}$ form an interesting group of elements to study from a chemical perspective. While $\mathrm{Zn}$ and $\mathrm{Cu}$ are metal cations, $\mathrm{B}$ is a metalloid that is neutral or negatively charged in soils ${ }^{146}$ and this might lead to different processes that control solid-solution partitioning. As already mentioned, the use of a multisurface modelling approach has not yet been applied for natural soil B, creating an opportunity to test this approach for $\mathrm{B}$, and to enhance the knowledge on the soil chemical processes that control the solid-solution partitioning of $B$ in soils. While previous multi-surface modeling calculations have resulted in relatively good predictions of the solid-solution partitioning of $\mathrm{Cu}^{31,95}$, the opposite is true for $\mathrm{Zn}$ and more research is needed.

Considering all the above, three main objectives are formulated, and the experimental and modelling chapters (Chapters 2-7) are organized according to these objectives, as shown by Table 2. An overview of the experimental and modelling chapters is also shown in Figure 2.

Table 2: Summary of the experimental chapters in this thesis, ordered according to the three main objectives.

\begin{tabular}{|c|c|c|}
\hline Objective & Chapter & Main topic \\
\hline \multirow{2}{*}{$\begin{array}{l}\text { Reactivity of tropical } \\
\text { soils }\end{array}$} & 2 & $\begin{array}{l}\text { Surface reactivity of the natural metal (hydr)oxide } \\
\text { fraction in tropical soils from SSA }\end{array}$ \\
\hline & 3 & $\begin{array}{l}\text { The role of the metal (hydr)oxides in controlling } \\
\text { capacity of soils to store organic carbon }\end{array}$ \\
\hline \multirow{2}{*}{$\begin{array}{l}\text { Parametrization of the } \\
\text { CD model for Fh }\end{array}$} & 4 & $\begin{array}{l}\text { The interaction of } \mathrm{Zn} \text { with Fh nanoparticles in the } \\
\text { absence and presence of } \mathrm{PO}_{4} \text { : Experiments and } \\
\text { CD-MUSIC modelling }\end{array}$ \\
\hline & 5 & $\begin{array}{l}\text { The interaction of B with Fh nanoparticles in the } \\
\text { absence and presence of } \mathrm{PO}_{4} \text { : Experiments and } \\
\text { CD-MUSIC modelling }\end{array}$ \\
\hline \multirow{2}{*}{$\begin{array}{l}\text { 3. Modelling solid- } \\
\text { solution partitioning in } \\
\text { tropical soils }\end{array}$} & 6 & $\begin{array}{l}\text { Boron speciation and extractability in temperate } \\
\text { and tropical soils: a multi-surface modeling } \\
\text { approach }\end{array}$ \\
\hline & 7 & $\begin{array}{l}\text { The solid-solution partitioning of } \mathrm{Zn}, \mathrm{Cu} \text { and } \mathrm{B} \text { in } \\
\text { tropical soils: mechanistic and empirical models }\end{array}$ \\
\hline
\end{tabular}

A first objective of this thesis is to better understand the reactivity of the most important adsorption surfaces for $\mathrm{Zn}, \mathrm{Cu}$ and $\mathrm{B}$, in tropical soils from SSA, i.e. metal (hydr)oxides and organic matter. 
In Chapter 2, the reactivity of the natural metal (hydr)oxide fraction will be measured for a series of tropical agricultural topsoils from Kenya and Burundi. To do so, we will use a novel method in which phosphate is used as probe-ion method for assessing the reactive surface area of the natural oxide fraction ${ }^{133,134}$. The experimental results will be interpreted with the CD-MUSIC model, using either goethite or ferrihydrite as model oxide to assess which model oxide describes best the surface reactivity of these tropical soils.

In Chapter 3 the experimental and modelling results from Chapter 2 with regard to the natural metal (hydr)oxide fraction, will be used to understand and conceptualize the capacity of soils to store soil organic carbon.

A second objective of this thesis is to use a consistent modeling approach for describing the adsorption of $\mathrm{Zn}, \mathrm{Cu}$ and $\mathrm{B}$ to ferrihydrite with the CD-MUSIC model. In Chapter 4 and 5, experimental adsorption data of $\mathrm{Zn}, \mathrm{Cu}$ and $\mathrm{B}$ with freshly prepared ferrihydrite particles will be interpreted with a consistent modelling approach. The consistent modelling approach consists of (1) a characterization of the freshly prepared ferrihydrite nanoparticles in terms of reactive surface area, using phosphate as probe-ion ${ }^{147}$ (2) An independent derivation of the charge distribution values based on a bond valence analysis of the optimized geometry of the formed surface complexes with molecular orbital calculations and (3) The use of the MUSIC model based on the most recent insights into the surface structure of ferrihydrite ${ }^{85}$. This modelling approach results in a parameterization of the CDMUSIC model that is consistent with previously derived parameters ${ }^{85,122,124,125}$. In addition, the cooperative and competitive effects of phosphate on the $\mathrm{Zn}, \mathrm{Cu}$ and $\mathrm{B}$ adsorption will be assessed. Phosphate is an oxyanion that is omnipresent in soils and has been shown to enhance metal cation adsorption to ferrihydrite, due to electrostatic effects or via the formation of ternary complexes ${ }^{123,148}$. By contrast, it can significantly reduce the adsorption of anionic species, such as carbonate ${ }^{122}$ or silicate $^{125}$.

The third objective of this thesis is to apply a multi-surface model for calculating the solid-solution partitioning of $\mathrm{Zn}, \mathrm{Cu}$, and $\mathrm{B}$ in tropical soils. Chapter 6 of this thesis focuses on $B$. In this chapter, a multi-surface model based on the adsorption of $B$ on dissolved and solid organic matter, ferrihydrite, and clay mineral edges will be applied to to assess the important processes that control the solid-solution partitioning of $\mathrm{B}$ in five temperate and five tropical topsoils. In addition, the performance of previously proposed extraction methods for measuring reactive $\mathrm{B}$ will be evaluated based on the $\mathrm{B}$ measurements in a $0.01 \mathrm{M} \mathrm{CaCl}_{2}$ soil extraction. In Chapter 7, a similar multi-surface modelling approach will be used for calculating the solid-solution partitioning of $\mathrm{Zn}, \mathrm{Cu}$, and $\mathrm{B}$ in 172 soils from Burundi, Kenya and Rwanda. Using these results as benchmark, empirical partition relations will be developed to predict the soluble concentrations based on general soil properties and the reactive content, which can be used to predict the soluble micronutrient concentrations based on existing soil data as illustrated in Chapter 8. 
Finally, in Chapter 8, a summary is given of the main results from the abovementioned chapters, together with a discussion of the limitations of this study in relation to future research. Finally, I will end with an example on how partition relations can be used as accessible tools for making spatial predictions of micronutrient solubility based on existing spatial soil data for a selection of SSA countries.

\section{REFERENCES}

1. Kazibwe, S. W., Rabbinge, R., Swaminathan, M. S. \& Others, A. Realizing the Promise and Potential of African Agriculture. https://www.interacademies.org/node/49419 (2004).

2. Sánchez, P. A. Tripling crop yields in tropical Africa. Nature Geoscience vol. 3 299-300 (2010).

3. FAO. FAO Regional statistical yearbook. ttp://www.fao.org/faostat/en/\#data/QC (2019).

4. Vanlauwe, B. et al. Sustainable intensification and the African smallholder farmer. Curr. Opin. Environ. Sustain. 8, 15-22 (2014).

5. Andriesse, W. \& Giller, K. E. The state of soil fertility in sub-Saharan Africa. Agric. Dev. 32 36 (2015).

6. Vanlauwe, B. et al. Integrated Soil Fertility Management: Operational Definition and Consequences for Implementation and Dissemination. Outlook Agric. 39, 17-24 (2010).

7. Rurinda, J. et al. Science-based decision support for formulating crop fertilizer recommendations in sub-Saharan Africa. Agric. Syst. 180, 102790 (2020).

8. Marschner, H. \& Marschner, P. Marschner's mineral nutrition of higher plants. (Academic Press, 2012).

9. Vanlauwe, B. et al. Integrated soil fertility management in sub-Saharan Africa: unravelling local adaptation. SOIL 1, 491-508 (2015).

10. Kihara, J. et al. Application of secondary nutrients and micronutrients increases crop yields in sub-Saharan Africa. Agron. Sustain. Dev. (2017) doi:10.1007/s13593-017-0431-0.

11. Stoorvogel, J. J., Smaling, E. M. A. \& Janssen, B. H. Calculating soil nutrient balances in Africa at different scales - I Supra-national scale. Fertil. Res. 35, 227-235 (1993).

12. Mutsaers, H. et al. Soil and soil fertility management research in sub-Saharan Africa: Fifty years of shifting visions and chequered achievements. Soil and Soil Fertility Management Research in Sub-Saharan Africa: Fifty years of shifting visions and chequered achievements (Taylor and Francis, 2017). doi:10.4324/9781315518855.

13. Kihara, J. et al. Understanding variability in crop response to fertilizer and amendments in sub-Saharan Africa. Agric. Ecosyst. Environ. 229, 1-12 (2016).

14. Sillanpää, M. Micronutrients and the nutrient status of soils: a global study. (1982).

15. Manzeke, G. M., Mtambanengwe, F., Nezomba, H. \& Mapfumo, P. Zinc fertilization influence on maize productivity and grain nutritional quality under integrated soil fertility management in Zimbabwe. F. Crop. Res. 166, 128-136 (2014).

16. Van Asten, P. J. A., Barro, S. E., Wopereis, M. C. S. \& Defoer, T. Using farmer knowledge to combat low productive spots in rice fields of a sahelian irrigation scheme. L. Degrad. Dev. 15, 383-396 (2004).

17. Kang, B. T. \& Osiname, O. A. Micronutrient problems in tropical Africa. in Micronutrients in Tropical Food Crop Production 131-150 (Springer Netherlands, 1985). doi:10.1007/978-94009-5055-9_5.

18. Mehri, A. Trace elements in human nutrition (II) - An update. International Journal of Preventive Medicine vol. 11 (2020).

19. Donoiu, I. et al. Effects of boron-containing compounds on cardiovascular disease risk factors - A review. Journal of Trace Elements in Medicine and Biology vol. 50 47-56 (2018).

20. Cakmak, I. Agronomic approaches in biofortification of food crops with micronutrients. Proc. Int. Plant Nutr. Colloq. XVI (2009). 
21. Grember, K. von et al. Global hunger index, the challenge of hidden hunger. (2014).

22. Black, R., Allen, L., Bhutta, Z. \& Caulfield, L. Maternal and child undernutrition: global and regional exposures and health consequences. Lancet 371, 243-260 (2008).

23. Cakmak, I. Identification and correction of widespread zinc deficiency in Turkey : a success story. in 28 (International Fertiliser Society, 2004).

24. Dimkpa, C. O. \& Bindraban, P. S. Fortification of micronutrients for efficient agronomic production: a review. Agron. Sustain. Dev. 36, 7 (2016).

25. Berkhout, E. D., Malan, M. \& Kram, T. Better soils for healthier lives? An econometric assessment of the link between soil nutrients and malnutrition in Sub-Saharan Africa. PLoS One 14, e0210642 (2019).

26. Ichami, S. M., Shepherd, K. D., Sila, A. M., Stoorvogel, J. J. \& Hoffland, E. Fertilizer response and nitrogen use efficiency in African smallholder maize farms. Nutr. Cycl. Agroecosystems 113, 1-19 (2019).

27. Janssen, B. H. et al. A system for quantitative evaluation of the fertility of tropical soils (QUEFTS). Geoderma 46, 299-318 (1990).

28. Alloway BJ. Zinc in Soils and Crop Nutrition. (IZA Publications, 2008).

29. Kabata-Pendias, A. \& Pendias, H. Trace Elements in Soils and Plants, Third Edition. www.crcpress.com (2001).

30. de Vries, W., Curlík, J., Murányi, A., Alloway, B. \& Groenenberg, B. J. Assessment of relationships between total and reactive concentrations of cadmium, copper, lead and zinc in Hungarian and Slovakian soils. Ekológia (Bratislava) 24, 152-169 (2005).

31. Groenenberg, J. E., Römkens, P. F. A. M., Zomeren, A. Van, Rodrigues, S. M. \& Comans, R. N. J. Evaluation of the Single Dilute $(0.43 \mathrm{M})$ Nitric Acid Extraction to Determine Geochemically Reactive Elements in Soil. Environ. Sci. Technol. 51, 2246-2253 (2017).

32. Towett, E. K. et al. Total elemental composition of soils in Sub-Saharan Africa and relationship with soil forming factors. Geoderma Reg. 5, 157-168 (2015).

33. Towett, E. K., Shepherd, K. D. \& Cadisch, G. Quantification of total element concentrations in soils using total X-ray fluorescence spectroscopy (TXRF). Sci. Total Environ. 463-464, 374-388 (2013).

34. Gaudino, S. et al. The role of different soil sample digestion methods on trace elements analysis: A comparison of ICP-MS and INAA measurement results. Accredit. Qual. Assur. 12, 84-93 (2007).

35. Nolan, A. L., Lombi, E. \& McLaughlin, M. J. Metal bioaccumulation and toxicity in soils Why bother with speciation? Aust. J. Chem. 56, 77-91 (2003).

36. Allen, H., McGrath, S., McLaughlin, M. \& Peijnenburg, W. Bioavailability of metals in terrestrial ecosystems: importance of partitioning for bioavailability to invertebrates, microbes, and plants. (2001).

37. Harmsen, J. Measuring Bioavailability: From a Scientific Approach to Standard Methods. J. Environ. Qual. 36, 1420-1428 (2007).

38. ISO. Soil quality 17586:2016 -- Extraction of trace elements using dilute nitric acid. https://www.iso.org/standard/60060.html (2016).

39. Degryse, F., Buekers, J. \& Smolders, E. Radio-labile cadmium and zinc in soils as affected by pH and source of contamination. Eur. J. Soil Sci. 55, 113-122 (2004).

40. Degryse, F., Voegelin, A., Jacquat, O., Kretzschmar, R. \& Smolders, E. Characterization of zinc in contaminated soils: complementary insights from isotopic exchange, batch extractions and XAFS spectroscopy. Eur. J. Soil Sci. 62, 318-330 (2011).

41. Nawara, S. et al. A comparison of soil tests for available phosphorus in long-term field experiments in Europe. Eur. J. Soil Sci. 68, 873-885 (2017).

42. ISO 17402. Soil quality -- Requirements and guidance for the selection and application of methods for the assessment of bioavailability of contaminants in soil and soil materials. https://www.iso.org/standard/38349.html (2008).

43. Römkens, P. F. et al. Characterization of soil heavy metal pools in paddy fields in Taiwan: chemical extraction and solid-solution partitioning. J. Soils Sediments 9, 216-228 (2009).

44. Marzouk, E. R., Chenery, S. R. \& Young, S. D. Measuring reactive metal in soil: A comparison 
of multi-element isotopic dilution and chemical extraction. Eur. J. Soil Sci. 64, 526-536 (2013).

45. Smolders, E., Brans, K., Földi, A. \& Merckx, R. Cadmium Fixation in Soils Measured by Isotopic Dilution. Soil Sci. Soc. Am. J. 63, 78-85 (1999).

46. Young, S. D., Tye, A., Carstensen, A., Resende, L. \& Crout, N. Methods for determining labile cadmium and zinc in soil. Eur. J. Soil Sci. 51, 129-136 (2000).

47. Lindsay, W. L. \& Norvell, W. A. Development of a DTPA Soil Test for Zinc, Iron, Manganese, and Copper1. Soil Sci. Soc. Am. J. 42, 421 (1978).

48. Streck, T. \& Richter, J. Heavy Metal Displacement in a Sandy Soil at the Field Scale: I. Measurements and Parameterization of Sorption. J. Environ. Qual. 26, 49-56 (1997).

49. Sinaj, S., Dubois, A. \& Frossard, E. Soil isotopically exchangeable zinc: A comparison between e and L values. Plant Soil 261, 17-28 (2004).

50. Keren, R. Boron. in Methods of Soil Analyses. Part 3: Chemical Methods. (eds. Sparks, D. L. et al.) 603-626 (Soil Science Society of America Book Series, 1996). doi:10.2136/sssabookser5.3.c21.

51. Hou, J., Evans, L. J. \& Spiers, G. A. Chemical fractionation of soil boron: 1. Method development. Can. J. Soil. Sci. 76, 485-491 (1995).

52. Goldberg, S. \& Suarez, D. L. A New Soil Test for Quantitative Measurement of Available and Adsorbed Boron. Soil Sci. Soc. Am. J. 78, 480 (2014).

53. Bingham, F. T. Boron. in Methods of soil analysis Part 2 Chemical and Mineralogical properties (eds. Page, A. L., Miller, M. H. \& Keeny, D. R.) 431-448 (American Society of Agronomy, 1982).

54. Mehlich, A. New extractant for soil test evaluation of phosphorus, potassium, magnesium, calcium, sodium, manganese and zinc. Commun. Soil Sci. Plant Anal. 9, 477-492 (1978).

55. Penn, C. et al. A Discussion on Mehlich-3 Phosphorus Extraction from the Perspective of Governing Chemical Reactions and Phases: Impact of Soil pH. Agriculture 8, 106 (2018).

56. Mylavarapu, R. S., Sanchez, J. F., Nguyen, J. H. \& Bartos, J. M. Evaluation of Mehlich-1 and Mehlich-3 extraction procedures for plant nutrients in acid mineral soils of Florida. Commun. Soil Sci. Plant Anal. 33, 807-820 (2002).

57. Cropnuts. Technical Methods \& References. https://cropnuts.com/wpcontent/uploads/2019/11/Technical-Method-References-for-ISO-accredited-tests-forwebsite.pdf (2021).

58. Hengl, T. et al. Soil nutrient maps of Sub-Saharan Africa: assessment of soil nutrient content at $250 \mathrm{~m}$ spatial resolution using machine learning. Nutr. Cycl. Agroecosystems 109, 77-102 (2017).

59. Martínez Cuesta, N. et al. Determining Mehlich-3 and DTPA extractable soil zinc optimum economic threshold for maize. Soil Use Manag. (2020) doi:10.1111/sum.12657.

60. Mehlich, A. Mehlich 3 soil test extractant: A modification of Mehlich 2 extractant. Commun. Soil Sci. Plant Anal. 15, 1409-1416 (1984).

61. Campbell, D. J. The soil solution chemistry of some Oxfordshire soils: temporal and spatial variability. Journalof Soil Science vol. 40 (1989).

62. Duffner, A., Hoffland, E., Weng, L. \& van der Zee, S. E. A. T. M. Predicting zinc bioavailability to wheat improves by integrating $\mathrm{pH}$ dependent nonlinear root surface adsorption. Plant Soil 373, 919-930 (2013).

63. Houba, V. J. G. G., Novozamsky, I., Lexmond, T. M., van der Lee, J. J. \& Jvnn Der, L. Applicability Of $0.01 \mathrm{M}$ Cacl2 As a Single Extraction Solution for the Assessment of the Nutrient Status of Soils and Other Diagnostic Purposes. Commun. Soil Sci. Plant Anal. 21, 2281-2290 (1990).

64. Degryse, F., Broos, K., Smolders, E. \& Merckx, R. Soil solution concentration of Cd and Zn canbe predicted with a $\mathrm{CaCl} 2$ soil extract. Eur. J. Soil Sci. 54, 149-158 (2003).

65. Fest, E. P. M. J. M. J., Temminghoff, E. J. M. J. M., Comans, R. N. J. N. J. \& van Riemsdijk, W. H. H. Partitioning of organic matter and heavy metals in a sandy soil: Effects of extracting solution, solid to liquid ratio and pH. Geoderma 146, 66-74 (2008).

66. de Abreu, C. A., de Abreu, M. F., de Andrade, J. C. \& van Raij, B. Restrictions in the use of 
correlation coefficients in comparing methods for the determination of the micronutrients in soils. Commun. Soil Sci. Plant Anal. 29, 1961-1972 (1998).

67. Maskina, M. S., Randhawa, N. S. \& Sinha, M. K. Relation of growth and zinc uptake of rice to quantity, intensity and buffering capacity factors of zinc in soils. Plant Soil 54, 195-205 (1980).

68. Wu, X. \& Aasen, I. Models for predicting soil zinc availability for barley. Plant Soil 163, 279285 (1994).

69. Kim, R.-Y. et al. Bioavailability of heavy metals in soils: definitions and practical implementation - a critical review. Environ. Geochem. Health 37, 1041-1061 (2015).

70. Smolders, E., Wagner, S., Prohaska, T., Irrgeher, J. \& Santner, J. Sub-millimeter distribution of labile trace element fluxes in the rhizosphere explains differential effects of soil liming on cadmium and zinc uptake in maize. Sci. Total Environ. 738, 140311 (2020).

71. Nolan, A. L., Zhang, H. \& McLaughlin, M. J. Prediction of zinc, cadmium, lead, and copper availability to wheat in contaminated soils using chemical speciation, diffusive gradients in thin films, extraction, and isotopic dilution techniques. J. Environ. Qual. 34, 496-507 (2005).

72. Hengl, T. et al. SoilGrids250m: Global gridded soil information based on machine learning. PLoS One 12, e0169748 (2017).

73. Hengl, T. et al. African Soil Properties and Nutrients Mapped at 30-m Spatial Resolution using Two-scale Ensemble Machine Learning. Sci Rep 11, 6130 (2021).

74. Hengl, T. et al. Mapping Soil Properties of Africa at $250 \mathrm{~m}$ Resolution: Random Forests Significantly Improve Current Predictions. PLoS One 10, e0125814 (2015).

75. Duffner, A., Weng, L., Hoffland, E. \& van der Zee, S. E. A. T. M. Multi-surface modeling to predict free zinc ion concentrations in low-zinc soils. Environ. Sci. Technol. 48, 5700-5708 (2014).

76. Benjamin, M. M. \& Leckie, J. O. Multiple-site adsorption of $\mathrm{Cd}, \mathrm{Cu}, \mathrm{Zn}$, and $\mathrm{Pb}$ on amorphous iron oxyhydroxide. J. Colloid Interface Sci. 79, 209-221 (1981).

77. Degryse, F., Smolders, E. \& Parker, D. R. Partitioning of metals (Cd, Co, Cu, Ni, Pb, Zn) in soils: concepts, methodologies, prediction and applications - a review. Eur. J. Soil Sci. 60, 590-612 (2009).

78. Catlett, K. M., Heil, D. M., Lindsay, W. L. \& Ebinger, M. H. Soil Chemical Properties Controlling Zinc Activity in 18 Colorado Soils. Soil Sci. Soc. Am. J. 66, 1182 (2002).

79. Groenenberg, B. et al. Transfer functions for solid-solution partitioning of cadmium, copper, nickel, lead and zinc in soils: derivation of relationships for free metal ion activities and validation with independent data. Eur. J. Soil Sci. 61, 58-73 (2010).

80. Groenenberg, J. E., Dijkstra, J. J., Bonten, L. T. C., De Vries, W. \& Comans, R. N. J. Evaluation of the performance and limitations of empirical partition-relations and process based multisurface models to predict trace element solubility in soils. Environ. Pollut. 166, 98-107 (2012).

81. Elzinga, E. J., Van Grinsven, J. J. M. \& Swartjes, F. A. General purpose Freundlich isotherms for cadmium, copper and zinc in soils. Eur. J. Soil Sci. 50, 139-149 (1999).

82. Sauvé, S., Hendershot, W. \& Allen, H. E. Solid-Solution Partitioning of Metals in Contaminated Soils: Dependence on pH, Total Metal Burden, and Organic Matter. Environ. Sci. Technol. 34, 1125-1131 (2000).

83. Groenenberg, J. E. \& Lofts, S. The use of assemblage models to describe trace element partitioning, speciation, and fate: a review. Environ. Toxicol. Chem. 33, 2181-96 (2014).

84. Van Riemsdijk, W. H., Koopal, L. K., Kinniburgh, D. G., Benedetti, M. F. \& Weng, L. Modeling the interactions between humics, ions, and mineral surfaces. Environmental Science and Technology vol. 40 7473-7480 (2006).

85. Hiemstra, T. \& Zhao, W. Reactivity of ferrihydrite and ferritin in relation to surface structure, size, and nanoparticle formation studied for phosphate and arsenate. Environ. Sci. Nano 3, 1265-1279 (2016).

86. Dijkstra, J. J., Meeussen, J. C. L. \& Comans, R. N. J. Leaching of Heavy Metals from Contaminated Soils: An Experimental and Modeling Study. Environ. Sci. Technol. 38, 43904395 (2004). 


\section{Chapter 1}

87. Dijkstra, J. J., Meeussen, J. C. L. \& Comans, R. N. J. Evaluation of a Generic Multisurface Sorption Model for Inorganic Soil Contaminants. Environ. Sci. Technol. 43, 6196-6201 (2009).

88. Mao, L. C. C., Young, S. D. D., Tye, A. M. M. \& Bailey, E. H. H. Predicting trace metal solubility and fractionation in Urban soils from isotopic exchangeability. Environ. Pollut. 231, 1529-1542 (2017).

89. Gustafsson, J. P. \& Tiberg, C. Molybdenum binding to soil constituents in acid soils: An XAS and modelling study. Chem. Geol. 417, 279-288 (2015).

90. Tiberg, C., Sjöstedt, C. \& Gustafsson, J. P. Metal sorption to Spodosol Bs horizons: Organic matter complexes predominate. Chemosphere 196, 556-565 (2018).

91. Verbeeck, M., Hiemstra, T., Thiry, Y.\& Smolders, E. Soil organic matter reduces the sorption of arsenate and phosphate: a soil profile study and geochemical modelling. Eur. J. Soil Sci. 68, 678-688 (2017).

92. Warrinnier, R. et al. Investigation on the control of phosphate leaching by sorption and colloidal transport: Column studies and multi-surface complexation modelling. Appl. Geochemistry 100, 371-379 (2019).

93. Weng, L., Temminghoff, E. J. M. \& Van Riemsdijk, W. H. Contribution of Individual Sorbents to the Control of Heavy Metal Activity in Sandy Soil. Environ. Sci. Technol. 35, 4436-4443 (2001).

94. Meima, J. A. \& Comans, R. N. J. Application of surface complexation/precipitation modeling to contaminant leaching from weathered municipal solid waste incinerator bottom ash. Environ. Sci. Technol. 32, 688-693 (1998).

95. Klinkert, S. \& Comans, R. N. J. Geochemical Multisurface Modeling of Reactive Zinc Speciation in Compost as Influenced by Extraction Conditions. Environ. Sci. Technol. 54, 2467-2475 (2020).

96. Schenkeveld, W. D. C. et al. Metal mobilization from soils by phytosiderophores - experiment and equilibrium modeling. Plant Soil 383, 59-71 (2014).

97. Geelhoed, J. S., Van Riemsdijk, W. H. \& Findenegg, G. R. Simulation of the effect of citrate exudation from roots on the plant availability of phosphate adsorbed on goethite. Eur. J. Soil Sci. 50, 379-390 (1999).

98. Schenkeveld, W. D. C., Weng, L. P., Reichwein, A. M., Temminghoff, E. J. M. \& van Riemsdijk, W. H. Evaluation of the potential impact of $\mathrm{Cu}$ competition on the performance of o,o-FeEDDHA in soil applications. Eur. J. Soil Sci. 66, 277-285 (2015).

99. Goldberg, S. Reanalysis of boron adsorption on soils and soil minerals using the constant capacitance model. Soil Sci. Soc. Am. J. 63, 823-829 (1999).

100. Goldberg, S. \& Glaubig, R. Boron adsorption on California soils. Soil Sci. Soc. Am. 50, 1173 1176 (1986).

101. Goldberg, S., Lesch, S. M. \& Suarez, D. L. Predicting Boron Adsorption by Soils Using Soil Chemical Parameters in the Constant Capacitance Model. Soil Sci. Soc. Am. J. 64, 1356 (2000).

102. Sarret, G. et al. Zn speciation in the organic horizon of a contaminated soil by micro-X-ray fluorescence, micro- and powder-EXAFS spectroscopy, and isotopic dilution. Environ. Sci. Technol. 38, 2792-2801 (2004).

103. Rahman, N. \& Schoenau, J. Response of wheat, pea, and canola to micronutrient fertilization on five contrasting prairie soils. Sci. Rep. 10, 18818 (2020).

104. Strawn, D. G. \& Baker, L. L. Speciation of $\mathrm{Cu}$ in a contaminated agricultural soil measured by XAFS, $\mu$-XAFS, and $\mu$-XRF. Environ. Sci. Technol. 42, 37-42 (2008).

105. Goli, E., Hiemstra, T. \& Rahnemaie, R. Interaction of boron with humic acid and natural organic matter: Experiments and modeling. Chem. Geol. 515, 1-8 (2019).

106. Lemarchand, E., Schott, J. \& Gaillardet, J. Boron isotopic fractionation related to boron sorption on humic acid and the structure of surface complexes formed. Geochim. Cosmochim. Acta 69, 3519-3533 (2005).

107. Goli, E., Rahnemaie, R., Hiemstra, T. \& Malakouti, M. J. The interaction of boron with goethite: Experiments and CD-MUSIC modeling. Chemosphere 82, 1475-1481 (2011).

108. Dzombak, D. A. \& Morel, F. F. M. M. Surface Complexation Modeling: Hydrous Ferric 
Oxide. (John Wiley \& Sons, 1990).

109. Hiemstra, T. \& Van Riemsdijk, W. H. A Surface Structural Approach to Ion Adsorption: The Charge Distribution (CD) Model. J. Colloid Interface Sci. 179, 488-508 (1996).

110. Hiemstra, T., Barnett, M. O. \& van Riemsdijk, W. H. Interaction of silicic acid with goethite. J. Colloid Interface Sci. 310, 8-17 (2007).

111. Hiemstra, T. \& van Riemsdijk, W. H. Biogeochemical speciation of Fe in ocean water. Mar. Chem. 102, 181-197 (2006).

112. Hiemstra, T. \& Van Riemsdijk, W. H. A surface structural model for ferrihydrite I: Sites related to primary charge, molar mass, and mass density. Geochim. Cosmochim. Acta 73, 4423-4436 (2009).

113. Mathur, S. S. \& Dzombak, D. A. Chapter 16 Surface complexation modeling: goethite. in Interface Science and Technology vol. 11 443-468 (Elsevier, 2006).

114. Karamalidis, A. K. \& Dzombak, D. A. Surface Complexation Modeling. Surface Complexation Modeling: Gibbsite (John Wiley \& Sons, Inc., 2010). doi:10.1002/9780470642665.

115. Tonkin, J. W., Balistrieri, L. S. \& Murray, J. W. Modeling sorption of divalent metal cations on hydrous manganese oxide using the diffuse double layer model. Appl. Geochemistry 19, 29-53 (2004).

116. Venema, P., Hiemstra, T., Weidler, P. G. \& Van Riemsdijk, W. H. Intrinsic Proton Affinity of Reactive Surface Groups of Metal (Hydr)oxides: Application to Iron (Hydr)oxides. J. Colloid Interface Sci. 198, 282-295 (1998).

117. Ponthieu, M., Juillot, F., Hiemstra, T., van Riemsdijk, W. H. \& Benedetti, M. F. Metal ion binding to iron oxides. Geochim. Cosmochim. Acta (2006) doi:10.1016/j.gca.2006.02.021.

118. Weng, L., Van Riemsdijk, W. H. \& Hiemstra, T. Cu2+ and Ca2+adsorption to goethite in the presence of fulvic acids. Geochim. Cosmochim. Acta 72, 5857-5870 (2008).

119. Michel, F. M. et al. The structure of ferrihydrite, a nanocrystalline material. Science (80-. ). 316, 1726-1729 (2007).

120. Hiemstra, T. Surface and mineral structure of ferrihydrite. Geochim. Cosmochim. Acta 105, 316-325 (2013).

121. Hiemstra, T. Surface structure controlling nanoparticle behavior: Magnetism of ferrihydrite, magnetite, and maghemite. Environ. Sci. Nano 5, 752-764 (2018).

122. Mendez, J. C. \& Hiemstra, T. Carbonate Adsorption to Ferrihydrite: Competitive Interaction with Phosphate for Use in Soil Systems. ACS Earth Sp. Chem. 3.1, 129-141 (2018).

123. Mendez, J. C. \& Hiemstra, T. Ternary complex formation of phosphate with $\mathrm{Ca}$ and $\mathrm{Mg}$ ions binding to ferrihydrite: Experiments and mechanisms. ACS Earth Sp. Chem. 4.4, acsearthspacechem.9b00320 (2020).

124. Mendez, J. C. \& Hiemstra, T. High and low affinity sites of ferrihydrite for metal ion adsorption: Data and modeling of the alkaline-earth ions $\mathrm{Be}, \mathrm{Mg}, \mathrm{Ca}, \mathrm{Sr}, \mathrm{Ba}$, and $\mathrm{Ra}$. Geochim. Cosmochim. Acta (2020) doi:10.1016/j.gca.2020.07.032.

125. Hiemstra, T. Ferrihydrite interaction with silicate and competing oxyanions: Geometry and Hydrogen bonding of surface species. Geochim. Cosmochim. Acta 238, 453-476 (2018).

126. Hiemstra, T. \& Van Riemsdijk, W. H. On the relationship between charge distribution, surface hydration, and the structure of the interface of metal hydroxides. J. Colloid Interface Sci. 301, $1-18$ (2006).

127. Chen, C. \& Thompson, A. Ferrous Iron Oxidation under Varying pO2 Levels: The Effect of $\mathrm{Fe}(\mathrm{III}) / \mathrm{Al}(\mathrm{III})$ Oxide Minerals and Organic Matter. Environ. Sci. Technol. 52, 597-606 (2018).

128. Perret, D., Gaillard, J. F., Dominik, J. \& Atteia, O. The diversity of natural hydrous iron oxides. Environ. Sci. Technol. 34, 3540-3546 (2000).

129. van der Zee, C., Roberts, D. R., Rancourt, D. G. \& Slomp, C. P. Nanogoethite is the dominant reactive oxyhydroxide phase in lake and marine sediments. Geology 31, 993-996 (2003).

130. Lal, R., Sanchez, P. A., Sanchez, P. A. \& Logan, T. J. Myths and Science about the Chemistry and Fertility of Soils in the Tropics. 1971-1979 (1992) doi:10.2136/sssaspecpub29.c3.

131. Basile-Doelsch, I. et al. Mineralogical control of organic carbon dynamics in a volcanic ash soil on La Reunion. Eur. J. Soil Sci. 0, 050912034650042 (2005). 
132. Mikutta, R. et al. Biogeochemistry of mineral-organic associations across a long-term mineralogical soil gradient (0.3-4100 kyr), Hawaiian Islands. Geochim. Cosmochim. Acta 73, 2034-2060 (2009).

133. Mendez, J. C., Hiemstra, T. \& Koopmans, G. F. Assessing the Reactive Surface Area of Soils and the Association of Soil Organic Carbon with Natural Oxide Nanoparticles Using Ferrihydrite as Proxy. Environ. Sci. Technol. 54, 11990-12000 (2020).

134. Hiemstra, T., Antelo, J., Rahnemaie, R. \& van Riemsdijk, W. H. Nanoparticles in natural systems I: The effective reactive surface area of the natural oxide fraction in field samples. Geochim. Cosmochim. Acta 74, 41-58 (2010).

135. Koopal, L. K., Saito, T., Pinheiro, J. P. \& Riemsdijk, W. H. van. Ion binding to natural organic matter: General considerations and the NICA-Donnan model. Colloids Surfaces A Physicochem. Eng. Asp. 265, 40-54 (2005).

136. Kinniburgh, D. G. et al. Ion binding to natural organic matter: competition, heterogeneity, stoichiometry and thermodynamic consistency. Colloids Surfaces A Physicochem. Eng. Asp. 151, 147-166 (1999).

137. Rusch, U., Borkovec, M., Daicic, J. \& Van Riemsdijk, W. H. Interpretation of competitive adsorption isotherms in terms of affinity distributions. J. Colloid Interface Sci. 191, 247-255 (1997).

138. Benedetti, M. ., van Riemsdijk, W. H. \& Koopal, L. K. Humic Substances Considered as a Heterogeneous Donnan Gel Phase. Environ. Sci. Technol 30, 1905-1913 (1996).

139. Milne, C. J. C. J., Kinniburgh, D. G. D. G. D. G. \& Tipping, E. Generic NICA-Donnan Model Parameters for Proton Binding by Humic Substances. Environ. Sci. Technol. 35, 2049-2059 (2001).

140. Milne, C. J., Kinniburgh, D. G., van Riemsdijk, W. H. \& Tipping, E. Generic NICA-Donnan Model Parameters for Metal-Ion Binding by Humic Substances. Environ. Sci. Technol. 37, 958-971 (2003).

141. Hoffland, E., Kuyper, T. W., Comans, R. N. J. \& Creamer, R. E. Eco-functionality of organic matter in soils. Plant Soil (2020) doi:10.1007/s11104-020-04651-9.

142. Lehmann, J. \& Kleber, M. The contentious nature of soil organic matter. Nature 528, 60-68 (2015).

143. Rasmussen, C. et al. Beyond clay: towards an improved set of variables for predicting soil organic matter content. Biogeochemistry 137, 297-306 (2018).

144. Kaiser, K. \& Guggenberger, G. The role of DOM sorption to mineral surfaces in the preservation of organic matter in soils. in Organic Geochemistry vol. 31 711-725 (Pergamon, 2000).

145. Hiemstra, T., Antelo, J., Rahnemaie, R. \& van Riemsdijk, W. H. Nanoparticles in natural systems I: The effective reactive surface area of the natural oxide fraction in field samples. Geochim. Cosmochim. Acta 74, 41-58 (2010).

146. Bassett, R. L. L. A critical evaluation of the thermodynamic data for boron ions, ion pairs, complexes, and polyanions in aqueous solution at $298.15 \mathrm{~K}$ and 1 bar. Geochim. Cosmochim. Acta 44, 1151-1160 (1980).

147. Mendez, J. C. \& Hiemstra, T. Surface area of ferrihydrite consistently related to primary surface charge, ion pair formation, and specific ion adsorption. Chem. Geol. 532, (2020).

148. Tiberg, C., Sjöstedt, C., Persson, I. \& Gustafsson, J. P. Phosphate effects on copper(II) and lead(II) sorption to ferrihydrite. Geochim. Cosmochim. Acta 120, 140-157 (2013). 


\section{Chapter 2}

Surface reactivity of the natural metal (hydr)oxides in weathered tropical soils

Juan C. Mendez *, Elise Van Eynde*, Tjisse Hiemstra, and Rob N.J. Comans

*These authors contributed equally

Submitted for publication 


\begin{abstract}
Assessing the surface reactivity of metal (hydr)oxides in soils is essential for quantifying ion adsorption phenomena that control the availability of nutrients and pollutants. Despite the high natural abundance of $\mathrm{Fe}$ and $\mathrm{Al}$ (hydr)oxides in intensively weathered environments, the surface reactivity of these pedogenic materials has not been consistently characterized for weathered tropical soils. Here, we used a novel probe-ion methodology combined with state-of-the-art surface complexation modeling (SCM) to derive the reactive surface area (RSA) of the soils, as well as the amount of phosphate (PO4) reversibly adsorbed $\left(\mathrm{R}-\mathrm{PO}_{4}\right)$ to the metal (hydr)oxide fraction. We applied this methodology to a series of weathered topsoils from the Sub-Saharan region. The results showed that nanocrystalline ferrihydrite (Fh) is a better proxy than well-crystallized goethite for describing with SCM the reactivity of the metal (hydr)oxides, even though well-crystallized materials dominate the mass fraction of metal (hydr)oxide of these soils. Using Fh as a proxy, the RSA ranged from $\sim 2$ to $40 \mathrm{~m}^{2} \mathrm{~g}^{-1}$ soil. Nanoparticles with a mean diameter of $\sim 1.5-5.0$ $\mathrm{nm}$ dominate the reactive fraction of metal (hydr)oxides in these topsoils. We found that a substantial fraction of the total PO4 associated with the metal (hydr)oxides, on average $\sim 64 \%$, is occluded in the crystalline $\mathrm{Fe}$ and $\mathrm{Al}$ oxide fraction and not available for solid-solution partitioning of $\mathrm{PO}_{4}$. Overall, this research provides new insights into the reactivity of the metal (hydr)oxide fraction in weathered tropical soils and highlights the relevance of these pedogenic materials in determining the speciation and availability of $\mathrm{PO}_{4}$.
\end{abstract}




\section{INTRODUCTION}

The bioavailability, mobility, and consequently the risk of deficiencies, toxicities, and leaching of ions in soils is determined by adsorption to the fraction of natural metal (hydr)oxides. The reactive metal (hydr)oxide materials are particularly relevant for controlling the solid-solution partitioning of oxyanions such as phosphate $\left(\mathrm{PO}_{4}\right)$, arsenate $\left(\mathrm{AsO}_{4}\right)$, selenite $\left(\mathrm{SeO}_{3}\right)$, and others ${ }^{1-3}$. These reactive metal (hydr)oxides are also important for adsorbing soil organic matter (SOM $)^{4,5}$, thereby contributing to the long-term stabilization of organic carbon in soils by forming chemically stable mineral-organic associations ${ }^{6,7}$.

The fraction of natural metal (hydr)oxide comprises a series of minerals with variable chemical composition, mineral structure, and crystallinity, implying also different adsorption interactions with oxyanions and SOM. The dominant mass fraction and mineral composition of metal (hydr)oxides often vary across different environments ${ }^{8-}$ 10. In the humid tropics, soils are often intensively weathered, which results in a high abundance of $\mathrm{Fe}$ and $\mathrm{Al}$ (hydr)oxides, mainly present as well-crystallized metal (hydr)oxide material ${ }^{11,12}$.

The adsorption of ions to the natural metal (hydr)oxides of soils is a complex process influenced by the amount and surface properties of the mineral phases, the chemistry of the soil solution (e.g. pH, ionic strength, type, and concentration of coexisting ions), and the competitive or cooperative interaction of negatively charged $\mathrm{SOM}^{13}$. In this context, surface complexation models (SCMs) are envisioned as promising tools for analyzing how these multiple factors interact and affect the overall ion adsorption behavior of soil systems. A successful application of SCMs in soils requires a consistent characterization of the surface reactivity of the metal (hydr)oxide fraction. However, despite the naturally high content of $\mathrm{Fe}$ and $\mathrm{Al}$ (hydr)oxides in weathered soils from the tropics, a consistent characterization of the surface reactivity of the natural metal (hydr)oxides is not available for this type of soils.

The reactive surface area (RSA, in $\mathrm{m}^{2} \mathrm{~g}^{-1}$ soil) of the pedogenic metal (hydr)oxides is a key feature determining the capacity of soils for adsorbing oxyanions and for interacting with SOM ${ }^{14-16}$. A consistent assessment of this important soil property is not trivial, and multiple challenges are faced when using traditional analytical approaches. Gas adsorption analysis using for instance $\mathrm{N}_{2}$ or $\mathrm{Ar}$ as probe molecules, and subsequent interpretation with the Brunauer-Emmett-Teller (BET) equation ${ }^{17}$, is a classical approach commonly used for assessing the surface area of synthetic oxides. It can also be applied to soils ${ }^{18-20}$. However, this approach has a number of disadvantages ${ }^{21}$. Drying and outgassing the samples prior to the gas adsorption analysis provoke irreversible aggregation of colloidal nanoparticles, which leads to an underestimation of the RSA ${ }^{16,22}$. Moreover, part of the mineral surface area is covered by adsorbed SOM and might not be accessible for the probe gas molecules $^{23}$. Attempts to remove the SOM before the gas adsorption analysis, for 
instance by oxidizing the $\mathrm{SOM}^{18}$, may change the physical-chemical properties of the mineral surfaces, also affecting the RSA ${ }^{24}$.

An alternative to gas adsorption is the use of ethylene glycol monoethyl ether (EGME) as a probe molecule for assessing the RSA of soils ${ }^{25}$, but this approach provides mainly an estimation of the surface area of clay minerals ${ }^{26}$. Humic acids adsorption has been proposed for assessing the surface area contribution of kaolinite and goethite in the fine fraction $(<45 \mu \mathrm{M})$ of river sediments, but this method only provides an estimation of the relative surface area of the components in binary mineral systems (e.g. kaolinite-goethite), rather than offering the overall RSA for the whole metal (hydr)oxide fraction ${ }^{27}$.

Another alternative approach is based on measuring the amount of $\mathrm{Fe}$ and $\mathrm{Al}$ with selective dissolution extractions. Using these data, the RSA of the soil is then calculated by applying a set of "standard" values for the specific surface area (SSA) and molar mass of the crystalline and nanocrystalline metal (hydr)oxide fraction ${ }^{28-31}$. However, the SSA of metal (hydr)oxides greatly varies across different soil samples ${ }^{24,32}$. Moreover, the molar mass, mass density, and surface curvature of metal (hydr)oxide nanoparticles are size-dependent ${ }^{16}$ and the use of single values will therefore introduce inconsistencies.

Recently, it has been proposed to calculate the RSA of soils using $\mathrm{PO}_{4}$ as a probeion ${ }^{24}$. It is natively present in all soils and has a high affinity for $\mathrm{Fe}$ and $\mathrm{Al}$ (hydr)oxides. In this methodology, the competitive adsorption interaction between $\mathrm{CO}_{3}$ and $\mathrm{PO}_{4}$ is measured in a $0.5 \mathrm{M} \mathrm{NaHCO}_{3}$ solution $(\mathrm{pH}=8.5)$. Simultaneously, this method allows to estimate the amount of $\mathrm{PO}_{4}$ that is reversibly bound to the metal (hydr)oxide surfaces (R-PO $\mathrm{P}_{4}$ ). In the probe-ion approach, a desorption curve is established by applying a range of solution-to-solid ratios (SSR) in the soil extractions. The results are then interpreted with the charge-distribution (CD) model ${ }^{33}$, using a chosen metal (hydr)oxide material as reference for which the competitive adsorption isotherms of $\mathrm{PO}_{4}$ have been well parameterized in synthetic systems in the presence of $\mathrm{CO}_{3}$ ions. Recently, we have shown, for a set of agricultural topsoils from a temperate climate, that the reactivity of the natural metal (hydr)oxides is better described when nanocrystalline ferrihydrite $(\mathrm{Fh})$, rather than well-crystallized goethite, is used as a reference oxide material in the CD model ${ }^{32}$.

In highly weathered soils from the humid tropics, crystalline $\mathrm{Fe}$ - and $\mathrm{Al}$-(hydr)oxides (e.g. goethite, hematite, gibbsite) generally dominate the fraction of pedogenic metal (hydr)oxides ${ }^{34,35}$, while the contribution of oxide nanoparticles (i.e. Fh-like materials) may be relatively small on a mass basis. Nevertheless, it is still possible that these nano-sized materials greatly contribute to the overall reactivity of soils, even at low concentrations, because the SSA of these nanoparticles is substantially higher than the SSA of the crystalline metal (hydr)oxides. Therefore, the question arises of how well the overall behavior of the metal (hydr)oxide fractions of weathered tropical soils 
can be represented using synthetic materials as proxies. One of the objectives of the present study is to answer this question.

In soils, phosphorus $(\mathrm{P})$ can be categorized in pools with different chemical behavior and bioavailability. Generally, $\mathrm{PO}_{4}$ fractions are operationally defined with selective extraction methods ${ }^{36}$. The separation between organic and inorganic $P$ pools is one of the most common distinctions made in the literature. For the inorganic $\mathrm{P}$ pool, different $\mathrm{PO}_{4}$ fractions with different lability can be also distinguished, i.e. soluble phosphate, reversibly adsorbed phosphate, and phosphate that is occluded within metal (hydr) oxide particles. The relative contribution of the different $P$ pools might change according to the soil weathering stage and the composition of the metal (hydr)oxide fraction ${ }^{36,37}$, and therefore, it may vary significantly for intensively weathered soils from the tropics, compared to soils from temperate climates.

In the present study, we will discuss the results of the probe-ion method applied to a series of weathered topsoil samples from Sub-Saharan African countries. The results will be interpreted with the $C D$ model using Fh or goethite as reference metal (hydr)oxides, to evaluate which material represents best the overall behavior of the natural metal (hydr)oxide fraction in these soils. The RSA values obtained by the probe-ion method in combination with experimental data of selective dissolution extractions will be used to assess the properties of the reactive metal (hydr)oxides of these topsoils. To do so, a novel approach is applied that estimates, in a selfconsistent manner, the size-dependent values of specific surface area (SSA), molar mass $\left(M_{\text {nano }}\right)$, and mass density $\left(\rho_{\text {nano }}\right)$ of the metal (hydr)oxide nanoparticles in soils $^{32}$. Regarding the different $P$ pools, we will assess the potential contribution of occluded $\mathrm{PO}_{4}$ to the total pool of $\mathrm{PO}_{4}$ associated with the metal (hydr)oxide minerals. For this purpose, we will measure the amount of $\mathrm{PO}_{4}$ in the dithionite-citrate extracts and compare the results to the amount of reversibly bound $\mathrm{PO}_{4}\left(\mathrm{R}-\mathrm{PO}_{4}\right)$. Additionally, we will analyze the relative contribution of the organic and $\mathrm{P}$ and inorganic $\mathrm{PO}_{4}$ pools in the ammonium oxalate $(\mathrm{AO})$ extracts and will compare the results with those of the series of topsoils from a temperate climate that has been characterized previously with regard to these properties ${ }^{32}$.

\section{MATERIAL AND METHODS}

\subsection{General soil characteristics}

From a larger set of soil samples from Sub-Saharan African countries, 18 samples were selected based on differences in $\mathrm{pH}$, soil organic carbon (SOC) content, acid ammonium oxalate (AO) extractable $\mathrm{PO}_{4}$, and $\mathrm{Fe}$ and $\mathrm{Al}$-(hydr)oxides content. The soils originate from Burundi (samples 1-15) and Kenya (samples 16-18). General characteristics of the selected soil samples are shown in Table 1. According to the Soil Grids system ${ }^{38}$, based on the World Reference Base (WRB) soil classification system, the majority of the soils are classified as Acrisols and Ferralsols, which are 
typically highly weathered soils from the humid tropics, generally known for their inherent low soil fertility and high $\mathrm{P}$ adsorption capacity.

\subsection{Chemical analyses for samples characterization}

Total SOC content of the soils was analyzed using a wet oxidation method according to the Kurmies procedure and measured with a spectrophotometer ${ }^{39}$. The clay content was measured by laser-diffraction after pre-treatment with $\mathrm{H}_{2} \mathrm{O}_{2}$ and $\mathrm{HCl}$ while standing in a warm water bath. The volume percentage of the particle fraction smaller than $2 \mu \mathrm{m}$ was re-calculated to the mass percentage of clay using a particle density of $2.6 \mathrm{~g} \mathrm{~cm}^{-3}$ and a bulk density of $1.3 \mathrm{~g} \mathrm{~cm}^{-3}$. The $\mathrm{pH}$ was measured with a glass electrode in a $0.01 \mathrm{M} \mathrm{CaCl}_{2}$ soil extract ${ }^{40}$.

The nanocrystalline fractions of $\mathrm{Fe}$ and $\mathrm{Al}$-(hydr)oxides of the soils were estimated using an acid ammonium oxalate (AO) extraction. Dried soil samples were extracted with a solution ( $\mathrm{pH} 3.0 \pm 0.1$ ) containing $0.1 \mathrm{M}$ di-ammonium oxalate and $0.1 \mathrm{M}$ oxalic acid at a solution-to-solid ratio (SSR) of $20 \mathrm{~L} \mathrm{~kg}^{-1}$. Samples were equilibrated in the dark at a constant temperature of $20^{\circ} \mathrm{C}$ and continuously shaken using an end-overend shaker. After equilibration for $4 \mathrm{~h}$, samples were centrifuged at $2100 \mathrm{~g}$ for $30 \mathrm{~min}$ and an aliquot of the supernatant was filtered over a $0.45 \mu \mathrm{m}$ membrane filter. The filtrates were measured for $\mathrm{Al}(\mathrm{AO}-\mathrm{Al}), \mathrm{Fe}(\mathrm{AO}-\mathrm{Fe})$, and total $\mathrm{P}\left(\mathrm{AO}-\mathrm{P}_{\text {tot }}\right)$ using an ICP-OES (Thermo Scientific iCAP6500). In a separate batch of AO soil extracts, the inorganic ortho-phosphate $\left(\mathrm{AO}-\mathrm{PO}_{4}\right)$ was analyzed using a segmented flow analyzer (SFA), applying the colorimetric molybdenum-blue method ${ }^{41}$. For the $\mathrm{AO}^{-\mathrm{PO}_{4}}$ analysis, the samples were diluted 100 times with demi-water to eliminate the interference of oxalate in the colorimetric reaction ${ }^{42,43}$. Preliminary tests showed no differences in the $\mathrm{PO}_{4}$ concentration measured with either 10- or 100-times dilution of the AO soil extracts. We applied the ISO protocol for AO extractions which prescribes an extraction time of $4 \mathrm{~h}^{44}$, while this method was originally established using an extraction time of $2 \mathrm{~h}^{45}$. We assessed the effect of using either 4 or $2 \mathrm{~h}$ as extraction time on the measured concentrations of $\mathrm{AO}$ extractable $\mathrm{P}_{\text {tot }}, \mathrm{PO}_{4}, \mathrm{Al}$, and Fe. For inorganic $\mathrm{PO}_{4}$ measurements, the concentration increased on average only with $\sim 10 \%$ when increasing the equilibration time from 2 to $4 \mathrm{hrs}$, while this increment was substantially larger for $\mathrm{P}_{\text {tot }}(22 \%), \mathrm{Fe}_{\mathrm{AO}}(40 \%)$ and $\mathrm{Al}_{\mathrm{AO}}(27 \%)$.

Dithionite-citrate (DC) extractions were performed to assess the crystalline metal (hydr)oxide fraction. The DC extractions were done using the ISO protocol ${ }^{46}$. Briefly, dried soil samples were extracted with a mixture of $0.3 \mathrm{M}$ sodium acetate, $0.2 \mathrm{M}$ trisodium citrate, and $0.3 \mathrm{M}$ sodium dithionite, adjusted to $\mathrm{pH} 4.8$ with sodium acetate, at an SSR of $20 \mathrm{~L} \mathrm{~kg}^{-1}$. Samples were equilibrated for $3.5 \mathrm{~h}$ in a water bath at $60{ }^{\circ} \mathrm{C}$. After equilibration, samples were centrifuged at $3000 \mathrm{~g}$ for $30 \mathrm{~min}$ and an aliquot of the supernatant was filtered over a $0.45 \mu \mathrm{m}$ membrane filter. The filtrates were subsequently analyzed for $\mathrm{Fe}$ (DC-Fe), $\mathrm{Al}(\mathrm{DC}-\mathrm{Al})$, and total $\mathrm{P}$ (DC-P tot) by ICP$\mathrm{OES}$ and for inorganic ortho- $\mathrm{PO}_{4}\left(\mathrm{DC}-\mathrm{PO}_{4}\right)$ by SFA using the colorimetric molybdenum-blue method ${ }^{41}$. For the samples $16-18$ of the Sub-Saharan series, the 
$\mathrm{DC}-\mathrm{PO}_{4}$ pool was not analyzed. Similar to the $\mathrm{AO}$ extracts, the DC extracts were diluted (100 times) with demi-water prior to the colorimetric analyses of $\mathrm{PO}_{4}$. The fraction of crystalline $\mathrm{Fe}$ and $\mathrm{Al}$ (hydr)oxides was estimated as the difference between the DC- and AO-extractable amounts of $\mathrm{Fe}$ and $\mathrm{Al}$.

\subsection{Equilibrium $\mathrm{NaHCO}_{3}$ extractions}

$\mathrm{PO}_{4}$ desorption data were collected applying the probe-ion method proposed by Hiemstra et al..$^{24}$ In this method, soil extractions with a $0.5 \mathrm{M} \mathrm{NaHCO}_{3}$ solution $(\mathrm{pH}$ 8.5) are performed to promote $\mathrm{PO}_{4}$ desorption from the soil mineral surfaces ${ }^{47}$. First, a fresh $0.5 \mathrm{M} \mathrm{NaHCO}_{3}$ solution was prepared by dissolving $21.2 \mathrm{~g}$ of $\mathrm{NaHCO}_{3}$ in 0.5 $\mathrm{L}$ of demi-water. The $\mathrm{pH}$ of the $\mathrm{NaHCO}_{3}$ solution was then adjusted to $\mathrm{pH} 8.5$ using a $2 \mathrm{M} \mathrm{NaOH}$ solution. Dried soil samples were extracted with the $0.5 \mathrm{M} \mathrm{NaHCO}_{3}$ solution at six different SSR of 10, 20,50, 80, 100, and $200 \mathrm{~L} \mathrm{~kg}^{-1}$. For the three lowest SSRs, soils were extracted with $20 \mathrm{~mL}$ of the $\mathrm{NaHCO}_{3}$ solution in $50 \mathrm{~mL}$ polyethylene tubes, whereas for the three highest SSRs, soils were extracted with $90 \mathrm{~mL}$ of $\mathrm{NaHCO}_{3}$ in polyethylene $225 \mathrm{~mL}$ bottles. These conditions led to a constant gas-to-solution volume ratio of $1.5 \mathrm{~L} \mathrm{~L}^{-1}$ among all SSR.

In soils, the presence of $\mathrm{SOM}$ may affect the $\mathrm{PO}_{4}-\mathrm{CO}_{3}$ interaction due to competition with $\mathrm{PO}_{4}$ for the binding sites at the soil surfaces. To suppress this possible effect during the $\mathrm{NaHCO}_{3}$ extractions and facilitate the measurement of $\mathrm{PO}_{4}$, an excess of powdered activated carbon (AC) was added $\left(0.40 \mathrm{~g} \mathrm{~g}^{-1}\right.$ soil) to the soil suspensions. Because the $A C$ might contain small amounts of $\mathrm{PO}_{4}$, the $A C$ was pre-cleaned with AO-solution ${ }^{48}$. An additional rinsing step with $0.5 \mathrm{M} \mathrm{NaHCO}_{3}$ was included before drying the $\mathrm{AC}$ at $40^{\circ} \mathrm{C}$ for 3 days. For each extraction batch, blank samples were included containing only $\mathrm{AC}$ and the $\mathrm{NaHCO}_{3}$ solution. The $\mathrm{PO}_{4}$ concentration in these blank samples ranged from 0.01 to $0.05 \mathrm{mg} \mathrm{L}^{-1}$, which was relatively low compared to the $\mathrm{PO}_{4}$ concentration measured in the supernatants of the soil samples $\left(0.13-12.45 \mathrm{mg} \mathrm{L}^{-1} \mathrm{PO}_{4}\right)$.

In a pre-experiment, we evaluated the kinetics of $\mathrm{PO}_{4}$ desorption in the $\mathrm{NaHCO}_{3}$ solution using three soil samples from the Sub-Saharan series at two different SSR $\left(10\right.$ and $\left.100 \mathrm{~L} \mathrm{~kg}^{-1}\right)$. The samples were shaken for 24, 168, 336, 504, and $672 \mathrm{~h}$ in an end-over-end shaker at 30 cycles per min. Based on the kinetic experiment, a final equilibration time of 504 hours ( 21 days) was used for the main experiment. After equilibration, the $\mathrm{pH}$ was measured with a glass electrode and the samples were centrifuged at $3000 \mathrm{~g}$ for $10 \mathrm{~min}$ and filtered over $0.45 \mu \mathrm{m}$ membrane filters. Before measuring the $\mathrm{PO}_{4}$ concentration using the colorimetric molybdenum-blue method $^{41}$, the filtrate was diluted ( 3 times) with a $0.3 \mathrm{M} \mathrm{HCl}$ solution to adjust the $\mathrm{pH}$ value to $\mathrm{pH} 2$, and the excess $\mathrm{CO}_{2}$ was removed from the filtrate by degassing these solutions in an ultrasonic bath for $\sim 10 \mathrm{~min}$. 


\subsection{Assessment of reactive surface area and reactive phosphate pool}

The charge distribution $(C D)$ mode ${ }^{33}$ was used to derive the reactive surface area (RSA in $\mathrm{m}^{2} \mathrm{~g}^{-1}$ soil) of each soil sample. The calculated $\mathrm{PO}_{4}$ concentrations were fitted to the concentrations measured in the $0.5 \mathrm{M} \mathrm{NaHCO}_{3}$ extraction solution at different SSRs. In this approach, the amount of reactive $\mathrm{PO}_{4}\left(\mathrm{R}-\mathrm{PO}_{4}\right.$ in $\left.\mathrm{mol} \mathrm{kg}^{-1}\right)$ that is reversibly adsorbed in soils is simultaneously calculated by modeling. A detailed modeling description is found elsewhere ${ }^{24,32}$.

The RSA and R-PO $\mathrm{PO}_{4}$ of the soils were calculated using either ferrihydrite (Fh) or goethite as proxies for the fraction of reactive natural metal (hydr)oxides. In the modeling, the surface interaction between $\mathrm{PO}_{4}$ and $\mathrm{CO}_{3}$ is described using an internally consistent thermodynamic database derived previously in model systems with freshly prepared Fh nanoparticles ${ }^{49}$ and well-crystallized goethite ${ }^{50}$. The CD model is combined with state-of-the-art multi-site ion complexation (MUSIC) models for the specific model (hydr)oxide considered ${ }^{33,51}$. The model calculations were done using the ECOSAT program (version 4.9$)^{52}$ in combination with the FIT program (version 2.581) ${ }^{53}$ for optimization of the RSA and $\mathrm{R}-\mathrm{PO}_{4}$ values.

To identify which reference material, either Fh or goethite, is a better proxy for the natural metal (hydr)oxide fraction in our set of soils, the amount of $\mathrm{PO}_{4}$ extracted by the selective dissolution extractions (i.e. $\mathrm{AO}$ and $\mathrm{DC}$ ) was compared to the $\mathrm{R}-\mathrm{PO}_{4}$ pool obtained by modeling the $\mathrm{PO}_{4}$ desorption curves measured in $0.5 \mathrm{M} \mathrm{NaHCO}_{3}$. 
Table 1: Chemical characteristics and modeling results of the soil samples selected for the present study.

\begin{tabular}{|c|c|c|c|c|c|c|c|c|c|c|c|c|c|c|c|c|}
\hline \multirow[b]{2}{*}{ Soil } & \multirow[b]{2}{*}{ OC } & \multirow[b]{2}{*}{$<2 \mu \mathrm{m}$} & \multirow[b]{2}{*}{$\begin{array}{l}\text { AO- } \\
\mathrm{Fe}^{a}\end{array}$} & \multirow[b]{2}{*}{$\begin{array}{l}\text { AO- } \\
\text { Al }^{a}\end{array}$} & \multirow[b]{2}{*}{$\begin{array}{l}\text { AO- } \\
P_{\text {tot }}{ }^{a}\end{array}$} & \multirow[b]{2}{*}{$\begin{array}{l}\mathrm{AO}- \\
\mathrm{PO}_{4}{ }^{\mathrm{a}}\end{array}$} & \multirow[b]{2}{*}{$\begin{array}{l}\text { DC- } \\
\mathrm{Fe}^{b}\end{array}$} & \multirow[b]{2}{*}{$\begin{array}{l}\text { DC- } \\
\text { Al }^{b}\end{array}$} & \multirow[b]{2}{*}{$\begin{array}{l}\text { DC- } \\
\mathrm{P}_{\text {tot }}{ }^{b}\end{array}$} & \multirow[b]{2}{*}{$\begin{array}{c}\text { DC- } \\
\mathrm{PO}_{4}{ }^{b}\end{array}$} & \multicolumn{2}{|c|}{$\mathrm{CaCl}_{2}$} & \multicolumn{2}{|c|}{$R-\mathrm{PO}_{4}$} & \multicolumn{2}{|c|}{ RSA } \\
\hline & & & & & & & & & & & $\mathrm{pH}^{\mathrm{c}}$ & $\mathrm{PO}_{4}{ }^{\mathrm{c}}$ & Gt ${ }^{d}$ & Fhe & Gt ${ }^{d}$ & Fhe \\
\hline & & & \multicolumn{9}{|c|}{ 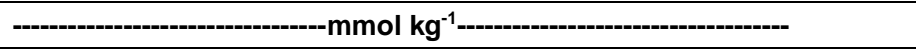 } & $\mu \mathrm{M}$ & \multicolumn{2}{|c|}{----------mmol kg-1.--------- } & \multicolumn{2}{|c|}{--------------m² g $^{-1}$------------- } \\
\hline 1 & 1.4 & 33 & 20.1 & 42.3 & 4.4 & 2.4 & 332.1 & 98.3 & 11.3 & 7.8 & 4.4 & 0.39 & $3.94 \pm 0.10$ & $2.09 \pm 0.30$ & $3.89 \pm 0.08$ & $8.24 \pm 0.70$ \\
\hline 2 & 1.5 & 7 & 20.8 & 50.7 & 4.7 & 2.5 & 289.9 & 97.2 & 9.9 & 6.7 & 4.1 & 0.40 & $3.80 \pm 0.07$ & $1.94 \pm 0.07$ & $3.73 \pm 0.07$ & $6.62 \pm 0.39$ \\
\hline 3 & 5 & 7 & 156.2 & 314.3 & 11.4 & 4.3 & 458.7 & 328.7 & 10.6 & 4.3 & 4.3 & 0.09 & $9.25 \pm 0.125$ & $2.96 \pm 0.08$ & $12.29 \pm 0.07$ & $15.50 \pm 0.05$ \\
\hline 4 & 2 & 71 & 48.9 & 132.8 & 2.5 & 1.5 & 996.1 & 186.8 & 8.1 & 5.2 & 4.1 & 0.05 & $2.45 \pm 0.09$ & $1.26 \pm 0.15$ & $4.80 \pm 0.19$ & $23.04 \pm 2.83$ \\
\hline 5 & 2.8 & 10 & 70.7 & 168.2 & 7.5 & 3.2 & 492.1 & 244.7 & 11.9 & 6.1 & 4.4 & 0.07 & $6.25 \pm 0.07$ & $2.71 \pm 0.09$ & $8.25 \pm 0.12$ & $16.88 \pm 0.58$ \\
\hline 6 & 2.4 & 12 & 41.2 & 138.3 & 7.5 & 5.2 & 510.1 & 202.5 & 11.0 & 7.9 & 5.0 & 0.25 & $15.89 \pm 0.13$ & $7.22 \pm 0.25$ & $16.36 \pm 0.07$ & $24.74 \pm 0.26$ \\
\hline 7 & 1.9 & 12 & 27.8 & 76.3 & 3.1 & 1.3 & 373.7 & 180.7 & 9.7 & 5.6 & 5.1 & 0.09 & $2.34 \pm 0.02$ & $1.38 \pm 0.04$ & $3.15 \pm 0.02$ & $12.54 \pm 0.44$ \\
\hline 8 & 1.4 & 7 & 20.7 & 51.3 & 2.6 & 1.7 & 310.1 & 134.9 & 7.4 & 5.1 & 4.5 & 0.19 & $3.94 \pm 0.14$ & $2.01 \pm 0.12$ & $4.57 \pm 0.10$ & $11.24 \pm 0.51$ \\
\hline 9 & 2.1 & 2 & 77.7 & 94.5 & 7.6 & 2.9 & 342.2 & 215.0 & 15.4 & 9.5 & 4.5 & 0.10 & $5.94 \pm 0.064$ & $2.98 \pm 0.08$ & $6.29 \pm 0.06$ & $12.09 \pm 0.14$ \\
\hline 10 & 2.2 & 17 & 57.6 & 147.5 & 3.9 & 1.7 & 646.7 & 253.0 & 8.7 & 4.8 & 4.2 & 0.04 & $3.034 \pm 0.08$ & $1.59 \pm 0.03$ & $5.77 \pm 0.18$ & $27.83 \pm 0.08$ \\
\hline 11 & 2.2 & 4 & 59.5 & 152.1 & 4.0 & 1.5 & 615.9 & 245.1 & 8.7 & 4.5 & 4.3 & 0.04 & $3.20 \pm 0.07$ & $1.54 \pm 0.03$ & $7.06 \pm 0.13$ & $39.13 \pm 0.47$ \\
\hline 12 & 1.4 & 4 & 37.3 & 63.3 & 3.4 & 1.4 & 327.6 & 139.5 & 8.0 & 4.7 & 4.2 & 0.06 & $2.57 \pm 0.02$ & $1.15 \pm 0.05$ & $3.58 \pm 0.02$ & $11.072 \pm 0.24$ \\
\hline 13 & 1.2 & 5 & 37.8 & 63.6 & 3.3 & 1.6 & 327.8 & 141.1 & 8.4 & 4.8 & 4.1 & 0.08 & $2.46 \pm 0.02$ & $1.32 \pm 0.03$ & $3.36 \pm 0.02$ & $12.59 \pm 0.37$ \\
\hline 14 & 1.5 & 7 & 41.5 & 70.9 & 4.1 & 2.1 & 325.2 & 141.3 & 8.3 & 4.9 & 4.2 & 0.08 & $2.72 \pm 0.04$ & $1.54 \pm 0.03$ & $3.48 \pm 0.03$ & $12.99 \pm 0.18$ \\
\hline 15 & 3.2 & 8 & 65.7 & 135.9 & 4.0 & 1.5 & 730.3 & 171.7 & 9.1 & 4.8 & 4.9 & 0.09 & $2.53 \pm 0.05$ & $1.20 \pm 0.07$ & $3.88 \pm 0.08$ & $10.34 \pm 0.17$ \\
\hline 16 & 0.9 & 7 & 56.9 & 56.1 & 22.3 & 16.9 & 289.5 & 90.7 & 32.5 & - & 5.3 & 2.23 & $25.85 \pm 0.18$ & $14.25 \pm 0.22$ & $15.14 \pm 0.05$ & $9.69 \pm 0.03$ \\
\hline 17 & 0.3 & 20 & 7.8 & 24.1 & 2.1 & 2.2 & 67.0 & 39.4 & 3.2 & - & 6.8 & 1.61 & $2.39 \pm 0.05$ & $1.57 \pm 0.04$ & $1.43 \pm 0.05$ & $1.70 \pm 0.06$ \\
\hline 18 & 0.6 & 53 & 32.9 & 40.4 & 1.7 & 1.6 & 634.9 & 58.2 & 6.1 & - & 6.3 & 0.16 & $1.51 \pm 0.13$ & $0.92 \pm 0.05$ & $1.51 \pm 0.14$ & $3.47 \pm 0.14$ \\
\hline
\end{tabular}

${ }^{a}$ Measured with acid ammonium oxalate extraction $(\mathrm{pH}=3)^{44}$

${ }^{b}$ Measured with dithionite citrate extraction ${ }^{46}$

${ }^{c}$ Measured in $0.01 \mathrm{M} \mathrm{CaCl}_{2}$ (solution-to-solid ratio SSR $=10 \mathrm{~L} \mathrm{~kg}^{-1}$, time $=2 \mathrm{~h}$ ) 40

${ }^{d}$ Pool of reversely bound $\mathrm{PO}_{4}\left(R-\mathrm{PO}_{4}\right)$ and reactive surface area (RSA) derived with the results of the probe-ion method, using goethite as reference oxide material in the CD modeling.

e Pool of reversely bound $\mathrm{PO}_{4}\left(R-\mathrm{PO}_{4}\right)$ and reactive surface area (RSA) derived with the results of the probe-ion method, using ferrihydrite as reference oxide material in the CD modeling. 


\section{RESULTS AND DISCUSSION}

\subsection{Phosphate desorption in $0.5 \mathrm{M} \mathrm{NaHCO}_{3}$ extractions}

In Figure 1, the equilibrium $\mathrm{PO}_{4}$ concentration in the $0.5 \mathrm{M} \mathrm{NaHCO}_{3}$ soil extracts as a function of the SSR is given for six selected samples. For all SSRs, the measured $\mathrm{PO}_{4}$ concentrations were considerably higher than our detection limit for measuring $\mathrm{PO}_{4}$ colorimetrically in the $0.5 \mathrm{M} \mathrm{NaHCO}_{3}$ soil extracts (dotted horizontal line in Figure 1). From a practical perspective, this observation is important because the probe-ion method was first developed and tested for agricultural topsoils from temperate climates, which generally have high concentrations of soluble $\mathrm{PO}_{4}{ }^{24}$.

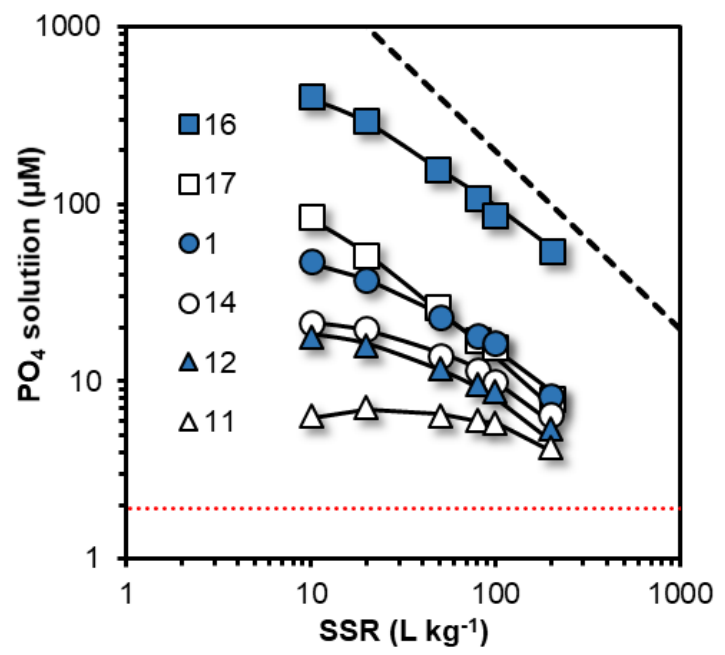

Figure 1: Phosphate $\left(\mathrm{PO}_{4}\right)$ concentration in the $0.5 \mathrm{M} \mathrm{NaHCO}_{3}$ solution at a pH value near 8.5 as a function of the solution-to-solid ratio (SSR) shown here for six selected soil samples. The full lines are the CD model calculations using ferrihydrite as reference metal (hydr)oxide material. In the modeling, RSA and $\mathrm{R}-\mathrm{PO}_{4}$ are defined as adjustable parameters. The model parameters used to calculate the competitive interaction of $\mathrm{CO}_{3}-\mathrm{PO}_{4}$ were taken from Mendez and Hiemstra ${ }^{49}$. The dashed diagonal line represents a theoretical linear dilution curve, i.e. a 10 times decrease in SSR results in a 10 times lower $\mathrm{PO}_{4}$ concentration in solution. The experimental data shows a lower slope than the linear dilution curve, which is due to the release of $\mathrm{PO}_{4}$ from the soil surfaces (i.e. $\mathrm{PO}_{4}$ buffering) when the SSR increases. The red dotted horizontal line represents the lowest measurable concentration of $\mathrm{PO}_{4}$ in the $0.5 \mathrm{M} \mathrm{NaHCO}_{3}$ solution with our analytical procedure.

As expected, the highest $\mathrm{PO}_{4}$ concentrations in the soil extracts are found at the lowest SSR. When the SSR increases (i.e. at further dilution of the soil sample with $\mathrm{NaHCO}_{3}$ solution), the equilibrium $\mathrm{PO}_{4}$ concentrations decrease but less than expected from the imposed dilution factor, because additional $\mathrm{PO}_{4}$ is released from the soil surfaces. The $\mathrm{PO}_{4}$ buffering capacity of the soil leads to the non-linearity of the dilution curves. In Figure 1, the slope of the $\mathrm{PO}_{4}$ dilution curves is therefore related to the $\mathrm{PO}_{4}$ buffer capacity of the soil, which is determined by its reactive surface area ${ }^{24}$. Soils with less steep dilution curves are expected to have a higher RSA. If soil does not release any additional $\mathrm{PO}_{4}$ upon soil sample dilution with 
$\mathrm{NaHCO}_{3}$, the experimental $\mathrm{PO}_{4}$ concentration will decrease linearly with the SSR (dashed line in Figure 1). As inferred from Figure 1, the $\mathrm{PO}_{4}$ buffer capacity (slope) and consequently the RSA, greatly differs among the soil samples.

\subsection{Phosphate fractions associated with the metal (hydr)oxides}

\subsubsection{Reactive pool of adsorbed phosphate}

The pool of reactive $\mathrm{PO}_{4}$ reversibly adsorbed to the soil surfaces $\left(\mathrm{R}-\mathrm{PO}_{4}\right)$ can be revealed by interpreting the results from the probe-ion method with the CD model. This R-PO $\mathrm{P}_{4}$ pool controls the solid-solution partitioning of $\mathrm{PO}_{4}$ in the $0.5 \mathrm{M} \mathrm{NaHCO}_{3}$ soil extractions at different SSRs. The calculated $\mathrm{R}-\mathrm{PO}_{4}$ depends on the properties of the metal (hydr)oxide chosen as reference, in our study either goethite or $\mathrm{Fh}^{32,49}$. In this section, we compare the modeled $\mathrm{R}-\mathrm{PO}_{4}$ values with the amount of inorganic $\mathrm{PO}_{4}$ that is measured in the soil extracts of a selective dissolution methodology. Specifically, our benchmark will be finding agreement between the experimental $\mathrm{PO}_{4}$ pool measured by the $\mathrm{AO}$ extraction and the modeled $\mathrm{R}-\mathrm{PO}_{4}$ pool. $\mathrm{AO}$ extractions are often applied to selectively dissolve and quantify the nanocrystalline fraction of $\mathrm{Fe}$ and $\mathrm{Al}$ (hydr)oxides in soils ${ }^{54-56}$. In the $\mathrm{AO}$ soil extracts, the total pool of released $\mathrm{P}\left(\mathrm{AO}-\mathrm{P}_{\mathrm{tot}}\right)$ can also be conveniently analyzed by ICP-OES, simultaneously with the analysis of $\mathrm{Fe}$ and Al. These results are often used for assessing the degree of $\mathrm{P}$ saturation in soils ${ }^{57-59}$. Long-term experiments have shown that nearly all $P$ that is extracted with $\mathrm{AO}$ is potentially desorbable when the soil is exposed to an "infinite" sink condition for $\mathrm{P}^{60}$. In line with these results, others have found that $A O-P_{\text {tot }}$ was largely desorbable in a long-term P-mining pot experiment ${ }^{57}$.

In the original probe-ion approach developed by Hiemstra et al. ${ }^{24}$, the calculated R$\mathrm{PO}_{4}$ was compared with the AO-P $\mathrm{P}_{\text {tot }}$ pool measured by ICP-OES, rather than with ortho- $\mathrm{PO}_{4} \quad\left(\mathrm{AO}-\mathrm{PO}_{4}\right)$ measured using the colorimetric molybdenum-blue methodology $41,43,61$. However, the AO-P tot pool may also include other $P$ species than ortho- $\mathrm{PO}_{4}$, especially organic $\mathrm{P}\left(\mathrm{P}_{\text {org }}\right)^{62}$, whereas the probe-ion method is based on the measurement of the equilibrium concentration of ortho- $\mathrm{PO}_{4}$ in the $0.5 \mathrm{M}$ $\mathrm{NaHCO}_{3}$ extracts. For instance, inositol phosphates, the most abundant organic $\mathrm{P}$ form in soils, can be effectively extracted with $\mathrm{AO}$ solutions ${ }^{63}$. Therefore, we have used in the present study $\mathrm{AO}$-extractable $\mathrm{PO}_{4}$ rather than $\mathrm{P}_{\text {tot }}$, as a validation criterion in our evaluation of the $\mathrm{R}-\mathrm{PO}_{4}$ values obtained by $\mathrm{CD}$ modeling. For a series of agricultural topsoils from the Netherlands, we have recently shown that the experimental $A O-\mathrm{PO}_{4}$ values agree well with the $\mathrm{R}-\mathrm{PO}_{4}$ revealed by $\mathrm{CD}$ modeling using Fh as a proxy ${ }^{32}$.

Figure 2a shows the modeled $\mathrm{R}-\mathrm{PO}_{4}$ values using $\mathrm{Fh}$ as reference (hydr)oxide in relation to the experimental $\mathrm{AO}-\mathrm{PO}_{4}$ pool. In general, a good agreement (1:1 line) is observed between both model and experimental $\mathrm{PO}_{4}$ pools, while this is not the case when goethite is used as reference metal (hydr)oxide in the CD modeling. In the latter case, as is also shown in Figure 2a, the $\mathrm{R}-\mathrm{PO}_{4}$ values predicted by the model 
are $\sim 2$ times higher than the experimental $\mathrm{AO}-\mathrm{PO}_{4}$ pool. Therefore, based on the $\mathrm{AO}-\mathrm{PO}_{4}$ data as the validation criterion, we conclude that $\mathrm{Fh}$ is a better proxy for the reactive fraction of $\mathrm{Fe}$ and $\mathrm{Al}$ (hydr)oxides of these weathered tropical topsoils from sub-Saharan Africa. This observation is remarkable considering that, on a mass basis, well-crystallized materials are clearly the dominant fraction of metal (hydr)oxides in our set of tropical soil samples (Table 1).
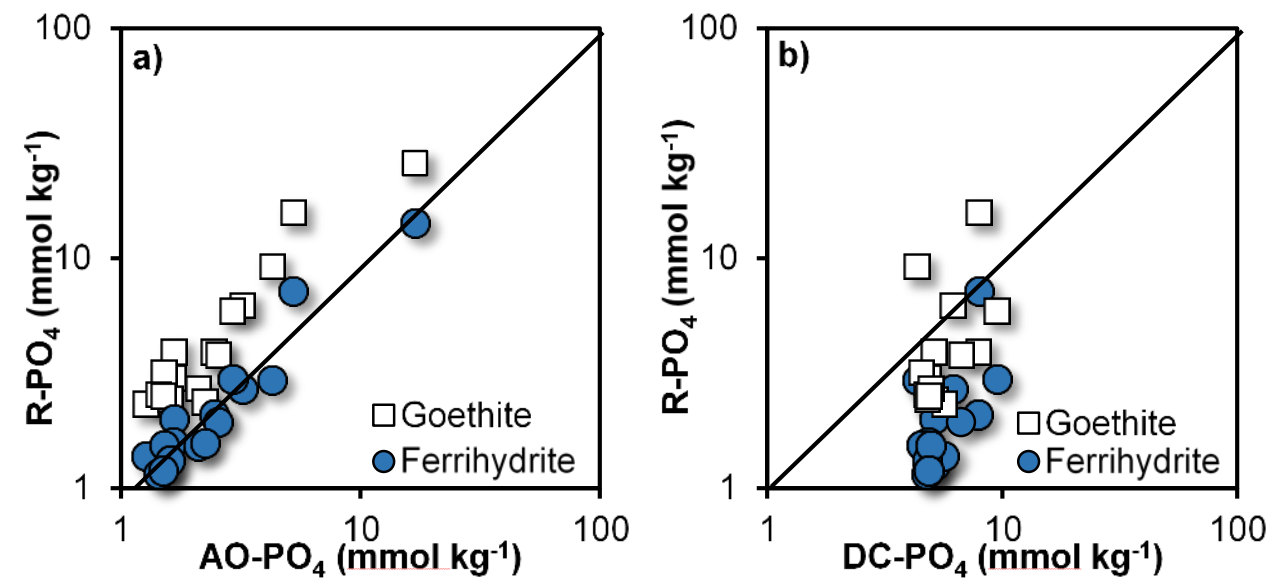

Figure 2: Comparison between the amount of reactive $\mathrm{PO}_{4}$ that is reversibly bound to the soil surfaces $\left(\mathrm{R}-\mathrm{PO}_{4}\right)$, calculated with the $\mathrm{CD}$ model using either goethite or $\mathrm{Fh}$ as reference metal (hydr)oxide, and experimental measurements of $\mathrm{PO}_{4}$ in the (a) ammonium oxalate (AO) extracts or (b) in the dithionitecitrate (DC) extracts of the series of Sub-Saharan soil samples. Both $x$-and $y$-axes are shown based on $\log _{10}$ scale. In panel b, samples $16-18$ are not included because DC-PO ${ }_{4}$ data are not available for these samples.

\subsubsection{Phosphate occluded in the crystalline metal (hydr)oxides}

In addition to the soil extractions with $\mathrm{AO}$ solution, soils can also be extracted with a dithionite-citrate (DC) solution to assess the total content of Fe and Al (hydr)oxides, comprising both the fraction of nanocrystalline and well-crystallized metal (hydr)oxides ${ }^{64,65}$. Because our present set of tropical topsoils have a relatively high content of crystalline metal (hydr)oxides (Table 1), we have also measured the pool of inorganic $\mathrm{PO}_{4}$ extracted with $\mathrm{DC}\left(\mathrm{DC}-\mathrm{PO}_{4}\right)$ for a subset of soils.

The $\mathrm{DC}-\mathrm{PO}_{4}$ pool (Figure $2 \mathrm{~b}$ ) is considerably larger than the corresponding $\mathrm{R}-\mathrm{PO}_{4}$ values calculated by $C D$ modeling using $\mathrm{Fh}$ as a proxy (Figure 2a). Using goethite as reference material did also not provide a good relationship between DC-PO 4 and $\mathrm{R}-\mathrm{PO}_{4}$ (Figure 2b). In both cases, the lack of agreement between experimental and modeled values suggests that an important fraction of $\mathrm{DC}-\mathrm{PO}_{4}$ is not desorbable and does not play a significant role in the solid-solution partitioning of $\mathrm{PO}_{4}$ in the soil extractions with $0.5 \mathrm{M} \mathrm{NaHCO}_{3}$ solution. 
The amount of $\mathrm{PO}_{4}$ that is associated with the crystalline metal (hydr)oxide fraction $\left(\mathrm{PO}_{4, \text { crys }}\right)$, can be defined operationally as the difference between $\mathrm{DC}$ and $\mathrm{AO}$ extractable $\mathrm{PO}_{4}$. This excess amount $\left(\mathrm{PO}_{4, \text { crys }}\right)$ is not part of a fast process of equilibration. Likely, it is bound internally as occluded $\mathrm{PO}_{4}$, similarly as found in synthetic systems at recrystallization of $\mathrm{Fh}$ to hematite and goethite in the presence of $\mathrm{PO}_{4}{ }^{66}$. In our samples with soil 3 as an exception, the occluded pool of $\mathrm{PO}_{4}$ is 64 $\pm 11 \%$ of total DC-extractable $\mathrm{PO}_{4}$.

\subsubsection{Organic phosphorus bound to thed reactive metal (hydr)oxides}

In AO-extracts, the total amount of phosphorus $\mathrm{P}_{\text {tot }}$ is systematically higher than the amount of orthophosphate. This is shown in Figure 3a. It highlights the importance of distinguishing analytically between the ortho- $\mathrm{PO}_{4}$ fraction and the total $\mathrm{P}$ pool measured in the $A O$ soil extracts of both tropical and temperate soils. At a low value of $\mathrm{P}$-total in the $\mathrm{AO}$ extracts, the contribution of the inorganic fraction is relatively lower. This is particularly for the series of tropical topsoils (Figure S1). For our set of weathered tropical topsoils, the fraction of ortho- $\mathrm{PO}_{4}$ is on average only about 0.55 while for the topsoils from a temperate climate, this contribution is higher (on average 0.74 , see Table 2). The difference between $\mathrm{P}$-total and $\mathrm{P}_{-} \mathrm{PO}_{4}$ is likely due to the presence of organic $\mathrm{P}\left(\mathrm{P}_{\text {org }}\right)$ in the $\mathrm{AO}$-extracts ${ }^{62,63}$ because the difference is related to the total SOC content of the soil samples as shown in Figure $3 \mathrm{~b}$. These results suggest that $\mathrm{SOM}$ contributes to the extraction of $\mathrm{P}_{\text {org }}$ in topsoils. In combination, the data of figures $3 a, b$ underline the importance of $P_{\text {org }}$ and SOM in the availability of $P$ and the overall $P$ cycle in these tropical topsoils 67,68 . 

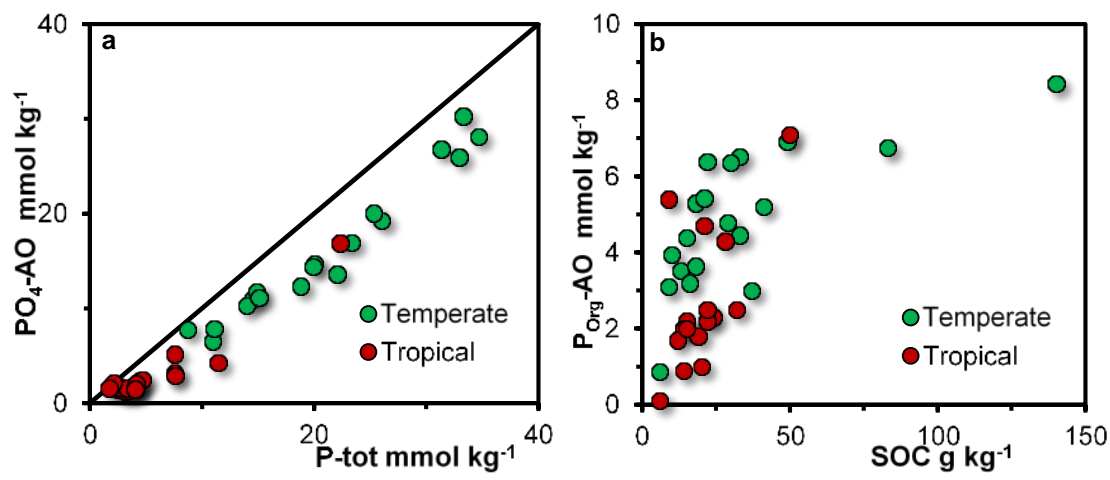

Figure 3: Relationship between the total $\mathrm{P}$ and the amount of ortho $\mathrm{PO}_{4}$ that is extracted from temperate and tropical soils with an acid ammonium oxalate $(\mathrm{AO})$ solution $(\mathrm{pH}=3$ ) (panel a). Operationally, the difference between AO- $\mathrm{P}_{\text {tot }}$ and $\mathrm{AO}-\mathrm{PO}_{4}$ can be defined as the $\mathrm{P}_{\text {org }}$ pool as this pool is related to the amount of soil organic carbon (SOC) (panel b). The red symbols are for the samples collected from agricultural tropical topsoils from sub-Saharan countries. The green symbols are for a series of agricultural topsoils from a temperate region analyzed previously ${ }^{32,69}$ and this information is shown here for comparison. The diagonal in panel $a$ is the $1: 1$ line.

In other SCM studies 2,70 , the use of $A O-\mathrm{P}_{\text {tot }}$ as a proxy for $\mathrm{R}-\mathrm{PO}_{4}$ has led to model overestimations of the $\mathrm{PO}_{4}$ concentration in soil leachates ${ }^{70}$ and soil extraction solutions ${ }^{2}$. Based on those results, the use of isotopically exchangeable $\mathrm{PO}_{4}(E-$ value) has been proposed as a proxy for $\mathrm{R}_{-} \mathrm{PO}_{4}$ in SCM. However, the contribution of organic $\mathrm{P}$ species to the AO- $\mathrm{P}_{\text {tot }}$ was not explicitly considered, whereas it can significantly contribute to the total pool of $\mathrm{P}$ measured in $\mathrm{AO}$ extractions, as we have shown here. In addition, measurement of the isotopically exchangeable $\mathrm{PO}_{4}$ pool is strongly influenced by the kinetics of $\mathrm{P}$ exchange, the predefined evaluation time ${ }^{71}$, as well as by the properties of the solution in which it is determined.

\subsection{Reactive metal (hydr)oxides in weathered tropical soils}

\subsubsection{The reactive surface area}

The reactive surface area (RSA) of our sub-Saharan African soils, calculated with Fh as a proxy, varies by a factor of $\sim 20$, over the range $\sim 2-40 \mathrm{~m}^{2} \mathrm{~g}^{-1}$ soil (Table 1 ). In the case of using goethite as reference metal (hydr)oxide, the variation is less, over the range $\sim 1.5-15 \mathrm{~m}^{2} \mathrm{~g}^{-1}$ soil (Table 1 ). The RSA values calculated with goethite as reference are generally lower than the values obtained by using $\mathrm{Fh}$ as reference metal (hydr)oxide. The opposite has been found for the set of P-rich soils from temperate climates analyzed in our previous study 32 .

The remarkable difference behavior of both categories of soils with respect to the calculation of the RSA can be understood from the difference in shape of the competitive $\mathrm{PO}_{4}$ isotherms of goethite and $\mathrm{Fh}$ in $0.5 \mathrm{M} \mathrm{NaHCO}_{3}$. Both isotherms are shown in Figure S2. The high-affinity character of $\mathrm{PO}_{4}$ adsorption to goethite is much better preserved at relatively low concentrations compared to $\mathrm{Fh}^{32}$. This leads to a 
higher $\mathrm{PO}_{4}$ buffering of the solution by goethite compared to $\mathrm{Fh}^{32,49}$. At higher concentrations, this behavior reverses. The $\mathrm{PO}_{4}$ isotherm of goethite flattens down more strongly than the isotherm of Fh. Consequently, the $\mathrm{PO}_{4}$ buffering is in thase case higher for Fh. In the temperate soils, the loading is relatively high, while in the tropical soils, it is relatively less. This difference results in an opposite outcome of the CD modeling ${ }^{32}$.

In Section 3.2.1, we have concluded, based on the comparison between $\mathrm{R}_{-} \mathrm{PO}_{4}$ and AO- $\mathrm{PO}_{4}$, that the interaction between the reactive fraction of the metal (hydr)oxides and $\mathrm{PO}_{4}$ in our set of tropical topsoils can be best described using $\mathrm{Fh}$ as reference metal (hydr)oxide in the CD modeling. The use of the latter leads to relatively large RSA values as discussed above. However, the question arises whether the fraction of well-crystallized $\mathrm{Fe}$ and $\mathrm{Al}$ (hydr)oxides also contributes to the soil reactivity toward $\mathrm{PO}_{4}$. This will be discussed next.

Figure 4 shows that the calculated RSA of our tropical soils is positively correlated to the amount of $\mathrm{Fe}$ and $\mathrm{Al}$, either extracted with $\mathrm{AO}$ (spheres) or DC (squares). The slope of the regression lines approximates the mean specific surface area (SSA) of the metal (hydr)oxide fraction. It leads to a mean value of SSA $=110 \pm 22 \mathrm{~m}^{2} \mathrm{mmol}^{-}$ ${ }^{1}$ when the RSA values are scaled to the content of $[\mathrm{Fe}+\mathrm{Al}]_{\mathrm{AO}}$ and $\mathrm{SSA}=23 \pm 5 \mathrm{~m}^{2}$ $\mathrm{mmol}^{-1}$ in case of scaling to the content of $[\mathrm{Fe}+\mathrm{Al}] \mathrm{Dc}$. This large difference is due to the significant contribution of well-crystallized Fe and $\mathrm{Al}$ (hydr)oxides ([Fe+Al $]_{\text {cryst }}$ ) to the total Fe and $\mathrm{Al}$ measured in the DC soil extracts. For the tropical topsoils, the mean SSA value obtained at scaling of the RSA to $[\mathrm{Fe}+\mathrm{Al}]_{\mathrm{AO}}$ is about two-fold higher than found for the agricultural topsoils from a temperate climate ${ }^{32}$.

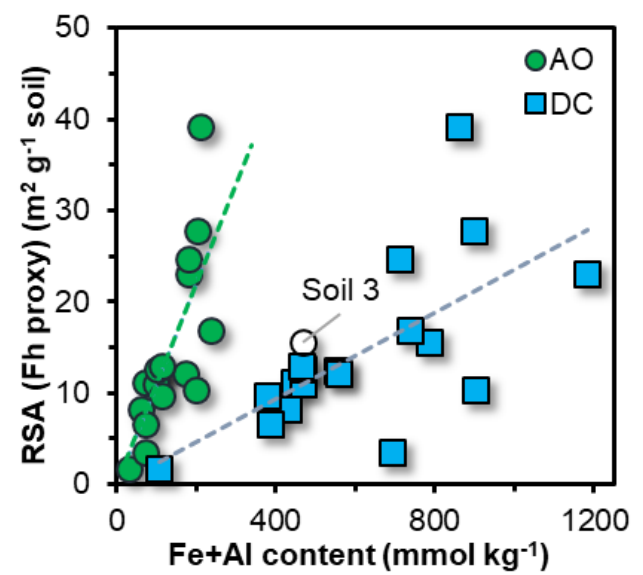

Figure 4: Relationship between the reactive surface area (RSA), calculated by interpreting the results of the probe-ion method using $\mathrm{Fh}$ as reference (hydr)oxide in the $\mathrm{CD}$ model calculations, and the $\mathrm{Fe}+\mathrm{Al}$ content measured either in the ammonium oxalate (AO) or in the dithionite-citrate (DC) soil extracts. Sample 3 largely deviates from the relationship between $[\mathrm{Fe}+\mathrm{Al}]_{\mathrm{AO}}$ and RSA, which is likely due to an important contribution of $\mathrm{Al}^{3+}$ or Al-polymers in this sample that can be complexed by SOM in the AO extraction $^{72}$ as sample 3 has a relatively high content of SOM (5\%). 
The data in Figure 4 show a large scattering around the regression lines. This implies that the values of SSA largely vary amongst the different soils. Expressed in terms of the more conventional unit of $\mathrm{m}^{2} \mathrm{~g}^{-1}$, the SSA varies between $\sim 400-1750 \mathrm{~m}^{2} \mathrm{~g}^{-1}$ when the RSA is scaled to the fraction of [Fe+Al] $]_{A O}$ (see Section 3.3.2). In SCM studies applied to soil samples, the RSA is commonly estimated based on the amount of $\mathrm{Fe}$ and $\mathrm{Al}$ extracted with $\mathrm{AO}$ and an assumed "standard" values for the SSA, i.e. $600 \mathrm{~m}^{2} \mathrm{~g}^{-1}$ and $100 \mathrm{~m}^{2} \mathrm{~g}^{-1}$ for the nano-and crystalline fraction of the metal (hydr)oxides respectively $70,73,74,75$. For about half of our samples, the use of the latter approach leads to a strong deviation compared to the presently measured RSA values (Figure S3).

The molar ratio $[\mathrm{Fe}+\mathrm{Al}]_{\mathrm{AO}} /[\mathrm{Fe}+\mathrm{Al}]_{\text {Cryst }}$ is on average $\sim 0.4$ in our set of tropical soils. In other words, on a mass basis the crystalline fraction dominates. However, in terms of surface reactivity, this will be different because of the relatively large difference in SSA. Nanocrystalline (hydr)oxides typically have a $\sim 10$ times higher SSA than wellcrystallized (hydr)oxides ${ }^{69}$. When performing a multiple regression analysis using $[\mathrm{Fe}+\mathrm{Al}]_{\mathrm{AO}}$ and $[\mathrm{Fe}+\mathrm{Al}] \mathrm{cryst}$ as two independent variables, only $[\mathrm{Fe}+\mathrm{Al}]_{\mathrm{AO}}$ is found to be significant for explaining the modeled values of $\mathrm{RSA}\left(\mathrm{R}^{2}=0.87\right.$, with $p<0.05$ for $[\mathrm{Fe}+\mathrm{Al}]_{\mathrm{AO}}$ and $p>0.5$ for $\left.[\mathrm{Fe}+\mathrm{Al}]_{\text {Cryst }}\right)$. The statistical evaluation suggests that despite the large mass fraction of crystalline $\mathrm{Fe}$ and $\mathrm{Al}$ (hydr)oxides, the presence of nanocrystalline (hydr)oxides is the major factor that determines the reactivity of the metal (hydr)oxides in this set of highly weathered soils.

Table 2: Comparison of experimental and modeling results for the tropical topsoils from this study and the set of topsoils from temperate climate studied previously ${ }^{32}$. Fe and $\mathrm{Al}$ were measured in acid ammonium oxalate (AO) and dithionite-citrate (DC) extractions. $\mathrm{P}_{\text {tot }}$ and $\mathrm{PO}_{4}$ were measured in the $\mathrm{AO}$ extracts by ICP-OES and the colorimetric molybdenum blue method, respectively. Based on the probeion method described by Hiemstra et al. ${ }^{24}$, the reactive surface area (RSA), reactive $\mathrm{PO}_{4}$ pool $\left(\mathrm{R}-\mathrm{PO}_{4}\right)$, and surface loading of $\mathrm{PO}_{4}\left(\Gamma_{\mathrm{PO}}\right)$ were calculated using $\mathrm{Fh}$ as reference metal (hydr)oxide ${ }^{32}$.

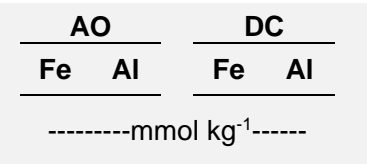

\begin{tabular}{|c|c|c|c|}
\hline \multicolumn{2}{|c|}{ AO/DC } & \multicolumn{2}{|c|}{ AO } \\
\hline $\mathrm{Fe}$ & Al & $\mathrm{PO}_{4} / \mathrm{P}_{\text {tot }}$ & $\mathrm{PO}_{4} /(\mathrm{Fe}+\mathrm{Al})$ \\
\hline
\end{tabular}

\begin{tabular}{ccc}
\multicolumn{3}{c}{ Probe-ion method } \\
\hline $\mathbf{R}^{-P}{ }_{4}$ & $\mathbf{R S A}$ & $\Gamma_{\mathrm{PO} 4}$ \\
$\mathrm{mmol}$ & $\mathrm{m}^{2}$ & $\mu \mathrm{mol}$ \\
$\mathrm{kg}^{-1}$ & $\mathrm{~g}^{-1}$ & $\mathrm{~m}^{-2}$ \\
\hline
\end{tabular}

\begin{tabular}{lccccccccccc}
\hline \multicolumn{10}{c}{ Weathered tropical topsoils (Sub-Sahara African soils) } \\
mean & 49 & 101 & 448 & 165 & 0.12 & 0.59 & 0.55 & 0.03 & 2.8 & 14.3 & 0.36 \\
$\min$ & 8 & 24 & 67 & 39 & 0.05 & 0.38 & 0.37 & 0.01 & 0.9 & 1.7 & 0.04 \\
$\max$ & 156 & 314 & 996 & 329 & 0.34 & 0.96 & 1.00 & 0.15 & 14.3 & 39.1 & 1.47 \\
\hline \multicolumn{10}{c}{ Temperate climate topsoils (The Netherlands) } \\
mean & 102 & 29 & 188 & 34 & 0.58 & 0.80 & 0.76 & 0.13 & 12.5 & 9.0 & 1.64 \\
$\min$ & 11 & 3 & 16 & 6 & 0.28 & 0.50 & 0.60 & 0.03 & 3.2 & 2.1 & 0.51 \\
$\max$ & 342 & 58 & 852 & 59 & 0.95 & $1.30^{*}$ & 0.91 & 0.48 & 27.9 & 19.5 & 3.44 \\
\hline
\end{tabular}

For the soil from the temperate regions, soil extractions with dithionite-citrate-bicarbonate (DCB) solution were performed, rather than the dithionite-citrate (DC) extraction used in the present study. Details of the DCB extraction procedure are described in Hiemstra et al. ${ }^{24}$

"For one soil sample, the measured Al content in the AO soil extracts was higher than in the DCB extracts. 
According to literature, differences in the pedogenic conditions and weathering stages of soils may affect both the composition of the metal (hydr)oxides ${ }^{11,12}$ and the relative distribution of the various $P$ pools ${ }^{36,37}$. To illustrate this, we have summarized for our soils the results of the selective dissolution extractions and the CD modeling in Table 2, and compared the present results with previous ones obtained for soils from soil temperate climate ${ }^{32}$.

The weathered tropical topsoils have, on a molar basis, a larger contribution of crystalline metal (hydr)oxides, which is reflected in the lower average AO/DC ratio of Fe and Al. Remarkably, as shown in Section 3.2, the reactivity of these topsoils can nevertheless be best described by using nanocrystalline $\mathrm{Fh}$ as reference metal (hydr)oxide. In this respect, our tropical topsoils do not differ from the previously studied set of topsoils from a temperate climate. A clear difference between both types of soils is the molar ratio $\mathrm{PO}_{4} /(\mathrm{Fe}+\mathrm{Al})$, measured in $\mathrm{AO}$ extracts. For the tropical topsoils, this molar ratio is on average only $\sim 0.03$, which is substantially lower than the average ratio of $\sim 0.13$ found for the temperate climate topsoils ${ }^{32}$. The difference is likely due to the different origin and history of $\mathrm{PO}_{4}$ fertilization since the sum of $\mathrm{Fe}$ and $\mathrm{Al}$ in $\mathrm{AO}$ is similar between the two soil sets. The difference in the $\mathrm{PO}_{4}$ status between both soil series is also revealed by the $C D$ model results. The average surface loading of $\mathrm{PO}_{4}\left(\Gamma_{\mathrm{PO}}\right)$, calculated as $\Gamma_{\mathrm{PO}}=\mathrm{R}-\mathrm{PO}_{4} / \mathrm{RSA}$, is on average $\sim 5$ times lower in the weathered topsoil from the tropics, in comparison with the topsoils from a temperate climate.

\subsubsection{Size-dependent properties of the reactive metal (hydr)oxides}

As mentioned above, the slope of the regression lines in Figure 4 approximates the mean SSA of the metal (hydr)oxide fraction expressed in $\mathrm{m}^{2} \mathrm{mmol}^{-1}$. For expressing these SSAs in a more conventional unit of $\mathrm{m}^{2} \mathrm{~g}^{-1}$ of metal (hydr)oxides, additional information about the molar mass of the oxide $\left(M_{\text {nano }}\right.$ in $\mathrm{g} \mathrm{mol}^{-1} \mathrm{Fe}$ or $\left.\mathrm{Al}\right)$ is required. Subsequently, the SSA can be translated into an equivalent particle diameter (d), using the mass density $\left(\rho_{\text {nano }}\right.$ in $\mathrm{g} \mathrm{m}^{-3}$ ) of the metal (hydr)oxide particles. In these calculations, the values of $M_{\text {nano, }}$, and $\rho_{\text {nano }}$ are not constant but particle sizedependent, because of the nano nature of the metal (hydr) oxide particles. However, these values can be calculated iteratively. The resulting particle diameter is consistently applied to calculate the Stern layer capacitances which change with surface curvature ${ }^{16,76}$.

The above-sketched approach for scaling the RSA to the amount Fe and Al extracted with $\mathrm{AO}^{32}$ has been applied here. In the present approach, the natural oxide fraction is considered as a solid-solution of two constituting endmembers, represented by $\mathrm{Fh}$ $\left(\mathrm{FeO}_{1.4}(\mathrm{OH})_{0.2} \cdot n \mathrm{H}_{2} \mathrm{O}\right)$ and nano-sized Al hydroxide $\left(\mathrm{Al}(\mathrm{OH})_{3} \cdot n \mathrm{H}_{2} \mathrm{O}\right)$ in which $n \mathrm{H}_{2} \mathrm{O}$ is the excess amount of chemisorbed water that is due to the presence of surface groups. The overall molar mass of the natural oxide fraction can then be calculated from the weighted sum of the molar masses of the endmembers, according to: 


$$
M_{\text {soil (hydr)oxide }}=\frac{\mathrm{Fe}}{\mathrm{Fe}+\mathrm{Al}} M_{\mathrm{Fh}}+\frac{\mathrm{Al}}{\mathrm{Fe}+\mathrm{Al}} M_{\text {nanogibbsite }} \quad \text { Eq. } 1
$$

The molar ratios of $\mathrm{Fe}$ and $\mathrm{Al}$ used in equation Eq. 1 are measured in the $\mathrm{AO}$ extract. The overall mass density ( $\rho_{\text {soil }}$ (hydr)oxide $)$ can be calculated from the volume-weighted mass densities of both endmembers:

$$
\rho_{\text {soil (hydr)oxide }}=\frac{m_{1}+m_{2}}{m_{1 / \rho_{\text {nano1 }}}+m_{2 / \rho_{\text {nano } 2}}}
$$

in which $\rho_{\text {nano1 }}$ and $\rho_{\text {nano2 }}$ are the mass densities and $m_{1}$ and $m_{2}$ the masses of the constituting endmembers, i.e. Fh and nanogibbsite. The latter values $(m)$ follow from the measured metal ion concentrations in the $\mathrm{AO}$ extract and the molar masses of both constituting endmembers, i.e. $M_{\text {Fh }}$ and $M_{\text {nanogibbsite. }}$

In our approach, the iterative calculations start by considering a particle with a diameter ( $d$ ) for which $M_{\text {soil (hydroxide }}$ and $\rho_{\text {soil (hydr)oxide }}$ are calculated using the values $M_{\text {nano }}$ and $\rho_{\text {nano }}$ of both nano-endmembers that are derived applying a set of mathematical relationships given by Hiemstra. ${ }^{77}$ Next, the corresponding SSA of the soil metal (hydr) oxide fraction follows from:

$S S A=\frac{6}{\rho_{\text {soil (hydr)oxide }} d}$

and the value obtained can be translated to a corresponding RSA of soil according to:

$R S A=m_{\text {soil (hydr)oxide }} S S A$

Eq. 3

in which the mass of the soil metal (hydr) oxide fraction is calculated from the metal ion concentrations measured in the $\mathrm{AO}$ extract using as molar masses ( $\left.M_{\text {nano }}\right)$ the values of the corresponding metal (hydr)oxide endmembers (Eq. 1). By adapting the mean particle diameter $(d)$, the calculated value of RSA can be brought in line with the experimental value obtained by the probe-ion method. The details of these calculations are described in Mendez et al. ${ }^{32}$, and the final results for each soil are given in Table S1.

Using the above-given set of equations, one can calculate the average particle diameter of the metal (hydr)oxide fraction. Our tropical topsoils have a high fraction of $\mathrm{Al}$ in the $\mathrm{AO}$ extracts ( $67 \pm 8$ molar \%, as derived from Table 2). Excluding soil 3 , the mean particle diameter of the metal oxides is $d \sim 2.3 \mathrm{~nm}$. This particle size is similar to the particle size of $\mathrm{Fh}$ freshly prepared in the laboratory ${ }^{78}$. Such $\mathrm{Fh}$ particles have an SSA of only $A \sim 700 \mathrm{~m}^{2} \mathrm{~g}^{-1}$, while we calculate for $\mathrm{Al}(\mathrm{OH})_{3} . n \mathrm{H}_{2} \mathrm{O}$ nanoparticles with the same mean size, a significantly higher SSA of $\sim 1200 \mathrm{~m}^{2} \mathrm{~g}^{-1}$. The reason for the much higher value of SSA is the lower mass density of $\mathrm{Al}(\mathrm{OH})_{3} . n \mathrm{H}_{2} \mathrm{O}$ nanoparticles. In the lattice of the Al hydroxide, the oxygen charge is compensated by light $(\mathrm{Al})$ and very light $(\mathrm{H})$ cations, whereas in $\mathrm{Fh}$, the dominant 
cation $(\mathrm{Fe})$ is much heavier, which creates a large difference in mass density ${ }^{32}$ and consequently in SSA (Eq. 3).

It is essential to acknowledge the differences in the size-dependent values of $M_{\text {nano }}$ and $\rho_{\text {nano }}$ between the $\mathrm{Fe}$ and $\mathrm{Al}$ (hydr)oxides, particularly for soils with such high molar Al/Fe ratios as our tropical topsoils. Neglecting this, and assuming instead fixed and equal $M_{\text {nano }}$ and $\rho_{\text {nano }}$ for both representative oxide phases, as the Fh core for instance, would lead to smaller (unrealistic) mean particle sizes and corresponding higher values of SSA (Figure S4).

For our tropical topsoils, the representative spherical particle size is in the range of $1.4-5.5 \mathrm{~nm}$ (Table S2). A similar range has been found for the topsoils from temperate regions studied previously, covering the range $1.5-5.1 \mathrm{~nm}^{32}$. At the same size, Al-dominated nanoparticles will contain much fewer metal ions than $\mathrm{Fe}$ dominated nanoparticles (Table S1). If both types of particles are loaded with $\mathrm{PO}_{4}$ at the same surface density $\left(\mu \mathrm{mol} / \mathrm{m}^{2}\right)$, the Al-dominated particles will have a much higher $\mathrm{PO}_{4}$ adsorption capacity when the value is expressed per mol metal ion. Such a difference in adsorption capacity has been reported for nanocrystalline $\mathrm{Al}$ hydroxide compared to $\mathrm{Fh}^{79,80}$ and differences in mass density may play an important role in that. So, tropical soils may have a higher phosphate adsorption capacity at the same particle size compared to soils that are Fe-oxide dominated.

For the tropical soils, Fh has been used as a proxy whereas the reactive oxide fraction is Al-dominated. This may influence our results. Data collection for pure materials shows that the adsorption isotherms of $\mathrm{PO}_{4}$ for $\mathrm{Fh}$ and $\mathrm{Al}$ hydroxide have a similar shape ${ }^{79,80}$. It suggests similarity in affinity. If this similarity in affinity and shape of the isotherm also exists for the $\mathrm{PO}_{4}$ adsorption in $0.5 \mathrm{M} \mathrm{NaHCO}_{3}$, the outcome of our modeling may be rather realistic. The good agreement between R$\mathrm{PO}_{4}$ and $\mathrm{AO}-\mathrm{PO}_{4}$ (Figure 2a) supports this.

\section{CONCLUSIONS}

In this contribution, we have presented new insights into the surface reactivity of the metal (hydr)oxides in weathered tropical soils.

In our approach, we use a probe-ion method that is based on measuring the competitive adsorption of $\mathrm{PO}_{4}-\mathrm{CO}_{3}$ in $0.5 \mathrm{M} \mathrm{NaHCO}_{3}$ with a succeeding $\mathrm{CD}$ model interpretation. The probe ion method has been developed and tested previously for P-rich topsoils from temperate climates. Here, we show that this method can also be successfully applied without major methodological adaptations to sub-Saharan African topsoils that are low in $\mathrm{P}$ and relatively rich in metal (hydr)oxide. The surface reactivity of the oxide fraction of these soils is best explained by using Fh rather than goethite as a proxy. The same conclusion was drawn for agricultural topsoils from a 
temperate region analyzed in our previous study ${ }^{32}$. Considering that the soils from temperate and tropical climates have contrasting chemical and mineralogical properties, this finding is remarkable. The sub-Saharan African soils are relatively rich in crystalline $\mathrm{Fe}$ and $\mathrm{Al}$ (hydr)oxides while the amount of nanocrystalline metal (hydr)oxide (i.e. Fh-like materials) is relatively small. On a molar basis, only $\sim 25 \pm$ $10 \%$ of the oxide is present as nanoparticles which is $\sim 60 \pm 15 \%$ for the topsoils from a temperate climate. Despite the relatively small amount of nano-sized material, this fraction largely controls the reactivity of the pedogenic metal (hydr)oxides, even if present at low molar concentrations. This is likely due to a large difference in SSA of nanocrystalline metal (hydr) oxide fraction and the fraction of well-crystallized oxides.

According to our data and modeling, a significant amount of $\mathrm{PO}_{4}$ does not take part in equilibrium reactions. On average about $\sim 60 \%$ of total $\mathrm{PO}_{4}$ is occluded in the crystalline oxide fraction and can be released at a chemical reduction during a DC extraction. During the AO extraction, a considerable amount of $P$ is released as organic $P$. In our tropical soils, this amount can be as high as $\sim 60 \%$. The contribution of $\mathrm{P}_{\text {org }}$ is relatively high because of the rather low surface loading with inorganic $\mathrm{PO}_{4}$. At increase of fertilization, the relative amount of $P_{\text {org }}$ will decrease.

Scaling of the RSA to the content of AO-extractable Fe and Al (hydr)oxides reveals a large variation in specific surface area (SSA) of the reactive metal (hydr)oxide fraction. In our tropical soils, the SSA is in the range of $\sim 400-1750 \mathrm{~m}^{2} \mathrm{~g}^{-1}$. The corresponding mean particle size is between $\sim 1.4-5.5 \mathrm{~nm}$. It illustrates that nanosized particles dominate the reactive fraction of metal (hydr)oxides in the studied set of tropical topsoils.

The nano oxide fraction of the tropical soils is dominated by Al. Nevertheless, we found that Fh can be used well as a proxy for describing the surface reactivity of phosphate. This success suggests that, within limits, soil metal (hydr)oxides have rather similar adsorption behavior. The present probe-ion method can be considered as a state-of-the-art approach and is a large improvement compared to traditional methods of quantifying the RSA of soils. It will be interesting for future research to compare the $\mathrm{PO}_{4}$ adsorption behavior to $\mathrm{Fe}$ and $\mathrm{Al}$ (hydr)oxides in the presence of $0.5 \mathrm{M} \mathrm{NaHCO}_{3}$, and how possible differences can be used to improve the probe-ion method.

\section{ACKNOWLEDGEMENTS}

This work was supported by NWO (grant number 14688, "Micronutrients for better yields") and by the University of Costa Rica. We highly appreciate the work of Koen Dijkstra in collecting part of the experimental data. 


\section{SUPPORTING INFORMATION}

\section{A. Orthophosphate vs total phosphorus in the ammonium oxalate soil} extracts

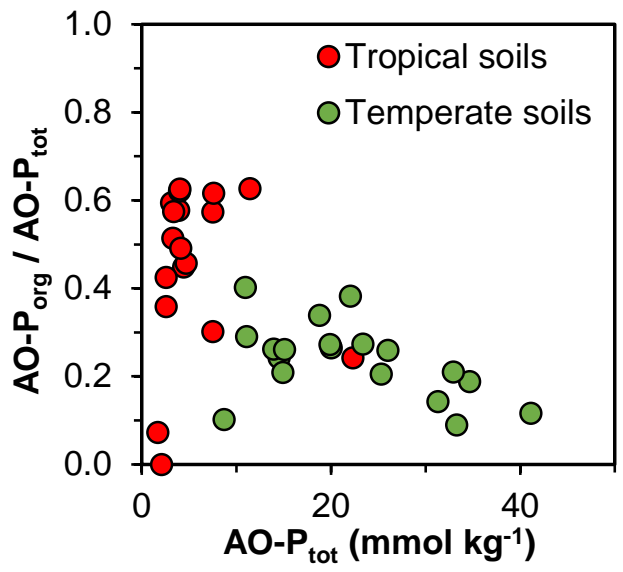

Figure S1: Relationship between the total $\mathrm{P}$ pool $\left(\mathrm{AO}-\mathrm{P}_{\text {tot }}\right)$ that is extracted by an acid ammonium oxalate solution $(\mathrm{pH}=3)$ and the soil organic phosphorus $\left(\mathrm{P}_{\text {org }}\right)$ expressed as fraction of the total $\mathrm{P}$ pool. The $\mathrm{P}_{\text {org }}$ pool is estimated as the difference between AO- $\mathrm{P}_{\text {tot }}$ and $\mathrm{AO}-\mathrm{PO}_{4}$. The red symbols are for the samples collected from agricultural tropical topsoils from sub-Saharan countries. The green symbols are for a series of agricultural topsoils from a temperate region analyzed previously ${ }^{32,69}$ and this information is shown here for comparison.

\section{B. $\mathrm{PO}_{4}$ adsorption isotherms of goethite and ferrihydrite}

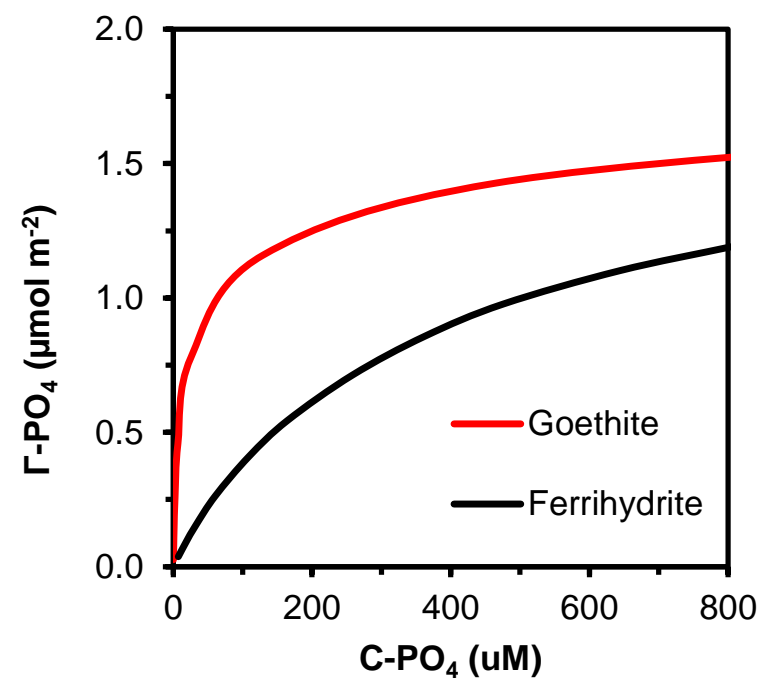

Figure S2: The modeled $\mathrm{PO}_{4}$ adsorption isotherms to goethite and ferrihydrite in systems with $0.5 \mathrm{M}$ $\mathrm{NaHCO}_{3}$ at $\mathrm{pH}$ 8.7. The combination of SSAA $\left(\mathrm{m}^{2} \mathrm{~g}^{-1}\right)$ and solution-to-solid ratio $\left(\mathrm{kg} \mathrm{L}^{-1}\right)$ leads to a total amount of reactive surface area of $375 \mathrm{~m}^{2} \mathrm{~L}^{-1}$ for both cases. The CD model calculations were performed with the parameters for Fh from Mendez et al. ${ }^{49}$, and for goethite from Rahmenaie et al. ${ }^{50}$ 


\section{Comparison between calculated and modeled reactive surface area}

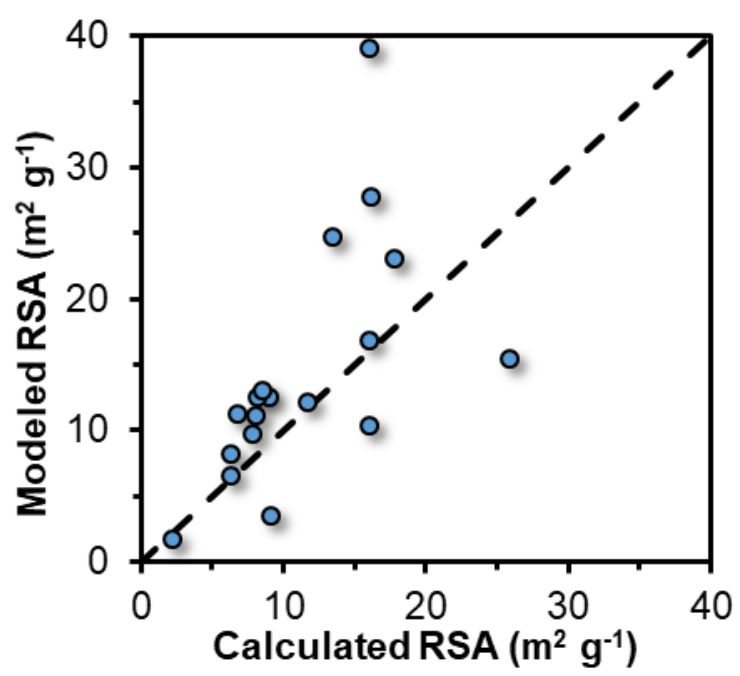

Figure S3: The comparison between the calculated reactive surface area (RSA) and the RSA obtained from the probe-ion method and subsequent $C D$ modeling. Commonly, in modeling studies applied to soil samples, the RSA is estimated based on the Fe and Al measurements by selective dissolution extractions, and an assumed 'standard' value for the molar mass and specific surface area (SSA). The calculated RSA was based on these assumptions, namely that the metal (hydr)oxide fraction based on the AOextractable Fe and Al have a molar mass of $89 \mathrm{~g} \mathrm{~mol}^{-1}$, and a specific surface area of $600 \mathrm{~m}^{2} \mathrm{~g}^{-1}$, and that the crystalline oxide fraction (i.e. estimated by the difference in $\mathrm{Fe}$ and $\mathrm{Al}$ in the dithionite and the $\mathrm{AO}$ extract) has a molar mass of 89 and $78 \mathrm{~g} \mathrm{~mol}^{-1} \mathrm{Fe}$ and Al respectively, and a specific surface area of 100 $\mathrm{m}^{2} \mathrm{~g}^{-1} \cdot{ }^{75}$ 


\section{Size-dependent properties of the reactive metal (hydr)oxides}

Table S1: Size-dependent properties of the reactive metal (hydr)oxides. The approach described by Mendez et al. ${ }^{32}$ was used for scaling the modeled values of RSA to the amount of Fe and Al extracted by ammonium oxalate. The approach is based on iterative calculations, in which an initial estimate of particle diameter $d$ is used to calculate the set of specific surfaces areas $(A)$, the molar mass $\left(M_{\text {nano }}\right)$ and the density $\left(\rho_{\text {nano }}\right)$ for the Fe and Al metal (hydr)oxides in soils. For calculating the total RSA, the values for the SSA of both Fe and Al (hydr)oxide particles are mass-weighted summed, based on the contents of $\mathrm{Fe}$ and $\mathrm{Al}$ measured in the ammonium oxalate extractions. The calculations are repeated until the calculated RSA is equal to the RSA derived by the probe-ion method.

\begin{tabular}{ccccccccccc} 
Soil & $\begin{array}{c}\boldsymbol{d} \\
(\mathbf{n m})\end{array}$ & \multicolumn{2}{c}{$\boldsymbol{M}_{\text {nano }}\left(\mathbf{g} \mathbf{~ m o l}^{-1}\right)$} & \multicolumn{2}{c}{$\boldsymbol{\rho}_{\text {nano }}\left(\mathbf{g ~ m}^{-3}\right)$} & \multicolumn{2}{c}{$\boldsymbol{A}\left(\mathbf{m}^{2} \mathbf{~ g}^{-1}\right)$} & \multicolumn{2}{c}{$\mathbf{S S A}$} & \multicolumn{2}{c}{$\mathbf{n}$ atoms } \\
& & $\mathbf{F e}$ & $\mathbf{A l}$ & $\mathbf{F e}$ & $\mathbf{A l}$ & $\mathbf{F e}$ & $\mathbf{A l}$ & $\left.\mathbf{m}^{2} \mathbf{g}^{-1}\right)$ & $\mathbf{F e}$ & $\mathbf{A l}$ \\
\hline 1 & 1.76 & 106.15 & 94.55 & $3.35 \mathrm{E}+06$ & $2.24 \mathrm{E}+06$ & 1018 & 1519 & 1345 & 54 & 41 \\
2 & 2.30 & 97.24 & 89.79 & $3.69 \mathrm{E}+06$ & $2.29 \mathrm{E}+06$ & 707 & 1140 & 1006 & 146 & 98 \\
3 & 5.46 & 86.66 & 82.42 & $4.31 \mathrm{E}+06$ & $2.36 \mathrm{E}+06$ & 255 & 465 & 393 & 2551 & 1469 \\
4 & 1.83 & 104.45 & 93.72 & $3.41 \mathrm{E}+06$ & $2.25 \mathrm{E}+06$ & 962 & 1456 & 1313 & 63 & 46 \\
5 & 2.85 & 93.04 & 87.13 & $3.89 \mathrm{E}+06$ & $2.31 \mathrm{E}+06$ & 540 & 910 & 795 & 307 & 194 \\
6 & 1.74 & 106.71 & 94.81 & $3.33 \mathrm{E}+06$ & $2.24 \mathrm{E}+06$ & 1035 & 1539 & 1413 & 52 & 39 \\
7 & 1.90 & 103.00 & 92.99 & $3.46 \mathrm{E}+06$ & $2.26 \mathrm{E}+06$ & 914 & 1399 & 1260 & 73 & 53 \\
8 & 1.58 & 111.73 & 97.04 & $3.19 \mathrm{E}+06$ & $2.22 \mathrm{E}+06$ & 1187 & 1703 & 1540 & 36 & 29 \\
9 & 2.72 & 93.83 & 87.66 & $3.85 \mathrm{E}+06$ & $2.31 \mathrm{E}+06$ & 572 & 956 & 777 & 261 & 167 \\
10 & 1.74 & 106.64 & 94.78 & $3.34 \mathrm{E}+06$ & $2.24 \mathrm{E}+06$ & 1033 & 1537 & 1383 & 52 & 39 \\
11 & 1.42 & 119.64 & 100.08 & $3.01 \mathrm{E}+06$ & $2.20 \mathrm{E}+06$ & 1400 & 1915 & 1751 & 23 & 20 \\
12 & 1.98 & 101.49 & 92.20 & $3.51 \mathrm{E}+06$ & $2.26 \mathrm{E}+06$ & 862 & 1337 & 1150 & 85 & 60 \\
13 & 1.82 & 104.71 & 93.85 & $3.40 \mathrm{E}+06$ & $2.25 \mathrm{E}+06$ & 971 & 1466 & 1269 & 62 & 46 \\
14 & 1.91 & 102.72 & 92.84 & $3.47 \mathrm{E}+06$ & $2.26 \mathrm{E}+06$ & 904 & 1388 & 1198 & 75 & 54 \\
15 & 3.70 & 89.71 & 84.78 & $4.09 \mathrm{E}+06$ & $2.34 \mathrm{E}+06$ & 396 & 694 & 593 & 728 & 440 \\
16 & 2.30 & 97.32 & 89.84 & $3.68 \mathrm{E}+06$ & $2.29 \mathrm{E}+06$ & 710 & 1144 & 917 & 144 & 97 \\
17 & 3.68 & 89.76 & 84.82 & $4.09 \mathrm{E}+06$ & $2.34 \mathrm{E}+06$ & 398 & 698 & 622 & 717 & 433 \\
18 & 3.77 & 89.53 & 84.65 & $4.11 \mathrm{E}+06$ & $2.34 \mathrm{E}+06$ & 388 & 682 & 546 & 772 & 465
\end{tabular}




\section{E. Simple approach for estimating $d$}

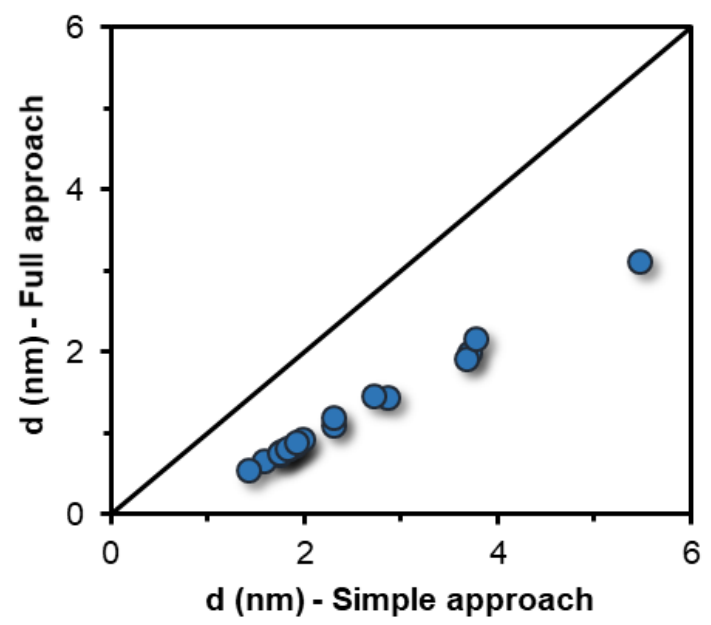

Figure S4: The comparison between the calculated particle diameter $d$ when using a simple and full approach. The full approach is based on the differences in size-dependent values of $M_{\text {nano }}$ and $\rho_{\text {nano }}$ between the $\mathrm{Fe}$ and $\mathrm{Al}$ (hydr)oxides, while the simple approach used a fixed value for $M_{\text {nano }}$ and $\rho_{\text {nano }}$ based on the core of a ferrihydrite particle (i.e. $81.65 \mathrm{~g} \mathrm{~mol}^{-1}$ and $\left.4.77 \mathrm{E}+06 \mathrm{~g} \mathrm{~m}^{-3}\right)^{32}$.

\section{REFERENCES}

1. Hiemstra, T., Antelo, J., van Rotterdam, A. M. D. \& van Riemsdijk, W. H. Nanoparticles in natural systems II: The natural oxide fraction at interaction with natural organic matter and phosphate. Geochim. Cosmochim. Acta 74, 59-69 (2010).

2. Verbeeck, M., Hiemstra, T., Thiry, Y. \& Smolders, E. Soil organic matter reduces the sorption of arsenate and phosphate: a soil profile study and geochemical modelling. Eur. J. Soil Sci. 68, 678-688 (2017).

3. Zhang, Y. et al. Modes of selenium occurrence and LCD modeling of selenite desorption/adsorption in soils around the selenium-rich core, Ziyang County, China. Environ. Sci. Pollut. Res. 25, 14521-14531 (2018).

4. Kaiser, K. \& Guggenberger, G. The role of DOM sorption to mineral surfaces in the preservation of organic matter in soils. in Organic Geochemistry vol. 31 711-725 (Pergamon, 2000).

5. Wiseman, C. L. S. S., Püttmann, W. \& Puttmann, W. Soil organic carbon and its sorptive preservation in central Germany. Eur. J. Soil Sci. 56, 65-76 (2005).

6. Kleber, M. et al. Mineral-Organic Associations: Formation, Properties, and Relevance in Soil Environments. Adv. Agron. 130, 1-140 (2015).

7. Lalonde, K., Mucci, A., Ouellet, A. \& Gélinas, Y. Preservation of organic matter in sediments promoted by iron. Nature 483, 198-200 (2012).

8. Chen, C. \& Thompson, A. Ferrous Iron Oxidation under Varying pO2 Levels: The Effect of $\mathrm{Fe}(\mathrm{III}) / \mathrm{Al}(\mathrm{III})$ Oxide Minerals and Organic Matter. Environ. Sci. Technol. 52, 597-606 (2018).

9. Perret, D., Gaillard, J. F., Dominik, J. \& Atteia, O. The diversity of natural hydrous iron oxides. Environ. Sci. Technol. 34, 3540-3546 (2000).

10. van der Zee, C., Roberts, D. R., Rancourt, D. G. \& Slomp, C. P. Nanogoethite is the dominant reactive oxyhydroxide phase in lake and marine sediments. Geology 31, 993-996 (2003).

11. Basile-Doelsch, I. et al. Mineralogical control of organic carbon dynamics in a volcanic ash soil on La Reunion. Eur. J. Soil Sci. 0, 050912034650042 (2005).

12. Mikutta, R. et al. Biogeochemistry of mineral-organic associations across a long-term mineralogical soil gradient (0.3-4100 kyr), Hawaiian Islands. Geochim. Cosmochim. Acta 73, 2034-2060 (2009).

13. Weng, L., Vega, F. A. \& Van Riemsdijk, W. H. Competitive and Synergistic Effects in pH Dependent Phosphate Adsorption in Soils: LCD Modeling. Environ. Sci. Technol. 45, 8420-8428 (2011). 
14. Eusterhues, K., Rumpel, C. \& Kögel-Knabner, I. Organo-mineral associations in sandy acid forest soils: Importance of specific surface area, iron oxides and micropores. Eur. J. Soil Sci. 56, 753-763 (2005).

15. Mayer, L. M. Surface area control of organic carbon accumulation in continental shelf sediments. Geochim. Cosmochim. Acta 58, 1271-1284 (1994).

16. Mendez, J. C. \& Hiemstra, T. Surface area of ferrihydrite consistently related to primary surface charge, ion pair formation, and specific ion adsorption. Chem. Geol. 532, (2020).

17. Brunauer, S., Emmett, P. H. \& Teller, E. Adsorption of Gases in Multimolecular Layers. J. Am. Chem. Soc. 60, 309-319 (1938).

18. Coward, E. K., Thompson, A. \& Plante, A. F. Contrasting Fe speciation in two humid forest soils: Insight into organomineral associations in redox-active environments. Geochim. Cosmochim. Acta 238, 68-84 (2018).

19. Eisazadeh, A., Kassim, K. A. \& Nur, H. Morphology and BET surface area of phosphoric acid stabilized tropical soils. Eng. Geol. 154, 36-41 (2013).

20. Fontes, M. P. F. \& Weed, S. B. Phosphate adsorption by clays from Brazilian Oxisols: relationships with specific surface area and mineralogy. Geoderma vol. 72 (1996).

21. Heister, K. The measurement of the specific surface area of soils by gas and polar liquid adsorption methods-Limitations and potentials. Geoderma 216, 75-87 (2014).

22. Dzombak, D. A. \& Morel, F. F. M. M. Surface Complexation Modeling: Hydrous Ferric Oxide. (John Wiley \& Sons, 1990).

23. Mödl, C., Wörmann, H. \& Amelung, W. Contrasting effects of different types of organic material on surface area and microaggregation of goethite. Geoderma 141,167-173 (2007).

24. Hiemstra, T., Antelo, J., Rahnemaie, R. \& van Riemsdijk, W. H. Nanoparticles in natural systems I: The effective reactive surface area of the natural oxide fraction in field samples. Geochim. Cosmochim. Acta 74, 41-58 (2010).

25. Cihacek, L. J. \& Bremner, J. M. A Simplified Ethylene Glycol Monoethyl Ether Procedure for Assessment of Soil Surface Area1. Soil Sci. Soc. Am. J. 43, 821 (1979).

26. Kennedy, M. J., Pevear, D. R. \& Hill, R. J. Mineral surface control of organic carbon in black shale. Science (80-. ). 295, 657-660 (2002).

27. Dong, W. \& Wan, J. Additive surface complexation modeling of uranium(VI) adsorption onto quartz-sand dominated sediments. Environ. Sci. Technol. 48, 6569-6577 (2014).

28. Dijkstra, J. J., Meeussen, J. C. L. \& Comans, R. N. J. Evaluation of a Generic Multisurface Sorption Model for Inorganic Soil Contaminants. Environ. Sci. Technol. 43, 6196-6201 (2009).

29. Groenenberg, J. E., Römkens, P. F. A. M., Zomeren, A. Van, Rodrigues, S. M. \& Comans, R. N. $\mathrm{J}$. Evaluation of the Single Dilute $(0.43 \mathrm{M})$ Nitric Acid Extraction to Determine Geochemically Reactive Elements in Soil. Environ. Sci. Technol. 51, 2246-2253 (2017).

30. Tiberg, C., Sjöstedt, C. \& Gustafsson, J. P. Metal sorption to Spodosol Bs horizons: Organic matter complexes predominate. Chemosphere 196, 556-565 (2018).

31. Weng, L., Temminghoff, E. J. M. \& Van Riemsdijk, W. H. Contribution of Individual Sorbents to the Control of Heavy Metal Activity in Sandy Soil. Environ. Sci. Technol. 35, 4436-4443 (2001).

32. Mendez, J. C., Hiemstra, T. \& Koopmans, G. F. Assessing the Reactive Surface Area of Soils and the Association of Soil Organic Carbon with Natural Oxide Nanoparticles Using Ferrihydrite as Proxy. Environ. Sci. Technol. 54, 11990-12000 (2020).

33. Hiemstra, T. \& Van Riemsdijk, W. H. A Surface Structural Approach to lon Adsorption: The Charge Distribution (CD) Model. J. Colloid Interface Sci. 179, 488-508 (1996).

34. Kleber, M., Schwendenmann, L., Veldkamp, E., Rößner, J. \& Jahn, R. Halloysite versus gibbsite: Silicon cycling as a pedogenetic process in two lowland neotropical rain forest soils of La Selva, Costa Rica. Geoderma 138, 1-11 (2007).

35. Xu, R. K., Qafoku, N. P., Van Ranst, E., Li, J. Y. \& Jiang, J. Adsorption Properties of Subtropical and Tropical Variable Charge Soils: Implications from Climate Change and Biochar Amendment. in Advances in Agronomy vol. 135 1-58 (2016).

36. Weihrauch, C. \& Opp, C. Ecologically relevant phosphorus pools in soils and their dynamics: The story so far. Geoderma 325, 183-194 (2018).

37. Yang, X. \& Post, W. M. Phosphorus transformations as a function of pedogenesis: A synthesis of soil phosphorus data using Hedley fractionation method. Biogeosciences 8, 2907-2916 (2011).

38. Hengl, T. et al. SoilGrids250m: Global Gridded Soil Information Based on Machine Learning. PLoS One (2016).

39. Nelson, D. W. \& Sommers, L. E. Total Carbon, Organic Carbon, and Organic Matter. in Methods of soil analysis Part 3-Chemical Methoods 961-1010 (1996). doi:10.2136/sssabookser5.3.c34. 
40. Houba, V. J. G. J. G., Temminghoff, E. J. M. J. M., Gaikhorst, G. A. A. \& van Vark, W. Soil analysis procedures using $0.01 \mathrm{M}$ calcium chloride as extraction reagent. Commun. Soil Sci. Plant Anal. 31, 1299-1396 (2000).

41. Murphy, J. \& Riley, J. P. A modified single solution method for the determination of phosphate in natural waters. Anal. Chim. Acta 27, 31-36 (1962).

42. Cui, Y. \& Weng, L. Arsenate and phosphate adsorption in relation to oxides composition in soils: LCD modeling. Environ. Sci. Technol. 47, 7269-7276 (2013).

43. Hass, A., Loeppert, R. H., Messina, M. G. \& Rogers, T. D. Determination of phosphate in selective extractions for soil iron oxides by the molybdenum blue method in an automated continuance flow injection system. Commun. Soil Sci. Plant Anal. 42, 1619-1635 (2011).

44. ISO. ISO 12782-3:2012 Soil quality -- Parameters for geochemical modelling of leaching and speciation of constituents in soils and materials -- Part 3: Extraction of aluminium oxides and hydroxides with ammonium oxalate/oxalic acid. (2012).

45. Schwertmann, U. Differenzierung der Eisenoxide des Bodens durch Extraktion mit Ammoniumoxalat-Lösung. Zeitschrift für Pflanzenernährung, Düngung, ... (1964).

46. ISO. ISO 12782-2:2012 - Soil quality -- Parameters for geochemical modelling of leaching and speciation of constituents in soils and materials -- Part 2: Extraction of crystalline iron oxides and hydroxides with dithionite. (2012).

47. Barrow, N. J. \& Shaw, T. C. Sodium bicarbonate as an extractant for soil phosphate, II. Effect of varying the conditions of extraction on the amount of phosphate initially displaced and on the secondary adsorption. Geoderma 16, 109-123 (1976).

48. Koopmans, G. F. et al. Use of iron oxide nanoparticles for immobilizing phosphorus in-situ: Increase in soil reactive surface area and effect on soluble phosphorus. Sci. Total Environ. 711, 135220 (2020).

49. Mendez, J. C. \& Hiemstra, T. Carbonate Adsorption to Ferrihydrite: Competitive Interaction with Phosphate for Use in Soil Systems. ACS Earth Sp. Chem. 3, 129-141 (2019).

50. Rahnemaie, R., Hiemstra, T. \& van Riemsdijk, W. H. Carbonate adsorption on goethite in competition with phosphate. J. Colloid Interface Sci. 315, 415-425 (2007).

51. Hiemstra, T. \& Zhao, W. Reactivity of ferrihydrite and ferritin in relation to surface structure, size, and nanoparticle formation studied for phosphate and arsenate. Environ. Sci. Nano 3, 12651279 (2016).

52. Keizer, M. G. \& Van Riemsdijk, W. H. ECOSAT, a Computer Program for the Calculation of Chemical Speciation and Transport in Soil-Water Systems. (1995).

53. Kinniburgh, D. G. FIT User Guide, Technical Report WD/93/23. (1993).

54. Borggaard, O. K. Dissolution of Poorly Crystalline Iron Oxides in Soils by EDTA and Oxalate. Zeitschrift für Pflanzenernährung und Bodenkd. 155, 431-436 (1992).

55. Schwertmann, U. Use of oxalate for the Fe extraction from soils. Can. J. Soil Sci. 53, 244-246 (1973).

56. Schwertmann, U., Schulze, D. G. \& Murad, E. Identification of Ferrihydrite in Soils by Dissolution Kinetics, Differential X-ray Diffraction, and Mössbauer Spectroscopy1. Soil Sci. Soc. Am. J. 46, 869-875 (1982).

57. Koopmans, G. F., Oenema, O. \& Van Riemsdijk, W. H. Characterization, desorption, and mining of phosphorus in noncalcareous sandy soils. (2004).

58. Maguire, R. O., Foy, R. H., Bailey, J. S. \& Sims, J. T. Estimation of the phosphorus sorption capacity of acidic soils in Ireland. Eur. J. Soil Sci. 52, 479-487 (2001).

59. van der Zee, S. E. A. T. M. \& van Riemsdijk, W. H. Model for Long-term Phosphate Reaction Kinetics in Soil. J. Environ. Qual. 17, 35-41 (1988).

60. Lookman, R., Freese, D., Merckx, R., Vlassak, K. \& Van Riemsdijk, W. H. Long-Term Kinetics of Phosphate Release from Soil. Environ. Sci. Technol. 29, 1569-1575 (1995).

61. Worsfold, P., McKelvie, I. \& Monbet, P. Determination of phosphorus in natural waters: A historical review. Analytica Chimica Acta vol. 918 8-20 (2016).

62. Wolf, A. M. \& Baker, D. E. Colorimetric Method for Phosphorus Measurement in Ammonium Oxalate Soil Extracts. Commun. Soil Sci. Plant Anal. 21, 2257-2263 (1990).

63. Jørgensen, C., Turner, B. L. \& Reitzel, K. Identification of inositol hexakisphosphate binding sites in soils by selective extraction and solution 31P NMR spectroscopy. Geoderma 257-258, 22-28 (2015).

64. Aguilera, N. H. \& Jackson, M. L. Iron Oxide Removal from Soils and Clays. Soil Sci. Soc. Am. J. 17, 359-364 (1953).

65. Mehra, O. P. \& Jackson, M. L. Iron Oxide Removal from Soils and Clays by a Dithionite-Citrate System Buffered with Sodium Bicarbonate. Clays Clay Miner. 7, 317-327 (1958). 
66. Gálvez, N., Barrón, V. \& Torrent, J. Effect of phosphate on the crystallization of hematite, goethite, and lepidocrocite from ferrihydrite. Clays Clay Miner. 47, 304-311 (1999).

67. Turner, B. L. Organic phosphorus in Madagascan rice soils. Geoderma 136, 279-288 (2006).

68. Nziguheba, G. \& Bünemann, E. K. Organic phosphorus dynamics in tropical agroecosystems. in Organic phosphorus in the environment 243-268 (CABI, 2004). doi:10.1079/9780851998220.0243.

69. Hiemstra, T., Antelo, J., Rahnemaie, R. \& van Riemsdijk, W. H. Nanoparticles in natural systems I: The effective reactive surface area of the natural oxide fraction in field samples. Geochim. Cosmochim. Acta 74, 41-58 (2010).

70. Warrinnier, R. et al. Investigation on the control of phosphate leaching by sorption and colloidal transport: Column studies and multi-surface complexation modelling. Appl. Geochemistry 100, 371-379 (2019).

71. Frossard, E. \& Sinaj, S. The isotope exchange kinetic technique: A method to describe the availability of inorganic nutrients. Applications to K, P, S and Zn. Isotopes Environ. Health Stud. 33, 61-77 (1997).

72. Jansen, B., Tonneijck, F. H. \& Verstraten, J. M. Selective Extraction Methods for Aluminium, Iron and Organic Carbon from Montane Volcanic Ash Soils. Pedosphere 21, 549-565 (2011).

73. Bonten, L. T. C., Groenenberg, J. E., Weng, L. \& van Riemsdijk, W. H. Use of speciation and complexation models to estimate heavy metal sorption in soils. Geoderma 146, 303-310 (2008).

74. Dijkstra, J. J., Meeussen, J. C. L. \& Comans, R. N. J. Leaching of heavy metals from contaminated soils: An experimental and modeling study. Environ. Sci. Technol. 38, 4390-4395 (2004).

75. Groenenberg, J. E., Dijkstra, J. J., Bonten, L. T. C., De Vries, W. \& Comans, R. N. J. Evaluation of the performance and limitations of empirical partition-relations and process based multisurface models to predict trace element solubility in soils. Environ. Pollut. 166, 98-107 (2012).

76. Hiemstra, T. \& Van Riemsdijk, W. H. A surface structural model for ferrihydrite I: Sites related to primary charge, molar mass, and mass density. Geochim. Cosmochim. Acta 73, 4423-4436 (2009).

77. Hiemstra, T. Ferrihydrite interaction with silicate and competing oxyanions: Geometry and hydrogen bonding of surface species. Geochim. Cosmochim. Acta 238, $453-476$ (2018).

78. Hiemstra, T., Mendez, J. C. \& Li, J. Evolution of the reactive surface area of ferrihydrite: Time, $\mathrm{pH}$, and temperature dependency of growth by Ostwald ripening. Environ. Sci. Nano 6, 820-833 (2019).

79. Liu, Y. T. \& Hesterberg, D. Phosphate bonding on noncrystalline Al/Fe-hydroxide coprecipitates. Environ. Sci. Technol. 45, 6283-6289 (2011).

80. Tiberg, C., Sjöstedt, C., Eriksson, A. K., Klysubun, W. \& Gustafsson, J. P. Phosphate competition with arsenate on poorly crystalline iron and aluminum (hydr)oxide mixtures. Chemosphere 255, 126937 (2020). 
Chapter 2 


\section{Chapter 3}

The role of metal (hydr)oxides in controlling organic carbon storage of soils

Elise Van Eynde*, Juan C. Mendez*, Tjisse Hiemstra, and Rob N.J. Comans

* These authors contributed equally 


\section{ABSTRACT}

Soil organic carbon (SOC) is the largest terrestrial carbon pool that is in exchange with the atmosphere, thereby playing a crucial role in the global carbon cycle and strategies for mitigation of climate change. SOC interacts with mineral surfaces forming intimate associations. For the ability of carbon storage, insight is required into the reactive surface area (RSA) of soils. Here, we show that in soils, SOC is not only well related to the RSA but also to the mean particle size of the natural oxide fraction. With a core-shell approach, we found that the mean thickness of the SOC layer $(L)$ around the metal oxide core is linearly related to the mean size $(d)$ of the oxide nanoparticles involved. Excellent relationships between $L$ and $d$ are found when studying soils with very contrasting chemical properties, pedogenesis, climate conditions, soil depth, and land use $\left(\mathrm{R}^{2}>0.97\right)$. According to our study, soil organic carbon is predominantly stored in primary organo-oxide aggregates that are additionally organized by association with larger mineral particles. Our study quantifies the ability of soils to store long-term SOC. 


\section{INTRODUCTION}

Soil organic carbon $(\mathrm{SOC})$ represents the largest terrestrial carbon pool that is in exchange with the atmosphere, thereby playing a crucial role in the global carbon cycle and strategies for climate change mitigation ${ }^{1}$. At the global scale, SOC content is mainly controlled by climatic variables such as temperature and precipitation, due to their effects on soil carbon inputs (i.e. primary productivity) and outputs (i.e. decomposition rates) $)^{2,3}$. At regional scales $^{4}$, the SOC content in mineral soils is mainly governed by processes of stabilization through interactions with the soil mineral matrix ${ }^{5}$.

The capacity of soil to store SOC is currently implemented in global C models using solely the fraction of clay-sized particles (i.e. soil particles $<2 \mu \mathrm{m}$ diameter) ${ }^{6,7}$ as abiotic soil variable ${ }^{8}$. This soil fraction has a variable composition among soil types and climates, and it can contain a wide variety of minerals with different chemical compositions, particle sizes, and surface reactivities. However, the importance of $\mathrm{Fe}$ - and Al-(hydr)oxides for SOC stabilization by these clay-sized fractions has been suggested by numerous authors in studies ${ }^{9-14}$ as a positive relationship between $\mathrm{SOC}$ and the $\mathrm{Fe}$ - and $\mathrm{Al}$-(hydr)oxide content was found. In particular, the fraction of nanocrystalline $\mathrm{Fe}$ - and $\mathrm{Al}$-(hydr)oxides has become a major focal point in recent studies of SOC stabilization ${ }^{12,14,15}$.

Despite the recognized importance of natural metal-(hydr)oxide nanoparticles for stabilizing SOC in soils from different climatic and pedogenic conditions ${ }^{12,14,15}$, it remains unclear which features of these nanoparticles determine the actual capacity of a soil to store SOC. The Fe- and Al-(hydr)oxide nanoparticles have a high affinity for interacting with $\mathrm{SOC}^{15}$ forming stable organo-mineral associations at a nanometer scale ${ }^{16}$ via adsorption ${ }^{17}$ or co-precipitation processes ${ }^{18,19}$. Due to their ultra-small size, being typically $<\sim 2-5 \mathrm{~nm}$, metal (hydr)oxide nanoparticles can create a large reactive surface area (RSA) and ion adsorption capacity ${ }^{20}$, but its ultimate role in stabilizing SOC is subject of debate ${ }^{21-24}$.

Besides RSA, the mean particle size ( $d$ ) of the $\mathrm{Fe}$ - and $\mathrm{Al}$-(hydr)oxide fraction has also given a role in determining the SOC content of soils ${ }^{25}$. Here, we show for soils with contrasting chemical, pedogenic, and climatic conditions that their capacity to store SOC can be understood from the combination of RSA and mean particle size of the metal (hydr) oxide fraction. For this, we analyzed different soil series that include temperate and tropical arable topsoils taken from one European and two African countries, next to a series of soil samples from soil profiles in forest and arable land, located in the Netherlands. Based on our experimental data and advanced geochemical modeling, we propose a conceptual model for the structural arrangement at the nanoscale of the organo-oxide associations for individual and aggregated particles, which explains the variation in SOC content in a wide range of soils from different climates and soil depths. 


\section{MATERIAL AND METHODS}

\subsection{Soil samples}

Soil samples from different geographical regions and climatic zones were used in this study. A first set of samples $(n=18)$ was selected from a larger series of representative agricultural topsoils from the Netherlands, referred to as the Copernicus series, with the exception of a peat soil (sample 11 in Table S1). ${ }^{26}$ The general chemical properties and the surface reactivity of the natural metal (hydr)oxide fraction of these soil samples have been analysed in previous studies 25,27 and the results are shown in Table S1.

A second set of samples represents agricultural topsoils from Sub-Saharan African countries, namely Burundi $(n=15)$ and Kenya $(n=3)$. According to the soil grid system using the World Reference Base soil classification system ${ }^{28}$, the majority of these soils are classified as Acrisol and Ferralsol. The general chemical properties and the characteristics of the natural metal (hydr)oxides fraction of these African tropical soils are presented in Table S2.

A third set of samples $(n=15)$ consists of samples taken from soil 5 profiles in the Netherlands. The soil profiles were located under different land use systems, namely forest (Regentesselaan (Reg), Everzwijnsgoed (Ever) and De Steeg (Steeg)), and arable land (Groesbeek (Groes), Stockum (Stock)). The general chemical properties and the characteristics of the natural metal (hydr)oxides fraction of these Dutch soil profiles are presented in Table S3.

\subsection{Chemical analyses for soil characterization}

All soil samples were analyzed for their oxide content, the total soil organic carbon (SOC), clay content and soil $\mathrm{pH}\left(\mathrm{CaCl}_{2}\right)$. The experimental procedures for characterizing the soil samples from the Copernicus and the African topsoil series have been described in detail in previous publications ${ }^{25,27,29}$ and the results are summarized in Tables S1-S3. The soil characteristics of the samples taken within Dutch soil profiles were analyzed according to the same methods as for the other Dutch topsoils, and the results are shown in Table S3. We note that the equilibration time of the ammonium oxalate extraction differ between the African topsoils and the Dutch topsoils and soil profile samples, which may have implications on the conclusions drawn in this study, with regard to the relationship between particle diameter and organic matter storage. However, in section 4 of the supplementary information, it is shown that the relations exist and remain the same, irrespective of equilibration time applied for the ammonium oxalate soil extraction.

\subsection{Assessment of reactive surface area of the metal (hydr)oxide fraction}

The reactive surface area (RSA) of the metal (hydr)oxide fraction was determined by applying the probe-ion method proposed by Hiemstra et al. ${ }^{27}$. In this method, soil samples are extracted with $0.5 \mathrm{M} \mathrm{NaHCO}_{3}(\mathrm{pH} 8.5)$ to promote phosphate $\left(\mathrm{PO}_{4}\right)$ 
desorption from the soil mineral surfaces. The charge distribution (CD) model ${ }^{30}$ was used to calculate the reactive surface area (RSA in $\mathrm{m}^{2} \mathrm{~g}^{-1}$ soil) of each soil sample, based on the experimental $\mathrm{PO}_{4}$ concentrations measured in the $0.5 \mathrm{M} \mathrm{NaHCO}_{3}$ extraction solution at different solution-to-solid ratios. A detailed modeling description is found elsewhere ${ }^{25,27}$. We have shown recently that the reactivity of the natural oxide fraction in temperate ${ }^{25}$ and tropical ${ }^{29}$ soils can be described accurately using $\mathrm{Fh}$ as model oxide rather than goethite. Therefore, the CD model in combination with a structural multisite ion complexation model (MUSIC) for $\mathrm{Fh}^{31}$ was used to interpret the $\mathrm{PO}_{4}-\mathrm{CO}_{3}$ interaction using an internally consistent thermodynamic database calibrated previously in model systems with freshly prepared Fh nanoparticles ${ }^{32}$.

\subsection{Calculation of size-dependent properties of the metal (hydr)oxide nanoparticles}

Key properties of Fh nanoparticles, such as the specific surface area (SSA, $\mathrm{m}^{2} \mathrm{~g}^{-1}$ oxide), the molar mass $\left(M_{\text {nano }}, \mathrm{g} \mathrm{mol}^{-1}\right.$ metal ion), and the mass density $\left(\rho_{\text {nano }}, \mathrm{g} \mathrm{m}^{-}\right.$ $\left.{ }^{3}\right)$, are particle-size dependent. A consistent set of these variables can be derived iteratively by using a set of mathematical relationships ${ }^{25,33}$ and the $\mathrm{Fe}$ and $\mathrm{Al}$ extracted with $\mathrm{AO}$.

In these calculations, the natural oxide fraction is considered as a mixture of two phases, namely $\mathrm{Fh}\left(\mathrm{FeO}_{1.4} \mathrm{OH}_{0.2} \cdot \mathrm{nH}_{2} \mathrm{O}\right)$ and $\mathrm{Al}$-(hydr)oxide $\left(\mathrm{Al}(\mathrm{OH})_{3} \cdot \mathrm{nH}_{2} \mathrm{O}\right)$ nanoparticles with specific characteristics (Table 1).

Using an initial estimate of the particle diameter $(d)$ in the calculations as starting point, the mass density of both materials can be calculated as follows:

$$
\rho_{\text {nano }}=\left(\frac{M_{\text {core }}}{n_{O} V_{0}}\right)-\left(\frac{M_{\text {core }}}{n_{\mathrm{O}}}-M_{\mathrm{H}_{2} \mathrm{O}}\right) \times \frac{6}{d} N_{\mathrm{H}_{2} \mathrm{O}}
$$

in which $M_{\text {core }}$ is the molar mass of the mineral $\left(\mathrm{g} \mathrm{mol}^{-1}\right), n_{O}$ is the number of oxygen atoms expressed as the molar ratio of oxygen over metal ions in the mineral core, $V_{O}$ is the lattice volume $\left(\mathrm{m}^{3}\right)$ per moles of oxygen, and $N_{H 2 O}$ is the excess water density in moles per unit of surface area. The variable $M_{\text {H2O }}$ in Eq. 1 is the molar mass of water, i.e. $18 \mathrm{~g} \mathrm{~mol}^{-1}$.

Table 1: Parameters of the mineral core and the density of water $\left(N_{\mathrm{H} 2 \mathrm{O}}\right)$ of ferrihydrite (Fh) and $\mathrm{Al}$ (hydr)oxide nanoparticles that are used in the mathematical expressions for calculating the sizedependent particle properties (Eq. 1-3) ${ }^{25}$.

\begin{tabular}{lcc} 
& $\mathrm{Fh}$ & $\mathrm{Al}-\left(\right.$ hydr)oxide $\left(\mathrm{Al}(\mathrm{OH})_{3} . \mathrm{nH}_{2} \mathrm{O}\right)$ \\
\hline$M_{\text {core }}\left(\mathrm{g} \mathrm{mol}^{-1}\right.$ metal ion $)$ & $\left(\mathrm{FeO}_{1.4} \mathrm{OH}_{0.2} \cdot \mathrm{nH}_{2} \mathrm{O}\right)$ & 78.00 \\
$n_{\mathrm{O}}\left(\mathrm{mol} \mathrm{O} \mathrm{mol}^{-1}\right.$ metal ion $)$ & 81.65 & 3.0 \\
$V_{\mathrm{O}}\left(\mathrm{m}^{3} \mathrm{~mol}^{-1} \mathrm{O}\right)$ & 1.6 & $1.07 \times 10^{-5}$ \\
$N_{\text {H2O }}\left(\mathrm{mol} \mathrm{H}_{2} \mathrm{O} \mathrm{m}^{-2}\right)$ & $1.07 \times 10^{-5}$ & $6.3 \times 10^{-6}$ \\
$\rho_{\text {core }}\left(\mathrm{g} \mathrm{m}^{-3}\right)$ & $12.6 \times 10^{-6}$ & $2.42 \times 10^{6}$ \\
\hline
\end{tabular}


When the mass density is calculated with Eq. 1 based on an initial estimate of the particle diameter $d$, the molar mass (Eq. 2) and the corresponding particle SSA (Eq. 3) can be calculated for the Fh and nano-sized Al hydroxide with the following equations:

$$
\begin{gathered}
M_{\text {nano }}=\frac{M_{\text {core }}}{\left(1-\left(\frac{6}{\rho_{\text {nano } x d}}\right) \times N_{\mathrm{H}_{2} \mathrm{O}} \times M_{\mathrm{H}_{2} \mathrm{O}} \mathrm{O}\right.} \\
S S A_{p}=\frac{6}{\rho_{\text {nano } \mathrm{x}} \mathrm{d}}
\end{gathered}
$$

From Eq. 1-3, we obtain the density, molar mass and SSA for Fe and Al metal (hydr)oxides that correspond to a specific diameter.

Based on the above, we can estimate the overall SSA for the soil samples, i.e. SSA soil $_{\text {s. }}$ $\left(\mathrm{m}^{2} \mathrm{~g}^{-1}\right.$ oxides) as the weighted value of the two constituting nano-oxide phase.:

$$
S S A_{\text {soil }}=S S A_{p, F e} *\left(\frac{F e_{A O}}{F e_{A O}+A l_{A O}}\right)+S S A_{p, A l} *\left(\frac{A l_{A O}}{F e_{A O}+A l_{A O}}\right)
$$

The $\mathrm{Fe}_{\mathrm{AO}}$ and $\mathrm{Al}_{\mathrm{AO}}$ refer to the molar $\mathrm{Fe}$ and $\mathrm{Al}$ concentrations in the ammonium oxalate extractions, and the $\mathrm{SSA}_{\mathrm{p}}$ stands for the SSA for the Fe and Al oxide particle with a specific diameter as calculated with Eq. 3.

Secondly, the SSA soil can be obtained by scaling the effective RSA that is obtained by the probe-ion method to the mass of metal (hydr)oxide constituents, based on the $\mathrm{Fe}$ and $\mathrm{Al}$ oxide content and their corresponding size-dependent molar mass:

$$
S S A_{\text {soil }}=\frac{R S A}{\left(M_{\text {nano }, F e^{*}} F e_{A O}\right)+\left(M_{\text {nano }, A l} * A l_{A O}\right)}
$$

The calculations are done iteratively, and the particle diameter $d$ is changed until Eq. 4 and Eq. 5 give the same results.

\subsection{Core-shell model and volumetric ratio}

The core-shell model considers all SOC to be stored in a homogeneous layer surrounding a metal (hydr)oxide nanoparticle. ${ }^{25}$ To calculate the representative layer thickness $\mathrm{L}$, the volume of soil organic matter (SOM) that covers the oxide nanoparticles is calculated based on the size dimensions of the latter as the difference between a spheric particle with $d+2 L$ as diameter, and the volume of the oxide particle itself:

$$
V_{\text {SOM }}=V_{d / 2+L}-V_{d / 2}=4 / 3 \times \pi \times\left\{(d / 2+L)^{3}-(d / 2)^{3}\right\}
$$

From the volume SOM that is stored in the layer with thickness $L$, the corresponding mass $M_{S O M}$ can be calculated using a constant mass density ${ }^{27}$ of $1250 \mathrm{~kg} \mathrm{~m}^{-3}$ :

$$
M_{\text {SOM }}=\rho_{\text {SOM }} x V_{\text {SOM }}
$$

The layer thickness $L$ is changed until the $M_{S O M}$ equals the experimental SOM content, with a carbon content of $58 \%$. 
At a higher hierarchical level, the core-shell-like particles aggregate into larger entities. The amount of SOM on a volume basis in these organo-oxide associations is calculated as follows:

$$
V_{S O M}=\frac{\operatorname{SOC} \times 1.58}{\rho_{S O M}}
$$

In which SOC is the measured soil organic carbon and $\rho_{S O M}$ the SOM density as noted above.

The volume of the $\mathrm{Fe}$ and $\mathrm{Al}$ (hydr)oxide nanoparticles in these organo-oxide associations, can be calculated based on oxide mass in the soil ( $\left.m_{o x, s o i l}\right)$ and the overall size-dependent mass density of the oxide particle in the soil ( $\rho_{o x, \text { soii }}$ :

$$
V_{o x}=\frac{m_{o x, \text { soil }}}{\rho_{\text {ox }, \text { soil }}}=\frac{F e_{A O} M_{\text {nano }, F e}+A l_{A O} M_{\text {nano }, A l}}{\frac{F e_{A O}}{F e_{A O}+A l_{A O}} \rho_{\text {nano }, F e}+\frac{A l}{F e_{A O}+A l_{A O}} \rho_{\text {nano }, A l}}
$$

in which $\mathrm{Fe}_{\mathrm{AO}}$ and $\mathrm{Al}_{\mathrm{AO}}$ are the molar concentrations measured in the acid ammonium oxalate, $M_{\text {nano }}$ is the size-dependent molar mass of the metal oxide particle, calculated as written in Eq. 2, and $\rho_{\text {nano }}$ is the size-dependent mass density of the metal oxide particle, calculated as shown in Eq. 1.

The mass density of the formed organo-mineral association ( $\rho_{\text {som-ox }}$ can be estimated using the volume ( $V_{o x}$, Eq. 9$)$ and overall mass density of the natural metal (hydr)oxide particles that is calculated based on the $\rho_{\text {nano }}$ and the relative contribution of the $\mathrm{Fe}$ and $\mathrm{Al}$ (hydr)oxide particles, in combination with the volume ( $V_{S O M}$, Eq. 8) and the mass density of soil organic matter $\left(\rho_{S O M}=1250 \mathrm{~kg} \mathrm{~m}^{-3}\right)$ :

$$
\rho_{\text {SOM }-O X}=\rho_{\text {SOM }} *\left(\frac{V_{S O M}}{V_{S O M}+V_{O X}}\right)+\rho_{O X} *\left(\frac{V_{O X}}{V_{S O M}+V_{O X}}\right)
$$




\section{RESULTS AND DISCUSSION \\ 3.1 SOC and reactive surface area}

The RSA, expressed in $\mathrm{m}^{2} \mathrm{~g}^{-1}$ soil, was measured with a novel approach that uses native phosphate as probe-ion for the surface area of the occurring metal (hydr)oxides, in combination with state-of-the-art geochemical modeling ${ }^{20,25}$. In addition, SOC, extractable contents of metal (hydr)oxide as well as several general soil characteristics were measured (Tables S1-S3).

In soils, metal (hydr)oxides are the most reactive mineral phases for interaction with SOC as is frequently observed in studies using soil samples from a single climatic region and similar land use system $9,10,12,13,21$. For all our arable topsoils, a very good correlation is indeed found between the content of $\mathrm{Fe}$ and $\mathrm{Al}$ extractable with ammonium oxalate $(\mathrm{AO})$ and $\mathrm{SOC}$, irrespective of climate region and soil chemical properties. The relationships between SOC and the crystalline metal (hydr)oxide fraction (Figure S1) and total content of clay size particles $(<2 \mu \mathrm{m})$ (Figure S2) are much less pronounced.

The observed intimate relation between SOC and the fraction of nanocrystalline oxides (Figure 1, upper panels) suggests that SOC binds to its surfaces. However, the scaling of SOC to the RSA measured for our soils shows a much less pronounced relationship (Figure 1 lower panels). This suggests that, apart from RSA, another property of the oxide fraction is involved in this interplay. Moreover, the slopes of the relationships are different for the tropical and temperate soils, pointing to a contribution of another additional factor which might be for instance biologically driven. 


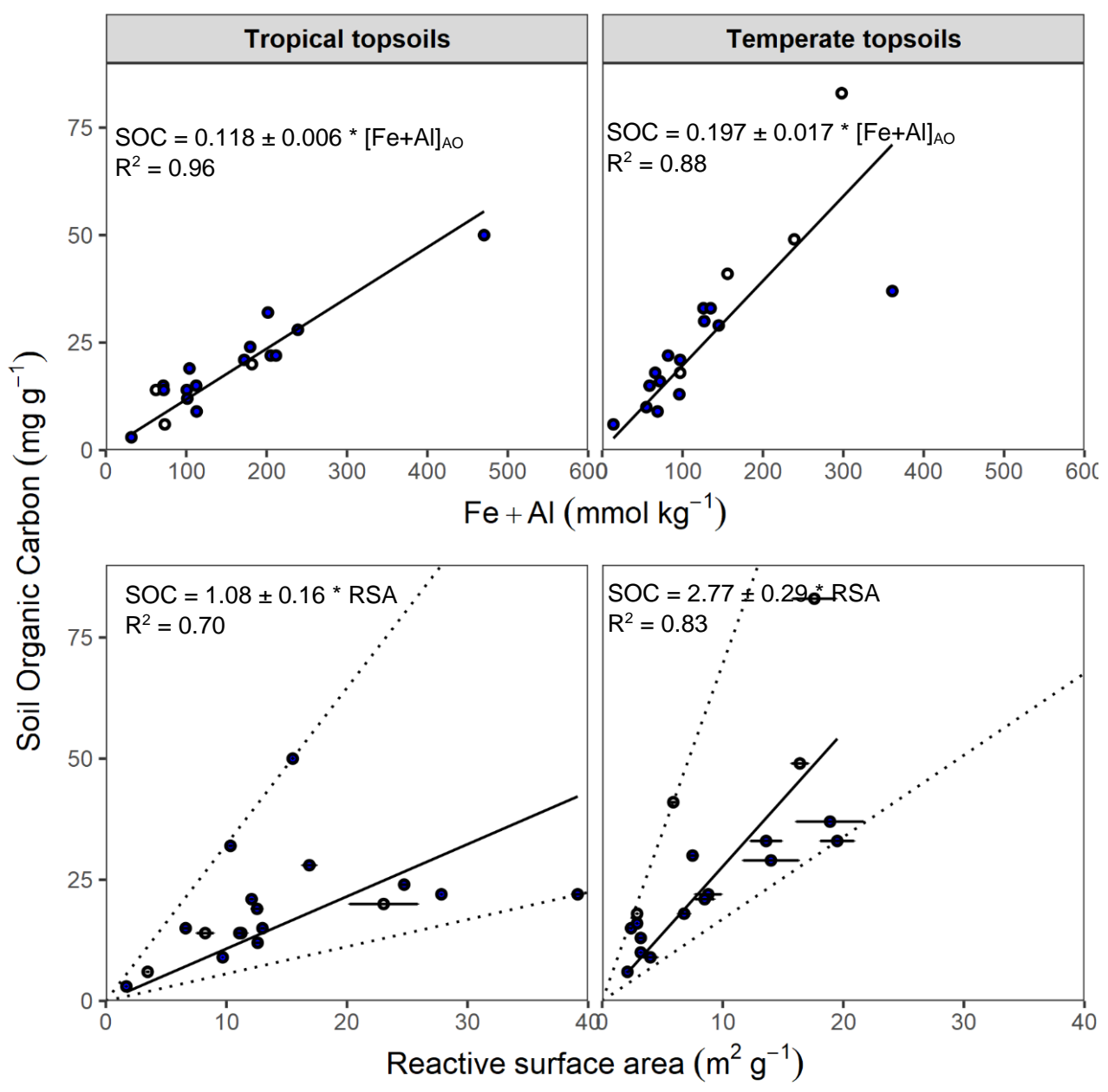

Figure 1: Relationship between the bulk soil organic carbon (SOC) and ammonium oxalate extractable $\mathrm{Fe}$ and $\mathrm{Al}$ content (upper panel) and the reactive surface area (lower panel) for the tropical topsoils (left side) and the temperate topsoils (right panels). All equations and corresponding $\mathrm{R}^{2}$ values were calculated for regression lines passing through the origin. The white markers show the soil samples with clay content $>20 \%$ to illustrate that clay does not play a role for SOC content ${ }^{14}$. In the lower panel, the error bars show the standard error in the reactive surface area that is obtained with the probe-ion method ${ }^{27}$, the dotted lines show the minimal and maximal slopes found for the relationship between the reactive surface area and the soil organic carbon.

\subsection{Organo-oxide nanoparticles}

The amount of SOC $\left(\mathrm{mg} \mathrm{C} \mathrm{g}^{-1}\right)$ can be expressed per unit surface area $\left(\mathrm{m}^{2} \mathrm{~g}^{-1}\right)$ using the above derived RSA. This quantity $\left(\mathrm{mg} \mathrm{C} \mathrm{m}^{-2}\right)$ is the slope of a line that can be drawn through any data point in Figure 1 (lower panels) and these slopes are highly variable. Analysis shows that the amount of available SOC per unit surface area is related to the mean diameter of the metal (hydr)oxide nanoparticle as is illustrated 
in Figure 2. It is found in both tropical and temperate soils. In our approach, the mean size of the oxide particles has been calculated based on the RSA in a consistent manner accounting for the size dependency of molar mass ( $\left.M_{\text {nano }}\right)$ and mass density ( $\left.\rho_{\text {nano }}\right)$, as described recently ${ }^{25,29}$. The observed relationship between mean particle size and SOC loading implies for soils with the same RSA but different amounts of metal (hydr)oxides, that soils with the higher metal (hydr)oxide content, and consequently larger oxide particles, will store more SOC per unit of surface area.

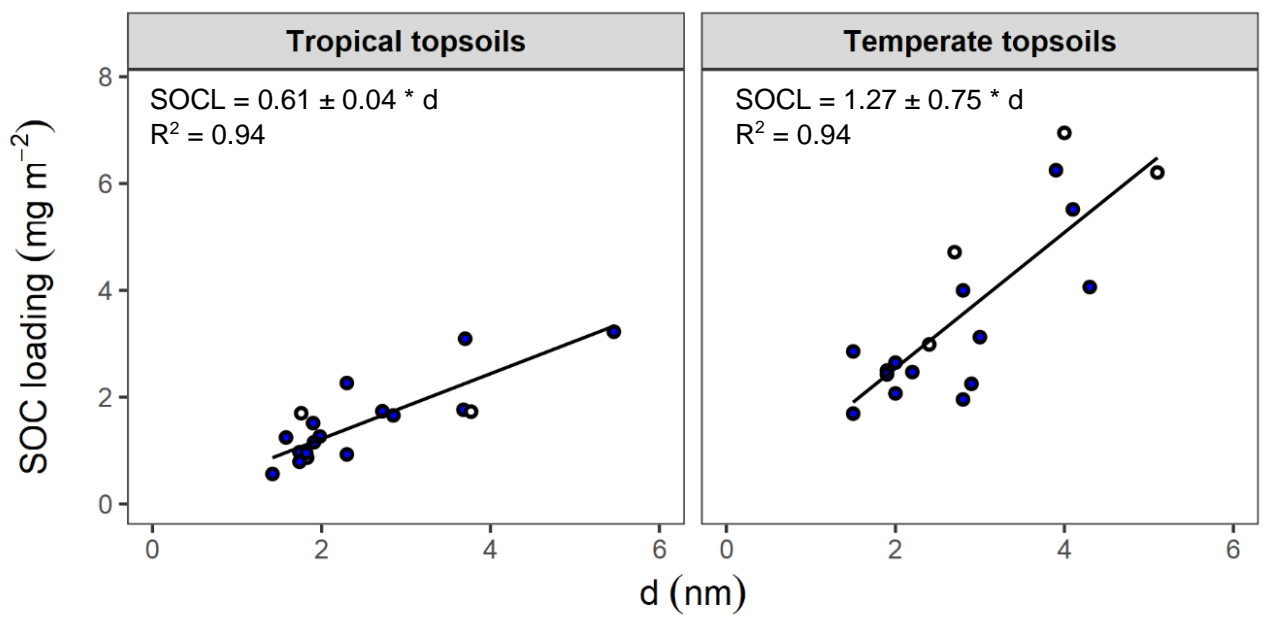

Figure 2: Relationship between the bulk soil carbon (SOC) loading, and the mean metal (hydr)oxide particle diameter. The organic matter loading per unit surface area is not constant but increases remarkably with the mean particle size. The surface loading differs by about a factor of 2 between the temperate and tropical soil series. This difference is likely due to a different steady state between the input (growth) and output (decomposition) of organic matter. The white markers show the soil samples with clay content $>20 \%$.

To explore the SOC-size relationship further, one can evaluate the structural arrangement of the organo-oxide associations conceptually with a core-shell model ${ }^{25}$, that is given as inset in Figure 3 (upper panel). In the approach, all soil organic matter (SOM) is accommodated in a layer with thickness $L$ around the metal (hydr)oxide particles. Experimental studies based on different fractionation schemes have indeed shown that SOM is predominantly found in association with minerals ${ }^{34}$, especially in humid regions with mild to warm temperatures ${ }^{4}$ and in deeper soil layers ${ }^{35}$. In the calculations, a mass density of $\rho_{\mathrm{som}}=1250 \mathrm{~kg} \mathrm{~m}^{-3}$ was used and a generic carbon content of $58 \%$ for $\mathrm{SOM}^{36}$.

As shown in Figure 3 (upper panels), we found for both data sets that the calculated thickness $(L)$ of the organic matter layer is linearly related to the mean particle diameter ( $d$ ) of the metal (hydr)oxide particles. It implies that larger particles are surrounded by a thicker layer of SOM. The approach shows that the SOM content of 
soils not only depends on the RSA but also on the mean particle diameter of the reactive metal (hydr)oxide fraction. In both soil series studied, the clay content is variable, but this does not affect the observed relationships when SOM is scaled with the core-shell model. The basic input variables for the applied core-shell model are only the metal (hydr)oxide content and RSA of soils. As shown here, the model can be applied successfully to soils from very different climate regions and pedogenic origins.

According to the core-shell model, most organic matter is not directly linked to the oxide surfaces. This follows from the upper panels of Figure 3 considering the thickness of the compact part of the electrical double layer around the metal (hydr)oxide particles (dotted line at $\sim 0.7 \mathrm{~nm}^{37}$ ) in relation to the equivalent layer thickness of SOM $(L)$ that varies between $\sim 1-4 \mathrm{~nm}$. On flat iron oxide surfaces, the maximum adsorption of SOM molecules is typically $\sim 1-3 \mathrm{mg} \mathrm{m}^{-2}$, which is equivalent to a layer thicknesses of $1-3 \mathrm{~nm}^{38}$. This range is well comparable with the layer thickness values found here for natural oxide nanoparticles in soils. These layer thickness values mean that on average $\sim 30 \%$ of the SOM lies outside the Stern layer. It implies that stabilization of SOC by association is likely not only due to protection by direct chemical bonding to the oxide surfaces, but also by protection as a result of aggregation into organo-mineral associations. 

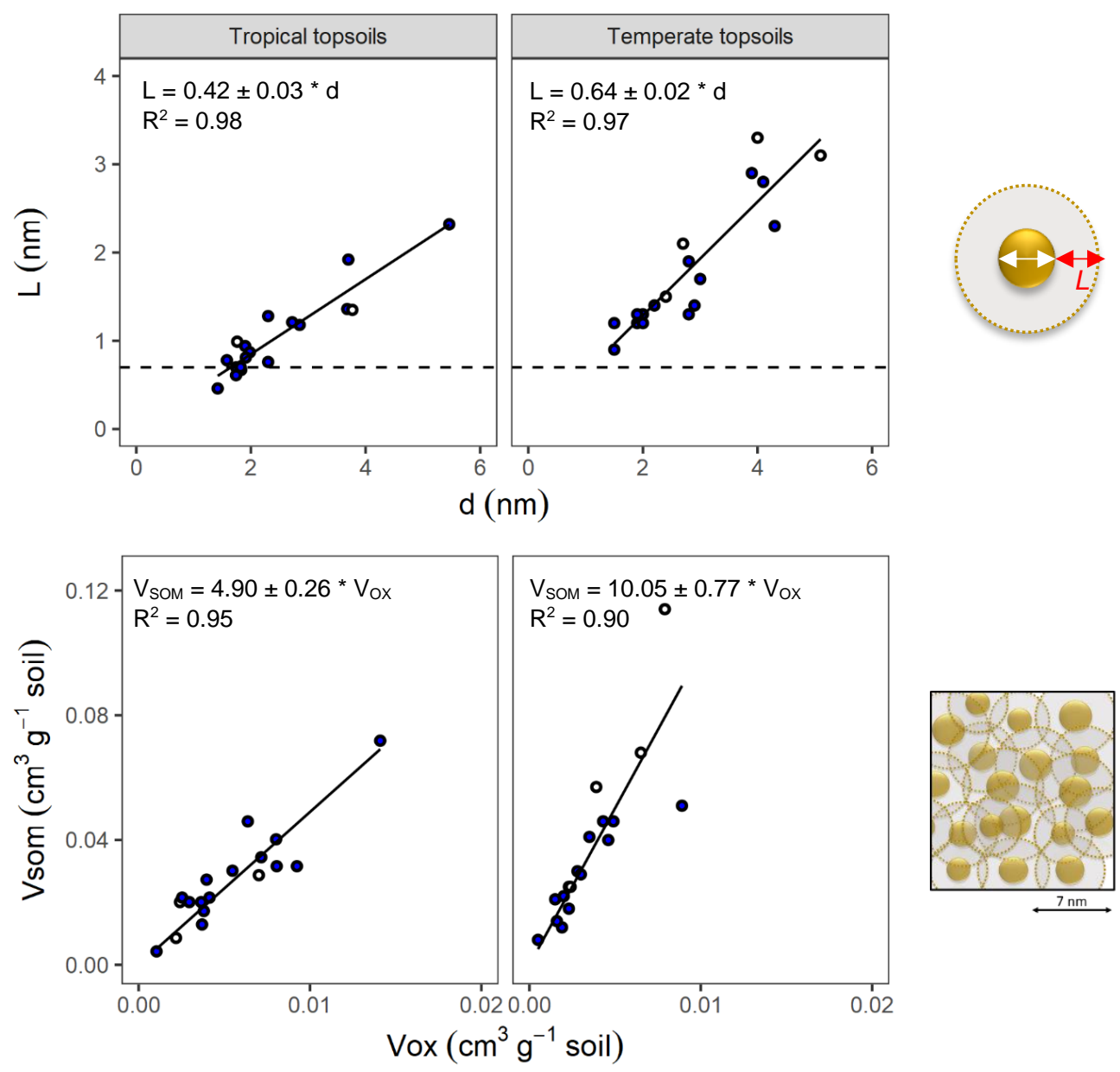

Figure 3: Upper panels: Relationship between the layer thickness $L$ of the soil organic matter (SOM) that surrounds an individual metal (hydr)oxide nanoparticle, according to a core-surface layer model (inset), and the mean metal (hydr)oxide particle diameter. The value of $L$ was calculated based on the measured SOC content, assuming a carbon content of $58 \%$ in the SOM and a mass density of $\rho_{\text {SOM }} 1250$ $\mathrm{g} \mathrm{cm}^{3}$. The horizontal (dash) lines represent the thickness of the compact part of the electrical double layer, being $\sim 0.7 \mathrm{~nm} .{ }^{37}$ Lower Panels: At a higher hierarchical level, the core-shell-like particles aggregate into larger entities (inset) that can additionally associate with micrometer-scaled particles. The relationship between the volumes of soil organic matter (SOM) in these microdomains and the volumes of the $\mathrm{Fe}$ and $\mathrm{Al}$ (hydr)oxide fraction extractable with ammonium oxalate are related. All figures are given for the tropical topsoils (left) and the temperate topsoils (right). The white markers show the samples with clay content $>20 \%$. The regression equations are given for the different relations, together with the multiple coefficients of determination calculated for the regression lines that go through the origin.

\subsection{Organo-mineral associations}

In the above structural view presented in the upper panels of Figure 3, organo-oxide particles are considered as separate entities. However, organo-oxide associations 
may be considered as a collection of organo-oxide particles, which are formed by self-assembly that is driven by the mutual interaction between both types of particles that have the same order of size $(\mathrm{nm})$. The individual core-shell units are aggregated into domains that consist of many SOM and metal (hydr)oxides particles ${ }^{39,40}$. In the collection of particles, the oxide nanoparticles are surrounded by organic molecules (inset Figure 3 lower panel) and vice versa. In such a structural view, the volume of the total SOM content will be related to the volume of the natural metal-(hydr)oxide nanoparticles. Indeed, such a relationship is found in the topsoils studied as shown in the lower panels of Figure 3. In the approach, the metal (hydr)oxide volume was calculated from the amount of AO-extractable $\mathrm{Al}$ and $\mathrm{Fe}$ using a set of consistent values for the molar mass $M_{\text {nano }}$ and mass density $\rho_{\text {nano, }}$ which both depend on the chemical composition ( $\mathrm{Al}$ and $\mathrm{Fe}$ ) of the metal (hydr)oxide particles and on the particle diameter (Eq. 1 in Methods and Mendez et al. ${ }^{25}$ ).

The intimate association of organic matter with $\mathrm{Fe}$ and Al oxides occurs at a primary level of hierarchy and leads to particle sizes in the single-digit nanometer range. These primary associates are attached, linked, and bound to the surfaces of larger particles such as clay minerals, crystalline metal (hydr)oxide domains, and other fine-grained crystalline silicates ${ }^{41,42}$, jointly forming microscale organo-mineral associations.

With our core-shell model, one may assess the values for mass density of the primary organo-oxide particles (Eq. 10 in the Methods section). The overall mass density will depend on the size of the oxide core (d), its chemical composition ( $\mathrm{Al} / \mathrm{Fe})$, and layer thickness with NOM $(L)$. Calculations show that for both soil series, the mass density of the primary organo-oxide associates is mostly between 1400 and $1600 \mathrm{~kg} \mathrm{~m}^{-3}$. These calculated mass densities of the organo-mineral association are typical for what has been defined operationally as the "light" fraction of SOM that is measured by common density fractionation procedures ${ }^{34,43}$. However, as discussed above, the primary organo-oxide associates will jointly form these secondary microscale organo-mineral associates, which will have a higher overall mass density. At physical mass density fractionation supplemented with ultra-sonic dispersion, these microscale structures can be broken ${ }^{41,44}$, allowing some of the primary organooxide particles to become part of the 'light' fraction, resulting in an underestimation of the real fraction of mineral-associated organic carbon. To the best of our knowledge, no study has explicitly evaluated that the "light" SOM fraction measured in density fractionation schemes is indeed free of mineral metal (hydr)oxide nanoparticles, while such analyses could corroborate our density calculations.

\subsection{Implications for predicting SOC content}

For predicting the content of $\mathrm{SOC}$ in a more absolute sense, it should be realized that the net amount of SOC is a result of the metal (hydr)oxide properties and the tradeoff between the input and output of organic matter. Both fluxes are determined by a large number of soil factors such as the availability of water and nutrients, 
temperature, $\mathrm{pH}$, and other conditions. In Figures 2 and 3 , the slopes of the linear relationships differ for both types of topsoils. It suggests a different condition of steady-state between input and output due to climatic origin ${ }^{2}$. As a result, in the tropical topsoils, less $\mathrm{OC}$ is available per unit surface area at the same particle size compared to the temperate topsoils.

Similarly to the above, a difference in the condition of steady-state may also be expected within a soil profile. In the topsoils, the net input of organic carbon will be higher than in the subsoils, and top and subsoils may also have a different mean rate of decomposition. Similarly, one may expect a difference between the topsoils under forest versus arable soils. But do we see at a comparable condition of steady state still the relation between mean particle size and layer thickness of organic matter? To answer this intriguing question, we have sampled three soil profiles under forest and two profiles of arable soil under temperate climatic conditions. We applied the same method as for the main topsoil series. The results are shown in Figure 4.

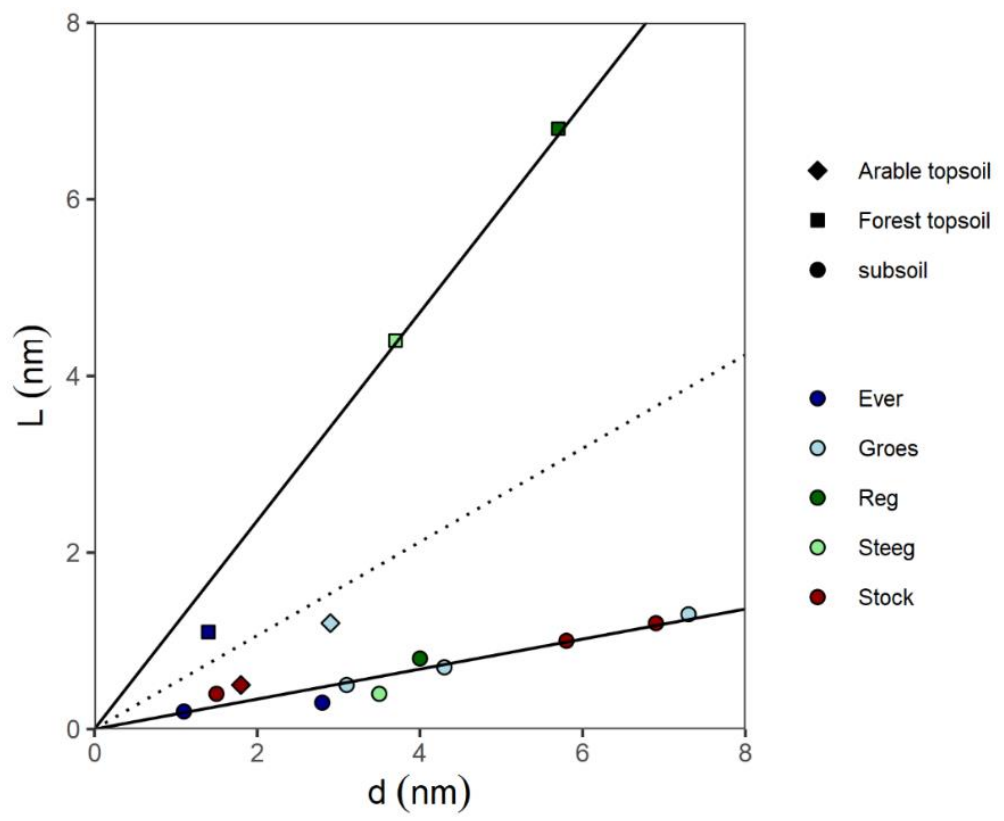

Figure 4: Relationship between the bulk soil organic carbon loading expressed in a mean layer thickness (L) around an oxide with a measured mean metal (hydr)oxide particle diameter for three profiles of forest soils (Regentesselaan, Everwijnsgoed, DeSteeg) and two arable soil profiles (Groesbeek and Stockum), located in the Netherlands. The subsoil samples are given as spheres. The mineral topsoil samples are given as squares (Forest) and as diamonds (Arable land). The dotted line gives the averaged relation found for the topsoils of the tropical and temperate soil series (Figure 3). The lower full line ( $L=0.17 d, n$ $\left.=10 R^{2}=0.97\right)$ describes the average relation for all subsoil samples. The upper line $(L=1.18 d, n=3$ $\left.R^{2}=0.99\right)$ gives the relation for the forest soil between the mean SOM layer thickness and the oxide particle size. These topsoils are very acid $(\mathrm{pH} 3-3.5)$, which will contribute to conditions of a low rate of decomposition leading to enhanced storage by interaction with the oxide fraction. 
In Figure 4, a linear relationships exist between SOM layer thickness $(L)$ and mean oxide particle size (d). These differ with land use and soil depth. For the mineral topsoils under forest (squares), the slope of the line $(s=1.18)$ largely deviates from the mean slope $(s \sim 0.53)$ found for both series of arable topsoils presented in the upper panels of Figures 3. This difference is likely due to the relatively low rate of decomposition of SOM in the forest soils for instance because of acidic conditions (pH 3-3.5, Table S3). In the subsoils (spheres), we do also find a linear relation, but the slope is very low $(s=0.17)$. This can be attributed to the low input of SOM by root production. Compared to the topsoils, about 3-7 times less carbon is stored in subsoils samples with the same metal (hydr)oxide content and mean oxide particle size.

\section{CONCLUSIONS}

With our experimental data and modeling, we have shown that SOC content is related to the RSA of soils and metal (hydr)oxide content. More importantly, we have shown how this information can be translated to a mean particle size of the natural oxide fraction ( $d$ ) or equivalently to its specific surface area (SSA), and how this can be further interpreted with the core-shell model to obtain the averaged layer thickness $(L)$ of associated SOM. Our evaluation strongly suggests the presence of nanocrystalline $\mathrm{Fe}$ and $\mathrm{Al}$ (hydr)oxides as the primary factor in the storage of natural organic matter, at a given condition of steady-state between input and output of organic matter. These interactions between natural metal (hydr)oxide nanoparticles and SOM are fundamental and omnipresent processes that must be considered in global geochemical models to evaluate the capacity of soils to store SOC and their potential contribution to climate change mitigation.

\section{ACKNOWLEDGEMENTS}

This work was supported by NWO (grant number 14688, "Micronutrients for better yields") and by the University of Costa Rica. 


\section{SUPPORTING INFORMATION}

\section{A. Properties of the soil samples}

Table S1: Soil characteristics of the Dutch top soil samples, taken from Mendez et al. ${ }^{25}$

\begin{tabular}{|c|c|c|c|c|c|c|c|c|c|c|c|c|c|c|c|c|c|c|c|}
\hline \multirow[b]{2}{*}{ Soil } & \multirow[b]{2}{*}{$\mathrm{pH}^{\mathrm{a}}$} & \multirow[b]{2}{*}{$O C^{b}$} & \multirow[b]{2}{*}{ Clay } & \multicolumn{2}{|c|}{$\mathrm{DC}^{\mathrm{c}}$} & \multicolumn{2}{|c|}{$A O^{d}$} & \multirow[b]{2}{*}{$\mathrm{RSA}^{\mathrm{e}}$} & \multirow[b]{2}{*}{$d^{f}$} & \multicolumn{2}{|c|}{ SSAp $^{f}$} & \multicolumn{2}{|c|}{$M_{\text {nano }}{ }^{f}$} & \multicolumn{2}{|c|}{$\rho_{\text {nano }}{ }^{f}$} & \multirow[b]{2}{*}{ SSA $_{\text {soil }}{ }^{f}$} & \multirow[b]{2}{*}{$\mathbf{L}^{g}$} & \multirow[b]{2}{*}{$V_{O M}^{h}$} & \multirow[b]{2}{*}{$V_{o x}^{i}$} \\
\hline & & & & $\mathrm{Fe}$ & $\mathrm{Al}$ & $\mathrm{Fe}$ & Al & & & $\mathrm{Fe}$ & Al & $\mathrm{Fe}$ & $\mathrm{Al}$ & $\mathrm{Fe}$ & $\mathrm{Al}$ & & & & \\
\hline & & $\mathrm{mg} \mathrm{g}^{-1}$ & $\%$ & \multicolumn{2}{|c|}{$\mathrm{mmol} \mathrm{kg}^{-1}$} & \multicolumn{2}{|c|}{ mmol kg-1 } & $\mathrm{m}^{2} \mathrm{~g}^{-1}$ soil & $\mathrm{nm}$ & $m^{2} g^{-1}$ & oxide & \multicolumn{2}{|c|}{$\mathrm{g} \mathrm{mol}^{-1}$ metal ion } & \multicolumn{2}{|c|}{$\mathrm{g} \mathrm{cm}^{-3}$} & $m^{2} g^{-1}$ & $\mathrm{~nm}$ & \multicolumn{2}{|c|}{$\mathrm{cm}^{3} \mathrm{~g}^{-1}$ soil } \\
\hline 1 & 4.3 & 18 & 5 & 48 & 41 & 32 & 34 & $6.8 \pm 0.5$ & 2.0 & 823 & 1288 & 100.4 & 91.6 & 3.55 & 2.27 & 1060 & 1.3 & 0.025 & 0.0023 \\
\hline 2 & 5.2 & 22 & 7 & 119 & 39 & 49 & 33 & $8.8 \pm 1.1$ & 1.9 & 907 & 1391 & 102.8 & 92.9 & 3.46 & 2.26 & 1099 & 1.3 & 0.03 & 0.0028 \\
\hline 3 & 5.6 & 37 & 8 & 852 & 27 & 342 & 19 & $18.9 \pm 2.8$ & 2.8 & 544 & 915 & 93.1 & 87.2 & 3.89 & 2.31 & 563 & 1.3 & 0.051 & 0.0089 \\
\hline 4 & 5.6 & 33 & 11 & 125 & 34 & 93 & 33 & $19.5 \pm 1.4$ & 1.5 & 1277 & 1796 & 114.9 & 98.3 & 3.11 & 2.22 & 1406 & 0.9 & 0.046 & 0.0049 \\
\hline 5 & 4.9 & 13 & 15 & 168 & 30 & 76 & 20 & $3.2 \pm 0.2$ & 4.3 & 333 & 594 & 88.3 & 83.7 & 4.19 & 2.35 & 387 & 2.3 & 0.018 & 0.0023 \\
\hline 6 & 5 & 15 & 9 & 68 & 25 & 43 & 16 & $2.4 \pm 0.1$ & 3.9 & 378 & 666 & 89.3 & 84.5 & 4.12 & 2.34 & 457 & 2.9 & 0.021 & 0.0015 \\
\hline 7 & 5.7 & 9 & 11 & 135 & 27 & 50 & 19 & $4.0 \pm 0.6$ & 2.9 & 536 & 904 & 93.0 & 87.1 & 3.90 & 2.31 & 637 & 1.4 & 0.012 & 0.0019 \\
\hline 8 & 5 & 30 & 14 & 101 & 32 & 96 & 31 & $7.5 \pm 0.3$ & 2.8 & 553 & 929 & 93.4 & 87.3 & 3.88 & 2.31 & 645 & 1.9 & 0.041 & 0.0035 \\
\hline 9 & 4.6 & 49 & 21 & 242 & 36 & 211 & 28 & $16.4 \pm 0.7$ & 2.4 & 672 & 1094 & 96.3 & 89.2 & 3.73 & 2.29 & 720 & 1.5 & 0.068 & 0.0065 \\
\hline 10 & 4.9 & 83 & 25 & 318 & 59 & 252 & 46 & $17.6 \pm 1.8$ & 2.7 & 574 & 959 & 93.9 & 87.7 & 3.85 & 2.31 & 634 & 2.1 & 0.114 & 0.0079 \\
\hline 11 & 5.5 & 140 & 28 & 146 & N.D. & 132 & 58 & $12.5 \pm 0.8$ & 2.6 & 593 & 985 & 94.3 & 88.0 & 3.83 & 2.30 & 712 & 3.1 & 0.193 & 0.0055 \\
\hline 12 & 4.5 & 33 & 13 & 233 & 29 & 114 & 21 & $13.6 \pm 1.3$ & 1.9 & 917 & 1402 & 103.1 & 93.0 & 3.45 & 2.26 & 990 & 1.2 & 0.046 & 0.0043 \\
\hline 13 & 7.2 & 18 & 26 & 178 & 32 & 65 & 32 & $2.9 \pm 0.1$ & 5.1 & 273 & 497 & 87.0 & 82.7 & 4.28 & 2.36 & 347 & 3.1 & 0.025 & 0.0024 \\
\hline 14 & 6.8 & 6 & 3 & 16 & 6 & 11 & 3 & $2.1 \pm 0.1$ & 1.5 & 1294 & 1812 & 115.6 & 98.6 & 3.10 & 2.21 & 1403 & 1.2 & 0.008 & 0.0005 \\
\hline 15 & 6.4 & 41 & 28 & 200 & 30 & 117 & 39 & $5.9 \pm 0.2$ & 4.0 & 362 & 641 & 89.0 & 84.2 & 4.14 & 2.34 & 432 & 3.3 & 0.057 & 0.0039 \\
\hline 16 & 6 & 10 & 6 & 84 & 34 & 31 & 24 & $3.2 \pm 0.1$ & 3.0 & 502 & 855 & 92.2 & 86.5 & 3.95 & 2.32 & 654 & 1.7 & 0.014 & 0.0016 \\
\hline 17 & 7.1 & 29 & 12 & 277 & 32 & 116 & 29 & $14 \pm 2.3$ & 2.0 & 873 & 1350 & 101.8 & 92.4 & 3.50 & 2.26 & 968 & 1.2 & 0.04 & 0.0046 \\
\hline 18 & 5.6 & 21 & 12 & 116 & 36 & 68 & 29 & $8.5 \pm 0.8$ & 2.2 & 773 & 1225 & 99.0 & 90.8 & 3.61 & 2.28 & 906 & 1.4 & 0.029 & 0.0030 \\
\hline 19 & 6.3 & 16 & 17 & 153 & 59 & 42 & 30 & $2.9 \pm 0.1$ & 4.1 & 349 & 620 & 88.7 & 84.0 & 4.16 & 2.34 & 461 & 2.8 & 0.022 & 0.0020 \\
\hline MIN & 4.3 & 6 & 3 & 16 & 6 & 11 & 3 & 2.1 & 1.5 & 273 & 497 & 87.0 & 82.7 & 3.10 & 2.21 & 347 & 0.9 & 0.008 & 0.0005 \\
\hline MAX & 7.2 & 140 & 28 & 852 & 59 & 342 & 58 & 19.5 & 5.1 & 1294 & 1812 & 115.6 & 98.6 & 4.28 & 2.36 & 1406 & 3.3 & 0.193 & 0.0089 \\
\hline MEAN & 5.6 & 32.8 & 14.3 & 18 & 34 & 102 & 29 & 9.0 & 2.8 & 660 & 1049 & 96.6 & 88.9 & 3.77 & 2.30 & 762 & 1.9 & 0.045 & 0.0037 \\
\hline
\end{tabular}


Table S2: Soil properties of the African tropical soils, 1-15 and 16-18 are topsoils from agricultural fields in respectively Burundi and Kenya.

\begin{tabular}{|c|c|c|c|c|c|c|c|c|c|c|c|c|c|c|c|c|c|c|c|}
\hline \multirow[b]{2}{*}{ Soil } & \multirow[b]{2}{*}{$\mathrm{pH}^{\mathrm{a}}$} & \multirow[b]{2}{*}{$O C^{b}$} & \multirow[b]{2}{*}{ Clay } & \multicolumn{2}{|c|}{$\mathrm{DC}^{\mathrm{c}}$} & \multicolumn{2}{|c|}{$A O^{d}$} & \multirow[b]{2}{*}{$\mathrm{RSA}^{\mathrm{e}}$} & \multirow[b]{2}{*}{$d^{f}$} & \multicolumn{2}{|c|}{ SSAp } & \multicolumn{2}{|c|}{$M_{\text {nano }}{ }^{f}$} & \multicolumn{2}{|c|}{$\rho_{\text {nano }}{ }^{f}$} & & \multirow[b]{2}{*}{$L^{g}$} & \multirow[b]{2}{*}{$\mathrm{V}_{\text {OM }}{ }^{\mathrm{h}}$} & \multirow[b]{2}{*}{$V_{o x^{i}}$} \\
\hline & & & & $\mathrm{Fe}$ & Al & $\mathrm{Fe}$ & Al & & & $\mathrm{Fe}$ & Al & $\mathrm{Fe}$ & Al & $\mathrm{Fe}$ & Al & & & & \\
\hline & & $\mathrm{mg} \mathrm{g}^{-1}$ & $\%$ & \multicolumn{2}{|c|}{$\mathrm{mmol} \mathrm{kg}^{-1}$} & \multicolumn{2}{|c|}{ mmol kg-1 } & $\mathrm{m}^{2} \mathrm{~g}^{-1}$ soil & $\mathrm{nm}$ & \multicolumn{2}{|c|}{$\mathrm{m}^{2} \mathrm{~g}^{-1}$ oxide } & \multicolumn{2}{|c|}{$\mathrm{g} \mathrm{mol}^{-1}$ metal ion } & \multicolumn{2}{|c|}{$\mathrm{g} \mathrm{cm}^{-3}$} & $\mathbf{m}^{2} \mathbf{g}^{-1}$ & $\mathrm{~nm}$ & $\mathrm{~cm}^{3}$ & soil \\
\hline 1 & 4.43 & 14 & 33 & 332 & 98 & 20 & 42 & $8.24 \pm 0.70$ & 1.8 & 1018 & 1519 & 106.15 & 94.55 & 3.35 & 2.24 & 1358 & 0.99 & 0.020 & 0.0024 \\
\hline 2 & 4.12 & 15 & 7 & 290 & 97 & 21 & 51 & $6.62 \pm 0.39$ & 2.3 & 707 & 1140 & 97.24 & 89.79 & 3.69 & 2.29 & 1014 & 1.28 & 0.022 & 0.0025 \\
\hline 3 & 4.28 & 50 & 7 & 459 & 329 & 156 & 314 & $15.50 \pm 0.05$ & 5.5 & 255 & 465 & 86.66 & 82.42 & 4.31 & 2.36 & 395 & 2.32 & 0.072 & 0.0141 \\
\hline 4 & 4.11 & 20 & 71 & 996 & 187 & 49 & 133 & $23.04 \pm 2.83$ & 1.8 & 962 & 1456 & 104.45 & 93.72 & 3.41 & 2.25 & 1323 & 0.67 & 0.029 & 0.0070 \\
\hline 5 & 4.38 & 28 & 10 & 492 & 245 & 71 & 168 & $16.88 \pm 0.58$ & 2.9 & 540 & 910 & 93.04 & 87.13 & 3.89 & 2.31 & 800 & 1.18 & 0.040 & 0.0080 \\
\hline 6 & 5.02 & 24 & 12 & 510 & 203 & 41 & 138 & $24.74 \pm 0.26$ & 1.7 & 1035 & 1539 & 106.71 & 94.81 & 3.33 & 2.24 & 1424 & 0.70 & 0.034 & 0.0072 \\
\hline 7 & 5.09 & 19 & 12 & 374 & 181 & 28 & 76 & $12.54 \pm 0.44$ & 1.9 & 914 & 1399 & 103.00 & 92.99 & 3.46 & 2.26 & 1270 & 0.94 & 0.027 & 0.0040 \\
\hline 8 & 4.54 & 14 & 7 & 310 & 135 & 21 & 51 & $11.24 \pm 0.51$ & 1.6 & 1187 & 1703 & 111.73 & 97.04 & 3.19 & 2.22 & 1555 & 0.78 & 0.020 & 0.0030 \\
\hline 9 & 4.46 & 21 & 2 & 342 & 215 & 78 & 94 & $12.09 \pm 0.14$ & 2.7 & 572 & 956 & 93.83 & 87.66 & 3.85 & 2.31 & 783 & 1.21 & 0.030 & 0.0055 \\
\hline 10 & 4.24 & 22 & 17 & 647 & 253 & 58 & 147 & $27.83 \pm 0.08$ & 1.7 & 1033 & 1537 & 106.64 & 94.78 & 3.34 & 2.24 & 1395 & 0.61 & 0.032 & 0.0081 \\
\hline 11 & 4.25 & 22 & 4 & 616 & 245 & 60 & 152 & $39.13 \pm 0.47$ & 1.4 & 1400 & 1915 & 119.64 & 100.08 & 3.01 & 2.20 & 1770 & 0.46 & 0.032 & 0.0092 \\
\hline 12 & 4.15 & 14 & 4 & 328 & 140 & 37 & 63 & $11.07 \pm 0.24$ & 2.0 & 862 & 1337 & 101.49 & 92.20 & 3.51 & 2.26 & 1161 & 0.87 & 0.020 & 0.0036 \\
\hline 13 & 4.1 & 12 & 5 & 328 & 141 & 38 & 64 & $12.59 \pm 0.37$ & 1.8 & 971 & 1466 & 104.71 & 93.85 & 3.40 & 2.25 & 1281 & 0.70 & 0.017 & 0.0038 \\
\hline 14 & 4.15 & 15 & 7 & 325 & 141 & 41 & 71 & $12.99 \pm 0.18$ & 1.9 & 904 & 1388 & 102.72 & 92.84 & 3.47 & 2.26 & 1209 & 0.81 & 0.022 & 0.0041 \\
\hline 15 & 4.91 & 32 & 8 & 730 & 172 & 66 & 136 & $10.34 \pm 0.17$ & 3.7 & 396 & 694 & 89.71 & 84.78 & 4.09 & 2.34 & 597 & 1.92 & 0.046 & 0.0064 \\
\hline 16 & 5.33 & 9 & 7 & 289 & 91 & 57 & 56 & $9.69 \pm 0.03$ & 2.3 & 710 & 1144 & 97.32 & 89.84 & 3.68 & 2.29 & 925 & 0.76 & 0.013 & 0.0037 \\
\hline 17 & 6.81 & 3 & 20 & 67 & 39 & 8 & 24 & $1.70 \pm 0.06$ & 3.7 & 398 & 698 & 89.76 & 84.82 & 4.09 & 2.34 & 625 & 1.36 & 0.004 & 0.0010 \\
\hline 18 & 6.3 & 6 & 53 & 635 & 58 & 33 & 40 & $3.48 \pm 0.14$ & 3.8 & 388 & 682 & 89.53 & 84.65 & 4.11 & 2.34 & 550 & 1.35 & 0.009 & 0.0022 \\
\hline MIN & 4.1 & 3 & 2 & 67 & 39 & 8 & 24 & 1.7 & 1.4 & 255 & 465 & 86.7 & 82.4 & 3.01 & 2.20 & 395 & 0.46 & 0.004 & 0.0010 \\
\hline MAX & 6.81 & 50 & 71 & 996 & 329 & 156 & 314 & 39.13 & 5.5 & 1400 & 1915 & 119.6 & 100.1 & 4.31 & 2.36 & 1770 & 2.32 & 0.072 & 0.0141 \\
\hline MEAN & 4.7 & 18.9 & 16 & 448 & 165 & 49 & 101 & 14.4 & 2.5 & 792 & 1219 & 100.2 & 91.0 & 3.62 & 2.28 & 1080 & 1.1 & 0.027 & 0.0053 \\
\hline
\end{tabular}


Table S3: Soil properties of the Dutch soil profiles.

\begin{tabular}{|c|c|c|c|c|c|c|c|c|c|c|c|c|c|c|c|c|c|c|c|c|}
\hline \multirow[b]{2}{*}{ Soil } & \multirow[b]{2}{*}{$\begin{array}{r}\text { Start } \\
\text { depth }\end{array}$} & \multirow[b]{2}{*}{$\mathrm{pH}^{\mathrm{a}}$} & \multirow[b]{2}{*}{$O C^{b}$} & \multirow[b]{2}{*}{ Clay } & \multicolumn{2}{|c|}{$\mathrm{DC}^{\mathrm{c}}$} & \multicolumn{2}{|c|}{$\mathrm{AO}^{\mathrm{d}}$} & \multirow[b]{2}{*}{$\mathrm{RSA}^{\mathrm{e}}$} & \multirow[b]{2}{*}{$d^{f}$} & \multirow{2}{*}{$\frac{\text { SSAp }^{f}}{F e}$} & \multicolumn{3}{|c|}{$M_{\text {nano }}{ }^{f}$} & \multicolumn{2}{|l|}{$\rho_{\text {nano }}{ }^{f}$} & \multirow[b]{2}{*}{ SSA $_{\text {soil }}{ }^{f}$} & \multirow[b]{2}{*}{$L^{g}$} & \multirow[b]{2}{*}{$\mathrm{V}_{\mathrm{OM}^{\mathrm{h}}}{ }^{\mathrm{h}}$} & \multirow[b]{2}{*}{$V_{o x}^{i}$} \\
\hline & & & & & $\mathrm{Fe}$ & Al & $\mathrm{Fe}$ & Al & & & & Al & $\mathrm{Fe}$ & Al & $\mathrm{Fe}$ & Al & & & & \\
\hline & & & $\mathrm{mg} \mathrm{g}^{-1}$ & $\%$ & \multicolumn{2}{|c|}{$\mathrm{mmol} \mathrm{kg}^{-1}$} & \multicolumn{2}{|c|}{$\mathrm{mmol} \mathrm{kg}^{-1}$} & $\mathrm{~m}^{2} \mathbf{g}^{-1}$ soil & $\mathrm{nm}$ & $\mathrm{m}^{2} \mathrm{~g}^{-1}$ & oxide & \multicolumn{2}{|c|}{$\mathrm{g} \mathrm{mol}^{-1}$ metal ion } & \multicolumn{2}{|c|}{$\mathrm{g} \mathrm{cm}^{-3}$} & $m^{2} g^{-1}$ & $\mathrm{~nm}$ & \multicolumn{2}{|c|}{$\mathrm{cm}^{3} \mathrm{~g}^{-1}$ soil } \\
\hline$\overline{\operatorname{Reg}}$ & 0 & 3.04 & 20.59 & $<5$ & 70 & 35 & 31 & 25 & $1.56 \pm 0.49$ & 5.70 & 243 & 443 & 86.41 & 82.12 & 4.33 & 2.38 & 329.18 & 6.84 & 0.0568 & 0.0015 \\
\hline Reg & -80 & 4.48 & 0.44 & $<5$ & 22 & 13 & 3 & 19 & $1.11 \pm 0.56$ & 4.10 & 352 & 621 & 88.73 & 83.91 & 4.16 & 2.36 & 581.47 & 0.76 & 0.0012 & 0.0008 \\
\hline Ever & 0 & 3.35 & 19.66 & $<5$ & 70 & 43 & 38 & 39 & $15.02 \pm 0.84$ & 1.35 & 1520 & 2014 & 124.63 & 101.07 & 2.92 & 2.21 & 1745.78 & 1.06 & 0.0542 & 0.0034 \\
\hline Ever & -30 & 4.01 & 4.98 & $<5$ & 95 & 101 & 54 & 110 & $53.29 \pm 0.22$ & 1.07 & 2309 & 2616 & 171.43 & 110.90 & 2.43 & 2.15 & 2482.88 & 0.19 & 0.0137 & 0.0095 \\
\hline Ever & -130 & 4.36 & 0.20 & $<5$ & 18 & 16 & 4 & 19 & $1.758 \pm 0.23$ & 2.77 & 560 & 933 & 93.53 & 87.23 & 3.87 & 2.32 & 869.89 & 0.26 & 0.0006 & 0.0008 \\
\hline Steeg & 0 & 3.05 & 42.43 & $\sim 10$ & 100 & 64 & 56 & 55 & $5.126 \pm 0.95$ & 3.72 & 394 & 687 & 89.65 & 84.59 & 4.10 & 2.35 & 534.32 & 4.38 & 0.1171 & 0.0032 \\
\hline Steeg & -95 & 3.91 & 1.12 & $\sim 10$ & 133 & 48 & 58 & 60 & $5.995 \pm 0.70$ & 3.45 & 430 & 743 & 90.47 & 85.17 & 4.05 & 2.34 & 584.23 & 0.41 & 0.0031 & 0.0035 \\
\hline Groes & 0 & 5.35 & 7.30 & $\sim 10$ & 83 & 57 & 48 & 67 & $7.73 \pm 0.35$ & 2.85 & 540 & 905 & 93.05 & 86.92 & 3.89 & 2.32 & 746.73 & 1.23 & 0.0201 & 0.0037 \\
\hline Groes & -30 & 4.72 & 1.37 & $\sim 10$ & 86 & 43 & 37 & 48 & $1.99 \pm 0.61$ & 7.12 & 191 & 353 & 85.34 & 81.25 & 4.42 & 2.39 & & 1.34 & 0.0038 & 0.0024 \\
\hline Groes & -50 & 4.85 & $0 . \varepsilon$ & $\sim 10$ & 43 & 21 & 26 & 3( & 2.21 & 4.31 & 33 & $5 \varsigma$ & 88.30 & 83 & 4.19 & 2.36 & 71 & 0.73 & 0.0022 & 0.0016 \\
\hline Groes & -90 & 4.12 & 0.6 & $\sim 10$ & 98 & 32 & 24 & 29 & $3.14 \pm$ & 3.07 & 49 & 839 & 91.95 & 86.19 & 3.96 & 2.33 & 676.47 & 0.46 & 0.0019 & 0.0016 \\
\hline Stock & 0 & 4.04 & 5.24 & $<5$ & 63 & 59 & 50 & 76 & $15.40 \pm 0.32$ & 1.81 & 975 & 1461 & 104.83 & 93.50 & 3.39 & 2.26 & 1255.08 & 0.55 & 0.0145 & 0.0047 \\
\hline Stock & -25 & 4.25 & 3.56 & $<5$ & 56 & 58 & 39 & 62 & $16.45 \pm 0.67$ & 1.52 & 1266 & 1773 & 114.53 & 97.62 & 3.12 & 2.23 & 1556.75 & 0.38 & 0.0098 & 0.0042 \\
\hline Stock & -70 & 5.28 & 0.94 & $<5$ & 27 & 30 & 14 & 42 & $1.50 \pm 1.36$ & 6.85 & 199 & 367 & 85.51 & 81.39 & 4.40 & 2.39 & 323.97 & 1.23 & 0.0026 & 0.0017 \\
\hline Stock & -100 & 5.43 & 0.19 & $<5$ & 24 & 10 & 6 & 8 & $0.39 \pm 0.17$ & 5.80 & 238 & 435 & 86.32 & 82.05 & 4.34 & 2.38 & 347.33 & 0.97 & 0.0005 & 0.0004 \\
\hline $\mathrm{MIN}$ & & 3.04 & 0.19 & & 18 & 10 & 3 & 8 & 0.39 & 1.07 & 1901 & 353 & 85.34 & 81.25 & 2.43 & 2.15 & 280.41 & 0.19 & 0.0005 & 0.0004 \\
\hline MAX & & 5.43 & 42.43 & & 133 & 101 & 58 & 110 & 53.29 & 7.12 & 2309 & 2616 & 171.43 & 110.90 & 4.42 & 2.39 & 2482.88 & 6.84 & 0.1171 & 0.0095 \\
\hline MEAN & & 4.28 & 7.30 & & 66 & 42 & 32 & 46 & 8.84 & 3.70 & 670 & 985 & 99.65 & 88.50 & 3.84 & 2.32 & 852.08 & 1.39 & 0.0201 & 0.0028 \\
\hline
\end{tabular}




\section{B. Relationship between crystalline oxides and SOC}

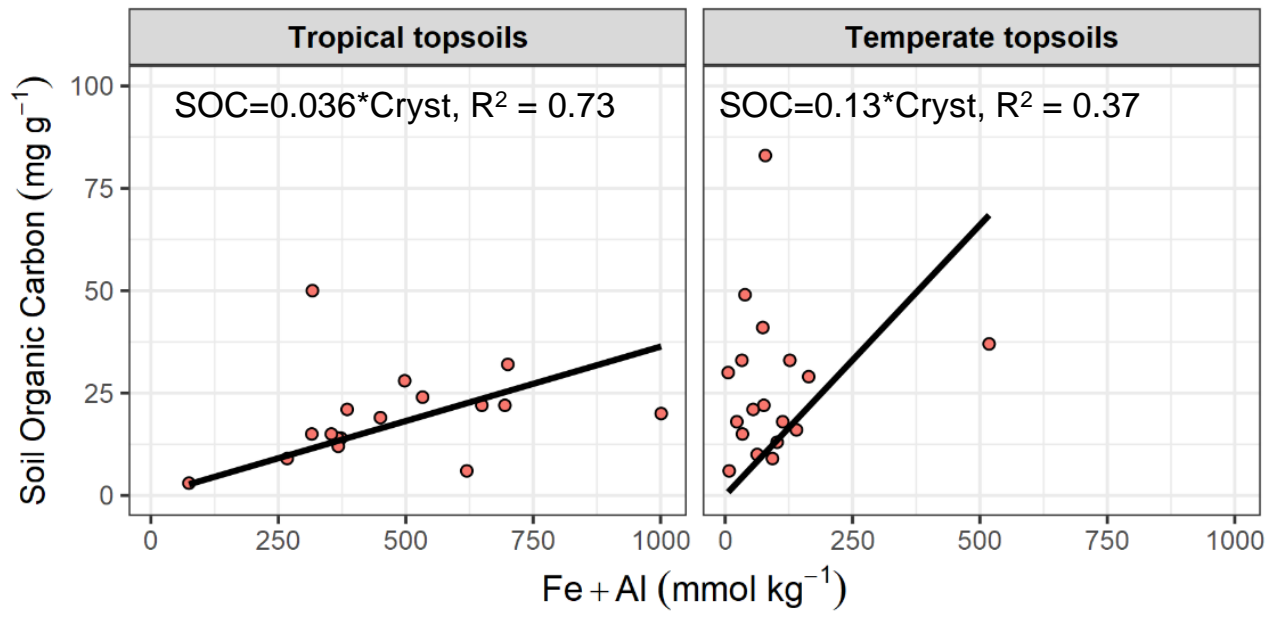

Figure S1: The relationship between the crystalline $\mathrm{Fe}$ and $\mathrm{Al}$ (hydr)oxides, estimated as the difference between the concentrations measured in dithionite (DC) and ammonium oxalate (AO) extractions, and the total soil organic carbon (SOC) in the African top tropical soils (left panel), the Dutch topsoils (middle panel) and (c) the Dutch profiles from natural land use systems (right panel). 
C. Relationship between clay content and SOC

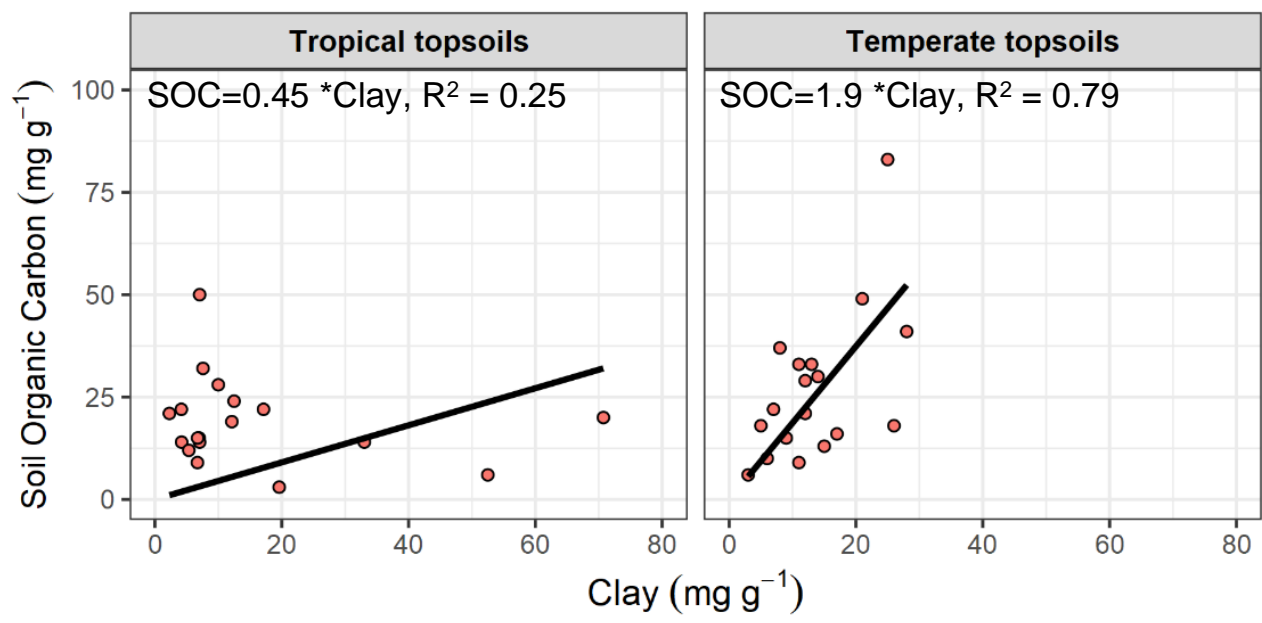

Figure S2: The relationship between the clay content and the total soil organic carbon (SOC) in the African top tropical soils (left panel), the Dutch topsoils (middle panel) and (c) the Dutch profiles from natural land use systems (right panel). 


\section{Effect of equilibration time on the relationship between particle diameter and SOC}

We note that the equilibration time used for the ammonium oxalate $(\mathrm{AO})$ extraction differ between the African topsoils ${ }^{48}$, and the Dutch topsoils and soil profiles ${ }^{27}$, namely $4 \mathrm{~h}$ and $2 \mathrm{~h}$ respectively. For a selection of African topsoils ( $\mathrm{n}=8)$, we have tested the effect of a shorter equilibration time (i.e. $2 \mathrm{~h}$ ), on the particle diameter and SOM layer thickness. The results show that with a decrease in equilibration time, the lower $\mathrm{Fe}$ and $\mathrm{Al}$ concentrations measured imply a smaller mean oxide particle diameter (d), and the difference is on average $20 \%$ (see Figure S3). However, Figure $\mathrm{S} 4$ shows that the relationship between the mean oxide particle diameter and the layer thickness of SOM according to the core-shell model ${ }^{25}$ is not affected by the absolute difference in particle size, and thus by the difference in equilibration time applied for the ammonium oxalate extraction for measuring the reactive metal (hydr)oxide content

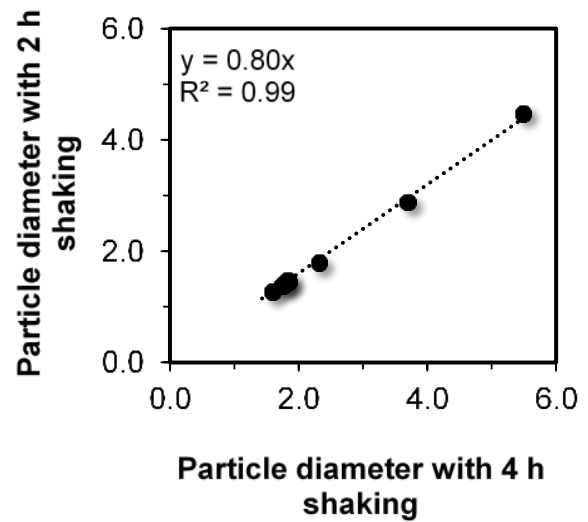

Figure S3: The comparison between the calculated diameter ( $d$ ) of the metal (hydr)oxides particles for a subset of African topsoils, when applying either $2 \mathrm{~h}$ or $4 \mathrm{~h}$ equilibration time for the ammonium oxalate extraction.
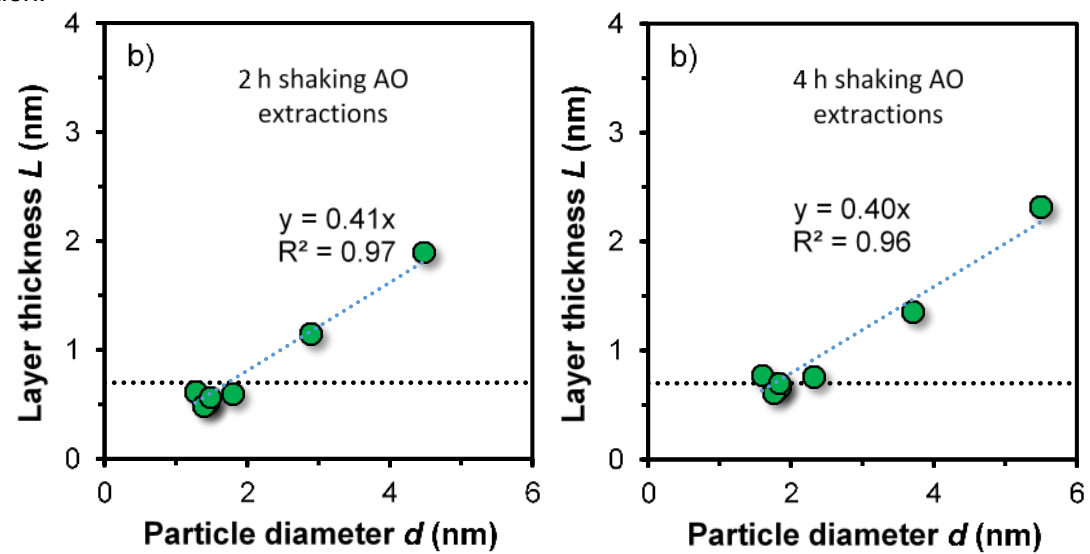

Figure S4: The relationship between particle diameter $d$ and the soil organic matter layer thickness $L$ according to the core-shell model, when the particle diameter is calculated based on either $2 \mathrm{~h}$ or $4 \mathrm{~h}$ shaking time of the ammonium oxalate extraction. 


\section{REFERENCES}

1. Lal, R. Soil Carbon Sequestration Impacts on Global Climate Change and Food Security. 304, 1623-1628 (2004).

2. Wiesmeier, M. et al. Soil organic carbon storage as a key function of soils - A review of drivers and indicators at various scales. Geoderma vol. 333 149-162 (2019).

3. Shi, Z. et al. The age distribution of global soil carbon inferred from radiocarbon measurements. Nat. Geosci. 13, 555-559 (2020).

4. Doetterl, S. et al. Soil carbon storage controlled by interactions between geochemistry and climate. Nat. Geosci. 8, 780-783 (2015).

5. Lehmann, J. \& Kleber, M. The contentious nature of soil organic matter. Nature 528, 60-68 (2015).

6. Hassink, J. The capacity of soils to preserve organic $\mathrm{C}$ and $\mathrm{N}$ by their association with clay and silt particles. Plant Soil 191, 77-87.

7. Six, J., Conant, R. T., Paul, E. A. \& Paustian, K. Stabilization mechanisms of soil organic matter: Implications for C-saturation of soils. Plant Soil 241, 155-176 (2002).

8. Abramoff, R. et al. The Millennial model: in search of measurable pools and transformations for modeling soil carbon in the new century. Biogeochemistry 137, 51-71 (2018).

9. Kaiser, K., Eusterhues, K., Rumpel, C., Guggenberger, G. \& Kögel-Knabner, I. Stabilization of organic matter by soil minerals - Investigations of density and particle-size fractions from two acid forest soils. in Journal of Plant Nutrition and Soil Science vol. 165 451-459 (John Wiley \& Sons, Ltd, 2002).

10. Eusterhues, K., Rumpel, C., Kogel-Knabner, I. \& Kögel-Knabner, I. Organo-mineral associations in sandy acid forest soils: Importance of specific surface area, iron oxides and micropores. Eur. J. Soil Sci. 56, 753-763 (2005).

11. Wiseman, C. L. S. \& Püttmann, W. Interactions between mineral phases in the preservation of soil organic matter. Geoderma 134, 109-118 (2006).

12. Kleber, M., Mikutta, R., Torn, M. S. \& Jahn, R. Poorly crystalline mineral phases protect organic matter in acid subsoil horizons. Eur. J. Soil Sci. 56, 717-725 (2005).

13. Souza, I. F. et al. Al-/Fe-(hydr)oxides-organic carbon associations in Oxisols - From ecosystems to submicron scales. Catena 154, 63-72 (2017).

14. Rasmussen, C. et al. Beyond clay: towards an improved set of variables for predicting soil organic matter content. Biogeochemistry 137, 297-306 (2018).

15. Kaiser, K. \& Guggenberger, G. The role of DOM sorption to mineral surfaces in the preservation of organic matter in soils. in Organic Geochemistry vol. 31 711-725 (Pergamon, 2000).

16. Possinger, A. R. et al. Organo-organic and organo-mineral interfaces in the soil at the nanometer scale. Nat. Commun. 11, (2020).

17. Eusterhues, K., Neidhardt, J., Hädrich, A., Küsel, K. \& Totsche, K. U. Biodegradation of ferrihydrite-associated organic matter. Biogeochemistry 119, 45-50 (2014).

18. Mikutta, R., Lorenz, D., Guggenberger, G., Haumaier, L. \& Freund, A. Properties and reactivity of $\mathrm{Fe}$-organic matter associations formed by coprecipitation versus adsorption: Clues from arsenate batch adsorption. Geochim. Cosmochim. Acta 144, 258-276 (2014).

19. Eusterhues, K. et al. Fractionation of organic matter due to reaction with ferrihydrite: Coprecipitation versus adsorption. Environ. Sci. Technol. 45, 527-533 (2011).

20. Hiemstra, T., Antelo, J., Rahnemaie, R. \& van Riemsdijk, W. H. Nanoparticles in natural systems I: The effective reactive surface area of the natural oxide fraction in field samples. Geochim. Cosmochim. Acta 74, 41-58 (2010).

21. Wiseman, C. L. S. \& Püttmann, W. Soil organic carbon and its sorptive preservation in central Germany. Eur. J. Soil Sci. 56, 65-76 (2005).

22. Beare, M. H. et al. Estimating the organic carbon stabilisation capacity and saturation deficit of soils: A New Zealand case study. Biogeochemistry 120, 71-87 (2014).

23. Coward, E. K., Thompson, A. \& Plante, A. F. Contrasting Fe speciation in two humid forest soils: Insight into organomineral associations in redox-active environments. Geochim. Cosmochim. Acta 238, 68-84 (2018).

24. Mayer, L. M. Surface area control of organic carbon accumulation in continental shelf sediments. Geochim. Cosmochim. Acta 58, 1271-1284 (1994).

25. Mendez, J. C., Hiemstra, T. \& Koopmans, G. F. Assessing the Reactive Surface Area of Soils and the Association of Soil Organic Carbon with Natural Oxide Nanoparticles Using Ferrihydrite as Proxy. Environ. Sci. Technol. 54, 11990-12000 (2020).

26. Van Erp, P. J., Houba, V. J. G. \& Van Beusichem, M. L. One hundredth molar calcium chloride extraction procedure. Part I: A review of soil chemical, analytical, and plant nutritional aspects. 
in Communications in Soil Science and Plant Analysis vol. 29 1603-1623 (Marcel Dekker Inc., 1998).

27. Hiemstra, T., Antelo, J., Rahnemaie, R. \& van Riemsdijk, W. H. Nanoparticles in natural systems I: The effective reactive surface area of the natural oxide fraction in field samples. Geochim. Cosmochim. Acta 74, 41-58 (2010).

28. Hengl, T. et al. SoilGrids250m: Global Gridded Soil Information Based on Machine Learning. PLoS One (2016).

29. Mendez, J. C., Van Eynde, E., Hiemstra, T. \& Comans, R. N. J. Surface reactivity of the natural metal (hydr)oxides in weathered tropical soils. In preparation

30. Hiemstra, T. \& Van Riemsdijk, W. H. A Surface Structural Approach to Ion Adsorption: The Charge Distribution (CD) Model. J. Colloid Interface Sci. 179, 488-508 (1996).

31. Hiemstra, T. \& Zhao, W. Reactivity of ferrihydrite and ferritin in relation to surface structure, size, and nanoparticle formation studied for phosphate and arsenate. Environ. Sci. Nano 3, 12651279 (2016).

32. Mendez, J. C. \& Hiemstra, T. Carbonate Adsorption to Ferrihydrite: Competitive Interaction with Phosphate for Use in Soil Systems. ACS Earth Sp. Chem. 3, 129-141 (2019).

33. Hiemstra, T. Ferrihydrite interaction with silicate and competing oxyanions: Geometry and hydrogen bonding of surface species. Geochim. Cosmochim. Acta 238, 453-476 (2018).

34. Poeplau, C. et al. Isolating organic carbon fractions with varying turnover rates in temperate agricultural soils - A comprehensive method comparison. Soil Biol. Biochem. 125, 10-26 (2018).

35. Rumpel, C. \& Kögel-Knabner, I. Deep soil organic matter-a key but poorly understood component of terrestrial C cycle. Plant Soil 338, 143-158 (2011).

36. Hiemstra, T., Antelo, J., van Rotterdam, A. M. D. \& van Riemsdijk, W. H. Nanoparticles in natural systems II: The natural oxide fraction at interaction with natural organic matter and phosphate. Geochim. Cosmochim. Acta 74, 59-69 (2010).

37. Hiemstra, T. \& Van Riemsdijk, W. H. On the relationship between charge distribution, surface hydration, and the structure of the interface of metal hydroxides. J. Colloid Interface Sci. 301, 118 (2006).

38. Weng, L. P., Van Riemsdijk, W. H. \& Hiemstra, T. Adsorption of humic acids onto goethite: Effects of molar mass, pH and ionic strength. J. Colloid Interface Sci. 314, 107-118 (2007).

39. Kopittke, P. M. et al. Soil organic matter is stabilized by organo-mineral associations through two key processes: The role of the carbon to nitrogen ratio. Geoderma 357, 113974 (2020).

40. Inagaki, T. M. et al. Subsoil organo-mineral associations under contrasting climate conditions. Geochim. Cosmochim. Acta 270, 244-263 (2020).

41. Asano, M. et al. In Search of a Binding Agent: Nano-Scale Evidence of Preferential Carbon Associations with Poorly-Crystalline Mineral Phases in Physically-Stable, Clay-Sized Aggregates. Soil Syst. 2, 32 (2018).

42. Basile-Doelsch, I., Balesdent, J. \& Rose, J. Are interactions between organic compounds and nanoscale weathering minerals the key drivers of carbon storage in soils? Environmental Science and Technology vol. 49 3997-3998 (2015).

43. Lavallee, J. M., Soong, J. L. \& Cotrufo, M. F. Conceptualizing soil organic matter into particulate and mineral-associated forms to address global change in the 21 st century. Glob. Chang. Biol. 26, 261-273 (2020).

44. Asano, M. \& Wagai, R. Evidence of aggregate hierarchy at micro- to submicron scales in an allophanic andisol. Geoderma 216, 62-74 (2014).

45. Houba, V. J. G. J. G., Temminghoff, E. J. M. J. M., Gaikhorst, G. A. A. \& van Vark, W. Soil analysis procedures using $0.01 \mathrm{M}$ calcium chloride as extraction reagent. Commun. Soil Sci. Plant Anal. 31, 1299-1396 (2000).

46. Nelson, D. W. \& Sommers, L. E. Total Carbon, Organic Carbon, and Organic Matter. in Methods of soil analysis Part 3-Chemical Methoods 961-1010 (1996). doi:10.2136/sssabookser5.3.c34.

47. ISO. ISO 12782-2:2012 - Soil quality -- Parameters for geochemical modelling of leaching and speciation of constituents in soils and materials -- Part 2: Extraction of crystalline iron oxides and hydroxides with dithionite. (2012).

48. ISO. ISO 12782-3:2012 Soil quality -- Parameters for geochemical modelling of leaching and speciation of constituents in soils and materials -- Part 3: Extraction of aluminium oxides and hydroxides with ammonium oxalate/oxalic acid. (2012). 
Chapter 3 


\section{Chapter 4}

\section{Boron Adsorption to Ferrihydrite with Implications for Surface Speciation in Soils: Experiments and Modeling}

Elise Van Eynde, Juan C. Mendez, Tjisse Hiemstra, and Rob N.J. Comans

Published as:

Van Eynde, E., Mendez, J. C., Hiemstra, T., \& Comans, R. N. (2020). Boron adsorption to ferrihydrite with implications for surface speciation in soils: Experiments and modeling. ACS Earth and Space Chemistry, 4(8), 1269-1280. doi.org/10.1021/acsearthspacechem.0c00078 


\section{ABSTRACT}

The adsorption and desorption of boric acid onto reactive materials such as metal (hydr)oxides and natural organic matter are generally considered to be controlling processes for the leaching and bioavailability of boron (B). We studied the interaction of $B$ with ferrihydrite (Fh), a nanosized iron (hydr)oxide omnipresent in soil systems, using batch adsorption experiments at different $\mathrm{Ph}$ values and in the presence of phosphate as a competing anion. Surface speciation of B was described with a recently developed multisite ion complexation (MUSIC) and charge distribution (CD) approach. To gain insight into the $B$ adsorption behavior in whole-soil systems, and in the relative contribution of $\mathrm{Fh}$ in particular, the $\mathrm{pH}$-dependent $\mathrm{B}$ speciation was evaluated for soils with representative amounts of ferrihydrite, goethite, and organic matter. The $\mathrm{pH}$-dependent $\mathrm{B}$ adsorption envelope of ferrihydrite is bell-shaped with a maximum around $\mathrm{pH}$ 8-9. In agreement with spectroscopy, modeling suggests formation of a trigonal bidentate complex and an additional outer-sphere complex at low to neutral $\mathrm{pH}$ values. At high $\mathrm{pH}$, a tetrahedral bidentate surface species becomes important. In the presence of phosphate, B adsorption decreases strongly and only formation of the outer-sphere surface complex is relevant. The $\mathrm{pH}$ dependent $\mathrm{B}$ adsorption to $\mathrm{Fh}$ is rather similar to that of goethite. Multisurface modeling predicts that ferrihydrite may dominate the $B$ binding in soils at low to neutral $\mathrm{pH}$ and that the relative contribution of humic material increases significantly at neutral and alkaline $\mathrm{pH}$ conditions. This study identifies ferrihydrite and natural organic matter (i.e. humic substances) as the major constituents that control the $\mathrm{B}$ adsorption in topsoils. 


\section{INTRODUCTION}

Boron (B) is an essential micronutrient to sustain plant growth and development ${ }^{1}$. Plants take up $B$ from the soil solution as boric acid $\left(B(O H)_{3}{ }^{0}\right)$, which is the dominant $B$ species in the soil solution at $\mathrm{pH}$ values below $9^{2}$. The concentration of $B$ in the soil solution usually varies in the range between 1 and $300 \mu \mathrm{M}^{3}$. Boron deficiencies in crop production have been reported in many parts of the world, especially in strongly weathered acidic soils and alkaline soils ${ }^{4}$. However, boron toxicity has also been reported ${ }^{5,6}$ to reduce crop growth.

The bioavailability, toxicity, and mobility of boron, and its loss by leaching ${ }^{7-9}$ are related to the solution concentration of $B$. It has been stated that the $B$ concentration in the soil solution depends on the interaction of $B$ with soil mineral surfaces as well as with natural organic matter ${ }^{10-13}$. In literature, B adsorption has been studied for various $\mathrm{Fe}$ (hydr)oxides including goethite ${ }^{14}$ and ferrihydrite $(\mathrm{Fh})^{15-17}$. The latter material was found to be a key variable in explaining the $\mathrm{pH}$-dependent $\mathrm{B}$ adsorption of soils ${ }^{18}$. Ferrihydrite is a nanomaterial that is ubiquitous in natural soil systems ${ }^{19}$. Due to its small particle size and corresponding large specific surface area, it has a significantly higher adsorption capacity than other Fe (hydr)oxides, making this mineral particularly relevant for elucidating the $B$ adsorption properties of soils.

Surface complexation modeling (SCM) can be used to understand, identify, and predict interactions of ions with reactive mineral and organic surfaces, and when applied in a multi-surface approach, to comprehend the speciation of major and trace elements in soil environments and the processes that affect their bioavailability 20 . Until now, B adsorption to $\mathrm{Fh}$ has been modeled by using the diffuse layer model $(\mathrm{DLM})^{21}$, or more commonly, the constant capacitance model $(\mathrm{CCM})^{15,22}$. Both models simplify the mineral-solution interface to a single surface plane in which inner-sphere complexes reside, while outer-sphere complexation cannot explicitly be considered $^{23}$. A more realistic physical representation of the interface is a double layer model in which surface charge and counter charge are separated by a Stern layer ${ }^{24}$. This approach can be further refined by introducing for inner-sphere surface complexes the concept of charge distribution ${ }^{25}$. In combination, this leads to an improved calculation of the electrostatic energy of ion adsorption. For Fh, surface complexation modeling can be further improved by using a realistic set of reactive sites and corresponding site densities that are based on a surface structural approach ${ }^{26,27}$, rather than using a set of hypothetical reactive sites with a virtual site density. Such an advancement is presently possible due to our improved insights into the structure of $\mathrm{Fh}^{28-30}$.

Another aspect of improving our understanding of the ion adsorption behavior to $\mathrm{Fh}$ is the scaling of ion adsorption data to the surface area of Fh. The primary data refer to the molar ion/Fe ratios that can be translated to adsorption per unit mass and unit surface area, for which the values of molar mass $\left(M_{\text {nano }}\right)$ and specific surface area (SSA) of Fh are needed. A consistent scaling of ion adsorption data allows the 
comparison of experimental results obtained for different Fh preparations. For this purpose, consistent assessment of the actual specific surface area (SSA) of Fh is required, as this property is highly variable amongst different Fh preparations ${ }^{27,31}$ and changes over time ${ }^{32}$.

In the presently applied methodology to assess the SSA, the particle size dependency of the molar mass, mass density, and double layer capacitance of Fh will be considered in an internally consistent manner ${ }^{27}$. This approach enables the development of an internally consistent thermodynamic database with intrinsic ion affinity constants that can be generally used for SCM applications. Our methodology of measuring the SSA differs from traditional analytical techniques for wellcrystallized $\mathrm{Fe}$ (hydr)oxides because these are not suitable for Fh suspensions kept in the wet state. For instance, $\mathrm{N}_{2}$ adsorption analysis using the BET equation requires sample drying and outgassing ${ }^{21,33,34}$, leading to irreversible particles aggregation and lower values of SSA compared to the values found by transition electron microscopy (TEM) measurements ${ }^{35}$. The BET surface area can be $50 \%$ lower than the SSA according to TEM, ${ }^{27}$ while a similar reduction is also found when comparing the BET surface area with the SSA derived from interpretation of the primary surface charge of $\mathrm{Fh}$ with $\mathrm{SCM}^{27}$. To overcome this limitation, we have adopted in this study a recently developed systematic approach for assessing the SSA of $\mathrm{Fh}$ in which $\mathrm{PO}_{4}$ serves as a probe ion ${ }^{27}$.

Finally, ion adsorption modeling can be improved by using interfacial charge distribution coefficients that have been derived independently with a bond valence analysis ${ }^{36,37}$ of the optimized geometry of the formed surface complexes with molecular orbital (MO) calculations using density function theory (DFT). Such a SCM approach has been successfully applied previously to the modeling of the adsorption of ions to Fh comprising protons and electrolyte ion pairs $\left(\mathrm{Cl}^{-}, \mathrm{ClO}_{4}^{-}, \mathrm{NO}_{3}^{-}\right)^{27}, \mathrm{PO}_{4}^{3-}$ and $\mathrm{AsO}_{4}{ }^{3-}, 26 \mathrm{CO}_{3}{ }^{2-}, 38 \mathrm{H}_{4} \mathrm{SiO}_{4}{ }^{0}$ and $\mathrm{As}(\mathrm{OH})_{3}{ }^{0},{ }^{39}$ as well as $\mathrm{Ca}^{2+}$ and $\mathrm{Mg}^{2+}$ ions and their ternary complexes with $\mathrm{PO}_{4}^{3-} \cdot 40,41$

In the present study, we will measure the boron adsorption for freshly-prepared Fh. By using the above SCM approach, we intend to derive a set of adsorption reactions that can accurately describe our adsorption data in agreement with realistic $B$ surface species that have previously been identified by spectroscopy $13,16,17,42,43$. In addition, we will use the same approach to describe other experimental data reported in literature ${ }^{15-17}$ by consistently accounting for differences in the specific surface area of $\mathrm{Fh}$ in these studies. In this approach, the B adsorption behavior will be measured and described for mono-component systems with Fh. However, one may expect a different $B$ adsorption behavior in natural systems in which omnipresent and strongly adsorbing ions such as phosphate compete with the relatively weakly bound boron. To assess these competition effects, we will also measure the B adsorption to Fh in systems with increasing concentrations of added phosphate. The final goal of our study is to gain insight in the B speciation in natural systems, and in the relative 
contribution of $\mathrm{Fh}$ in particular, by using a multi-component and multi-surface modeling approach. Therefore, we will evaluate with that approach the $\mathrm{pH}$ dependent $\mathrm{B}$ adsorption behavior for model systems containing in addition to Fh also crystalline Fe oxide (goethite) and natural organic matter. For the latter two materials, we will rely on a generic set of model parameters derived previously ${ }^{14,44}$. In the multisurface approach, we will evaluate the importance of $\mathrm{PO}_{4}{ }^{3-}$ and $\mathrm{Ca}^{2+}$ ions that are omnipresent in natural soils and interact with the Fe (hydr)oxide surfaces simultaneously with $\mathrm{B}$.

\section{MATERIAL AND METHODS}

\subsection{Fh synthesis}

Fh suspensions were made by adding $0.02 \mathrm{M} \mathrm{NaOH}$ to a solution of $\sim 3.7 \mathrm{mM}$ of $\mathrm{Fe}\left(\mathrm{NO}_{3}\right)_{3}$ dissolved in $0.01 \mathrm{M} \mathrm{HNO}_{3}$ as previously described ${ }^{27}$. Initially, the $\mathrm{NaOH}$ was added in steps of $100 \mathrm{~mL}$ until a $\mathrm{pH}$ of $\sim 3.2$ was reached. Subsequently, the $\mathrm{NaOH}$ was added in steps of $5 \mathrm{~mL}$ until a final $\mathrm{pH}$ of 6.0 was reached $(\mathrm{pH}$ stable for 15 minutes). The suspension was left for 4 hours to let the particles settle down. Afterwards, the suspension was centrifuged for 45 minutes at $3500 \mathrm{~g}$. The supernatant was removed, and the Fh particles were collected and re-suspended in a background solution of $0.01 \mathrm{M} \mathrm{NaNO}_{3}$ to a final volume of 300 and $225 \mathrm{~mL}$ for the suspensions used in the mono-component and competition systems, respectively.

To reduce possible interference of atmospheric $\mathrm{CO}_{2}(\mathrm{~g})$ in the adsorption experiments, the stock Fh suspensions were left overnight and purged under moist $\mathrm{N}_{2}$ (g) until the final aging time of 24 hours. For each prepared Fh suspension, we measured the total Fe content in a matrix of $0.8 \mathrm{M} \mathrm{H}_{2} \mathrm{SO}_{4}$, using ICP-OES. Total Fe content was on average $20.5 \pm 0.8 \mathrm{mM}$.

The specific surface area of Fh depends much on the preparation protocol ${ }^{32}$. Since the surface area is an important parameter for consistently scaling and modeling ion adsorption data, we determined the specific surface area independently for each Fh preparation using $\mathrm{PO}_{4}$ as a probe ion. This approach is a good alternative to overcome the limitations of the BET method ${ }^{27}$, as discussed in the Introduction. Our method has been applied previously to adsorption studies with $\mathrm{Fh}^{38}$ and is described in detail by Mendez and Hiemstra ${ }^{27}$. In this approach, the $\mathrm{PO}_{4}$ adsorption data are interpreted with the parametrized CD model for $\mathrm{Fh}^{26}$ and the specific surface area is defined as the only adjustable parameter. In the data treatment, the primary data (molar $\mathrm{PO}_{4} / \mathrm{Fe}$ ratios) are scaled to an adsorption per unit mass $\left(\mathrm{mol} \mathrm{g}^{-1}\right)$ and surface area $\left(\mathrm{mol} \mathrm{m} \mathrm{m}^{-2}\right)$, using the particle size dependent molar mass ( $M_{\text {nano }}$ in $\left.\mathrm{g} \mathrm{Fh} \mathrm{mol}^{-1} \mathrm{Fe}\right)$ and mass density $\left(\rho_{\text {nano }}\right.$ in $\mathrm{g} \mathrm{m}^{-3}$ ) calculated with a consistent set of mathematical relationships ${ }^{27,32}$. The yet unknown surface area is then derived iteratively by $C D$ modeling. 
The specific surface area of the Fh preparations used for studying the monocomponent $\mathrm{B}$ adsorption was typically in the range $A_{\mathrm{PO}_{4}}=665-677 \mathrm{~m}^{2} \mathrm{~g}^{-1}$, being similar to values found previously ${ }^{27}$. The molar masses corresponding to these SSA values are $96.15-96.45 \mathrm{~g} \mathrm{~mol}^{-1} \mathrm{Fe}$. For the Fh used in the multi-component $\mathrm{B}$ and $\mathrm{P}$ adsorption experiment, the specific surface area was slightly lower, being $A_{\mathrm{PO}_{4}}=$ $628 \mathrm{~m}^{2} \mathrm{~g}^{-1}$ with a molar mass of $95.22 \mathrm{~g} \mathrm{~mol}^{-1} \mathrm{Fe}$.

\subsection{Boron adsorption to $\mathrm{Fh}$ in mono-component systems}

The interaction between $\mathrm{B}$ and $\mathrm{Fh}$ was assessed by collecting $\mathrm{pH}$-dependent adsorption data in systems with four different total $B$ concentrations $(0.05 \mathrm{mM}, 0.1$ $\mathrm{mM}, 0.25 \mathrm{mM}, 0.4 \mathrm{mM})$, one concentration of $\mathrm{Fh}(\sim 8 \mathrm{mM} \mathrm{Fe})$ and a constant ionic strength $\left(0.01 \mathrm{M} \mathrm{NaNO}_{3}\right)$. All systems were made with a total volume of $40 \mathrm{~mL}$.

First, an appropriate volume of $0.01 \mathrm{M} \mathrm{NaNO}_{3}$ was added to a $50 \mathrm{~mL}$ polypropylene tube. Next, $16 \mathrm{~mL}$ of $\mathrm{Fh}$ stock suspension in $0.01 \mathrm{M} \mathrm{NaNO}_{3}$ was pipetted into the tube and the $\mathrm{pH}$ was adjusted by adding pre-determined amounts of $0.01 \mathrm{M} \mathrm{HNO}_{3}$ or $\mathrm{NaOH}$. Finally, $2 \mathrm{~mL}$ of a freshly prepared $\mathrm{B}(\mathrm{OH})_{3}$ stock solution was pipetted into the tubes.

The $\mathrm{pH}$ was adjusted to values between 4 and 11 . Systems with $\mathrm{pH}$ values lower than $\mathrm{pH} 4$ were avoided to prevent dissolution of the Fh particles. The adsorption systems were equilibrated for 21 hours in a horizontal shaker (125 oscillations min$1)$ at a constant temperature of $20{ }^{\circ} \mathrm{C}$. After equilibration, the samples were centrifuged for 20 minutes at $3500 \mathrm{~g}$, and a subsample was subsequently filtered through a membrane filter $(0.45 \mu \mathrm{m})$. An aliquot of $10 \mathrm{~mL}$ was taken and acidified with $\mathrm{HNO}_{3}$ for $\mathrm{B}$ measurement by ICP-OES. The equilibrium $\mathrm{pH}$ was measured in the leftover suspension and this $\mathrm{pH}$ value was used as input for the model calculations. An overview of the B adsorption experiments with four different total B concentrations is given in Table S1 (Supplementary information).

\subsection{Competitive adsorption of boron and phosphate to $\mathrm{Fh}$}

The competitive effect of phosphate on the $\mathrm{B}$ adsorption to Fh was assessed by measuring the adsorption at a fixed $\mathrm{pH}$ in a $0.010 \mathrm{M} \mathrm{NaNO}_{3}$ system with a constant amount of added $\mathrm{B}$ and $\mathrm{Fh}$, but with increasing levels of total added phosphate (0$1.0 \mathrm{mM}$ ). For designing the competitive adsorption experiment, preliminary modeling was done using provisional adsorption parameters calculated based on the data from the monocomponent adsorption experiment. This modeling exercise showed that it is difficult to assess accurately the amount of adsorbed $B$ with the traditional method of determining the adsorption from the difference between the initial and the final equilibrium ion concentration, because in the presence of $\mathrm{PO}_{4}$, most of the added $B$ remains in solution due to its relatively low binding affinity. Therefore, we modified our methodology to allow a direct measurement of the amount of $B$ adsorbed by the solid phase. 
Our adsorption systems were prepared in $50 \mathrm{~mL}$ polypropylene tubes by adding variable volumes of $0.010 \mathrm{M} \mathrm{NaNO}_{3}$ and $16 \mathrm{~mL}$ of Fh stock suspension - prepared in $0.01 \mathrm{M} \mathrm{NaNO}_{3}$ - followed by adjustment of the $\mathrm{pH}$ to 7 using a $0.010 \mathrm{M}$ solution of either $\mathrm{NaOH}$ or $\mathrm{HNO}_{3}$. Subsequently, $2 \mathrm{~mL}$ of a $1 \mathrm{mM} \mathrm{B}(\mathrm{OH})_{3}$ stock solution were added together with variable volumes $(0-5 \mathrm{~mL})$ of a $0.008 \mathrm{M}$ stock solution of $\mathrm{NaH}_{2} \mathrm{PO}_{4}$. The $\mathrm{pH}$ was checked and adjusted after 3,5 , and 7 hours of equilibration. Finally, the total volume was increased to $40.0 \mathrm{~mL}$ with $0.01 \mathrm{M} \mathrm{NaNO}_{3}$ and the total equilibration time was set at 21 hours. One hour before the end of the experiment, so after 20 hours of shaking, the $\mathrm{pH}$ was rechecked and adjusted if needed. The corresponding maximum change in volume was $1 \%$ or less. The total equilibration time chosen for this study is similar to the time used in previous competitive adsorption experiments with ferrihydrite ${ }^{39,41,45}$.

For the above competitive adsorption experiments, two consecutive centrifugation steps were used in the sampling methodology. After a first centrifugation at $3500 \mathrm{~g}$ for $20 \mathrm{~min}$, most of the supernatant was removed and used for chemical analysis of $\mathrm{B}, \mathrm{P}$, and $\mathrm{Fe}$ with ICP-OES. In the second step, ultracentrifugation was applied ( $14300 \mathrm{~g}$ for $5 \mathrm{~min}$ ) to maximize the removal of the solution phase from the Fh pellet. With a dispensable pipet, most of the remaining liquid could be removed carefully, and the mass of the remaining paste was measured gravimetrically, showing an average mass of $0.58 \mathrm{~g}$ left. Next, the Fh pellet was dissolved by adding $7 \mathrm{ml}$ of 0.8 $\mathrm{M} \mathrm{H}_{2} \mathrm{SO}_{4}$. This extract was measured for $\mathrm{B}, \mathrm{P}$, and Fe with ICP-OES.

For calculation of the $B$ adsorption, the $B$ concentration measured in the $0.8 \mathrm{M} \mathrm{H}_{2} \mathrm{SO}_{4}$ extract was corrected for boron present in the solution left in the Fh paste. The latter can be calculated from the mass of the wet paste $(\sim 0.58 \mathrm{~g})$, the mass of the originally added Fh $(\sim 0.030 \mathrm{~g})$ and the equilibrium concentration measured in the supernatant collected after the first centrifugation. The adsorbed amount was finally re-calculated to the percentage of initially added $B$ in the $40 \mathrm{~mL}$ suspension. An overview of the specific experimental conditions of the competitive experiment can be found in Table $\mathrm{S} 1$. Since $\mathrm{P}$ in the equilibrated solution was extremely low, the $\mathrm{PO}_{4}$ adsorption is directly found from the $\mathrm{P}$ measured after dissolving the Fh paste and scaled to the experimental concentration of Fe measured in the sulfuric acid solution. We modeled adsorbed $\mathrm{PO}_{4}$ for the specific conditions using parameters from Hiemstra and $\mathrm{Zhao}^{26}$ and by comparing it with the experimental $\mathrm{PO}_{4}$ data we validated the used approach for measuring the adsorbed $\mathrm{P}$ directly.

\subsection{Modeling adsorption data}

The adsorption of boric acid to Fh has been modeled using the CD model ${ }^{25}$ in combination with the extended Stern layer approach ${ }^{46}$ to describe the compact part of the electrical double layer. A multi-site ion adsorption approach was used, as developed recently for $\mathrm{Fh}^{26}$. This structural model defines two types of singly coordinated surface groups and a set of triply coordinated groups. The triply 
coordinated groups present at the Fh surface vary largely in their proton affinity, which leads to an internal charge compensation ${ }^{26}$, making the effective site density for these surface groups smaller. By assuming the same proton affinity for the triply coordinated surface groups as determined for the singly coordinated groups $(\log K$ $=8.1)^{26}$, an effective site density of $N_{s}=1.4 \mathrm{~nm}^{-2}$ was found by fitting phosphate adsorption data ${ }^{26}$. The triply coordinated groups are assumed to be only involved in the development of surface charge and interactions with ions through outer-sphere complexation. Similarly as for proton adsorption, the binding affinity of these groups for (electrolyte) ions is assumed to be the same as for the singly coordinated groups ${ }^{26}$.

With respect to bidentate complex formation, only a fraction of the singly coordinated groups $\left(N_{s}=2.8 \mathrm{~nm}^{-2}\right)$ is able to form binuclear complexes (double corner complexes). Formation of monodentate surface complexes is possible with both types of singly coordinated surface groups $\left(N_{s}=5.8 \mathrm{~nm}^{-2}\right)$. The primary surface charge was described with the parameters set presented in the publication by Hiemstra and Zhao ${ }^{26}$ and given in Table S2 (SI). The parameters to describe phosphate adsorption were taken from the same study. This parameter set is consistent with the adsorption behavior of Fh measured with the probe ion method using either protons or phosphate ${ }^{27}$.

As mentioned, Fh is a nanoparticle with a strong surface curvature which implies that the capacitance values of the Stern layers $\left(C_{1}\right.$ and $\left.C_{2}\right)$ are size-dependent, for which we consistently account ${ }^{47}$ by using the capacitance values for a flat surface as reference, i.e. $C_{1}=0.90 \mathrm{~F} \mathrm{~m}^{-2}$ and $C_{2}=0.74 \mathrm{~F} \mathrm{~m}^{-2}$. Modeling and parameter optimizations were done using the ECOSAT program, version $4.9^{48}$ in combination with FIT, version $2.581^{49}$. Input variables were $\mathrm{pH}$, electrolyte ion concentrations, and the total $\mathrm{B}(\mathrm{OH})_{3}$ and $\mathrm{PO}_{4}$ concentrations, as well as the concentration of $\mathrm{Fh}$ (based on the measured Fe content) and its specific surface area (based on the experiments using $\mathrm{PO}_{4}$ as probe ion). The aqueous $\mathrm{B}$ speciation reactions applied in the modeling are given in Table S3 (SI). The only fitted parameters were the log $K$ values, since $C D$ values were calculated based on a Brown valence analysis of the surface species geometry obtained with MO/DFT optimization, as explained by Goli et $\mathrm{al}^{14}$.

\subsection{Multicomponent model applications}

The $B$ speciation in soils consisting of multiple types of reactive surfaces has been evaluated with a multi-surface modeling approach. As crystalline $\mathrm{Fe}$ (hydr)oxides may also contribute to the adsorption of $\mathrm{B}$, we considered goethite as a representative crystalline $\mathrm{Fe}$ (hydr)oxide in the assessment of the difference in reactivity relative to nanocrystalline $\mathrm{Fh}$. For boron adsorption to goethite, the parameter set from Goli et al. ${ }^{14}$ was used for calculations with the CD-MUSIC model. The parameters for $\mathrm{H}^{+}, \mathrm{PO}_{4}{ }^{3-}$, and $\mathrm{Na}^{+}$and $\mathrm{NO}_{3}{ }^{-}$were taken from Hiemstra et al. ${ }^{50}$ Boron may also bind to soil organic matter. ${ }^{10-13}$ Therefore, its possible contribution 
to the $B$ adsorption has been evaluated using humic acids ( $\mathrm{HA})$ as model compound for which generic modeling parameters for $B$ have been derived recently ${ }^{44}$.

In our simulations, the chosen amounts of reactive $\mathrm{Fe}$ (hydr)oxides and organic matter are representative for Dutch top soils as reported previously ${ }^{50}$. From the soil data reported by Hiemstra et al. ${ }^{50}$, we choose 3 contrasting soils that have low and high amounts of organic matter and phosphate. The soil characteristics are given in Table S6 in the Supporting Information. The amount of nanocrystalline oxide, represented by $\mathrm{Fh}$ in the modeling, was calculated based on the amount of Fe and Al measured in ammonium oxalate $(\mathrm{AO})$ extracts. The goethite concentration was calculated from the excess amount of Fe found by extraction with dithionite; i,e. Fedithionite $-\mathrm{Fe}_{\mathrm{AO}}$. Provisionally, the specific surface area of Fh was taken as $600 \mathrm{~m}^{2}$ $\mathrm{g}^{-1}$, and $100 \mathrm{~m}^{2} \mathrm{~g}^{-1}$ for goethite 51,52 . For calculating the equivalent amount of $\mathrm{Fh}$, molar masses of 94 and $84 \mathrm{~g} \mathrm{~mol}^{-1} \mathrm{Fe}$ and Al were used, typical for particles with an SSA of $600 \mathrm{~m}^{2} \mathrm{~g}^{-1},{ }^{26}$ and for goethite we used $89 \mathrm{~g} \mathrm{~mol}^{-1} \mathrm{Fe}$. Soil organic matter was represented by humic acid assuming that $30 \%$ of the total organic matter is reactive and has a carbon content of $50 \%{ }^{53}$.

In our modeling, $0.01 \mathrm{M} \mathrm{CaCl}_{2}$ solution was used as a proxy for the soil solution, similarly as used in studies for validating a multi-surface model for ion partitioning $52,54,55$. The equilibrium concentration of $B$ in the solution phase was set at $1 \mu \mathrm{M}$, which is the average concentration that was found for 100 Dutch soils in $0.01 \mathrm{M} \mathrm{CaCl}_{2}$ by Novozamsky et al. ${ }^{56}$ Parameters for $\mathrm{Ca}^{2+}$ adsorption to humic acids were used from Milne et al. ${ }^{57}$, together with the structural parameters for the site density and Donnan phase. The $\mathrm{Ca}^{2+}$ adsorption parameters for goethite were taken from Hiemstra et al. ${ }^{50}$ and for ferrihydrite from Mendez and Hiemstra. ${ }^{41}$ In our modeling, we used a linear additivity approach as commonly done in multi-surface SCM applications to soils ${ }^{51,53,58}$, which considers distinct individual contributions of the different reactive surfaces to the total ion adsorption. Following this approach, we did not assume competitive adsorption of organic matter to the Fe (hydr)oxide surfaces nor ion complexation with dissolved organic matter.

\section{RESULTS AND DISCUSSION}

\section{1 $\mathrm{pH}$ dependency of B adsorption to $\mathrm{Fh}$ in mono-component systems}

For Fh, the measured $\mathrm{pH}$-dependent boron adsorption envelopes are given in Figure 1. From the relatively low values for the $B$ adsorption in Figure $1 b$, it is obvious that the $\mathrm{pH}$-dependent $\mathrm{B}$ adsorption is rather weak. The adsorption behavior of $\mathrm{B}$ shows a bell-shaped curve with a maximum adsorption around $\mathrm{pH} 8-9$, which is typical for $\mathrm{B}$ adsorption to metal oxides ${ }^{14,15,42}$.

The $\mathrm{pH}$ dependency of the $\mathrm{B}$ adsorption is determined by the solution speciation of boron and the interaction of the adsorbing species with protons at the surface, as follows from the thermodynamic consistency equation $39,46,59,60$ : 


$$
\left(\chi_{\mathrm{H}}-n_{\mathrm{H}}\right)_{\mathrm{pH}}=\left(\frac{\delta \log \left[\mathrm{B}_{\mathrm{T}}\right]}{\delta(\mathrm{pH})}\right)_{\Gamma_{\mathrm{B}}}
$$

This equation shows that the $\mathrm{pH}$ dependency of the total solution concentration of boron $\left[\mathrm{B}_{\mathrm{T}}\right]$ at a constant $\mathrm{B}$ loading $\left(\Gamma_{\mathrm{B}}\right)$, given at the right side of the equation, depends on a) the molar ratio of excess proton co-adsorption upon $B$ adsorption $(\chi \mathrm{H})$, and $\mathrm{b}$ ) on the solution speciation presented by $n_{\mathrm{H}}$ which is the excess number of protons bound to dissolved boron relative to a chosen reference species, for instance $\mathrm{B}(\mathrm{OH})_{3}(\mathrm{aq})$.

Model calculations show that the proton co-adsorption at acidic $\mathrm{pH}$ conditions is around $\chi_{\mathrm{H}} \sim-0.1$. At sufficiently low $\mathrm{pH}$, when $\mathrm{B}(\mathrm{OH})_{3}{ }^{0}$ is the predominant solution species, the value of $n_{H}$ approaches zero, which implies that $\chi_{H}-n_{H}$ will be negative, so will be the term at the right-hand side of Equation 1. Consequently, the change of the equilibrium $\mathrm{B}$ concentration with $\mathrm{pH}$ will be negative, which agrees with the negative slope at the low $\mathrm{pH}$ range for the relationship between the logarithm of the boron concentration in solution and the $\mathrm{pH}$, as shown in Figure $1 \mathrm{a}$. When $\mathrm{B}(\mathrm{OH})_{4}$ dominates the solution speciation of $\mathrm{B}$ at high $\mathrm{pH}$ values (i.e. $\mathrm{pH}>\sim 9$ ), $\chi_{\mathrm{H}}-n_{\mathrm{H}}$ becomes larger than 0 due to the negative value of $n_{\mathrm{H}}$ approaching -1 at high $\mathrm{pH}$. In that case, the solution concentration of $\mathrm{B}$ will increase with $\mathrm{pH}$, as is found in Figure 1a. Our thermodynamic analysis demonstrates that the bell shape of the adsorption envelope is predominantly due to a change of the $B$ solution speciation. The $\mathrm{pH}$ dependency of the $\mathrm{B}$ adsorption by $\mathrm{Fh}$ has large similarities with the $\mathrm{pH}$-dependent silicate adsorption to Fh that has been discussed by Hiemstra ${ }^{39}$.

The proton co-adsorption value that we obtained for $\mathrm{Fh}$ is similar to the value obtained when using goethite to calculate the proton co-adsorption with parameters from Goli et al. ${ }^{14}$ This finding implies that for both Fe (hydr)oxides, the adsorption of $\mathrm{B}$ has a similar $\mathrm{pH}$ dependency, and suggests the existence of similar types of adsorption complexes. This mechanistic interpretation of B-adsorption on $\mathrm{Fh}$ and goethite will be further elaborated in Section 3.4. 

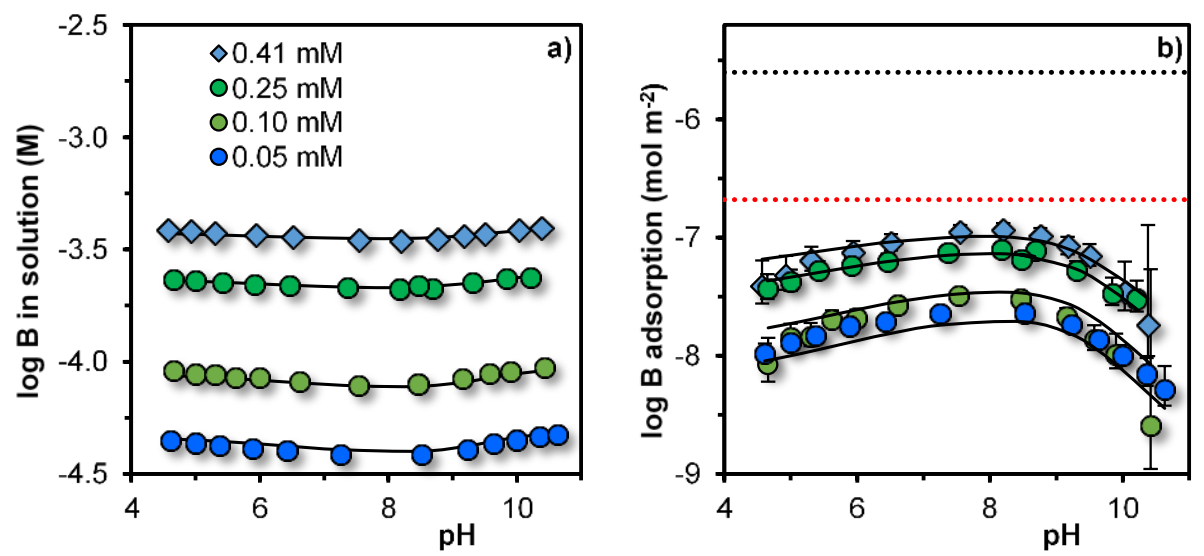

Figure 1: Logarithm of the equilibrium concentration (a) and adsorption (b) of $\mathrm{B}$ to $\mathrm{Fh}$ as a function of $\mathrm{pH}$ in a background solution of $0.01 \mathrm{M} \mathrm{NaNO}_{3}$ at four different total $\mathrm{B}$ concentrations. Solid lines represent the modeling results assuming adsorption to singly coordinated groups $\mathrm{Fe}(\mathrm{OH})(\mathrm{bh})$ with a high affinity, having a fitted surface density of $0.25 \pm 0.08 \mathrm{~nm}^{-2}$. The corresponding maximum adsorption of boron to these sites as a bidentate complex is given as the red-dotted line in figure b). The black dotted line represents the theoretical maximum adsorption density if the low affinity sites would contribute to the $B$ adsorption too. Our collected adsorption data are typically within the experimental window with high affinity sites, which implies that binding to the low affinity sites cannot be resolved by analysis of our adsorption data with SCM (Table 1). See discussion in the text. The error bars in figure b) represent the variation in adsorbed $B$ when assuming an uncertainty of only $2 \%$ in the measured B concentration in solution. The specific experimental conditions can be found in Table S1 (supporting information).

\section{2 $\quad \mathrm{PO}_{4}$-dependent $\mathrm{B}$ adsorption in multicomponent systems}

Figure 2 shows the adsorption of $\mathrm{PO}_{4}$ and $\mathrm{B}$ at $\mathrm{pH} 7.06 \pm 0.10$ for Fh systems with increasing levels of total added $\mathrm{PO}_{4}$ and a constant total $\mathrm{B}$ concentration. The adsorbed $\mathrm{B}$ and $\mathrm{PO}_{4}$ shown in Figure 2 are based on a direct measurement of the adsorbed quantities after dissolution in $0.8 \mathrm{M} \mathrm{H}_{2} \mathrm{SO}_{4}$ of the solid phase (i.e. Fh paste, see Experimental Section). Increase in the $\mathrm{PO}_{4}$ adsorption (orange spheres) significantly reduces the $\mathrm{B}$ adsorption in the system (green diamonds). By modeling the adsorption of $\mathrm{PO}_{4}$ under the same chemical conditions but in the absence of $\mathrm{B}$, we calculated the same $\mathrm{PO}_{4}$ adsorption as measured in our experiment with $\mathrm{B}$. The presence of $\mathrm{B}$ has no significant effect on the $\mathrm{PO}_{4}$ adsorption, since both oxyanions have a very large difference in affinity. At the highest $P$ content $(\sim 1 \mathrm{mM})$, our data reveal that only $5 \%$ of the total $\mathrm{B}$ is still adsorbed to $\mathrm{Fh}$, compared to $13 \%$ in the absence of phosphate. 


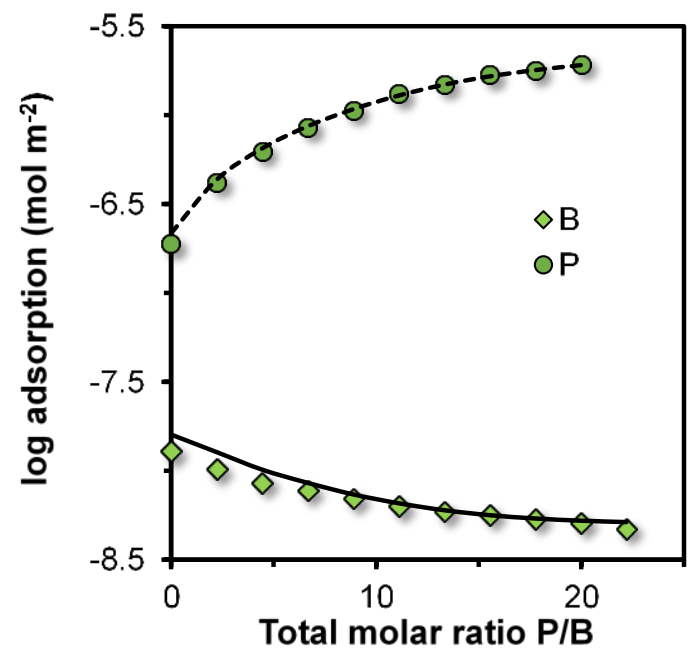

Figure 2: Logarithm of the $\mathrm{B}$ and $\mathrm{PO}_{4}$ adsorption as a function of the molar ratio of total $\mathrm{P}$ and $\mathrm{B}$ in a multi-component Fh system at pH $7.06 \pm 0.10$ in $0.01 \mathrm{M} \mathrm{NaNO}_{3}$. The total $\mathrm{B}$ concentration was kept constant but increasing levels of $\mathrm{P}$ were added, which leads to an increasing $\mathrm{PO}_{4}$ adsorption while the $\mathrm{B}$ adsorption decreases due to removal by adsorbed $\mathrm{PO}_{4}$. The total $\mathrm{B}$ concentration was $0.05 \mathrm{mM}$, the total $\mathrm{Fe}$ concentration was $7.88 \mathrm{mM}$, and the specific surface area of $\mathrm{Fh}$ was $A_{\mathrm{PO} 4}=628 \mathrm{~m}^{2} \mathrm{~g}^{-1}$. The solid line is the prediction of the $B$ adsorption to the high affinity sites with a site density of $0.25 \pm 0.08 \mathrm{~nm}^{-2}$ (Table 1 , option B). The dashed line has been calculated using the parameters from Hiemstra and Zhao. ${ }^{26}$ According to $\mathrm{CD}$ modeling, the $\mathrm{PO}_{4}$ adsorption is not affected by the presence of boron in the system which is due to the high intrinsic affinity of phosphate over that of boron.

Based on the results of Figure 2, it can be expected that the B adsorption to nanocrystalline $\mathrm{Fe}$ (hydr)oxides will be limited in natural systems since these often contain substantial amounts of adsorbed phosphate. This strong competition of phosphate will reduce the contribution of $\mathrm{Fe}$ (hydr)oxides, including $\mathrm{Fh}$, to the surface speciation of $B$ in soils. Consequently, organic matter may become relatively more important for controlling B speciation in soils. This will be further evaluated and discussed in Section 3.5.

\subsection{Modeling B adsorption data}

\subsubsection{B surface complex formation}

Boron speciation on oxide surfaces has been studied previously at the microscopic scale using different spectroscopic techniques, such as infrared ${ }^{16,17,42}$ and X-ray absorption spectroscopy ${ }^{61}$, as well as NMR analysis ${ }^{13,43}$. Using ATR-FTIR, Su and Suare $z^{16}$ found both trigonal and tetrahedral coordination for B with an increasing importance of the tetrahedral species at higher $\mathrm{pH}$ values. With ATR-FTIR, Peak et al. ${ }^{17}$ assigned two peaks (1330 and $1250 \mathrm{~cm}^{-1}$ ) to the presence of a trigonal innersphere surface species, while a third peak at $1395 \mathrm{~cm}^{-1}$ was assigned to an additional trigonal species with similar symmetry to the aqueous boric acid, which was 
interpreted as an outer-sphere complex. This latter peak was only found at near neutral $\mathrm{pH}$, but not at high $\mathrm{pH}(\sim 10)$. Formation of outer-sphere complexes for $\mathrm{B}$ have also been identified for manganese oxide ${ }^{42}$, silica gel, and illite ${ }^{43}$. With the above spectroscopic information, no distinction can be made between the presence of monodentate or bidentate surface species of $B$. However, based on isotopic fractionation, Lemarchand et al. ${ }^{42}$ concluded that boron binds likely as a bidentate complex to the goethite surface.

Based on the above, we formulated for Fh two inner-sphere bidentate complexation reactions with the formation of a trigonal (equation 3 ) and tetragonal (equation 4) surface species:

$$
\begin{aligned}
& 2 \equiv \mathrm{FeOH}(\mathrm{b})^{-0.5}+\mathrm{B}(\mathrm{OH})_{3}(\mathrm{aq}) \leftrightarrow \equiv(\mathrm{FeO})_{2} \mathrm{~B}(\mathrm{OH})+2 \mathrm{H}_{2} \mathrm{O}(\mathrm{l}) \\
& 2 \equiv \mathrm{FeOH}(\mathrm{b})^{-0.5}+\mathrm{B}(\mathrm{OH})_{3}(\mathrm{aq}) \leftrightarrow \equiv(\mathrm{FeO})_{2} \mathrm{~B}(\mathrm{OH})_{2}+1 \mathrm{H}_{2} \mathrm{O}(\mathrm{l})+\mathrm{H}^{+}(\mathrm{aq})
\end{aligned}
$$

in which $\equiv \mathrm{FeOH}(\mathrm{b})^{-1 / 2}$ represents the type of singly coordinated surface groups that may form double corner $\left({ }^{2} \mathrm{C}\right)$ bidentate complexes at the surface of Fh. Other singly coordinated groups ( $\left.=\mathrm{FeOH}(\mathrm{a})^{-1 / 2}\right)$ may form single edge $\left({ }^{1} \mathrm{E}\right)$ bidentate complexes on the ferrihydrite surface as found for uranyl ${ }^{62}$. From literature, it is not known whether edge-sharing complexes might be important for $\mathrm{B}$, and we will assume, therefore, only the formation of bidentate corner-sharing complexes. The site density of $\equiv \mathrm{FeOH}(\mathrm{b})^{-1 / 2}$ is $2.8 \mathrm{~nm}^{-2}$ according to a structural analysis of the Fh surface ${ }^{26}$.

The above described multi-site model of $\mathrm{Fh}$ has also been used previously to describe the adsorption of oxyanions such as $\mathrm{PO}_{4}{ }^{3-}, \mathrm{H}_{4} \mathrm{SiO}_{4}{ }^{0}$, and $\mathrm{CO}_{3}{ }^{2-}$ to $\mathrm{Fh}^{26,38,39}$. More recently, the model has been extended to describe the adsorption of alkalineearth metal cations ${ }^{40,41}$. Analysis of the adsorption data of this metal cation series shows that the adsorption can only be described over a vast range of surface loading by distinguishing both high affinity $\left(\equiv \mathrm{FeOH}(\mathrm{bh})^{-1 / 2}\right)$ and low affinity $\left(\equiv \mathrm{FeOH}(\mathrm{bl})^{-1 / 2}\right)$ sites. In the data analysis below, we will discuss whether this surface site heterogeneity is also important for the binding of boron.

As spectroscopy data also suggests the formation of outer-sphere complexes, the following reactions were formulated for binding of $\mathrm{B}$ to singly $\left(\equiv \mathrm{FeOH}^{-1 / 2}\right)$ and triply $\left(\equiv \mathrm{Fe}_{3} \mathrm{O}^{-1 / 2}\right.$ ) coordinated surface groups:

$$
\begin{aligned}
& \equiv \mathrm{FeOH}^{-0.5}+\mathrm{H}^{+}(\mathrm{aq})+\mathrm{B}(\mathrm{OH})_{3}(\mathrm{aq}) \leftrightarrow \equiv \mathrm{FeOH}_{2} \cdots \mathrm{B}(\mathrm{OH})_{3} \\
& \equiv \mathrm{Fe}_{3} \mathrm{O}^{-0.5}+\mathrm{H}^{+}(\mathrm{aq})+\mathrm{B}(\mathrm{OH})_{3}(\mathrm{aq}) \leftrightarrow \equiv \mathrm{Fe}_{3} \mathrm{OH} \cdots \mathrm{B}(\mathrm{OH})_{3}
\end{aligned}
$$

in which $\equiv \mathrm{FeOH}^{-1 / 2}$ represents the full collection of singly coordinated groups $\left(\mathrm{FeOH}^{-}\right.$ ${ }^{1 / 2}(\mathrm{a})$ and $\left.\mathrm{FeOH}^{-1 / 2}(\mathrm{~b})\right)$ with a total site density of $\mathrm{NS}_{\mathrm{S}}=5.8 \mathrm{~nm}^{-2}$ and $=\mathrm{Fe}_{3} \mathrm{O}^{-1 / 2}$ the triply 
coordinated groups with site density of $1.4 \mathrm{~nm}^{-2}$. The affinity of the singly and triply coordinated groups for B outer-sphere complexation is assumed to be equal, similarly as for the electrolyte ions and for the development of charge at the Fh surface ${ }^{26}$.

For the above bidentate inner-sphere surface species that share corners with Fe(III) octahedra (Eqs. 3,4), Goli et al. ${ }^{14}$ have optimized the geometry using molecular orbital (MO) calculations applying density functional theory (DFT). These geometries were used to calculate the $C D$ coefficients by applying a Brown bond valence analysis ${ }^{36}$ with a correction for dipole orientation of water molecules ${ }^{46}$. In our study, the values for these CD coefficients were used (Table 1) and only the corresponding $\log K$ values of the adsorption reactions were fitted.

\subsubsection{Initial modeling}

For our parameter optimization, we used the experimental data from both the $\mathrm{B}$ mono-component systems and the multi-component system with $\mathrm{PO}_{4}$. The $\log K$ values were derived by evaluating the adsorption data rather than the equilibrium boron concentrations in solution, because the variation in the equilibrium solution concentration with $\mathrm{pH}$ is smaller than that of adsorbed $\mathrm{B}$ (Figure 1). The final $\log K$ values were taken as the average of values fitted on the basis of the \% adsorbed and of the log of adsorbed B in $\mathrm{mol} \mathrm{m}^{-2}$. In the modeling, we used for each $\mathrm{Fh}$ preparation the specific surface area that was measured independently with $\mathrm{PO}_{4}$ surface probing and employed the corresponding size-dependent capacitance values, which were derived by applying spherical double layer theory to the compact part of the electrical double layer (EDL $)^{47}$.

In a first attempt to model the collected data accurately, it was assumed that all $\mathrm{FeOH}(\mathrm{b})$ sites contributed equally to the binding of boron, forming double corner $\left({ }^{2} \mathrm{C}\right)$ bidentate complexes. When using all data points $(n=59)$ from the mono- and multicomponent systems, formation of inner-sphere and outer-sphere complexes could be revealed. The corresponding $\log K$ values for each evaluation scale (i.e. \% B adsorbed and $\log B$ adsorbed in $\mathrm{mol} \mathrm{m}^{-2}$ ) are given in the supplementary information (Table S4), together with the quality of the fit $\left(R^{2}\right)$. The model predictions are given in Figures S1 and S2. The use of either evaluation scale resulted in similar $\log K$ values for all surface species. The $\log K$ value derived for the formation of outersphere complexes by $\mathrm{Fh}$ is equal to the $\log K$ found for goethite, taking as a reference the positively charged $\equiv \mathrm{FeOH}_{2}+1 / 2$ group and writing the adsorption reaction as $=\mathrm{FeOH}_{2}{ }^{+1 / 2}+\mathrm{B}(\mathrm{OH})_{3}(\mathrm{aq}) \Leftrightarrow \equiv \mathrm{FeOH}_{2}{ }^{1 / 2}-\mathrm{B}(\mathrm{OH})_{3}$. The fitted value is $\log K=1.24 \pm$ 0.06 for $\mathrm{Fh}$ and $\log K=1.22 \pm 0.21$ for goethite. The log $K$ of this outer-sphere complex is significantly higher than found for classical electrolyte ions such as $\mathrm{NO}_{3}{ }^{-}(\log K=-$ $0.68), \mathrm{Cl}^{-}(\log K=-0.45)$, and $\mathrm{Na}^{+}(\log \mathrm{K}=-0.60)^{46}$. This might suggest the formation of a relatively stronger $\mathrm{H}$ bond between $\equiv \mathrm{FeOH}_{2}+1 / 2$ and the adsorbed $\mathrm{B}(\mathrm{OH})_{3}$ species that might affect the assumed charge distribution. We have tried to optimize the geometry of a cluster representing the hydrated $\equiv \mathrm{FeOH}_{2}{ }^{1 / 2}-\mathrm{B}(\mathrm{OH})_{3}$ surface species 
with MO/DFT calculations, using the B3LYP model in Spartan'14 parallel software of Wavefunction. In the optimization, the boric acid was initially attached to a $\equiv \mathrm{FeOH}_{2}{ }^{+1 / 2}$ group with a strong $\mathrm{H}$-bond. However, the hydrated $\mathrm{B}(\mathrm{OH})_{3}$ species drifted away, suggesting no extra shift of proton charge (i.e. relative to our originally assumed charge distribution of $\Delta \mathrm{z}_{0}=+1 \mathrm{v} . \mathrm{u}$. and $\Delta \mathrm{z}_{1}=0 \mathrm{v}$.u.) is expected in the interface upon interaction of $B$ in an outer-sphere manner. Based on this result, we conclude that the original CD values from Table 1 used in the formation reaction of the outer sphere complexes can be considered as reasonable.

In a recent study ${ }^{39}$, it has been shown that silicic acid, $\mathrm{Si}(\mathrm{OH})_{4}(\mathrm{aq})$, may bind to $\mathrm{Fh}$ as a monodentate complex that reacts with an adjacent singly coordinated surface group, forming a strong $\mathrm{H}$ bond $(\equiv \mathrm{FeHO} \cdots \mathrm{H}-\mathrm{OSi})$. To explore this possibility for boric acid, we have performed several MO/DFT/B3LYP calculations, searching for the formation of a strong $\mathrm{H}$-bond if a monodentate $\equiv \mathrm{FeOB}(\mathrm{OH})_{2}$ complex interacts with an adjacent singly coordinate group, being either $\equiv \mathrm{FeOH}_{2}+1 / 2$ or $\equiv \mathrm{FeOH}^{-1 / 2}$. The optimizations show that the $\mathrm{B}-\mathrm{OH}$ ligand does not form a strong $\mathrm{H}$-bond with the adjacent surface group, i.e. its formation is less likely than the formation of a classical bidentate complex $\equiv(\mathrm{FeO})_{2} \mathrm{BOH}$ with the $\mathrm{CD}$ as calculated (Table 1 ).

Our initial modeling attempt showed a systematically larger concentration dependency of the $B$ adsorption than observed experimentally (Figure S2). At low B concentrations, the adsorption was underestimated by the model and at high concentrations, it was overestimated. The experimental data suggest a less linear adsorption behavior than suggested by the model. When the adsorption data $(Q)$ are fitted to a Freundlich model, $Q=K_{F} c^{n}$, the coefficient $n$ for the concentration dependency is $n=0.77$ (Figure 3). With the predicted adsorption using the initial model option (Table 1, option A) it is equivalent to $n=0.91$ (not shown), which means that the model in that case predicts a more linear adsorption behavior than shown by the experimental data. As pointed out by Benjamin and Leckie ${ }^{63}$, deviation from linear adsorption behavior $(n=1)$ can be caused by the existence of multiple types of binding sites with different affinities if not due to ignoring electrostatic energy contributions. The presence of high and low affinity sites has been used frequently in modeling surface complexation of ions by ferrihydrite, particularly for metal cations $21,41,45,64,65$. 
Table 1: Surface species of boron with Fh, using a modeling approach either without (A) or with (B) high affinity sites. The interfacial CD values $\left(\Delta z_{0}\right.$ and $\left.\Delta z_{1}\right)$ are from Goli et al. ${ }^{14}$ The applied Extended Stern layer model uses two capacitance values consistent with the specific surface area of Fh given in Table S1. The surface reactions defining the primary charge are given in Table S2. The values for $\log K$ are taken as the average and standard deviation of the fitted values using two evaluation scales (i.e. \% B adsorbed and $\log \mathrm{mol} \mathrm{m}^{-2} \mathrm{~B}$ adsorbed). Fitted values, $\mathrm{R}^{2}$ and RMSE for each evaluation scale are given in Table S4 and S5 (SI). Site densities of $\equiv \mathrm{Fe}_{3} \mathrm{O}$, $\equiv \mathrm{FeOH}(\mathrm{a})$ and $\equiv \mathrm{FeOH}(\mathrm{b})$ were taken as 1.4, 3.0, 2.8 sites $\mathrm{nm}^{-2}$ for scenario $A^{26}$. For scenario $B$, the inner-sphere complexes are only formed with high affinity sites that are a subtype of the $=\mathrm{FeOH}(\mathrm{b})$ surface groups with a fitted site density of $0.25 \pm 0.08$ sites nm 2 .

\begin{tabular}{|c|c|c|c|c|c|}
\hline Species & $\Delta \mathbf{z}_{\mathrm{o}}$ & $\Delta \mathbf{z}_{1}$ & $\Delta \mathrm{z}_{2}$ & $\log K^{\mathrm{A}}$ & $\log K^{B}$ \\
\hline$\equiv(\mathrm{FeO}(\mathrm{b}))_{2}{ }_{2}^{-0.82} \mathrm{~B}(\mathrm{OH})^{-0.18}$ & $0.18^{\#}$ & $-0.18^{\#}$ & 0 & $2.17 \pm 0.13$ & $3.39 \pm 0.18$ \\
\hline$\left.\equiv(\mathrm{FeO}(\mathrm{b}))_{2}\right)^{-1.25} \mathrm{~B}(\mathrm{OH}) 2^{-0.75}$ & $-0.25^{\#}$ & $-0.75^{\#}$ & 0 & $-5.85 \pm 0.08$ & $-4.68 \pm 0.20$ \\
\hline$\equiv \mathrm{Fe}_{3} \mathrm{OH}^{0.5} \quad \ldots \mathrm{B}(\mathrm{OH})_{3}{ }^{0}$ & 1 & 0 & 0 & $9.33 \pm 0.08$ & $9.32 \pm 0.07$ \\
\hline$\equiv \mathrm{FeOH}_{2}(\mathrm{a})^{0.5} \ldots \mathrm{B}(\mathrm{OH})_{3}{ }^{0}$ & 1 & 0 & 0 & $9.33 \pm 0.08$ & $9.32 \pm 0.07$ \\
\hline$\equiv \mathrm{FeOH}_{2}(\mathrm{~b})^{0.5} \ldots \mathrm{B}(\mathrm{OH})_{3}{ }^{0}$ & 1 & 0 & 0 & $9.33 \pm 0.08$ & $9.32 \pm 0.07$ \\
\hline
\end{tabular}

\subsubsection{Introducing high affinity sites}

Based on the above, we have explored the possible role of the presence of a limited number of surface sites with a higher affinity for binding $B$, forming inner-sphere bidentate surface complexes. In this modeling option, the $\equiv \mathrm{FeOH}(\mathrm{b})^{-1 / 2}$ sites were initially divided into two classes, each class with a different intrinsic affinity $(\log K)$ for boron. The site densities were fitted with the restriction that the sum of the densities of $\equiv \mathrm{FeOH}(\mathrm{bh})^{-1 / 2}$ and $\equiv \mathrm{FeOH}(\mathrm{bl})^{-1 / 2}$ equals the overall density of $\equiv \mathrm{FeOH}(\mathrm{b})^{-1 / 2}$. Formation of outer-sphere complexes was considered as explained before. No log $K$ value for $\mathrm{B}$ binding to the low affinity sites $\left(=\mathrm{FeOH}(\mathrm{bl})^{-1 / 2}\right)$ could be found by fitting under these boundary conditions and only the set of surface groups with higher affinity $\left(\equiv \mathrm{FeOH}(\mathrm{bh})^{-1 / 2}\right)$ played a role in $\mathrm{B}$ binding. With this approach, the quality of our data description substantially improved (Figure 1 vs Figure S2). Also important was the outcome of the fitted value for the site density of the high affinity sites, being $N_{s}(b h)=0.25 \pm 0.08 \mathrm{~nm}^{-2}$. This value is very similar to the value that has been found recently for the adsorption of alkaline-earth metal ions $\left(\mathrm{Ca}^{2+}, \mathrm{Sr}^{2+}\right)$, also forming double corner bidentate complexes with singly coordinated surface groups ${ }^{40}$, being in the order of $N_{s}(b h)=0.30 \pm 0.02 \mathrm{~nm}^{-2}$. 
Mendez and Hiemstra ${ }^{40}$ have identified a possible structural feature at the surface of Fh that might explain the observed surface density of the high affinity sites, and in addition, gives a rationale for the high affinity character of the singly coordinated groups of those sites. It is suggested that for the pair of $\mathrm{Fe}(\mathrm{III})$ octahedra involved in the high affinity sites, the oxygen charge of the ligands common with the mineral bulk are collectively slightly undersaturated resulting in some redistribution of charge within this moiety, making both singly-coordinated groups of this unit more reactive, thus increasing their affinity for an adsorbing ion. Per particle, about three pairs of such $\equiv \mathrm{FeOH}(\mathrm{bh})$ sites could be found when Fh particles were constructed according to the surface depletion model29. For our Fh suspensions, this number is equivalent with a site density of about $0.3 \mathrm{~nm}^{-2}$, which is consistent with the number found here by fitting the $\mathrm{B}(\mathrm{OH})_{3}$ adsorption data $\left(0.25 \pm 0.08 \mathrm{~nm}^{-2}\right)$.

A major difference with the alkaline-earth metal ion adsorption is that for boron, no binding to low affinity sites $\left(\equiv \mathrm{FeOH}(\mathrm{bl})^{-1 / 2}\right)$ could be established, while for the metal cations of the alkaline-earth series, this is clearly found. To understand this difference, we have plotted the maximum possible $\mathrm{B}(\mathrm{OH})_{3}$ adsorption in case of only binding to high affinity sites (red dotted line in Figure 1b). This representation indicates that our experimental surface B loadings are significantly lower. The affinity of boron is too low to saturate these sites to a sufficient level at which additional adsorption to low affinity sites starts to occur. For this reason, binding to low affinity sites cannot be resolved with our present data set. This contrasts with the adsorption of metal ions that may adsorb to Fh over a larger range of surface loadings ${ }^{40}$. In literature, the presence of high affinity sites has been particularly associated with the binding of cations, but this concept has not been applied so far to the modeling of the adsorption data of oxyanions. One plausible explanation for this difference might be that oxyanions such as $\mathrm{PO}_{4}$ and $\mathrm{AsO}_{4}$ are bound strongly to ferrihydrite, which does not enable identification of the relatively small number of high affinity sites. Another possibility can be that the anion adsorption is dominated by the formation of other types of surface complexes such as outer sphere complexes, inner-sphere monodentate complexes or bidentate edge complexes, which do not bind to high affinity sites.

With the introduction of high affinity sites for the adsorption of boron, a significantly smaller concentration dependency is predicted by the model compared to the initial modeling option that considers the binding of $\mathrm{B}$ to all $\mathrm{FeOH}(\mathrm{b})$ sites. When the adsorption isotherm is presented in a double log plot (Figure 3), the slope from the modelling predictions based on high affinity sites (Table 1, option $\mathrm{B}$ ) is $n=0.76$, which is much closer to the fitted slope of the data $(n=0.77)$ than when using the complete set of sites for B binding (Table 1 , option $A, n=0.91$ ). This analysis underlines the improvement of the data description when considering binding of boron only to a limited number of surface sites with a high affinity character. 


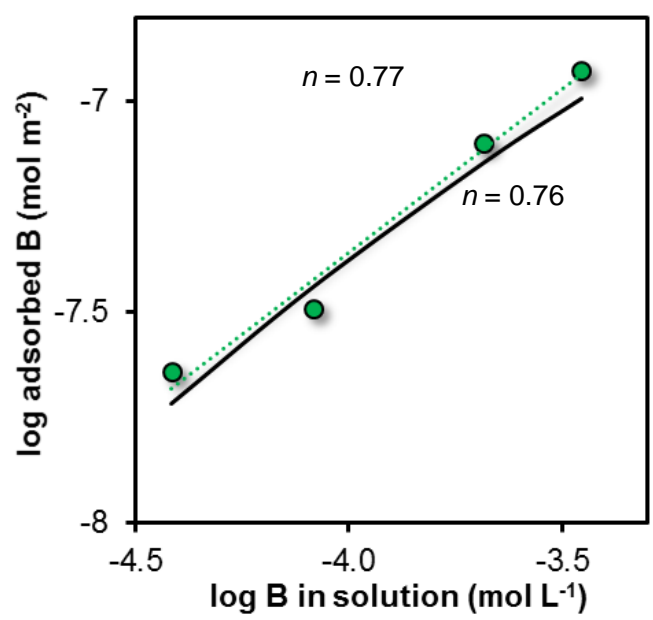

Figure 3: Adsorption isotherm for boron binding to Fh at pH 8.0 in $0.01 \mathrm{M} \mathrm{NaNO}_{3}$. The data points (green symbols) were derived by interpolation of the $\mathrm{pH}$-dependent $\mathrm{B}$ adsorption data from Figure 1 . The full line is the $C D$ modeling result, considering bidentate inner-sphere complex formation by reaction of $B$ with high affinity sites having a site density of $0.25 \pm 0.08$ sites $\mathrm{nm}^{-2}$. The CD model parameters are given in Table 1, option $\mathrm{B}$. The dotted green line is the Freundlich adsorption isotherm $\left(\log Q=\log K_{\mathrm{F}}+n \log C\right)$ with a fitted slope of $n=0.77$ in a double logarithmic plot that is close to mean slope $(n=0.76)$ predicted by the CD model.

In a study of the B adsorption to amorphous Al oxide, Xu and Peak ${ }^{61}$ have found a significant reduction of the $B$ adsorption due to the presence of carbonate in an open experimental system with continuous input of $\mathrm{CO}_{2}$. Presently, we have worked with closed systems, but nevertheless, we have assessed the possible effect of $\mathrm{CO}_{2}$ in our experiments on the fitted $\log K$ values. In our analysis, we assumed that the aqueous solutions used to prepare our closed systems were initially in equilibrium with $\mathrm{CO}_{2}$ at an atmospheric partial pressure of $0.4 \mathrm{mbar}$ (i.e. $\sim 20 \mu_{\mathrm{M} \mathrm{CO}}{ }^{-2}, \mathrm{pH} \sim 5.6$ ). With CD model calculations using the parameters from Mendez and Hiemstra ${ }^{38}$, we did not find any significant effect of the presence of the dissolved $\mathrm{CO}_{2}$ on the $\mathrm{B}$ adsorption. The same has been concluded by Goli et al. ${ }^{14}$ for the $\mathrm{B}$ adsorption to goethite in closed systems.

We evaluated our parameters for the B adsorption to Fh for other experimental data reported in literature, using the specific surface area of $\mathrm{Fh}$ as an adjustable parameter ${ }^{15-17}$. The results are shown and discussed in the supplementary information (Figure S3 and S4). The B adsorption at low $\mathrm{pH}$ was underestimated for the experimental data from Goldberg and Glaubig ${ }^{15}$ and Peak et al. ${ }^{17}$, but the experimental data from Su and Suare ${ }^{16}$ were reasonably well described.

\subsection{Surface speciation of $B$}

Using the $C D$ model parameters from Table 1 (scenario $B$, high affinity sites), we calculated for Fh the B surface speciation as a function of $\mathrm{pH}$ (Figure $4 \mathrm{a}$ ) and as a 102 
function of total $\mathrm{PO}_{4}$ concentration at constant $\mathrm{pH}$ value of 7.0 (Figure $4 \mathrm{~b}$ ). The chosen conditions are representative for our experiments. In the mono-component system of Figure $4 \mathrm{a}$, the trigonal inner-sphere complex is the most important surface species below $\mathrm{pH} \sim 8$. At higher $\mathrm{pH}$, the tetrahedral species becomes more relevant. Outer-sphere complexation is predicted to contribute only at low $\mathrm{pH}$ values, while above $\mathrm{pH} 9$, it is practically absent.

In the presence of $\mathrm{PO}_{4}$, the speciation changes drastically (Figure $4 \mathrm{~b}$ ). The innersphere complexation is strongly reduced and becomes negligible at total $\mathrm{P}$ concentrations of just $0.1 \mathrm{mM}$, in contrast to the presence of outer-sphere complexation that remains unaffected by the increasing levels of $\mathrm{PO}_{4}$ in the system. This difference in surface speciation of $\mathrm{B}$ in the absence and presence of $\mathrm{PO}_{4}$ can be attributed to electrostatic interactions. In general, competition by electrostatic interactions mainly occurs via charge attribution to the Stern plane $\left(\Delta z_{1}\right)$. Upon adsorption, the inner-sphere complexes of $\mathrm{B}$ and $\mathrm{P}$ both introduce negative charge to the 1-plane of the Stern layer. Due to the higher affinity of $\mathrm{PO}_{4}$ for the surface, the B inner-sphere complexes with $\Delta z_{1}=-0.18$ and $\Delta z_{1}=-0.75$ (Table 1) therefore decrease rapidly when phosphate concentrations increase. On the other hand, $\mathrm{B}(\mathrm{OH})_{3}$ bound as an outer-sphere complex does not introduce in our model any charge in the 1-plane $\left(\Delta z_{1}=0\right.$, see Table 1). Consequently, the formation of this complex is unaffected by electrostatic competition in the 1 -plane.
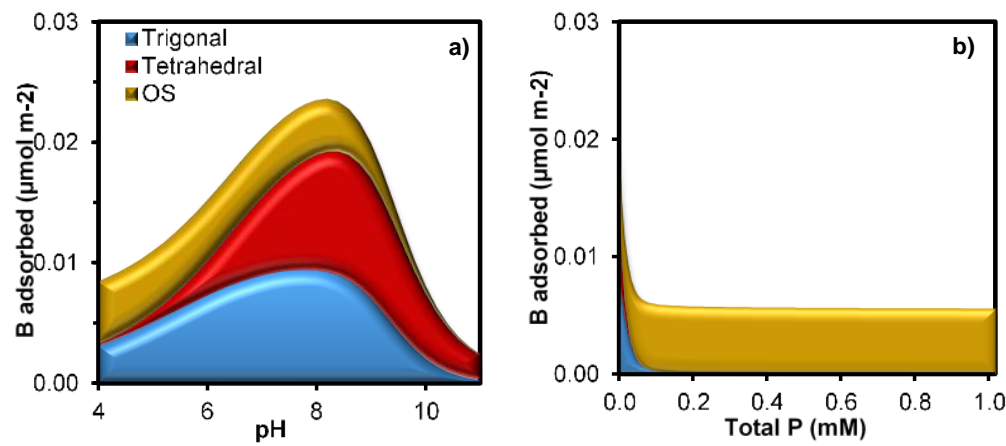

Figure 4: Surface speciation of $\mathrm{B}$ calculated in relation to $\mathrm{pH}(\mathrm{a})$ and to total $\mathrm{P}(\mathrm{b})$ in a Fh system. Boron can be bound to Fh as a trigonal or a tetrahedral inner-sphere bidentate complex, or as an outer-sphere (OS) species. Model calculations were done using the CD model (Table 1) with high affinity sites for the $\mathrm{B}$ adsorption. Parameters for the $\mathrm{PO}_{4}$ adsorption were taken from Hiemstra and Zhao ${ }^{26}$. Total $\mathrm{B}$ concentration was $0.05 \mathrm{mM}$ with a Fh concentration of $0.8 \mathrm{~g} \mathrm{~L}^{-1}$. The used surface area of Fh was $A=$ $668 \mathrm{~m}^{2} \mathrm{~g}^{-1}$. Background electrolyte was $0.01 \mathrm{M} \mathrm{NaNO}_{3}$. In Figure 4b, the $\mathrm{pH}$ was fixed at 7.

The above conclusion with respect to the relative importance of outer-sphere complexation is supported by our observations during a step-wise modeling approach. When using only the data from the mono-component systems, no $\log K$ value could be derived with certainty for the outer-sphere surface species. In the 
mono-component systems (Figure 4a), this species only contributes in the low to neutral $\mathrm{pH}$ range, and only in a minor proportion, making it difficult to distinguish this surface species in the fitting procedure from the dominating inner-sphere complex. However, including the data from the competitive adsorption experiment with $\mathrm{PO}_{4}$ made it possible to derive a log $K$ value for the outer-sphere complex with a relatively low uncertainty $( \pm 0.07)$, because at these conditions the B outer-sphere complex is the dominant species (Figure 4b). This indicates that the inclusion of competitive adsorption experiments in the derivation of binding constants can be helpful to identify and increase certainty about specific surface species and the quality of their $\log K$ values.

\subsection{Comparing $\mathrm{B}$ adsorption to $\mathrm{Fh}$ and goethite}

Natural systems consist of multiple reactive surfaces, which can adsorb B and consequently affect the $B$ speciation ${ }^{10,13,66}$. Boron may interact with the short-rangeorder iron oxides of soils (i.e. Fh) as well as crystalline iron oxides (i.e. goethite). Both ferrihydrite and goethite show for boric acid a similar bell-shaped adsorption envelope (Figure 5a) and surface loading. However, the affinity constant of trigonal bidentate species is substantially higher for ferrihydrite. We found $\log K=3.4 \pm 0.05$ for ferrihydrite (Table 1), while for goethite the value is lower, being $\log K=2.6 \pm$ $0.1^{14}$. This difference in affinity might be due to the difference in the types of reactive sites involved. The difference in affinity does not lead to a higher adsorption, probably because of the much lower reactive site density for B binding to Fh $(0.25 \pm$ $\left.0.08 \mathrm{~nm}^{-2}\right)$ compared to that of goethite $\left(3.45 \mathrm{~nm}^{-2}\right)$. For the tetragonal surface species, the $\log K$ is also significantly higher for ferrihydrite $(\log K=-5.3 \pm 0.6)$ than for goethite $(\log K=-7.7 \pm 0.4)$. This contrasts with the affinity constants for outersphere complex formation which are very similar if we correct for the difference in proton affinity of both materials, as discussed above in Section 3.4.2. The observed differences as well as similarities are consistent with the different types of sites involved in B complexation, namely ordinary (goethite) and high affinity (Fh) sites.

From Figure 5a, it is evident that Fh and goethite have a similar $\mathrm{B}$ adsorption when scaled to the surface area. This finding suggests that in terms of SCM applications to natural systems, the surface area of the metal oxide fraction is a more important parameter than the type of $\mathrm{Fe}$ (hydr)oxide used as model oxide material. However, for other oxyanions, both minerals may differ in reactivity and this may lead to another boron adsorption as the result of a different competitive behavior. If we compare the effect of $\mathrm{PO}_{4}$ on the $\mathrm{B}$ adsorption of goethite and $\mathrm{Fh}, \mathrm{CD}$ modeling predicts that the presence of $\mathrm{PO}_{4}$ decreases the $\mathrm{B}$ adsorption to goethite more strongly (Figure $5 b$ ). Although the log $K$ value for the outer-sphere $B$ surface species for both model oxides is the same, the total site density for the formation of outersphere complexes is higher for Fh, resulting in a higher $\mathrm{B}$ adsorption to Fh than to goethite at high $\mathrm{PO}_{4}$ concentrations. This observation underlines that in evaluations of the boron adsorption of soils, differentiation between both types of Fe (hydr) 
oxides may be relevant for unveiling the surface speciation of $B$. This will be further discussed in the next section.
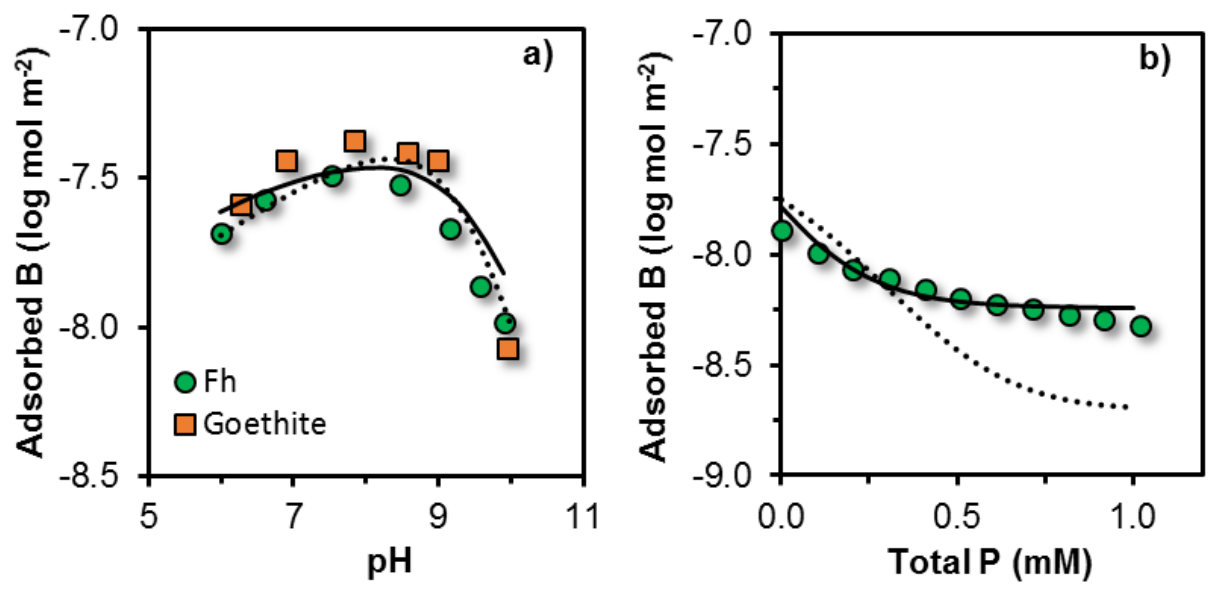

Figure 5: a) Logarithm of the $\mathrm{B}$ adsorption ( $\log \mathrm{mol} \mathrm{m}^{-2}$ ) as a function of $\mathrm{pH}$. Our data for $\mathrm{Fh}$ (circles) refer to a system with $0.1 \mathrm{mM} \mathrm{B}$ in $0.01 \mathrm{M} \mathrm{NaNO}_{3}$. The data for goethite (squares) are from Goli et al. ${ }^{14}$ and refer to a system with the same $B$ concentration but a slightly higher background electrolyte concentration ( $0.05 \mathrm{M} \mathrm{NaNO}_{3}$, which does not significantly affect the $\mathrm{B}$ adsorption, as shown by these authors). Dotted lines show the modeling results for goethite, using the $C D$ model parameters from Table 2 in Goli et al. ${ }^{14} \mathrm{~g}$, a concentration of $6 \mathrm{~g} \mathrm{~L}^{-1}$ goethite with a specific surface area of $94 \mathrm{~m}^{2} \mathrm{~g}^{-1}$. Full lines show the modeling results for Fh using the parameters from Table 1 and using high affinity sites together with a concentration of $0.81 \mathrm{~g} \mathrm{~L}^{-1} \mathrm{Fh}$ with a specific surface area of $665 \mathrm{~m}^{2} \mathrm{~g}^{-1}$. b) Logarithm of the $\mathrm{B}$ adsorption as a function of the initial $\mathrm{P}$ concentration in the Fh system at pH 7 and with $0.05 \mathrm{mM} \mathrm{B}$ in $0.01 \mathrm{M} \mathrm{NaNO}_{3}$. The data points for Fh are the same as in Figure 2. The model lines were calculated with $0.80 \mathrm{~g} \mathrm{~L}^{-1}$ and $628 \mathrm{~m}^{2} \mathrm{~g}^{-1}$ for Fh (full line), and $5 \mathrm{~g} \mathrm{~L}^{-1}$ and $100 \mathrm{~m}^{2} \mathrm{~g}^{-1}$ for goethite (dotted line), in order to have in both systems the same available surface area $\left(\mathrm{m}^{2} \mathrm{~L}^{-1}\right)$.

\subsection{Surface speciation of $B$ in soil systems}

Besides Fe (hydr)oxides, boron may also bind to natural organic matter ${ }^{10,13,44,66}$. To estimate the relative importance of organic matter for $\mathrm{B}$ adsorption, we have calculated the binding of $\mathrm{B}$ for a system containing organic matter in addition to nanocrystalline and crystalline $\mathrm{Fe}$ (hydr)oxide loaded with $\mathrm{PO}_{4}$. The $\mathrm{B}$ binding by natural organic matter has been assessed recently using the NICA-Donnan model ${ }^{44}$. For modeling the adsorption of $\mathrm{B}$ to the crystalline $\mathrm{Fe}$ (hydr)oxides, we rely on the $\mathrm{CD}$ model parameters reported for goethite ${ }^{14}$. For $\mathrm{Fh}$, the modeling results are based on the adsorption parameters obtained in this study, as given in Table 1, option B. Our model simulations were done for three agricultural soils from the Netherlands for which the data regarding reactive surfaces and $\mathrm{PO}_{4}$ loading were collected in a previous study ${ }^{50}$. The three soils differ in the amount of organic matter, $\mathrm{Fe}$ and $\mathrm{Al}$ (hydr)oxides, and phosphate loading (Table S6 (SI)). Importantly, our calculations have been done for systems with a (for soil solution more representative) background concentration of $0.01 \mathrm{M} \mathrm{CaCl}_{2}$. 

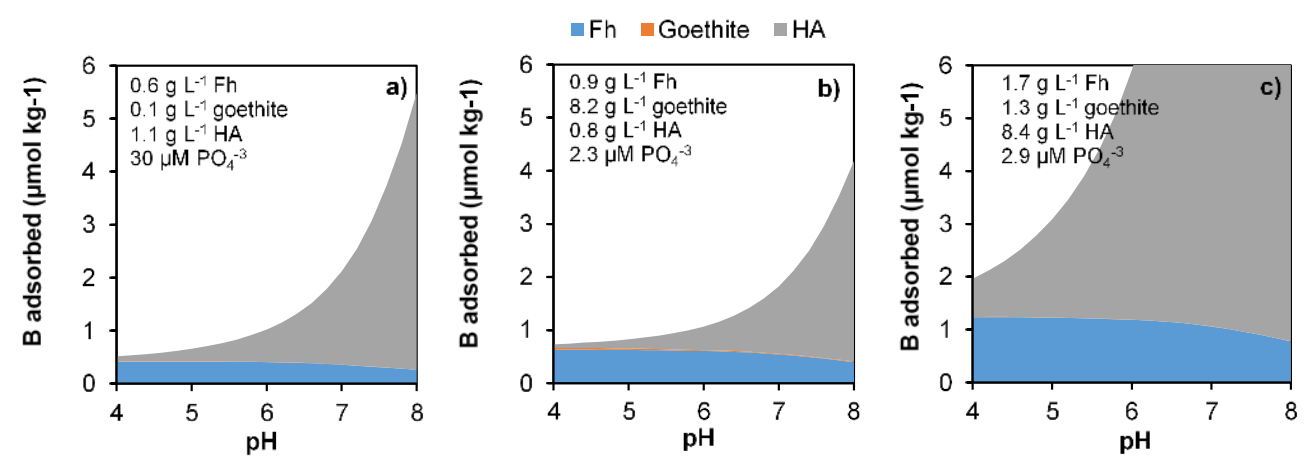

Figure 6: $\mathrm{pH}$-dependent $\mathrm{B}$ adsorption expressed in $\mu \mathrm{mol}$ per $\mathrm{kg}$ of soil. Calculations were done for a system containing natural organic matter ( $\mathrm{HA})$, nanocrystalline iron-(hydr)oxide (Fh), and crystalline $\mathrm{Fe}$ (hydr)oxide (goethite), in a background solution of $0.01 \mathrm{M} \mathrm{CaCl}_{2}$ at a solid-to-solution ratio of 1:10. The modeling scenarios are for systems with a low $(a, b)$ and high (c) organic matter content having a low (b, c) and a high (a) level of $\mathrm{PO}_{4}$ in solution. The soil oxide fraction is represented by Fh and goethite having a specific surface area of respectively 600 and $100 \mathrm{~m}^{2} \mathrm{~g}^{-1}$. Organic matter (OM) is represented by humic acids. The $B$ concentration in the solution phase is kept constant as $1 \mu \mathrm{M}$. For more details about modeling input, we refer to the supplementary information (Table S6). The B adsorption modeling to Fh was done with parameters from Table 1, using high affinity sites. The B adsorption parameters for goethite and $\mathrm{HA}$ were taken from Goli et al. ${ }^{14,44}$.

Our modeling shows that the $\mathrm{Fe}$ (hydr)oxides dominate the boron binding at low $\mathrm{pH}$. For all three soils, the contribution of goethite to the $B$ adsorption is minor. In soils with similar mass fractions of the nanocrystalline and crystalline $\mathrm{Fe}$ (hydr)oxide (Figure $5 \mathrm{c}$ ), the binding is dominated by ferrihydrite because for this oxide, the specific surface area is much higher, and the competitive effect of $\mathrm{PO}_{4}$ lower compared to goethite. There is relatively little $\mathrm{pH}$ dependency for the $\mathrm{B}$ binding to the $\mathrm{Fe}$ (hydr)oxides in the sub neutral $\mathrm{pH}$ range, the typical bell shape of the adsorption envelope observed in the mono-component oxide systems is lost for these materials due to the presence of $\mathrm{PO}_{4}$.

For organic matter, the boron envelope remains bell-shaped when extrapolated to higher $\mathrm{pH}$ values than shown in Figure 6 , as was found before ${ }^{44}$, since it is not affected by the presence of $\mathrm{PO}_{4}$. Consequently, the boron adsorption may not change importantly in alkaline soils by the addition of $\mathrm{PO}_{4}$ which was observed by Majidi et al. ${ }^{67}$ Because of this difference in $\mathrm{pH}$ dependency of the $\mathrm{B}$ adsorption, the contribution of organic matter becomes increasingly important at increasing $\mathrm{pH}$.

In soils rich in organic matter (Figure $5 \mathrm{c}$ ), the binding of $\mathrm{B}$ to organic matter is predicted to dominate the adsorption in nearly the entire $\mathrm{pH}$ range. In soils with less organic matter (Figure $5 \mathrm{a}$ and $5 \mathrm{~b}$ ), the natural metal (hydr)oxide fraction may contribute significantly at low $\mathrm{pH}$ in case of a sufficiently high content of ammonium oxalate extractable $\mathrm{Fe}$ and $\mathrm{Al}$ (i.e. similar concentration of $\mathrm{Fh}$ in terms of mass 
compared to $\mathrm{HA}$ ). In soils with a high content of organic matter (Figure $5 \mathrm{c}$ ), Fh can still contribute to about half of the $\mathrm{B}$ adsorption between $\mathrm{pH} 4$ and 5 . Our comparison of the $\mathrm{B}$ adsorption to the various reactive surfaces provide a mechanistic explanation for the suggestion from Gu and Lowe ${ }^{11}$ that in soil systems, oxides may contribute to $\mathrm{B}$ binding at a relatively low $\mathrm{pH}$.

\section{CONCLUSIONS}

In this study, we evaluated the boron adsorption by ferrihydrite nanoparticles, collecting experimental data and performing state-of-the-art surface complexation modeling. Interestingly, we found that the data could be best described when using a set of singly coordinated groups that act as high affinity sites for B adsorption to Fh. The adsorption densities that were found in our experimental window are clearly below the saturation level of these high affinity sites, and as a result, no binding to surface sites with lower affinity could be detected.

The $\mathrm{B}$ adsorption data were described using a set of surface species identified previously by spectroscopy. The adsorption at low $\mathrm{pH}$ is dominated by the formation of a trigonal bidentate surface species with a contribution of an outer-sphere complex. At high $\mathrm{pH}$, a tetrahedral bidentate complex becomes increasingly important. Our experimental data have shown that $\mathrm{PO}_{4}$ can strongly reduce the $\mathrm{B}$ adsorption on $\mathrm{Fh}$. In the presence of $\mathrm{PO}_{4}$, the only remaining surface species was found to be an outer-sphere complex, because this complex does not introduce charge into the $1^{\text {st }}$ Stern plane and therefore, it is little affected by the electrostatic repulsion induced by the adsorption of $\mathrm{PO}_{4}$.

We found that the $\mathrm{pH}$-dependent $\mathrm{B}$ adsorption density of $\mathrm{Fh}$ is similar to that of goethite, which was shown both experimentally as well as by CD modeling. However, modeling showed that the effect of $\mathrm{PO}_{4}$ on the $\mathrm{B}$ binding differs between Fh and goethite, with a larger reduction in $\mathrm{B}$ binding to goethite due to the presence of $\mathrm{PO}_{4}$. These findings imply that for natural systems the choice of a reference oxide (i.e. Fh or goethite) for describing the $B$ adsorption is important as competitive ions such as $\mathrm{PO}_{4}$ are usually present.

The derived adsorption parameters for B binding to Fh are internally consistent with those previously derived for other ions, using a common modeling framework. This work thus contributes to the enlargements of the consistent thermodynamic database for SCM using Fh as model oxide.

To gain insights into $B$ speciation in soil systems, we have assessed the importance of $\mathrm{B}$ binding to oxides relatively to organic matter, in a multi-surface modeling simulation. In the low $\mathrm{pH}$ range, the boron adsorption by organic matter is relatively low and under these conditions, the natural metal (hydr)oxide fraction and especially Fh may significantly contribute to the total boron binding if the oxalate extractable fraction of $\mathrm{Fe}$ and $\mathrm{Al}$ is sufficiently high (i.e. equal mass of $\mathrm{Fh}$ and solid humic acids). Even in soils rich in organic matter, Fh can still contribute to about half of the total $B$ 
adsorption when the $\mathrm{pH}$ is between $\mathrm{pH} 4$ and 5 . In the presence of $\mathrm{PO}_{4}$, the typical bell-shaped $\mathrm{pH}$ dependent $\mathrm{B}$ adsorption to oxides is lost, and as a result, the $\mathrm{B}$ speciation at high $\mathrm{pH}$ is predicted to be controlled by the adsorption to organic matter.

Our new adsorption parameters and state-of-the-art model predictions have important implications for understanding the B availability and mobility in soil systems at different $\mathrm{pH}$ values and with variable input of phosphate, due to for instance liming and fertilization.

\section{ACKNOWLEDGEMENTS}

This work was supported by NWO (grant number 14688, "Micronutrients for better yields"). All experiments and analyses were done in the CBLB laboratory at Wageningen University and Research. We greatly appreciate the help from Peter Nobels and Wobbe Schuurmans for the ICP-measurements. 


\section{SUPPORTING INFORMATION}

\section{A. Experimental conditions of the monocomponent and competitive batch adsorption systems}

Table S1: Overview of the experimental conditions of the four monocomponent $B$ adsorption experiments and one competitive adsorption experiment with $\mathrm{B}$ and $\mathrm{PO}_{4}$. All experiments were conducted in a background of $0.01 \mathrm{M} \mathrm{NaNO}_{3}$. For each experiment, the measured Fe concentration, the fitted SSA based on $\mathrm{PO}_{4}^{-3}$ adsorption data and the corresponding molar mass and Fh concentration are given. The $\mathrm{pH}$ values of the monocomponent systems ranged between 4.61 and 10.63 , the $\mathrm{pH}$ in the competitive adsorption experiments was $7.06 \pm 0.10$.

\begin{tabular}{cccccc}
\hline $\begin{array}{c}\text { Total B(OH) } \\
(\mathbf{m M})\end{array}$ & $\begin{array}{c}\text { Total PO }_{4} \\
(\mathbf{m M})\end{array}$ & $\begin{array}{c}\text { Total Fe } \\
(\mathbf{m M})\end{array}$ & $\begin{array}{c}\text { SSA } \\
\left(\mathbf{m}^{2} \mathbf{~ g}^{-1}\right)\end{array}$ & $\begin{array}{c}\mathbf{M}_{\text {nano }} \\
\left(\mathbf{g ~ m o l}^{-1} \mathbf{F e}\right)\end{array}$ & $\begin{array}{c}\mathbf{F h} \\
\left(\mathbf{g ~ l}^{-1}\right)\end{array}$ \\
\hline 0.41 & 0 & 7.88 & $665 \pm 17$ & 96.15 & 0.76 \\
0.25 & 0 & 8.31 & $665 \pm 13$ & 96.15 & 0.80 \\
0.10 & 0 & 8.38 & $665 \pm 9$ & 96.15 & 0.81 \\
0.05 & 0 & 7.81 & $677 \pm 15$ & 96.46 & 0.75 \\
0.05 & $0-1$ & 7.88 & $628 \pm 14$ & 95.22 & 0.75 \\
\hline
\end{tabular}


B. Thermodynamic database with $\log K$ and $C D$ values used in the $C D$ modeling

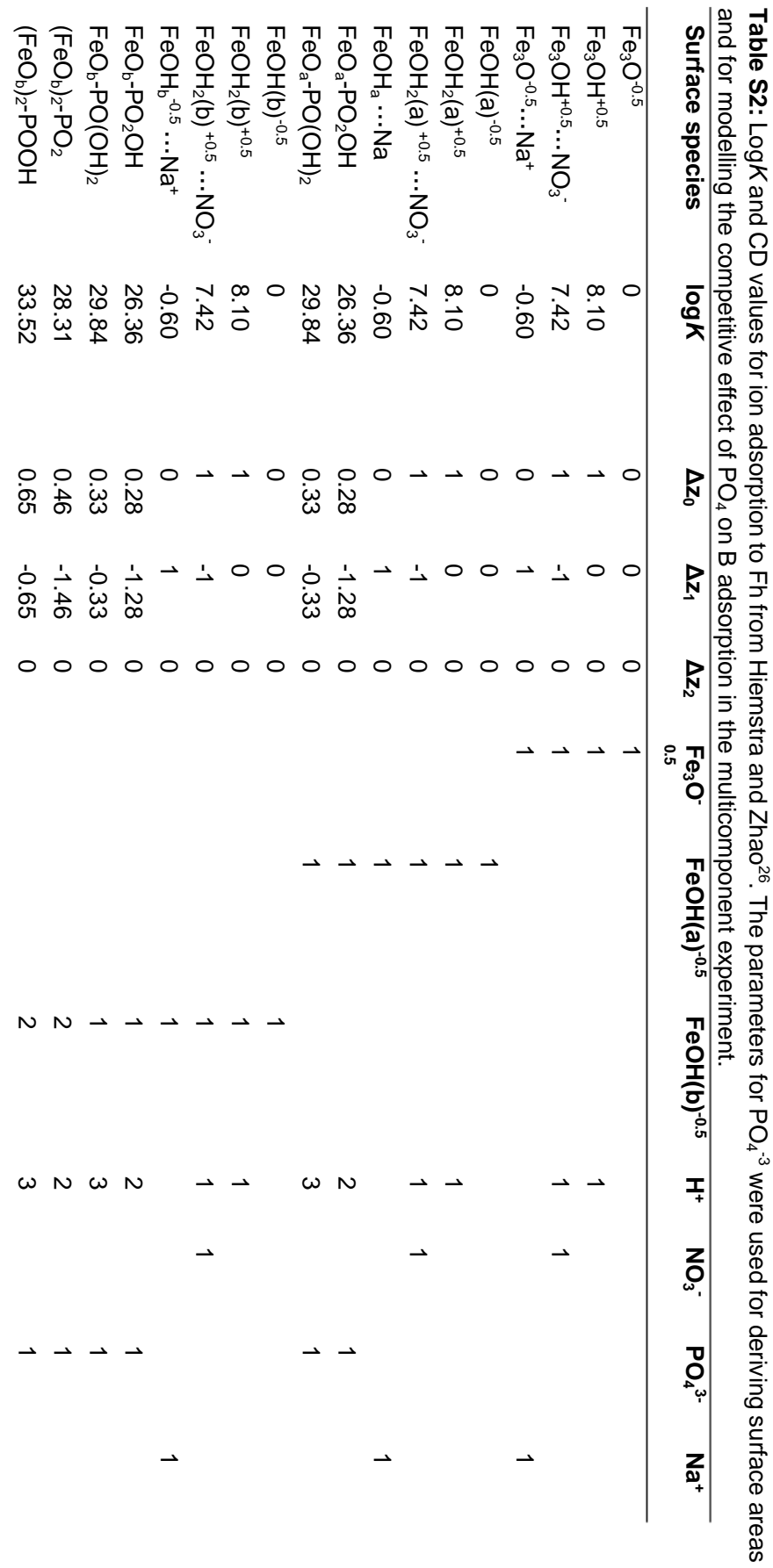




\section{Aqueous boron species used in the CD modeling}

Table S3: Aqueous Boron species included in the modelling

\begin{tabular}{lll}
\hline Species & Reaction & log $\boldsymbol{K}$ \\
\hline $\mathrm{B}(\mathrm{OH})_{4}$ & $\mathrm{~B}(\mathrm{OH})_{3} \leftrightarrow \mathrm{B}(\mathrm{OH})_{4}^{-}+\mathrm{H}^{+}$ & $-9.24^{2}$ \\
$\mathrm{~B}_{2} \mathrm{O}(\mathrm{OH})_{5}^{-}$ & $2 \mathrm{~B}(\mathrm{OH})_{3} \leftrightarrow \mathrm{B}_{2} \mathrm{O}(\mathrm{OH})_{5}+\mathrm{H}^{+}$ & $-9.31^{2}$ \\
$\mathrm{~B}_{3} \mathrm{O}_{3}(\mathrm{OH})_{4}^{-}$ & $3 \mathrm{~B}(\mathrm{OH})_{3} \leftrightarrow \mathrm{B}_{3} \mathrm{O}_{3}(\mathrm{OH})_{4}^{-}+\mathrm{H}^{+}$ & $-7.31^{2}$ \\
$\mathrm{~B}_{4} \mathrm{O}_{5}(\mathrm{OH})_{4}^{-2}$ & $4 \mathrm{~B}(\mathrm{OH})_{3} \leftrightarrow \mathrm{B}_{4} \mathrm{O}_{5}(\mathrm{OH})^{-2}+2 \mathrm{H}^{+}$ & $-15.06^{2}$ \\
$\mathrm{NaB}(\mathrm{OH})_{4}$ & $\mathrm{Na}^{+}+\mathrm{B}(\mathrm{OH})_{3} \leftrightarrow \mathrm{NaB}(\mathrm{OH})_{4}+\mathrm{H}^{+}$ & $-8.96^{68}$ \\
\hline
\end{tabular}

\section{D. $\log K$ values found by fitting at different scales when using complete set of sites for complexation with B}

Table S4: Log $K$ values found by fitting when using 59 data points, for which the conditions are described in Table S1. The evaluation scale used were $\%$ adsorbed and log $\mathrm{mol} \mathrm{m}^{-2}$. Site densities were taken as $\mathrm{Fe}_{3} \mathrm{O}=1.4, \mathrm{FeOH}(\mathrm{a})=3$ and $\mathrm{Fe}(\mathrm{OH})(\mathrm{b})=2.8$ sites $\mathrm{nm}^{-2}{ }_{2}^{26}$ For the competitive experiment, the $\mathrm{PO}_{4}$ adsorption parameters were taken from Hiemstra and $\mathrm{Zhao}^{26}$.

\begin{tabular}{|c|c|c|c|c|c|}
\hline Species & $\Delta \mathbf{z}_{\mathrm{o}}$ & $\Delta \mathbf{z}_{1}{ }^{\mathrm{a}}$ & $\Delta \mathbf{z}_{2}$ & $\begin{array}{c}\log K \\
(\% \text { adsorbed })^{a}\end{array}$ & $\begin{array}{c}\log K \\
\left(\log \mathrm{mol} \mathrm{m} \mathrm{m}^{-2}\right)^{\mathrm{b}}\end{array}$ \\
\hline$\equiv(\mathrm{FeO}(\mathrm{bh}))_{2}^{-0.82} \mathrm{~B}(\mathrm{OH})^{-0.18}$ & $0.18^{\#}$ & $-0.18^{\#}$ & 0 & $2.21 \pm 0.13$ & $2.13 \pm 0.13$ \\
\hline$\equiv(\mathrm{FeO}(\mathrm{bh}))_{2}^{-1.25} \mathrm{~B}(\mathrm{OH})_{2}^{-0.75}$ & $-0.25^{\#}$ & $-0.75^{\#}$ & 0 & $-5.85 \pm 0.09$ & $-5.84 \pm 0.07$ \\
\hline 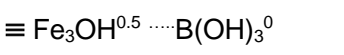 & 1 & 0 & 0 & $9.32 \pm 0.09$ & $9.34 \pm 0.06$ \\
\hline 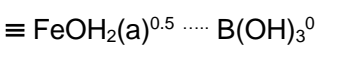 & 1 & 0 & 0 & $9.32 \pm 0.09$ & $9.34 \pm 0.06$ \\
\hline 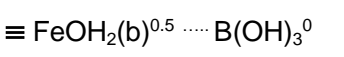 & 1 & 0 & 0 & $9.32 \pm 0.09$ & $9.34 \pm 0.06$ \\
\hline \multicolumn{6}{|c|}{$\begin{array}{l}\text { \# CD-values based on MO/DFT calculations of Goli et al. }{ }^{14} \\
\text { a When using } \% \text { adsorbed as evaluation scale, } R^{2}=0.73 \text { and RMSE }=2.60 \\
\text { b When using log mol m-2 adsorbed as evaluation scale, } R^{2}=0.91 \text { and } R M S E=0.13\end{array}$} \\
\hline
\end{tabular}




\section{E. $\log K$ values found by fitting at different scales when using a subset of high affinity sites for complexation with $B$}

Table S5: Log $K$ values found by fitting B adsorption data using 59 data points, from which the conditions are described in Table S1. The evaluation scale used were \% adsorbed and log $\mathrm{mol} \mathrm{m}^{-2}$. For the competitive experiment, the $\mathrm{PO}_{4}$ adsorption parameters were taken from Hiemstra and $\mathrm{Zhao}^{26}$. Site densities were taken as $\mathrm{Fe}_{3} \mathrm{O}=1.4, \mathrm{FeOH}(\mathrm{a})=3$ and total $\mathrm{Fe}(\mathrm{OH})(\mathrm{b})=2.8$ sites $\mathrm{nm}^{-2}{ }^{26}$ The site density of $\mathrm{FeOH}$ (bh) was found by fitting as $0.24 \pm 0.06$ when using $\%$ as evaluation scale, and $0.25 \pm 0.09$ when using log $\mathrm{mol} \mathrm{m}^{-2}$ adsorbed as evaluation scale.

\begin{tabular}{|c|c|c|c|c|c|}
\hline Species & $\Delta \mathbf{z}_{\mathrm{o}}$ & $\Delta \mathbf{z}_{1}^{\mathrm{a}}$ & $\Delta \mathbf{z}_{2}$ & $\begin{array}{c}\log K(\% \\
\text { adsorbed) a }\end{array}$ & $\begin{array}{c}\log K \\
\left(\log \mathrm{mol} \mathrm{m}^{-2}\right)^{b}\end{array}$ \\
\hline$\equiv(\mathrm{FeO}(\mathrm{bh}))_{2}^{-0.82} \mathrm{~B}(\mathrm{OH})^{-0.18}$ & $0.18^{\#}$ & $-0.18^{\#}$ & 0 & $3.42 \pm 0.14$ & $3.35 \pm 0.22$ \\
\hline$\equiv(\mathrm{FeO}(\mathrm{bh}))_{2}{ }^{-1.25} \mathrm{~B}(\mathrm{OH})_{2}^{-0.75}$ & $-0.25^{\#}$ & $-0.75^{\#}$ & 0 & $-4.64 \pm 0.18$ & $-4.71 \pm 0.21$ \\
\hline$\equiv \mathrm{Fe}_{3} \mathrm{OH}^{0.5 \ldots . .} \mathrm{B}(\mathrm{OH})_{3}{ }^{0}$ & 1 & 0 & 0 & $9.31 \pm 0.08$ & $9.32 \pm 0.06$ \\
\hline 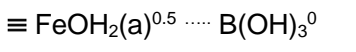 & 1 & 0 & 0 & $9.31 \pm 0.08$ & $9.32 \pm 0.06$ \\
\hline$\equiv \mathrm{FeOH}_{2}(\mathrm{~b})^{0.5 \cdots \cdots} \mathrm{B}(\mathrm{OH})_{3}{ }^{0}$ & 1 & 0 & 0 & $9.31 \pm 0.08$ & $9.32 \pm 0.06$ \\
\hline 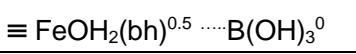 & 1 & 0 & 0 & $9.31 \pm 0.08$ & $9.32 \pm 0.06$ \\
\hline \multicolumn{6}{|c|}{$\begin{array}{l}\text { \# CD-values based on MO/DFT calculations of Goli et al. }{ }^{14} \\
\text { a When using } \% \text { adsorbed as evaluation scale, } R^{2}=0.81 \text { and RMSE }=2.21 \\
{ }^{b} \text { When using log mol m-2 adsorbed as evaluation scale, } R^{2}=0.92 \text { and } R M S E=0.12\end{array}$} \\
\hline
\end{tabular}




\section{F. Soil properties of representative top soils of the Netherlands for multi- surface modeling of B considering metal (hydr)oxides and organic matter}

Table S6: Soil properties for modelling the $\mathrm{pH}$-dependent $\mathrm{B}$ speciation. The amount of nanocrystalline oxide represented by $\mathrm{Fh}$ in the modeling was calculated based on the $\mathrm{Fe}$ and $\mathrm{Al}$ measured in ammonium oxalate extracts, using a molar mass of 95 and $84 \mathrm{~g} \mathrm{~mol}^{-1}$ respectively, which is consistent with a surface area of $600 \mathrm{~m}^{2} \mathrm{~g}^{-1}$. Goethite concentration was calculated based on the difference of $\mathrm{Fe}$ in dithionite and ammonium oxalate extraction, using a molar mass of $89 \mathrm{~g} \mathrm{~mol}^{-1}$ and SSA of $100 \mathrm{~m}^{2} \mathrm{~g}^{-1}$. The Fe and Al concentrations originate from a study by Hiemstra et al..$^{50}$ for top soils representative for the Netherlands. The $\mathrm{PO}_{4}$ concentration in solution was kept constant according to the concentration measured in $0.01 \mathrm{M}$ $\mathrm{CaCl}_{2}$ as reported by Hiemstra et al. ${ }^{50}$ The amounts of humic acid were calculated based on the estimation that $30 \%$ of total SOM is reactive humic acid, and assuming $50 \%$ carbon in the organic matter. Modelling of the adsorption by humic acids was done with the NICA-Donnan model. For the oxides, the $\mathrm{CD}$ and MUSIC model approach was used for the respective oxide. $\mathrm{PO}_{4}$ adsorption on ferrihydrite and goethite was modelled with the parameters from Hiemstra and $\mathrm{ZhaO}^{26}$ and Hiemstra et al ${ }^{50}$. Ca adsorption by humic acids was modelled with parameters from Milne et al. ${ }^{57}$. For ferrihydrite, the model parameters from Mendez and Hiemstra ${ }^{41}$ were used comprising the formation of $\mathrm{Ca}$ and $\mathrm{Mg}$ surface complexes as well as the formation of ternary surface complexes of $\mathrm{PO}_{4}$ with $\mathrm{Ca}$ and $\mathrm{Mg}$. The $\mathrm{Ca}$ adsorption parameters for goethite were taken from Hiemstra et al. ${ }^{50}$

\begin{tabular}{llllll}
\hline & $\begin{array}{l}\text { Ferrihydrite } \\
\left(\mathrm{kg} \mathrm{l}^{-1}\right)\end{array}$ & $\begin{array}{l}\text { Goethite } \\
\left(\mathrm{kg} \mathrm{l}^{-1}\right)\end{array}$ & $\begin{array}{l}\text { SHA } \\
\left(\mathrm{kg} \mathrm{l}^{-1}\right)\end{array}$ & $\begin{array}{l}\mathbf{P O}_{4}^{-3} \\
(\mu \mathrm{M})\end{array}$ & $\begin{array}{l}\mathrm{H}_{3} \mathrm{BO}_{3} \\
(\mu \mathrm{M})\end{array}$ \\
\hline $\begin{array}{l}\text { Low OM, } \\
\text { high } \boldsymbol{P}^{\mathbf{a}}\end{array}$ & $5.97 \mathrm{E}-04$ & $1.39 \mathrm{E}-04$ & $1.08 \mathrm{E}-03$ & 30 & 1 \\
$\begin{array}{l}\text { Low OM, } \\
\text { low } \boldsymbol{P}^{\mathbf{b}}\end{array}$ & $8.89 \mathrm{E}-04$ & $8.21 \mathrm{E}-03$ & $7.80 \mathrm{E}-04$ & 2.3 & 1 \\
$\begin{array}{l}\text { High OM, } \\
\text { low } \boldsymbol{P}^{\mathbf{c}}\end{array}$ & $1.74 \mathrm{E}-03$ & $1.26 \mathrm{E}-03$ & $8.40 \mathrm{E}-03$ & 2.9 & 1 \\
\hline
\end{tabular}




\section{G. Modeling calculations when assuming B innersphere complex formation by the entire set of $\mathrm{FeOH}(\mathrm{b})$ sites}
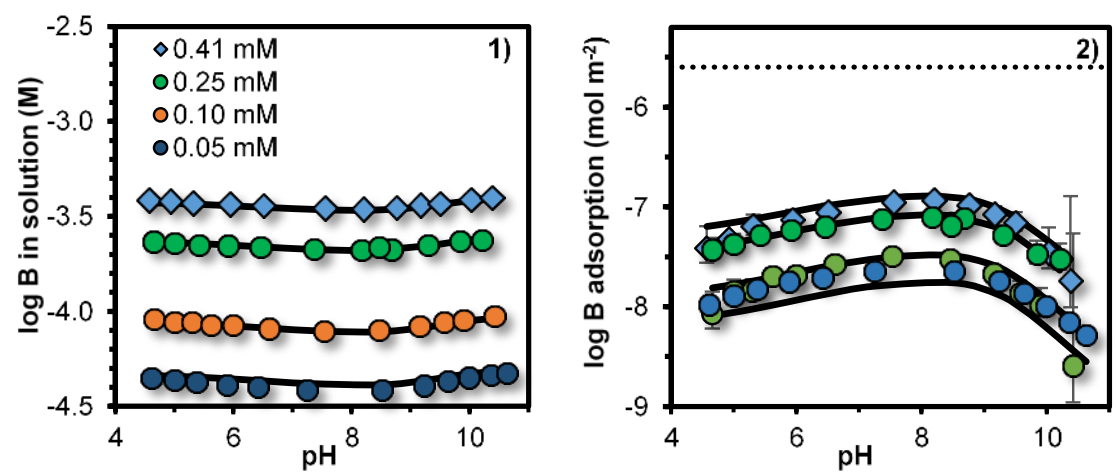

Figure S1 and S2: Logarithm of the equilibrium concentration (1) and adsorption of B to Fh (2) in a background solution of $0.01 \mathrm{M} \mathrm{NaNO}_{3}$. Solid lines represent the modelling results assuming adsorption to the singly coordinated groups $\mathrm{Fe}(\mathrm{OH})(\mathrm{b})$ with site density of $2.8 \mathrm{~nm}^{-2}$. The corresponding maximum adsorption of boron to these sites as a bidentate complex is given as the black-dotted line in figure S2. The error bars in figure S2 represent the variation in adsorbed B when assuming an uncertainty of just $2 \%$ in the measured B concentration in solution. The specific experimental conditions for systems can be found in Table S1. 


\section{H. Adsorption data of $B$ to ferrihydrite from literature}
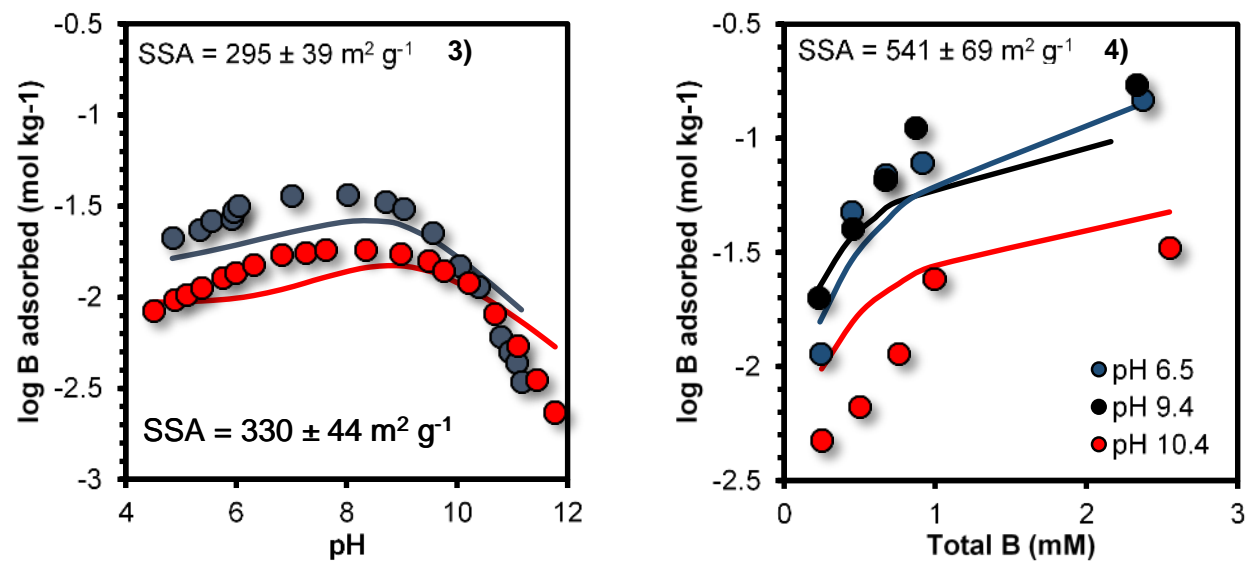

Figure S3 and S4: Evaluation of the experimental data from Goldberg and Glaubig ${ }^{15}$ (blue markers in Figure S3), from Su and Suarez ${ }^{16}$ (red markers in Figure S3) and Peak et al. ${ }^{17}$ (Figure S4). For specific experimental conditions, we refer to the corresponding publications. In the modeling, the specific surface area of the material was taken as the only adjustable parameter and the adsorption parameters for $\mathrm{B}$ were taken from our study (Table 1 in the main text, scenario B with high affinity sites). It has been shown that the mode of preparing and storing the Fh affects the particle size and consequently the specific surface area $^{27}$.

The Fh material of Goldberg and Glaubig ${ }^{15}$ was freeze-dried. The specific surface area found here for this Fh material, based on the $\mathrm{B}$ adsorption data, agrees with the surface area of found by interpreting $\mathrm{H}$ adsorption data of other freeze-dried Fh materials $\left(\sim 300 \mathrm{~m}^{2} \mathrm{~g}^{-1}\right)^{1}$.

The Fh preparation from Su and Suare ${ }^{16}$ was air-dried, but subsequently ground to pass a $50 \mu \mathrm{m}$ sieve. It is not clear how this could affect surface area and other surface properties, but a similar SSA was found here as for a frieze-dried Fh product.

The Fh from Peak et al. ${ }^{17}$ was made based on the protocol from Schwertmann and Cornell ${ }^{69}$ and kept in suspension until the $B$ adsorption experiment was done, but the aging time before the actual experiment was not reported. This can explain the fitted specific surface area to be lower than $600 \mathrm{~m}^{2} \mathrm{~g}^{-1}$.

At low $\mathrm{pH}$, our modelling parameters seem to under estimate the $\mathrm{B}$ adsorption that was experimentally measured by Goldberg and Glaubig ${ }^{15}$ and Su and Suarez ${ }^{16}$ (Figure S3). The experimental data from Peak et al. ${ }^{17}$ is reasonably well described with the adsorption parameters for B to Fh that we found in our study.

\section{REFERENCES}

1. Uluisik, I., Karakaya, H. C. \& Koc, A. The importance of boron in biological systems. J. Trace Elem. Med. Biol. 45, 156-162 (2018).

2. Bassett, R. L. L. A critical evaluation of the thermodynamic data for boron ions, ion pairs, complexes, and polyanions in aqueous solution at $298.15 \mathrm{~K}$ and 1 bar. Geochim. Cosmochim. Acta 44, 1151-1160 (1980).

3. Kabata-Pendias, A. \& Pendias, H. Trace Elements in Soils and Plants, Third Edition. www.crcpress.com (2001).

4. Shorrocks, V. M. The occurrence and correction of boron deficiency. Plant Soil 193, 121-148 (1997).

5. Howe, P. D. A Review of Boron Effects in the Environment. Biol. Trace Elem. Res. 66, 153-166 (1998).

6. Gupta, U. C., Jame, Y. W., Campbell, C. A., Leyshon, A. J. \& Nicholaichuk, An. W. Boron toxicity and deficiency: a review. Can. J. Soil Sci. 65, 381-409 (1985).

7. Goldberg, S. Reactions of boron with soils. Plant Soil 193, 35-48 (1997).

8. Keren, R., Bingham, F. T. \& Rhoades, J. D. Plant Uptake of Boron as Affected by Boron Distribution Between Liquid and Solid Phases in Soil. Soil Sci. Soc. Am. J. 49, 297-302 (1985).

9. Kot, F. S. Boron sources, speciation and its potential impact on health. vol. 8 3-28 (Springer 
Netherlands, 2009).

10. Goldberg, S. Chemical modeling of boron adsorption by humic materials using the constant capacitance model. Soil Sci. 179, 561-567 (2014).

11. Gu, B. \& Lowe, L. E. E. Studies on the adsorption of boron on humic acids. Can. J. Soil Sci. 70, 305-311 (1990).

12. Keren, R. \& Communar, G. Boron Sorption on Wastewater Dissolved Organic Matter: pH Effect. Soil Sci. Soc. Am. J. 73, 2021-2025 (2009).

13. Lemarchand, E., Schott, J. \& Gaillardet, J. Boron isotopic fractionation related to boron sorption on humic acid and the structure of surface complexes formed. Geochim. Cosmochim. Acta 69, 3519-3533 (2005).

14. Goli, E., Rahnemaie, R., Hiemstra, T. \& Malakouti, M. J. The interaction of boron with goethite: Experiments and CD-MUSIC modeling. Chemosphere 82, 1475-1481 (2011).

15. Goldberg, S. \& Glaubig, R. A. Boron Adsorption on Aluminum and Iron Oxide Minerals 1. Soil Sci. Soc. Am. J. 49, 1374-1379 (1985).

16. Su, C. \& Suarez, D. L. Coordination of Adsorbed Boron: A FTIR Spectroscopic Study. Environ. Sci. Technol. 29, 302-311 (1995).

17. Peak, D., Luther, G. W. \& Sparks, D. L. ATR-FTIR spectroscopic studies of boric acid adsorption on hydrous ferric oxide. Geochim. Cosmochim. Acta 67, 2551-2560 (2003).

18. Kurokawa, R. \& Kamura, K. Adsorption of Boron by Volcanic Ash Soils Distributed in Japan. Soil Sci. Soc. Am. J. 82, 671-677 (2018).

19. Jambor, J. L. \& Dutrizac, J. E. Occurrence and Constitution of Natural and Synthetic Ferrihydrite, a Widespread Iron Oxyhydroxide. Chem. Rev. 98, 2549-2585 (1998).

20. Groenenberg, J. E. \& Lofts, S. The use of assemblage models to describe trace element partitioning, speciation, and fate: a review. Environ. Toxicol. Chem. 33, 2181-96 (2014).

21. Dzombak, D. A. \& Morel, F. F. M. M. Surface Complexation Modeling: Hydrous Ferric Oxide. (John Wiley \& Sons, 1990).

22. Goldberg, S. Reanalysis of boron adsorption on soils and soil minerals using the constant capacitance model. Soil Sci. Soc. Am. J. 63, 823-829 (1999).

23. Goldberg, S. Use of Surface Complexation Models in Soil Chemical Systems. Adv. Agron. 47, 233-329 (1992).

24. Stern, O. ZUR THEORIE DER ELEKTROLYTISCHEN DOPPELSCHICHT. Zeitschrift für Elektrochemie und Angew. Phys. Chemie 30, 508-516 (1924).

25. Hiemstra, T. \& Van Riemsdijk, W. H. A Surface Structural Approach to Ion Adsorption: The Charge Distribution (CD) Model. J. Colloid Interface Sci. 179, 488-508 (1996).

26. Hiemstra, T. \& Zhao, W. Reactivity of ferrihydrite and ferritin in relation to surface structure, size, and nanoparticle formation studied for phosphate and arsenate. Environ. Sci. Nano 3, 12651279 (2016).

27. Mendez, J. C. \& Hiemstra, T. Surface area of ferrihydrite consistently related to primary surface charge, ion pair formation, and specific ion adsorption. Chem. Geol. 532, (2020).

28. Michel, F. M. et al. The structure of ferrihydrite, a nanocrystalline material. Science (80-. ). 316, 1726-1729 (2007).

29. Hiemstra, T. Surface and mineral structure of ferrihydrite. Geochim. Cosmochim. Acta 105, 316325 (2013).

30. Hiemstra, T. Surface structure controlling nanoparticle behavior: Magnetism of ferrihydrite, magnetite, and maghemite. Environ. Sci. Nano 5, 752-764 (2018).

31. Bompoti, N., Chrysochoou, M. \& Machesky, M. Surface structure of ferrihydrite: Insights from modeling surface charge. Chem. Geol. 464, 34-45 (2017).

32. Hiemstra, T., Mendez, J. C. \& Li, J. Evolution of the reactive surface area of ferrihydrite: Time, $\mathrm{pH}$, and temperature dependency of growth by Ostwald ripening. Environ. Sci. Nano 6, 820-833 (2019).

33. Davis, J. A. \& Leckie, J. O. Surface ionization and complexation at the oxide/water interface II. Surface properties of amorphous iron oxyhydroxide and adsorption of metal ions. J. Colloid Interface Sci. 67, 90-107 (1978).

34. Weidler, P. G. BET sample pretreatment of synthetic ferrihydrite and its influence on the determination of surface area and porosity. J. Porous Mater. 4, 165-169 (1997).

35. van der Giessen, A. A. The structure of iron (III) oxide-hydrate gels. J. Inorg. Nucl. Chem. 28, 2155-2159 (1966).

36. Brown, I. D. \& Altermatt, D. Bond-valence parameters obtained from a systematic analysis of the Inorganic Crystal Structure Database. Acta Crystallogr. Sect. B Struct. Sci. 41, 244-247 (1985).

37. Brown, I. D. Recent developments in the methods and applications of the bond valence model. 
Chem. Rev. 109, 6858-6919 (2009).

38. Mendez, J. C. \& Hiemstra, T. Carbonate Adsorption to Ferrihydrite: Competitive Interaction with Phosphate for Use in Soil Systems. ACS Earth Sp. Chem. 3.1, 129-141 (2018).

39. Hiemstra, T. Ferrihydrite interaction with silicate and competing oxyanions: Geometry and Hydrogen bonding of surface species. Geochim. Cosmochim. Acta 238, 453-476 (2018).

40. Mendez, J. C. \& Hiemstra, T. High and low affinity sites of ferrihydrite for metal ion adsorption: Data and modeling of the alkaline-earth ions $\mathrm{Be}, \mathrm{Mg}, \mathrm{Ca}, \mathrm{Sr}, \mathrm{Ba}$, and $\mathrm{Ra}$. Geochim. Cosmochim. Acta (2020) doi:10.1016/j.gca.2020.07.032.

41. Mendez, J. C. \& Hiemstra, T. Ternary complex formation of phosphate with $\mathrm{Ca}$ and $\mathrm{Mg}$ ions binding to ferrihydrite: Experiments and mechanisms. ACS Earth Sp. Chem. 4.4, acsearthspacechem.9b00320 (2020).

42. Lemarchand, E., Schott, J. \& Gaillardet, J. How surface complexes impact boron isotope fractionation: Evidence from $\mathrm{Fe}$ and $\mathrm{Mn}$ oxides sorption experiments. Earth Planet. Sci. Lett. 260, 277-296 (2007).

43. Kim, Y. \& Kirkpatrick, R. J. 11B NMR investigation of boron interaction with mineral surfaces: Results for boehmite, silica gel and illite. Geochim. Cosmochim. Acta 70, 3231-3238 (2006).

44. Goli, E., Hiemstra, T. \& Rahnemaie, R. Interaction of boron with humic acid and natural organic matter: Experiments and modeling. Chem. Geol. 515, 1-8 (2019).

45. Tiberg, C., Sjöstedt, C., Persson, I. \& Gustafsson, J. P. Phosphate effects on copper(II) and lead(II) sorption to ferrihydrite. Geochim. Cosmochim. Acta 120, 140-157 (2013).

46. Hiemstra, T. \& Van Riemsdijk, W. H. On the relationship between charge distribution, surface hydration, and the structure of the interface of metal hydroxides. J. Colloid Interface Sci. 301, 118 (2006).

47. Hiemstra, T. \& Van Riemsdijk, W. H. A surface structural model for ferrihydrite I: Sites related to primary charge, molar mass, and mass density. Geochim. Cosmochim. Acta 73, 4423-4436 (2009).

48. Keizer, M. G. \& Van Riemsdijk, W. H. ECOSAT, a Computer Program for the Calculation of Chemical Speciation and Transport in Soil-Water Systems. (1995).

49. Kinniburgh, D. G. FIT User Guide, Technical Report WD/93/23. (1993).

50. Hiemstra, T., Antelo, J., Rahnemaie, R. \& van Riemsdijk, W. H. Nanoparticles in natural systems I: The effective reactive surface area of the natural oxide fraction in field samples. Geochim. Cosmochim. Acta 74, 41-58 (2010).

51. Dijkstra, J. J., Meeussen, J. C. L. \& Comans, R. N. J. Evaluation of a Generic Multisurface Sorption Model for Inorganic Soil Contaminants. Environ. Sci. Technol. 43, 6196-6201 (2009).

52. Groenenberg, J. E., Römkens, P. F. A. M., Zomeren, A. Van, Rodrigues, S. M. \& Comans, R. N. $\mathrm{J}$. Evaluation of the Single Dilute (0.43 M) Nitric Acid Extraction to Determine Geochemically Reactive Elements in Soil. Environ. Sci. Technol. 51, 2246-2253 (2017).

53. Weng, L., Temminghoff, E. J. M. \& Van Riemsdijk, W. H. Contribution of Individual Sorbents to the Control of Heavy Metal Activity in Sandy Soil. Environ. Sci. Technol. 35, 4436-4443 (2001).

54. Duffner, A., Weng, L., Hoffland, E. \& van der Zee, S. E. A. T. M. Multi-surface modeling to predict free zinc ion concentrations in low-zinc soils. Environ. Sci. Technol. 48, 5700-5708 (2014).

55. Degryse, F., Broos, K., Smolders, E. \& Merckx, R. Soil solution concentration of Cd and Zn canbe predicted with a $\mathrm{CaCl} 2$ soil extract. Eur. J. Soil Sci. 54, 149-158 (2003).

56. Novozamsky, I., Barrera, L. L., Houba, V. J. G., van der Lee, J. J. \& van Eck, R. Comparison of a hot water and cold $0.01 \mathrm{M} \mathrm{Cacl}_{2}$ extraction procedures for the determination of boron in soil. Commun. Soil Sci. Plant Anal. 21, 2189-2195 (1990).

57. Milne, C. J., Kinniburgh, D. G., van Riemsdijk, W. H. \& Tipping, E. Generic NICA-Donnan Model Parameters for Metal-Ion Binding by Humic Substances. Environ. Sci. Technol. 37, 958-971 (2003).

58. Tiberg, C., Sjöstedt, C. \& Gustafsson, J. P. Metal sorption to Spodosol Bs horizons: Organic matter complexes predominate. Chemosphere 196, 556-565 (2018).

59. Rietra, R. P. J. . J. J., Hiemstra, T. \& van Riemsdijk, W. H. The relationship between molecular structure and ion adsorption on variable charge minerals. Geochim. Cosmochim. Acta 63, 30093015 (1999).

60. Perona, M. . \& Leckie, J. . Proton stoichiometry for the adsorption of cations on oxide surfaces. J. Colloid Interface Sci. 106, 64-69 (1985).

61. Xu, D. \& Peak, D. Adsorption of Boric Acid on Pure and Humic Acid Coated am-Al(OH)3: A Boron K-Edge XANES Study. Environ. Sci. Technol. 41, 903-908 (2007).

62. Hiemstra, T., Riemsdijk, W. H. Van, Rossberg, A. \& Ulrich, K.-U. A surface structural model for ferrihydrite II: Adsorption of uranyl and carbonate. Geochim. Cosmochim. Acta 73, 4437-4451 (2009). 


\section{Chapter 4}

63. Benjamin, M. M. \& Leckie, J. O. Multiple-site adsorption of $\mathrm{Cd}, \mathrm{Cu}, \mathrm{Zn}$, and $\mathrm{Pb}$ on amorphous iron oxyhydroxide. J. Colloid Interface Sci. 79, 209-221 (1981).

64. Swedlund, $\mathrm{P}$. Modelling $\mathrm{Cu}, \mathrm{Zn}, \mathrm{Cd}$ and $\mathrm{Pb}$ Adsorption by Iron Oxyhydroxides in $\mathrm{SO} 4$-rich Systems Simulating Acid Mine Drainage. (2004).

65. Tiberg, C. \& Gustafsson, J. P. Phosphate effects on cadmium(II) sorption to ferrihydrite. J. Colloid Interface Sci. 471, 103-111 (2016).

66. Gu, B. \& Lowe, L. E. Studies on the ad sorption of boron on humic acids. Can. J. Soil Sci. 70, 305-311 (1990).

67. Majidi, A., Rahnemaie, R., Hassani, A. \& Malakouti, M. J. Adsorption and desorption processes of boron in calcareous soils. Chemosphere 80, 733-739 (2010).

68. Pokrovski, G. S., Schott, J. \& Sergeyev, A. S. Experimental determination of the stability constants of NaSO4- and $\mathrm{NaB}(\mathrm{OH}) 40$ in hydrothermal solutions using a new high-temperature sodium-selective glass electrode - Implications for boron isotopic fractionation. Chem. Geol. 124, 253-265 (1995).

69. Schwertmann, U. \& Cornell, R. M. Iron Oxides in the Laboratary: Preparation and Characterization. vol. 56 (Wiley-VCH Verlag GmbH, 2000). 


\section{Chapter 5}

Interaction of $\mathrm{Zn}$ with ferrihydrite and its cooperative binding in the presence of $\mathrm{PO}_{4}$

Elise Van Eynde, Tjisse Hiemstra, Rob N.J. Comans

Submitted for publication 


\section{ABSTRACT}

In nature, zinc $(\mathrm{Zn})$ ions interact with natural organic matter and soil metal (hydr)oxides. The resulting solid-solution partitioning is of major importance for the geochemical cycling and environmental risks of toxicity and deficiency. Ferrihydrite (Fh) is an important proxy for the natural metal (hydr)oxide fraction in soils. At its surfaces, $\mathrm{Zn}^{2+}$ ions have multi-component interactions, for instance with phosphate $\left(\mathrm{PO}_{4}{ }^{3-}\right)$. In nature, the latter is particularly relevant in topsoils since $\mathrm{PO}_{4}$ is omnipresent and has a high affinity for Fh. Zinc and phosphate ions may bind cooperatively by electrostatic forces only and/or by ternary complex formation. This was studied presently with batch adsorption experiments using freshly prepared and well-characterized $\mathrm{Fh}$ nanoparticles. The data have been interpreted using the charge distribution (CD) model combined with a surface structural model for Fh that includes site heterogeneity. The CD coefficients have been derived independently by optimization of the surface geometries with molecular orbital calculations applying density functional theory (MO/DFT/B3LYP/6-31+G**). These computations confirm that upon adsorption $\mathrm{Zn}$ can change spontaneously its coordination number from 6 to 4. In agreement with X-ray absorption spectroscopy (XAS), $\mathrm{Zn}$ is bound at low loading as a double-corner bidentate complex. At higher loading, the number of ions in the second shell of $\mathrm{Zn}$ decreases according to XAS. Our model with the formation of surface complexes with single corner-sharing can quantitatively predict this decrease. $\mathrm{Zn}$ polymerization only occurs at a very high molar $\mathrm{Zn} / \mathrm{Fe}$ ratio $(>0.1)$, which can be described using a neutral, hydrolyzed $\mathrm{Zn}$-dimer species. The presence of $\mathrm{PO}_{4}$ enhances the $\mathrm{Zn}$ adsorption, especially in the $\mathrm{pH}$ range 5-6. At the $\mathrm{Zn}$ and $\mathrm{PO}_{4}$ levels studied, no ternary $\mathrm{Zn}-\mathrm{P}$ surface complexes can be revealed. For comparison, we re-interpreted $\mathrm{Cu}-\mathrm{PO}_{4}-\mathrm{Fh}$ adsorption literature data with similar metal and $\mathrm{PO}_{4}$ loadings. In line with the results for $\mathrm{Zn}$, no ternary Cu-P surface complexes were found. We conclude that electrostatic interactions explain metal adsorption at background levels in multi-component systems, and that ternary complexes may form only at rather extreme metal or $\mathrm{PO}_{4}$ loading conditions. 


\section{INTRODUCTION}

Elaboration of the mechanisms that control zinc (Zn) speciation in soils is of major importance to understand the fate and cycling of this element in nature. It is also crucial for optimizing various management practices since it is estimated that $1 / 3^{\text {rd }}$ of the soils worldwide contain suboptimal levels of plant-available $\mathrm{Zn}^{1}$. Moreover, $\mathrm{Zn}$ contaminated soils can lead to serious environmental problems ${ }^{2,3}$.

Predicting the risk of deficiencies and toxicities of trace elements such as $\mathrm{Zn}$ in the environment requires a thorough understanding of their interaction with reactive surfaces that control the solid-solution partitioning. Surface complexation modeling (SCM) and spectroscopic analyses have shown that, next to soil organic matter, iron (hydr)oxides can be important for controlling soil $\mathrm{Zn}$ speciation in contaminated sites $^{4-6}$ and in agricultural fields with low $\mathrm{Zn}$ levels ${ }^{7}$.

Natural systems have a wide variety of metal (hydr)oxides with variable chemical composition and crystallinity, which results in different adsorption interactions with nutrients and pollutants. Within this heterogenic natural oxide fraction, ferrihydrite (Fh) is the most reactive material due to its very small size and the corresponding very high specific surface area ${ }^{8}$. Moreover, it has been shown recently that Fh is a good proxy for describing the overall reactivity of the natural oxide fraction for a set of Dutch soils ${ }^{9}$ as well as soils from tropical climates ${ }^{10}$.

The interaction between $\mathrm{Zn}$ and ferrihydrite nanoparticles has been studied with adsorption experiments $\mathrm{s}^{11,12}$ and with spectroscopy ${ }^{13-16}$. Surface complexation modeling can be used to quantify the variation of the adsorption with the solution conditions. To date, $\mathrm{Zn}$ adsorption to Fh has mostly been interpreted with the general two-layer model (GTLM) ${ }^{17}$. This model approach uses a hypothetical site density that cannot be related to the surface structure of Fh nanoparticles. Additionally, the GTLM simplifies the interface to a single plane in which inner-sphere adsorbed complexes exist as point charges. A more realistic physical representation of the interface is a double layer model in which surface charge and counter-charge are separated by a Stern layer ${ }^{18}$. The combination of the Charge Distribution model (CD) and a Multisite Ion Complexation model (MUSIC) can constrain thermodynamic approaches by defining reactive surface sites based on a surface structural analysis of the oxide particle (Hiemstra and Van Riemsdijk, 1999), and considering inner-sphere surface complexes as spatial moieties that distribute the corresponding charge over more than one electrostatic position by introducing one or more Stern layers in the interface model ${ }^{20}$.

The charge distribution of surface complexes over different electrostatics planes is determined by the specific type of surface species. A major factor is the relative distribution of the ligands in the interface, and secondly, the corresponding bond lengths within the adsorption complex. For the $\mathrm{Zn}$ ion, the coordination state can vary between 2 and 8 , with tetrahedral and octahedral coordination being most 
common ${ }^{21}$. In an aqueous solution, $\mathrm{Zn}$ is present in octahedral coordination, as it is surrounded by 6 water molecules as $\mathrm{Zn}\left(\mathrm{OH}_{2}\right)_{6}{ }^{2+}{ }^{22}$. Spectroscopic measurements have shown that upon adsorption, the coordination number can change depending on the type of adsorbent, the $\mathrm{pH}$, and the $\mathrm{Zn}$ loading at the surface. For example, the coordination of $\mathrm{Zn}$ adsorbed to goethite is octahedral with a typical bond length of $d z n-0=210 p m^{23}$, while the $Z n$ adsorbed to magnetite and hematite is a mixture of tetrahedrally and octahedrally coordinated adsorbed $\mathrm{Zn}^{24,25}$. Tetrahedrally coordinated surface species with $d z n-0=197 \mathrm{pm}$ have been proposed for the $\mathrm{Zn}$ adsorption to ferrihydrite based on spectroscopic measurements ${ }^{13-16,25}$. Depending on the loading, one or two second-shell $\mathrm{Fe}$ or $\mathrm{Zn}$ ions can be present in the coordination shell of $\mathrm{Zn}^{14}$. The Fe-Zn distances are in the range of 340-350 pm, which has been attributed to the formation of double corner complexes. Cismasu et al. ${ }^{16}$ resolved the contribution of an additional surface complex with an $\mathrm{Fe}-\mathrm{Zn}$ distance of $325 \mathrm{pm}$ that has about 1 neighbor in the second shell. No solid explanation has yet been put forward, particularly with respect to the low number of second neighbors. At a high $\mathrm{Zn}$ loading, $\mathrm{Zn}$ may form polymers and ultimately a $\mathrm{Zn}(\mathrm{OH})_{2}$ network and precipitate ${ }^{13,14,16}$.

The above illustrates the large variety of possible $\mathrm{Zn}$ species that may be found in $\mathrm{Zn}$-Fh systems. The delineation between the various types of $\mathrm{Zn}$ surface species is not evident. Therefore, this study aims to improve our insight into the speciation of $\mathrm{Zn}$ adsorption to Fh by integrating results previously collected by $\mathrm{X}$-ray absorption spectroscopy (XAS), together with information from quantum chemical calculations and SCM.

In natural environments, the adsorption of metal ions to the natural oxide fraction will not only be governed by the primary adsorption process but also influenced by coexisting ions. For $\mathrm{Zn}$, and other metal cations such as $\mathrm{Cu}$, it has been shown that the adsorption is enhanced by the presence of anions such as sulfate ${ }^{12}$, arsenate ${ }^{26}$, and phosphate 27,28 . In general, the cooperative binding between cations and anions to iron (hydr)oxides has been explained by electrostatic effects ${ }^{29}$ and/or by the formation of ternary complexes ${ }^{12,27,28,30}$.

Studying the cooperative binding of $\mathrm{Zn}$ and phosphate $\left(\mathrm{PO}_{4}\right)$ with ferrihydrite nanoparticles is particularly relevant since $\mathrm{PO}_{4}$ is omnipresent in soil systems and used in agricultural fertilization practices. Phosphate exhibits a large affinity for adsorption to $\mathrm{Fh}^{31-33}$, thereby strongly changing the interfacial properties. The use of $\mathrm{PO}_{4}$ fertilizers is widespread and enhanced $\mathrm{Zn}$ adsorption to metal (hydr)oxide particles by $\mathrm{PO}_{4}$ fertilization has been suggested for explaining the occurrence of $\mathrm{Zn}$ deficiencies in crops $^{34,35}$.

Based on the above, the objective of the present study is to assess with experiments, quantum chemical calculations, and surface complexation modeling the interaction of $\mathrm{Zn}^{2+}$ ions with freshly precipitated and shortly aged $\mathrm{Fh}$ in monocomponent $\mathrm{Zn}$ as well as multi-component $\mathrm{Zn}-\mathrm{PO}_{4}$ systems. For comparison, we will also re-interpret 
$\mathrm{Cu}$ adsorption data from literature to assess possible similarities in the effect of $\mathrm{PO}_{4}$ on the metal adsorption to Fh. The geometry of various surface complexes will be optimized with molecular orbital (MO) calculations using density functional theory (DFT). For surface complexation modeling, we will use a state-of-the-art modeling framework that is based on the recent insights into the surface structure of $\mathrm{Fh}^{31}$. In the approach, the charge distribution will be based on bond valence analysis ${ }^{36,37}$ of the MO/DFT optimized structures. Importantly, our experimental adsorption data will be scaled to the reactive surface area of the prepared Fh that will be measured independently with surface ion probing ${ }^{8}$. Consequently, our data will become consistent with earlier data collected in recent studies to derive the adsorption parameters for a large range of other ions, comprising the major monovalent electrolyte ions $\left(\mathrm{K}^{+}, \mathrm{Na}^{+}, \mathrm{Li}^{+}, \mathrm{Cl}^{-}, \mathrm{NO}_{3}{ }^{-}, \mathrm{ClO}_{4}{ }^{-}\right)$as well as the alkaline earth metal cations $\left(\mathrm{Mg}^{2+}, \mathrm{Ca}^{2+}, \mathrm{Sr}^{2+}, \mathrm{Ba}^{2+}, \mathrm{Ra}^{2+}\right)$ and additionally $\mathrm{PO}_{4}{ }^{3-}, \mathrm{As}(\mathrm{V})$ and $\mathrm{As}(\mathrm{III}), \mathrm{CO}_{3}{ }^{2-}$ , $\mathrm{H}_{4} \mathrm{SiO}_{4}$ and $\mathrm{B}(\mathrm{OH})_{3}{ }^{30-33,38,39}$.

\section{MATERIAL AND METHODS}

\section{$2.1 \quad$ Ferrihydrite synthesis}

For each adsorption experiment, a ferrihydrite suspension was freshly prepared by adding $0.02 \mathrm{M} \mathrm{NaOH}$ to a solution of $\sim 3.7 \mathrm{mM}$ of $\mathrm{Fe}\left(\mathrm{NO}_{3}\right)_{3}$ dissolved in $0.01 \mathrm{M} \mathrm{HNO}_{3}$, as described previously ${ }^{38}$. The initial addition of $\mathrm{NaOH}$ was done in steps of $200 \mathrm{~mL}$ until a pH of 3.2 was reached. Afterward, the $\mathrm{NaOH}$ was added in steps of $5 \mathrm{~mL}$ until a final $\mathrm{pH}$ of 8.2 was reached. When the $\mathrm{pH}$ was kept stable for at least 15 minutes, the suspension was decanted into large centrifuge bottles, and subsequently centrifuged for 45 minutes at $3270 \mathrm{~g}$. Afterwards, the supernatant in each bottle was carefully removed and the ferrihydrite particles were re-suspended in a $0.01 \mathrm{M}$ $\mathrm{NaNO}_{3}$ background solution. This final suspension was used for the adsorption experiment, after an aging time of four hours that started from the moment that a $\mathrm{pH}$ of 8.2 was reached.

Each ferrihydrite preparation was characterized for its Fe content, and its specific surface area (SSA), as it was done in previous studies ${ }^{32,38,39}$. Briefly, the Fe content of each batch of Fh was measured by dissolving an aliquot of the ferrihydrite suspension in a matrix of $0.8 \mathrm{M} \mathrm{H}_{2} \mathrm{SO}_{4}$. The Fe content was measured in this solution by Inductively Coupled Plasma Optical Emission Spectroscopy (ICP-OES). The SSA was derived by using $\mathrm{PO}_{4}$ as a probe ion ${ }^{8}$. For each $\mathrm{Fh}$ preparation, adsorption data were collected with a constant initial $\mathrm{PO}_{4}$ concentration at four different $\mathrm{pH}$ values in a background of $0.01 \mathrm{M} \mathrm{NaNO}_{3}$. With SCM, the SSA was fitted iteratively based on the $\mathrm{PO}_{4}$ adsorption data with an initial estimate of the SSA-dependent molar mass $\left(M_{\text {nano, }} \mathrm{g} \mathrm{Fh} \mathrm{mol}{ }^{-1} \mathrm{Fe}\right)$ to calculate the $\mathrm{Fh}$ concentration based on the measured $\mathrm{Fe}$ concentration of the suspension ${ }^{40}$. The measured Fe concentrations and SSA values derived for each Fh preparation can be found in Table S1. 


\subsection{Adsorption experiments}

The interaction between $\mathrm{Zn}$ and $\mathrm{Fh}$ was assessed by collecting $\mathrm{pH}$-dependent adsorption data in systems with different initial $\mathrm{Zn}$ concentrations $(0.3-94 \mu \mathrm{M})$, different Fh concentrations $\left(0.15-0.36 \mathrm{~g} \mathrm{~L}^{-1}\right)$, and different concentrations of the background electrolyte $\left(0.01,0.1\right.$, and $\left.1 \mathrm{M} \mathrm{NaNO}_{3}\right)$. The specific conditions for the different adsorption series can be found in Table $\mathrm{S} 1$.

All systems were made with a total volume of $40 \mathrm{~mL}$. First, an appropriate volume of $0.01 \mathrm{M} \mathrm{NaNO}_{3}$ was added into a $50 \mathrm{~mL}$ polypropylene tube. For the experiments with higher salt levels, higher concentrations of $\mathrm{NaNO}_{3}$ and ultra-pure water were added to reach the pre-defined ionic strength. Next, a specific volume of the freshly prepared Fh stock suspension was pipetted into the tube and the $\mathrm{pH}$ was adjusted by adding predetermined amounts of $0.01 \mathrm{M} \mathrm{HNO}_{3}$ or $\mathrm{NaOH}$. Finally, an amount of freshly prepared $\mathrm{Zn}\left(\mathrm{NO}_{3}\right)_{2}$ stock solution was added. The $\mathrm{pH}$ was adjusted to values between 4 and 11. For studying the adsorption of $\mathrm{Zn}$ to $\mathrm{Fh}$ in the presence of $\mathrm{PO}_{4}$, the systems were prepared similarly, but now also $4.0 \mathrm{~mL}$ of a freshly prepared $\mathrm{NaH}_{2} \mathrm{PO}_{4}$ stock solution was added right after the $\mathrm{Zn}$ addition. During the experiments, contact between solutions and air was minimized to avoid the interference of $\mathrm{CO}_{2(\mathrm{~g})}$.

The prepared systems were placed in a reciprocal shaker (120 strokes minute $\left.{ }^{-1}\right)$ in a conditioned room at $20{ }^{\circ} \mathrm{C}$ for 21 hours. Afterward, the suspensions were centrifuged for 20 minutes at $3750 \mathrm{~g}$. An aliquot of the supernatant was filtered over a $0.45 \mu \mathrm{m}$ membrane, acidified to $0.14 \mathrm{M} \mathrm{HNO}_{3}$, and analyzed for $\mathrm{Zn}$ and $\mathrm{PO}_{4}$ using Inductively Coupled Plasma Mass Spectrometry (ICP-MS) or optical emission spectroscopy (ICP-OES), depending on the concentrations. The $\mathrm{Zn}$ and $\mathrm{PO}_{4}$ concentrations of the stock solutions were also measured. The adsorbed $\mathrm{Zn}$ and $\mathrm{PO}_{4}$ were calculated as the difference between the initial and final concentrations in the adsorption systems. After sampling, the remaining volume was re-suspended by shortly shaking before measuring the $\mathrm{pH}$ with a glass electrode. This final $\mathrm{pH}$ was used for modeling the adsorption data.

\subsection{Modeling adsorption data}

The adsorption of $\mathrm{Zn}$ to ferrihydrite, with or without the addition of $\mathrm{PO}_{4}$, was modeled using the $C D$ model ${ }^{20}$. The surface sites and corresponding site densities were set in correspondence with the multi-site ion complexation model (MUSIC) for $\mathrm{Fh}{ }^{31}$, which was recently developed based on knowledge of the bulk and surface structure of $\mathrm{Fh}^{41,42}$. This structural model defines different surface groups based on the coordination number with $\mathrm{Fe}$, namely singly coordinated groups $\left(\equiv \mathrm{FeOH}^{-0.5}\right)$, doubly coordinated groups $\left(\equiv \mathrm{Fe}_{2} \mathrm{OH}^{0}\right)$, and a representative set of triply coordinated groups $\left(\equiv \mathrm{Fe}_{3} \mathrm{OH}^{-0.5}\right)$. The singly coordinated groups are divided into surface groups with the ability to form bidentate edge-sharing surface complexes $\left(=\mathrm{FeOH}(\mathrm{a})^{-0.5}\right)$ or bidentate double corner surface complexes $\left(\equiv \mathrm{FeOH}(\mathrm{b})^{-0.5}\right)$ with site densities of respectively 3.0 and 2.8 sites $\mathrm{nm}^{-2}{ }^{31}$. Both types of singly coordinated groups can also form 124 
monodentate corner-sharing surface complexes. The representative triply coordinated groups, with a site density of 1.4 sites $\mathrm{nm}^{-2}$, react with protons and electrolyte ions and therefore, contribute to the surface primary charge development. The solution speciation and the surface reactions for the development of the primary charge ${ }^{8}$, together with the formation reactions of $\mathrm{PO}_{4}$ adsorption complexes ${ }^{31}$, are given in Tables S2 and S3.

The compact part of the electrical double layer of ferrihydrite was described with the extended Stern layer approach ${ }^{43}$. Since $\mathrm{Fh}$ is a nanoparticle with a strong surface curvature, the capacitance values of the Stern layers $\left(C_{1}\right.$ and $\left.C_{2}\right)$ are sizedependent. This was taken into account using as reference the capacitance values of a flat $\mathrm{Fe}$ (hydr)oxide surface, i.e. $C_{1}=0.90$ and $C_{2}=0.74 \mathrm{~F} \mathrm{~m}^{-2}{ }^{40}$. For each $\mathrm{Fh}$ preparation, the SSA and the corresponding size-dependent molar mass and capacitance values were established using $\mathrm{PO}_{4}$ as part of the probe-ion method ${ }^{8}$.

CD modeling was done with ECOSAT, version 4.944. Parameter optimization was done using FIT, version $2.581^{45}$. The log $K$ values for the surface complexes were optimized using $C D$ values that were derived independently by optimization of the geometry of the $\mathrm{Zn}$ surface species, using molecular orbital (MO) calculations with density functional theory (DFT). The optimization of the geometry of inner-sphere $\mathrm{Zn}$ complexes was done using Spartan'14 and '18 parallel software of Wavefunction.

\section{RESULTS}

\subsection{Zn adsorption data}

\subsection{1 pH-dependency and surface loading}

The $\mathrm{pH}$-dependent $\mathrm{Zn}$ adsorption by $\mathrm{Fh}$ is shown in Figure 1 for Fh systems with a variation in the initial solution concentration, studied for two different $\mathrm{Zn}$ concentration ranges. With increasing $\mathrm{pH}$, the $\mathrm{Zn}$ adsorption increases especially between $\mathrm{pH} 5$ and 6.5, going from 0 to $100 \%$ adsorption. As the $\mathrm{pH}$-dependency is thermodynamically related to the proton exchange ratio, the high $\mathrm{pH}$ dependency of $\mathrm{Zn}$ implies a strong release of protons, in agreement with $\mathrm{H} / \mathrm{Zn}$ exchange data collected by Kinniburgh ${ }^{46}$. It points to a strong interaction of the adsorbed $\mathrm{Zn}^{2+}$ ions with protons at the surface and/or to hydrolysis of adsorbed $\mathrm{Zn}$ ions. 

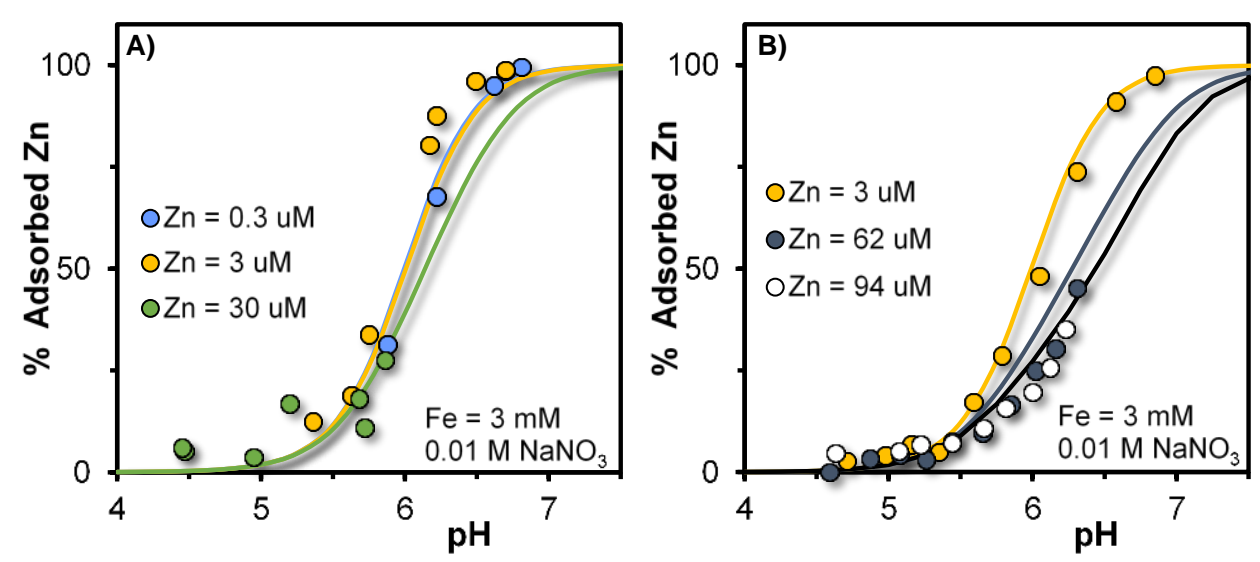

Figure 1: pH-dependent $\mathrm{Zn}$ adsorption by ferrihydrite in single ion systems with $0.01 \mathrm{M} \mathrm{NaNO}_{3}$ as background, as defined in Table S1. The markers are the experimental data, and the lines are the CD model calculations obtained with the parameter set of Table 1. The initial $\mathrm{Zn}$ concentration was varied with a constant Fh concentration of about $3 \mathrm{mM} \mathrm{Fe}$ (see details in Table S1). In the systems with a low $\mathrm{Zn}$ content $(\mathrm{A})$, the specific surface area is $691 \pm 6 \mathrm{~m}^{2} \mathrm{~g}^{-1}$ with a corresponding molar mass $M_{\text {nano }}=96.8 \pm$ $0.2 \mathrm{~g} \mathrm{~mol}^{-1} \mathrm{Fe}$. In the other systems (B) specific surface area of the Fh in the various batches is $684 \pm 49$ $\mathrm{m}^{2} \mathrm{~g}^{-1}$ with a corresponding molar mass $M_{\text {nano }}=96.6 \pm 1.3 \mathrm{~g} \mathrm{~mol}^{-1} \mathrm{Fe}$. More details can be found in Table S1.

An increase of the initial $\mathrm{Zn}$ concentration does not lead to a significant shift of the adsorption edges when the total added concentration is relatively low (Figure 1a). This is typical for systems with a linear adsorption isotherm. When the total $\mathrm{Zn}$ concentration is substantially raised, the adsorption edges switch to higher $\mathrm{pH}$ values (Figure 1b), due to the non-linear concentration dependency of the $\mathrm{Zn}$ adsorption. Similar behavior is observed for the adsorption of e.g. calcium ${ }^{39}$, and this is typical for cation adsorption in general.

\subsubsection{Variation solid concentration}

The effect of the concentration of $F$ in the suspension was studied with two experiments (Figures 2a,b). A lower concentration of $\mathrm{Fh}$ at the same initial $\mathrm{Zn}$ concentration will lead to a higher equilibrium concentration of $\mathrm{Zn}$ and a smaller fraction of adsorbed $\mathrm{Zn}$. Consequently, the adsorption edge will shift to higher $\mathrm{pH}$ values when less $\mathrm{Fh}$ is added to the system. 

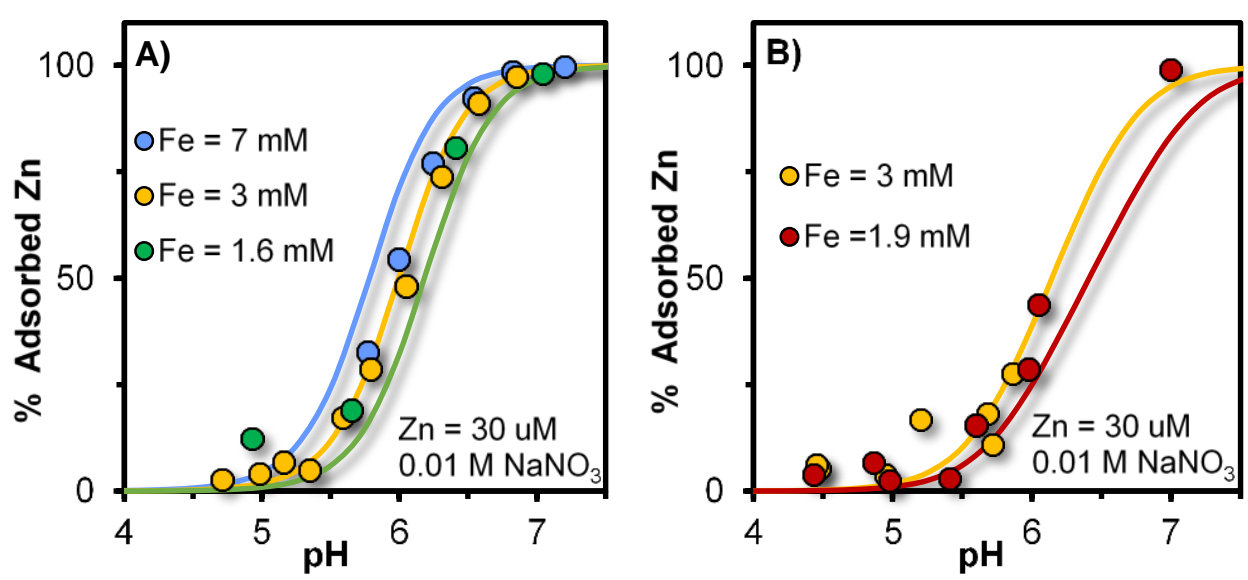

Figure 2: $\mathrm{pH}$-dependent $\mathrm{Zn}$ adsorption to ferrihydrite in single ion systems with $0.01 \mathrm{M} \mathrm{NaNO}_{3}$ as background, as defined in Table S1. The markers are the experimental data, and the lines are the CD model calculations obtained with the parameter set of Table 1. The initial Zn concentration was kept constant at about $30 \mu \mathrm{M}$ (Precise values are given in Table S1), and the amount of Fh was varied having a specific surface area of $745 \pm 11 \mathrm{~m}^{2} \mathrm{~g}^{-1}$ and corresponding molar mass of $M_{\text {nano }}=98.3 \pm 0.3 \mathrm{~g} \mathrm{~mol}^{-1} \mathrm{Fe}$ A), or with a surface area of $688 \pm 14 \mathrm{~m}^{2} \mathrm{~g}^{-1}$ and corresponding molar mass of $M_{\text {nano }}=96.7 \pm 0.4 \mathrm{~g} \mathrm{~mol}^{-1}$ $\mathrm{Fe}(\mathrm{B})$.

\subsubsection{Effect of lonic Strength}

Figure 3 shows the $\mathrm{pH}$-dependent $\mathrm{Zn}$ adsorption for systems with a constant $\mathrm{Zn}$ and Fh concentration but a different concentration of the $\mathrm{NaNO}_{3}$ background solution. The variation of the adsorption with the ionic strength is relatively small as follows from the figure.

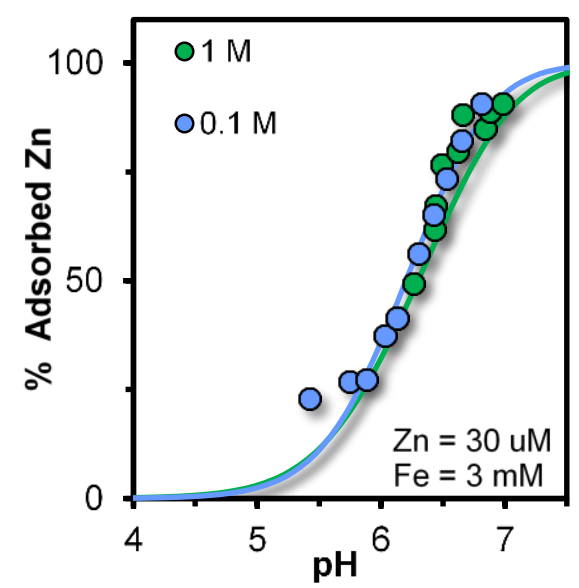

Figure 3: $\mathrm{pH}$-dependent $\mathrm{Zn}$ adsorption to $\mathrm{Fh}$ in single ion systems as defined in Table S1, at different $\mathrm{NaNO}_{3}$ background concentrations. The initial $\mathrm{Zn}$ concentration was $34.7 \mu \mathrm{M}$. The added ferrihydrite has a specific surface area of $724 \mathrm{~m}^{2} \mathrm{~g}-1$ and a corresponding molar mass of $M_{\text {nano }}=97.7 \mathrm{~g} \mathrm{~mol}^{-1} \mathrm{Fe}$. Its concentration was $0.33 \mathrm{~g} / \mathrm{L}$, equivalent to $3.4 \mathrm{mM}$ Fe. The symbols represent the adsorption data and the lines are the results of the CD model calculations with the parameters from Table 1. 


\section{2 $\mathrm{Zn}-\mathrm{PO}_{4}$ adsorption data}

As shown in Figure 4, the presence of $\mathrm{PO}_{4}$ in the $\mathrm{Zn}$-Fh systems enhances the $\mathrm{Zn}$ adsorption. The effect is most evident at $\mathrm{pH}$ values between 5 and 6 .
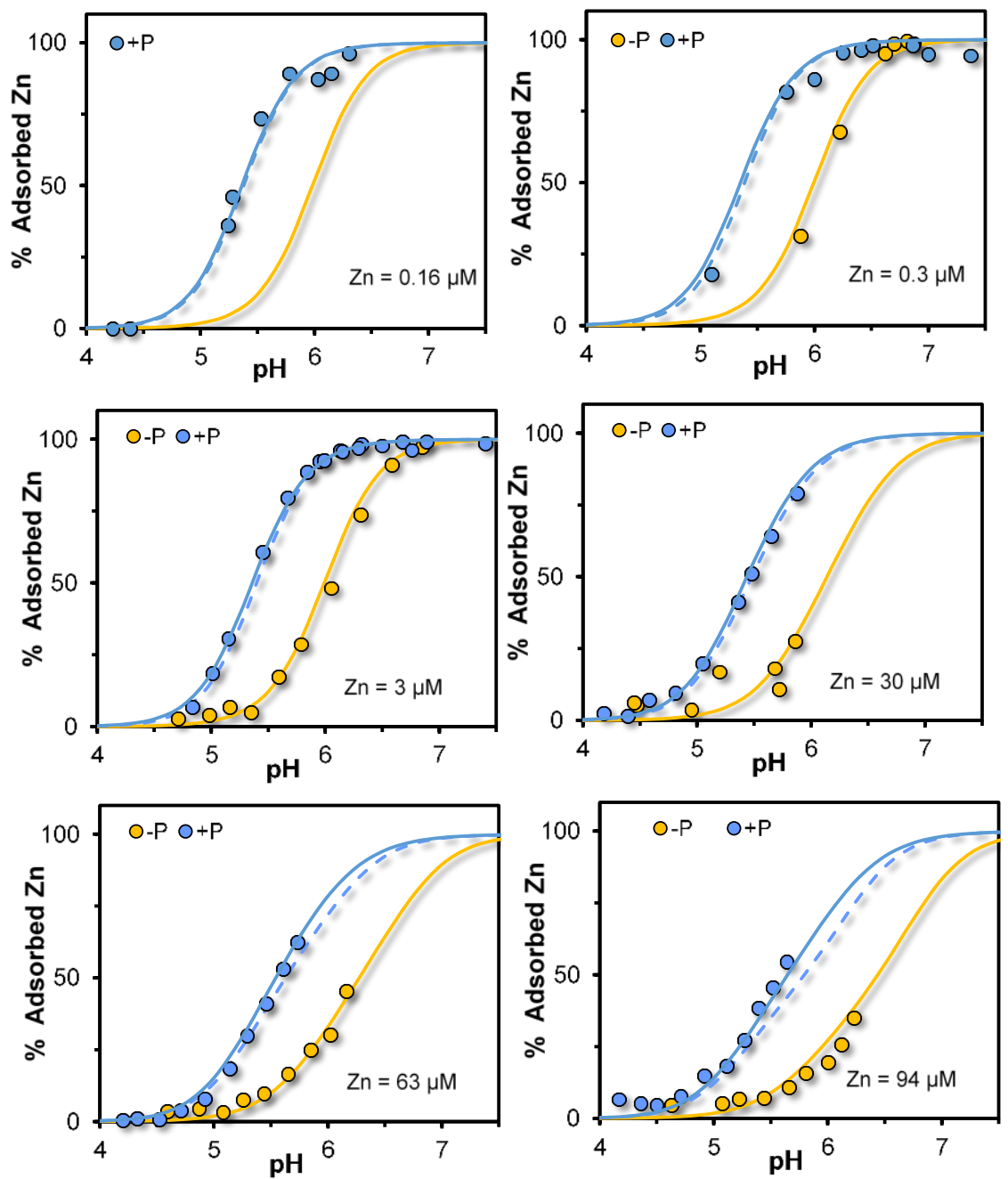

Figure 4: $\mathrm{pH}$-dependent $\mathrm{Zn}$ adsorption to $\mathrm{Fh}$ in single ion systems (-P) and in the presence of $600 \mu \mathrm{M}$ $\mathrm{PO}_{4}(+\mathrm{P})$, with $0.01 \mathrm{M} \mathrm{NaNO}_{3}$ as background. Fh was added at a concentration of $3 \mathrm{mM}$, with a specific surface area of $716 \pm 14 \mathrm{~m}^{2} \mathrm{~g}^{-1}$ (See Table S1 for the specific conditions of the batch experiments). The symbols represent the adsorption data, and the full lines are the CD model calculations with parameters from Table 1. The dashed lines give the predicted adsorption assuming solely an electrostatic interaction between adsorbed $\mathrm{Zn}$ and $\mathrm{PO}_{4}$, indicating that in these systems, a contribution of ternary $\mathrm{ZnPO}{ }_{4}$ surface complexes is absent or negligible. 


\section{DISCUSSION}

\subsection{Modeling $\mathrm{Zn}$ adsorption}

\subsubsection{Adsorption mechanisms}

The adsorption of $\mathrm{Zn}$ by freshly prepared $\mathrm{Fh}$ samples has been studied with $\mathrm{X}$-ray absorption spectroscopy (XAS) applying extended fine structure spectroscopy (EXAFS) and X-ray absorption near-edge spectroscopy (XANES) ${ }^{13-16}$. From this spectroscopic analysis, it was concluded that the coordination state of $\mathrm{Zn}$ changes from octahedral $(\mathrm{CN}=6)$ in solution into mainly tetrahedral coordination $(\mathrm{CN}=4)$ upon adsorption on the Fh surface in agreement with a relatively low value of $197 \mathrm{pm}$ for the average $\mathrm{Zn}-\mathrm{O}$ distance ${ }^{14,15}$. The observed average $\mathrm{Zn}$-Fe distances fall in the range of $340-350 \mathrm{pm}^{14,15}$. In later work ${ }^{16}$, a small contribution of an additional complex with a shorter Fe-Zn distance (325 \pm 4 pm) was found. The dominant complex with a larger distance was attributed to double corner-sharing ${ }^{14,16}$, while the additional $\mathrm{Zn}$ surface complex with smaller Fe-Zn distance was explained by a decrease in Fe-O-Zn angle of the double-corner sharing $\mathrm{Zn}$ complex ${ }^{16}$.

EXAFS as well as XANES can also give information about the number of ions in the second shell $(N)$. According to the analysis of Waychunas et al. ${ }^{13,14}$, the number of neighbors decreases from $N \sim 2$ at a low $\mathrm{Zn}$ loading to $N \sim 1$ at a high $\mathrm{Zn}$ loading. The former is typical for double-corner sharing and the latter might be due to edgeand/or single corner-sharing.

For the present study, the geometry of the above-mentioned Zn surface species (i.e. double corner, single corner, and edge-sharing complexes) has been optimized with $\mathrm{MO} / \mathrm{DFT} / 6-31+\mathrm{G}^{* *} / \mathrm{B} 3 \mathrm{LYP}$ calculations (Figure 5 and Table S4). The $\mathrm{Zn}$ complexes were defined using a representative predefined $\mathrm{Fe}_{2}(\mathrm{OH})_{6}\left(\mathrm{OH}_{2}\right)_{2}$ template ${ }^{31,38,47}$ with fixed atomic positions. On the top of the moiety, two $\mathrm{OH}_{2}$ ligands were placed. Depending on the type of $Z n$ complex, these water ligand(s) were exchanged against an $\mathrm{OH}$ ligand when the $\mathrm{Zn}^{2+}$ ion was attached either as a monodentate (single corner) or bidentate (double corner) complex. To form a complex by edge-sharing, $\mathrm{Zn}$ was attached to two singly coordinated $\mathrm{OH}$ groups of one of the Fe octahedra of the template. The coordination sphere of the attached $\mathrm{Zn}^{2+}$ ion was completed to form a $\mathrm{Zn}$ species with a coordination number of either $\mathrm{CN}=4$ and/or $\mathrm{CN}=6$. Next, a shell of secondary hydration water was defined. During the optimizations, the $\mathrm{Zn}$ ion, its primary ligands as well as the water molecules added for hydration, could relax. The remaining ligands of the basic template were always fixed, but depending on the option, the $\mathrm{Fe}$ in both polyhedra were able to relax or not. To study the effect of hydrolysis of the hydrated $\mathrm{Zn}^{2+}$ ion, i.e. the formation of a $\mathrm{ZnOH}^{+}$species, a proton was removed from one of the $\mathrm{OH}_{2}$ ligands of the primary coordination sphere of $\mathrm{Zn}$, followed by re-optimization.

Table S4 shows the results from the MO/DFT calculations and gives the average distances and charge distribution values obtained for the various $\mathrm{Zn}$ complexes. The 
number of water molecules added to form the second hydration shell was usually about $n_{\mathrm{H} 2 \mathrm{O}} \sim 10$. The predefined $\mathrm{Zn}$ complexes with $\mathrm{CN}=6$ spontaneously released water ligands to change to $\mathrm{CN}=4$. For all the single and double-corner $\mathrm{Zn}$ complexes, the calculated mean $\mathrm{Zn}-\mathrm{O}$ distance was $\sim 198 \mathrm{pm}$. This relatively short distance is in good agreement with a tetrahedral configuration, and within the uncertainty, this calculated value is equal to the mean $\mathrm{Zn}-\mathrm{O}$ distance obtained with EXAFS for $\mathrm{Zn}$ adsorption complexes at the surface of $\mathrm{Fh}^{14-16}$. The calculated mean $\mathrm{Fe}-\mathrm{Zn}$ distances for the bidentate double corner $\left({ }^{2} \mathrm{C}\right)$ surface species were between 340 and $350 \mathrm{pm}$ and fall in the range of values derived by fitting of EXAFS data ${ }^{14,16}$.

In addition, we have optimized a monodentate single corner $\left({ }^{1} \mathrm{C}\right)$ complex, showing a Zn-Fe distance of either 376 or $343 \mathrm{pm}$, depending on whether it is complexed with $\mathrm{FeOH}(\mathrm{a})$ or $\mathrm{FeOH}$ (b) surface groups respectively. The latter falls within the range of values $(340-350 \mathrm{pm})$ reported by Waychunas et al. ${ }^{14}$. This finding illustrates that a fitted distance alone may not be conclusive enough to differentiate between single and double corner-sharing surface species if these have a similar distance, and it implies that the number of Fe atoms present in the second shell $(N)$ then needs to be considered as well.

As mentioned above, the mean number of $\mathrm{Fe}$ ions in the second shell decreases with increased $\mathrm{Zn}$ adsorption from about $N=2$ to $N=1^{14}$, suggesting at a high loading, the formation of $\mathrm{Zn}$ complexes by for instance edge-sharing or single-corner sharing. The Fe-Zn distance, calculated with MO/DFT optimizations of the edgesharing complex, is small, i.e. $303 \pm 1 \mathrm{pm}$ (Table S4). This would mean that the mean $\mathrm{Fe}-\mathrm{Zn}$ distance decreases with increasing $\mathrm{Zn}$ loading, which was not observed by spectroscopy ${ }^{14,16}$. Instead, the formation of single-corner complexes is more likely.

According to the EXAFS analysis of Cismasu et al. ${ }^{16}$, there may be a contribution of an adsorbed $Z n$ surface species with $d_{F e-Z n}=325 \pm 5 \mathrm{pm}$ and $N=1 \pm 0.5$. Again, edge complex formation cannot explain this observation as its Fe-Zn distance is too small (Table S4). Cismasu et al. ${ }^{16}$ proposed that the shorter distance is due to a slightly stronger bending of the Fe-O-Zn angle for a certain fraction of the double corner-sharing $\left({ }^{2} \mathrm{C}\right) \mathrm{Zn}$ complexes at the Fh surface. However, this view with only bidentate surface complexes would result in a constant number of second shell neighbors (i.e. $N=2$ ) in contrast to what has been observed with EXAFS ${ }^{14,16}$.

Alternatively, one may explain the data of Cismasu et al. ${ }^{16}$ assuming monodentate complex formation. These complexes are more flexible in bending the $\mathrm{Fe}-\mathrm{O}-\mathrm{Zn}$ angle compared to bidentate double corner complexes, as a set of MO/DFT calculations showed. Furthermore, our calculations showed that the Fe- $\mathrm{Zn}$ distance in a $\mathrm{Zn}$ monodentate complex may vary depending on the number of hydration water molecules involved. With little secondary water, the observed distance from Cismasu et al. ${ }^{16}$ can be reproduced. However, adding more water molecules leads to larger Fe-Zn distances and the distance also depends on which type of singly coordinated 
groups are involved (Table S4), showing that definite conclusions cannot yet be drawn. In our approach, we propose monodentate complex formation most likely, and assumed that this complex is formed based on the reaction with all singly coordinated $\mathrm{FeOH}$ groups (see section 4.2.1).

For the optimized double-corner $\mathrm{Zn}$ complex, the coordination sphere is slightly asymmetrical. The bonds of $\mathrm{Zn}$ with the $\mathrm{OH}$ ligands common with the Fe polyhedra are relatively short, resulting in a corresponding larger contribution of charge. This shift is most pronounced for the $\mathrm{Zn}^{2+}$ present as a non-hydrolyzed double-corner complex $\equiv(\mathrm{FeOH})_{2} \mathrm{Zn}\left(\mathrm{OH}_{2}\right)_{2}$, being about 0.21 v.u (Table S4). Formation of a $\mathrm{ZnOH}$ double-corner surface complex by hydrolysis of one of the outer $-\mathrm{OH}_{2}$ ligands of the $\mathrm{Zn}^{2+}$ ion leads to a redistribution of charge of about $0.13 \mathrm{v} . \mathrm{u}$. (Table S4). The interfacial charge distribution becomes more symmetrical and overall closer to a Pauling distribution ${ }^{48}$. This is also found for the monodentate $\mathrm{ZnOH}$ complex with $n_{0}$ $=0.56 \pm 0.01$ and $n_{1}=0.44 \pm 0.01$ v.u.

At a sufficiently high concentration of $\mathrm{Zn}$, the solution will get oversaturated with

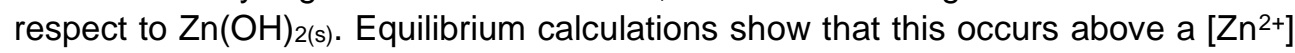
concentration of $10^{-1} \mathrm{M}$ at pH $6.5(I=0.1 \mathrm{M})^{49}$. At lower concentrations, $\mathrm{Zn}$ polymers may form at the surface ${ }^{13,14,16}$, which may contribute to a high sorption density ${ }^{11,14,17}$. As will be illustrated in the next section, our modeling of the data of Kinniburgh and Jackson ${ }^{11}$ suggests that $\mathrm{Zn}$ polymers contribute to the surface speciation at a concentration of $\sim 10^{-2} \mathrm{M}$ and higher at $\mathrm{pH} 6.5$.

Any $\mathrm{Zn}$ polymerization at the ferrihydrite surface appears to be in the form of tetrahedral $Z n$ complexes, but it changes towards octahedral coordination when an amorphous precipitate is formed at $\mathrm{Zn} /(\mathrm{Zn}+\mathrm{Fe})>>0.1^{14}$. We have performed $\mathrm{MO} / \mathrm{DFT}$ calculations assuming a $\mathrm{Zn}$ dimer that is attached as monodentate surface species (Table S5). The CD value derived has been used in our data analysis of the next section (Table 1).

To summarize, based on published XAS studies in combination with our MO/DFT calculations, the formation of $Z n$ surface species at the surface of Fh by single and double corner sharing is proposed, in combination with the formation of $\mathrm{Zn}$ polymers at a high surface loading (Figure 5). In the following section, SCM will be used to further evaluate the formation and the importance of these surface species in explaining the adsorption of $\mathrm{Zn}$ to $\mathrm{Fh}$. 


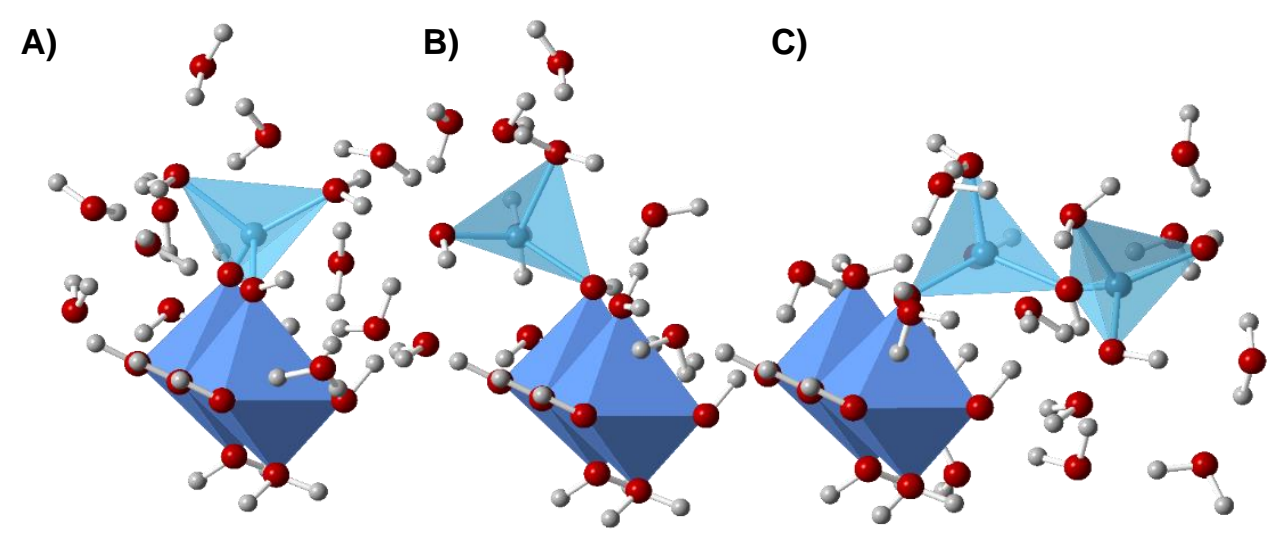

Figure 5: MO/DFT/6-31+G** optimized geometries of hydrated clusters in which Zn tetrahedra (light blue) attached to a cluster of two edge-shared Fe polyhedra (dark blue), forming a double corner complex $=\mathrm{Fe}_{2}(\mathrm{OH})_{2} \mathrm{Zn} \quad$ (A), a single-corner complex $\equiv \mathrm{Fe}(\mathrm{OH}) \mathrm{ZnOH} \quad$ (B) or an adsorbed dimer $\equiv \mathrm{Fe}(\mathrm{OH}) \mathrm{Zn}(\mathrm{OH})_{2} \mathrm{Zn}(\mathrm{OH})_{2}(\mathrm{C})$. The red and white spheres represent the oxygen and hydrogen atoms, respectively.

\subsubsection{Surface complexation modeling}

Based on the above analysis, a range of reactions have been formulated for evaluating the $\mathrm{Zn}$ adsorption to $\mathrm{Fh}$ with surface complexation modeling. In the reactions, two types of single coordinated surface groups are considered, being either $=\mathrm{FeOH}(\mathrm{a})^{-0.5}$ or $\equiv \mathrm{FeOH}(\mathrm{b})^{-0.5}$. The latter can form double-corner complexes. The former can form single-edge complexes. Both types of groups can react with $\mathrm{Zn}$ to form monodentate complexes ${ }^{31}$.

The reaction for the formation of a bidentate double-corner surface species with the possibility of being hydrolyzed was defined as:

$2 \equiv \mathrm{FeOH}(\mathrm{b})^{-0.5}+\mathrm{Zn}^{2+}(\mathrm{aq}) \leftrightarrow \equiv(\mathrm{FeOH}) 2^{-1+\Delta z o} \mathrm{Zn}^{\Delta z 1}$

$2 \equiv \mathrm{FeOH}(\mathrm{b})^{-0.5}+\mathrm{Zn}^{2+}(\mathrm{aq})+\mathrm{H}_{2} \mathrm{O}(\mathrm{I}) \leftrightarrow \equiv(\mathrm{FeOH})^{-1+\Delta z o} \mathrm{ZnOH}^{\Delta z 1}+\mathrm{H}^{+}(\mathrm{aq})$

in which the sum of $\Delta z_{0}+\Delta z_{1}$ is respectively 2 and 1 .

Monodentate single-corner sharing complex formation may occur by the reaction of $\mathrm{Zn}$ ions with either $\equiv \mathrm{FeOH}(\mathrm{a})^{-0.5}$ or $\equiv \mathrm{FeOH}(\mathrm{b})^{-0.5}$, leading, in the case of hydrolysis, to:

$1 \equiv \mathrm{FeOH}(\mathrm{a})^{-0.5}+\mathrm{Zn}^{2+}(\mathrm{aq})+\mathrm{H}_{2} \mathrm{O}(\mathrm{I}) \leftrightarrow \equiv \mathrm{FeOH}^{-0.5+\Delta z o} \mathrm{ZnOH}^{\Delta z 1}+\mathrm{H}^{+}(\mathrm{aq})$
$1 \equiv \mathrm{FeOH}(\mathrm{b})^{-0.5}+\mathrm{Zn}^{2+}(\mathrm{aq})+\mathrm{H}_{2} \mathrm{O}(\mathrm{I}) \leftrightarrow \equiv \mathrm{FeOH}^{-5+\Delta z o} \mathrm{ZnOH}^{\Delta z 1}+\mathrm{H}^{+}(\mathrm{aq})$

To account for $\mathrm{Zn}$ polymerization at the surface, the formation of a $\mathrm{Zn}$ oligomer is defined as: 


$$
\begin{aligned}
& 1 \equiv \mathrm{FeOH}(\mathrm{a})^{-0.5}+2 \mathrm{Zn}^{2+}(\mathrm{aq})+4 \mathrm{H}_{2} \mathrm{O}(\mathrm{l}) \leftrightarrow \\
& \equiv \mathrm{FeOH}^{-0.5+\Delta z o} \mathrm{Zn}(\mathrm{OH})_{2}{ }^{\Delta z 1} \mathrm{Zn}(\mathrm{OH})_{2}{ }^{\Delta z 2}+4 \mathrm{H}^{+}(\mathrm{aq}) \\
& 1 \equiv \mathrm{FeOH}(\mathrm{b})^{-0.5}+2 \mathrm{Zn}^{2+}(\mathrm{aq})+4 \mathrm{H}_{2} \mathrm{O}(\mathrm{l}) \leftrightarrow \\
& \equiv \mathrm{FeOH}^{-0.5+\Delta z o} \mathrm{Zn}(\mathrm{OH})_{2} \Delta z 1 \mathrm{Zn}(\mathrm{OH})_{2} \Delta z 2+4 \mathrm{H}^{+}(\mathrm{aq})
\end{aligned}
$$

Based on the assumption that for the $\mathrm{Zn}$ polymer the exterior part of the dimer is close to a neutral $\mathrm{Zn}(\mathrm{OH})_{2}$ precipitate $^{14}$, the value of $\Delta z_{2}$ was set to 0 (Table 1).

For all surface species, the values for $\Delta z_{0}$ and $\Delta z_{1}$ (Table 1) were defined based on MO/DFT calculations (Tables S4 and S5).

Kinniburgh and Jackson ${ }^{11}$ have studied the $\mathrm{Zn}$ adsorption of Fh for a very wide range of solution concentrations corresponding to a change of the surface loading by more than 6-orders of magnitude. For the adsorption isotherms measured at a given $\mathrm{pH}$, they noticed that from the perspective of modeling, the adsorption was relatively high at low solution concentrations and relatively low at high solution concentrations. Similar behavior has been found for $\mathrm{Ca}^{2+}$ ions as well as for divalent metal ions in genera ${ }^{39}$. For the modeling of this type of adsorption behavior, surface heterogeneity, i.e. the existence of sites with a different intrinsic affinity, has frequently been suggested ${ }^{12,17,28,39,50,51}$, and this concept was applied here too.

In our modeling approach for describing the $\mathrm{Zn}$ adsorption to $\mathrm{Fh}$, we have interpreted our own data set simultaneously with the data set of Kinniburgh and Jackson ${ }^{11}$, using the adsorption reactions, defined in Equations 1-3. Various optimization approaches were explored for describing these $\mathrm{Zn}$ adsorption data. Excluding site-heterogeneity resulted in a systematic deviation for describing the adsorption data: the adsorption was underestimated at low loading and overestimated at a high loading (Figure S1). The introduction of high-affinity sites improved the description of the adsorption data (Figure 6). The $\equiv \mathrm{FeOH}(\mathrm{b})$ sites were divided into two classes of affinities. For the high-affinity sites, the site density was set to the value previously obtained in the evaluation of the $\mathrm{Ca}^{2+}$ adsorption of the same Fh preparation, i.e. $0.29 \pm 0.02$ sites $\mathrm{nm}^{-2}{ }^{39}$, which was found to be similar to the density of high-affinity sites necessary for explaining boron adsorption data ${ }^{32}$. The remaining $\equiv \mathrm{FeOH}(\mathrm{b})$ sites then have a site density of $2.8-0.29=2.51 \mathrm{~nm}^{-2}$.

In Figure 6, the description of the data Kinniburgh and Jackson ${ }^{11}$ is shown using all the above-defined species (eqs.1-3). Applying the above set of species, generally, a good description of the adsorption data was found. Relatively large deviations are only found at low $\mathrm{pH}$ in the system with $10^{-3} \mathrm{M} \mathrm{Zn}$ (Figure 6b). 

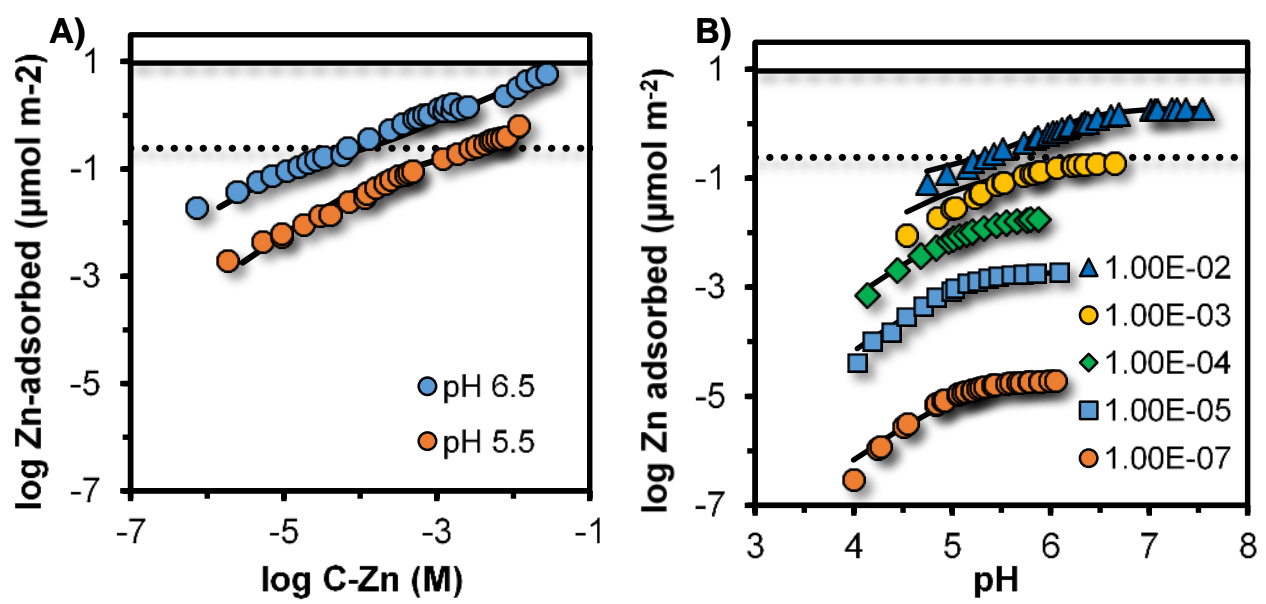

Figure 6: a) $\mathrm{Zn}$ adsorption isotherms for $\mathrm{Fh}$ at $\mathrm{pH} 5.5$ and 6.5 in $1.0 \mathrm{M} \mathrm{NaNO}_{3}$. b) $\mathrm{pH}$-dependent adsorption edges of $\mathrm{Zn}$ in $1.0 \mathrm{M} \mathrm{NaNO}_{3}$ at different initial $\mathrm{Zn}$ concentrations (M) for Fh systems with 93 $\mathrm{mM}$ Fe. Data (symbols) are from Kinniburgh and Jackson ${ }^{11}$; lines are modelled (Table 1, option A) using SSA $=585 \mathrm{~m}^{2} \mathrm{~g}^{-1}$, a consistent molar mass of $M_{\text {nano }}=94.1 \mathrm{~g} \mathrm{~mol}^{-1} \mathrm{Fe}$, and capacitance values of $C_{1, \text { nano }}=$ 1.14 and $C_{2 \text {,nano }}=0.92 \mathrm{~F} \mathrm{~m}^{-28}$. The dashed upper line indicates the maximum adsorption by monomeric $\mathrm{Zn}$ bound as a monodentate complex. The dotted line gives the maximum $\mathrm{Zn}$ binding to the high-affinity sites as a bidentate complex.

For the modeling of the adsorption of the $\mathrm{Zn}$ oligomer, the degree of hydrolysis was tested. As polymerization only occurs at very high $\mathrm{Zn}$ concentrations, the number of relevant data points is limited to $n=5$ for a set measured at a single $\mathrm{pH}$. Therefore, no clear difference in the quality of the fit could be revealed assuming the hydrolysis of 3,4 , or 5 water ligands. Fortunately, the level of hydrolysis of the $\mathrm{Zn}$ ions in the dimer hardly affects the log $K$ values fitted for the monomeric $Z n$ species (Results not shown). According to our model calculations, $\mathrm{Zn}$ polymerization starts to play a role when the $\mathrm{Zn}$ adsorption becomes higher than $\sim 1 \mu \mathrm{mol} \mathrm{m} \mathrm{m}^{-2}$ or above a molar $\mathrm{Zn} / \mathrm{Fe}$ ratio of 0.1 . Such a high loading is usually not relevant for natural systems but can be present in highly polluted environments and industrial settings. 
Table 1: $\mathrm{Zn}$ surface species binding to various sites of $\mathrm{Fh}$. The CD values $\left(\Delta \mathrm{z}_{0}\right)$ have been calculated from MO/DFT-optimized structures (Tables S4 and S5). The log $K$ values have been fitted expressing the data from Kinniburgh and Jackson ${ }^{11}$ and the data collected for this study $(n=311)$ in \% adsorbed (RMSE $=6.54 \%, R^{2}=0.957$ ). The data from Kinniburgh and Jackson ${ }^{11}$ have been modeled using SSA $=585 \mathrm{~m}^{2}$ $\mathrm{g}^{-18}$, a consistent molar mass of $M_{\text {nano }}=94.1 \mathrm{~g} \mathrm{~mol}^{-1} \mathrm{Fe}$, and the corresponding capacitance values of $\mathrm{C}_{1, \text { nano }}=1.14$ and $\mathrm{C}_{2, \text { nano }}=0.92 \mathrm{~F} \mathrm{~m}^{-2}$. The data collected in this study for various $\mathrm{Fh}$ preparations were modeled using SSA values measured with ion probing, applying a consistent value for $M_{\text {nano }}$ and the capacitances (Table S1). In the $\mathrm{Zn}-\mathrm{PO}_{4}$ systems, a ternary complex may form at rather high concentrations of $\mathrm{PO}_{4}$ The log $K$ value and charge distribution have been fitted $(n=89)$, but no definite conclusion can be drawn with respect to its structure (see text).

\begin{tabular}{|c|c|c|c|c|c|c|c|c|c|c|}
\hline Species & $\equiv \mathrm{FeOH}_{\mathrm{b} . \mathrm{h}}$ & $\equiv \mathrm{FeOH}_{\mathrm{b}, \mathrm{I}}$ & $\equiv \mathrm{FeOH}_{\mathrm{a}}$ & $\mathrm{Zn}^{2+}$ & $\mathrm{H}^{+}$ & $\mathrm{PO}_{4}$ & $\Delta \mathbf{z}_{0}$ & $\Delta \mathbf{z}_{1}$ & $\Delta \mathbf{z}_{2}$ & $\log K$ \\
\hline$\equiv(\mathrm{FeOH}(\mathrm{b}, \mathrm{h}))_{2} \mathrm{Zn}$ & 2 & 0 & 0 & 1 & 0 & 0 & 1.17 & 0.83 & 0 & $7.97 \pm 0.02$ \\
\hline$\equiv(\mathrm{FeOH}(\mathrm{b}, \mathrm{h}))_{2} \mathrm{ZnOH}$ & 2 & 0 & 0 & 1 & -1 & 0 & 1.06 & -0.06 & 0 & $0.69 \pm 0.05$ \\
\hline$\equiv \mathrm{FeOH}(\mathrm{a}) \mathrm{ZnOH}^{\#}$ & 0 & 0 & 1 & 1 & -1 & 0 & 0.57 & 0.43 & 0 & $-2.40 \pm 0.03$ \\
\hline$\equiv \mathrm{FeOH}(\mathrm{b}, \mathrm{l}) \mathrm{ZnOH}^{\#}$ & 0 & 1 & 0 & 1 & -1 & 0 & 0.57 & 0.43 & 0 & $-2.40 \pm 0.03$ \\
\hline $\begin{array}{l}\equiv \mathrm{FeOH}(\mathrm{a}) \mathrm{Zn}(\mathrm{OH})_{2} \mathrm{Zn} \\
(\mathrm{OH})_{2}\end{array}$ & 0 & 0 & 1 & 2 & -4 & 0 & 0.52 & -0.52 & 0 & $-21.02 \pm 0.27$ \\
\hline $\begin{array}{l}\equiv \mathrm{FeOH}(\mathrm{bl}) \mathrm{Zn}(\mathrm{OH})_{2} \mathrm{Zn} \\
(\mathrm{OH})_{2}\end{array}$ & 0 & 1 & 0 & 2 & -4 & 0 & 0.52 & -0.52 & 0 & $-21.02 \pm 0.27$ \\
\hline$\equiv \mathrm{FeOH}(\mathrm{a}) \mathrm{ZnH}_{2} \mathrm{PO}_{4}$ & 1 & 0 & 0 & 1 & +2 & 1 & $0.70^{*}$ & 0.54 & $-0.24^{*}$ & $27.94 \pm 1.03$ \\
\hline$\equiv \mathrm{FeOH}(\mathrm{b}, \mathrm{l}) \mathrm{ZnH}_{2} \mathrm{PO}_{4}$ & 0 & 1 & 0 & 1 & +2 & 1 & $0.70^{*}$ & 0.54 & $-0.24^{*}$ & $27.94 \pm 1.03$ \\
\hline$\equiv \mathrm{FeOH}(\mathrm{b}, \mathrm{h}) \mathrm{ZnH}_{2} \mathrm{PO}_{4}$ & 0 & 0 & 1 & 1 & +2 & 1 & $0.70^{*}$ & 0.54 & $-0.24^{*}$ & $27.94 \pm 1.03$ \\
\hline
\end{tabular}

\# Formation of a monodentate $\mathrm{Zn}^{2+}$ complex without hydrolysis could not be revealed.

${ }^{*}$ Fitted $\Delta z_{0}=0.70 \pm 0.32$ and $\Delta z_{2}=-0.24 \pm 0.42\left(R M S E=3.16 \%, R^{2}=0.993\right)$.

According to EXAFS, the increase of the $\mathrm{Zn}$ adsorption at $\mathrm{pH} 6.5$ leads to a decrease in the mean number of ions in the second shell ${ }^{14}$. This decrease is predicted well with our model as shown in Figure 7a. At a low Zn loading, the adsorption is dominated by the high-affinity sites that form double corner complexes (Eqs.1a, b). This leads to $N=2$ in agreement with the data. At an increase of the $\mathrm{Zn}$ loading, the number of ions in the second shell decreases due to the formation of a monodentate surface complex (Eqs.2a,b) with $N=1$. At a very high loading $(\mathrm{Zn} / \mathrm{Fe}>0.1)$, the number of ions in the second shell increases again due to $\mathrm{Zn}$ polymerization (Figures $7 \mathrm{a}$ and $7 \mathrm{~b})$.

The relative contribution of the various species as a function of the $\mathrm{Zn} / \mathrm{Fe}$ ratio is given in Figure $7 \mathrm{~b}$. At low loading, the bidentate complex is dominant. This complex is bound to high-affinity sites only. At higher $\mathrm{Zn} / \mathrm{Fe}$ ratios, the monodentate complex becomes dominant. This species can be formed by the interaction with the other singly coordinated surface groups $(\equiv \mathrm{FeOH}(\mathrm{a})$ and $\equiv \mathrm{FeOH}(\mathrm{b}, \mathrm{l})$. As the density of these surface sites is large $\left(N_{s}=5.5 \mathrm{~nm}^{-2}=9.1 \mu \mathrm{mol} \mathrm{m} \mathrm{m}^{-2}\right), \mathrm{Zn}$ can potentially reach a very high surface loading. Further increase of the $\mathrm{Zn}$ concentration in solution will likely lead to the attachment of $\mathrm{Zn}$ ions to the adsorbed monodentate $\mathrm{Zn}$ species, forming a polymer. According to our model, this occurs above a molar ratio $\mathrm{Zn} / \mathrm{Fe}$ of 0.1 (Figure $7 \mathrm{~b}$ ). At $\mathrm{pH} 6.5$, this is at an equilibrium concentration of about $10^{-2} \mathrm{M}$. The contribution of the polymers to the overall $\mathrm{Zn}$ adsorption is not more than $\sim 25 \%$, as can be seen in Figure 7b. 

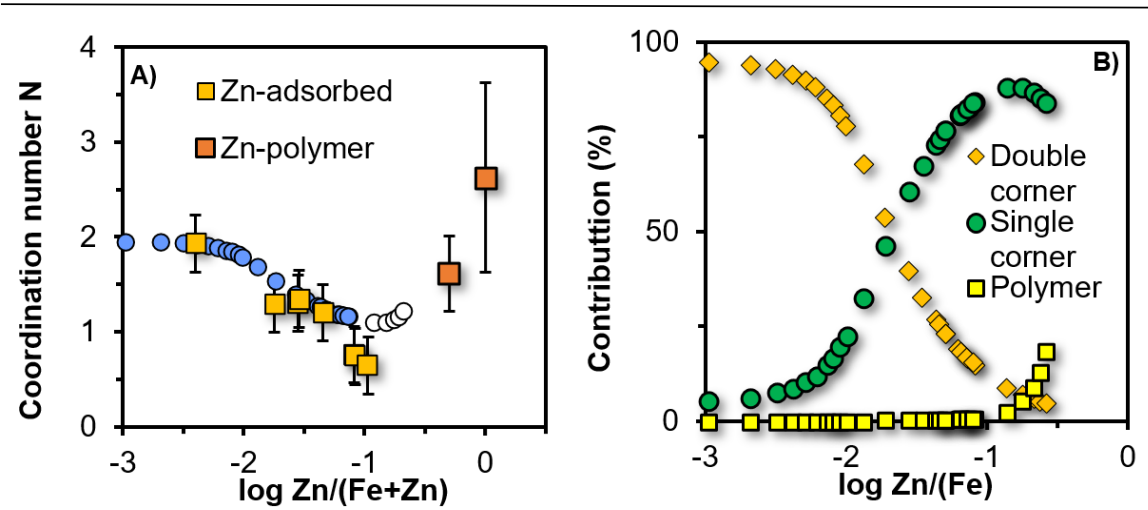

Figure 7: A) Number of ions $(M)$ in the second shell of $\mathrm{Zn}$. Experimental EXAFS data at $\mathrm{pH} \sim 6.5$ and I $=0.1 \mathrm{M} \mathrm{NaNO}_{3}$ (squares) from Waychunas et al. (2002). The model results (spheres) without (blue) and with polymerization (white) were calculated for the experiment of Kinniburgh and Jackson ${ }^{11}$ at pH 6.5 in 1 $\mathrm{M} \mathrm{NaNO}_{3}$ using the parameters of Table 1. B) Surface speciation of $\mathrm{Zn}$ at pH 6.5 in $1 \mathrm{M} \mathrm{NaNO}_{3}$ showing that the contribution of double-corner complexes is gradually overshadowed by the formation of singlecorner complexes. Only at a very high $\mathrm{Zn} / \mathrm{Fe}$ ratio $(>0.1)$, $\mathrm{Zn}$-polymers are formed that compete with the monodentate single-corner complex. The formation of the monodentate complex explains the decrease of the mean coordination number.

\subsubsection{Description of our $\mathrm{Zn}-\mathrm{Fh}$ data}

Our $\mathrm{Zn}$ adsorption data have been fitted simultaneously with the data collected by Kinniburgh and Jackson ${ }^{11}$, accounting for the difference in surface area. The results are shown in Table 1. These parameters were used to calculate the model lines in Figures 1-3. With the parameters from Table 1, our own $\mathrm{Zn}$ adsorption data could be reasonably well described (RMSE $=6.8 \%$ ), and no systematic deviations between model predictions and measured $\mathrm{Zn}$ adsorption data were found.

\subsection{Modeling $\mathrm{Zn}-\mathrm{PO}_{4}-\mathrm{Fh}$ interactions}

As shown in Figure 4, the introduction of $\mathrm{PO}_{4}$ in the $\mathrm{Fh}$ systems enhances $\mathrm{Zn}$ adsorption. This can be due to either electrostatics and/or the formation of additional surface species. Modeling calculations have shown that the formation of $\mathrm{Zn}_{3}\left(\mathrm{PO}_{4}\right)_{2}$ precipitates $\left(\log K_{s o}=-35.3\right.$ from Lindsay $\left.{ }^{49}\right)$ did not occur in the concentration range used in this study.

Using the thermodynamic parameters that are derived based on the monocomponent adsorption data (Table 1 and Table S3), we have calculated the $\mathrm{Zn}$ and $\mathrm{PO}_{4}$ adsorption in the multi-component systems. For all systems of Figure 4, our model calculations show that the enhanced $\mathrm{Zn}$ adsorption in the presence of $\mathrm{PO}_{4}$ is well-predicted (dotted lines) using solely the parameters from the monocomponent systems. It indicates that electrostatic interactions dominate the enhanced $\mathrm{Zn}$ adsorption due to the presence of $\mathrm{PO}_{4}$. Our calculations further show that there is no significant effect of $\mathrm{Zn}$ on the adsorbed $\mathrm{PO}_{4}$ (Figure S2), most likely due to the 
low concentrations of $\mathrm{Zn}$ compared to the added $\mathrm{PO}_{4}$. Liu et al. ${ }^{27}$ used X-ray photoelectron spectroscopy (XPS) and in-situ attenuated total reflectance Fourier transform infrared spectroscopy (ATR-FTIR) for studying the $\mathrm{Zn}-\mathrm{PO}_{4}$ interaction in systems with dried and ground Fh. For systems with a total initial concentration of $1.5 \mathrm{mM} \mathrm{Zn}$, in the absence and presence of $3.2 \mathrm{mM} \mathrm{PO}_{4}(1 \mathrm{mM} \mathrm{NaCl}, \mathrm{pH} 5)$, they found two peaks in the $\mathrm{Zn} 2 \mathrm{p}$ XPS spectra. In the absence of $\mathrm{PO}_{4}$, one of the peaks $\left(1021.6 \mathrm{~cm}^{-1}\right)$ was close to the value for $\mathrm{ZnFe}_{2} \mathrm{O}_{4}\left(1021.3 \mathrm{~cm}^{-1}\right)$ and shifted slightly towards the peak found for $\mathrm{Zn}_{3} \mathrm{PO}_{4}\left(1022.7 \mathrm{~cm}^{-1}\right)$ in the presence of $\mathrm{PO}_{4}$. Resolving the band using the peaks of $\mathrm{Zn}_{3} \mathrm{PO}_{4}$ and adsorbed $\mathrm{Zn}$ suggests the dominance of a $\mathrm{ZnPO}$ interaction. For $\mathrm{PO}_{4}$, XPS showed two contributions $\left(133.4 \mathrm{~cm}^{-1}\right.$ and 134.4 $\mathrm{cm}^{-1}$ ) that did not shift upon $\mathrm{Zn}$ addition, suggesting no or a minor interaction of $\mathrm{PO}_{4}$ with Zn. As XPS is an ex-situ technique, definite conclusions cannot be made per se because the forced-drying and ultra-high vacuum may have influenced the interaction of $\mathrm{Zn}$ and $\mathrm{PO}_{4}$.

With in-situ ATR-FTIR, Liu et al. ${ }^{27}$ measured a difference spectrum for a system with $3.2 \mathrm{mM} \mathrm{PO}_{4}$ equilibrium concentration without $\mathrm{Zn}$ and in the presence of a high $\mathrm{Zn}$ concentration of $1.6 \mathrm{mM}$. Three spectral bands related to the formation of innersphere $\mathrm{PO}_{4}$ complexes were found, pointing to additional adsorption of $\mathrm{PO}_{4}$ in the presence of $\mathrm{Zn}$. This can be expected from the perspective of electrostatic interactions. However, a contribution by ternary complex formation may contribute too, as the $\mathrm{Zn}$ and $\mathrm{PO}_{4}$ concentrations used in their ATR analysis were high very compared to the conditions in most of our $\mathrm{PO}_{4}-\mathrm{Zn}$ experiments. According to our model, at least $0.4 \mu \mathrm{mol} \mathrm{m}^{-2} \mathrm{Zn}$ and $4 \mu \mathrm{mol} \mathrm{m}^{-2} \mathrm{PO}_{4}$ will be adsorbed in the systems of Liu et al. ${ }^{27}$, while our data in the systems of Figure 4 refer to a much lower $\mathrm{Zn}$ adsorption being in the range of 0.001 to $0.1 \mu \mathrm{mol} \mathrm{m}{ }^{-2}$ and our mean $\mathrm{PO}_{4}$ loading was $2.5 \pm 0.15 \mu \mathrm{mol} \mathrm{m}{ }^{-2}$. For this reason, an additional experiment was done.

In addition to the experiments shown in Figure 4, we have studied a system in which we almost doubled the $\mathrm{Zn} / \mathrm{Fe}$ and $\mathrm{PO}_{4} / \mathrm{Fe}$ ratio, which resulted in an increase of the solution concentration of $\mathrm{PO}_{4}$ by a factor of about 20. In this system, the measured $\mathrm{Zn}$ adsorption was significantly higher than predicted assuming only an electrostatic interaction (Figure 8). The formation of an additional ternary $\mathrm{Zn}-\mathrm{PO}_{4}$ complex at these conditions may explain the difference. The structure of such a complex is not known. It might be similar to the dominant $\mathrm{PO}_{4}$-bridged ternary complex found for $\mathrm{Ca}$ and $\mathrm{Mg}$ ions ${ }^{30}$ or a shared monodentate complex as suggested for $\mathrm{Cu}$ ions ${ }^{28}$. We have explored the possible description of the data by formulating a reaction in which we varied the ion stoichiometry and fitted the charge distribution. This exploration showed that a reaction in which $2 \mathrm{H}^{+}$per $\mathrm{Zn}$ and $\mathrm{PO}_{4}$ resulted in the best description of the data. The formulated reaction is:

$$
\equiv \mathrm{FeOH}(\mathrm{a}, \mathrm{b}, \mathrm{h})^{-0.5}+2 \mathrm{H}^{+}(\mathrm{aq})+\mathrm{PO}_{4}^{3-}(\mathrm{aq})+\mathrm{Zn}^{2+}(\mathrm{aq}) \leftrightarrow \equiv \mathrm{FeOHZnH}_{2} \mathrm{PO}_{4}
$$


The resulting $C D$ values and $\log K$ are shown in Table 1. The fitted charge distribution suggested the presence of some negative charge in the second Stern plane, which is quite different from the ternary $\equiv \mathrm{FeOPO}_{3} \mathrm{Ca}$ and $\equiv \mathrm{FeOPO}_{3} \mathrm{Mg}$ complexes ${ }^{30}$. A possible explanation might be the formation of a ternary complex in which an $\mathrm{H}_{2} \mathrm{PO}_{4}$ ion is attached to a monodentate $=\mathrm{FeOHZn}$ surface species (Figure S4). This could be in line with the unique IR bands at $\sim 1045$ and $1067 \mathrm{~cm}^{-1}$ in the difference spectrum measured by Liu et al. ${ }^{27}$ These bands have been attributed to the presence of distorted, loosely bound, phosphate molecules. Our MO/DFT calculations of such a ternary complex resulted in an optimized Fe-Zn distance of $362 \mathrm{pm}$, and a corresponding charge distribution of 0.49 v.u. to the surface plane (Table S4). This $C D$ value is, within the uncertainty, similar as what has been found by fitting the adsorption data (Table 1). However, we acknowledge that our present data set is too limited to draw definite conclusions.

In Figure 8, the adsorption data (symbol) for the system with a high $\mathrm{Zn} / \mathrm{Fe}$ and $\mathrm{P} / \mathrm{Fe}$ ratio have been given (symbols) as well as the model line including ternary complex formation (full line). The dotted line is the $\mathrm{Zn}$ adsorption in the system without phosphate that serves as a reference, and the dashed line is the predicted adsorption assuming only an electrostatic interaction. The difference with the full line gives the contribution of the ternary complex. This contribution is minor but evident. In all other systems (Figure 4), the contribution of the ternary complex is practically absent.

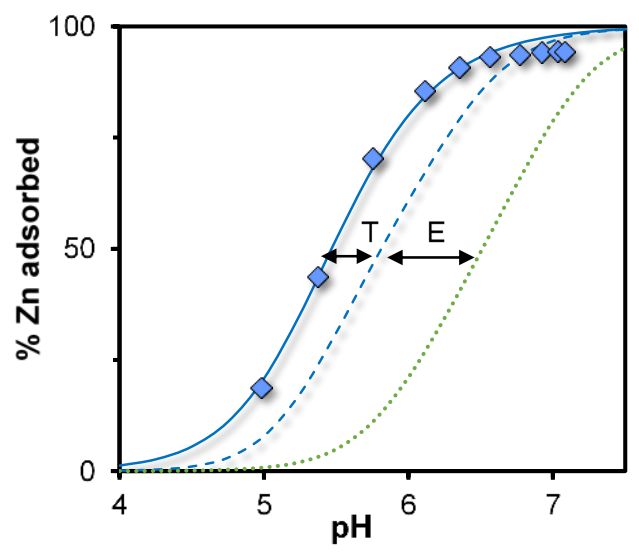

Figure 8: $\mathrm{pH}$-dependent adsorption of $\mathrm{Zn}$ by Fh $\left(1.78 \mathrm{~g} \mathrm{~L}^{-1}, A=574 \mathrm{~m}^{2} \mathrm{~g}^{-1}\right)$ in $0.01 \mathrm{M} \mathrm{NaNO}_{3}$ (colored spheres) with initial $\mathrm{Zn}$ and $\mathrm{PO}_{4}$ concentration of $30 \mu \mathrm{M}$ and $600 \mu \mathrm{M}$ respectively. The dotted line (green) is the calculated adsorption edge in the reference system with only $\mathrm{Zn}$. The full and dashed lines are the predictions with and without the formation of a ternary complex. Electrostatic $(E)$ interactions dominate the cooperative binding of $\mathrm{Zn}$ and $\mathrm{PO}_{4}$. A secondary contribution comes from the formation of the ternary (T) complex, but this will only occur at a high concentration of $\mathrm{PO}_{4}$ and /or $\mathrm{Zn}$ in solution (see text).

In general, competitive and cooperative effects by electrostatic interactions mainly occur via charge present in first Stern plane $\left(\Delta z_{1}\right)$. The introduction of $\mathrm{PO}_{4}$ in the 
system changes the electrostatic potential in the 1-plane dramatically from a positive to a negative value (Figure S3). This is due to the introduction of negative charge by the $\mathrm{PO}_{4}$ surface complexes (Table S3). The created electrostatic field becomes attractive for the positively charged $\mathrm{Zn}$ ions whereas it is repulsive in the absence of $\mathrm{PO}_{4}$. The adsorption of the non-hydrolyzed bidentate $\mathrm{Zn}$ surface species introduces positive charge in the 1-plane in contrast to the hydrolyzed $\mathrm{ZnOH}$ bidentate species, as follows from the $\Delta z_{1}$ values given in Table 1 . In addition, the positive charge of the bidentate surface species in the 1-plane is higher than the positive charge introduced by the monodentate hydrolyzed surface species. Consequently, in the presence of $\mathrm{PO}_{4}$, the non-hydrolyzed bidentate double corner-sharing $\mathrm{Zn}$ surface species is more preferred and becomes the dominant adsorption species according to our calculations (Figure 9) and the $\mathrm{Zn}$ edge switches to lower $\mathrm{pH}$ values (Figure 4).
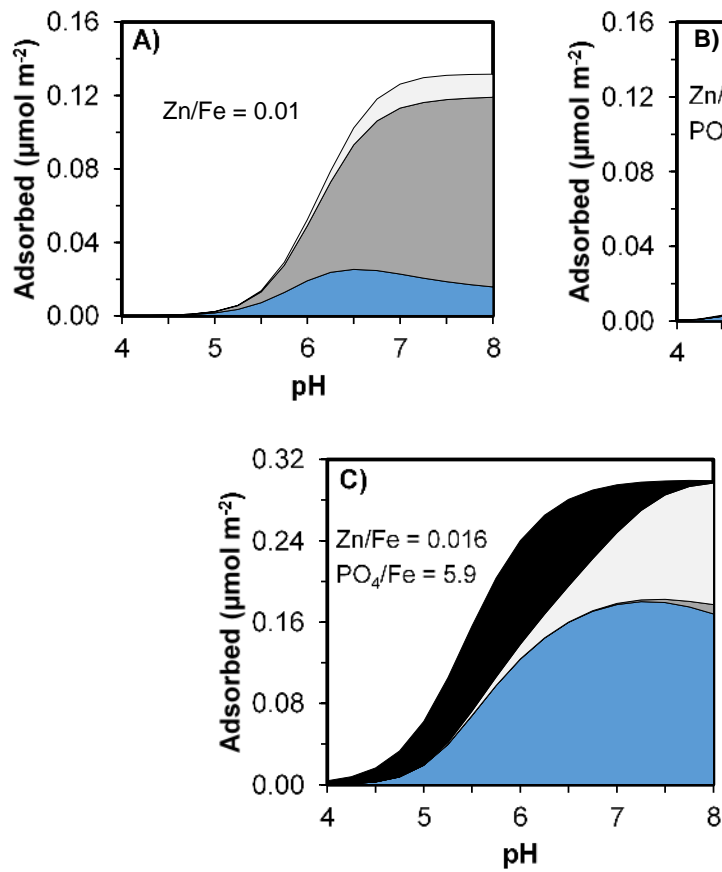

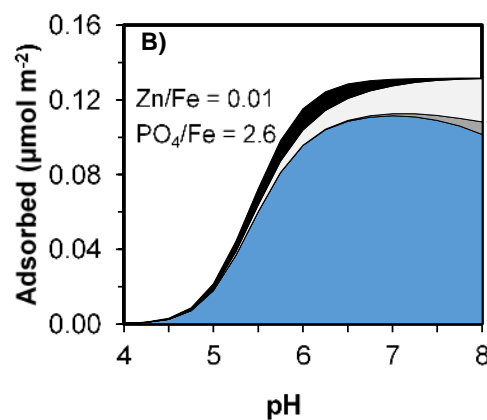

- MPZn

$\square \mathrm{MZnOH}$

$\square \mathrm{BhZnOH}$

$\square \mathrm{BhZn}$

Figure 9: $\mathrm{pH}$-dependent surface speciation of $\mathrm{Zn}$ calculated with the parameters from Table 1, in the absence $(A)$ and presence of $\mathrm{PO}_{4}(\mathrm{~B}$ and $\mathrm{C})$ in systems with $\mathrm{Fh}$ in $0.01 \mathrm{M} \mathrm{NaNO}_{3}$. The conditions are similar to those of the adsorption experiments shown in Figure 4. The calculations were done for systems with total initial concentrations of $30 \mu \mathrm{M} \mathrm{Zn}$ and $600 \mu \mathrm{M} \mathrm{PO}$ in the case of Figure $\mathrm{B}$ and $\mathrm{C}, 3 \mathrm{mM} \mathrm{Fe}(\mathrm{A}$ and $\mathrm{B}$ ) and $1.9 \mathrm{mM} \mathrm{Fe}(\mathrm{C})$. At the surface of $\mathrm{Fh}, \mathrm{Zn}$ is present as a bidentate corner complex that binds to high-affinity sites $(\mathrm{BhZn})$, with the option of being hydrolyzed $(\mathrm{BhZnOH})$, or as a hydrolyzed monodentate single-corner surface species that adsorbs to the low-affinity sites $(\mathrm{MZnOH})$. In the presence of $\mathrm{PO}_{4}, \mathrm{Zn}$ can also adsorb at ternary complexes (MPZn) which is only relevant at higher $\mathrm{PO}_{4}$ loadings as shown in Figure $\mathrm{C}$.

Upon higher $\mathrm{Zn}$ or $\mathrm{PO}_{4}$ loadings, the ternary $\mathrm{Zn}-\mathrm{P}$ complex becomes important and electrostatic interactions alone are not enough to explain the enhanced $\mathrm{Zn}$ 
adsorption in the presence of $\mathrm{PO}_{4}$. This resulted already from the batch adsorption experiments, and is again shown in Figure 9.

\subsection{Interpretation of $\mathrm{Cu}-\mathrm{PO}_{4}-\mathrm{Fh}$ interactions}

In contrast to what we have found here for $\mathrm{Zn}$, Tiberg et al. ${ }^{28}$ found that the inclusion of ternary complexes was necessary for explaining $\mathrm{Cu}$ adsorption to $\mathrm{Fh}$ in the presence of $\mathrm{PO}_{4}$, at surface loadings similar to our $\mathrm{Zn}-\mathrm{PO}_{4}-\mathrm{Fh}$ adsorption experiments from Figure 4. The surface speciation of $\mathrm{Cu}$ on Fh differs from $\mathrm{Zn}$, since $\mathrm{Cu}$ adsorbs as a bidentate edge-sharing complex with a mean $\mathrm{Cu}-\mathrm{Fe}$ distance of about $\sim 300 \mathrm{pm}^{28,52,53}$. According to the MUSIC model for Fh from Hiemstra and $\mathrm{Zhao}^{31}$, the surface sites that are involved for $\mathrm{Cu}$ adsorption are consequently different compared to the sites for $\mathrm{Zn}$ adsorption, and this may have implications on the electrostatic interactions with $\mathrm{PO}_{4}$. However, Tiberg et al. ${ }^{28}$ did not differentiate between singly coordinated groups that can form double corner-sharing and edgesharing complexes. In addition, they fitted the $C D$ values of the various surface complexes together with the $\log K$ values, which may have major effects on the calculated electrostatic interactions. Therefore, we re-interpreted the $\mathrm{Cu}$ adsorption data to Fh of Tiberg et al. ${ }^{28}$ in the absence and presence of $\mathrm{PO}_{4}$, to assess whether ternary complexes are necessary or if electrostatic interactions alone explain the multi-component $\mathrm{Cu}$ adsorption data, in line with what we have found here for most of the $\mathrm{Zn}-\mathrm{PO}_{4}$ adsorption systems. First, we derived adsorption parameters for the $\mathrm{Cu}-\mathrm{Fh}$ monocomponent systems. We used therefore the adsorption data from Swedlund and Webster ${ }^{12}$ and Tiberg et al. ${ }^{28}$. To make the $\mathrm{Cu}$ adsorption data internally consistent, we derived the SSA for the Fh preparations from Swedlund and Webster ${ }^{12}$ based on the $\mathrm{Zn}$ adsorption data that was collected in the same study, using our adsorption parameters from Table 1 (Figure S5). For the Fh preparations from Tiberg et al. ${ }^{28}$, we used an SSA of $611 \mathrm{~m}^{2} \mathrm{~g}^{-1}$ based on previous analysis of $\mathrm{PO}_{4}$ adsorption data ${ }^{31}$. 
Table 2: Cu adsorption to Fh as edge-sharing or monodentate single corner-sharing surface species. The $\mathrm{CD}$ values $\left(\Delta \mathrm{z}_{0}\right)$ have been calculated from MO/DFT/B3LYP/6-31+G** optimized structures. Option $A$ $\left(\log K^{a}\right)$ : Cu adsorption only as edge-sharing surface species. The log $K$ values have been fitted expressing the data from Swedlund and Webster (2001) and the monocomponent data from Tiberg et al. ${ }^{28}$, in $\%$ adsorbed, with $\mathrm{R}^{2}=0.98$ and RMSE of $5.21 \%$ (2 surface species). Option $\mathrm{B}\left(\log K^{b}\right)$ : Cu adsorption as edge-sharing and monodentate single-corner sharing surface species. The $\log K$ values have been fitted based on the monocomponent and multicomponent $\left(+\mathrm{PO}_{4}\right)$ adsorption data (\%) from Swedlund and Webster ${ }^{12}$ and Tiberg et al. ${ }^{28}$ with $\mathrm{R}^{2}=0.974$ and RMSE $=6.11 \%$ adsorbed (3 surface species). The data from Swedlund and Webster ${ }^{12}$ have been modeled using SSA $=582 \mathrm{~m}^{2} \mathrm{~g}^{-1}$, a consistent molar mass of $M_{\text {nano }}=94.1 \mathrm{~g} \mathrm{~mol}^{-1} \mathrm{Fe}$, and the corresponding capacitance values of $C_{1, \text { nano }}=1.14$ and $C_{2, \text { nano }}=0.89 \mathrm{~F} \mathrm{~m}^{-}$ 2. The value of SSA was fitted using the $\mathrm{Zn}$ adsorption data and the $\mathrm{Zn}$ adsorption parameters derived in the present study (see main text and Figure S5). The data from Tiberg et al. ${ }^{28}$ have been modeled using an SSA of $611 \mathrm{~m}^{2} \mathrm{~g}^{-1}$ and corresponding molar mass of $94.8 \mathrm{~g} \mathrm{~mol}^{-1} \mathrm{Fe}$.

\begin{tabular}{|c|c|c|c|c|c|c|c|c|c|}
\hline Species & $\equiv \mathrm{FeOH}_{\mathrm{a}}$ & $\equiv \mathrm{FeOH}_{\mathrm{b}}$ & $\mathrm{Cu}^{2+}$ & $\mathrm{H}^{+}$ & $\Delta \mathrm{z}_{0}$ & $\Delta \mathbf{z}_{1}$ & $\Delta \mathrm{z}_{2}$ & $\log K^{a}$ & $\log K^{b}$ \\
\hline $\begin{array}{l}\equiv(\mathrm{FeOH}(\mathrm{a}))_{2} \\
\mathrm{Cu}^{*}\end{array}$ & 2 & 0 & 1 & 0 & $\begin{array}{l}1.03 \pm \\
0.00\end{array}$ & 0.97 & 0 & $\begin{array}{l}8.21 \pm \\
0.05\end{array}$ & $\begin{array}{c}7.60 \pm \\
0.08\end{array}$ \\
\hline $\begin{array}{l}\equiv(\mathrm{FeOH}(\mathrm{a}))_{2} \\
\mathrm{CuOH}^{\star *}\end{array}$ & 2 & 0 & 1 & -1 & $\begin{array}{l}1.00 \pm \\
0.00\end{array}$ & 0.00 & 0 & $\begin{array}{l}1.85 \pm \\
0.04\end{array}$ & $\begin{array}{c}1.83 \pm \\
0.09\end{array}$ \\
\hline $\begin{array}{l}\equiv \mathrm{FeOH}(\mathrm{a}) \\
\mathrm{CuOH}^{\star \star \star}\end{array}$ & 1 & 0 & 1 & -1 & $\begin{array}{c}0.58 \pm \\
0.00\end{array}$ & 0.42 & 0 & - & $\begin{array}{c}0.23 \pm \\
0.17\end{array}$ \\
\hline $\begin{array}{l}\equiv \mathrm{FeOH}(\mathrm{b}) \\
\mathrm{CuOH}^{* * *}\end{array}$ & 0 & 1 & 1 & -1 & $\begin{array}{c}0.58 \pm \\
0.00\end{array}$ & 0.42 & 0 & - & $\begin{array}{c}0.23 \pm \\
0.17\end{array}$ \\
\hline
\end{tabular}

${ }^{*} n-\mathrm{H}_{2} \mathrm{O}=6 ; \quad d(\mathrm{Cu}-\mathrm{Fe})=302 \pm 1 \mathrm{pm} ; \mathrm{CN}=5$ with $d(\mathrm{Cu}-\mathrm{O})_{\mathrm{eq}}=200 \pm 7 \mathrm{pm}, d(\mathrm{Cu}-\mathrm{O})_{\mathrm{ax}}=235 \pm 2$ $\mathrm{pm}$;

** $n-\mathrm{H}_{2} \mathrm{O}=7 ; \quad d(\mathrm{Cu}-\mathrm{Fe})=306 \pm 1 \mathrm{pm} ; \mathrm{CN}=4$ with $d(\mathrm{Cu}-\mathrm{O})_{\text {eq }}=197 \pm 7 \mathrm{pm}$;

${ }^{* * *} n-\mathrm{H}_{2} \mathrm{O}=11 ; d(\mathrm{Cu}-\mathrm{Fe})=369 \pm 1 \mathrm{pm} ; \mathrm{CN}=4$ with $d(\mathrm{Cu}-\mathrm{O})_{\text {eq }}=197 \pm 11 \mathrm{pm}$;

NB The variation in $d(\mathrm{Cu}-\mathrm{O})$ is the variation between the different lengths within the Cu coordination sphere. In case of only monodentate formation at the $=\mathrm{FeOH}(\mathrm{a})$ groups, the values are: $\log K_{1}=7.62$ $\pm 0.08, \log K_{2}=1.91 \pm 0.07$, and $\log K_{3}=0.34 \pm 0.22$

The resulting parameters are shown in are shown in Table 2. As shown by Figures S6-S7, both sets of $\mathrm{Cu}$ adsorption data could be generally well described using a bidentate edge-sharing surface species (Figure 10a) in line with spectroscopic results $^{28}$. Both the hydrolyzed and non-hydrolyzed species was found to be important (Table 2). This finding is not in agreement with the results from Tiberg et al. ${ }^{28}$, who found that only the hydrolyzed edge-sharing surface species played a role for $\mathrm{Cu}$ adsorption based on a different structural model for Fh and different CD values. 

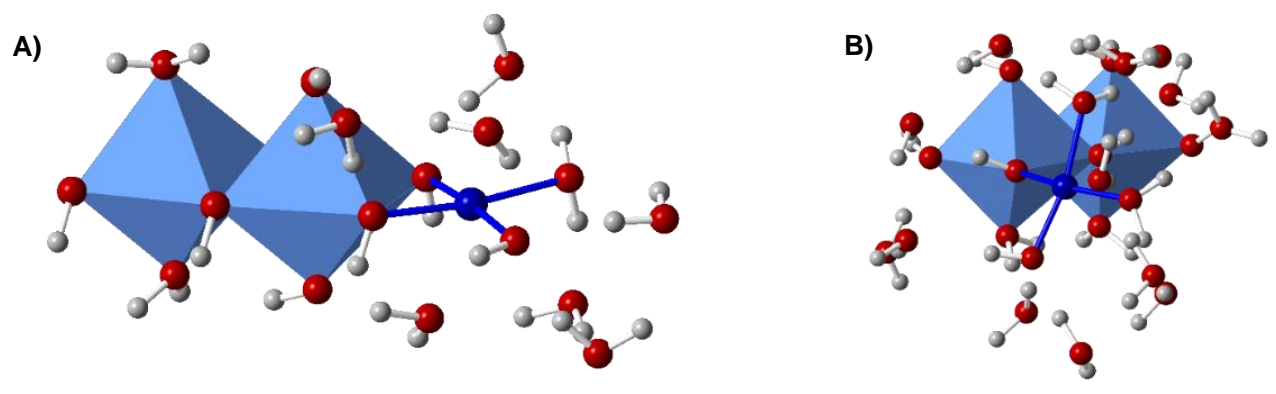

Figure 10: A) Hydrated edge complex of $\mathrm{CuOH}(\mathrm{CN}=4)$ with $d(\mathrm{Fe}-\mathrm{Cu})=306 \mathrm{pm}$, optimized with $\mathrm{MO} / \mathrm{DFT} / \mathrm{B} 3 \mathrm{LYP} / 6-31+\mathrm{G}^{* *}$. B) Hydrated single corner complex of $\mathrm{CuOH}(\mathrm{CN}=4) d(\mathrm{Fe}-\mathrm{Cu})=369 \mathrm{pm}$, optimized with MO/DFT/B3LYP/6-31+G**

As shown for $\mathrm{Zn}$, the presence of a non-hydrolyzed species may have serious implications for the electrostatic interactions. Indeed, we found that the increased $\mathrm{Cu}$ adsorption due to the presence of $\mathrm{PO}_{4}$ as found by Tiberg et al. ${ }^{28}$, can be explained by electrostatic effects only if these newly derived adsorption parameters are used (Figure S7), in line with what we found for $\mathrm{Zn}$. Tiberg et al. ${ }^{28}$ did not only interpret $\mathrm{Cu}$ adsorption data with SCM, but also with EXAFS analysis. The Fourier transforms of the EXAFS spectra for $\mathrm{Cu}$, showed in the presence of $\mathrm{PO}_{4}$ an additional signal that was attributed to a Cu surface complex with a larger distance of around $\sim 360 \mathrm{pm}^{28}$. The spectra showed that this additional scattering was particularly present for one $\mathrm{Cu}-\mathrm{PO}_{4}$-Fh sample with the highest $\mathrm{Cu}$ loading. Tiberg et al. ${ }^{28}$ proposed a ternary complex where both the $\mathrm{Cu}$ and $\mathrm{PO}_{4}$ are attached to the Fh surface. However, when combining both mono-and multicomponent $\mathrm{Cu}$ adsorption data, we could not reveal such a species. In addition, our MO/DFT calculations suggested a Fe-Cu distance of $345 \mathrm{pm}$ for such a species, which is not completely in line with the observed distance by EXAFS ${ }^{28}$. Alternatively, a monodentate species was also suggested by Tiberg et al. ${ }^{28}$ to explain the additional Cu surface species. Based on our modeling, the inclusion of a monodentate $\mathrm{Cu}$ surface species next to the bidentate edgesurface species (Figure 10), leads to a good description of the $\mathrm{Cu}$ adsorption data with and without $\mathrm{PO}_{4}$ (Figure S7 and Table 2). Importantly, the contribution of this monodentate surface species to the total $\mathrm{Cu}$ adsorption is in general low (between $10-30 \%$ ), except for the system with the highest Cu loading (Figure S8). This finding is in agreement with the Fourier transform of the Cu spectra measured by Tiberg et al. ${ }^{28}$ for the Fh-Cu-P system with high $\mathrm{Cu}$ loading. The $\mathrm{Fe}-\mathrm{Cu}$ distance of this monodentate surface species is according to MO/DFT calculations $369 \pm 1 \mathrm{pm}$ (Table 2), which is reasonably in agreement with the observed distance by EXAFS ${ }^{28}$. Thus, we propose that this monodentate surface complex does more likely explain the observed species with larger $\mathrm{Fe}-\mathrm{Cu}$ distance than the ternary complex suggested by Tiberg et al. ${ }^{28}$.

Based on our results for $\mathrm{Zn}$ and $\mathrm{Cu}$, we conclude that electrostatic interactions explain metal adsorption at background levels in multi-component systems, and that ternary complexes may form only at rather extreme metal or $\mathrm{PO}_{4}$ loading conditions. 142 
We have illustrated that conclusions with regard to ternary complex formation in multi-component systems, depend on the electrostatic model, the surface species and $C D$ values. If the electrostatic part of the ion adsorption model is weakly described, for example when the GTLM is used $^{12}$, caution is needed for the interpretation of ternary complexes for describing multi-component data.

\section{CONCLUSIONS}

The adsorption of $\mathrm{Zn}$ to $\mathrm{Fh}$ consists of various surface species, from which the contribution depends on the $\mathrm{pH}$ and surface loading. Interpretation of the $\mathrm{Zn}$ adsorption data confirms the existence of site heterogeneity, namely that a set of singly coordinated groups act as high-affinity sites for bidentate double-corner sharing $\mathrm{Zn}$ surface complexes similar to what has been previously identified for the alkaline earth metals and weakly adsorbing anions. In addition, a monodentate single-corner sharing complex was necessary to describe the decrease in $\mathrm{Zn}-\mathrm{Fe}$ coordination number with increasing loading that has been observed previously with X-ray absorption spectroscopy. The distances of both surface species that were calculated by quantum chemical calculations are in line with the distances previously derived by spectroscopy.

The addition of $\mathrm{PO}_{4}$ results in increased metal adsorption to Fh. Based on our CD modeling, we conclude that both $\mathrm{Zn}$ and $\mathrm{Cu}$ adsorption to $\mathrm{Fh}$ in the presence of $\mathrm{PO}_{4}$ can be described without the formation of ternary complexes for most of the systems studied. We have shown that at high metal and/or $\mathrm{PO}_{4}$ loadings, additional surface species may become important. In the case of $\mathrm{Zn}$, we suggest the formation of a monodentate $\mathrm{Zn}$ species to which a $\mathrm{H}_{2} \mathrm{PO}_{4}$ species is attached at high $\mathrm{PO}_{4}$ and $\mathrm{Zn}$ loadings, which is also in line with previously collected ATR spectra. We could not find the presence of a similar ternary complex for $\mathrm{Cu}-\mathrm{PO}_{4}-\mathrm{Fh}$ systems. Based on our results, we conclude that the cooperative effect of $\mathrm{PO}_{4}$ on the adsorption of metal cations such as $\mathrm{Zn}$ and $\mathrm{Cu}$ is mainly the result of electrostatic interactions.

\section{ACKNOWLEDGEMENTS}

This work was supported by NWO (grant number 14688, "Micronutrients for better yields"). All experiments and analyses were done in the CBLB laboratory at Wageningen University and Research. We greatly appreciate the help from Stef Heijink who collected part of the $\mathrm{Zn}$ adsorption data, and from Peter Nobels and Wobbe Schuurmans with the ICP measurements. 


\section{SUPPORTING INFORMATION}

\section{A. Overview of the batch adsorption experiments}

Table S1: Characteristics of the different ferrihydrite $(\mathrm{Fh})$ preparations and the adsorption experiments that were done in this study.

\begin{tabular}{ccccc|}
$\mathbf{F e}$ & $\mathbf{S S A}$ & $\boldsymbol{M}_{\text {nano }}$ & $\boldsymbol{C}_{\mathbf{1}}$ & $\boldsymbol{C}_{\mathbf{2}}$ \\
$\mathbf{m g}$ & $\mathbf{m}^{2} \mathbf{~ g}^{-1}$ & $\mathbf{g ~ m o l}^{-1}$ & $\mathbf{F ~ m}^{-2}$ & $\mathbf{F ~ m}^{-2}$ \\
$\mathbf{L}^{-1}$ & & & & \\
118 & $688^{*}$ & 96.74 & 1.17 & 0.94 \\
118 & $688^{*}$ & 96.74 & 1.17 & 0.94 \\
116 & 698 & 97.00 & 1.17 & 0.94 \\
110 & 678 & 96.48 & 1.16 & 0.93 \\
116 & 732 & 97.90 & 1.18 & 0.94 \\
116 & 752 & 98.43 & 1.18 & 0.94 \\
116 & 752 & 98.43 & 1.19 & 0.94 \\
117 & 724 & 97.70 & 1.18 & 0.94 \\
117 & 724 & 97.70 & 1.18 & 0.94 \\
122 & 684 & 96.65 & 1.17 & 0.92 \\
129 & 634 & 95.37 & 1.15 & 0.92 \\
& & & & \\
106 & 574 & 93.86 & 1.13 & 0.91 \\
116 & 664 & 96.12 & 1.16 & 0.93 \\
116 & 664 & 96.12 & 1.16 & 0.93 \\
116 & 708 & 97.26 & 1.17 & 0.94 \\
115 & 736 & 98.01 & 1.18 & 0.94 \\
126 & 652 & 95.83 & 1.16 & 0.93 \\
126 & 652 & 95.83 & 1.16 & 0.93 \\
126 & 652 & 95.83 & 1.16 & 0.93
\end{tabular}

----------Adsorption experiment

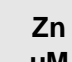

$\mu \mathrm{M}$

0.3

3.3

33.2

33.2

3.3

3.4

3.4

34.7

34.7

62.6

94.1

$\mathrm{PO}_{4}$

$\mu \mathrm{M}$

Fh

$\mathrm{g} \mathrm{L}^{-1}$

0.33

0.33

0.33

0.19

0.33

0.15

0.77

0.33

0.33

0.34

0.36

0.18

0.32

0.32

0.32

0.33

0.34

0.35

0.35

Figure in text

Figure 1a

Figure 1a

Figure $1 \mathrm{a}$, Figure $2 \mathrm{~b}$

Figure $2 b$

Figure $1 b$, Figure $2 a$

Figure 2a

Figure 2a

Figure 3

Figure 3

Figure 1b

Figure 1b

Figure 4

Figure 4

Figure 4

Figure 4

Figure 4

Figure 4

Figure 4

Figure 4

'The SSA of these Fh preparations were not measured with the probe-ion method but estimated as the average of the Fh preparations for the monocomponent systems. 


\section{B. Thermodynamic database used in CD modelling}

Table S2: Aqueous species and their formation reactions considered in the modelling, with affinity constants taken from Lindsay ${ }^{49}$.

\begin{tabular}{|c|c|c|}
\hline Aqueous species & Reaction & $\log K$ \\
\hline $\mathrm{HPO}_{4}^{-2}$ & $\mathrm{PO}_{4}^{-3}+\mathrm{H}^{+} \leftrightarrow \mathrm{HPO}_{4}^{-2}$ & 12.375 \\
\hline $\mathrm{H}_{2} \mathrm{PO}_{4}^{-}$ & $\mathrm{PO}_{4}^{-3}+2 \mathrm{H}^{+} \leftrightarrow \mathrm{H}_{2} \mathrm{PO}^{-2}$ & 19.573 \\
\hline $\mathrm{H}_{3} \mathrm{PO}_{4}$ & $\mathrm{PO}_{4}^{-3}+3 \mathrm{H}^{+} \leftrightarrow \mathrm{H}_{3} \mathrm{PO}_{4}$ & 21.721 \\
\hline $\mathrm{NaOH}$ & $\mathrm{Na}^{+}+\mathrm{OH}^{-} \leftrightarrow \mathrm{NaOH}$ & -0.2 \\
\hline $\mathrm{Zn}\left(\mathrm{NO}_{3}\right)_{2}$ & $\mathrm{Zn}^{+2}+2 \mathrm{NO}_{3}^{-} \leftrightarrow \mathrm{Zn}\left(\mathrm{NO}_{3}\right)_{2}$ & -0.3 \\
\hline $\mathrm{ZnNO}_{3}^{+}$ & $\mathrm{Zn}^{+2}+\mathrm{NO}_{3}^{-} \leftrightarrow \mathrm{ZnNO}_{3}^{+}$ & 0.4 \\
\hline $\mathrm{ZnOH}^{+}$ & $\mathrm{Zn}^{+2}+\mathrm{OH}^{-} \leftrightarrow \mathrm{ZnOH}^{+}$ & 6.31 \\
\hline $\mathrm{Zn}(\mathrm{OH})_{2}$ & $\mathrm{Zn}^{+2}+2 \mathrm{OH}^{-} \leftrightarrow \mathrm{Zn}(\mathrm{OH})_{2}$ & 11.20 \\
\hline $\mathrm{Zn}(\mathrm{OH})_{3}^{-}$ & $\mathrm{Zn}^{+2}+3 \mathrm{OH}^{-} \leftrightarrow \mathrm{Zn}(\mathrm{OH})_{3}^{-}$ & 14.32 \\
\hline $\begin{array}{l}\mathrm{Zn}(\mathrm{OH})_{4}^{-2} \\
\mathrm{ZnH}_{2} \mathrm{PO}_{4}^{+}\end{array}$ & $\begin{array}{l}\mathrm{Zn}^{+2}+4 \mathrm{OH}^{-} \leftrightarrow \mathrm{Zn}(\mathrm{OH})_{4}^{-2} \\
\mathrm{Zn}^{+2}+2 \mathrm{H}^{+}+\mathrm{PO}_{4}^{-3} \leftrightarrow \mathrm{ZnH}_{2} \mathrm{PO}_{4}^{+}\end{array}$ & $\begin{array}{l}17.71 \\
21.15\end{array}$ \\
\hline $\mathrm{ZnHPO}_{4}{ }^{+2}$ & $\mathrm{Zn}^{+2}+\mathrm{H}^{+}+\mathrm{PO}_{4}^{-3} \leftrightarrow \mathrm{ZnHPO}_{4}^{+}$ & 15.65 \\
\hline
\end{tabular}




\section{Adsorption reactions for ferrihydrite}

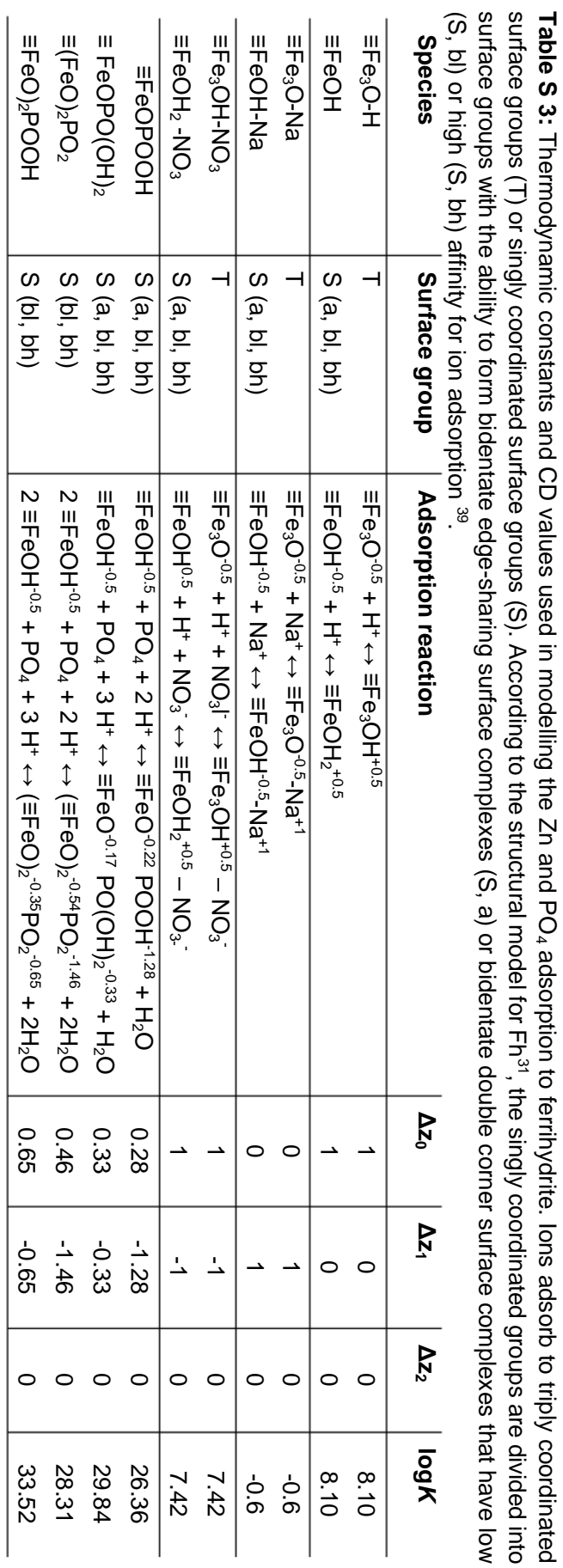




\section{Results of the optimized geometries for the $\mathrm{Zn}$ monomers and polymers}

Table S4: Properties of MO/DFT/B3LYP/6-31+G** optimized Zn-Fe $2(\mathrm{OH})_{6}\left(\mathrm{OH}_{2}\right)_{2}$ clusters with bidentate double corner $\left({ }^{2} \mathrm{C}\right)$, bidentate edge sharing $\left({ }^{1} \mathrm{E}\right)$ and monodentate single corner sharing $\left({ }^{1} \mathrm{C}\right)$ complex formation for the $\mathrm{Zn}$ ion. Given is the excess number of water molecules $\left(n_{\mathrm{H} 2 \mathrm{O}}\right)$ added for secondary hydration, the coordination number of $\mathrm{Zn}(\mathrm{CN})$, optimized mean $d(\mathrm{Zn}-\mathrm{O}), d(\mathrm{Zn}-\mathrm{Fe})$ distances in pm, the charge attribution of $\mathrm{Zn}^{2+}$ to the common $\left(\mathrm{n}_{0}\right)$ and non-common $\left(\mathrm{n}_{1}\right)$ ligands in its primary coordination sphere, and the corresponding interfacial charge distribution coefficients with $\Delta z_{0}, \Delta z_{1}$, and $\Delta z_{2}$. The standard deviation is given behind the \pm signs. For the Fe-Zn-P species, the optimized mean $d(Z n-P)$ distance is given.

\begin{tabular}{|c|c|c|c|c|c|c|c|c|c|c|c|}
\hline Species $^{\text {a) }}$ & ID & $n_{\mathrm{H} 2 \mathrm{O}}$ & $\begin{array}{l}\mathrm{C} \\
\mathrm{N}\end{array}$ & $\begin{array}{c}d(\mathrm{Zn}- \\
\mathrm{O}) \\
\mathrm{pm}\end{array}$ & $\begin{array}{c}d(\mathrm{Zn}- \\
\mathrm{Fe}) \\
\mathrm{pm}\end{array}$ & $\begin{array}{c}d(\mathrm{Zn}- \\
\mathrm{P}) \\
\mathrm{pm}\end{array}$ & $n_{0}$ & $n_{1}$ & $\Delta \mathrm{z}_{0}$ & $\Delta \mathrm{z}_{1}$ & $\Delta \mathrm{z}_{2}$ \\
\hline$\equiv \mathrm{Fe}_{2}(\mathrm{OH})_{2} \mathrm{Zn}$ & ${ }^{2} \mathrm{C}$ & $7-10$ & 4 & $\begin{array}{l}198 \\
\pm 1\end{array}$ & $\begin{array}{l}340 \\
\pm 9\end{array}$ & - & $\begin{array}{c}1.21 \pm \\
0.01\end{array}$ & $\begin{array}{c}0.7 \\
9\end{array}$ & $\begin{array}{c}1.17 \pm \\
0.01\end{array}$ & 0.83 & 0 \\
\hline$\equiv \mathrm{Fe}_{2}(\mathrm{OH})_{2} \mathrm{ZnOH}$ & ${ }^{2} \mathrm{C}$ & $8-11$ & 4 & $\begin{array}{l}199 \\
\pm 1\end{array}$ & $\begin{array}{l}344 \\
\pm 7\end{array}$ & - & $\begin{array}{c}1.08 \pm \\
0.01\end{array}$ & $\begin{array}{c}0.9 \\
2\end{array}$ & $\begin{array}{c}1.06 \pm \\
0.01\end{array}$ & -0.06 & 0 \\
\hline$\equiv \mathrm{Fe}_{1} \mathrm{OHZnOH}$ & ${ }^{1} \mathrm{C}$ & $10-12$ & 4 & $\begin{array}{r}200 \\
\pm 0\end{array}$ & $\begin{array}{c}343- \\
376^{\star}\end{array}$ & - & $\begin{array}{c}0.56 \pm \\
0.01\end{array}$ & $\begin{array}{c}0.4 \\
4\end{array}$ & $\begin{array}{c}0.57 \pm \\
0.01\end{array}$ & 0.43 & 0 \\
\hline$\equiv \mathrm{Fe}_{1}(\mathrm{OH})_{2} \mathrm{ZnOH}$ & ${ }^{1} \mathrm{E}$ & 14 & 4 & $\begin{array}{l}199 \\
\pm 0\end{array}$ & $\begin{array}{c}303 \pm \\
1\end{array}$ & - & $\begin{array}{c}0.93 \pm \\
0.01\end{array}$ & $\begin{array}{c}0.0 \\
7\end{array}$ & $\begin{array}{c}0.94 \pm \\
001\end{array}$ & 0.06 & 0 \\
\hline$\equiv \mathrm{Fe}_{1} \mathrm{OHZn \textrm {H } _ { 2 }} \mathrm{PO}_{4}$ & ${ }^{1} \mathrm{C}$ & 10 & 4 & 199 & 362 & 309 & 0.48 & $\begin{array}{c}0.5 \\
2\end{array}$ & 0.49 & 0.51 & 0 \\
\hline
\end{tabular}

"This distance depends whether $\mathrm{FeOHa}$ or $\mathrm{FeOHb}$ is taken to calculate the surface species geometry.

Table S5: Properties of MO/DFT/B3LYP/6-31+G* optimized hydrated $\mathrm{Zn}_{2}(\mathrm{OH})_{4} \mathrm{Fe}_{2}(\mathrm{OH})_{6}\left(\mathrm{OH}_{2}\right)_{2}$ clusters. The $\mathrm{Zn}$ dimer is attached as monodentate single corner $\left({ }^{1} \mathrm{C}\right)$ complex to one of the Fe polyhedra. Given is the excess number of water molecules $\left(n \mathrm{H}_{2} \mathrm{O}\right)$ added for secondary hydration, the coordination number of $\mathrm{Zn}(\mathrm{CN})$, the optimized mean $\mathrm{d}(\mathrm{Zn}-\mathrm{O}), \mathrm{d}(\mathrm{Zn}-\mathrm{Fe})$, and $\mathrm{d}(\mathrm{Zn}-\mathrm{Zn})$ distances in $\mathrm{pm}$, the interfacial charge distribution coefficients $\Delta z_{0}, \Delta z_{1}$, and $\Delta z_{2}$. The standard deviation is given behind the \pm signs.

\begin{tabular}{ccccccccc}
\hline Species ${ }^{\mathrm{a})}$ & $n_{\mathrm{H} 2 \mathrm{O}}$ & $\mathrm{CN}^{\mathrm{b})}$ & $\begin{array}{c}d(\mathrm{Zn}-\mathrm{O}) \\
\mathrm{pm}\end{array}$ & $\begin{array}{c}d(\mathrm{Zn}-\mathrm{Fe}) \\
\mathrm{pm}\end{array}$ & $\begin{array}{c}d(\mathrm{Zn}-\mathrm{Zn}) \\
\mathrm{pm}\end{array}$ & $\Delta \mathrm{z}_{0}$ & $\Delta \mathrm{z}_{1}$ & $\Delta \mathrm{z}_{2}$ \\
\hline$\equiv \mathrm{FeOHZn}(\mathrm{OH})_{2} \mathrm{Zn}(\mathrm{OH})_{2}$ & \multirow{2}{*}{10} & $4+4$ & $198 \pm 0$ & $343 \pm 1$ & $\begin{array}{c}355 \pm \\
0.11\end{array}$ & 0.52 & -0.52 & 0 \\
\hline
\end{tabular}

\footnotetext{
a) The common ligand between $\mathrm{Fe}$ and $\mathrm{Zn}$ as well between $\mathrm{Zn}$ and $\mathrm{Zn}$ are hydroxyl. Each $\mathrm{Zn}$ has
} an additional $\mathrm{OH}$ ligand

b) Both $\mathrm{Zn}$ ions have a tetrahedral coordination sphere 


\section{E. Zn adsorption without site heterogeneity}
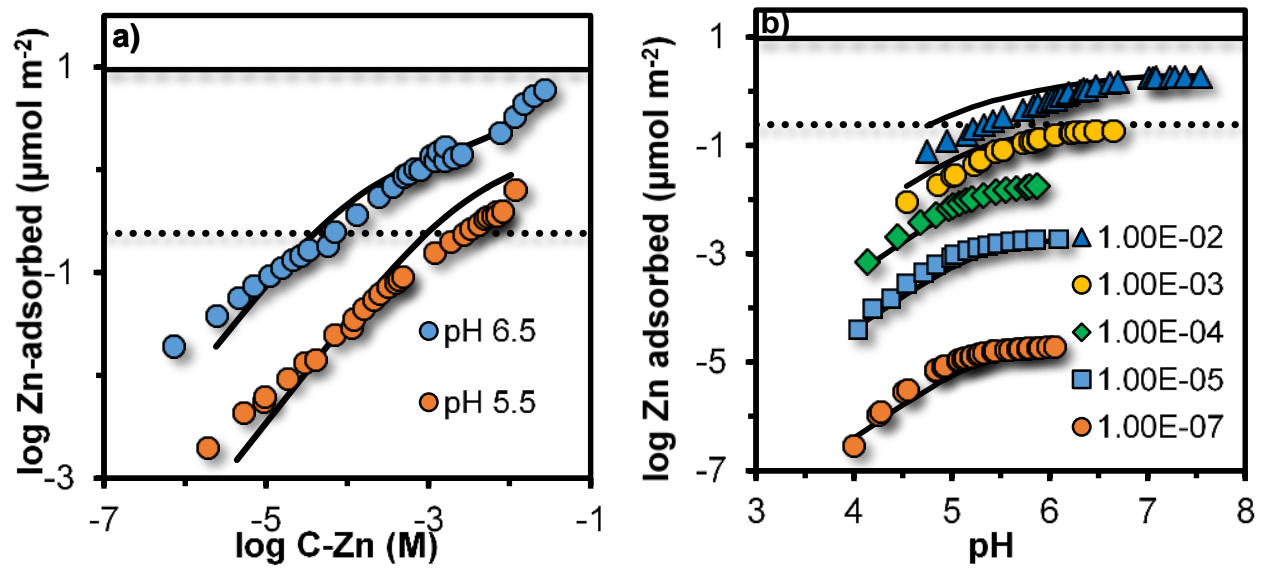

Figure S1: a) The $\mathrm{Zn}$ adsorption to $\mathrm{Fh}$ at $\mathrm{pH} 5.5$ and 6.5 in $1.0 \mathrm{M} \mathrm{NaNO}_{3}$. b) $\mathrm{pH}$ dependent adsorption edges of $\mathrm{Zn}$ to $\mathrm{Fh}$ in $1.0 \mathrm{M} \mathrm{NaNO}_{3}$ at different initial $\mathrm{Zn}$ concentration (M) for Fh systems with $93 \mathrm{mM} \mathrm{Fe}$. Data (symbols) are from Kinniburgh and Jackson (1982). The lines have been calculated with the CD model assuming corner sharing bidentate $\mathrm{Zn}$ complexes to all $=\mathrm{FeOH}(\mathrm{b})$ sites $\left(\mathrm{N}_{\mathrm{s}}=2.8\right.$ sites $\left.\mathrm{nm}^{-2}\right)$. The SSA is set to $585 \mathrm{~m}^{2} \mathrm{~g}^{-1}$ and the consistent molar mass is $M_{\text {nano }}=94.1 \mathrm{~g} \mathrm{~mol}^{-1} \mathrm{Fe}$. The applied capacitance values are $C_{1, \text { nano }}=1.14$ and $C_{2, \text { nano }}=0.92 \mathrm{~F} \mathrm{~m}^{-2}$. 


\section{F. $\mathrm{PO}_{4}$ adsorption in systems with $\mathrm{Zn}$}

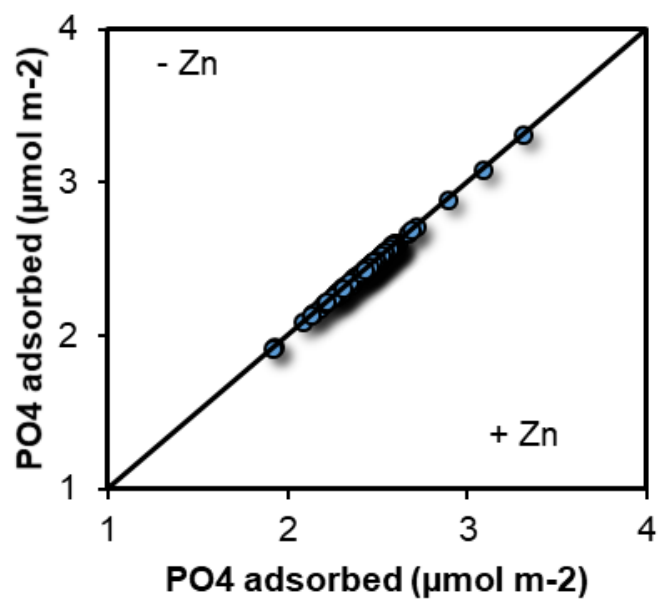

Figure S2: The calculated $\mathrm{PO}_{4}$ adsorption in $\mu \mathrm{mol} \mathrm{m} \mathrm{m}^{-2}$ to Fh for systems with $\mathrm{Zn}$ (x-axis) and without $\mathrm{Zn}$ (y-axis) for the conditions shown in Figure 4 in the main text. The line represents the 1:1 line. The modelling calculations were done with parameters from Table S2 and Table 1.

\section{G. Effect of $\mathrm{PO}_{4}$ on the surface electrostatic potentials}

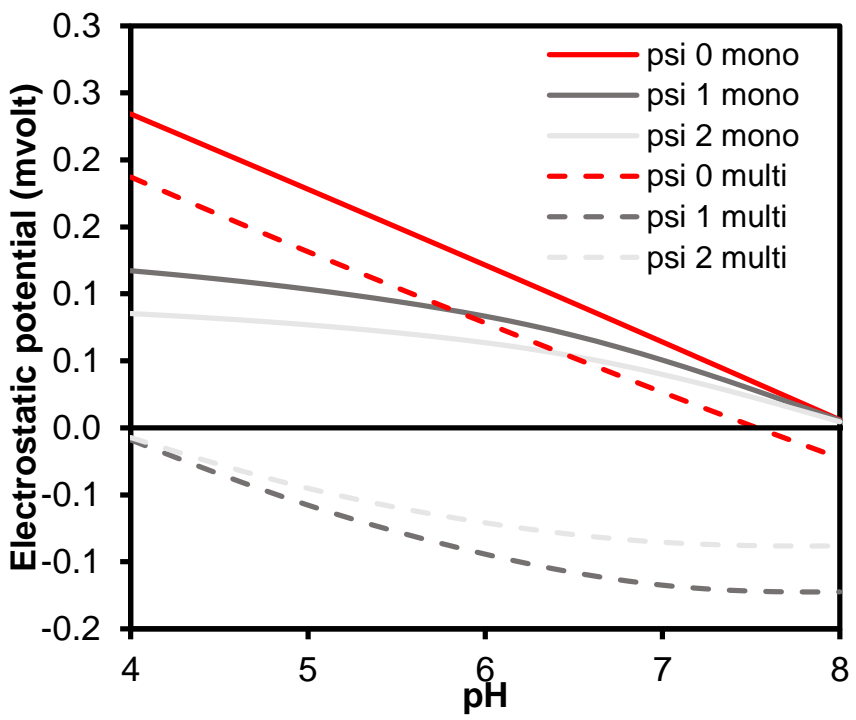

Figure S3: The electrostatic potentials (psi) calculated for the 3 different Stern planes with the CD models in mono-component systems $(\mathrm{Zn}+\mathrm{Fh})$ and multi-component systems $\left(\mathrm{Zn}+\mathrm{PO}_{4}+\mathrm{Fh}\right)$. Calculations were done using parameters from Table 1, option $\mathrm{C}$ in the main text for $\mathrm{Zn}$, and parameters from Table $\mathrm{S} 2$ for $\mathrm{PO}_{4}, \mathrm{H}, \mathrm{Na}$ and $\mathrm{NO}_{3}$. Calculations were done for systems with total concentrations of $33 \mu \mathrm{M} \mathrm{Zn}$ and 600 $\mu \mathrm{M} \mathrm{PO}_{4}, 3 \mathrm{~g} \mathrm{~L}^{-1} \mathrm{Fh}$ with a specific surface area of $698 \mathrm{~m}^{2} \mathrm{~g}^{-1}$. 


\section{H. Fe-Zn-P ternary complex}

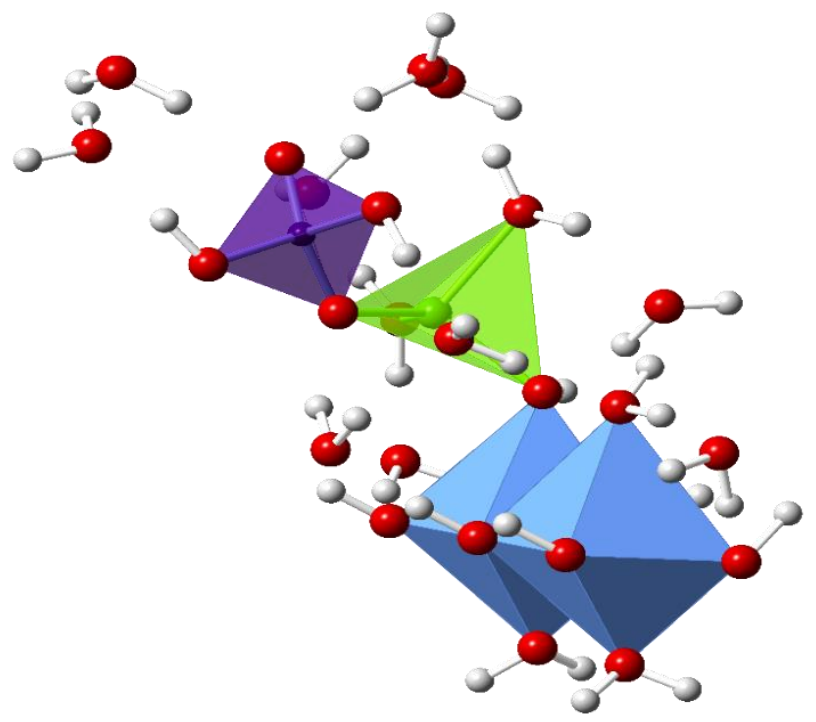

Figure S4: Hydrated monodentate $\mathrm{ZnH}_{2} \mathrm{PO}_{4}$ complex, optimized with MO/DFT/B3LYP/6-31+G** (Green tetrahedron $=\mathrm{Zn}$; Purple tetrahedron $=\mathrm{H}_{2} \mathrm{PO}_{4} ;$ Blue octahedra $=\mathrm{Fe}$ )

\section{Specific surface area of Fh preparations from literature based on $\mathrm{Zn}$ adsorption data}
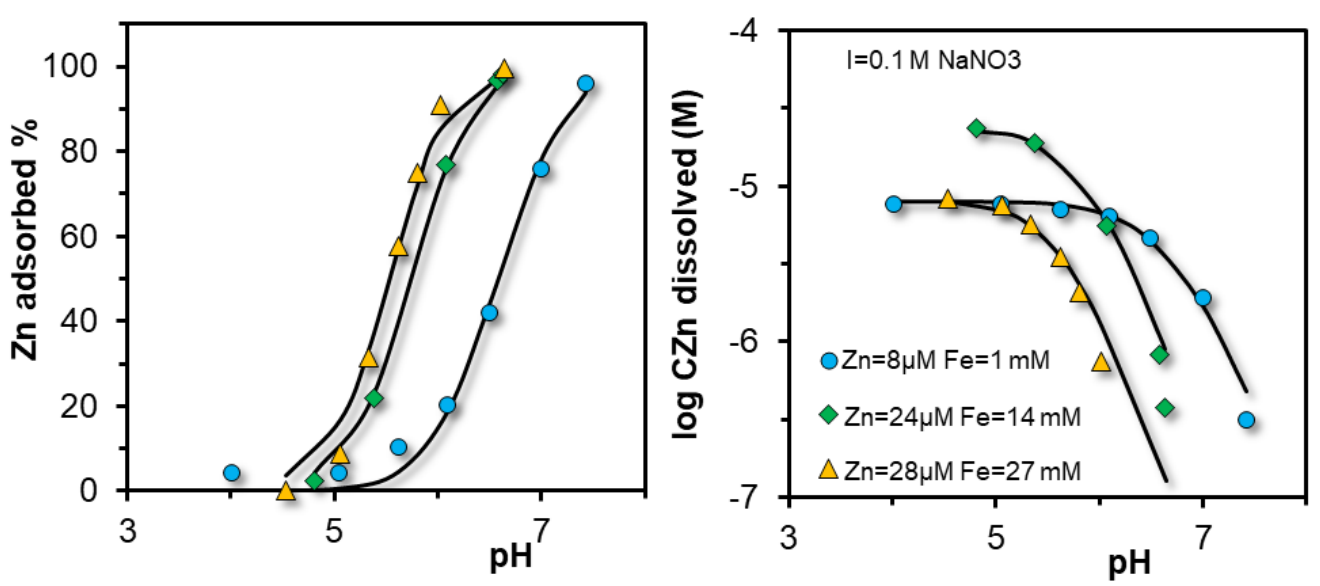

Figure S5: The $\mathrm{pH}$ dependent $\mathrm{Zn}$ adsorption data from Swedlund and Webster (2001) shown by the \% adsorbed and the equilibrium concentration. Markers show the experimental data, and the lines are the modeling calculations. The $\mathrm{Zn}$ adsorption was modeled with the parameters from Table $1 \mathrm{in}$ the main text, and the specific surface area was consequently fitted based on iteration with changing molar mass and capacitance values. The SSA was found to be $582 \pm 29 \mathrm{~m}^{2} \mathrm{~g}^{-1}$, with corresponding molar mass of $M_{\text {nano }}=94.1 \mathrm{~g} \mathrm{~mol}^{-1} \mathrm{Fe}$ and capacitance values of $C_{1}=1.14 \mathrm{~F}$ and $C_{2}=0.89 \mathrm{~F} \mathrm{~m}^{-2}$. The quality of fit is given by $R^{2}$ of 0.992 and a root mean squared error (RMSE) of $3.34 \%$. 


\section{J. $\mathrm{Cu}$ adsorption to $\mathrm{Fh}$ in the absence and presence of $\mathrm{PO}_{4}$}

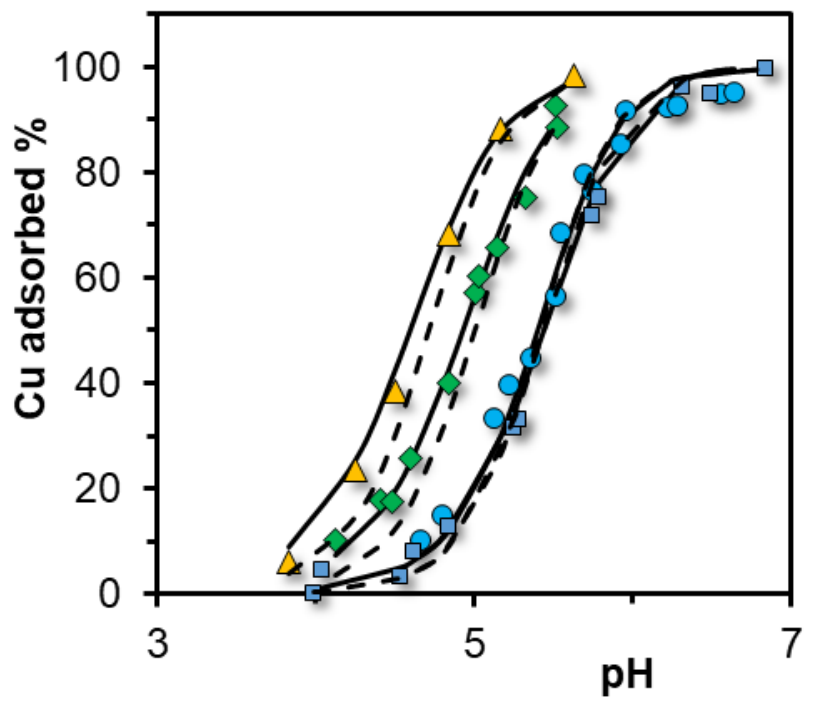

Figure S6: The $\mathrm{pH}$ dependent $\mathrm{Cu}$ adsorption data from Swedlund and Webster (2001). Markers show the experimental data, and the solid lines are the modeling calculations with parameters from Table 2 in the main text, option A (bidentate edge-sharing $\mathrm{Cu}$ complex), and the dashed lines are the modeling calculations with parameters from Table 2 in the main text, option B (bidentate edge-sharing and monodentate single corner hydrolyzed Cu complex). The data have been modeled using SSA $=582 \mathrm{~m}^{2} \mathrm{~g}$ 1 , a consistent molar mass of Mnano $=94.1 \mathrm{~g} \mathrm{~mol}^{-1} \mathrm{Fe}$, and the corresponding capacitance values of $C_{1, \text { nano }}=1.14$ and $C_{2, \text { nano }}=0.89 \mathrm{~F} \mathrm{~m}^{-2}$. 

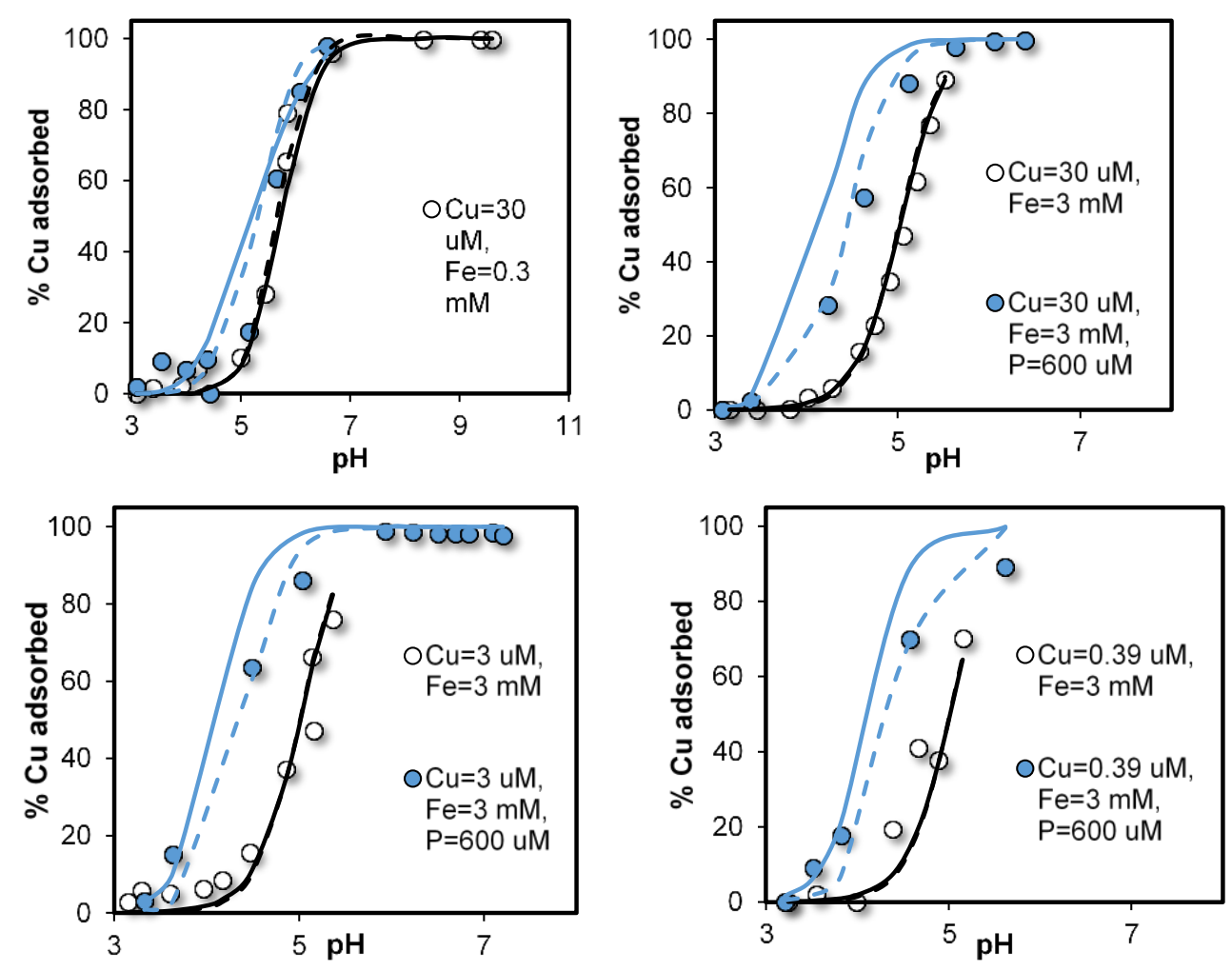

Figure S7: $\mathrm{pH}$ dependent $\mathrm{Cu}$ adsorption in the absence (white markers) and presence (blue markers) of $\mathrm{PO}_{4}$. Data from Tiberg et al. ${ }^{28}$ The lines show the modeling calculations for the mono-component (black lines) and multi-component (blue lines) systems, with parameters from Table 2 in the main text, option $A$ (solid lines) and B (dashed lines). The data have been modeled using SSA $=611 \mathrm{~m}^{2} \mathrm{~g}^{-1}$, a consistent molar mass of Mnano $=94.8 \mathrm{~g} \mathrm{~mol}^{-1} \mathrm{Fe}$, and the corresponding capacitance values of $C_{1, \text { nano }}=1.15$ and $C_{2, \text { nano }}=0.90 \mathrm{~F} \mathrm{~m}^{-2}$. 


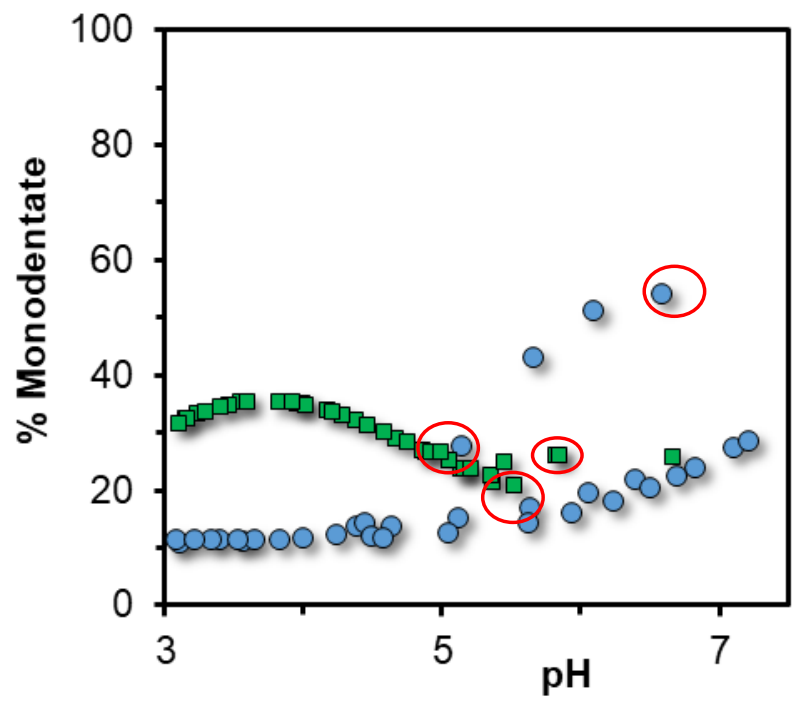

Figure S8: The $\mathrm{pH}$ dependent contribution of the $\mathrm{Cu}$ monodentate species to the overall $\mathrm{Cu}$ adsorption, calculated with the $\mathrm{CD}$ model with parameters from Table 2 in the main text, option $\mathrm{B}$. Calculations are shown for the samples from Tiberg et al. ${ }^{28}$ without $\mathrm{PO}_{4}$ (green markers) and with $\mathrm{PO}_{4}$ (blue markers). The samples for which Tiberg et al. ${ }^{28}$ performed EXAFS analysis, are encircled in red: one at $\mathrm{pH} 5$ and pH 5.85 without $\mathrm{PO}_{4}$, others with $\mathrm{PO}_{4}$ at pH 5.40, and 6.58. The EXAF sample with the highest pH was from the series with the highest $\mathrm{Cu}$ loading, and for these samples a higher contribution of the monodentate species was found by modeling the data with parameters from Table 2 in the main text, option B.

\section{REFERENCES}

1. Sillanpää, M. Micronutrients and the nutrient status of soils: a global study. (1982).

2. Degryse, F. \& Smolders, E. Mobility of $\mathrm{Cd}$ and $\mathrm{Zn}$ in polluted and unpolluted Spodosols. Eur. J. Soil Sci. 57, 122-133 (2006).

3. Boekhold, A. E. \& Van Der Zee, S. E. A. T. M. Field scale variability of cadmium and zinc in soil and barley. Environ. Monit. Assess. 29, 1-15 (1994).

4. Kirpichtchikova, T. A. et al. Speciation and solubility of heavy metals in contaminated soil using X-ray microfluorescence, EXAFS spectroscopy, chemical extraction, and thermodynamic modeling. Geochim. Cosmochim. Acta 70, 2163-2190 (2006).

5. Buekers, J., Degryse, F., Maes, A. \& Smolders, E. Modelling the effects of ageing on Cd, Zn, Ni and Cu solubility in soils using an assemblage model. Eur. J. Soil Sci. 59, 1160-1170 (2008).

6. Mao, L. C. C., Young, S. D. D., Tye, A. M. M. \& Bailey, E. H. H. Predicting trace metal solubility and fractionation in Urban soils from isotopic exchangeability. Environ. Pollut. 231, 1529-1542 (2017).

7. Duffner, A., Weng, L., Hoffland, E. \& van der Zee, S. E. A. T. M. Multi-surface modeling to predict free zinc ion concentrations in low-zinc soils. Environ. Sci. Technol. 48, 5700-5708 (2014).

8. Mendez, J. C. \& Hiemstra, T. Surface area of ferrihydrite consistently related to primary surface charge, ion pair formation, and specific ion adsorption. Chem. Geol. 532, (2020).

9. Mendez, J. C., Hiemstra, T. \& Koopmans, G. F. Assessing the Reactive Surface Area of Soils and the Association of Soil Organic Carbon with Natural Oxide Nanoparticles Using Ferrihydrite as Proxy. Environ. Sci. Technol. 54, 11990-12000 (2020).

10. Mendez, J. C. Ion complexation modelling of ferrihydrite: From fundamentals of metal (hydr)oxide nanoparticles to applications in soils systems. (Wageningen University, 2020). doi:10.18174/525623.

11. Kinniburgh, D. G. \& Jackson, M. L. Concentration and pH Dependence of Calcium and Zinc Adsorption by Iron Hydrous Oxide Gel1. Soil Sci. Soc. Am. J. 46, 56 (1982).

12. Swedlund, P. . \& Webster, J. . Cu and Zn ternary surface complex formation with $\mathrm{SO} 4$ on ferrihydrite and schwertmannite. Appl. Geochemistry 16, 503-511 (2001). 
13. Waychunas, G. A., Fuller, C. C., Davis, J. A. \& Rehr, J. J. Surface complexation and precipitate geometry for aqueous $\mathrm{Zn}$ (II) sorption on ferrihydrite: II. XANES analysis and simulation. Geochim. Cosmochim. Acta 67, 1031-1043 (2003).

14. Waychunas, G. A., Fuller, C. C. \& Davis, J. A. Surface complexation and precipitate geometry for aqueous $\mathrm{Zn}$ (II) sorption on ferrihydrite I: X-ray absorption extended fine structure spectroscopy analysis. Geochim. Cosmochim. Acta 66, 1119-1137 (2002).

15. Trivedi, P., Dyer, J. A., Sparks, D. L. \& Pandya, K. Mechanistic and thermodynamic interpretations of zinc sorption onto ferrihydrite. J. Colloid Interface Sci. 270, 77-85 (2004).

16. Cismasu, A. C., Levard, C., Michel, F. M. \& Brown, G. E. Properties of impurity-bearing ferrihydrite II: Insights into the surface structure and composition of pure, Al- and Si-bearing ferrihydrite from Zn(II) sorption experiments and Zn K-edge X-ray absorption spectroscopy. Geochim. Cosmochim. Acta 119, 46-60 (2013).

17. Dzombak, D. A. \& Morel, F. F. M. M. Surface Complexation Modeling: Hydrous Ferric Oxide. (John Wiley \& Sons, 1990).

18. Stern, O. ZUR THEORIE DER ELEKTROLYTISCHEN DOPPELSCHICHT. Zeitschrift für Elektrochemie und Angew. Phys. Chemie 30, 508-516 (1924).

19. Hiemstra, T. \& Van Riemsdijk WH. Surface Structural Ion Adsorption Modeling of Competitive Binding of Oxyanions by Metal (Hydr)oxides. J. Colloid Interface Sci. 210, 182-193 (1999).

20. Hiemstra, T. \& Van Riemsdijk, W. H. A Surface Structural Approach to Ion Adsorption: The Charge Distribution (CD) Model. J. Colloid Interface Sci. 179, 488-508 (1996).

21. Burgess, J. \& Prince, R. H. Zinc: Inorganic \& Coordination Chemistry. Encycl. Inorg. Bioinorg. Chem. (2011) doi:10.1002/9781119951438.eibc0242.

22. Pavlov, M., Siegbahn, P. E. M. \& Sandström, M. Hydration of beryllium, magnesium, calcium, and zinc ions using density functional theory. J. Phys. Chem. A 102, 219-228 (1998).

23. Schlegel, M. L., Manceau, A. \& Charlet, L. EXAFS study of $\mathrm{Zn}$ and ZnEDTA sorption at the goethite $(\alpha-\mathrm{FeOOH}) /$ water interface. J. Phys. IV JP 7, (1997).

24. Bochatay, L. \& Persson, P. Metal ion coordination at the water-manganite $(\mathrm{Y}-\mathrm{MnOOH})$ interface: II. An EXAFS study of zinc(II). J. Colloid Interface Sci. 229, 593-599 (2000).

25. Ha, J., Trainor, T. P., Farges, F. \& Brown, G. E. Interaction of aqueous Zn(II) with hematite nanoparticles and microparticles. Part 1. EXAFS study of $\mathrm{Zn}(\mathrm{II})$ adsorption and precipitation. Langmuir 25, 5574-5585 (2009).

26. Gräfe, M., Nachtegaal, M. \& Sparks, D. L. Formation of metal-arsenate precipitates at the goethite-water interface. Environ. Sci. Technol. 38, 6561-6570 (2004).

27. Liu, J. et al. Co-adsorption of phosphate and zinc(II) on the surface of ferrihydrite. Chemosphere 144, 1148-1155 (2016).

28. Tiberg, C., Sjöstedt, C., Persson, I. \& Gustafsson, J. P. Phosphate effects on copper(II) and lead(II) sorption to ferrihydrite. Geochim. Cosmochim. Acta 120, 140-157 (2013).

29. Rietra, R. P. J. J., Hiemstra, T. \& Van Riemsdijk, W. H. Interaction between calcium and phosphate adsorption on goethite. Environ. Sci. Technol. 35, 3369-3374 (2001).

30. Mendez, J. C. \& Hiemstra, T. Ternary complex formation of phosphate with $\mathrm{Ca}$ and $\mathrm{Mg}$ ions binding to ferrihydrite: Experiments and mechanisms. ACS Earth Sp. Chem. 4.4, acsearthspacechem.9b00320 (2020).

31. Hiemstra, T. \& Zhao, W. Reactivity of ferrihydrite and ferritin in relation to surface structure, size, and nanoparticle formation studied for phosphate and arsenate. Environ. Sci. Nano 3, 12651279 (2016).

32. Van Eynde, E., Mendez, J. C., Hiemstra, T. \& Comans, R. N. J. Boron adsorption to ferrihydrite with implications for surface speciation in soils: Experiments and modeling. ACS Earth Sp. Chem. acsearthspacechem.0c00078 (2020) doi:10.1021/acsearthspacechem.0c00078.

33. Hiemstra, T. Ferrihydrite interaction with silicate and competing oxyanions: Geometry and Hydrogen bonding of surface species. Geochim. Cosmochim. Acta 238, $453-476$ (2018).

34. Agbenin, J. O. Phosphate-induced zinc retention in a tropical semi-arid soil. Eur. J. Soil Sci. 49, 693-700 (1998).

35. Pérez-Novo, C., Bermúdez-Couso, A., López-Periago, E., Fernández-Calviño, D. \& AriasEstévez, M. Zinc adsorption in acid soils. Influence of phosphate. Geoderma 162, 358-364 (2011).

36. Brown, I. D. \& Altermatt, D. Bond-valence parameters obtained from a systematic analysis of the Inorganic Crystal Structure Database. Acta Crystallogr. Sect. B Struct. Sci. 41, 244-247 (1985).

37. Brown, I. D. Recent developments in the methods and applications of the bond valence model. Chem. Rev. 109, 6858-6919 (2009).

38. Mendez, J. C. \& Hiemstra, T. Carbonate Adsorption to Ferrihydrite: Competitive Interaction with Phosphate for Use in Soil Systems. ACS Earth Sp. Chem. 3.1, 129-141 (2018). 
39. Mendez, J. C. \& Hiemstra, T. High and low affinity sites of ferrihydrite for metal ion adsorption: Data and modeling of the alkaline-earth ions $\mathrm{Be}, \mathrm{Mg}, \mathrm{Ca}, \mathrm{Sr}, \mathrm{Ba}$, and $\mathrm{Ra}$. Geochim. Cosmochim. Acta (2020) doi:10.1016/j.gca.2020.07.032.

40. Hiemstra, T. \& Van Riemsdijk, W. H. A surface structural model for ferrihydrite I: Sites related to primary charge, molar mass, and mass density. Geochim. Cosmochim. Acta 73, 4423-4436 (2009).

41. Michel, F. M. et al. The structure of ferrihydrite, a nanocrystalline material. Science (80-. ). 316, 1726-1729 (2007).

42. Hiemstra, T. Surface and mineral structure of ferrihydrite. Geochim. Cosmochim. Acta 105, 316325 (2013).

43. Hiemstra, T. \& Van Riemsdijk, W. H. On the relationship between charge distribution, surface hydration, and the structure of the interface of metal hydroxides. J. Colloid Interface Sci. 301, 118 (2006).

44. Keizer, M. G. \& Van Riemsdijk, W. H. ECOSAT, a Computer Program for the Calculation of Chemical Speciation and Transport in Soil-Water Systems. (1995).

45. Kinniburgh, D. G. FIT User Guide, Technical Report WD/93/23. (1993).

46. Kinniburgh, D. G. The $\mathrm{H}+/ \mathrm{M} 2+$ exchange stoichiometry of calcium and zinc adsorption by ferrihydrite. J. Soil Sci. 34, 759-768 (1983).

47. Rahnemaie, R., Hiemstra, T. \& van Riemsdijk, W. H. Carbonate adsorption on goethite in competition with phosphate. J. Colloid Interface Sci. 315, 415-425 (2007).

48. Pauling, L. The principles determining the structure of complex ionic crystals. J. Am. Chem. Soc. 51, 1010-1026 (1929).

49. Lindsay, W. L. Chemical equilibria in soils. (Wiley, 1979).

50. Tiberg, C. \& Gustafsson, J. P. Phosphate effects on cadmium(II) sorption to ferrihydrite. J. Colloid Interface Sci. 471, 103-111 (2016).

51. Swedlund, $\mathrm{P}$. Modelling $\mathrm{Cu}, \mathrm{Zn}, \mathrm{Cd}$ and $\mathrm{Pb}$ Adsorption by Iron Oxyhydroxides in $\mathrm{SO} 4$-rich Systems Simulating Acid Mine Drainage. (2004).

52. Moon, E. M. \& Peacock, C. L. Adsorption of $\mathrm{Cu}(\mathrm{II})$ to ferrihydrite and ferrihydrite-bacteria composites: Importance of the carboxyl group for Cu mobility in natural environments. Geochim. Cosmochim. Acta 92, 203-219 (2012).

53. Scheinost, A. C., Abend, S., Pandya, K. I. \& Sparks, D. L. Kinetic controls on Cu and Pb sorption by ferrihydrite. Environ. Sci. Technol. 35, 1090-1096 (2001). 
Chapter 5 


\section{Chapter 6 \\ Boron speciation and extractability in temperate and tropical soils: a multi-surface modeling approach}

Elise Van Eynde, Liping Weng, and Rob N.J. Comans

Published as:

Van Eynde, E., Weng, L., \& Comans, R. N. (2020). Boron speciation and extractability in temperate and tropical soils: A multi-surface modeling approach. Applied Geochemistry, 123, 104797.

doi.org/10.1016/j.apgeochem.2020.104797 


\begin{abstract}
Boron is an essential micronutrient for plants, but can also be toxic when present in excess in the soil solution. A multi-surface geochemical model was used to assess the important processes that affect the distribution of the geochemically reactive $B$ in soils over the solution and solid phase. The multi-surface model was based on the adsorption of $B$ on dissolved and solid humic acids, representing reactive organic matter, ferrihydrite, representing the Fe and Al (hydr)oxides, and clay mineral edges. In addition, the performance of previously proposed extraction methods for measuring reactive $B$ was evaluated. Based on $B$ measured in $0.01 \mathrm{M} \mathrm{CaCl}_{2}$ soil extracts $\left(7-85 \mu \mathrm{mol} \mathrm{kg}{ }^{-1}\right.$ soil), we calculated the reactive boron concentration for 5 temperate and 5 tropical soils $\left(8-106 \mu \mathrm{mol} \mathrm{kg}{ }^{-1}\right.$ soil). We found that extractions with $0.43 \mathrm{M} \mathrm{HNO}_{3}$ or with $0.2 \mathrm{M}$ mannitol $+0.1 \mathrm{M}$ triethanolamine buffer extract on average 240 and $177 \%$ of the reactive $B$ predicted by the model, thus releasing additional $B$ that is assumed to be not or only very slowly available for exchange with the soil solution. Reactive $\mathrm{B}$ calculated by the model corresponded best to the $\mathrm{B}$ measured in a $0.05 \mathrm{M} \mathrm{KH}_{2} \mathrm{PO}_{4}(\mathrm{pH}$ 4.5) extraction. In general, the multi-surface modeling showed that $68 \%$ or more of reactive boron was present in the solution phase for the soils in this study and that the adsorption was dominated by oxides in the tropical soils, while solid organic matter was the main adsorbent in the temperate soils. When changing the soil $\mathrm{pH}\left(\mathrm{CaCl}_{2}\right)$, B concentration was found to decrease with increasing $\mathrm{pH}$, and both experimental data and modelling suggests that this effect is mainly due to increased binding of $B$ to organic matter.
\end{abstract}




\section{INTRODUCTION}

Boron (B) is an essential micronutrient for plant growth and development ${ }^{1}$. Boron deficiencies are widespread around the globe, often in weathered acidic soils or calcareous soils with high $\mathrm{pH}^{2}$. On the other hand, $\mathrm{B}$ can also be toxic when present in excess in the soil solution ${ }^{3,4}$. Therefore, $B$ requires accurate nutrient management, which demands a thorough understanding of $B$ speciation in soils and the processes that control $B$ bioavailability.

Total B content in soils can go up to $8000 \mu \mathrm{mol} \mathrm{kg}{ }^{-1}, 5$ of which a large part is present in primary minerals, or occluded in secondary minerals ${ }^{6}$. There are also studies showing that most of the total $B$ in soils is part of the organic matter fractions ${ }^{7}$. These total pools of $B$ are not directly available for plants, as there is no relation between $\mathrm{B}$ uptake by plants and total $\mathrm{B}$ in soils ${ }^{8,9}$. Plants take up $\mathrm{B}$ from the soil solution as boric acid $\left(\mathrm{B}(\mathrm{OH})_{3}\right)^{10}$ and $\mathrm{B}$ concentrations in soil solutions often range between 1 and $300 \mu \mathrm{mol} \mathrm{L}^{-1} .^{5}$ The $B$ that originates from mineralization of fresh organic matter, weathering of minerals or atmospheric deposition ${ }^{11}$ enters the reactive $\mathrm{B}$ pool and is distributed over the solid and solution phase by adsorption/desorption processes. Despite the fact that $\mathrm{B}$ is relatively mobile compared to the other micronutrients ${ }^{5}$, it has been stated by various authors that the adsorption onto reactive soil surfaces controls the $B$ in solution, and therefore regulates $B$ bioavailability, potential toxicity and leaching ${ }^{12,13}$. Adsorption studies of $B$ have focused on organic matter ${ }^{14-17}$, clay minerals ${ }^{18-22}$, poorly crystalline $\mathrm{Al}$ and $\mathrm{Fe}$ oxides ${ }^{20,23-25}$ and crystalline oxides ${ }^{20,26}$. In general, it has been found that $\mathrm{B}$ adsorption on these mineral and organic reactive surfaces increases with increasing $\mathrm{pH}$, and that the maximum adsorption occurs at $\mathrm{pH}$ between 8 and 10, after which the adsorption decreases again. A similar $\mathrm{pH}$ dependent adsorption behavior has been found for $\mathrm{B}$ adsorption in soils ${ }^{27-30}$. Van Eynde et al. ${ }^{25}$ have shown with a multi-surface modeling simulation that $\mathrm{pH}$ can also determine the relative importance of each of these reactive surfaces for $B$ adsorption: in the acidic region, oxides may be more important whereas organic matter may bind most of the $B$ in the alkaline region. However, these modeling simulations have not yet been validated for soil samples.

Geochemical surface complexation models (SCM) are powerful tools to enhance the quantitative understanding of the speciation of trace elements in the soil environment, and processes that affect their bioavailability. The constant capacitance model (CC) has been used mostly to model B adsorption behavior in soils ${ }^{20,29}$. Goldberg et al. ${ }^{31-33}$ have used the $\mathrm{CC}$ model to describe $\mathrm{pH}$-dependent $\mathrm{B}$ adsorption in soil systems, considering the soil as one type of plane for surface reactions in a generalized composite approach ${ }^{34}$. Binding constants were fitted to $\mathrm{pH}$ dependent $\mathrm{B}$ adsorption data for the soil-specific assemblage ${ }^{31}$, and as a result are not generically applicable to other soils. To make the constants more widely 
usable, Goldberg et al. ${ }^{32}$ used regression models to relate these binding constants for $\mathrm{B}$ adsorption in soils to the surface area, organic carbon, inorganic carbon and Al content, which suggests that different surfaces are playing a role in B adsorption among soil types. The use of the $\mathrm{CC}$ model for modeling ion speciation in soils does not take into account the specific characteristics (i.e., binding capacities, affinities, heterogeneity of surface sites) of each of the reactive surfaces, which makes it difficult to assign relative importance to the different reactive surfaces for $B$ adsorption. Conversely, a multi-surface or assemblage modeling approach combines separate models for ion binding to the different reactive surfaces ${ }^{35,36}$. Examples of such models that have been recently parameterized for $B$ are the Charge Distribution and Multi Site Complexation model (CD-MUSIC) for modeling adsorption on oxides ${ }^{25,26}$, or the Non-ideal Competitive Adsorption (NICA) Donnan model for modeling adsorption on organic matter ${ }^{37}$.

No attempts have yet been made to model the speciation of naturally occurring $B$ in soils instead of modeling adsorption experiments with added $\mathrm{B}^{20,29}$. Modeling the partitioning of natural $B$ over the solid and solution phase requires an estimation of the geochemically reactive concentration of $B$ in soils. The reactive concentration is considered as potentially available for uptake by plants, and is the concentration that is distributed over the solid and solution phase through adsorption/desorption and precipitation/dissolution equilibria ${ }^{38}$. The actual available pool in solution then depends on the reactive concentration, $\mathrm{pH}$, content of reactive surfaces and competing ions, and is often approximated by extraction with weak salts ${ }^{39}$ such as $\mathrm{CaCl}_{2}{ }^{40}$. Recently, an ISO standard has been developed to extract the geochemical reactive pool of trace elements, using a dilute nitric acid solution at approximately $\mathrm{pH} 0.5^{41}$. The method has been evaluated for a wide range of trace elements based on geochemical multi-surface modeling, but not for $\mathrm{B}^{38}$. Others have used alternative extraction solutions as part of fractionation schemes to quantify the adsorbed $\mathrm{B}$, such as $\mathrm{KH}_{2} \mathrm{PO}_{4}{ }^{6,42}$ that is based on ligand exchange between $\mathrm{B}$ and phosphate $\left(\mathrm{PO}_{4}\right)$ on reactive surfaces, or polyols that are known to form soluble complexes with $B^{8,43,44}$.

In this study, we will use a multi-surface modeling approach for simulating natural B speciation in both temperate and tropical soil samples. Boron speciation and extractability may differ between these two climatic regions due to differences in content of important reactive soil surfaces and mineralogy. The first aim is to evaluate the use of different previously proposed extraction methods $(0.05 \mathrm{M}$ $\mathrm{KH}_{2} \mathrm{PO}_{4}, 0.2 \mathrm{M}$ mannitol ( $\mathrm{pH} 7.3$ ) and $0.43 \mathrm{M} \mathrm{HNO}_{3}$ ), based on different $\mathrm{B}$-exchange principles, for estimating the reactive $B$. The calculated reactive $B$ with a multisurface model will be compared with the $B$ measured in the three different extraction methods. Secondly, we aim to evaluate which soil surfaces control B speciation in temperate and tropical soils in relation to soil properties and $\mathrm{pH}$. We will discuss the 
quality of the model prediction and possible explanations for model performance limitations in relation to soil properties.

\section{MATERIAL AND METHODS}

\subsection{Soil analyses}

Two sets of air dried top soil samples were used for extractions and modeling of the soil $B$. The first set consisted of 5 temperate top soil samples from the Netherlands. These are samples taken from sandy, clay or clay-peat arable fields. The second set consisted of 5 tropical top soil samples from arable fields in Burundi. These 5 samples were selected out of a larger set of soil samples $(n=15)$ that were taken from different locations in the country. All 5 soils are classified as Ferralsol based on their geo-referenced location ${ }^{45}$.

The solution concentration of $\mathrm{B}\left(\mathrm{B}-\mathrm{CaCl}_{2}\right)$, dissolved organic carbon $(\mathrm{DOC})$ and $\mathrm{pH}\left(\mathrm{CaCl}_{2}\right)$ were measured in extracts of $0.01 \mathrm{M} \mathrm{CaCl}^{40}$. Calcium chloride extractions have often been used as a proxy for the soil solution, and to validate multi-surface models that aim to model the solid-solution partitioning of ions $36,38,46,47$. A $0.01 \mathrm{M} \mathrm{CaCl}_{2}$ was freshly prepared in a plastic volumetric flask, in order to avoid $\mathrm{B}$ contamination from glassware. A fixed volume of this solution was added to the soil samples at a solution-to-solid ratio (SSR) of 10 in polypropylene centrifuge tubes. The suspensions were equilibrated in a horizontal shaker at 180 oscillations minute1 for 24 hours and were afterwards centrifuged for $10 \mathrm{~min}$ at $1800 \mathrm{~g}$ and filtered over a $0.45 \mu \mathrm{m}$ membrane filter. The $\mathrm{B}-\mathrm{CaCl}_{2}$ was measured in an acidified subsample of the supernatant, using High Resolution Inductively Coupled Plasma Mass Spectrometry (HR-ICP-MS, Element 2, Thermo Scientific) with a Teflon nebulizer and spray chamber instead of (boron-silicate) glass. All measurements were done in duplicate and average values were used as final estimates for $\mathrm{B}-\mathrm{CaCl}_{2}$ concentrations. The average difference between both $\mathrm{B}$ measurements was around $10 \%$ of the average concentration. The average $B$ concentrations of the blanks $(n=5)$ was $0.4 \pm 1.1 \mu \mathrm{g} \mathrm{L}^{-1}$.

The $\mathrm{pH}\left(\mathrm{CaCl}_{2}\right)$ was measured using a glass electrode, from which no significant $\mathrm{B}$ release was found (results not shown). The dissolved total carbon and dissolved inorganic carbon concentrations were measured in a non-acidified supernatant with an Segmented Flow Analyzer (SFA-TOC, San++, Skalar) equipped with an IR detector that measures the amount of $\mathrm{CO}_{2(\mathrm{~g})}$ after an internal acidification and destruction step, and the DOC concentrations were calculated as the difference between total and inorganic carbon. The inorganic phosphate $\left(\mathrm{PO}_{4}-\mathrm{CaCl}_{2}\right)$ concentration in the $\mathrm{CaCl}_{2}$ extraction was measured by the molybdenum-blue method $^{48}$ using a fully automated segmented flow analyzer (SFA-PO4). 
The clay content in the Burundi soils was measured by laser-diffraction analysis ${ }^{49}$. Soils (0.5-1.5 g) were pre-treated three times with $\mathrm{H}_{2} \mathrm{O}_{2}$ and once with $\mathrm{HCl}$, while standing in a warm water bath. Before analysis, the pre-treated samples were suspended in water $(\sim 200 \mathrm{ml})$ which was removed and replaced with fresh ultrapure water for multiple times to remove excess of salts. The measured volume percentage of the fraction smaller than $2 \mu \mathrm{m}$ was re-calculated to the mass percentage of clay using a particle density of $2.6 \mathrm{~g} \mathrm{~cm}^{-3}$ and a bulk density of $1.3 \mathrm{~g}$ $\mathrm{cm}^{-3}$. The clay content from the Dutch soils was derived by the accredited laboratory of Eurofins Agro Netherlands via Near-Infrared Spectroscopy measurements from which clay content was calculated via a spectral library that is calibrated against the sieve and pipet method ${ }^{50}$.

An ammonium oxalate $(A O)$ extraction ${ }^{51}$ was used for both soil sets to measure micro-crystalline $\mathrm{Fe}$ and $\mathrm{Al}$, and $\mathrm{P}$ (Fe-AO, Al-AO, $\mathrm{Pt}-\mathrm{AO})$. Iron (Fe-D) and $\mathrm{Al}(\mathrm{Al}-\mathrm{D})$ were also measured in a sodium-dithionite extraction (for the Dutch soils as described by Hiemstra et al. ${ }^{52}$; for the Burundian soils as described in $\mathrm{ISO}^{53}$ ), and crystalline $\mathrm{Fe}$ and $\mathrm{Al}$ were calculated as the difference between the $\mathrm{Fe}$ and $\mathrm{Al}$ measured in the dithionite and $\mathrm{AO}$ extraction. The $\mathrm{Fe}, \mathrm{Al}$ and $\mathrm{P}_{\mathrm{t}}$ in ammonium oxalate and $\mathrm{Fe}$ and $\mathrm{Al}$ in the dithionite extractions were analyzed using Inductively coupled plasma optical emission spectrometry (ICP-OES, iCAP6500, Thermo Scientific). The $\mathrm{P}_{\mathrm{t}}$ measured in $\mathrm{AO}$ extraction can include phosphorus species that are different from inorganic $\mathrm{PO}_{4}$, such as organic $\mathrm{P}^{54,55}$. The latter is expected to not control the inorganic $\mathrm{PO}_{4}$ concentration in the $\mathrm{CaCl}_{2}$ extraction. Therefore, for the Burundian soils, the inorganic $\mathrm{PO}_{4}$ concentration in the $\mathrm{AO}$ was measured using the molybdenum blue method ${ }^{48}$, using an SFA-PO ${ }_{4}$ instrument. The samples were diluted 100 times with ultra-pure water to eliminate the interference of oxalate for the colorimetric reaction ${ }^{56,57}$. For the Dutch soils, $\mathrm{PO}_{4}$ was not measured in the $\mathrm{AO}$ extraction, but instead, an empirical relation was used to calculate the contribution of organic $\mathrm{P}$ species to the $\mathrm{Pt}_{\mathrm{t}}$ measured in the $\mathrm{AO}$ based on the $\mathrm{OC}$ content in the soils ${ }^{58}$.

The humic and fulvic acid fractions of the organic matter in the solid phase were measured following the procedure as described by van Zomeren and Comans ${ }^{59}$. The soils were extracted with $\sim 0.1 \mathrm{M} \mathrm{HCl}(\mathrm{pH} 1)$, after which the pellet was re-dissolved in $\sim 0.1 \mathrm{M} \mathrm{NaOH}$ (pH 12). Subsequently, the supernatant of the acid extraction and the supernatant of the acidified base extract were combined and equilibrated with pre-cleaned DAX-8 resin. The fulvic acids were afterwards desorbed from the resin with $0.1 \mathrm{M} \mathrm{KOH}$. The HA were measured by re-dissolving the pellet in the acidified base extract with $0.1 \mathrm{M} \mathrm{KOH}$. 
In addition, total boron content was measured in the soils. Soil digestion was done with a mixture of $\mathrm{HNO}_{3}-\mathrm{HClO}_{4}-\mathrm{HF}$, in a ratio of $90: 5: 5$, under heating at $190^{\circ} \mathrm{C}$ for 10 hours. Element concentrations of $\mathrm{B}, \mathrm{Si}, \mathrm{Fe}$ and $\mathrm{Al}$ were measured using ICP-OES.

\section{$2.2 \quad \mathrm{pH}$ dependent B solubility}

The $\mathrm{B}-\mathrm{CaCl}_{2}$ concentration in the 10 soils was measured at different $\mathrm{pH}\left(\mathrm{CaCl}_{2}\right)$ levels, to assess the $\mathrm{pH}$ dependent $\mathrm{B}$ solubility. Two grams of soil was put into 50 $\mathrm{ml}$ polypropylene centrifuge tubes, together with $10 \mathrm{ml}$ of $0.02 \mathrm{M} \mathrm{CaCl}_{2}$ and $8 \mathrm{ml}$ of ultra-pure water (UPW). The $\mathrm{pH}$ was adjusted through addition of $0.5 \mathrm{M}$ $\mathrm{HNO}_{3} / \mathrm{NaOH}$. After 2 hours, the $\mathrm{pH}$ was checked and more acid or base was added to obtain the pre-determined $\mathrm{pH}$ value. The same was done after 6 hours, and ultrapure water was added in order to reach a total volume of $20 \mathrm{ml}$ with a final $\mathrm{CaCl}_{2}$ concentration of $0.01 \mathrm{M}$. After 24 hours, the final $\mathrm{pH}$ was measured, and samples were centrifuged and filtered over a $0.45 \mu \mathrm{m}$ filter for the analysis of $\mathrm{B}$ on HR-ICP$\mathrm{MS}$, and TOC/IC on the SFA.

\subsection{Reactive B extractions}

Three different extraction methods were tested to estimate the geochemically reactive fraction of $B$ in the soils. For all three soil extractions, no glassware was used throughout the extraction procedure to avoid B contamination.

Firstly, samples were suspended in a freshly prepared extraction solution with 0.05 $\mathrm{M} \mathrm{KH}_{2} \mathrm{PO}_{4}$ at a SSR of 20. The suspensions were shaken for 16 hours, and afterwards centrifuged and filtered over a $0.45 \mu \mathrm{m}$ membrane filter. The protocol was based on the design by Hou et al. ${ }^{42}$, who used this extraction solution as part of a fractionation scheme, to quantify specifically bound $B$ in soils on surfaces with variable charge. The $B$ concentration in the blanks $(n=2)$ was negative.

Secondly, a $0.43 \mathrm{M} \mathrm{HNO}_{3}$ extraction solution was used, with a SSR of 10 and an equilibration time of 4 hours, according to the ISO standard ${ }^{41}$. After centrifugation, the samples were filtered over a $0.45 \mu \mathrm{m}$ membrane filter. The $B$ concentration in the blanks $(n=4)$ was negative. This ISO protocol has been developed for measuring the reactive content of trace elements in the environment, and has been evaluated for a wide range of elements based on multi-surface modeling ${ }^{38}$. However, B was not included in that study, and the $0.43 \mathrm{M} \mathrm{HNO}_{3}$ extraction is therefore evaluated here for its suitability to measure reactive $B$.

Thirdly, we used a mannitol extraction to estimate reactive B. Bingham ${ }^{43}$ formulated an extraction protocol to measure $B$ by using $0.01 \mathrm{M}$ mannitol $+0.01 \mathrm{M} \mathrm{CaCl}_{2}$ solution. However, Goldberg and Suarez ${ }^{44}$ showed that the addition of $\mathrm{Ca}$ in the extraction solution resulted in a lower B concentration. The Ca was not included in 
their optimal extraction solution, since Goldberg and Suarez ${ }^{44}$ argued that the only reason to include $\mathrm{Ca}$ is to facilitate filtration because of coagulation of organic matter. Vaughan and $\mathrm{Howe}^{60}$ found that mannitol had similar effectiveness in extracting $B$ as sorbitol, another polyol, when buffering the $\mathrm{pH}$ at 7.3 in both extraction solutions. A mannitol and sorbitol extraction is based on the principle of complex formation between the polyol and borate ${ }^{61}$. Borate $\left(B(O H) 4^{-}\right)$is the anionic form of $B$ that is formed according to the reaction ${ }^{62}$ :

$\mathrm{B}(\mathrm{OH})_{3}^{0}+\mathrm{H}_{2} \mathrm{O} \Leftrightarrow \mathrm{B}(\mathrm{OH})_{4}^{-}+\mathrm{H}^{+} \quad \log K=-9.24$

The complexation of mannitol with boron then proceeds according to the reactions ${ }^{61}$ : $\mathrm{B}(\mathrm{OH})_{3}^{0}+\mathrm{H}_{2} \mathrm{O}+$ Mannitol $\Leftrightarrow \mathrm{B}(\mathrm{OH})_{4}^{-}-$Mannitol $+\mathrm{H}^{+} \quad \log \mathrm{K}=-6.45$

$\mathrm{B}(\mathrm{OH})_{3}^{0}+\mathrm{H}_{2} \mathrm{O}+2$ Mannitol $\Leftrightarrow \mathrm{B}(\mathrm{OH})_{4}^{-}-$Mannitol $_{2}+\mathrm{H}^{+} \quad \log \mathrm{K}=-4.26$

with the latter being the most important species. The formation of borate is only significant at high $\mathrm{pH}$ values. Since the polyol forms complexes with borate ${ }^{61}$, the $\mathrm{pH}$ of the extraction solution affects the concentration of borate and thus the final $\mathrm{B}$ measured in the extract ${ }^{63}$. In this study, the extraction was executed with a mannitol concentration of $0.2 \mathrm{M}$, together with $0.1 \mathrm{M}$ triethanolamine (TEA) buffer ( $\mathrm{pH} 7.3$ ), with an equilibration time of 24 hours and a SSR of 10 . This concentration, equilibration time and SSR were suggested by Goldberg and Suarez (2014) for their optimal sorbitol extraction. The samples were filtered over a $0.45 \mu \mathrm{m}$ filter after centrifugation. The average $B$ concentration of the blanks $(n=2)$ was $0.01 \pm 0.008$ $\mathrm{mg} \mathrm{L}^{-1}$.

For the mannitol extraction, the $\mathrm{B}$ was measured using ICP-OES. For the $\mathrm{HNO}_{3}$ extraction, ICP-OES was used for the Dutch soils, and HR-ICP-MS for the Burundi soils. For the $\mathrm{B}$ measurement in $\mathrm{CaCl}_{2}$ and $\mathrm{KH}_{2} \mathrm{PO}_{4}, \mathrm{HR}-\mathrm{ICP}-\mathrm{MS}$ was used. The concentrations of $\mathrm{Si}, \mathrm{Fe}$ and $\mathrm{Al}$ in the three extractions was measured using ICPOES, to assess the potential dissolution of minerals that could contain $B$.

\subsection{Multi-surface modeling set-up}

The multi-surface model included ion adsorption to organic matter, the edges of clay minerals and $\mathrm{Fe}$ and $\mathrm{Al}$ (hydr)oxides. For adsorption to the reactive surfaces, we used specific models for each surface. The aqueous species are given in Table S1 in the supporting information. All modeling and adsorption parameters are given in Tables S2-S3. Modeling calculations were performed in ECOSAT, version 4.964.

\section{Oxides}

The CD-MUSIC model was used to model adsorption to oxides. The content of poorly crystalline oxides was calculated based on the $\mathrm{Al}$ and $\mathrm{Fe}$ found in $\mathrm{AO}$ extraction. To transform the moles of $\mathrm{Al}$ and $\mathrm{Fe}$ in the $\mathrm{AO}$ extraction to the oxide 
mass, a molar mass of $95 \mathrm{~g} \mathrm{~mol}^{-1} \mathrm{Fe}$ and $84 \mathrm{~g} \mathrm{~mol}^{-1} \mathrm{Al}$ was used, which corresponds to particles with a specific surface area of $600 \mathrm{~m}^{2}$ per gram of oxide ${ }^{65}$. The amount of crystalline oxides was calculated based on the difference between the $\mathrm{Fe}$ and $\mathrm{Al}$ in the sodium-dithionite extraction and the $\mathrm{Fe}$ and $\mathrm{Al}$ measured in the ammoniumoxalate extraction, with a molar mass of respectively 89 and $78 \mathrm{~g} \mathrm{~mol}^{-1}$ for $\mathrm{Fe}$ (goethite) and $\mathrm{Al}$ (gibbsite) ${ }^{52}$. For the crystalline oxides, a specific surface area of $100 \mathrm{~m}^{2} \mathrm{~g}^{-1}$ was assumed ${ }^{47}$. Ferrihydrite (Fh) was used as model oxide for the natural oxide fraction of the soils. The total Fh content was calculated by summing the mass of poorly crystalline oxides and $1 / 6$ of the mass of crystalline oxides, and in the modelling calculations a specific surface area of $600 \mathrm{~m}^{2} \mathrm{~g}^{-1}$ was used for Fh.

Next to $\mathrm{B}(\mathrm{OH})_{3}$, adsorption of $\mathrm{H}^{+}, \mathrm{Ca}^{2+}, \mathrm{Cl}^{2}, \mathrm{CO}_{3}{ }^{2-}$ and $\mathrm{PO}_{4}{ }^{3-}$ on the oxide surface was included in the model. The $\mathrm{B}$ binding on oxide surfaces is $\mathrm{pH}$ dependent. Competitive anions such as $\mathrm{PO}_{4}{ }^{3-}$ and $\mathrm{CO}_{3}{ }^{2-}$ can reduce the $\mathrm{B}$ binding to oxides ${ }^{25,66}$. Binding of both ions is affected by the $\mathrm{Ca}^{2+}$ and $\mathrm{Cl}^{-}$ion adsorption due to mainly electrostatic effects and additional surface species. The $\mathrm{Ca}^{2+}, \mathrm{CO}_{3}{ }^{3-}$ and $\mathrm{Cl}^{-}$ concentrations in the $\mathrm{CaCl}_{2}$ extraction, and the inorganic $\mathrm{PO}_{4}$ in the $\mathrm{AO}$ extraction were given as input for modelling $B$ speciation. For modeling the adsorption of these ions to ferrihydrite, a consistent set of recently derived adsorption parameters was used $^{25,67-70}$ which is shown in Table S2.

In addition, experiments have shown that soil organic matter can be a competitor with $B$ for adsorption on the oxide surface ${ }^{71,72}$. To account for this effect, we have used the modelling approach from Hiemstra et al. ${ }^{73}$ to calculate the natural organic matter (NOM) loading on the oxide surface. The NOM surface species with corresponding charge distribution and $\log K$ values are given in Table S2. Before modelling $B$ speciation, the NOM loading was fitted for each soil sample based on the $\mathrm{PO}_{4}$ solid-solution partitioning. In these modelling calculations, the inorganic $\mathrm{PO}_{4}$ in the $\mathrm{AO}$ extraction was given as input, together with the $\mathrm{pH}\left(\mathrm{CaCl}_{2}\right)$, the $\mathrm{CO}_{3}{ }^{2-}, \mathrm{Ca}^{2+}$ and $\mathrm{Cl}^{-}$concentration in the $\mathrm{CaCl}_{2}$ extraction, the content of clay minerals and $\mathrm{Fh}$. The NOM loading was adjusted until the calculated dissolved $\mathrm{PO}_{4}$ corresponded to the measured $\mathrm{PO}_{4}$ concentration in the $\mathrm{CaCl}_{2}$ extraction, and this $\mathrm{NOM}$ loading was further used in modelling the $B$ speciation. The results from this modelling calculation for the different soils are given in Table S4.

The effect of including the different competitors for $\mathrm{B}$ adsorption on oxides, i.e. NOM, $\mathrm{CO}_{3}$ and $\mathrm{PO}_{4}$, on the modelling calculations is shown in Figure $\mathrm{S} 1$ in the supporting information.

\section{Organic matter}

Adsorption to organic matter was modelled by using the NICA-Donnan model ${ }^{74}$, assuming that the interaction between oxides and organic matter does not influence 
the $B$ adsorption to the organic matter. We considered the reactive organic matter as humic acids, since NICA parameters are only available for B adsorption on humic acids $^{37}$. For the dissolved organic matter, $50 \%$ was considered as humic acids for both Dutch and Burundian soils as it has been done previously ${ }^{38,47}$. The solid humic acids were calculated as the sum of the fulvic and humic acids that were measured according to the batch procedure of van Zomeren and Comans ${ }^{59}$. Next to B, we considered adsorption of $\mathrm{H}^{+}$and $\mathrm{Ca}^{2+}$ on humic acids. Goli et al. ${ }^{37}$ showed that next to $\mathrm{pH}$, the $\mathrm{B}$ binding to $\mathrm{HA}$ is affected by the presence of $\mathrm{Ca}^{2+}$ due to site competition and electrostatics. Except for the model parameters for B, all other NICA-Donnan parameters are based on the generic parameters for humic acid from Milne et al. ${ }^{75}$ including the site densities of the carboxylic and phenolic groups, as well as the bparameter to calculate the Donnan volume based on ionic strength (Table S3).

The NICA-Donnan model calculates total ion adsorption as the sum of the specifically and electrostatically adsorbed concentrations, with the latter being the Donnan species in the Donnan layer of the humic acids. For the neutral $\mathrm{B}(\mathrm{OH})_{3}{ }^{0}$ ion, which is the dominant ion in the $\mathrm{pH}$ range of these soil samples (Eq. 1), there is no electrostatic attraction. Therefore, we did not include the $\mathrm{B}(\mathrm{OH})_{3}{ }^{0}$ present in the Donnan layer for calculating the total adsorbed $B$ to humic acids. Calculations showed that the contribution of the electrostatically bound $B$ as Donnan species to total $\mathrm{B}$ adsorbed to humic acids in the $\mathrm{CaCl}_{2}$ extraction was less than $10 \%$.

\section{Clay}

In previous multi-surface modeling studies, ion adsorption to clay minerals has been considered as an electrostatic process, which has been modelled using a Donnan approach ${ }^{36,47}$. Since B will be present in solution mainly as a neutral species (Eq. 1), there will be no electrostatic attraction/repulsion. However, $B$ can bind to the reactive surface sites on the edges of clay minerals. Therefore, we included specific $B$ adsorption to the edges of the clay surfaces. The content of clay minerals was estimated as the measured clay content (i.e. particles smaller than $2 \mu \mathrm{m}$ ) minus the mass of oxides that were calculated as described above.

We assumed that the B adsorption behavior on clay edges can be modelled using the CD-MUSIC modeling parameters for ferrihydrite. This was tested by using the $B$ adsorption parameters for ferrihydrite from Van Eynde et al. ${ }^{25}$ for describing the $\mathrm{pH}$ dependent $\mathrm{B}$ adsorption to illite and kaolinite clays from Goldberg ${ }^{20}$, assuming an SSA of 15 and $5 \mathrm{~m}^{2} \mathrm{~g}^{-1}$ respectively. The results are shown in Figure S2. The $\mathrm{pH}$ dependent $B$ adsorption to clays can be described reasonably well with the parameters for ferrihydrite, especially for kaolinite. These results are consistent with the findings of Goldberg ${ }^{20}$ that the logK values for $\mathrm{pH}$-dependent $\mathrm{B}$ adsorption, fitted 
by using the CC model, did not differ significantly for iron oxides and clay minerals such as kaolinite and illite.

For the multi-surface modelling calculations, it was assumed that next to $\mathrm{B}(\mathrm{OH})_{3}$, adsorption of $\mathrm{H}^{+}, \mathrm{Ca}^{2+}, \mathrm{Cl}^{-}, \mathrm{CO}_{3}{ }^{2-}$ and $\mathrm{PO}_{4}{ }^{3-}$ to these clay edges can be also described using the consistent set of adsorption parameters for ferrihydrite ${ }^{67-70}$ as presented in Table S2. No interaction of NOM with the clay minerals was considered, based on previous findings that have shown that organic matter is mainly associated with the micro-crystalline oxide fraction in soils ${ }^{58}$.

An (edge) specific surface area of $5 \mathrm{~m}^{2} \mathrm{~g}^{-1}$ was considered in the modelling calculations ${ }^{76}$ since we did not have knowledge on the specific clay minerals being dominant in the different soil samples. Although the $5 \mathrm{~m}^{2} \mathrm{~g}^{-1}$ might be an underestimation of the edge surface area for clays such as illite, the modeling results were not greatly affected by the chosen edge surface area of the clay minerals (Figure S3).

\subsection{Multi-surface modeling calculations}

We firstly calculated the reactive $\mathrm{B}$ for the ten soil samples using a multi-surface model with the $\mathrm{B}$ measured in the $\mathrm{CaCl}_{2}$ at the original soil $\mathrm{pH}\left(\mathrm{CaCl}_{2}\right)$ as input. Because in the ECOSAT speciation software, only free ion concentration/activity or total amount of a compound can be used as the input, this calculation was carried out in two steps. Firstly, free $\mathrm{B}(\mathrm{OH})_{3}, \mathrm{CO}_{3}{ }^{2-}, \mathrm{Ca}^{2+}$ and $\mathrm{Cl}^{-}$concentrations were calculated based on the measured $\mathrm{B}$ concentration in $\mathrm{CaCl}_{2}$ extraction considering solution speciation and $\mathrm{B}$ and $\mathrm{Ca}$ adsorption to $\mathrm{DOC}$. Secondly, the calculated free ion concentrations of $\mathrm{B}(\mathrm{OH})_{3}, \mathrm{CO}_{3}^{2-}, \mathrm{Ca}^{2+}$ and $\mathrm{Cl}^{-}$were used as input together with $\mathrm{pH}\left(\mathrm{CaCl}_{2}\right), \mathrm{PO}_{4}-\mathrm{AO}$ as reactive $\mathrm{PO}_{4}$ and the reactive surfaces (including $\mathrm{DOC}$ ), to model the solid and solution distribution of $\mathrm{B}$. The NOM loading at the oxide surface, which was derived by preliminary modelling of the $\mathrm{PO}_{4}$ solubility, was also given as input. From these modelling calculations, the $B$ distribution over the different solid and solution phases could be extracted, and the total reactive $B$ concentration was then calculated as the sum of soluble and adsorbed B.

The calculated reactive $\mathrm{B}$ was subsequently compared with the $\mathrm{B}$ measured in the $\mathrm{HNO}_{3}, \mathrm{KH}_{2} \mathrm{PO}_{4}$ or mannitol extraction to assess the suitability of the different extraction methods to measure reactive $B$ in soils (Section 3.2). For this purpose, we calculated the mean error (ME) as the mean difference between the predicted and measured concentrations (in log $\mathrm{mol} \mathrm{kg}^{-1}$ ).

Additionally, we tested the capacity of the different extraction methods to dissolve reactive $B$, by modeling the solid and solution distribution of $B$ in the respective extraction method. Following the approach of Groenenberg et al. ${ }^{38}$, we accounted 
for the adsorption of B to the major reactive mineral (oxides and clay) and organic adsorbents in the soil as described above, and the chemical composition of the specific extract, including (1) $\mathrm{pH}$ of the extractant ( 7.3 for mannitol; 0.9 for $0.43 \mathrm{M}$ $\mathrm{HNO}_{3}{ }^{38} ; 4.5$ for $\mathrm{KH}_{2} \mathrm{PO}_{4}$ ), (2) concentration of the major dissolved ions in the extraction solution ( $0.43 \mathrm{M} \mathrm{NO}_{3} ; 0.05 \mathrm{M} \mathrm{K} ; 0.2 \mathrm{M}$ mannitol, respectively). The reactive $B$ calculated based on the $B$ measured in the $0.01 \mathrm{M} \mathrm{CaCl}_{2}$ at natural $\mathrm{pH}\left(\mathrm{CaCl}_{2}\right)$, was used in the model as input for total reactive $\mathrm{B}$. The capacity of the different extraction methods for extracting $B$ was calculated as the percentage of total $\mathrm{B}$ input that was predicted to remain in solution according to the model. The input of total reactive $\mathrm{P}$ for the $\mathrm{KH}_{2} \mathrm{PO}_{4}$ solution was given as the sum of the $\mathrm{PO}_{4}$ in the acid ammonium oxalate extraction and the $0.05 \mathrm{M} \mathrm{PO}_{4}$ from the extraction solution. For the $\mathrm{HNO}_{3}$ and mannitol extraction, input of total reactive $\mathrm{PO}_{4}$ was given as the $\mathrm{PO}_{4}$ in the ammonium oxalate extraction. The ionic strength of the mannitol extraction was fixed at $0.05 \mathrm{M}$, based on the presence of $\mathrm{H}-\mathrm{TEA}^{+}$of around $50 \%$ of total $\mathrm{TEA}^{77}$. In the $\mathrm{HNO}_{3}$ extraction, the oxide surface for ion adsorption will be reduced due to partial dissolution of the poorly crystalline oxides at $\mathrm{pH} 0.9$. This was taken into account, by subtracting the $\mathrm{Fe}$ and $\mathrm{Al}$ concentration in the $\mathrm{HNO}_{3}$ extractions from the concentrations in the $A O$ extraction to calculate the oxide content. No competition of NOM for B adsorption to oxides in the different extractions was considered in these modelling calculations. Preliminary modeling has shown that the presence/absence of measured DOC concentration did not lead to differences in the calculated extractability of the different extraction methods (Results not shown). Therefore, DOC was not included as a reactive surface in these calculations.

In a final modeling calculation, calculated concentration of $B$ in solution was compared with the measured data of the $\mathrm{pH}$ dependent $\mathrm{B}$ solubility experiment. In this calculation, reactive $\mathrm{B}, \mathrm{pH}$, total reactive $\mathrm{PO}_{4}^{-3}$, concentration of $\mathrm{CO}_{3}{ }^{2-}, \mathrm{Ca}^{2+}$ and $\mathrm{Cl}^{-}$in the extractant, total reactive surfaces (including DOC) were used as input. The NOM loading that was calculated based on modelling the $\mathrm{PO}_{4}$ solubility at the original soil $\mathrm{pH}\left(\mathrm{CaCl}_{2}\right)$ was used as input. The input value for reactive $\mathrm{B}$ was either the one calculated based on the $\mathrm{B}$ measured in $\mathrm{CaCl}_{2}$ extraction at natural soil $\mathrm{pH}$, or the $\mathrm{B}$ measured in each of the extraction methods (i.e. $\mathrm{HNO}_{3}, \mathrm{KH}_{2} \mathrm{PO}_{4}$, mannitol).

\section{RESULTS AND DISCUSSION}

\subsection{Soil characteristics}

Soil characteristics of the Dutch (D1-5) and Burundian soils (B1-5) are given in Table 1. The Dutch soils have higher B concentrations than the Burundian soils, in all extraction methods, except for soil D2 which has low total boron relatively to the other Dutch soils. In general, the tropical soil samples from Burundi are characterized 
by higher contents of oxides, more specifically oxalate-extractable Al and dithioniteextractable $\mathrm{Fe}$. The $\mathrm{pH}$ of the Burundian soil samples is similar for all samples, between 4.2 and 4.9 , whereas for the Dutch soils, the $\mathrm{pH}$ ranges from 4.7 to 7.1 .

Table 1: Soil characteristics. HA and FA are the humic and fulvic acids measured in the acid-base fractionation of the solid organic matter. The $\mathrm{P}_{\mathrm{t}}-\mathrm{AO}$, Fe-AO and Al-AO are measured in ammonium oxalate, whereas Fe-D and AI-D are measured in dithionite extraction. B was measured in $0.43 \mathrm{M} \mathrm{HNO}_{3}$, $0.05 \mathrm{M} \mathrm{KH}_{2} \mathrm{PO}_{4}, 0.2 \mathrm{M}$ mannitol and $0.01 \mathrm{M} \mathrm{CaCl}_{2}$. The B-reactive is the reactive $\mathrm{B}$ calculated with the multi-surface model using the $\mathrm{B}-\mathrm{CaCl}_{2}$ as input.

\begin{tabular}{|c|c|c|c|c|c|c|c|c|c|c|c|c|c|c|c|c|}
\hline \multirow{3}{*}{$\begin{array}{l}\text { Soil } \\
\text { D1' }\end{array}$} & \multirow{3}{*}{\begin{tabular}{|l|} 
pH \\
7.1
\end{tabular}} & \multirow{2}{*}{$\begin{array}{l}\text { Clay } \\
\%\end{array}$} & soc & $\mathrm{HA}+\mathrm{FA}$ & \multirow{2}{*}{$\begin{array}{c}\text { DOC } \\
\mathrm{mg} \\
\mathrm{L}^{-1}\end{array}$} & \multirow[t]{2}{*}{$\begin{array}{l}\text { Fe- } \\
\text { AO }\end{array}$} & \multirow[t]{2}{*}{$\begin{array}{l}\text { Al- } \\
\text { AO }\end{array}$} & \multirow[t]{2}{*}{$\begin{array}{c}\text { Fe- } \\
\text { D }\end{array}$} & \multirow[t]{2}{*}{$\begin{array}{c}\text { Al- } \\
\text { D }\end{array}$} & \multirow[t]{2}{*}{$\begin{array}{l}\mathbf{P t}_{\mathbf{t}^{-}} \\
\mathrm{AO}\end{array}$} & \multirow[t]{2}{*}{$\begin{array}{c}\text { B- } \\
\text { total }\end{array}$} & \multirow[t]{2}{*}{$\underset{\mathrm{HNO}_{3}}{\mathrm{~B}-}$} & \multirow[t]{2}{*}{$\underset{\mathrm{KH}_{2} \mathrm{PO}_{4}}{\mathrm{~B}-}$} & \multirow[t]{2}{*}{$\begin{array}{c}\text { B- } \\
\text { Mannitol }\end{array}$} & \multirow[t]{2}{*}{$\underset{\mathrm{CaCl}_{2}}{\mathrm{~B}-}$} & \multirow{2}{*}{$\begin{array}{c}\text { B- } \\
\text { Reactive }\end{array}$} \\
\hline & & & \multicolumn{2}{|c|}{----gC kg-1--- } & & & & & & & & & & & & \\
\hline & & 24 & 20 & 2 & 22 & 27 & 17 & 141 & 27 & 12.24 & 5943 & 480 & 274 & 271 & 77 & 85 \\
\hline D2 ${ }^{A}$ & 4.8 & 3 & 32 & 15 & 43 & 37 & 42 & 38 & 39 & 13.53 & 773 & 81 & 41 & 43 & 40 & 45 \\
\hline $\mathrm{D}^{\mathrm{A}}$ & 4.7 & 11 & 133 & $49^{+}$ & 131 & 136 & 89 & 107 & 63 & 26.38 & ** & 166 & 61 & ** & 77 & 101 \\
\hline D4 ${ }^{A}$ & 5.8 & 35 & 31 & 9 & 36 & 89 & 21 & 164 & 24 & 21.15 & 6090 & 332 & 162 & 156 & 85 & 100 \\
\hline D5 ${ }^{A}$ & 6.4 & 39 & 85 & 21 & 105 & 133 & 44 & 192 & 29 & 33.23 & 5950 & 393 & 142 & 188 & 72 & 106 \\
\hline$B 1^{B}$ & 4.3 & 7 & 50 & 25 & 11 & 156 & 314 & 456 & 328 & 11.43 & 1754 & 29 & 23 & 38 & 20 & 28 \\
\hline B2 $^{\mathrm{B}}$ & 4.4 & 10 & 28 & 10 & 9 & 71 & 168 & 493 & 244 & 7.52 & 1097 & 39 & 25 & 36 & 24 & 30 \\
\hline B3 $^{B}$ & 4.3 & 17 & 22 & 12 & 10 & 58 & 147 & 647 & 253 & 3.91 & 568 & 14 & 7 & 16 & 7 & 9 \\
\hline$B 4^{B}$ & 4.2 & 4 & 14 & 5 & 5 & 37 & 63 & 616 & 139 & 3.36 & 778 & 15 & 8 & 18 & 7 & 8 \\
\hline B5 $^{\mathrm{B}}$ & 4.9 & 8 & 32 & 15 & 3 & 66 & 136 & 328 & 171 & 4.00 & 1165 & 33 & 18 & 32 & 13 & 16 \\
\hline \multicolumn{17}{|c|}{$\begin{array}{l}\text { * Insufficient soil material was left for this analysis. Therefore, the average } \% \text { of total OC that was found to be HA and FA for } \\
\text { the other soil samples was taken as reactive OC. This was } 30 \% \mathrm{HA} \text { and } 6 \% \mathrm{FA} \text {. } \\
{ }^{* *} \text { Insufficient soil material was left for this analysis. This sample was not included in the discussion of the mannitol } \\
\text { extraction. } \\
\text { A Temperate soil samples from the Netherlands } \\
\text { B Tropical soil samples from Burundi }\end{array}$} \\
\hline
\end{tabular}

Total $B$ is higher than the $B$ concentrations found in the other extractions. The fraction of total $B$ found in the extractions used to measure reactive $B$ ranges from 2 to $5 \%$, depending on the extraction method. Highest $\mathrm{B}$ concentrations are found in the $\mathrm{HNO}_{3}$ and mannitol extractions, followed by $\mathrm{KH}_{2} \mathrm{PO}_{4}$ and $\mathrm{CaCl}_{2}$. The fraction of reactive $\mathrm{B}$ that is found in the $\mathrm{CaCl}_{2}$ extraction can be used as an indicator of the adsorption capacity of the soils, and this fraction is on average 42,51 and $77 \%$ when using respectively the $\mathrm{B}$ measured in $\mathrm{HNO}_{3}$, mannitol and $\mathrm{KH}_{2} \mathrm{PO}_{4}$ as reactive B. In two cases, the $\mathrm{CaCl}_{2}$ has extracted slightly more $\mathrm{B}$ than the $\mathrm{KH}_{2} \mathrm{PO}_{4}$ solution (i.e. D3 and B3). The B concentrations in all extractions were found to be significantly correlated among each other (Table S5).

The observed $\mathrm{B}$ concentrations in the $\mathrm{CaCl}_{2}$ extraction as a function of $\mathrm{pH}$ cannot be explained by the solubility of $\mathrm{B}$-containing $\mathrm{Ca}, \mathrm{Mg}$ and $\mathrm{Na}$ minerals, such as Pinnoite, Inderite, Colemanite, Inyoite, Borax, McAllisterite and Nobleite ${ }^{78}$. The solubility of B based on these minerals differ orders of magnitude from our observations, and show a reverse $\mathrm{pH}$ trend from what we have found here. These observations are 
consistent with the findings from Fruchter et al. ${ }^{79}$ who did not find B solubilitycontrolling phases in waste material.

The risk of boron deficiency is often estimated by a hot water soil extraction ${ }^{80}$. Critical $\mathrm{B}$ concentrations in hot water extractions for crop production are reported to be in

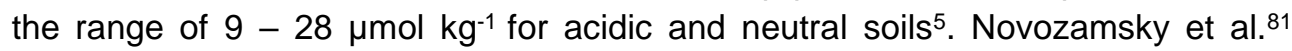
established a relation between $\mathrm{B}$ measured by hot water and $0.01 \mathrm{M} \mathrm{CaCl}_{2}$ extraction for 100 soils from the Netherlands, and they found that $\sim 26 \%$ of $B$ measured by hot water is extracted by the $\mathrm{CaCl}_{2}$ extraction. This would mean a critical concentration of $~ 7 \mu \mathrm{mol} \mathrm{kg}{ }^{-1}$ of $B$ in $0.01 \mathrm{M} \mathrm{CaCl}_{2}$. As can be seen in Table 1, the $B$ measured in $\mathrm{CaCl}_{2}$ is higher than this critical value except for soil B3 and B4.

\subsection{Reactive B}

Based on the $\mathrm{B}$ measured in $0.01 \mathrm{M} \mathrm{CaCl}_{2}$ in soils at their original soil $\mathrm{pH}\left(\mathrm{CaCl}_{2}\right)$, the total reactive $B$ was calculated by the multi-surface model as the sum of adsorbed and soluble B. The results of these calculations for each soil sample are shown in the last column of Table 1.

We tested the capacity to dissolve reactive B for the three different extraction methods. Multi-surface modelling calculations of boron speciation in the different extraction solutions show that more than $~ 90 \%$ of the reactive boron from Table 1 is dissolved by each of these solutions (Figure S4). The three different extraction solutions from this study are thus, based on these modeling calculations, efficient in extracting reactive boron from soils.

A $0.43 \mathrm{M} \mathrm{HNO}_{3}$ solution extracts metal cations by competitive desorption with protons and it dissolves oxyanions due to their protonation at low $\mathrm{pH}$ and partial dissolution of hydrous oxides to which anions are adsorbed ${ }^{38}$. Van Eynde et al. ${ }^{25}$ showed that at low $\mathrm{pH}$, oxides, and especially ferrihydrite exhibit larger adsorption affinity for $\mathrm{B}$ compared to organic matter. The model predicts that still up to $10 \% \mathrm{~B}$ is adsorbed to ferrihydrite, at $\mathrm{pH} 0.9$ in the $0.43 \mathrm{M} \mathrm{HNO}_{3}$ extract (Figure S4).

Similar results for boron speciation are found for the $\mathrm{KH}_{2} \mathrm{PO}_{4}$ and $\mathrm{HNO}_{3}$ extraction. The $\mathrm{KH}_{2} \mathrm{PO}_{4}$ extraction method is a milder extraction solution in terms of $\mathrm{pH}$ (4.5), but adds in turn a strong competing phosphate ligand to desorb the B from the solid phase ${ }^{42,82}$. Figure $S 4$ shows that for the soils with the highest organic matter and ferrihydrite content (B1 and D3), part of the boron is not in solution but is predicted to be adsorbed to oxides and organic matter.

The addition of $0.2 \mathrm{M}$ mannitol to the soil samples results in nearly $100 \%$ of all reactive boron in solution (Figure S4), according to the complexation constants from Knoeck and Taylor ${ }^{61}$ (Equation 2-3). Only the reaction of mannitol with $\mathrm{B}(\mathrm{OH})_{3}$ was 170 
included in the modelling. However, it is known from literature that mannitol can also form complexes with other anions and cations ${ }^{83-85}$, but no consistent $\log K$ values for complex formation with the uncharged mannitol are reported. The presence of other cations and anions in the soil system might therefore reduce the actual recovery of $B$ by a mannitol extraction.

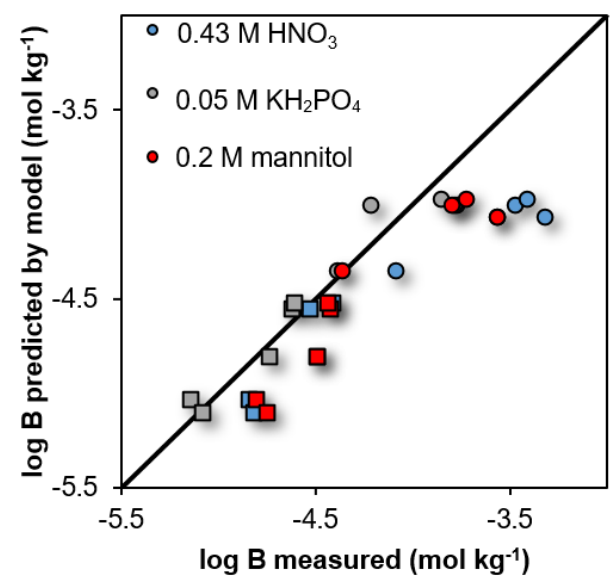

Figure 1: Comparison between the $\mathrm{B}$ measured in $\mathrm{HNO}_{3}, \mathrm{KH}_{2} \mathrm{PO}_{4}$ or mannitol with the $\mathrm{B}$ that is calculated by the model from soluble $\mathrm{B}$ in $\mathrm{CaCl}_{2}$ as total reactive $\mathrm{B}$ based on adsorption processes on humic acids, clay minerals and ferrihydrite for the different soil samples. The Dutch soils are shown by circles, the Burundian soil samples by squares and the color of the markers shows the different extraction for the $B$ measurement. The corresponding mean error based on log mol kg-1 concentrations are -0.32 for $\mathrm{HNO}_{3}(-$ 0.46 for temperate, -0.18 for tropical), -0.04 for $\mathrm{KH}_{2} \mathrm{PO}_{4}$ ( -0.12 for temperate, 0.04 for tropical), -0.22 for mannitol (-0.23 for temperate and -0.22 for tropical).

Figure 1 shows the comparison between calculated reactive $B$ with the multi-surface model, and the actual B concentrations measured in the three different extraction solutions. Although the model predicts that all three extraction methods are efficient in dissolving reactive $B$ (Figure S4), Figure 1 shows that for most soils more $B$ is extracted than the amount of reactive $B$ according to the multi-surface model.

For the temperate soils, the $\mathrm{HNO}_{3}$-extractable $\mathrm{B}$ deviated most from the prediction, followed by $\mathrm{B}$ in the mannitol and in $\mathrm{KH}_{2} \mathrm{PO}_{4}$ extracts, with $\mathrm{ME}$ of $-0.46,-0.23$ and 0.12 respectively. For the tropical soils, the $\mathrm{KH}_{2} \mathrm{PO}_{4}$ extraction also gave the best results with a $\mathrm{ME}$ of 0.04 . Using the mannitol and $\mathrm{HNO}_{3}$ extraction resulted in a $\mathrm{ME}$ of -0.22 and -0.18 respectively and both extraction methods result in an overestimation of reactive $B$ for all tropical soil samples.

As shown in Figure 1, the measured $\mathrm{B}-\mathrm{HNO}_{3}$ is larger than the reactive $\mathrm{B}$ predicted for all soil samples. The measured $B$ concentration in $\mathrm{HNO}_{3}$ is on average $240 \%$ of the calculated reactive concentration, indicating that $0.43 \mathrm{M} \mathrm{HNO}_{3}$ has dissolved non-adsorbed $\mathrm{B}$. A $0.43 \mathrm{M} \mathrm{HNO}_{3}$ extraction acidifies the soils to a $\mathrm{pH}$ between 0.5 
and 1, which can result in a dissolution of various minerals, including Fe oxides and silicate minerals ${ }^{38,86}$. Since most of the total soil $B$ can be a structural part of clay minerals ${ }^{6,87}$, this nitric acid extraction can solubilize non-reactive $B$. The $B$ in the $\mathrm{HNO}_{3}$ differs more from the calculated reactive $\mathrm{B}$ for the Dutch than for the Burundian soil samples. This difference might be explained by the difference in soil $\mathrm{pH}$, degree of weathering and clay type: The tropical Burundian soils have a relatively low $\mathrm{pH}$ compared to the Dutch soils, therefore the minerals present in the Dutch soils are more sensitive to acid extraction. In addition, the tropical Burundian soils are likely dominated by clay minerals such as kaolinite, due to prolonged intensive weathering, while illite-type clays are more representative for the less weathered temperate Dutch soils. Illite releases higher absolute concentrations of $\mathrm{Si}$ than kaolinite in acidic solutions ${ }^{88-90}$ and has been found to have a slightly higher dissolution rate at an acidic $\mathrm{pH}$, compared to kaolinite ${ }^{91}$. In addition, illite contains more $B$ than kaolinite ${ }^{92}$, which, next to the larger amount of clay minerals dissolved, might contribute to the higher $\mathrm{ME}$ for the Dutch soils when comparing the predicted reactive $B$ with the $B$ measured in $\mathrm{HNO}_{3}$.

For the temperate soil samples, mannitol results in a slightly more negative ME compared to $\mathrm{KH}_{2} \mathrm{PO}_{4}$, and both extractions overestimate reactive $B$ with extracted concentrations being on average $177 \%$ and $123 \%$ of the predicted reactive $B$ respectively. For the tropical soils the mannitol extraction also overestimated reactive $B$ (on average $170 \%$ ) compared to predictions by the multi-surface model (Figure 1), and unlike for the temperate soils, the $B$ extracted by mannitol is even slightly higher than the $\mathrm{B}$ extracted by $0.43 \mathrm{M} \mathrm{HNO}_{3}$. In line with the $\mathrm{B}$ measurements, mannitol extracts the largest fraction of total $\mathrm{Si}, \mathrm{Al}$ and $\mathrm{Fe}$ in most of the tropical soil samples (Table 2). Although the samples extracted with mannitol were filtered over a $0.45 \mu \mathrm{m}$ filter before analysis, the high $\mathrm{B}, \mathrm{Si}, \mathrm{Al}$ and $\mathrm{Fe}$ concentrations in the mannitol extraction can be an indication of colloidal particles $(<0.45 \mu \mathrm{m})$ in the extract because of the low salt level in the mannitol extraction. Although the ionic strength was not measured in the mannitol extraction, it was possibly lower in the extracts from the tropical soils, due to a lower concentration of charged ions because of prolonged weathering. Moreover, the larger increase in $\mathrm{pH}$ relative to the original soil $\mathrm{pH}$ upon addition of the (buffered, $\mathrm{pH} 7.3$ ) mannitol extraction solution for the tropical soils, can also favor the formation of colloids in the extract $^{93,94}$.

Additionally, the ligands in the mannitol extraction (mannitol, TEA buffer), can form soluble complexes with $\mathrm{Si}, \mathrm{Al}$ and $\mathrm{Fe}$, thus enhancing the solubility of B-containing minerals at alkaline $\mathrm{pH}$. Kinrade et al. ${ }^{95}$ found that poly-hydroxy alcohols, such as mannitol and sorbitol, another commonly used polyol for B extraction ${ }^{44}$, form concentrations of stable complexes with silicate anions. Moreover, these authors reported that the Si complexation with polyols enhances silica solubility. It has also 172 
been shown that mannitol can form complexes with metals ${ }^{85}$, such as $\mathrm{Fe}^{96}$. Similarly, the use of TEA buffer might also lead to enhanced Fe and Al mineral dissolution, since it has been reported that TEA can form stable complexes with $\mathrm{Fe}$ in alkaline solutions $^{97}$ as well as with $\mathrm{Al}^{98}$, which can possibly lead to dissolution of $\mathrm{Fe} / \mathrm{Al}$ minerals. Since the TEA buffer is also a typical component of the frequently used DTPA extraction for measuring bioavailable $\mathrm{Fe}^{99}$, this possible effect on mineral dissolution may be worth further investigation.

Modifications in the extraction protocols, such as shorter equilibration time, lower concentration of mannitol or increasing the salt level of the extraction, might reduce the risk of dissolution, or formation of colloids, of non-reactive B. Measurements of $\mathrm{Si}, \mathrm{Al}$ and $\mathrm{Fe}$ should thus be included in experiments testing different extraction protocols, to assess possible mineral dissolution, colloid formation, and associated mobilization of non-reactive $B$.

Overall, the $\mathrm{KH}_{2} \mathrm{PO}_{4}$ is the best extraction method to quantify reactive boron for both tropical and Dutch soils (Figure 1). For soils D2, D3, B1, B2 and B3, there seems to be either an under-estimation of reactive $\mathrm{B}$ by the $\mathrm{KH}_{2} \mathrm{PO}_{4}$ extraction or an overestimation of $\mathrm{B}$ adsorption by the multi-surface model. However, the differences between $\mathrm{B}-\mathrm{KH}_{2} \mathrm{PO}_{4}$ and calculated reactive $\mathrm{B}$ are relatively small (Table 1 ). These soils are characterized by a low $\mathrm{pH}$ and a high content of organic matter and ferrihydrite. Using the multi-surface model to calculate the $\mathrm{B}$ speciation in the $\mathrm{KH}_{2} \mathrm{PO}_{4}$ extraction has shown that $\mathrm{B}$ was still partly bound to organic matter and ferrihydrite for these soils (Figure S4). An twofold increase in $\mathrm{PO}_{4}$ concentration in the extraction solution would still lead to a fraction of $B$ that is bound to oxides instead of being extracted (results not shown).

For soils D1, D4 and D5, either the $\mathrm{KH}_{2} \mathrm{PO}_{4}$ solution extracts more $\mathrm{B}$ than is predicted by the model to be reactive, or the model under-estimates adsorption for these samples. These are the soil samples with the highest clay content and highest $\mathrm{pH}$. The $\mathrm{B}$ adsorption to clay particles was modelled using the adsorption parameters for ferrihydrite and a surface area of $5 \mathrm{~m}^{2} \mathrm{~g}^{-1}$. The latter could be an under-estimation of the actual specific surface area but changing the SSA of the clay edges did not have a large effect on the calculations (Figure S3). Another explanation might be that the $\mathrm{KH}_{2} \mathrm{PO}_{4}$ extraction potentially solubilizes part of the $\mathrm{B}$ that is occluded in the clay minerals ${ }^{6,100}$, however, this is unlikely at the $\mathrm{pH} 4.5$ of this extraction. The $\mathrm{Si}$ measured in the $\mathrm{KH}_{2} \mathrm{PO}_{4}$ extraction for soils D1, D4 and D5 is higher than for the other Dutch soils (Table 2), which might be due to competition with adsorbed Si by the added $\mathrm{PO}_{4}{ }^{101}$. Additionally, the multi-surface model might under-estimate $\mathrm{B}$ adsorption in these high $\mathrm{pH}$ soils. The model performance in describing $\mathrm{pH}$ dependent B solubility is further discussed in Section 3.4. 


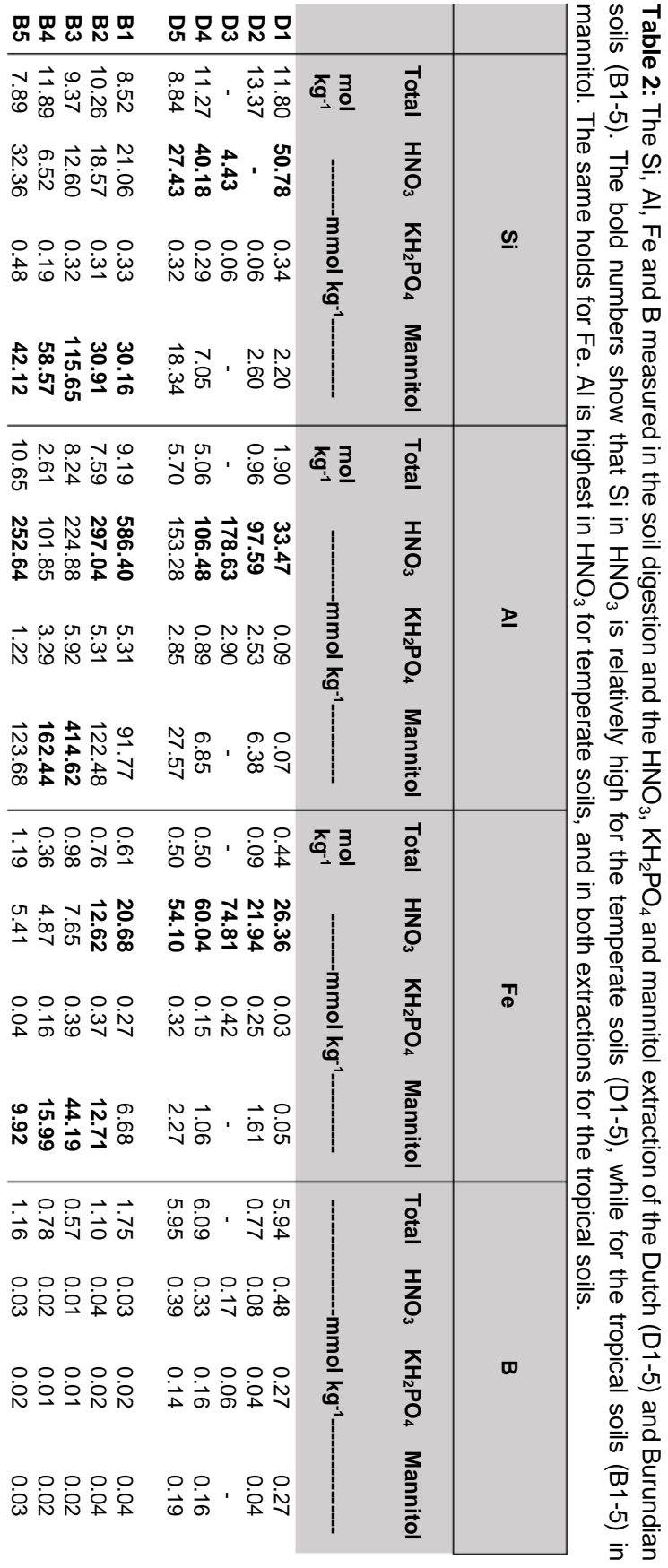




\subsection{Boron speciation in soils}

Based on the $B$ measured in $0.01 \mathrm{M} \mathrm{CaCl}_{2}$, the geochemical reactive $\mathrm{B}$ was calculated. In addition, from this modelling calculation the distribution of $B$ over the solid and solution phase can be obtained. This predicted $B$ speciation for the 10 soil samples is shown in 3 . The model predicts that most of the geochemical reactive $B$ is in the solution phase, with a minimum of $68 \%$ for soil D5. The adsorption to dissolved organic matter is negligible and maximal $1 \%$ of boron in solution was calculated to be bound to DHA. This finding is consistent with previous studies that have shown that $B$ is mainly present as free dissolved $B$ instead of being bound to dissolved organic matter102,103. From the inorganic aqueous species that were considered in the modelling (Table $\mathrm{S} 1$ ), $\mathrm{B}(\mathrm{OH})_{3}$ was predicted to be most important, representing $>99 \%$ of all $B$ in solution.

Our modeling results show that the contribution of the reactive surfaces for boron adsorption is different for the tropical and temperate soils: in the temperate soils, organic matter contributes mainly to $B$ adsorption, whereas oxides play the most important role in the tropical Burundian soils. The difference in solid phase B speciation between the temperate and tropical soils can be explained by $\mathrm{pH}$, the amount of reactive surfaces and $\mathrm{PO}_{4}{ }^{3-}$ loading. The tropical soils in our dataset are characterized by a low $\mathrm{pH}$, and it has been shown that under these conditions ferrihydrite exhibits the largest B adsorption capacity of all reactive surfaces considered, according to the multi-surface mode ${ }^{25}$. In addition, as shown in Figure $\mathrm{S} 1$, the exclusion of $\mathrm{PO}_{4}$ in the modelling led to a higher reactive $\mathrm{B}$ concentration calculated by the model, due to an increased $\mathrm{B}$ binding to oxides.

It has been previously shown that $B$ amended to a variety of soil systems binds only weakly to the solid phase ${ }^{104}$. Figure 2 shows that the same holds for the reactive $B$ that is in situ present in soils. It has been stated before that the interaction of $B$ with the soil solid phase controls the $B$ in solution and consequently $B$ bioavailability ${ }^{12,13}$. However, the multi-surface modelling calculations show that the interaction of natural reactive $B$ with the solid phase is limited, and that a substantial part of the bioavailable boron will be in the solution phase. Other processes than adsorption will therefore be more important for buffering bioavailable $B$ in the soil solution. Next to atmospheric input via precipitation and aerosol deposition, two potential processes in soils might be important for $B$ replenishment to the soil solution; B originating from mineral weathering ${ }^{105}$, and from mineralization of organic matter that originates from plant material ${ }^{100}$. Evidence for both processes have been found in previous studies. For example, isotopic measurements in forest stands have shown that the B in solution in the upper soil layers appears to be mainly originated from decomposing 
vegetation ${ }^{87,106}$. On the other hand, a similar study showed that for two soil profiles rich in clay minerals the $B$ behavior was not controlled by biological cycling but most probably by chemical weathering ${ }^{107}$. Kot et al. ${ }^{108}$ measured $B$ in leachates from litter and leaves, and found higher B concentrations and fluxes than the ones estimated from chemical weathering or atmospheric deposition for the global biogeochemical cycle of boron ${ }^{11}$. They concluded that the turnover of organic inputs is the main source for bioavailable B in soils.

The question arises how these results from forests stands can be translated to agricultural top soils, where above ground biomass is largely removed from the field after the growing season. Given that adsorption contributes only minor to the bioavailable boron pool and that both mineralization of organic matter and chemical weathering have been suggested as potential processes that resupply boron in the soil solution, we have performed a regression analysis between $\mathrm{B}$ concentrations in the $\mathrm{CaCl}_{2}$ and the SOC and clay content of the soils. These results are shown in Figure S5. For the Burundian soil samples, there is a tendency that the largest B in $\mathrm{CaCl}_{2}$ is found for those soils with the highest organic carbon content, while this is not the case for the Dutch soils. For the latter, the relation between $\mathrm{B}$ in $\mathrm{CaCl}_{2}$ and clay content seems to be more important. Even though organic matter represents the most important adsorption surface for the Dutch soils (Figure 2), chemical weathering might be more important for buffering bioavailable boron. Although the regression was done based on a small set of soils $(n=5)$, these results can be indicative that different processes such as mineralization and chemical weathering can be important for buffering bioavailability of $B$ in the different soil sets of this study. 


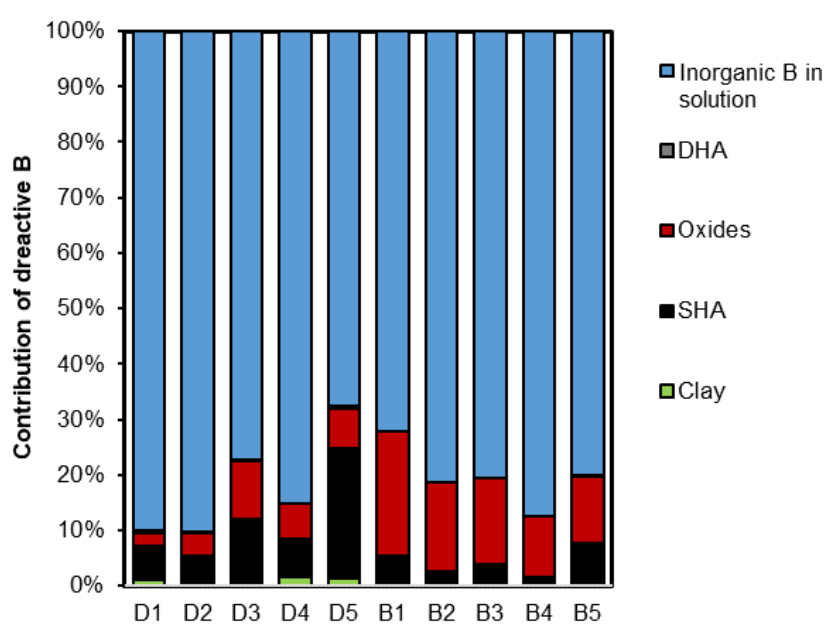

Figure 2: $B$ speciation in the 10 soil samples calculated by the multi-surface model based on the $B$ measured in the $0.01 \mathrm{M} \mathrm{CaCl}_{2}$ extraction. The $B$ is distributed in the solution phase as inorganic $B$ species $\left(>99 \%\right.$ as $\mathrm{B}(\mathrm{OH})_{3}$ ) and bound to dissolved organic matter (as humic acid, DHA), and in the solid phase bound to oxides, solid organic matter (as humic acid, SHA) and Clay. The soils D1-D5 are the 5 Dutch soils, while soils B1-B5 are the 5 Burundian soils (soil properties are shown in Table 1).

\subsection{Model performance to describe pH dependent B solubility in soils}

Adsorption experiments on reactive surfaces have shown that $\mathrm{B}$ adsorption increases with increasing $\mathrm{pH}$ up to a maximum at around $\mathrm{pH} 8-10^{15,25,109}$. In line with these results, we found for all soil samples a decreasing $B$ concentration in the 0.01 $\mathrm{M} \mathrm{CaCl}_{2}$ with increasing $\mathrm{pH}$ (Figure 3). The decrease in $\mathrm{B}$ with increasing $\mathrm{pH}$, calculated as $\Delta B / \Delta p H$ was between 0.1 (B4) and $6 \mu \mathrm{mol} \mathrm{L}^{-1}$ (D1) per unit of $\mathrm{pH}$. Except for soil D1 and D4, the experimental data shows that the decrease in $\mathrm{B}$ concentration with increasing $\mathrm{pH}$ is related to the soil organic carbon content (Figure S6). This is also predicted by the multi-surface model: a decrease in B concentration with increasing $\mathrm{pH}$ is due to an increase in $\mathrm{B}$ adsorption to soil organic matter (Figure S7) and for soils with higher SOC, a larger decrease in B concentration with increasing $\mathrm{pH}$ is consequently predicted by the model.

Both modelling predictions and experimental data show that from $\mathrm{pH}>6$, more than $50 \%$ of reactive boron is in the solid phase, mainly adsorbed to solid organic matter (Figure S7). 

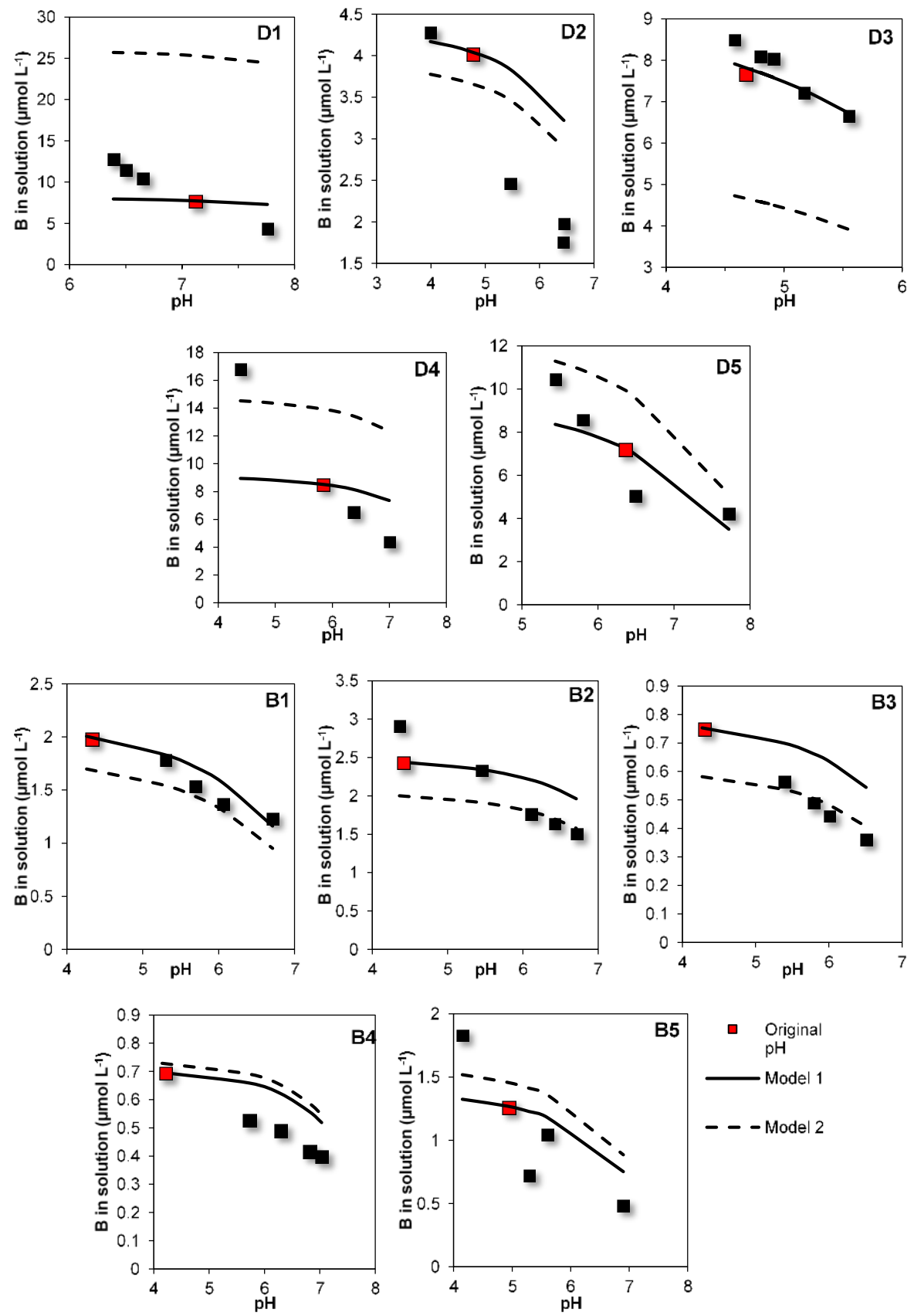

Figure 3: $\mathrm{pH}$ dependent $\mathrm{B}$ concentration for all 10 soil samples. Red markers show $\mathrm{B}$ concentration at natural soil $\mathrm{pH}$. $\mathrm{B}$ and $\mathrm{pH}$ were measured in $0.01 \mathrm{M} \mathrm{CaCl}_{2}$ after 24 hours. Modeling calculations were performed using either the calculated reactive $\mathrm{B}$ based on the measured $\mathrm{B}$ in $\mathrm{CaCl}_{2}$ at original $\mathrm{pH}\left(\mathrm{CaCl}_{2}\right)$ (Model 1) or using the $\mathrm{B}$ measured in $0.05 \mathrm{M} \mathrm{KH}_{2} \mathrm{PO}_{4}$ as input for reactive $\mathrm{B}$ (Model 2). The soils D1-D5 are the 5 Dutch soils, while soils B1-B5 are the 5 Burundian soils (soil properties are shown in Table 1). 
In Figure 3, the dashed line (model 2) shows model calculations in which the $\mathrm{B}$ solubility was calculated with $\mathrm{KH}_{2} \mathrm{PO}_{4}$-extracted $\mathrm{B}$ as input for reactive $\mathrm{B}$. The latter was chosen since the $B$ in this extraction method differed the least from the calculated reactive $B$ by the model (Figure 1). In terms of absolute prediction levels with $\mathrm{KH}_{2} \mathrm{PO}_{4}-\mathrm{B}$ as input, it can be seen that for some soils the $\mathrm{B}$ concentrations in the $\mathrm{CaCl}_{2}$ extraction are over-estimated by up to a factor of 5 (D1), and for some soils under-estimated by up to a factor of 2 (D3).

In order to assess model performance irrespective of the chosen extraction method, the $\mathrm{pH}$ dependent $\mathrm{B}$ solubility was calculated using as input the reactive $\mathrm{B}$ as it was calculated based on the $\mathrm{B}$ measured in $0.01 \mathrm{M} \mathrm{CaCl}_{2}$ at the original soil $\mathrm{pH}$. This is shown as the solid line (model 1) in Figure 3. Looking at these modeling calculations, it can be seen that in general, the model predicts for most soils a too weak $\mathrm{pH}$ dependent B solubility when compared to the experimental data. This is most pronounced for the soils with the lowest organic matter content, namely soil D1 and D4. With increasing $\mathrm{pH}$, the model calculates on average a decrease in $\mathrm{B}$ concentration of $0.65 \mu \mathrm{mol} \mathrm{L}^{-1} \mathrm{pH}^{-1}$ when all 10 soils are considered, which is lower than the $1.84 \mu \mathrm{mol} \mathrm{L}^{-1} \mathrm{pH}^{-1}$ found based on the experimental data. Based on the $\mathrm{pH}$ dependent $B$ speciation, it seems that $B$ adsorption to organic matter at high $\mathrm{pH}$ is underestimated by the model.

\section{CONCLUSIONS}

In this study, we used a multi-surface model to understand the chemical speciation of $B$ in soils, which includes $B$ in solution as boric acid and borate, and $B$ bound to dissolved humic acids, as well as $B$ adsorption on clay mineral edges, oxides and solid humic acids. Taking the $\mathrm{B}$ measured in $0.01 \mathrm{M} \mathrm{CaCl}_{2}$ as the starting point, reactive $B$ was calculated with the model as the sum of dissolved and adsorbed $B$. Our results show that these calculations are most comparable to the reactive $B$ concentration estimated by a $0.05 \mathrm{M} \mathrm{KH}_{2} \mathrm{PO}_{4}$ extraction for the soils in this study. The alternative $0.43 \mathrm{M} \mathrm{HNO}_{3}$ and 0.2 mannitol $+0.1 \mathrm{M}$ TEA buffer extractions resulted in higher estimates of reactive $B$ concentrations, probably due to dissolution of $\mathrm{B}$-containing $\mathrm{Si}, \mathrm{Fe}$ and $\mathrm{Al}$ minerals and/or due to the formation of colloidal particles because of the low salt level in the latter extraction solution. Caution should thus be taken when using these extraction methods to quantify reactive $B$ for determination of plant available B. For the mannitol extraction, changes in the extraction protocol (e.g. concentration, change of buffer solution, addition of salts) might lead to better measurements of reactive $B$ that is potentially available for plant uptake.

Measurements of $\mathrm{pH}$ dependent $\mathrm{B}$ solubility showed that the $\mathrm{B}$ in $0.01 \mathrm{M} \mathrm{CaCl}_{2}$ decreases with increasing $\mathrm{pH}$. Except for two soil samples, the largest decrease in 
$\mathrm{B}$ concentration with increasing $\mathrm{pH}$ was found for the soils with the highest organic carbon content. This is also predicted by the model, due to an increase in B adsorption with an increase in $\mathrm{pH}$. The multi-surface model tends to underestimate the adsorption of $\mathrm{B}$ by organic matter at higher $\mathrm{pH}$ values, resulting in a weaker $\mathrm{pH}$ dependency predicted by the model than shown by the experimental data.

Speciation calculations based on our model show that oxides are most important for $\mathrm{B}$ adsorption in acidic soil from the humid tropics, while organic matter is more important for temperate and high $\mathrm{pH}$ soils. However, the interaction of the natural reactive $B$ with the solid phase is limited for soils with $\mathrm{pH}\left(\mathrm{CaCl}_{2}\right)$ lower than 6 . Future studies should focus on non-equilibrium processes that buffer bioavailable $B$ in agricultural top soils, such as organic matter mineralization and mineral weathering. These results will further enhance the understanding of $B$ speciation and bioavailability in soils, and improve the development of $B$ management strategies.

\section{ACKNOWLEDGEMENTS}

This work was supported by NWO (grant number 14688, 'Micronutrients for better yields'). The Dutch soil samples were sampled and provided by Nutrient Management Institute, The Netherlands in the framework of the NWO project ' Micronutrient Management for Sustainable Agriculture and Environment' (grant number 10707). Soil samples from Burundi were collected by IFDC (the International Fertilizer Development Centre) in the framework of the PAPAB project, and sent to Wageningen for analyses. All analyses were done in the CBLB laboratory in Wageningen University and Research, except for the total element concentrations which were measured at ECN-TNO in Petten. We greatly appreciate the help and expertise of Peter Nobels for the ICP measurements of boron in the different extraction solutions. 


\section{SUPPORTING INFORMATION}

\section{A. Aqueous boron species used in modelling}

Table S1: Aqueous species considered in the modelling. The species which involves $\mathrm{K}^{+}$and mannitol were used only in modelling the recovery of the $\mathrm{KH}_{2} \mathrm{PO}_{4}$ and mannitol reaction, respectively. The aqueous species for $\mathrm{B}$ were taken the same as the ones used by Goli et al. ${ }^{37}$, except for the polymers.

\begin{tabular}{l} 
Aqueous species \\
\hline $\mathrm{B}(\mathrm{OH})_{4}^{-}$ \\
$\mathrm{CaB}(\mathrm{OH})_{4}^{+}$ \\
$\mathrm{CaPO}_{4}^{+}$ \\
$\mathrm{CaHPO}_{4}$ \\
$\mathrm{CaH}_{2} \mathrm{PO}_{4}^{+}$ \\
$\mathrm{HPO}_{4}^{-2}$ \\
$\mathrm{H}_{2} \mathrm{PO}_{4}^{-}$ \\
$\mathrm{H}_{3} \mathrm{PO}_{4}$ \\
$\mathrm{KOH}$ \\
$\mathrm{B}(\mathrm{OH})_{4}-$ Mannitol $^{-}$ \\
$\mathrm{B}(\mathrm{OH})_{4}-$ Mannitol $_{2}^{-}$
\end{tabular}

\begin{tabular}{ll} 
Reaction & log $K$ \\
\hline $\mathrm{B}(\mathrm{OH})_{3}+\mathrm{OH}^{-} \leftrightarrow \mathrm{B}(\mathrm{OH})_{4}^{-}$ & $4.76^{62}$ \\
$\mathrm{~B}(\mathrm{OH})_{3}+\mathrm{Ca}^{+2}+\mathrm{OH}^{-} \leftrightarrow \mathrm{CaB}(\mathrm{OH})_{4}^{+}$ & $6.59^{62}$ \\
$\mathrm{PO}_{4}^{-3}+\mathrm{Ca}^{+2} \leftrightarrow \mathrm{CaPO}_{4}^{-}$ & $6.46^{90}$ \\
$\mathrm{PO}_{4}^{-3}+\mathrm{H}^{+}+\mathrm{Ca}^{+2} \leftrightarrow \mathrm{CaHPO}_{4}^{-}$ & $15.035^{90}$ \\
$\mathrm{PO}_{4}^{-3}+2 \mathrm{H}^{+}+\mathrm{Ca}^{+2} \leftrightarrow \mathrm{CaH}_{2} \mathrm{PO}^{-}$ & $20.9230^{90}$ \\
$\mathrm{PO}_{4}^{-3}+\mathrm{H}^{+} \leftrightarrow \mathrm{HPO}_{4}^{-2}$ & $12.375^{90}$ \\
$\mathrm{PO}_{4}^{-3}+2 \mathrm{H}^{+} \leftrightarrow \mathrm{H}_{2} \mathrm{PO}^{-2}$ & $19.573^{90}$ \\
$\mathrm{PO}_{4}^{-3}+3 \mathrm{H}^{+} \leftrightarrow \mathrm{H}_{3} \mathrm{PO}_{4}$ & $21.721^{90}$ \\
$\mathrm{~K}^{+}+\mathrm{OH}^{-} \leftrightarrow \mathrm{KOH}$ & $-0.55^{90}$ \\
$\mathrm{~B}(\mathrm{OH})_{3}+\mathrm{OH}^{-}+$Mannitol $\leftrightarrow \mathrm{B}(\mathrm{OH})_{4}-$ Mannitol $^{-}$ & $7.55^{61}$ \\
$\mathrm{~B}(\mathrm{OH})_{3}+\mathrm{OH}^{-}+2$ Mannitol $\leftrightarrow \mathrm{B}(\mathrm{OH})_{4}-$ Mannitol $_{2}$ & $9.74^{61}$
\end{tabular}




\section{B. Thermodynamic database with log $K$ and $C D$ values used in $C D$ modelling for ferrihydrite}

Table S2: Formation of surface species in the $C D$ model for ferrihydrite and clay edges, in combination with an extended Stern layer with $C_{1}=1.15$ and $C_{2}=0.9$ $\mathrm{F} \mathrm{m}^{-2}{ }^{268}$ Formation reactions are with triply coordinated groups $(\mathrm{T}) \mathrm{Fe}_{3} \mathrm{O}\left(1.4 \mathrm{~nm}^{-2}\right)$ or singly coordinated groups (S) FeOH that can form only monodentate surface species (a) $\left(3 \mathrm{~nm}^{-2}\right)$, or that can form mono-and bidentate corner-sharing surface species with low ((bl), $\left.2.3 \mathrm{~nm}^{-2}\right)$ and high affinity for $\mathrm{Ca}^{2}$ and $\mathrm{H}_{3} \mathrm{BO}_{3}((\mathrm{bh}), 0.25$ $\left.\mathrm{nm}^{-2}\right)^{25,69,70}$. For modelling ion adsorption to ferrihydrite, the interaction with natural organic matter was also considered, using the approach and formation reactions from Hiemstra et al. ${ }^{73}$ that considers a virtual NOM component with site density that is fitted based on the $\mathrm{PO}_{4}$ measurements in acid ammonium oxalate and 0.01 $\mathrm{M} \mathrm{CaCl}_{2}$.

\begin{tabular}{|c|c|c|c|c|c|c|}
\hline Species & Surface group & Adsorption reaction & $\Delta \mathbf{z}_{0}$ & $\Delta \mathbf{z}_{1}$ & $\Delta \mathbf{z}_{2}$ & $\log K$ \\
\hline$\equiv \mathrm{Fe}_{3} \mathrm{O}-\mathrm{H}$ & $\mathrm{T}$ & $\equiv \mathrm{Fe}_{3} \mathrm{O}^{-0.5}+\mathrm{H}^{+} \leftrightarrow \equiv \mathrm{Fe}_{3} \mathrm{OH}^{+0.5}$ & 1 & 0 & 0 & 8.10 \\
\hline$\equiv \mathrm{FeOH}_{\mathrm{a}}$ & $\mathrm{S}(\mathrm{a}, \mathrm{bl}, \mathrm{bh})$ & $\equiv \mathrm{FeOH}^{-0.5}+\mathrm{H}^{+} \leftrightarrow \equiv \mathrm{FeOH}_{2}{ }^{+0.5}$ & 1 & 0 & 0 & 8.10 \\
\hline$\equiv \mathrm{Fe}_{3} \mathrm{OH}-\mathrm{Cl}$ & $\mathrm{T}$ & $\equiv \mathrm{Fe}_{3} \mathrm{O}^{-0.5}+\mathrm{H}^{+}+\mathrm{Cl}^{-} \leftrightarrow \equiv \mathrm{Fe}_{3} \mathrm{OH}^{+0.5}-\mathrm{Cl}^{-}$ & 1 & -1 & 0 & 7.65 \\
\hline$\equiv \mathrm{FeOH}_{2}-\mathrm{Cl}$ & $\mathrm{S}(\mathrm{a}, \mathrm{bl}, \mathrm{bh})$ & $\equiv \mathrm{FeOH}^{0.5}+\mathrm{H}^{+}+\mathrm{Cl}^{-} \leftrightarrow \equiv \mathrm{FeOH}_{2}{ }^{+0.5}-\mathrm{Cl}^{-}$ & 1 & -1 & 0 & 7.65 \\
\hline$\equiv \mathrm{Fe}_{3} \mathrm{OH}-\mathrm{NO}_{3}$ & $\mathrm{~T}$ & $\equiv \mathrm{Fe}_{3} \mathrm{O}^{-0.5}+\mathrm{H}^{+}+\mathrm{NO}_{3} \mathrm{I}^{-} \leftrightarrow=\mathrm{Fe}_{3} \mathrm{OH}^{+0.5}-\mathrm{NO}_{3}^{-}$ & 1 & -1 & 0 & 7.42 \\
\hline$\equiv \mathrm{FeOH} \mathrm{H}_{2}-\mathrm{NO}_{3}$ & $\mathrm{~S}(\mathrm{a}, \mathrm{bl}, \mathrm{bh})$ & $\equiv \mathrm{FeOH}^{0.5}+\mathrm{H}^{+}+\mathrm{NO}_{3}^{-} \leftrightarrow \equiv \mathrm{FeOH}_{2}{ }^{+0.5}-\mathrm{NO}_{3-}^{-}$ & 1 & -1 & 0 & 7.42 \\
\hline$\equiv \mathrm{Fe}_{3} \mathrm{O}-\mathrm{K}$ & $\mathrm{T}$ & $\equiv \mathrm{Fe}_{3} \mathrm{O}^{-0.5}+\mathrm{K}^{+} \leftrightarrow \equiv \mathrm{Fe}_{3} \mathrm{O}^{-0.5}-\mathrm{K}^{+}$ & 0 & 1 & 0 & -1.61 \\
\hline$\equiv \mathrm{FeOH}_{2}-\mathrm{K}$ & $\mathrm{S}(\mathrm{a}, \mathrm{bl}, \mathrm{bh})$ & $\equiv \mathrm{FeOH}^{-0.5}+\mathrm{K}^{+} \leftrightarrow \equiv \mathrm{FeOH}^{-0.5}-\mathrm{K}^{+}$ & 0 & 1 & 0 & -1.61 \\
\hline EFeOPOOH & $\mathrm{S}(\mathrm{a}, \mathrm{bl}, \mathrm{bh})$ & $\equiv \mathrm{FeOH}^{-0.5}+\mathrm{PO}_{4}+2 \mathrm{H}^{+} \leftrightarrow \mathrm{FeO}^{-0.22} \mathrm{POOH}^{-1.28}+\mathrm{H}_{2} \mathrm{O}$ & 0.28 & -1.28 & 0 & 26.36 \\
\hline$\equiv \mathrm{FeOPO}(\mathrm{OH})_{2}$ & $\mathrm{~S}(\mathrm{a}, \mathrm{bl}, \mathrm{bh})$ & $\equiv \mathrm{FeOH}^{-0.5}+\mathrm{PO}_{4}+3 \mathrm{H}^{+} \leftrightarrow \equiv \mathrm{FeO}^{-0.17} \mathrm{PO}(\mathrm{OH})_{2}{ }^{-0.33}+\mathrm{H}_{2} \mathrm{O}$ & 0.33 & -0.33 & 0 & 29.84 \\
\hline$\equiv(\mathrm{FeO})_{2} \mathrm{PO}_{2}$ & $\mathrm{~S}$ (bl, bh) & $2 \equiv \mathrm{FeOH}^{-0.5}+\mathrm{PO}_{4}+2 \mathrm{H}^{+} \leftrightarrow(\equiv \mathrm{FeO})_{2}{ }^{-0.54} \mathrm{PO}_{2}^{-1.46}+2 \mathrm{H}_{2} \mathrm{O}$ & 0.46 & -1.46 & 0 & 28.31 \\
\hline$\equiv \mathrm{FeO})_{2} \mathrm{POOH}$ & $\mathrm{S}(\mathrm{bl}, \mathrm{bh})$ & $2 \equiv \mathrm{FeOH}^{-0.5}+\mathrm{PO}_{4}+3 \mathrm{H}^{+} \leftrightarrow(\equiv \mathrm{FeO})_{2}^{-0.35} \mathrm{PO}_{2}^{-0.65}+2 \mathrm{H}_{2} \mathrm{O}$ & 0.65 & -0.65 & 0 & 33.52 \\
\hline$\equiv(\mathrm{FeOH})_{2} \mathrm{Ca}$ & $\mathrm{S}(\mathrm{bl})$ & $2 \equiv \mathrm{FeOH}^{-0.5}+\mathrm{Ca}^{2+} \leftrightarrow(=\mathrm{FeOH})_{2}^{-0.06} \mathrm{Ca}^{1.06}$ & 0.94 & 1.06 & 0 & 2.64 \\
\hline$\equiv\left(\mathrm{FeO}_{\mathrm{b}} \mathrm{H}\right)_{2}-\mathrm{Ca}$ & $\mathrm{S}$ (bh) & $2 \equiv \mathrm{FeOH}^{-0.5}+\mathrm{Ca}^{2+} \leftrightarrow(\equiv \mathrm{FeOH})_{2}^{-0.06} \mathrm{Ca}^{1.06}$ & 0.94 & 1.06 & 0 & 5.13 \\
\hline$\equiv \mathrm{FeOPO}{ }_{3} \mathrm{Ca}$ & $\mathrm{S}(\mathrm{a}, \mathrm{bl}, \mathrm{bh})$ & $\equiv \mathrm{FeOH}^{-0.5}+\mathrm{Ca}^{2+}+\mathrm{PO}_{4}{ }^{3-}+\mathrm{H}^{+} \leftrightarrow \mathrm{FeO}^{-0.26} \mathrm{PO}_{3}^{-1.30} \mathrm{Ca}^{-06}+\mathrm{H}_{2} \mathrm{O}$ & 0.24 & -1.30 & 1.06 & 22.27 \\
\hline$\equiv(\mathrm{FeOH})_{2} \mathrm{PO}_{2} \mathrm{Ca}$ & S (bl, bh) & $\begin{array}{l}2 \equiv \mathrm{FeOH}^{-0.5}+\mathrm{Ca}^{2+}+\mathrm{PO}_{4}^{3-}+2 \mathrm{H}^{+} \leftrightarrow(=\mathrm{FeO})_{2}{ }^{-0.38} \mathrm{PO}_{3}^{-1.08} \mathrm{Ca}^{1.46}+ \\
2 \mathrm{H}_{2} \mathrm{O}\end{array}$ & 0.62 & -1.08 & 1.46 & 30.09 \\
\hline$\equiv(\mathrm{FeO})_{2} \mathrm{~B}(\mathrm{OH})$ & $\mathrm{S}$ (bh) & $2=\mathrm{FeOH}^{-0.5}+\mathrm{B}(\mathrm{OH})_{3}{ }^{0} \leftrightarrow(\equiv \mathrm{FeO})_{2}{ }^{-0.82} \mathrm{~B}(\mathrm{OH})^{-0.18}+2 \mathrm{H}_{2} \mathrm{O}$ & 0.18 & -0.18 & 0 & 3.39 \\
\hline$\equiv(\mathrm{FeO})_{2} \mathrm{~B}(\mathrm{OH})_{2}$ & $\mathrm{~S}$ (bh) & $2 \equiv \mathrm{FeOH}^{-0.5}+\mathrm{B}(\mathrm{OH})_{3}{ }^{0} \leftrightarrow(=\mathrm{FeO})_{2}{ }^{-0.82} \mathrm{~B}(\mathrm{OH})_{2}{ }^{-0.18}+\mathrm{H}_{2} \mathrm{O}+\mathrm{H}^{+}$ & -0.25 & -0.75 & 0 & -4.68 \\
\hline$\equiv \mathrm{Fe}_{3} \mathrm{OH}-\mathrm{B}(\mathrm{OH})_{3}$ & & $\equiv \mathrm{Fe}_{3} \mathrm{O}^{-0.5}+\mathrm{B}(\mathrm{OH})_{3}{ }^{0}+\mathrm{H}^{+} \leftrightarrow \mathrm{FeO}_{3} \mathrm{H}^{+0.5}-\mathrm{B}(\mathrm{OH})_{3}{ }^{0}$ & 1 & 0 & 0 & 9.32 \\
\hline$\equiv \mathrm{FeOH}_{2}-\mathrm{B}(\mathrm{OH})_{3}$ & $\mathrm{~S}(\mathrm{a}, \mathrm{bl}, \mathrm{bh})$ & $\equiv \mathrm{FeOH}^{-0.5}+\mathrm{B}(\mathrm{OH})_{3}{ }^{0}+\mathrm{H}^{+} \leftrightarrow \mathrm{FeOH}_{2}{ }^{+0.5}-\mathrm{B}(\mathrm{OH})_{3}{ }^{0}$ & 1 & 0 & 0 & 9.32 \\
\hline$\equiv \mathrm{Fe}^{0} \mathrm{NOM}^{-1,-0.5}$ & $\mathrm{~S}(\mathrm{a}, \mathrm{bl}, \mathrm{bh}), \mathrm{NOM}$ & $\equiv \mathrm{FeOH}^{-0.5}+\mathrm{EHNOM}^{-1}$ & 1.5 & -1 & -0.5 & 0 \\
\hline$\equiv \mathrm{FeOH}_{2} \mathrm{NOM}$ & $\mathrm{S}(\mathrm{a}, \mathrm{bl}, \mathrm{bh}), \mathrm{NOM}$ & $\equiv \mathrm{FeOH}^{-0.5}+\equiv_{\mathrm{HNOM}^{-1}}$ & 2 & -1.5 & -0.5 & 0.6 \\
\hline EFeNOMH & $\mathrm{S}$ (a, bl, bh), NOM & $\equiv \mathrm{FeOH}^{-0.5}+\mathrm{EHNOM}^{-1}+1 \mathrm{H}^{+}$ & 1.5 & -0.5 & 0 & 3.4 \\
\hline
\end{tabular}




\section{Structural and adsorption parameters of the NICA-Donnan model}

Table S3: Structural and adsorption parameters of the NICA-DONNAN model for ion adsorption on dissolved and solid organic matter that have been used in modelling the soil systems. All parameters originate from Milne et al..$^{75,110}$, except the ones for B adsorption that are taken from Goli et al. ${ }^{37}$

\begin{tabular}{|c|c|c|}
\hline Site density & \multicolumn{2}{|l|}{$\begin{array}{l}\mathrm{S} 1=3.15 \mathrm{~mol} \mathrm{~kg}^{-1} \\
\mathrm{~S} 2=2.55 \mathrm{~mol} \mathrm{~kg}^{-1}\end{array}$} \\
\hline $\begin{array}{l}\text { Heterogeneity } \\
\text { parameter }(p)\end{array}$ & \multicolumn{2}{|l|}{$\begin{array}{l}\mathrm{p}_{1}=0.62 \\
\mathrm{p}_{2}=0.41\end{array}$} \\
\hline Donnan volume & \multicolumn{2}{|c|}{$\log V_{D}=b^{*} \log I-b-1$ with $b=-0.49$} \\
\hline Adsorption reactions & $\log K$ & $n_{i}$ \\
\hline $\mathrm{S} 1-\mathrm{H}$ & 2.93 & 0.81 \\
\hline S2-H & 8 & 0.63 \\
\hline $\mathrm{S} 1-\mathrm{Ca}$ & -1.37 & 0.78 \\
\hline $\mathrm{S} 2-\mathrm{Ca}$ & -0.43 & 0.75 \\
\hline $\mathrm{S} 1-\mathrm{B}(\mathrm{OH})_{3}$ & -0.08 & 0.91 \\
\hline $\mathrm{S} 2-\mathrm{B}(\mathrm{OH})_{3}$ & 1.90 & 0.61 \\
\hline
\end{tabular}

\section{Calculated NOM loading and $\mathrm{PO}_{4}$ solubility}

Table S4: Calculated NOM loading based on the $\mathrm{PO}_{4}$ solubility in the $\mathrm{CaCl}_{2}$ for the different soils. D1-D5 represent the 5 Dutch soils, B1-B5 are the 5 Burundian soils. The NOM loading is the sum of the different NOM species at the surface of ferrihydrite that are shown in Table S3. The NOM loading was adjusted until the soluble inorganic $\mathrm{PO}_{4}$ was the same as the concentration measured in the $\mathrm{CaCl}_{2}$ extraction, using inorganic $\mathrm{PO}_{4}$ in acid ammonium oxalate as input for reactive $\mathrm{PO}_{4}$, together with $\mathrm{pH}, \mathrm{Ca}, \mathrm{Cl}$ and $\mathrm{CO}_{3}$ concentrations, and the amount of ferrihydrite and clay as described in the main text.

\begin{tabular}{|c|c|}
\hline Soil & $\operatorname{NOM}\left(\mu \mathrm{mol} \mathrm{m} \mathrm{m}^{-2}\right)$ \\
\hline D1 & 3.06 \\
\hline D2 & 1.59 \\
\hline D3 & 1.64 \\
\hline D4 & 0.90 \\
\hline D5 & 1.15 \\
\hline B1 & 3.14 \\
\hline B2 & 2.66 \\
\hline B3 & 3.44 \\
\hline B4 & 3.17 \\
\hline B5 & 3.06 \\
\hline
\end{tabular}




\section{E. Effect of including $\mathrm{CO}_{3}, \mathrm{NOM}$ and $\mathrm{PO}_{4}$ as competitors for $\mathrm{B}$ adsorption to oxides}
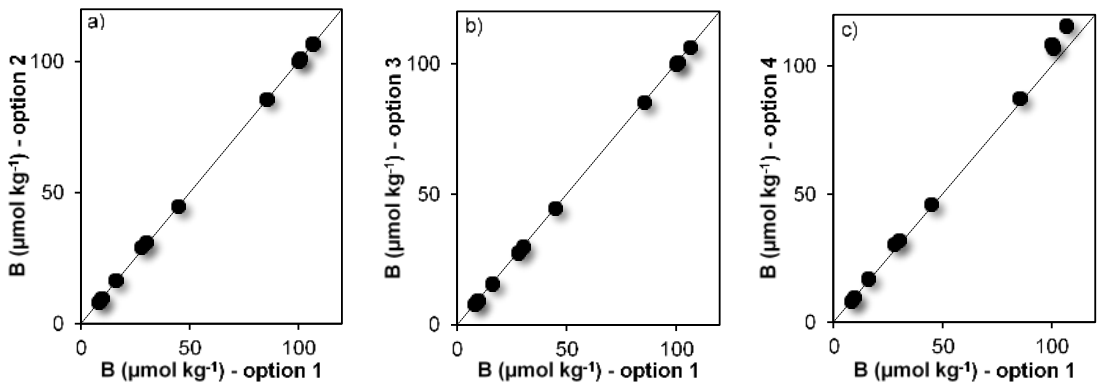

Figure S1: The reactive $\mathrm{B}$ was calculated with the multi-surface model from the soluble $\mathrm{B}$ in $\mathrm{CaCl}_{2}$ at the original soil $\mathrm{pH}\left(\mathrm{CaCl}_{2}\right)$ based on adsorption processes on humic acids, clay minerals and ferrihydrite for the different soil samples. The different options refer to the multi-surface model approach that includes different competitors for $\mathrm{B}$ adsorption to oxides. Option 1: next to $\mathrm{H}^{+}, \mathrm{Ca}^{2+}, \mathrm{Cl}^{-}$and $\mathrm{H}_{3} \mathrm{BO}_{3}$, the adsorption of $\mathrm{CO}_{3}{ }^{2-}, \mathrm{PO}_{4}{ }^{3-}$ and $\mathrm{NOM}$ is included in the model. This is the modelling approach that is described in the main text of the manuscript. Option 2: Same approach but without including NOM. Option 3: Same approach as option 1 but without including $\mathrm{CO}_{3}{ }^{2-}$. Option 4: Same as option 1 but without including NOM, $\mathrm{CO}_{3}{ }^{2-}$ and $\mathrm{PO}_{4}{ }^{3-}$.

\section{F. Literature data and modelling calculations for B adsorption on clay minerals}
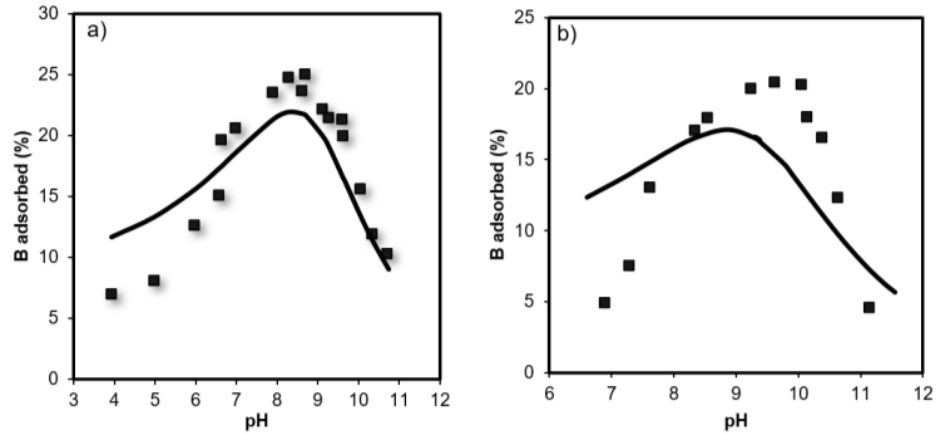

Figure S2: Data from Goldberg ${ }^{20}$ showing B adsorption on clay minerals. Figure a shows the data points from figure $3 \mathrm{c}$ from Goldberg ${ }^{20}$, that represents adsorption on a type of kaolinite clay. Figure $b$ shows the data points from figure $4 \mathrm{f}$ from Goldberg ${ }^{20}$, that represents adsorption on an illite clay. All adsorption experiments were carried out in a background electrolyte of $0.1 \mathrm{M} \mathrm{NaCl}$. For specific conditions of total clay content and total B content, we refer to Goldberg ${ }^{20}$. The lines represent modelling calculations using ferrihydrite as a proxy for clay minerals, with the structural and adsorption parameters from Table S2. We used a surface area of $5 \mathrm{~m}^{2} \mathrm{~g}^{-1}$ for kaolinite clays and $15 \mathrm{~m}^{2} \mathrm{~g}^{-1}$ for illite clays ${ }^{76}$, which we consider as the edge surface area of clay minerals that can perform specific adsorption of $\mathrm{B}$. We conclude that the $\mathrm{pH}$ adsorption trend can be reasonably well approached by using ferrihydrite adsorption parameters, especially for kaolinite clays. However, adsorption might be over-predicted at low pH values. 


\section{G. Effect of chosen SSA for the clay minerals on modelled reactive B}

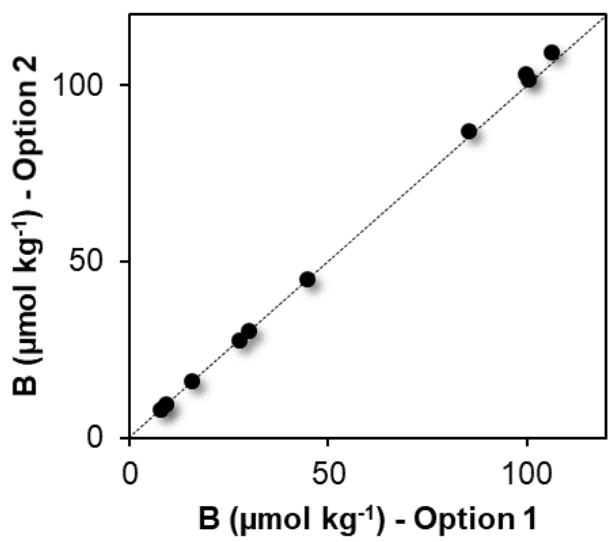

Figure S3: The calculated reactive B with the multi-surface model with different specific surface areas for the edges of clay minerals. Option 1: specific surface area is $5 \mathrm{~m}^{2} \mathrm{~g}^{-1}$ (modelling set-up as described in the main text). Option 2: specific surface area is $15 \mathrm{~m}^{2} \mathrm{~g}^{-1}$.

\section{H. Correlation between soil properties and B measurements}

Table S5: Pearson correlation coefficients between the different soil characteristics that are also shown in Table 1 of the manuscript. All data (except $\mathrm{pH}$ ) was log transformed for calculating the correlation coefficients. The asterisks show the significance level of the calculated correlation coefficients.

\begin{tabular}{|c|c|c|c|c|c|c|c|c|c|c|c|c|c|c|c|}
\hline & \multirow[t]{2}{*}{ pH } & Clay & soc & $\mathrm{HA}+\mathrm{FA}$ & \multirow{2}{*}{$\begin{array}{c}\text { DOC } \\
\log (m \\
\left.g^{-1}\right)\end{array}$} & $\begin{array}{l}\text { Fe- } \\
\text { AO }\end{array}$ & $\begin{array}{l}\text { Al- } \\
\text { AO }\end{array}$ & AL-D & Fe-D & \multirow[t]{2}{*}{$\mathrm{Pr}_{\mathrm{r}-\mathrm{AO}}$} & \multirow[t]{2}{*}{$\begin{array}{c}\text { B- } \\
\text { total }\end{array}$} & \multirow[t]{2}{*}{$\begin{array}{c}\mathrm{B}- \\
\mathrm{HNO}_{3}\end{array}$} & \multirow{2}{*}{$\begin{array}{c}\mathrm{B}- \\
\mathrm{KH}_{2} \mathrm{PO}_{4} \\
\log (\mu \mathrm{mol}\end{array}$} & \multirow{2}{*}{$\begin{array}{c}\text { B- } \\
\text { Mannito } \\
1\end{array}$} & \multirow[t]{2}{*}{$\begin{array}{c}\mathrm{B}- \\
\mathrm{CaCl}_{2}\end{array}$} \\
\hline & & $\log (\%)$ & \multicolumn{2}{|c|}{$\log \left(\mathrm{g} \mathrm{C} \mathrm{kg}^{-1}\right)$} & & $\ldots$ & 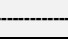 & $\log (\mathrm{mmol}$ & $\left(g^{-1}\right)-\ldots$ & & & & & & \\
\hline $\mathrm{pH}$ & 1.00 & & & & & & & & & & & & & & \\
\hline Clay & $0.70^{*}$ & 1.00 & & & & & & & & & & & & & \\
\hline soc & 0.06 & 0.21 & 1.00 & & & & & & & & & & & & \\
\hline $\mathrm{HA}+\mathrm{FA}$ & -0.44 & -0.13 & $0.84^{* * *}$ & 1.00 & & & & & & & & & & & \\
\hline DOC & 0.42 & 0.40 & $0.71^{\star *}$ & 0.39 & 1.00 & & & & & & & & & & \\
\hline $\mathrm{Fe}-\mathrm{AO}$ & -0.16 & 0.29 & $0.81^{* * *}$ & $0.81^{* * *}$ & 0.36 & 1.00 & & & & & & & & & \\
\hline Al-AO & $0.79^{\star \star *}$ & -0.42 & 0.17 & 0.55 & -0.45 & 0.46 & 1.00 & & & & & & & & \\
\hline Al-D & $0.79^{* \star *}$ & -0.46 & -0.19 & 0.22 & $0.71^{* *}$ & 0.16 & $0.93^{\star \star \star *}$ & 1.00 & & & & & & & \\
\hline Fe-D & -0.41 & 0.04 & -0.37 & -0.13 & $0.70^{\star *}$ & 0.12 & $0.58^{*}$ & $0.76^{* *}$ & 1.00 & & & & & & \\
\hline Pr-AO & 0.53 & 0.49 & $0.74^{* *}$ & 0.36 & $0.92^{* * *}$ & 0.50 & -0.42 & $-0.69^{\star *}$ & $-0.65^{\star *}$ & 1.00 & & & & & \\
\hline B-Total & $0.88^{* \star \star}$ & $0.77^{* *}$ & 0.41 & -0.22 & $0.61^{*}$ & 0.27 & $-0.63^{*}$ & $-0.71^{* *}$ & -0.32 & $0.79^{* *}$ & 1.00 & & & & \\
\hline B- $\mathrm{HNO}_{3}$ & $0.88^{* * *}$ & $0.65^{* \star}$ & 0.40 & -0.10 & $0.75^{\star *}$ & 0.11 & $0.76^{* *}$ & $-0.89^{* * *}$ & $-0.66^{* *}$ & & $0.91^{\star \star \star}$ & 1.00 & & & \\
\hline $\begin{array}{l}\mathrm{B}-\mathrm{KH}_{2} \mathrm{PO}_{4} \\
\text { B- }\end{array}$ & $0.89^{* \star *}$ & $0.63^{*}$ & 0.34 & -0.17 & $0.67^{\star \star}$ & 0.08 & $0.74^{* \star}$ & $-0.85^{\star \star \star}$ & $-0.62^{\star}$ & $\begin{array}{l}0.81^{* *} \\
0.83^{* *}\end{array}$ & $0.92^{* \star *}$ & $0.99^{* * *}$ & 1.00 & & \\
\hline Mannitol & $0.95^{\star \star \star \star}$ & $0.69^{\star *}$ & 0.37 & -0.29 & $0.70^{\star *}$ & 0.08 & $0.73^{\star \star}$ & $-0.82^{\star \star \star \star}$ & -0.54 & * & $0.95^{\star \star \star}$ & $0.99^{* \star *}$ & $0.99^{* \star *}$ & 1.00 & \\
\hline $\mathrm{B}-\mathrm{CaCl}_{2}$ & $0.71^{* *}$ & 0.53 & 0.54 & 0.08 & $0.82^{\star \star *}$ & 0.24 & $-0.62^{\star}$ & $-0.81^{\star \star *}$ & $-0.72^{\star *}$ & $0.92^{* *}$ & $0.85^{\star \star \star}$ & $0.96^{* * \star}$ & $0.95^{* \star *}$ & $0.94^{\star \star \star}$ & 1.00 \\
\hline
\end{tabular}




\section{B speciation in the 3 different extraction methods}
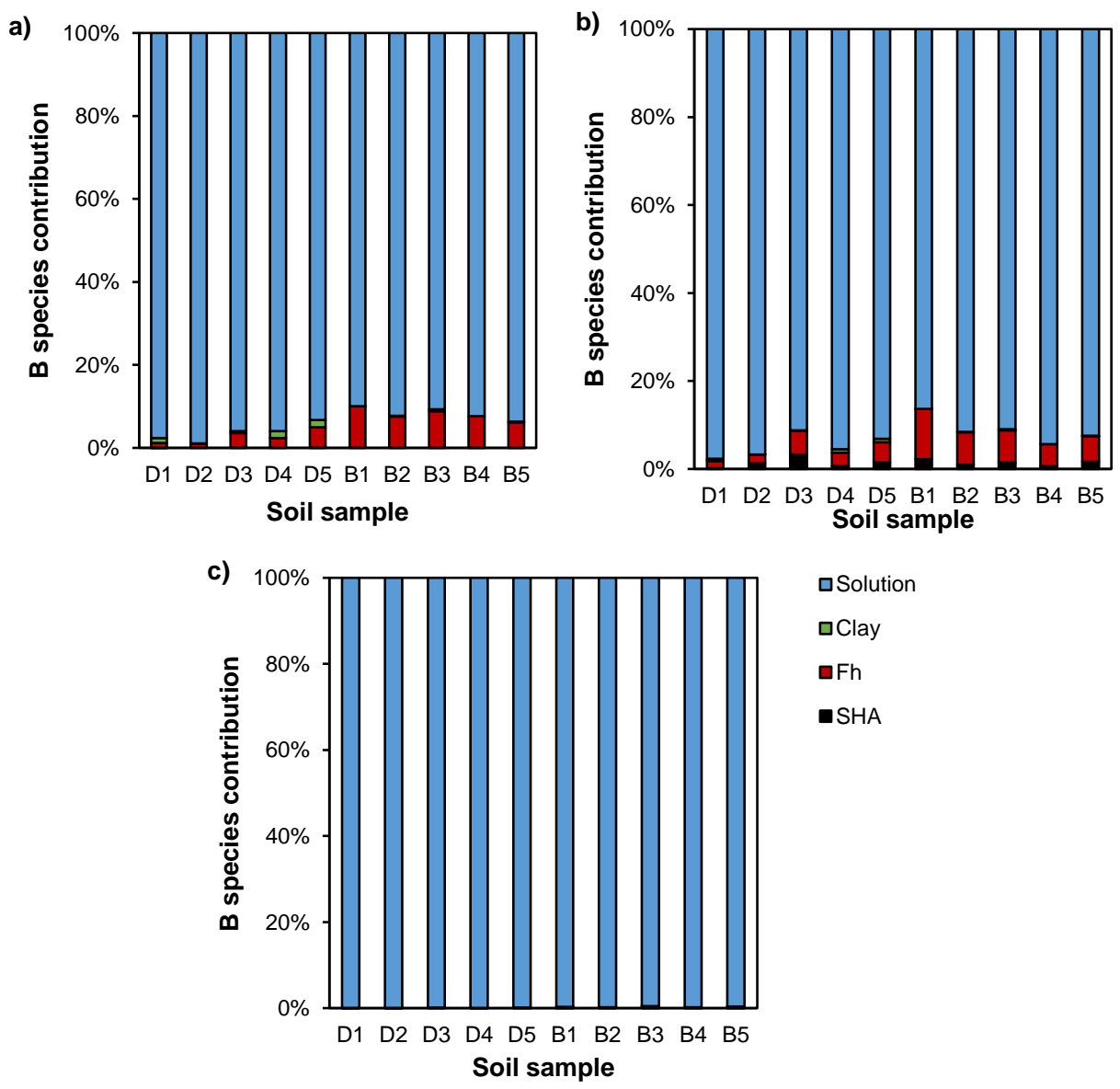

口Solution

口Clay

$\mathrm{aFh}$

- SHA

Figure S4: Calculated B speciation for the temperate (D1-D5) and tropical (B1-B5) soils in the extractions that were tested for measuring reactive boron. The extraction solutions were $0.43 \mathrm{M} \mathrm{HNO}_{3}(\mathrm{a}), 0.05 \mathrm{M}$ $\mathrm{KH}_{2} \mathrm{PO}_{4}$ (b) and $0.2 \mathrm{M}$ mannitol (c). The $\mathrm{B}$ is distributed over the solution phase (See Table $\mathrm{S} 1$ for the aqueous inorganic $\mathrm{B}$ species) and solid phase (bound to solid humic acids (SHA), the edges of clay minerals, and oxides modelled as ferrihydrite $(F h)$ ). 


\section{J. Relation between $\mathrm{B}-\mathrm{CaCl}_{2}$ and soil properties}
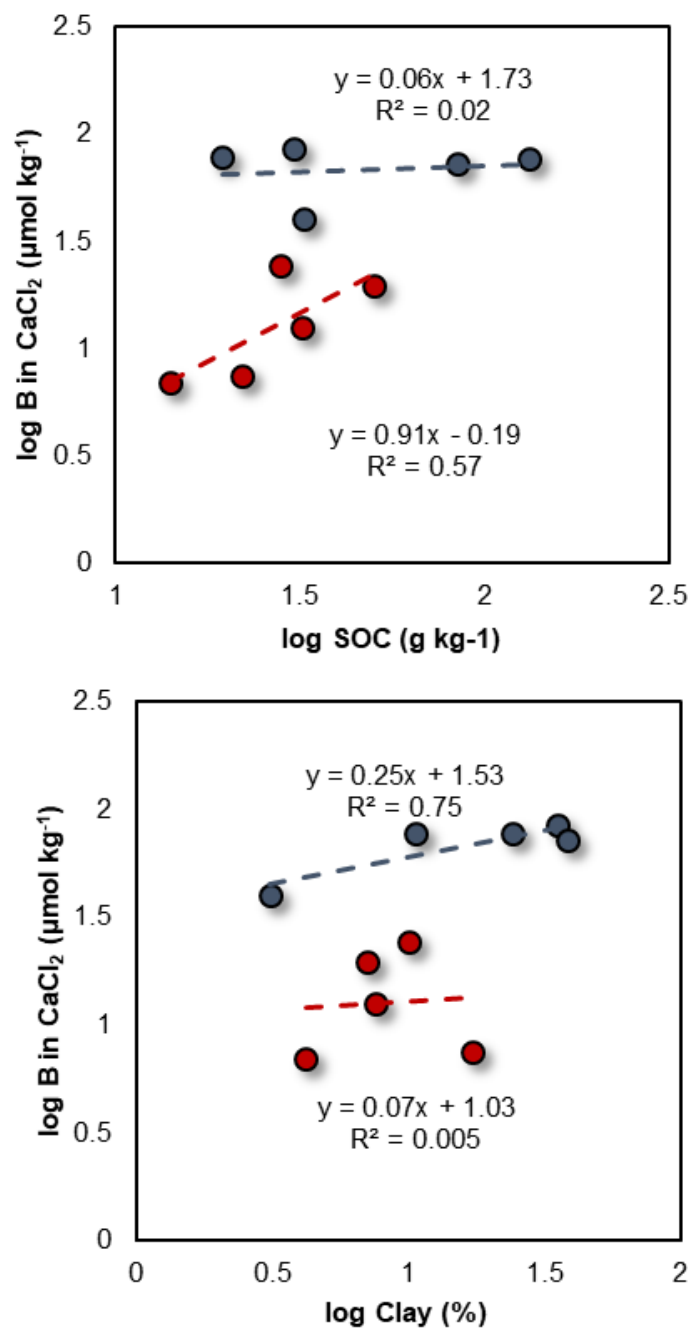

Figure S5: The relation between $\mathrm{B}$ in $\mathrm{CaCl}_{2}$ and soil organic carbon (a) and clay (b) for the Burundian soil samples (red markers) and Dutch soil samples (blue markers). The equation and $\mathrm{R}^{2}$ show the quality of the regression lines. The $\mathrm{P}$-values for the $\mathrm{x}$-variables in all regression equations were above 0.05 . 


\section{K. pH dependent B solubility}

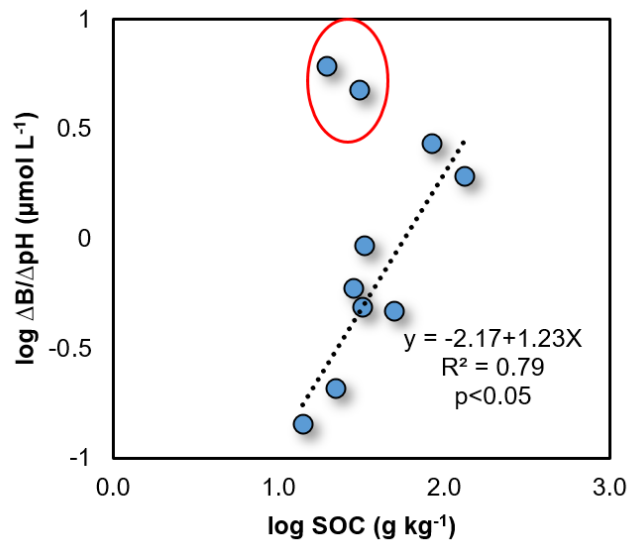

Figure S6: Relation between the soil organic carbon content (x-axis) and the experimentally observed decrease in boron concentration with increasing $\mathrm{pH}$, calculated as logarithm of $\triangle \boldsymbol{B}-\mathrm{CaCl}_{2} / \boldsymbol{p H}\left(\mathrm{CaCl2}_{2}\right)$ in $\mu \mathrm{mol} \mathrm{L}^{-1}$ (y-axis). The regression equation is calculated based on all samples except for D1 and D4, which are the two markers that are encircled. For these samples, the largest decrease in B solubility with increasing $\mathrm{pH}$ was observed, not corresponding with a high SOC content. 


\section{L. pH dependent $B$ speciation}
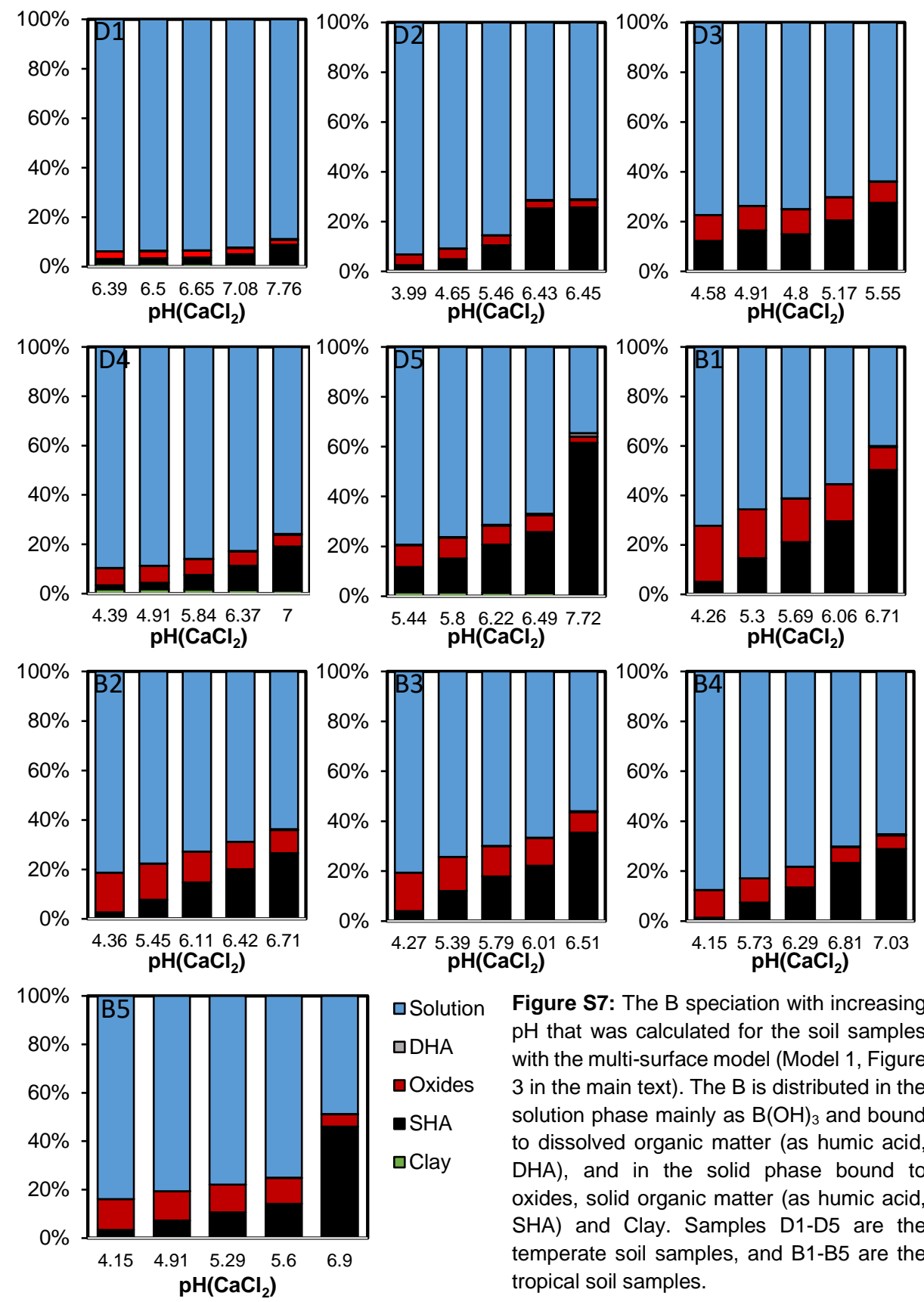

$\square$ Solution Figure S7: The B speciation with increasing 口DHA

-Oxides $\mathrm{pH}$ that was calculated for the soil samples with the multi-surface model (Model 1, Figure

- SHA 3 in the main text). The $B$ is distributed in the solution phase mainly as $\mathrm{B}(\mathrm{OH})_{3}$ and bound 口Clay to dissolved organic matter (as humic acid, DHA), and in the solid phase bound to oxides, solid organic matter (as humic acid, SHA) and Clay. Samples D1-D5 are the temperate soil samples, and B1-B5 are the tropical soil samples. 


\section{REFERENCES}

1. Uluisik, I., Karakaya, H. C. \& Koc, A. The importance of boron in biological systems. J. Trace Elem. Med. Biol. 45, 156-162 (2018).

2. Shorrocks, V. M. The occurrence and correction of boron deficiency. Plant Soil 193, 121-148 (1997).

3. Howe, P. D. A Review of Boron Effects in the Environment. Biol. Trace Elem. Res. 66, 153-166 (1998).

4. Gupta, U. C., Jame, Y. W., Campbell, C. A., Leyshon, A. J. \& Nicholaichuk, An. W. Boron toxicity and deficiency: a review. Can. J. Soil Sci. 65, 381-409 (1985).

5. Kabata-Pendias, A. \& Pendias, H. Trace Elements in Soils and Plants, Third Edition. www.crcpress.com (2001).

6. Hou, J., Evans, L. J. \& Spiers, G. A. Boron fractionation in soils. Commun. Soil Sci. Plant Anal. 25, 1841-1853 (1994).

7. Kot, F. S., Farran, R., Kochva, M. \& Shaviv, A. Boron in humus and inorganic components of Hamra and Grumosol soils irrigated with reclaimed wastewater. Soil Res. 50, 30-43 (2012).

8. Jin, J., Martens, D. C. \& Zelazny, L. W. Distribution and Plant Availability of Soil Boron Fractions. Soil Sci. Soc. Am. J. 51, 1228 (1987).

9. Chaudhary, D. R. \& Shukla, L. . Availability of soil boron fractions to mustard [Brassica juncea (L.) Czern.] in arid soils of Rajasthan (India). Agrochimica 173-179 (2003).

10. Dordas, C., Chrispeels, M. J. \& Brown, P. H. Permeability and channel-mediated transport of boric acid across membrane vesicles isolated from squash roots. Plant Physiol. 124, 1349-1361 (2000).

11. Park, H. \& Schlesinger, W. H. Global biogeochemical cycle of boron. Global Biogeochem. Cycles 16, 20-1-20-11 (2002).

12. Goldberg, S. Reactions of boron with soils. Plant Soil 193, 35-48 (1997).

13. Keren, R., Bingham, F. T. \& Rhoades, J. D. Plant Uptake of Boron as Affected by Boron Distribution Between Liquid and Solid Phases in Soil. Soil Sci. Soc. Am. J. 49, 297-302 (1985).

14. Gu, B. \& Lowe, L. E. Studies on the ad sorption of boron on humic acids. Can. J. Soil Sci. 70, 305-311 (1990).

15. Lemarchand, E., Schott, J. \& Gaillardet, J. Boron isotopic fractionation related to boron sorption on humic acid and the structure of surface complexes formed. Geochim. Cosmochim. Acta 69, 3519-3533 (2005).

16. Goldberg, S. Chemical modeling of boron adsorption by humic materials using the constant capacitance model. Soil Sci. 179, 561-567 (2014).

17. Keren, R. \& Communar, G. Boron Sorption on Wastewater Dissolved Organic Matter: pH Effect. Soil Sci. Soc. Am. J. 73, 2021-2025 (2009).

18. Goldberg, S. \& Glaubig, R. A. Boron Adsorption and Silicon Release by the Clay Minerals Kaolinite, Montmorillonite, and Illite1. Soil Sci. Soc. Am. J. 50, 1442 (1986).

19. Manning, B. A. \& Goldberg, S. Modeling Competitive Adsorption of Arsenate with Phosphate and Molybdate on Oxide Minerals. Repr. from Soil Sci. Soc. Am. J. Vol. GO South Segoe Rd 60, 121-131 (1996).

20. Goldberg, S. Reanalysis of boron adsorption on soils and soil minerals using the constant capacitance model. Soil Sci. Soc. Am. J. 63, 823-829 (1999).

21. Keren, R. \& Mezuman, U. Boron adsorption by clay minerals using a phenomenological equation. Clays Clay Miner. 29, 198-204 (1981).

22. Sims, J. R. \& Bingham, F. T. Retention of Boron by Layer Silicates, Sesquioxides, and Soil Materials: I. Layer Silicates1. Soil Sci. Soc. Am. J. 31, 728 (1967).

23. Peak, D., Luther, G. W. \& Sparks, D. L. ATR-FTIR spectroscopic studies of boric acid adsorption on hydrous ferric oxide. Geochim. Cosmochim. Acta 67, 2551-2560 (2003).

24. Su, C. \& Suarez, D. L. Coordination of Adsorbed Boron: A FTIR Spectroscopic Study. Environ. Sci. Technol. 29, 302-311 (1995).

25. Van Eynde, E., Mendez, J. C., Hiemstra, T. \& Comans, R. N. J. Boron adsorption to ferrihydrite with implications for surface speciation in soils: Experiments and modeling. ACS Earth Sp. Chem. acsearthspacechem.0c00078 (2020) doi:10.1021/acsearthspacechem.0c00078.

26. Goli, E., Rahnemaie, R., Hiemstra, T. \& Malakouti, M. J. The interaction of boron with goethite: Experiments and CD-MUSIC modeling. Chemosphere 82, 1475-1481 (2011).

27. Goldberg, S., Forster, H. S. \& Heick, E. L. Boron Adsorption Mechanisms on Oxides, Clay Minerals, and Soils Inferred from lonic Strength Effects. Soil Sci. Soc. Am. J. 57, 704 (1993). 
28. Goldberg, S. \& Glaubig, R. Boron adsorption on California soils. Soil Sci. Soc. Am. 50, 11731176 (1986).

29. Goldberg, S. Modeling Boron Adsorption Isotherms and Envelopes Using the Constant Capacitance Model. Vadose Zo. J. 3, 676-680 (2004).

30. Goldberg, S., Forster, H. S., Lesch, S. M. \& Heick, E. L. Influence of anion competition on Boron adsorption by clays and soils. Soil Sci. 161, 99-103 (1996).

31. Goldberg, S., Suarez, D. L., Basta, N. T. \& Lesch, S. M. Predicting Boron Adsorption Isotherms by Midwestern Soils using the Constant Capacitance Model. Soil Sci. Soc. Am. J. 68, 137-142 (2004).

32. Goldberg, S., Lesch, S. M. \& Suarez, D. L. Predicting Boron Adsorption by Soils Using Soil Chemical Parameters in the Constant Capacitance Model. Soil Sci. Soc. Am. J. 64, 1356 (2000).

33. Goldberg, S., Corwin, D. L., Shouse, P. J. \& Suarez, D. L. Prediction of boron adsorption by field samples of diverse textures. Soil Sci. Soc. Am. J. 69, 1379-1388 (2005).

34. Goldberg, S. Application of surface complexation models to anion adsorption by natural materials. Environ. Toxicol. Chem. 33, 2172-2180 (2014).

35. Groenenberg, J. E. \& Lofts, S. The use of assemblage models to describe trace element partitioning, speciation, and fate: a review. Environ. Toxicol. Chem. 33, 2181-96 (2014).

36. Weng, L., Temminghoff, E. J. M. \& Van Riemsdijk, W. H. Contribution of Individual Sorbents to the Control of Heavy Metal Activity in Sandy Soil. Environ. Sci. Technol. 35, 4436-4443 (2001).

37. Goli, E., Hiemstra, T. \& Rahnemaie, R. Interaction of boron with humic acid and natural organic matter: Experiments and modeling. Chem. Geol. 515, 1-8 (2019).

38. Groenenberg, J. E., Römkens, P. F. A. M., Zomeren, A. Van, Rodrigues, S. M. \& Comans, R. N. J. Evaluation of the Single Dilute $(0.43 \mathrm{M})$ Nitric Acid Extraction to Determine Geochemically Reactive Elements in Soil. Environ. Sci. Technol. 51, 2246-2253 (2017).

39. Degryse, F., Smolders, E. \& Parker, D. R. Partitioning of metals (Cd, Co, Cu, Ni, Pb, Zn) in soils: concepts, methodologies, prediction and applications - a review. Eur. J. Soil Sci. 60, 590-612 (2009).

40. Houba, V. J. G., Temminghoff, E. J. M., Gaikhorst, G. A. \& van Vark, W. Soil analysis procedures using $0.01 \mathrm{M}$ calcium chloride as extraction reagent. Commun. Soil Sci. Plant Anal. 31, 12991396 (2000).

41. ISO. Soil quality 17586:2016 -- Extraction of trace elements using dilute nitric acid. https://www.iso.org/standard/60060.html (2016).

42. Hou, J., Evans, L. J. \& Spiers, G. A. Chemical fractionation of soil boron: I. Method development. Can. J. Soil. Sci. 76, 485-491 (1995).

43. Bingham, F. T. Boron. in Methods of soil analysis Part 2 Chemical and Mineralogical properties (eds. Page, A. L., Miller, M. H. \& Keeny, D. R.) 431-448 (American Society of Agronomy, 1982).

44. Goldberg, S. \& Suarez, D. L. A New Soil Test for Quantitative Measurement of Available and Adsorbed Boron. Soil Sci. Soc. Am. J. 78, 480 (2014).

45. Hengl, T. et al. SoilGrids250m: Global gridded soil information based on machine learning. PLoS One 12, e0169748 (2017).

46. Degryse, F., Broos, K., Smolders, E. \& Merckx, R. Soil solution concentration of Cd and Zn canbe predicted with a CaCl 2 soil extract. Eur. J. Soil Sci. 54, 149-158 (2003).

47. Dijkstra, J. J., Meeussen, J. C. L. \& Comans, R. N. J. Evaluation of a Generic Multisurface Sorption Model for Inorganic Soil Contaminants. Environ. Sci. Technol. 43, 6196-6201 (2009).

48. Murphy, J. \& Riley, J. P. A modified single solution method for the determination of phosphate in natural waters. Anal. Chim. Acta 27, 31-36 (1962).

49. Konert, M. \& Vandenberghe, J. Comparison of laser grain size analysis with pipette and sieve analysis: a solution for the underestimation of the clay fraction. Sedimentology vol. 44 (1997).

50. NEN 5753:2018. Soil - Determination of clay content and particle distribution in soil and sediment by sieve and pipet. (2018).

51. ISO. ISO 12782-3:2012 Soil quality -- Parameters for geochemical modelling of leaching and speciation of constituents in soils and materials -- Part 3: Extraction of aluminium oxides and hydroxides with ammonium oxalate/oxalic acid. (2012).

52. Hiemstra, T., Antelo, J., Rahnemaie, R. \& van Riemsdijk, W. H. Nanoparticles in natural systems I: The effective reactive surface area of the natural oxide fraction in field samples. Geochim. Cosmochim. Acta 74, 41-58 (2010).

53. ISO. ISO 12782-2:2012 - Soil quality -- Parameters for geochemical modelling of leaching and speciation of constituents in soils and materials -- Part 2: Extraction of crystalline iron oxides and hydroxides with dithionite. https://www.iso.org/standard/51698.html (2012).

54. Wolf, A. M. \& Baker, D. E. Colorimetric Method for Phosphorus Measurement in Ammonium Oxalate Soil Extracts. Commun. Soil Sci. Plant Anal. 21, 2257-2263 (1990). 
55. Jørgensen, C., Turner, B. L. \& Reitzel, K. Identification of inositol hexakisphosphate binding sites in soils by selective extraction and solution 31P NMR spectroscopy. Geoderma 257-258, 22-28 (2015).

56. Cui, Y. \& Weng, L. Arsenate and phosphate adsorption in relation to oxides composition in soils: LCD modeling. Environ. Sci. Technol. 47, 7269-7276 (2013).

57. Hass, A., Loeppert, R. H., Messina, M. G. \& Rogers, T. D. Determination of phosphate in selective extractions for soil iron oxides by the molybdenum blue method in an automated continuance flow injection system. Commun. Soil Sci. Plant Anal. 42, 1619-1635 (2011).

58. Mendez, J. C., Hiemstra, T. \& Koopmans, G. F. Assessing the Reactive Surface Area of Soils and the Association of Soil Organic Carbon with Natural Oxide Nanoparticles Using Ferrihydrite as Proxy. Environ. Sci. Technol. 54, 11990-12000 (2020).

59. Van Zomeren, A. \& Comans, R. N. J. Measurement of humic and fulvic acid concentrations and dissolution properties by a rapid ratch procedure. Environ. Sci. Technol. (2007) doi:10.1021/es0709223.

60. Vaughan, B. \& Howe, \& J. Evaluation of boron chelates in extracting soil boron. Commun. Soil Sci. Plant Anal. 25, 1071-1084 (1994).

61. Knoeck, J. \& Taylor, J. K. Aqueous Boric Acid-Borate-Mannitol Equilibria. Anal. Chem. 41, 17301734 (1969).

62. Bassett, R. L. L. A critical evaluation of the thermodynamic data for boron ions, ion pairs, complexes, and polyanions in aqueous solution at $298.15 \mathrm{~K}$ and 1 bar. Geochim. Cosmochim. Acta 44, 1151-1160 (1980).

63. Aitken, R. L., Jeflrey, A. J. \& Compton, B. L. Evaluation of Selected Extractants for Boron in Some Queensland Soils. Aust. J. Soil Res vol. 25 https://eurekamag.com/pdf/001/001590009.pdf (1987).

64. Keizer, M. G. \& Van Riemsdijk, W. H. ECOSAT, a Computer Program for the Calculation of Chemical Speciation and Transport in Soil-Water Systems. (1995).

65. Hiemstra, T. \& Van Riemsdijk, W. H. A surface structural model for ferrihydrite I: Sites related to primary charge, molar mass, and mass density. Geochim. Cosmochim. Acta 73, 4423-4436 (2009).

66. Xu, D. \& Peak, D. Adsorption of Boric Acid on Pure and Humic Acid Coated am-Al(OH)3: A Boron K-Edge XANES Study. Environ. Sci. Technol. 41, 903-908 (2007).

67. Mendez, J. C. \& Hiemstra, T. Carbonate Adsorption to Ferrihydrite: Competitive Interaction with Phosphate for Use in Soil Systems. ACS Earth Sp. Chem. 3.1, 129-141 (2018).

68. Hiemstra, T. \& Zhao, W. Reactivity of ferrihydrite and ferritin in relation to surface structure, size, and nanoparticle formation studied for phosphate and arsenate. Environ. Sci. Nano 3, 12651279 (2016).

69. Mendez, J. C. \& Hiemstra, T. Ternary complex formation of phosphate with $\mathrm{Ca}$ and $\mathrm{Mg}$ ions binding to ferrihydrite: Experiments and mechanisms. ACS Earth Sp. Chem. 4.4, acsearthspacechem.9b00320 (2020).

70. Mendez, J. C. \& Hiemstra, T. High and low affinity sites of ferrihydrite for metal ion adsorption: Data and modeling of the alkaline-earth ions $\mathrm{Be}, \mathrm{Mg}, \mathrm{Ca}, \mathrm{Sr}, \mathrm{Ba}$, and $\mathrm{Ra}$. Geochim. Cosmochim. Acta (2020) doi:10.1016/j.gca.2020.07.032.

71. Marzadori, C., Antisari, L. V., Ciavatta, C. \& Sequi, P. Soil Organic Matter Influence on Adsorption and Desorption of Boron. Soil Sci. Soc. Am. J. 55, 1582 (1991).

72. Sarkar, D., De, D. K., Das, R. \& Mandal, B. Removal of organic matter and oxides of iron and manganese from soil influences boron adsorption in soil. Geoderma 214-215, 213-216 (2014).

73. Hiemstra, T., Mia, S., Duhaut, P.-B. B. \& Molleman, B. Natural and pyrogenic humic acids at goethite and natural oxide surfaces interacting with phosphate. Environ. Sci. Technol. 47, 91829189 (2013).

74. Kinniburgh, D. G. et al. Metal ion binding by humic acid: Application of the NICA-Donnan model. Environ. Sci. Technol. 30, 1687-1698 (1996).

75. Milne, C. J., Kinniburgh, D. G., van Riemsdijk, W. H. \& Tipping, E. Generic NICA-Donnan Model Parameters for Metal-lon Binding by Humic Substances. Environ. Sci. Technol. 37, 958-971 (2003).

76. Heidmann, I., Christl, I., Leu, C. \& Kretzschmar, R. Competitive sorption of protons and metal cations onto kaolinite: experiments and modeling. J. Colloid Interface Sci. 282, 270-282 (2005).

77. Scopes, R. K. Protein Purification: Principles and Practice. (Springer Science \& Business Media, 1994).

78. Parks, J. L. \& Edwards, M. Boron in the Environment. Crit. Rev. Environ. Sci. Technol. 35, 81114 (2005). 
79. Fruchter, J. S., Ral, D. \& Zachara, J. M. Identification of Solubility-Controlling Solid Phases in a Large Fly Ash Field Lysimeter. Environ. Sci. Technol vol. 24 https://pubs.acs.org/sharingguidelines (1990).

80. Sparks, D. L. et al. Methods of soil analysis. Part 3, Chemical methods. (Soil Science Society of America, 1996).

81. Novozamsky, I., Barrera, L. L., Houba, V. J. G., van der Lee, J. J. \& van Eck, R. Comparison of a hot water and cold $0.01 \mathrm{M} \mathrm{Cacl}_{2}$ extraction procedures for the determination of boron in soil. Commun. Soil Sci. Plant Anal. 21, 2189-2195 (1990).

82. Chao, T. T. \& Sanzolone, R. F. Fractionation of Soil Selenium by Sequential Partial Dissolution. Soil Sci. Soc. Am. J. 53, 385 (1989).

83. Kutus, B., Ozsvár, D., Varga, N., Pálinkó, I. \& Sipos, P. ML and ML 2 complex formation between $\mathrm{Ca}$ ( ii ) and d -glucose derivatives in aqueous solutions. Dalt. Trans. 46, 1065-1074 (2017).

84. Bourne, E. J., Hutson, D. H. \& Weigel, H. 7. Complexes between molybdate and acyclic polyhydroxy-compounds. J. Chem. Soc. 0, 35 (1961).

85. Doleźal, J., Klausen, K. S. \& Langymyhr, F. J. Studies in the complex formation of metal ions with sugars : Part I. The complex formation of cobalt(II), cobalt(III), copper(II) and nickel(II) with mannitol. Anal. Chim. Acta 63, 71-77 (1973).

86. Huertas, F. J., Chou, L. \& Wollast, R. Mechanism of kaolinite dissolution at room temperature and pressure Part II: kinetic study. Geochim. Cosmochim. Acta 63, 3261-3275 (1999).

87. Lemarchand, D., Cividini, D., Turpault, M.-P. \& Chabaux, F. Boron isotopes in different grain size fractions: Exploring past and present water-rock interactions from two soil profiles (Strengbach, Vosges Mountains). Geochim. Cosmochim. Acta 98, 78-93 (2012).

88. Bibi, I., Singh, B. \& Silvester, E. Dissolution of illite in saline-acidic solutions at $25^{\circ} \mathrm{C}$. Geochim. Cosmochim. Acta 75, 3237-3249 (2011).

89. Bibi, I., Singh, B. \& Silvester, E. Mineralogy and Acid Neutralisation Mechanisms in Inland Acid Sulfate Environments. (University of Sydney , 2012).

90. Lindsay, W. L. Chemical equilibria in soils. (Wiley, 1979).

91. Köhler, S. J., Dufaud, F. \& Oelkers, E. H. An experimental study of illite dissolution kinetics as a function of ph from 1.4 to 12.4 and temperature from 5 to $50^{\circ} \mathrm{C}$. Geochim. Cosmochim. Acta 67, 3583-3594 (2003).

92. Couch, E. L. Calculation of paleosalinities from boron and clay mineral data. Am. Assoc. Pet. Geol. Bull. 55, (1971).

93. Kaplan, D. I., Sumner, M. E., Bertsch, P. M. \& Adriano, D. C. Chemical Conditions Conducive to the Release of Mobile Colloids from Ultisol Profiles. Soil Sci. Soc. Am. J. 60, 269-274 (1996).

94. Kretzschmar, R. \& Sticher, H. Colloid Transport in Natural Porous Media: Influence of Surface Chemistry and Flow Velocity. Phys. Chem. Earth 23, 133-139 (1998).

95. Kinrade, S. D. et al. Stable Five-and Six-Coordinated Silicate Anions in Aqueous Solution. Science (80-. ). 285, 1542-1545 (1999).

96. Doleźal, J. \& Langmyhr, F. J. Some possibilities of redox titrations with iron(ii) sulphate in an alkaline mannitol medium. Anal. Chim. Acta 61, 73-81 (1972).

97. Mohr, S. \& Bechtold, T. Electrochemical behaviour of iron-complexes in presence of competitive ligands: A strategy for optimization of current density. J. Appl. Electrochem. 31, 363-368 (2001).

98. Karlsson, P. M., Postmus, B. R. \& Palmqvist, A. E. C. Dissolution and Protection of Aluminium Oxide in Corrosive Aqueous Media-An Ellipsometry and Reflectometry Study. J. Dispers. Sci. Technol. 30, 949-953 (2009).

99. Lindsay, W. L. \& Norvell, W. A. Development of a DTPA Soil Test for Zinc, Iron, Manganese, and Copper1. Soil Sci. Soc. Am. J. 42, 421 (1978).

100. Gaillardet, J. \& Lemarchand, D. Boron in the Weathering Environment. in 163-188 (Springer, Cham, 2018). doi:10.1007/978-3-319-64666-4_7.

101. Hiemstra, T. Ferrihydrite interaction with silicate and competing oxyanions: Geometry and Hydrogen bonding of surface species. Geochim. Cosmochim. Acta 238, 453-476 (2018).

102. Pédrot, M. et al. Insights into colloid-mediated trace element release at the soil/water interface. J. Colloid Interface Sci. 325, 187-197 (2008).

103. Pokrovsky, O. . \& Schott, J. Iron colloids/organic matter associated transport of major and trace elements in small boreal rivers and their estuaries (NW Russia). Chem. Geol. 190, 141-179 (2002).

104. Mertens, J., Van Laer, L., Salaets, P. \& Smolders, E. Phytotoxic doses of boron in contrasting soils depend on soil water content. Plant Soil 342, 73-82 (2011).

105. Su, C. \& Suarez, D. L. Boron Release from Weathering of Illites, Serpentine, Shales, and Illitic/Palygorskitic Soils. Soil Sci. Soc. Am. J. 68, 96-105 (2004).

106. Cividini, D., Lemarchand, D., Chabaux, F., Boutin, R. \& Pierret, M.-C. From biological to 
lithological control of the B geochemical cycle in a forest watershed (Strengbach, Vosges). Geochim. Cosmochim. Acta 74, 3143-3163 (2010).

107. Noireaux, J., Gaillardet, J., Sullivan, P. L. \& Brantley, S. L. Boron Isotope Fractionation in Soils at Shale Hills CZO. Procedia Earth Planet. Sci. 10, 218-222 (2014).

108. Kot, F. S. et al. On boron turnover in plant-litter-soil system. Geoderma 268, 139-146 (2016).

109. Goldberg, S. \& Glaubig, R. A. Boron Adsorption on Aluminum and Iron Oxide Minerals 1. Soil Sci. Soc. Am. J. 49, 1374-1379 (1985).

110. Milne, C. J. C. J., Kinniburgh, D. G. D. G. D. G. \& Tipping, E. Generic NICA-Donnan Model Parameters for Proton Binding by Humic Substances. Environ. Sci. Technol. 35, 2049-2059 (2001). 


\section{Chapter 7}

Solid-solution partitioning of micronutrients $\mathrm{Zn}, \mathrm{Cu}$ and $B$ in tropical soils: mechanistic and empirical models

Elise Van Eynde, Jan E. Groenenberg, Ellis Hoffland, Rob N.J. Comans

Submitted for publication 


\begin{abstract}
Trace elements such as zinc $(\mathrm{Zn})$, copper $(\mathrm{Cu})$ and boron $(\mathrm{B})$ are important micronutrients for crop production. Their bioavailability is essential to crops yield quantity and quality in many tropical soils. Nutrient bioavailability depends partly on the soil nutrient status, and in particular on the labile and soluble concentrations. Adsorption/desorption and precipitation/dissolution processes control the partitioning of the labile pool over the solid and solution phase. However, so far the solid-solution partitioning of trace elements has mostly been studied in temperate and often contaminated soils, to estimate the risk for leaching and toxicity towards plants and biota. We studied, therefore, the solid-solution partitioning of $\mathrm{Zn}, \mathrm{Cu}$ and B for 172 soils from Burundi, Rwanda and Kenya, using extensive soil characterization in combination with multi-surface modelling and two types of empirical Freundlich type partition relations. Our aim was to enhance the understanding of the soil chemical processes that control the solid-solution partitioning of the three micronutrients in these soils from the tropics with a multisurface model, and to use this knowledge as benchmark to develop partition relations that require less input data and are more convenient tools for predicting the soluble concentration based on existing soil data. We show that the generic multisurface model applied to these tropical soils performs similarly for $\mathrm{Zn}$ and $\mathrm{Cu}$ as in previous studies on temperate and contaminated soils. The $\mathrm{Zn}$ and $\mathrm{Cu}$ speciation was dominated by adsorption to soil organic matter, with an increased importance of metal (hydr)oxides with increasing $\mathrm{pH}$. Given its generally low concentrations in these soils, dissolved organic matter was found to be important only for the solution speciation of $\mathrm{Cu}$. The multi-surface model overestimated B solubility for most soil samples, which we attribute to an inaccurate estimation of reactive B. Interestingly, the observed and modeled solid-solution partitioning of $\mathrm{Cu}$ and $\mathrm{B}$ is rather constant among the soils, and the soluble concentration is consistently mainly controlled by the reactive concentration. Generally, the partition relations resulted in a smaller prediction error compared to the multi-surface models. The partition relations in which the soluble concentration was optimized, resulted in an average overestimation for the lowest observed concentrations, and an underestimation for highest concentrations of all three elements. Partition relations with optimized Freundlich parameters $K_{f}$ and $n$ resulted in more robust predictions since the prediction error was not related to the actual measured concentration. The partition relations from this study are easy-to-use tools for predicting the soluble concentrations of $\mathrm{Zn}, \mathrm{Cu}$ and $\mathrm{B}$ in soils from the tropics and can therefore enhance the usability of current existing soil information data for SSA.
\end{abstract}




\section{INTRODUCTION}

There is increasing awareness that micronutrients can play an important role in reducing crop yields and hampering yield responses to regular NPK-fertilizer in SubSaharan Africa (SSA) 1 . Among the micronutrients, especially zinc (Zn), but also copper $(\mathrm{Cu})$ and boron (B) are predominantly deficient in SSA soils ${ }^{2,3}$. Low soil levels of micronutrients such as $\mathrm{Zn}$ also lead to inadequate intake of these nutrients by humans via the consumption of crops, which can lead to severe health issues ${ }^{4-6}$.

Soils usually contain substantial total amounts of micronutrients. A large part that is present in the solid phase is however inert and not readily available for crop uptake, because it is occluded in the matrix of soil constituents such as (hydr)oxides and clay minerals 7,8 . This fraction is assumed to be released from the soil matrix only by very slow weathering processes. More relevant is the labile, reactive or potentially available pool that is distributed over the solid and solution phase, through sorption/desorption and precipitation/dissolution ${ }^{8}$. A fraction of this labile pool is present in the soil solution and is therefore directly available for plant uptake. Several chemical processes affect the speciation and the partitioning of the labile pool between the solid and solution phase. Ions can be in the solid phase when adsorbed to mineral and organic reactive surfaces (i.e. clay particles, organic matter, metal (hydr)oxides) and precipitated as minerals, or in the solution phase as free ion or in the form of inorganic and organic complexes. The solid-solution partitioning of $\mathrm{Zn}$ and $\mathrm{Cu}$ has been used to assess the risk for leaching ${ }^{9,10}$ or toxicity or deficiency towards crops ${ }^{11,12}$. In the context of plant nutrition, the difference in solid-solution partitioning has been used to explain why certain soil tests are more successful in predicting crop response to fertilization than others. For example, soil tests that approximate the soluble concentration have been found to be better in predicting the crop growth response to phosphate fertilization in tropical soils, while soil tests that approximate the labile pool work better for soils from temperate regions because phosphate adsorbs more strongly in tropical than in temperate soils ${ }^{13}$.

Different methods exist for assessing, understanding and predicting the solidsolution partitioning of elements in soils. One approach is geochemical multi-surface models that use thermodynamic constants for inorganic speciation together with an assembly of specialized models that describe ion adsorption to reactive surfaces in soils, such as clay, metal (hydr)oxides and organic matter ${ }^{14}$. Next to predicting the solid-solution partitioning of various elements, these models also provide insight in which soil chemical processes are most relevant in controlling solid-solution partitioning. These models have contributed to our understanding of the behavior of trace elements such as $\mathrm{Zn}$ and $\mathrm{Cu}$, often in polluted soils ${ }^{10,15-20}$ but have also been successfully applied to model the free $\mathrm{Zn}$ activity in a limited set of low $\mathrm{Zn}$ soils ${ }^{21}$. 
A generalized composite approach has been mainly used to model B adsorption behavior in soils, in which the soil is considered as a single plane for adsorption reactions ${ }^{22,23}$. This modelling approach does not take into account the specific characteristics (i.e. binding capacities, affinities, heterogeneity of surface sites) of each of the reactive surfaces, and does not give insights into the relative importance of the different reactive surfaces that control B speciation in soils. In a previous contribution, we applied successfully a multi-surface model for assessing the B speciation and extractability in a limited set of temperate and tropical soils ${ }^{24}$.

The application of geochemical multi-surface models on soil systems requires modelling parameters such as the thermodynamic constants and generic adsorption parameters. In addition, there are a number of decisions to be made regarding the input variables, which results in a certain conditionality of the multi-surface model. For example, the reactive content of the elements of interest is required as input. Examples for measuring the labile pool are the $0.43 \mathrm{M} \mathrm{HNO}_{3}$ soil extraction ${ }^{8}$ or the isotopically exchangeable fraction ${ }^{25}$. Secondly, the multi-surface model requires the fraction of soil organic matter (SOM), in the form of humic substances, that is reactive towards ion adsorption in the solid and solution phase ${ }^{17}$. In previous modelling studies, this fraction has either been estimated ${ }^{17}$, calculated based on the CEC and clay content ${ }^{21}$, fitted during the modelling calculations ${ }^{19}$ or measured by fractionation schemes $^{26}$. In general, for soils from the tropics, little is known about the reactivity of SOM for ion adsorption in comparison with soils from temperate regions. Previous fractionation studies suggest the existence of a lower fraction of humic and fulvic acids of SOM in tropical soils compared to soils from temperate regions ${ }^{27}$, while others have found opposite results ${ }^{28}$. Since it has been shown that soil organic matter is an important reactive surface for the adsorption of $\mathrm{Zn}, \mathrm{Cu}$ and $\mathrm{B}^{17,21,24}$, the fraction of reactive organic matter in the solid and solution phase will have great implications on the solubility and speciation of these micronutrients.

Another method for calculating the solid-solution partitioning of elements are partition relations in which the distribution of elements between the solid and solution phase is empirically related to soil properties. In this approach, the mechanistic soil chemical processes that are explicitly described in the multi-surface model, are lumped into empirical equations in the form of regression functions. A major advantage of such models is that they generally require less input data and less assumptions, making them suitable for large-scale applications and when limited data are available ${ }^{7,29}$. For SSA countries, partition relations have the potential to use micronutrient soil information based on currently available soil data ${ }^{30,31}$ for the formulation of site-specific micronutrient fertilizer recommendations. Previously, relations have been derived for the solid-solution partitioning of trace elements such as $\mathrm{Zn}$ and $\mathrm{Cu}$, often for contaminated soils from temperate regions ${ }^{17,32-36}$. These partition relations vary in their specific mathematical form, in the choice of 198 
independent and dependent variables and in their methodological specifications ${ }^{11,37}$. Relations for the solid-solution partitioning of $\mathrm{Zn}$ and $\mathrm{Cu}$ do not yet exist for soils from the tropics, with their frequently relatively low background levels. To the best of our knowledge, no partition relations exist for $\mathrm{B}$.

Based on the above, the aim of this study was to improve the understanding of soil chemical processes that control the solid-solution partitioning of $\mathrm{Zn}, \mathrm{Cu}$ and $\mathrm{B}$ in soils from tropical regions and thus are relevant in terms of nutrient availability. We therefore applied a geochemical multi-surface model to calculate the speciation of the above mentioned micronutrients. With this modelling approach, we also aimed to assess whether a generic multi-surface model works equally well for soils from the tropics for which it has not yet been used, compared to soils from temperate regions. Secondly, this knowledge was then used as benchmark to develop simplified partition relations to predict the soluble concentrations based on general soil properties in combination with an estimation of the labile pool.

\section{MATERIAL AND METHODS}

\subsection{Soil samples}

We used 172 soil samples originating from Burundi (74), Rwanda (50) and Kenya (48). The Burundi soil samples included 59 samples that originated from 29 locations in the south of the country, where samples were taken at $0-20 \mathrm{~cm}$ and $20-40 \mathrm{~cm}$ depth. The other 15 samples were topsoils $(0-20 \mathrm{~cm})$ originating from different regions in the country. The Rwanda (50) and Kenya soil samples (48) were from the topsoil and spatially distributed all over the countries, resulting in a large variety of soil types (Figure S1).

\subsection{Soil chemical analyses}

The solution concentration of $\mathrm{B}, \mathrm{Cu}, \mathrm{Zn}, \mathrm{Ca}, \mathrm{Mn}, \mathrm{Mg}, \mathrm{K}, \mathrm{Fe}, \mathrm{P}$ and $\mathrm{Al}$, together with the total and inorganic carbon and $\mathrm{pH}$ were measured in soil extracts with $0.01 \mathrm{M}$ $\mathrm{CaCl}_{2}{ }^{38}$. Calcium chloride extractions have often been used as a proxy for the soil solution, and to validate multi-surface models or calibrate partition relations that aim to model the solid-solution partitioning of elements in soils $16,17,33,39 . \mathrm{A} 0.01 \mathrm{M} \mathrm{CaCl}_{2}$ solution was freshly prepared in a plastic volumetric flask, in order to avoid element contamination from glassware. A fixed volume of the $\mathrm{CaCl}_{2}$ solution was added to the soil samples at a solution-to-solid ratio of $10 \mathrm{~L} \mathrm{~kg}^{-1}$ in polypropylene centrifuge tubes. The suspensions were equilibrated in a horizontal shaker at 180 oscillations minute $^{-1}$ for $2 \mathrm{~h}$ and were afterwards centrifuged for $10 \mathrm{~min}$ at $1800 \mathrm{~g}$ and filtered over a $0.45 \mu \mathrm{m}$ membrane filter. The concentrations of $\mathrm{Zn}, \mathrm{Cu}, \mathrm{B}, \mathrm{Fe}$, and $\mathrm{P}$ were measured in the acidified $\left(0.14 \mathrm{M} \mathrm{HNO}_{3}\right)$ subsample of the supernatant, using High 
Resolution Inductively Coupled Plasma Mass Spectrometry (HR-ICP-MS, Element 2, Thermo Scientific). The concentrations of $\mathrm{Al}, \mathrm{Ca}, \mathrm{Mg}, \mathrm{K}$ and $\mathrm{Mn}$ were measured using Inductively Coupled Plasma Optical Emission Spectroscopy (Thermo Scientific iCAP6500). Blank samples were included throughout the analyses and the average concentrations of the micronutrients of interest in the sixteen blank samples were: $0.20 \pm 0.14 \mu \mathrm{g} \mathrm{B} \mathrm{L}^{-1}, 0.21 \pm 0.04 \mu \mathrm{g} \mathrm{Cu} \mathrm{L}-1$ and $0.43 \pm 0.36 \mu \mathrm{Zn} \mathrm{L}^{-1}$. In addition, the $\mathrm{Zn}$, $\mathrm{Cu}$ and $\mathrm{B}$ concentrations of two reference samples were measured. The standard deviation among the eight measurements were $0.12 / 0.25 \mu \mathrm{g} \mathrm{L}^{-1}, 0.17 / 0.06 \mu \mathrm{g} \mathrm{Cu}$ $\mathrm{L}^{-1}$ and $0.80 / 1.06 \mu \mathrm{gn} \mathrm{L}^{-1}$ for the two reference samples respectively. Based on the concentrations measured in the blank samples, the limit of detection was set at the average blank concentration $+2 x$ the standard deviation ${ }^{40}$. Samples with measured concentrations below this value, were not included for the validation of the multisurface model and for the calibration of the partition relations.

The $\mathrm{pH}$ was measured in the $0.01 \mathrm{M} \mathrm{CaCl}_{2}$ using a glass electrode. The dissolved total carbon and dissolved inorganic carbon concentrations were measured in the supernatant with a Segmented Flow Analyzer (SFA-TOC, San++, Skalar) equipped with an IR detector that measures the amount of $\mathrm{CO}_{2}(\mathrm{~g})$ after an internal acidification and destruction step, and the dissolved organic carbon (DOC) concentrations were calculated as the difference between total and inorganic carbon.

The reactive or labile concentrations of $\mathrm{B}, \mathrm{Cu}$ and $\mathrm{Zn}$ were estimated by a $0.43 \mathrm{M}$ $\mathrm{HNO}_{3}$ extraction ${ }^{8}$. The samples were suspended in a freshly prepared extraction solution with $0.43 \mathrm{M} \mathrm{HNO}_{3}$ at a solution-to-solid ratio of $10 \mathrm{~L} \mathrm{~kg}^{-1}$. The suspensions were shaken for $4 \mathrm{~h}$, centrifugated and filtered over a $0.45 \mu \mathrm{m}$ membrane filter ${ }^{41}$. Afterwards, the filtrates were analyzed for $\mathrm{B}, \mathrm{Cu}$ and $\mathrm{Zn}$ using ICP-OES.

The clay content was measured by laser diffraction analysis ${ }^{42}$. Soils $(0.5-1.5 \mathrm{~g})$ were pre-treated three times with $\mathrm{H}_{2} \mathrm{O}_{2}$ and once with $\mathrm{HCl}$, while standing in a warm water bath. Before analysis, the pre-treated samples were suspended in water ( 200 ml) which was removed and replaced with fresh ultra-pure water for multiple times to remove excess of salts. The measured volume percentage of the fraction smaller than $2 \mu \mathrm{m}$ was re-calculated to the mass percentage of clay using a particle density of $2.6 \mathrm{~g} \mathrm{~cm}^{-3}$ and a bulk density of $1.3 \mathrm{~g} \mathrm{~cm}^{-3}$.

An ammonium oxalate $(\mathrm{AO})$ extraction ${ }^{43}$ was used to measure nano-crystalline $\mathrm{Fe}$ and $\mathrm{Al}$ (Fe-AO, Al-AO). Iron (Fe-D) and Al (Al-D) were also measured in a sodiumdithionite extraction ${ }^{44}$ and crystalline $\mathrm{Fe}$ and $\mathrm{Al}$ were calculated as the difference between the $\mathrm{Fe}$ and $\mathrm{Al}$ measured in the dithionite and $\mathrm{AO}$ extraction. The $\mathrm{Fe}$ and $\mathrm{Al}$ in the ammonium oxalate and in the dithionite extractions were analyzed using ICPOES. 
Total SOC content in the soils was analyzed using a wet oxidation method according to the Kurmies procedure and measured with a spectrophotometer ${ }^{45}$. For a selection of soil samples, the humic and fulvic acid fractions of the organic matter in the solid phase $(n=19)$ and solution phase $(n=12)$ were measured following the procedure as described by Van Zomeren and Comans ${ }^{46}$. For the analysis of the solid organic carbon, the soils were first suspended with $0.1 \mathrm{M} \mathrm{HCl}(\mathrm{pH} \mathrm{1})$ and centrifuged, after which the pellet was re-extracted with $0.1 \mathrm{M} \mathrm{NaOH}(\mathrm{pH} 12)$. This base solution was afterwards acidified to $\mathrm{pH} \mathrm{1,} \mathrm{followed} \mathrm{by} \mathrm{centrifugation.} \mathrm{Subsequently,} \mathrm{the}$ supernatant of the acid extraction and the supernatant of the acidified base extract were combined and equilibrated with pre-cleaned DAX-8 resin. The fulvic acids were afterwards desorbed from the resin with $0.1 \mathrm{M} \mathrm{KOH}$. The HA were measured by redissolving the pellet from the acidified base extract with $0.1 \mathrm{M} \mathrm{KOH}$. A similar procedure was followed for the fractionation of the dissolved organic carbon in the $0.01 \mathrm{M} \mathrm{CaCl}_{2}$ extraction.

\subsection{Multi-surface model}

The multi-surface model included ion adsorption to organic matter, electrostatic adsorption to the planar surfaces of clay minerals and ion adsorption to $\mathrm{Fe}$ and $\mathrm{Al}$ (hydr)oxides. The aqueous speciation and corresponding thermodynamic constants can be found in Table S1. Modeling calculations were performed in ECOSAT, version $4.9^{47}$. In order to calculate the solid-solution partitioning, the $\mathrm{B}, \mathrm{Cu}$ and $\mathrm{Zn}$ concentrations measured in $0.43 \mathrm{M} \mathrm{HNO}_{3}$ were used as input as the geochemically reactive concentration. To include competitive and synergistic adsorption effects from $\mathrm{Fe}^{3+}, \mathrm{Al}^{3+}, \mathrm{Ca}^{2+}, \mathrm{Cl}^{-}, \mathrm{K}^{+}, \mathrm{Mn}^{2+}, \mathrm{Mg}^{2+}$ and $\mathrm{PO}_{4}{ }^{3-}$, the measured concentrations of these elements in the $0.01 \mathrm{M} \mathrm{CaCl}_{2}$ extractions were used as input as total dissolved concentrations. Preliminary calculations showed that the inclusion of $\mathrm{Mg}, \mathrm{Mn}$ and $\mathrm{K}$ did not affect the calculated $\mathrm{Zn}, \mathrm{Cu}$ and B speciation.

In the ECOSAT speciation software, either the free ion concentration or activity in solution, or the total amount of an element can be used as the input. Therefore, the calculation was carried out in two steps. Firstly, free concentrations of the elements except $\mathrm{Cu}, \mathrm{Zn}$ and $\mathrm{B}$ were calculated based on the measured concentrations in the $\mathrm{CaCl}_{2}$ extractions considering solution speciation and the adsorption to DOC. Secondly, the calculated free ion concentrations were used as fixed input together with $\mathrm{pH}$, reactive $\mathrm{B}, \mathrm{Zn}$ and $\mathrm{Cu}$, and the reactive surfaces (including $\mathrm{DOC}$ ), to model the speciation of $\mathrm{Zn}, \mathrm{B}$ and $\mathrm{Cu}$. We corrected the total $\mathrm{P}$ measurements in the $\mathrm{CaCl}_{2}$ for organic species ${ }^{48}$ by taking $20 \%$ of total $\mathrm{P}$ as inorganic $\mathrm{PO}_{4}$. This was found to be the average \% of inorganic $\mathrm{PO}_{4}$, measured by a segmented flow analyzer applying a colorimetric molybdenum blue method ${ }^{49}$, relative to the total $\mathrm{P}$ measured in the $0.01 \mathrm{M} \mathrm{CaCl}_{2}$ by ICP-MS (results not shown). 
To test if mineral solubility controls the micronutrient concentration in the $\mathrm{CaCl}_{2}$ extraction, the free $\mathrm{Zn}, \mathrm{Cu}$ and $\mathrm{B}$ activity in solution was calculated using ECOSAT based on the measured concentrations in the $\mathrm{CaCl}_{2}$ extractions and compared with the activities that are expected based on the solubility of various $\mathrm{Zn}$ minerals ${ }^{21}, \mathrm{Cu}$ hydroxide ${ }^{50}$ and various $B$ minerals ${ }^{51}$. These calculations showed that the solubility of these minerals does not control the measured concentrations, except for the $\mathrm{Zn}$ concentration measured in the soil with the highest $\mathrm{pH}$ value in relation to $\mathrm{Zn}$ phyllosilicate (Figure S2).

\subsubsection{Adsorption to organic matter}

The adsorption to solid and dissolved organic matter was modelled with the Non Ideal Competitive Adsorption (NICA) model in combination with a Donnan model for electrostatic interactions ${ }^{52}$. Based on the measurements of humic substances in selected soil samples, the average percentage of $\mathrm{HA}$ and FA relative to the total SOC and DOC was calculated, and these percentages were used to estimate the $\mathrm{HA}$ and FA concentrations for all soil samples in the dissolved and particulate phase. The adsorption to SOM was modelled assuming the reactive SOM being HA and FA based on their estimated concentration, and the adsorption to DOM was modelled assuming the reactive DOM being FA.

For the neutral $\mathrm{B}(\mathrm{OH})_{3}{ }^{0}$ ion, which is the dominant $\mathrm{B}$ species in the $\mathrm{pH}$ range of these soil samples (Table S1), there is no electrostatic attraction. Therefore, we did not include the $\mathrm{B}(\mathrm{OH})_{3}{ }^{0}$ present in the Donnan layer for calculating the total adsorbed $\mathrm{B}$ to SOM and $D^{2} M^{24,53}$. Since there are only NICA parameters for B adsorption to HA, we considered the $B$ adsorption to $F A$ similar as the adsorption to $H A$ and used the same adsorption parameters for both $\mathrm{OM}$ fractions ${ }^{53}$.

The structural NICA-Donnan parameters for HA and FA were taken from Milne et al. ${ }^{54,55}$. Next to $\mathrm{Zn}, \mathrm{Cu}$ and $\mathrm{B}$, we included the specific adsorption of $\mathrm{Fe}, \mathrm{Al}$ and $\mathrm{Ca}$ in the NICA-Donnan model to account for possible competitive effects. We used for these ions the parameters from Milne et al. ${ }^{55}$, except for Fe complexation to $\mathrm{FA}^{56}$ and for $\mathrm{B}$ complexation to HA and FA53. The adsorption parameters for the NICADonnan model are given in Tables S2 and S3.

\subsubsection{Adsorption to clay.}

Cation adsorption to the planar surfaces of clay minerals due to the presence of a permanent negative charge was modelled with a Donnan ion exchange model. Illite was taken as a reference clay, with a constant Donnan volume of $1 \mathrm{~L} \mathrm{~kg}^{-1}$, and a surface charge of $0.25 \mathrm{eq} \mathrm{kg}^{-1} .{ }^{18,21}$ Using this approach, there will be no adsorption of $B$ to the clay surfaces because of the neutral charge of the boric acid. However, we have shown previously by modeling that adsorption to clay does not play an important role for $B$ speciation in soils ${ }^{24}$. Although illite may not be representative for 
the dominant clay minerals in the soils of this study, preliminary calculations showed that the choice of clay surface charge did not affect the modeling calculations because of the minor contribution of clay to the $\mathrm{Zn}$ and $\mathrm{Cu}$ adsorption in the solid phase.

\subsubsection{Adsorption to metal (hydr)oxides}

The Charge Distribution (CD) model ${ }^{57}$ was used, in combination with a multi-site ion adsorption complexation (MUSIC) model ${ }^{58}$ for calculating ion adsorption to the $\mathrm{Fe}$ and Al oxides.

The content of poorly crystalline oxides was calculated based on the $\mathrm{Al}$ and $\mathrm{Fe}$ measured in the $\mathrm{AO}$ extraction. To transform the moles of $\mathrm{Al}$ and $\mathrm{Fe}$ in the $\mathrm{AO}$ extraction to the oxide mass, a molar mass of $95 \mathrm{~g} \mathrm{~mol}^{-1} \mathrm{Fe}$ and $84 \mathrm{~g} \mathrm{~mol}^{-1} \mathrm{Al}$ was used, which corresponds to particles with a specific surface of $600 \mathrm{~m}^{2} \mathrm{~g}^{-1} .48,59$ The amount of crystalline oxides were calculated as the difference between the Fe and $\mathrm{Al}$ in the sodium-dithionite extraction and the $\mathrm{Fe}$ and $\mathrm{Al}$ measured in the $\mathrm{AO}$ extraction, using a molar mass of 89 and $78 \mathrm{~g} \mathrm{~mol}^{-1}$ for Fe (goethite) and Al (gibbsite) respectively ${ }^{60}$. In case no $\mathrm{Al}$ was measured in the dithionite extraction, we did not include crystalline $\mathrm{Al}$ (hydr)oxides in the modelling. For the crystalline oxides, a specific surface area of $100 \mathrm{~m}^{2} \mathrm{~g}^{-1}$ was assumed.

Ferrihydrite (Fh) was used as model oxide for the natural oxide fraction of the soils ${ }^{61}$, using the structural parameters from Hiemstra and Zhao ${ }^{58}$. The total Fh content was calculated by summing the mass of poorly crystalline and $1 / 6$ of the mass of crystalline oxides (assuming a specific surface area of $100 \mathrm{~m}^{2} \mathrm{~g}^{-1}$ ), and in the modelling calculations a specific surface area of $600 \mathrm{~m}^{2} \mathrm{~g}^{-1}$ was used for Fh. The CD-MUSIC parameters for modelling ion adsorption to $\mathrm{Fh}$ are given in Table S4.

Manganese (Mn) oxides have been suggested to be important for the adsorption of metal cations in soils ${ }^{62-64}$. For the subset of Kenyan soils, the Mn concentration was also measured in the $\mathrm{AO}$ extraction to test its importance for the calculation of $\mathrm{Zn}$ and $\mathrm{Cu}$ speciation. The total $\mathrm{Mn}$ oxide content was calculated based on this $\mathrm{Mn}$ concentration in the AO extraction, using a molar mass of $86.9 \mathrm{~g}_{\text {oxide }} \mathrm{mol}^{-1} \mathrm{Mn}^{65}$. The adsorption of $\mathrm{Zn}$ and $\mathrm{Cu}$ to the Mn oxides was modeled with the General Two Layer Model (GTLM) using a specific surface area of $746 \mathrm{~m}^{2} \mathrm{~g}^{-1}$ and the parameters from Tonkin et al. ${ }^{65}$ (Table S5).

\subsection{Partition relations}

Different forms of empirical models exist to describe the solid-solution partitioning of elements in soils. The most basic (linear adsorption) model uses a constant distribution coefficient:

$$
K_{d}=\frac{Q}{C}
$$


in which $K_{d}=$ the distribution constant $\left(\mathrm{L} \mathrm{kg}^{-1}\right), \mathrm{Q}=$ the concentration in the solid phase $\left(\mathrm{mol} \mathrm{kg}^{-1}\right)$ and $\mathrm{C}$ the concentration in the soil solution $\left(\mathrm{mol} \mathrm{L}^{-1}\right)$. Since values of $K_{d}$ can vary by different orders of magnitude among soil samples ${ }^{33}$, equation 1 is often extended with soil properties $\left(X_{i}\right)$ to account for this variation ${ }^{66}$ :

$$
\log K_{d}=\alpha_{0}+\sum a_{i} \log X_{i}
$$

In order to account for non-linear adsorption, Freundlich-type equations have been used as partition relations according to:

$$
Q=K_{f} C^{n}
$$

in which the exponential term $n$ shows the deviation of linear adsorption $(<1)$, and expresses the extent of decrease in bonding strength with increasing concentrations in solution.

Groenenberg et al. ${ }^{37}$ differentiated between three types of Freundlich-type partitioning relations (equation 3), depending on which variable is optimized during the derivation of the partition relation. Most commonly, the concentration in solution is optimized as follows ${ }^{67}$ :

$$
\log C=\alpha_{0}+\alpha_{1} \log Q+\sum \alpha_{i} \log X_{i}
$$

or the other way around, if $Q$ is optimized instead of $C^{68}$. Less common is the optimization of the Freundlich constants $K_{f}$ and $n$ from equation $3^{37,69}$ :

$$
\log K_{f}=\log Q-n \log C=\alpha_{0}+\sum \alpha_{i} \log X_{i}
$$

from which the concentration in solution can be calculated after optimization.

To assess the experimental variation in solid-solution partitioning of $\mathrm{Zn}, \mathrm{Cu}$ and $\mathrm{B}$, the $K_{d}$ values were calculated according to equation 1 , based on the concentrations measured in the $0.43 \mathrm{M} \mathrm{HNO}_{3}$ and the $\mathrm{CaCl}_{2}$ extracts. Next, the partition relations shown in equations 4 and 5 were derived. Input variables for the soil properties were SOM (\%) based on the total carbon measurements and assuming that SOM consists of $50 \%$ carbon, the clay content (\%), $\mathrm{FeAl}_{\mathrm{AO}}$ as the sum of $\mathrm{Fe}$ and $\mathrm{Al}$ measured in the AO extraction $\left(\mathrm{mmol} \mathrm{kg}^{-1}\right)$, the DOC measured in the $0.01 \mathrm{M} \mathrm{CaCl}_{2}$ extraction $\left(\mathrm{mg} \mathrm{L}^{-1}\right)$ and the $\mathrm{pH}$ measured in the $0.01 \mathrm{M} \mathrm{CaCl}_{2}$ extract.

The coefficients in equation 4 were derived by multiple linear regression analysis, using a stepwise regression approach in which all possible directions (i.e. forward, backward and both) were tested to select the variables in the regression model (MASS package in R, stepAIC function) based on the Akaike's Information Criterion (AIC) value ${ }^{70}$.

For equation 5 , the value of $n$ and the coefficients of the regression model were optimized simultaneously. In $\mathrm{R}$, the value of $\mathrm{n}$ was varied from 0.01 to 1 , in steps of 0.01 . For each $n$ and corresponding $K_{f}$ values, a regression model was calculated 
based on the soil properties. The final $n$ was selected for the minimal sum of squared differences in $K_{f}$. Afterwards, the regression model for the particular $n$ value was optimized in $\mathrm{R}$ with stepwise regression to select only the most important input variables for the final the equation.

The final regression equations were checked for multicollinearity between the independent variables, using the vif function in $\mathrm{R}$ (car package). For the final models, the variance inflation factor (vif) for a specific independent variable was found never to be larger than 5 . The contribution of the variables to the total variance explained by the model was calculated using the calc.relimp function in $\mathrm{R}$ (relaimpo package). The inclusion of interaction terms in the form of $\log X_{i}^{*} p H$ with $X_{i}$ one of the reactive surfaces (i.e. DOC, SOM, Clay, metal (hydr)oxides), did not improve the explained variance in the soluble concentrations or $K_{f}$ values by the regression models.

The R software (version 4.0.2) was used for derivation of the partition relations, the visualization of the results and the calculation of the correlation table ${ }^{71}$. The evaluation of the multi-surface model and the two different partition relations were done based on the mean error (ME: mean(predicted - measured)) and the root-

mean-squared error (RMSE: $\sqrt{\text { mean(predicted-measured })^{2}}$ ). The residuals of both model predictions were checked visually in relation with the independent and dependent variables.

\section{RESULTS AND DISCUSSION}

\subsection{Soil characteristics}

The concentrations of the micronutrients in the $\mathrm{CaCl}_{2}$ and the $\mathrm{HNO}_{3}$ extractions of the studied soils (Table 1) were relatively low, compared to previous measurements in temperate soils with natural background levels ${ }^{17}$. To illustrate, in the case for $\mathrm{Zn}$, around $40 \%$ of all samples had $\mathrm{Zn}$ concentrations in the $\mathrm{CaCl}_{2}$ extraction below 1 $\mu \mathrm{mol} \mathrm{kg}{ }^{-1}$, which is around the highest concentration for which Duffner et al. ${ }^{72}$ found a positive response of wheat biomass to $\mathrm{Zn}$ application. For $\mathrm{B}, 52 \%$ of all samples had measured $\mathrm{B}$ concentrations in the $\mathrm{CaCl}_{2}$ extraction below $7 \mu \mathrm{mol} \mathrm{kg}{ }^{-1}$. The latter

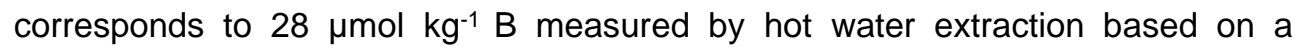
previously derived relationship ${ }^{73}$ and is assumed to be a critical value associated with $\mathrm{B}$ deficiency for plant production ${ }^{74}$.

The Kenyan soil samples have the largest variation in soil properties and micronutrient concentrations (Table 1), which coincides with the largest variation in soil types compared to Burundi and Rwanda (Figure S1).

As shown in Figures S3-S4, the fractions of reactive SOC and DOC, as defined by the content of humic and fulvic acids, were on average 32 and $21 \%$ of total SOC 
and DOC, respectively. These percentages were lower than the general assumptions that have been used before in multi-surface modelling studies ${ }^{14}$ or that have been measured previously in temperate soils ${ }^{75-77}$, especially for the DOC. These relatively low values will have implications for the micronutrient speciation calculated by the multi-surface modeling. As shown by Figure S5, the data show a tendency that the fraction of reactive $\mathrm{SOC}$ decreases with increasing $\mathrm{pH}$, and increases with metal (hydr)oxide content and total SOC. The latter is the opposite from what has been observed by Tipping et al. ${ }^{35}$ based on model fitting. However, these results were for specific upland soils with higher SOM levels. As shown by Figure S6, the fraction of reactive DOC also increases with total DOC, but increases with increasing $\mathrm{pH}$, and decreases with metal (hydr)oxide content. The relations between the reactive organic carbon fractions and $\mathrm{pH}$ and metal (hydr)oxides, suggests the importance of adsorption to metal (hydr)oxides for SOC stabilization ${ }^{78}$, resulting in a higher fraction of humic substances. Future studies with additional data are needed to confirm these trends and the underlying processes that explain the reactive organic matter fractions for ion adsorption in the particulate and solution phase.

Table 1: The median values and the range of soil properties, reactive concentrations $(Q)$ measured in the $0.43 \mathrm{M} \mathrm{HNO}_{3}$ extraction, and concentrations measured in the $0.01 \mathrm{M} \mathrm{CaCl}_{2}$ extractions (C) for the soil samples from Burundi $(n=74)$, Rwanda $(n=50)$ and Kenya $(n=48)$.

\begin{tabular}{|c|c|c|c|c|c|c|}
\hline & \multicolumn{2}{|c|}{ Burundi } & \multicolumn{2}{|c|}{ Rwanda } & \multicolumn{2}{|c|}{ Kenya } \\
\hline & Median & Range & Median & Range & Median & Range \\
\hline $\mathrm{pH}$ & 4.4 & $3.9-5.3$ & 4.9 & $3.9-6.2$ & 6.9 & $4.0-8.8$ \\
\hline $\mathrm{DOC}\left(\mathrm{mg} \mathrm{L}^{-1}\right)$ & 9.4 & $4.7-16.6$ & 13.9 & $7.2-33.9$ & 4.5 & $1.6-22.4$ \\
\hline $\operatorname{SOC}\left(\mathrm{g} \mathrm{kg}^{-1}\right)$ & 18 & $7-50$ & 16 & $9-50$ & 10 & $2-67$ \\
\hline Clay $\left(\mathbf{g ~ k g}^{-1}\right)$ & 94 & $23-708$ & 86 & $11-776$ & 290 & $1-813$ \\
\hline AO-Fe (mmol kg- & 43 & $11-156$ & 49 & $13-273$ & 20 & $2-183$ \\
\hline ÁO-Al $\left(\mathrm{mmol} \mathrm{kg}^{-1}\right)$ & 76 & $34-314$ & 50 & $14-459$ & 31 & $6-832$ \\
\hline $\mathrm{DC}-\mathrm{Fe}\left(\mathrm{mmol} \mathrm{kg}{ }^{-1}\right)$ & 536 & 261-995 & 459 & $124-919$ & 245 & $16-1273$ \\
\hline DC-Al (mmol kg-1) & 88 & $34-328$ & 50 & $14-459$ & 58 & $7-832$ \\
\hline$Q-Z n\left(\mu \mathrm{mol} \mathrm{kg}{ }^{-1}\right)$ & 15 & $3-171$ & 28 & $5-273$ & 37 & $8-1361$ \\
\hline $\mathrm{Q}-\mathrm{Cu}\left(\mu \mathrm{mol} \mathrm{kg}^{-1}\right)$ & 68 & $17-198$ & 51 & $10-346$ & 46 & $6-807$ \\
\hline Q-B $\left(\mu \mathrm{mol} \mathbf{k g}^{-1}\right)$ & 29 & $9-11$ & 22 & $2-159$ & 74 & $5-1748$ \\
\hline C-Zn $\left(\mu \mathrm{mol} \mathrm{kg}{ }^{-1}\right)$ & 1.99 & $<0.2-14.19$ & 1.53 & $0.26-13.65$ & 0.08 & $<0.2-25.05$ \\
\hline $\mathrm{C}-\mathrm{Cu}\left(\mu \mathrm{mol} \mathrm{kg}^{-1}\right)$ & 0.17 & $0.05-1.62$ & 0.13 & $0.08-0.43$ & 0.13 & $<0.04-1.08$ \\
\hline C-B $\left(\mu \mathrm{mol} \mathrm{kg}^{-1}\right)$ & 5.78 & $1.48-43.78$ & 5.45 & $<0.2-22.05$ & 13.26 & $<0.2-477.37$ \\
\hline
\end{tabular}




\subsection{Measured solid-solution partitioning}

The experimental solid-solution partitioning of $\mathrm{Zn}$, expressed as $K_{d}$ (equation 1) varied by a factor $\sim 1000$, and was mostly controlled by the soil pH (Figure 1). For $\mathrm{Cu}$, the $K_{d}$ values varied less among the soils and were mostly related to the relative soil organic carbon fractions, expressed as the ratio of SOC over DOC (Figure 1), showing that the interplay between these two organic matter pools controls the final Cu solubility. The variance in $K_{d}$ of $\mathrm{Cu}$ was generally less explained by soil properties than for $\mathrm{Zn}$. For B, most $K_{d}$ values were in the relatively narrow range between 10 and 100 (Figure S7). These $K_{d}$ values were low compared to $\mathrm{Zn}$ and $\mathrm{Cu}$, pointing towards a relatively high mobility of $B$ in soils ${ }^{74}$. In addition, there was no clear relation between the solid-solution partitioning of $B$ and any of the soil properties (Figure S7).
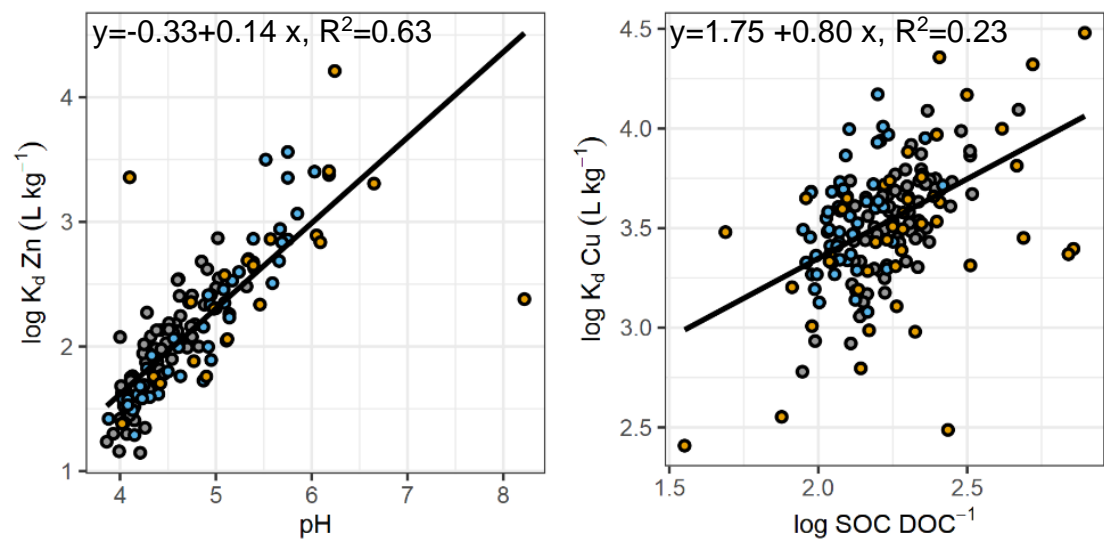

Country

- Burundi

- Kenya

- Rwanda

Figure 1: Relation between the $K_{d}$ value for $\mathrm{Zn}$ and $\mathrm{pH}$ (left) and for $\mathrm{Cu}$ and the ratio of soil organic carbon (SOC) over the dissolved organic carbon (DOC). The $K_{d}$ value was calculated based on the concentration measured in $0.43 \mathrm{M} \mathrm{HNO}_{3}$ minus the soluble concentration measured in $0.01 \mathrm{M} \mathrm{CaCl}_{2}\left(\mathrm{~mol} \mathrm{~kg}^{-1}\right)$ divided by the concentration measured in the $0.01 \mathrm{M} \mathrm{CaCl}_{2}\left(\mathrm{~mol} \mathrm{~L}^{-1}\right)$.

The correlation table in Figure S8 confirmed the observed relations between $K_{d}$ and soil properties. The strongest correlation for the $\mathrm{Zn}$ concentration in the $\mathrm{CaCl}_{2}$ extract was found with soil $\mathrm{pH}(-0.83, \mathrm{p}<0.001)$, while the concentrations of $\mathrm{B}$ and $\mathrm{Cu}$ were mostly correlated with the reactive content $(0.75$ and 0.49 respectively, $p<0.001)$.

\subsection{Multi-surface model calculations}

The accuracy of the geochemical multi-surface model predictions for the concentrations in a $0.01 \mathrm{M} \mathrm{CaCl}_{2}$ extract varied among $\mathrm{Zn}, \mathrm{Cu}$ and $\mathrm{B}$ (Figure 2). Based on the RMSE $E_{\log M}$, the model performed best for predicting the solubility of $\mathrm{Cu}$ (0.30), followed by $B(0.43)$ and $\mathrm{Zn}(0.47)$. The RMSE $E_{\log M}$ values of $\mathrm{Cu}$ and $\mathrm{Zn}$ for these tropical soils were in line with previous multi-surface modelling studies for temperate soils ${ }^{14}$, contaminated sites ${ }^{16,25}$, compost samples ${ }^{26}$ and waste materials ${ }^{79}$. This allows for the conclusion that these generic geochemical multi-surface models 
are performing equally well for soils from the tropics with lower levels of trace elements.

For $B$ we first assumed its concentration in the $0.43 \mathrm{M} \mathrm{HNO}_{3}$ extract represented the reactive $B$ fraction. This resulted in an overprediction of soluble $B$ for nearly all soils (Figure S9). We have previously evaluated the use of three different extraction methods to estimate reactive $B$ with a multi-surface model, for a limited set of tropical and temperate topsoils ${ }^{24}$. The results suggested that the $0.43 \mathrm{M} \mathrm{HNO}_{3}$ extraction may overestimate the reactive $B$ content, due to (partial) dissolution of $B$ containing minerals. We previously found that the $B$ measured in $0.05 \mathrm{M} \mathrm{KH}_{2} \mathrm{PO}_{4}$ was a better estimation for the reactive $B$ than $B$ measured in $0.43 \mathrm{M} \mathrm{HNO}_{3}$, based on modeling calculations. Based on the measurements of 10 soil samples, we found that the $\mathrm{KH}_{2} \mathrm{PO}_{4}$-extractable $\mathrm{B}$ was on average half of the concentration measured by the $0.43 \mathrm{M} \mathrm{HNO}_{3}$ extraction (Figure S10). So, in a next attempt to predict $\mathrm{B}$ solubility (i.e., the scenario shown in Figure 2), we used half of the $B$ concentration measured in the $0.43 \mathrm{M} \mathrm{HNO}_{3}$ soil extracts as input for the multi-surface model. This approach still overestimated $B$ solubility for most samples, resulting in a positive mean error of $0.27 \log M$ (Figure 2). Figure S11 shows that the modeling error was not related to any of the soil variables. A possible explanation may be that the ratio between $B$ measured in $0.43 \mathrm{M} \mathrm{HNO}_{3}$ and actual reactive $B$ varies among soil samples and may be larger in general, explaining the modeling deviation. 

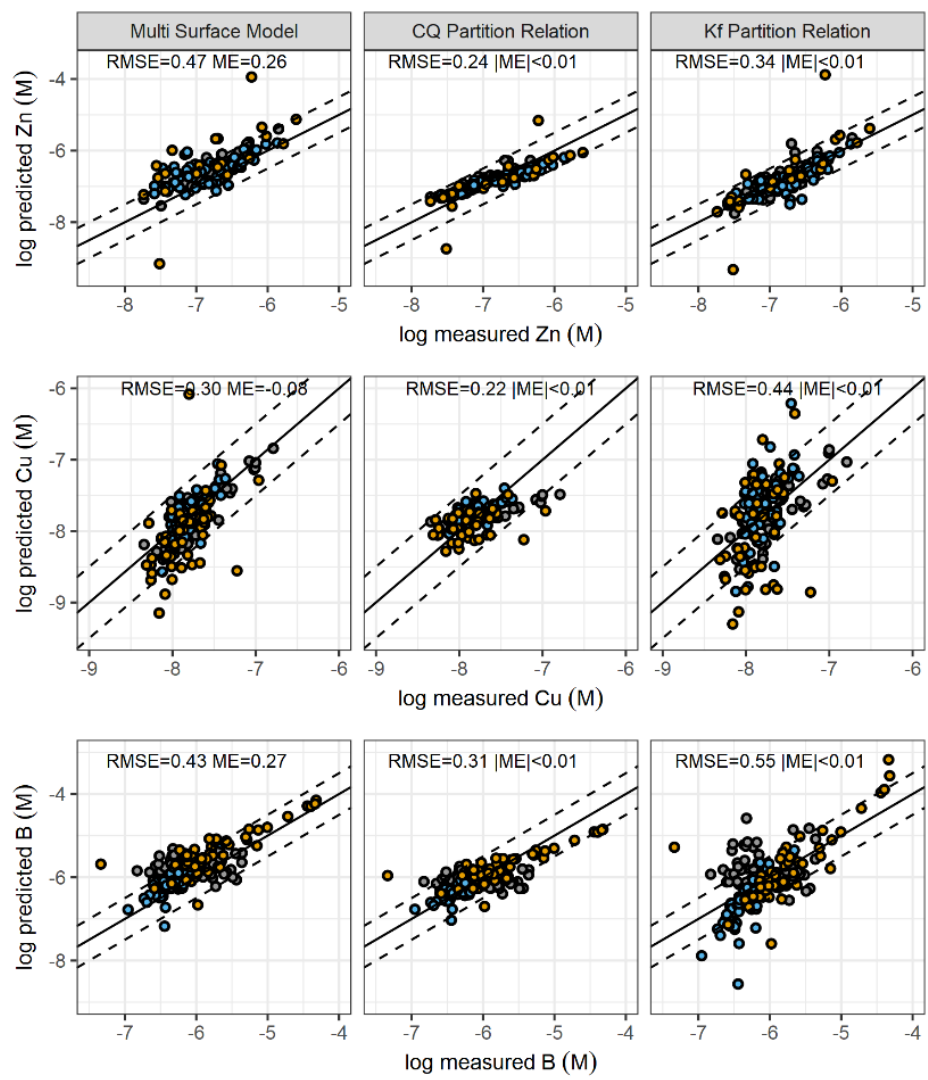

Country

- Burundi
- Kenya
- Rwanda

Figure 2:Comparison between measured and predicted concentration in a $0.01 \mathrm{M} \mathrm{CaCl}_{2}$ soil extraction, using multi-surface modelling (left column), C-Q partition relations (middle) or $K_{f}$ partition relations (right) for $\mathrm{Zn}$ (top row), $\mathrm{Cu}$ (middle) and B (lower). The numbers given in the graphs represent the root mean squared error (RMSE) and the mean error (ME) in log M. For the partition relations, the absolute value of the mean error $(|\mathrm{ME}|)$ was smaller than 0.01 . The solid line is the $1: 1$ line, points within the dashed lines are within $0.5 \log \mathrm{M}$ deviation from the measured concentrations.

For $\mathrm{Zn}$, the multi-surface model tended to overestimate $\mathrm{Zn}$ solubility with increasing $\mathrm{pH}$, with increasing reactive content and with increasing $\mathrm{Zn}$ loading, expressed as the reactive $\mathrm{Zn}$ concentration per mass of reactive SOM or metal (hydr)oxides (Figure S12). This resulted in an overall positive mean error of $0.26 \log M$ (Figure 2), suggesting an average overestimation of soluble $\mathrm{Zn}$ by the model. These relations between modelling deviations for $\mathrm{Zn}$ and soil variables, have been observed in previous multi-surface modeling studies that focused on contaminated and noncontaminated soils from temperate regions and composts $8,9,25,26$, so these deviations are not specific for soils from the tropics with low $\mathrm{Zn}$ concentration. 
The aforementioned studies have given various explanations for this systematic deviation in multi-surface modeling calculations for $\mathrm{Zn}$. For example, it has been shown that $\mathrm{HNO}_{3}$-extractable $\mathrm{Zn}$ overestimates the exchangeable pool in neutral to alkaline soils due to dissolution of minerals from which the $\mathrm{Zn}$ is not isotopically exchangeable $e^{8,80,81}$. Such an overestimation of reactive $\mathrm{Zn}$ as input may explain the over-prediction of $\mathrm{Zn}$ in solution in high $\mathrm{pH}$ soils by the multi-surface model as observed in this study, and in previous work ${ }^{8,82}$. Mao et al. ${ }^{25}$ attributed the increased overprediction of soluble $\mathrm{Zn}$ with increasing $\mathrm{pH}$ to the simplification of the wide variety in metal (hydr)oxides to one single model oxide in the multi-surface model, resulting in a weak description of the ion adsorption processes to the different metal (hydr)oxides. The use of the CD model in this study is expected to better represent ion adsorption processes to metal (hydr)oxides compared to the models used in previous studies $8,16,25$. Moreover, it has been shown recently that ferrihydrite nanoparticles are a good proxy for describing the metal (hydr)oxide surface reactivity, at least for phosphate, in temperate ${ }^{48}$ and tropical ${ }^{61}$ soils. In addition, by using the modeling parameters from Tonkin et al. ${ }^{65}$, our results do not suggest that the inclusion of other metal (hydr)oxides such as Mn oxides reduces this systematic modeling error (Figure S14 and S15). Related to the adsorption processes to the metal (hydr)oxides and other reactive surfaces, one may question the validity of the concept of linear additivity that is assumed in this study and in previous studies that modeled trace element speciation in soils $8,16,19,25$. The concept of linear additivity assumes that the different reactive surfaces in the multi-surface model do not interact in such a way that it affects ion adsorption processes ${ }^{14}$. For cations, such as $\mathrm{Cu}$ and $\mathrm{Ca}$, batch adsorption experiments have shown that the presence of organic matter compounds enhances the cation adsorption to metal (hydr)oxides such as goethite with increasing $\mathrm{pH}$, due to electrostatic effects and the formation of ternary complexes $^{83}$. The interaction between SOM and metal (hydr)oxides, which was not included in the multi-surface model, may lead to more $\mathrm{Zn}$ adsorption. Finally, others have questioned the validity of the NICA-donnan parameters, especially the electrostatic model for fulvic acids $26,56,84$ or the specific adsorption parameters for $\mathrm{Ca}^{85}$. Both aspects may have resulted in an overprediction of $\mathrm{Ca}$ competition on $\mathrm{Zn}$ adsorption, thereby leading to an overestimation of $\mathrm{Zn}$ solubility by the model. Future research should confirm which of the above mentioned limitations is most important for explaining the observed overprediction of soluble $\mathrm{Zn}$, which is according to our results a general modeling error irrespective of $\mathrm{Zn}$ levels or soil types.

For $\mathrm{Cu}$, the multi-surface model underestimated the soluble $\mathrm{Cu}$ concentrations especially in soils with low Cu loadings (Figure S13). Groenenberg et al. ${ }^{8}$ found similar results for a large set of Dutch soil samples and attributed this error to an incomplete recovery of $\mathrm{Cu}$ by the $0.43 \mathrm{M} \mathrm{HNO}_{3}$ extraction at low $\mathrm{Cu}$ loadings due to very strong binding. 

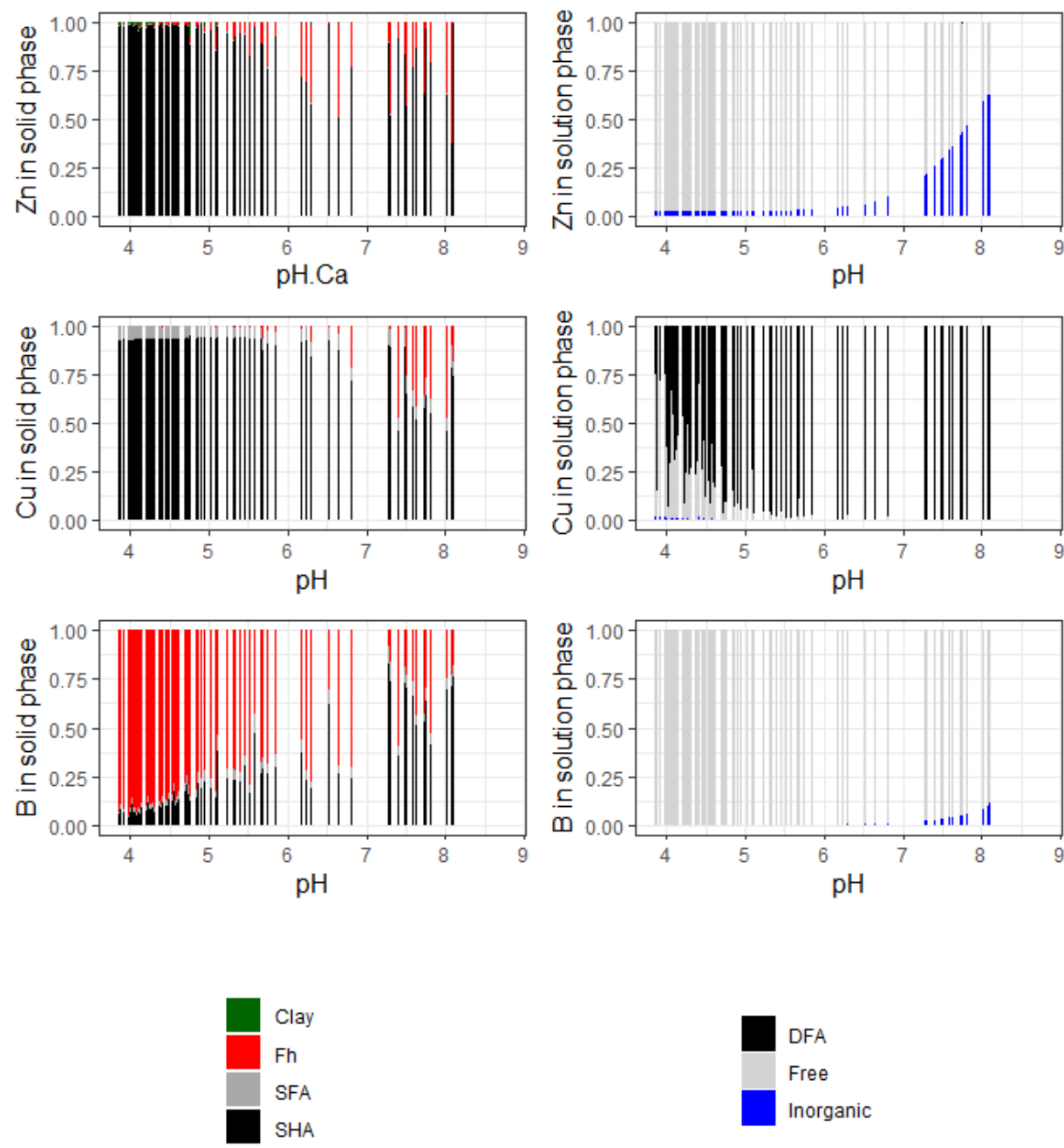

Figure 3: The chemical speciation of $\mathrm{Zn}, \mathrm{Cu}$ and $\mathrm{B}$ in the solid phase (left figures) and the solution phase (right figures) of all samples as it is predicted by the multi-surface model. The speciation is shown as a function of the soil pH measured in the $0.01 \mathrm{M} \mathrm{CaCl}_{2}$. In the solid phase, the ions are adsorbed by clay, ferrihydrite (Fh), solid humic acids (SHA) and solid fulvic acids (SFA). In the solution phase, the elements are present as free ion, inorganic complexes or adsorbed to dissolved fulvic acids (DFA).

The speciation of $\mathrm{Zn}, \mathrm{Cu}$ and $\mathrm{B}$ in the solid and solution phase according to the multisurface model is shown in Figure 3. In line with previous findings, soil organic matter was found to be the most important reactive surface for $\mathrm{Zn}$ and $\mathrm{Cu}$ adsorption in the solid phase $8,18,19$. However, with increasing $\mathrm{pH}$, the metal (hydr)oxides start to play an important role. For $\mathrm{Zn}$, this is already noticeable from $\mathrm{pH}$ 5.5-6, while for $\mathrm{Cu}$ above $\mathrm{pH}$ 7. Interestingly, for $\mathrm{B}$, the opposite trend was found as shown by Figure 2: the adsorption to metal (hydr)oxides dominates $\mathrm{B}$ adsorption in low pH soils, while soil 
organic matter becomes increasingly important with increasing $\mathrm{pH}$. In the solution phase, $\mathrm{Zn}$ and $\mathrm{B}$ are mainly present as the free $\mathrm{Zn}^{2+}$ ion, and as $\mathrm{B}(\mathrm{OH})_{3}$. Only for $\mathrm{Cu}, \mathrm{DOC}$ was found to play an important role for the solution speciation.

\subsection{Partition relations}

Two different types of partition relations were derived for calculating the solidsolution partitioning of the three micronutrients: $C-Q$ relations, in which the concentration in the $0.01 \mathrm{M} \mathrm{CaCl}_{2}$ extraction was optimized based on soil variables and the reactive content (Eq. 4) and $K_{f}$ relations in which the two Freundlich parameters and regression coefficients were optimized simultaneously (Eq. 5).

\subsubsection{C-Q partition relations}

The $C-Q$ partition relations based on the tropical soils from this study for the three different micronutrients are shown in Table 2. The soil pH explained most variation $(42 \%)$ in the $\mathrm{Zn}$ concentrations measured in the $\mathrm{CaCl}_{2}$ extraction, with a negative coefficient. This is consistent with the previously observed relation between $K_{d}$ and the concentration in the $\mathrm{CaCl}_{2}$ extraction with $\mathrm{pH}$ (Figure 1). For $\mathrm{B}$ and $\mathrm{Cu}$, for which we found a less clear relation between $K_{d}$ and soil properties, the reactive content appears to be most important variable to predict soluble $\mathrm{B}$ and $\mathrm{Cu}$ : respectively more than 51 and $23 \%$ of the variance in the dissolved concentration was due to the variation in the reactive content (Table 2).

Table 2: The C-Q partition relations as shown in equation 4 , derived based on $n$ soil samples for predicting the concentrations of $\mathrm{Zn}, \mathrm{Cu}$ and $\mathrm{B}$ in the $0.01 \mathrm{M} \mathrm{CaCl}_{2}(\log C$, in $\mathrm{M})$. The final input variables were selected based on stepwise regression: the concentration measured in the $0.43 \mathrm{M} \mathrm{HNO}_{3}$ of the specific element $(\mathrm{Q})$, the $\mathrm{pH}\left(\mathrm{CaCl}_{2}\right)$, the soil organic matter (SOM), the clay content, the sum of $\mathrm{Fe}$ and $\mathrm{Al}$ measured in the ammonium oxalate extraction (AO-FeAl) and the dissolved organic carbon concentration in the $0.01 \mathrm{M}$ $\mathrm{CaCl}_{2}$ extraction (DOC). For each soil variable, the coefficient in the model is given with the standard error between brackets, followed by the \% of variation that is explained by the model and attributed to the particular variable. The coefficient of determination $\left(R^{2}\right)$ is given to show the percentage of variation in $\log C$ that is explained by the model.

\begin{tabular}{|c|c|c|c|c|c|c|c|c|c|}
\hline & $\alpha_{0}$ & $\mathrm{pH}$ & $\begin{array}{l}\log Q \\
\mathrm{~mol}^{-1}\end{array}$ & $\begin{array}{c}\log S O M \\
\%\end{array}$ & $\begin{array}{c}\log F e A I \\
\mathrm{mmol} \\
\mathrm{kg}^{-1}\end{array}$ & $\begin{array}{c}\log \text { Clay } \\
\%\end{array}$ & $\begin{array}{c}\log D O C \\
\mathrm{mg} \mathrm{L}^{-1}\end{array}$ & $\mathbf{R}^{2}$ & $n$ \\
\hline $\log C(Z n)$ & $\begin{array}{c}0.86 \\
( \pm 0.53)\end{array}$ & $\begin{array}{c}-0.65 \\
( \pm 0.04 \\
42 \%)\end{array}$ & $\begin{array}{c}0.79 \\
( \pm 0.06 \\
22 \%)\end{array}$ & $\begin{array}{c}-0.37 \\
( \pm 0.17 \\
2 \%)\end{array}$ & $\begin{array}{c}-0.35 \\
( \pm 0.12 \\
2 \%)\end{array}$ & - & - & 0.68 & 143 \\
\hline $\log C(C u)$ & $\begin{array}{c}-5.79 \\
( \pm 0.26)\end{array}$ & $\begin{array}{c}-0.04 \\
( \pm 0.02 \\
3 \%)\end{array}$ & $\begin{array}{c}0.46 \\
( \pm 0.06 \\
23 \%)\end{array}$ & $\begin{array}{c}-0.52 \\
( \pm 0.11 \\
3 \%)\end{array}$ & - & - & $\begin{array}{c}0.40 \\
( \pm 0.10 \\
4 \%)\end{array}$ & 0.33 & 171 \\
\hline $\log C(B)$ & $\begin{array}{c}-2.82 \\
( \pm 0.23)\end{array}$ & - & $\begin{array}{c}0.72 \\
( \pm 0.05 \\
51 \%)\end{array}$ & $\begin{array}{c}-0.19 \\
( \pm 0.10 \\
6 \%)\end{array}$ & - & - & - & 0.57 & 170 \\
\hline
\end{tabular}

The partition relations, with their most important input variables and coefficients, were in agreement with the importance of the various reactive surfaces from the multi-surface modelling calculations (Figure 3 ). For $\mathrm{Zn}$ and $\mathrm{Cu}$, the multi-surface 212 
modelling calculations have shown that soil organic matter is the most important reactive surface for adsorption in the solid phase. In line with these results, SOM appears as a significant variable in both regression equations with a negative coefficient (Table 1). The multi-surface modelling has also shown that the metal (hydr)oxides are important for $\mathrm{Zn}$ adsorption at high $\mathrm{pH}$, which explains the presence of the oxides with a negative coefficient in the partition relation for $\mathrm{Zn}$.

Consistent with the prediction by the multi-surface model that $\mathrm{Cu}$ in the $\mathrm{CaCl}_{2}$ solution is mostly present as a complex with DOC (Figure 3), DOC concentration was found to be a significant variable with a positive coefficient explaining dissolved $\mathrm{Cu}$ in the partition relations (Table 2).

For $B$, the partition relation showed that the solubility of $B$ is mainly governed by the reactive content (Table 2). Initially, a significant contribution of DOC was found for $B$ solubility in these tropical soils, with a negative coefficient (results not shown). However, our multi-surface modeling calculations did not show an important role of DOC for B solubility (Figure 3). The negative contribution of DOC on B solubility cannot be explained by soil chemical processes. The DOC concentrations were found to be strongly related to the SOC content (Figure S8). As such, DOC may be a surrogate for $S O C$ in the partition relation. When the partition relation was recalibrated without DOC as possible input variable, SOM was selected as a significant variable next to the reactive concentration by stepwise regression (Table 2), and the negative coefficient for SOM is in line with the multi-surface modeling predictions that showed that SOM is an important reactive surface for B adsorption. The final model had the same $\mathrm{R}^{2}$ and prediction error as the model with $\mathrm{DOC}$.

The explained variance in Cu solubility by the partition relation (31\%) was relatively low (Table 2), probably because of a too small variation in the experimental solidsolution partitioning. For B (57\%) and $\mathrm{Zn}(68 \%)$, the explained variation was higher. However, the prediction error of the partition relations in the soluble concentrations was similar for all three elements, as illustrated by the RMSE (Figure 1), and was lower than the prediction error for the multi-surface models. Based on this observation, we can conclude that the partition relations are accurate prediction models of the soluble $\mathrm{Zn}, \mathrm{Cu}$ and $\mathrm{B}$ concentrations measured in a $0.01 \mathrm{M} \mathrm{CaCl}_{2}$ extraction for these soils from the tropics.

The prediction error of the $\mathrm{C}-\mathrm{Q}$ partition relations for all three micronutrients was not related to any input variable (Figures S11-S13), but it was related to the dependent variable namely the concentration measured in the $\mathrm{CaCl}_{2}$ extraction: The dissolved concentrations are overestimated in the lower range, and underestimated in the higher range by the $\mathrm{C}-\mathrm{Q}$ partition relation (Figure 1). As discussed in the next section, 
this systematic prediction error can be reduced when using $K_{f}$ instead of $\mathrm{C}-\mathrm{Q}$ partition relations.

\subsection{2 $K_{F}$ partition relations}

For the derivation of $K_{f}$ partition relations, the solid-solution partitioning itself was optimized instead of the concentration in solution ${ }^{37}$, according to equation 5 . The resulting equations are shown in Table 3 . For $\mathrm{Zn}$, this approach resulted in a partition relation that is similar to the $C-Q$ relation, only that now $D O C$ was retained in the final model. However, the contribution of DOC to the total variance explained by the model was only $1 \%$. Again, $\mathrm{pH}$ was the most important variable, similar as in the $\mathrm{C}$ $\mathrm{Q}$ partition relation, explaining $63 \%$ of the variance in $K_{f}$. Compared to the $\mathrm{C}-\mathrm{Q}$ partition relation, the total variance explained by the model is larger for the $K_{f}$ relation (79\%; Table 3), but the error in the Zn concentration is also slightly larger (see RMSE values in Figure 2). More importantly, the systematic underestimation of $\mathrm{Zn}$ in solution at highest concentrations and overestimation at lowest concentrations, as observed in the $\mathrm{C}-\mathrm{Q}$ relation, disappeared when using a $K_{f}$ partition relation (Figure 2).

Table 3: The $K_{f}$ partition relations as shown in equation 5, derived for predicting the Freundlich parameters for $\mathrm{Zn}, \mathrm{Cu}$ and $\mathrm{B}$ concentrations in the $0.01 \mathrm{M} \mathrm{CaCl}_{2}$ extract $(\log C$, in $\mathrm{M})$. The input variables were selected based on stepwise regression, using as possible input variables the concentration measured in the 0.43 $\mathrm{M} \mathrm{HNO}_{3}$ of the specific element $(\mathrm{Q})$, the $\mathrm{pH}\left(\mathrm{CaCl}_{2}\right)$, the soil organic matter (SOM), the clay content, the sum of $\mathrm{Fe}$ and $\mathrm{Al}$ measured in the ammonium oxalate extraction (AO-FeAl) and the dissolved organic carbon concentration in the $0.01 \mathrm{M} \mathrm{CaCl}_{2}$ extraction (DOC). For each soil variable, the coefficient in the model is given with the standard error between brackets, followed by the \% of variation that is attributed to the particular variable. The coefficient of determination $\left(R^{2}\right)$ is given to show the percentage of variation in $\log K_{f}$ that is explained by the model.

\begin{tabular}{|c|c|c|c|c|c|c|c|c|c|}
\hline & $\mathbf{n}$ & $\alpha_{0}$ & $\mathrm{pH}$ & $\begin{array}{c}\log S O M \\
\%\end{array}$ & $\begin{array}{c}\log F e A I \\
\underset{\mathrm{mmol}^{-1}}{\mathrm{~kg}^{-1}}\end{array}$ & $\begin{array}{c}\log C l a y \\
\%\end{array}$ & $\begin{array}{c}\log D O C \\
\mathrm{mg} \mathrm{L}^{-1}\end{array}$ & $\mathrm{R}^{2}$ & $n$ \\
\hline $\log K_{f}(Z n)$ & 0.66 & $\begin{array}{l}-3.82 \\
(0.28)\end{array}$ & $\begin{array}{l}0.61 \\
(0.03 \\
63 \%) \\
\end{array}$ & $\begin{array}{c}0.94 \\
(0.17 \\
8 \%) \\
\end{array}$ & $\begin{array}{c}0.29 \\
(0.11 \\
4 \%) \\
\end{array}$ & - & $\begin{array}{c}-0.31 \\
(0.11, \\
1 \%) \\
\end{array}$ & 0.79 & 143 \\
\hline $\log K_{f}(\mathrm{Cu})$ & 0.57 & $\begin{array}{l}-0.10 \\
(0.17)\end{array}$ & - & $\begin{array}{l}0.56 \\
(0.15, \\
11 \%)\end{array}$ & $\begin{array}{c}0.27 \\
(0.09 \\
12 \%)\end{array}$ & - & $\begin{array}{c}-0.57 \\
(0.11, \\
7 \%)\end{array}$ & 0.30 & 171 \\
\hline $\log K_{f}(\mathrm{~B})$ & & $\begin{array}{l}-2.72 \\
(0.14)\end{array}$ & $\begin{array}{l}0.20 \\
(0.02, \\
36 \%)\end{array}$ & $\begin{array}{c}0.26 \\
(0.10, \\
4 \%)\end{array}$ & - & - & - & 0.40 & 170 \\
\hline
\end{tabular}


For $\mathrm{Cu}$ and $\mathrm{B}$, a $K_{f}$ partition relation resulted in a lower variance explained by the model, and a higher prediction error in the soluble concentration compared to the C$\mathrm{Q}$ relations. The $K_{f}$ partition relation confirmed the previous result that the concentrations of $\mathrm{Cu}$ and $\mathrm{B}$ in the $\mathrm{CaCl}_{2}$ extractions were mainly determined by the reactive content (Table 2), and that the experimental $K_{d}$ values of both nutrients did not clearly relate to soil properties (Figure 1 and S7). As a result, optimization of the solid-solution partitioning in the form of $K_{f}$ relations did not give good results for B and $\mathrm{Cu}$.

\section{CONCLUSIONS}

Micronutrient availability depends on the soil nutrient status, and in particular on the labile and soluble concentrations. To improve the understanding of the processes that control the solid-solution partitioning of $\mathrm{Zn}, \mathrm{Cu}$ and $\mathrm{B}$ in tropical soils, a multisurface model was used to calculate the micronutrient speciation in a series of 172 soils samples from Burundi, Rwanda and Kenya. Next, partition relations were developed for predicting the soluble $\mathrm{Zn}, \mathrm{Cu}$ and $\mathrm{B}$ concentrations based on general soil properties.

Most of the labile $\mathrm{Cu}$ is present in the solid phase. Interestingly, we found that the solid-solution partitioning of $\mathrm{Cu}$ was rather constant among all 173 soil samples studied, and it was only partly explained by the soil organic matter in the solid and solution phase. The importance of soil organic matter (SOM) for Cu solid-solution partitioning was confirmed by the multi-surface model calculations, which showed that SOM was the dominant adsorbent for $\mathrm{Cu}$, except for soils with $\mathrm{pH}$ larger than 7 in which the metal (hydr)oxides also start to play an important role. The weak relation between the rather constant $\mathrm{Cu}$ solid-solution partitioning and soil variables, resulted in partition relations in which the variation in soluble $\mathrm{Cu}$ was mainly explained by the reactive concentration.

The same was found for $\mathrm{B}$. However, in contrast to $\mathrm{Cu}$, these results for $\mathrm{B}$ can be explained by the weak interaction of $B$ with the solid phase, which was shown by the relatively low solid-solution partitioning ratios and the multi-surface modeling calculations. Although small, the interaction of $B$ with the solid phase is dominated by adsorption to metal (hydr)oxides in low pH soils, and to soil organic matter in soils with $\mathrm{pH}$ above $\sim 6$.

The solid-solution partitioning of $\mathrm{Zn}$ in the studied soils varied the most, and this variation was mainly explained by soil $\mathrm{pH}$. The speciation of $\mathrm{Zn}$ in the solid phase was dominated by soil organic matter in low pH soils, but above pH 5.5-6, the metal (hydr)oxides started to play an important role. Due to the strong relation between the solid-solution partitioning of $\mathrm{Zn}$ and soil variables such as $\mathrm{pH}$, the partition relations resulted in a higher explained variance of soluble $\mathrm{Zn}$ compared to $\mathrm{Cu}$ and $\mathrm{B}$. 
Both the soluble and labile micronutrient pool are important in terms of nutrient availability. For B, the interaction of the solid phase is limited, resulting in a large fraction of the labile pool being present in the solution. For $\mathrm{Zn}$, the same was found for acidic soils with $\mathrm{pH}$ around 4 . This implies that the buffering capacity of $\mathrm{B}$ and $\mathrm{Zn}$ in these soils is low, while the soluble concentration may be relatively high due to limited adsorption. As a consequence, the nutrient buffering capacity may limit nutrient uptake, and in these cases soil tests that approximate the labile pool such as the $0.43 \mathrm{M} \mathrm{HNO}_{3}$ extraction, may relate better to the above-ground $\mathrm{Zn}$ uptake. For $\mathrm{B}$, differentiation between the labile and soluble pool by soil tests may be less relevant due to the strong relation between both $B$ concentrations, that was found by the $\mathrm{C}-\mathrm{Q}$ partition relations.

Two types of partition relations have been derived. We have shown that partition relations in which the concentration in solution is optimized, may result in a systematic modelling deviation in relation to the measured concentration. This can be improved for $\mathrm{Zn}$ when models are calibrated in which the solid-solution partitioning itself is optimized. The partition relations from this study are easy-to-use tools for predicting the soluble concentrations of $\mathrm{Zn}, \mathrm{Cu}$ and $\mathrm{B}$ in soils from the tropics with low contents of these micronutrients. These models can greatly enhance the usability of current existing soil information data for SSA and may thereby expand current soil information with data on micronutrient availability, thereby facilitating future decision support tools for micronutrient fertilization.

\section{ACKNOWLEDGEMENTS}

This work was supported by NWO (grant number 14688, "Micronutrients for better yields"). We want to thank the International Fertilizer Development Center for the soil samples from Burundi (PAPAB project) and from Rwanda (Catalist-2 project). We thank Wageningen Environmental Research for the soil samples from Burundi that have been collected in the framework of the 'Building on Fertile Grounds' project. We also want to thank AgroCares Research for the 48 Kenyan soil samples. All experiments and analyses were done in the CBLB laboratory at Wageningen University and Research and we greatly appreciate the help from the CBLB staff with the soil analyses. 


\section{SUPPORTING INFORMATION}

\section{A. Locations of the soil samples used in this study}
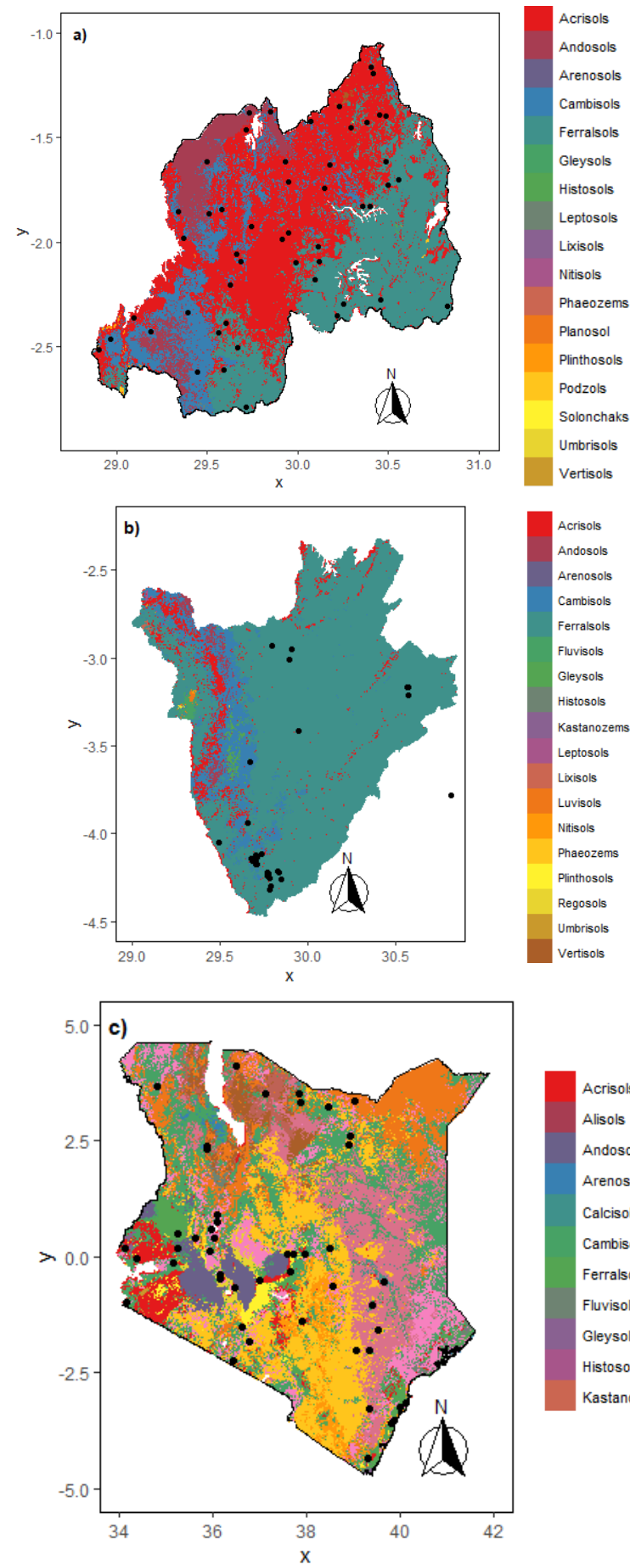

Figure S1: The locations of the soil samples used in this study, shown on the soil classifications map of a) Rwanda ( $n=50)$, b) Burundi ( $n=73)$ and c) Kenya $(n=48)$. The soil classification maps are based on the World Reference Base soil classification, taken from SoilGrids.org ${ }^{86}$.

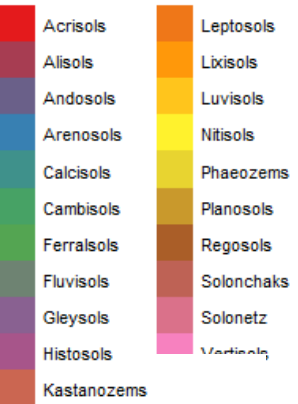




\section{B. Solution speciation}

Table S1: The aqueous species, the formation reactions and the corresponding $\log K$ values that were used in the multi-surface modelling ${ }^{50}$.

\begin{tabular}{|c|c|c|}
\hline Aqueous species & Reaction & $\log K$ \\
\hline $\mathrm{B}(\mathrm{OH})_{4}^{-}$ & $\mathrm{B}(\mathrm{OH})_{3}+\mathrm{OH}^{-} \leftrightarrow \mathrm{B}(\mathrm{OH})_{4}^{-}$ & $4.76^{\mathrm{a}}$ \\
\hline $\mathrm{CaB}(\mathrm{OH})_{4}^{+}$ & $\mathrm{B}(\mathrm{OH})_{3}+\mathrm{Ca}^{2+}+\mathrm{OH}^{-} \leftrightarrow \mathrm{CaB}(\mathrm{OH})_{4}^{+}$ & $6.59^{a}$ \\
\hline $\mathrm{CaPO}_{4}$ & $\mathrm{PO}_{4}^{3-}+\mathrm{Ca}^{2+} \leftrightarrow \mathrm{CaPO}_{4}^{-}$ & $6.46^{b}$ \\
\hline $\mathrm{CaHPO}_{4}$ & $\mathrm{PO}_{4}^{3-}+\mathrm{H}^{+}+\mathrm{Ca}^{2+} \leftrightarrow \mathrm{CaHPO}_{4}^{-}$ & $15.035^{b}$ \\
\hline $\mathrm{CaH}_{2} \mathrm{PO}_{4}^{+}$ & $\mathrm{PO}_{4}{ }^{3-}+2 \mathrm{H}^{+}+\mathrm{Ca}^{2+} \leftrightarrow \mathrm{CaH}_{2} \mathrm{PO}_{4}^{-}$ & $20.9230^{b}$ \\
\hline $\mathrm{HPO}_{4}{ }^{-2}$ & $\mathrm{PO}_{4}^{3-}+\mathrm{H}^{+} \leftrightarrow \mathrm{HPO}_{4}^{2-}$ & $12.375^{\mathrm{b}}$ \\
\hline $\mathrm{H}_{2} \mathrm{PO}_{4}^{-}$ & $\mathrm{PO}_{4}{ }^{3-}+2 \mathrm{H}^{+} \leftrightarrow \mathrm{H}_{2} \mathrm{PO}_{4}^{2-}$ & $19.573^{b}$ \\
\hline $\mathrm{H}_{3} \mathrm{PO}_{4}$ & $\mathrm{PO}_{4}^{3-}+3 \mathrm{H}^{+} \leftrightarrow \mathrm{H}_{3} \mathrm{PO}_{4}$ & $21.721^{b}$ \\
\hline $\mathrm{CaOH}$ & $\mathrm{Ca}^{2+}+\mathrm{OH}^{-} \leftrightarrow \mathrm{CaOH}^{+}$ & 1.3 \\
\hline $\mathrm{Ca}(\mathrm{OH})_{2}{ }^{+}$ & $\mathrm{Ca}^{2+}+2 \mathrm{OH}^{-} \leftrightarrow \mathrm{Ca}(\mathrm{OH})_{2}$ & 0.01 \\
\hline $\mathrm{CaCl}$ & $\mathrm{Ca}^{2+}+\mathrm{Cl}^{-} \leftrightarrow \mathrm{CaCl}^{+}$ & -1 \\
\hline $\mathrm{CaCl}_{2}$ & $\mathrm{Ca}^{2+}+2 \mathrm{Cl}^{-} \leftrightarrow \mathrm{CaCl}_{2}$ & 0 \\
\hline $\mathrm{Fe}(\mathrm{OH})_{2}$ & $\mathrm{Fe}^{3+}+2 \mathrm{OH}^{-} \leftrightarrow \mathrm{Fe}(\mathrm{OH})_{2}^{+}$ & 22.31 \\
\hline $\mathrm{Fe}(\mathrm{OH})_{3}$ & $\mathrm{Fe}^{3+}+3 \mathrm{OH}^{-} \leftrightarrow \mathrm{Fe}(\mathrm{OH})_{3}$ & 28.91 \\
\hline $\mathrm{Fe}(\mathrm{OH})_{4}$ & $\mathrm{Fe}^{3+}+4 \mathrm{OH}^{-} \leftrightarrow \mathrm{Fe}(\mathrm{OH})^{-}$ & 34.41 \\
\hline $\mathrm{Fe}_{2}(\mathrm{OH})_{2}$ & $2 \mathrm{Fe}^{3+}+2 \mathrm{OH}^{-} \leftrightarrow \mathrm{Fe}_{2}(\mathrm{OH})_{2}^{4+}$ & 25.1 \\
\hline $\mathrm{FeCl}$ & $\mathrm{Fe}^{3+}+\mathrm{Cl}^{-} \leftrightarrow \mathrm{FeCl}^{2+}$ & 1.48 \\
\hline $\mathrm{FeCl}_{2}$ & $\mathrm{Fe}^{3+}+2 \mathrm{Cl}^{-} \leftrightarrow \mathrm{FeCl}^{+}$ & 2.13 \\
\hline $\mathrm{FeCl}_{3}$ & $\mathrm{Fe}^{3+}+3 \mathrm{Cl}^{-} \leftrightarrow \mathrm{FeCl}_{3}$ & 0.77 \\
\hline $\mathrm{FeH}_{2} \mathrm{PO}_{4}$ & $\mathrm{PO}_{4}^{3-}+\mathrm{Fe}^{3+}+2 \mathrm{H}^{+} \leftrightarrow \mathrm{FeH}_{2} \mathrm{PO}_{4}^{2+}$ & 24.98 \\
\hline $\mathrm{FeHPO}_{4}$ & $\mathrm{PO}_{4}^{3-}+\mathrm{Fe}^{3+}+\mathrm{H}^{+} \leftrightarrow \mathrm{FeHPO}_{4}^{+}$ & 23.26 \\
\hline $\mathrm{Fe}(\mathrm{OH})_{4}$ & $\mathrm{Fe}^{3+}+\mathrm{OH}^{-} \leftrightarrow \mathrm{Fe}(\mathrm{OH})_{2}^{2+}$ & 49.7 \\
\hline $\mathrm{Al}(\mathrm{OH})_{2}$ & $\mathrm{Al}^{3+}+2 \mathrm{OH}^{-} \leftrightarrow \mathrm{Al}(\mathrm{OH})_{2}^{+}$ & 18.7 \\
\hline $\mathrm{Al}(\mathrm{OH})_{3}$ & $\mathrm{Al}^{3+}+3 \mathrm{OH}^{-} \leftrightarrow \mathrm{Fe}(\mathrm{OH})_{3}$ & 27.01 \\
\hline $\mathrm{Al}(\mathrm{OH})_{4}$ & $\mathrm{Al}^{3+}+4 \mathrm{OH}^{-} \leftrightarrow \mathrm{Fe}(\mathrm{OH})^{-}$ & 32.67 \\
\hline $\mathrm{Al}(\mathrm{OH})_{5}$ & $\mathrm{Al}^{3+}+5 \mathrm{OH}^{-} \leftrightarrow \mathrm{Al}(\mathrm{OH})^{2-}$ & 35.76 \\
\hline $\mathrm{Al}_{2}(\mathrm{OH})_{2}$ & $2 \mathrm{Al}^{3+}+2 \mathrm{OH}^{-} \leftrightarrow \mathrm{Al}_{2}(\mathrm{OH})_{2}^{4+}$ & 20.31 \\
\hline $\mathrm{AlOH}$ & $\mathrm{Al}^{3+}+\mathrm{OH}^{-} \leftrightarrow \mathrm{Fe}(\mathrm{OH})_{2}^{2+}$ & 8.98 \\
\hline $\mathrm{Cu}(\mathrm{OH})_{2}$ & $\mathrm{Cu}^{2+}+2 \mathrm{OH}^{-} \leftrightarrow \mathrm{Cu}(\mathrm{OH})_{2}$ & 11.8 \\
\hline $\mathrm{Cu}(\mathrm{OH})_{3}$ & $\mathrm{Cu}^{2+}+3 \mathrm{OH}^{-} \leftrightarrow \mathrm{Cu}(\mathrm{OH})_{2}^{-}$ & 15.25 \\
\hline $\mathrm{Cu}(\mathrm{OH})_{4}$ & $\mathrm{Cu}^{2+}+4 \mathrm{OH}^{-} \leftrightarrow \mathrm{Cu}(\mathrm{OH})_{2}^{2-}$ & 16.41 \\
\hline $\mathrm{Cu}_{2}(\mathrm{OH})_{2}$ & $2 \mathrm{Cu}^{2+}+2 \mathrm{OH}^{-} \leftrightarrow \mathrm{Cu}_{2}(\mathrm{OH})_{2}{ }^{2+}$ & 17.32 \\
\hline $\mathrm{CuCl}$ & $\mathrm{Cu}^{2+}+\mathrm{Cl}^{-} \leftrightarrow \mathrm{CuCl}^{+}$ & 0.4 \\
\hline $\mathrm{CuCl}_{2}$ & $\mathrm{Cu}^{2+}+2 \mathrm{Cl}^{-} \leftrightarrow \mathrm{CuCl}$ & -0.12 \\
\hline $\mathrm{CuCl}_{3}$ & $\mathrm{Cu}^{2+}+3 \mathrm{Cl}^{-} \leftrightarrow \mathrm{CuCl}^{-}$ & -1.57 \\
\hline $\mathrm{CuH}_{2} \mathrm{PO}_{4}$ & $\mathrm{PO}_{4}^{3-}+\mathrm{Cu}^{2+}+2 \mathrm{H}^{+} \leftrightarrow \mathrm{CuH}_{2} \mathrm{PO}_{4}^{+}$ & 21.14 \\
\hline $\mathrm{CuHPO}_{4}$ & $\mathrm{PO}_{4}^{3-}+\mathrm{Cu}^{2+}+\mathrm{H}^{+} \leftrightarrow \mathrm{CuH}_{2} \mathrm{PO}_{4}$ & 15.55 \\
\hline $\mathrm{CuOH}$ & $\left.\mathrm{Cu}^{2+}+\mathrm{OH}^{-} \leftrightarrow \mathrm{CuOH}\right)^{+}$ & 6.3 \\
\hline $\mathrm{Zn}(\mathrm{OH})_{2}$ & $\mathrm{Zn}^{2+}+2 \mathrm{OH}^{-} \leftrightarrow \mathrm{Zn}(\mathrm{OH})_{2}$ & 11.2 \\
\hline $\mathrm{Zn}(\mathrm{OH})_{3}$ & $\mathrm{Zn}^{2+}+3 \mathrm{OH}^{-} \leftrightarrow \mathrm{Zn}(\mathrm{OH})_{2}^{-}$ & 14.32 \\
\hline $\mathrm{Zn}(\mathrm{OH})_{4}$ & $\mathrm{Zn}^{2+}+4 \mathrm{OH}^{-} \leftrightarrow \mathrm{Zn}(\mathrm{OH})_{2}^{2-}$ & 17.71 \\
\hline $\mathrm{ZnCl}$ & $\mathrm{Zn}^{2+}+\mathrm{Cl}^{-} \leftrightarrow \mathrm{ZnCl}^{+}$ & 0.43 \\
\hline $\mathrm{ZnCl}_{2}$ & $\mathrm{Zn}^{2+}+2 \mathrm{Cl}^{-} \leftrightarrow \mathrm{ZnCl}_{2}$ & 0 \\
\hline $\mathrm{ZnCl}_{3}$ & $\mathrm{Zn}^{2+}+3 \mathrm{Cl}^{-} \leftrightarrow \mathrm{ZnCl}_{3}^{-}$ & 0.5 \\
\hline $\mathrm{ZnCl}_{4}$ & $\mathrm{Zn}^{2+}+4 \mathrm{Cl}^{-} \leftrightarrow \mathrm{ZnCl}_{4}^{2-}$ & 0.2 \\
\hline $\mathrm{ZnHPO}_{4}$ & $\mathrm{PO}_{4}^{3-}+\mathrm{Zn}^{2+}+2 \mathrm{H}^{+} \leftrightarrow \mathrm{ZnH}_{2} \mathrm{PO}_{4}^{+}$ & 21.15 \\
\hline $\mathrm{ZnHPO}_{4}$ & $\mathrm{PO}_{4}^{3-}+\mathrm{Zn}^{2+}+\mathrm{H}^{+} \leftrightarrow \mathrm{ZnH}_{2} \mathrm{PO}_{4}$ & 15.65 \\
\hline $\mathrm{ZnOH}$ & $\mathrm{Zn}^{2+}+\mathrm{OH}^{-} \leftrightarrow \mathrm{ZnOH}$ & 6.31 \\
\hline
\end{tabular}




\section{Solubility of minerals}

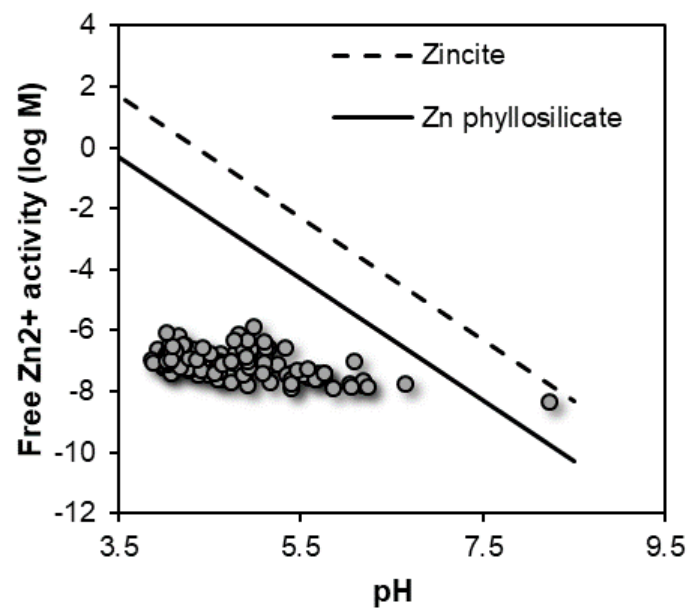

Figure S2: The free ion activity of $\mathrm{Zn}$ calculated based on the measured concentrations in the $0.01 \mathrm{M}$ $\mathrm{CaCl}_{2}$, the aqueous speciation as shown in Table S1 and the possibility of adsorption to the dissolved fulvic acids. The lines represent the $\mathrm{pH}$-dependent solubility of $\mathrm{Zn}$ minerals ${ }^{21}$, showing that the solubility of $\mathrm{Zn}$ minerals may control the $\mathrm{Zn}$ activity for the soil with the highest $\mathrm{pH}$.. 


\section{Parameters used in the multi-surface model}

Table S2: Structural and adsorption parameters of the NICA-DONNAN model for ion adsorption on fulvic acids, which was used as the reactive fraction of dissolved organic matter for ion adsorption in the multisurface modelling. All parameters originate from Milne et al. ${ }^{54,55}$, except the ones for $\mathrm{B}^{53}$ and $\mathrm{Fe}^{56}$ adsorption.

\begin{tabular}{|c|c|c|}
\hline $\begin{array}{l}\text { Site density } \\
\text { Heterogeneity } \\
\text { parameter }(p) \\
\text { Donnan volume } \\
\end{array}$ & \multicolumn{2}{|c|}{$\begin{array}{l}\mathrm{S} 1=5.88 \mathrm{~mol} \mathrm{~kg}^{-1} \\
\mathrm{~S} 2=1.86 \mathrm{~mol} \mathrm{~kg}^{-1} \\
\mathrm{p}_{1}=0.59 \\
\mathrm{p}_{2}=0.70 \\
\text { Log } \mathrm{V}_{\mathrm{D}}=\mathrm{b}^{*} \log \mathrm{I}-\mathrm{b}-1 \text { with } \mathrm{b}=-0.57\end{array}$} \\
\hline Adsorption reactions & $\log K$ & $\boldsymbol{n}_{i}$ \\
\hline $\mathrm{S} 1-\mathrm{H}$ & 2.34 & 0.66 \\
\hline $\mathrm{S} 2-\mathrm{H}$ & 8.60 & 0.76 \\
\hline $\mathrm{S} 1-\mathrm{Ca}$ & -2.13 & 0.85 \\
\hline $\mathrm{S} 2-\mathrm{Ca}$ & -3.0 & 0.80 \\
\hline $\mathrm{S} 1-\mathrm{B}(\mathrm{OH})_{3}$ & -0.08 & 0.91 \\
\hline $\mathrm{S} 2-\mathrm{B}(\mathrm{OH})_{3}$ & 1.90 & 0.61 \\
\hline$S 1-Z n$ & -3.84 & 0.67 \\
\hline $\mathrm{S} 2-\mathrm{Zn}$ & -0.73 & 0.61 \\
\hline $\mathrm{S} 1-\mathrm{Cu}$ & 0.26 & 0.53 \\
\hline $\mathrm{S} 2-\mathrm{Cu}$ & 8.26 & 0.36 \\
\hline $\mathrm{S} 1-\mathrm{Fe}$ & 2.70 & 0.36 \\
\hline $\mathrm{S} 2-\mathrm{Fe}$ & 8.30 & 0.23 \\
\hline S1-Al & -4.11 & 0.42 \\
\hline S2-Al & 12.16 & 0.31 \\
\hline
\end{tabular}

Table S3: Structural and adsorption parameters of the NICA-DONNAN model for ion adsorption on humic acids, which was used as the reactive fraction of solid organic matter for ion adsorption in the multi-surface modelling. All parameters originate from Milne et al. ${ }^{54,55}$, except the ones for $\mathrm{B}^{53}$ adsorption.

\begin{tabular}{|c|c|c|}
\hline $\begin{array}{l}\text { Site density } \\
\text { Heterogeneity } \\
\text { parameter ( } p) \\
\text { Donnan volume }\end{array}$ & $\begin{array}{l}\mathrm{S} 1=3.15 \mathrm{~mol} \mathrm{~kg}^{-1} \\
\mathrm{~S} 2=2.55 \mathrm{~mol} \mathrm{~kg}^{-1} \\
\mathrm{p}_{1}=0.62 \\
\mathrm{p}_{2}=0.41 \\
\text { Log } \mathrm{V}_{\mathrm{D}}=\mathrm{b}^{*} \log \mathrm{I}-\mathrm{b}-1 \text { with } \mathrm{b}=-0.49\end{array}$ & \\
\hline Adsorption reactions & $\log K$ & $n_{i}$ \\
\hline $\mathrm{S} 1-\mathrm{H}$ & 2.93 & 0.81 \\
\hline S2-H & 8 & 0.63 \\
\hline $\mathrm{S} 1-\mathrm{Ca}$ & -1.37 & 0.78 \\
\hline $\mathrm{S} 2-\mathrm{Ca}$ & -0.43 & 0.75 \\
\hline $\mathrm{S} 1-\mathrm{B}(\mathrm{OH})_{3}$ & -0.08 & 0.91 \\
\hline $\mathrm{S} 2-\mathrm{B}(\mathrm{OH})_{3}$ & 1.90 & 0.61 \\
\hline$S 1-Z n$ & 0.11 & 0.67 \\
\hline $\mathrm{S} 2-\mathrm{Zn}$ & 2.39 & 0.27 \\
\hline $\mathrm{S} 1-\mathrm{Cu}$ & 2.23 & 0.56 \\
\hline $\mathrm{S} 2-\mathrm{Cu}$ & 6.85 & 0.34 \\
\hline $\mathrm{S} 1-\mathrm{Fe}$ & 3.50 & 0.30 \\
\hline $\mathrm{S} 2-\mathrm{Fe}$ & 17.50 & 0.25 \\
\hline S1-Al & -1.05 & 0.40 \\
\hline S2-Al & 8.89 & 0.30 \\
\hline
\end{tabular}


Table S4: Formation of surface species and corresponding adsorption parameters for the CD model for ferrihydrite, which was taken as proxy for the total natural oxide content. The $\mathrm{CD}$ model was used in combination with an extended Stern layer with capacitance values of $C_{1}=1.14$ and $C_{2}=0.92 \mathrm{~F} \mathrm{~m}^{-2}$. Formation reactions are with triply coordinated groups $(\mathrm{T}) \mathrm{Fe}_{3} \mathrm{O}\left(1.4 \mathrm{~nm}^{-2}\right)$ or singly coordinated groups $(\mathrm{S}) \mathrm{FeOH}$ that can form only monodentate surface species $(\mathrm{a})\left(3 \mathrm{~nm}^{-2}\right)$, or that can form mono-and bidentate corner-sharing surface species with low ((bl), $\left.2.3 \mathrm{~nm}^{-2}\right)$ and high affinity for $\mathrm{Ca}, \mathrm{Zn}$ and $\mathrm{H}_{3} \mathrm{BO}_{3}\left((\mathrm{bh}), 0.25 \mathrm{~nm}^{-2}\right)^{39,58,87,88}$.

\begin{tabular}{|c|c|c|c|c|c|c|}
\hline Species & Surface group & Adsorption reaction & $\Delta \mathbf{z}_{0}$ & $\Delta \mathbf{z}_{1}$ & $\Delta \mathbf{z}_{2}$ & $\log K$ \\
\hline$\equiv \mathrm{Fe}_{3} \mathrm{O}-\mathrm{H}$ & $\mathrm{T}$ & $\equiv \mathrm{Fe}_{3} \mathrm{O}^{-0.5}+\mathrm{H}^{+} \leftrightarrow \equiv \mathrm{Fe}_{3} \mathrm{OH}^{+0.5}$ & 1 & 0 & 0 & $8.10^{\mathrm{a}}$ \\
\hline$\equiv \mathrm{FeOH}_{\mathrm{a}}$ & $\mathrm{S}(\mathrm{a}, \mathrm{bl}, \mathrm{bh})$ & $\equiv \mathrm{FeOH}^{-0.5}+\mathrm{H}^{+} \leftrightarrow \equiv \mathrm{FeOH}_{2}{ }^{+0.5}$ & 1 & 0 & 0 & $8.10^{\mathrm{a}}$ \\
\hline$\equiv \mathrm{Fe}_{3} \mathrm{OH}-\mathrm{Cl}$ & $\mathrm{T}$ & $\equiv \mathrm{Fe}_{3} \mathrm{O}^{-0.5}+\mathrm{H}^{+}+\mathrm{Cl}^{-} \leftrightarrow \equiv \mathrm{Fe}_{3} \mathrm{OH}^{+0.5}-\mathrm{Cl}^{-}$ & 1 & -1 & 0 & $7.65^{b}$ \\
\hline$\equiv \mathrm{FeOH}_{2}-\mathrm{Cl}$ & $\mathrm{S}(\mathrm{a}, \mathrm{bl}, \mathrm{bh})$ & $\equiv \mathrm{FeOH}^{0.5}+\mathrm{H}^{+}+\mathrm{Cl}^{-} \leftrightarrow \equiv \mathrm{FeOH}_{2}{ }^{+0.5}-\mathrm{Cl}^{-}$ & 1 & -1 & 0 & $7.65^{b}$ \\
\hline$\equiv \mathrm{Fe}_{3} \mathrm{O}-\mathrm{K}$ & $\mathrm{T}$ & $\equiv \mathrm{Fe}_{3} \mathrm{O}^{-0.5}+\mathrm{K}^{+} \leftrightarrow \mathrm{Fe}_{3} \mathrm{O}^{-0.5}-\mathrm{K}^{+}$ & 0 & 1 & 0 & $-1.61^{b}$ \\
\hline$\equiv \mathrm{FeOH}-\mathrm{K}$ & $\mathrm{S}(\mathrm{a}, \mathrm{bl}, \mathrm{bh})$ & $\equiv \mathrm{FeOH}^{-0.5}+\mathrm{K}^{+} \leftrightarrow \equiv \mathrm{FeOH}^{-0.5}-\mathrm{K}^{+}$ & 0 & 1 & 0 & $-1.61^{\mathrm{b}}$ \\
\hline EFeOPOOH & $\mathrm{S}(\mathrm{a}, \mathrm{bl}, \mathrm{bh})$ & $\equiv \mathrm{FeOH}^{-0.5}+\mathrm{PO}_{4}+2 \mathrm{H}^{+} \leftrightarrow \mathrm{FFeO}^{-0.22} \mathrm{POOH}^{-1.28}+\mathrm{H}_{2} \mathrm{O}$ & 0.28 & -1.28 & 0 & $26.36^{\mathrm{a}}$ \\
\hline$\equiv \mathrm{FeOPO}(\mathrm{OH})_{2}$ & $\mathrm{~S}(\mathrm{a}, \mathrm{bl}, \mathrm{bh})$ & $\equiv \mathrm{FeOH}^{-0.5}+\mathrm{PO}_{4}+3 \mathrm{H}^{+} \leftrightarrow \equiv \mathrm{FeO}^{-0.17} \mathrm{PO}(\mathrm{OH})_{2}^{-0.33}+\mathrm{H}_{2} \mathrm{O}$ & 0.33 & -0.33 & 0 & $29.84^{\mathrm{a}}$ \\
\hline$\equiv(\mathrm{FeO})_{2} \mathrm{PO}_{2}$ & $\mathrm{~S}(\mathrm{bl}, \mathrm{bh})$ & $2 \equiv \mathrm{FeOH}^{-0.5}+\mathrm{PO}_{4}+2 \mathrm{H}^{+} \leftrightarrow(\equiv \mathrm{FeO})_{2}{ }^{-0.54} \mathrm{PO}_{2}^{-1.46}+2 \mathrm{H}_{2} \mathrm{O}$ & 0.46 & -1.46 & 0 & $28.31 \mathrm{a}$ \\
\hline$\equiv \mathrm{FeO})_{2} \mathrm{POOH}$ & $\mathrm{S}(\mathrm{bl}, \mathrm{bh})$ & $2 \equiv \mathrm{FeOH}^{-0.5}+\mathrm{PO}_{4}+3 \mathrm{H}^{+} \leftrightarrow(\equiv \mathrm{FeO})_{2}^{-0.35} \mathrm{PO}_{2}^{-0.65}+2 \mathrm{H}_{2} \mathrm{O}$ & 0.65 & -0.65 & 0 & $33.52^{\mathrm{a}}$ \\
\hline$\equiv(\mathrm{FeOH})_{2} \mathrm{Ca}$ & $\mathrm{S}(\mathrm{bl})$ & $2=\mathrm{FeOH}^{-0.5}+\mathrm{Ca}^{2+} \leftrightarrow(=\mathrm{FeOH})_{2}^{-0.06} \mathrm{Ca}^{1.06}$ & 0.94 & 1.06 & 0 & $2.64^{c}$ \\
\hline$\equiv\left(\mathrm{FeO}_{\mathrm{b}} \mathrm{H}\right)_{2}-\mathrm{Ca}$ & $S(b h)$ & $2 \equiv \mathrm{FeOH}^{-0.5}+\mathrm{Ca}^{2+} \leftrightarrow(\equiv \mathrm{FeOH})_{2}^{-0.06} \mathrm{Ca}^{1.06}$ & 0.94 & 1.06 & 0 & $5.13^{\mathrm{c}}$ \\
\hline =FeOPO${ }_{3} \mathrm{Ca}$ & $\mathrm{S}(\mathrm{a}, \mathrm{bl}, \mathrm{bh})$ & $\equiv \mathrm{FeOH}^{-0.5}+\mathrm{Ca}^{2+}+\mathrm{PO}_{4}{ }^{3-}+\mathrm{H}^{+} \leftrightarrow \mathrm{F} \mathrm{Fe}^{-0.26} \mathrm{PO}_{3}{ }^{-1.30} \mathrm{Ca}^{-06}+\mathrm{H}_{2} \mathrm{O}$ & 0.24 & -1.30 & 1.06 & $22.27^{d}$ \\
\hline$\equiv(\mathrm{FeOH})_{2} \mathrm{PO}_{2} \mathrm{Ca}$ & $\mathrm{S}(\mathrm{bl}, \mathrm{bh})$ & $2 \equiv \mathrm{FeOH}^{-0.5}+\mathrm{Ca}^{2+}+\mathrm{PO}_{4}^{3-}+2 \mathrm{H}^{+} \leftrightarrow(\equiv \mathrm{FeO})_{2}^{-0.38} \mathrm{PO}_{3}^{-1.08} \mathrm{Ca}^{1.46}+2 \mathrm{H}_{2} \mathrm{O}$ & 0.62 & -1.08 & 1.46 & $30.09^{d}$ \\
\hline$\equiv(\mathrm{FeO})_{2} \mathrm{~B}(\mathrm{OH})$ & $\mathrm{S}(\mathrm{bh})$ & $2 \equiv \mathrm{FeOH}^{-0.5}+\mathrm{B}(\mathrm{OH})_{3}{ }^{0} \leftrightarrow(\equiv \mathrm{FeO})_{2}{ }^{-0.82} \mathrm{~B}(\mathrm{OH})^{-0.18}+2 \mathrm{H}_{2} \mathrm{O}$ & 0.18 & -0.18 & 0 & $3.39^{\mathrm{e}}$ \\
\hline$\equiv(\mathrm{FeO})_{2} \mathrm{~B}(\mathrm{OH})_{2}$ & $S(b h)$ & $2=\mathrm{FeOH}^{-0.5}+\mathrm{B}(\mathrm{OH})_{3}{ }^{0} \leftrightarrow(\equiv \mathrm{FeO})_{2}{ }^{-0.82} \mathrm{~B}(\mathrm{OH})_{2}{ }^{-0.18}+\mathrm{H}_{2} \mathrm{O}+\mathrm{H}^{+}$ & -0.25 & -0.75 & 0 & $-4.68^{e}$ \\
\hline$\equiv \mathrm{Fe}_{3} \mathrm{OH}-\mathrm{B}(\mathrm{OH})_{3}$ & $\mathrm{~T}$ & $=\mathrm{Fe}_{3} \mathrm{O}^{-0.5}+\mathrm{B}(\mathrm{OH})_{3}{ }^{0}+\mathrm{H}^{+} \leftrightarrow \equiv \mathrm{FeO}_{3} \mathrm{H}^{+0.5}-\mathrm{B}(\mathrm{OH})_{3}{ }^{0}$ & 1 & 0 & 0 & $9.32^{\mathrm{e}}$ \\
\hline$\equiv \mathrm{FeOH}_{2}-\mathrm{B}(\mathrm{OH})_{3}$ & $\mathrm{~S}(\mathrm{a}, \mathrm{bl}, \mathrm{bh})$ & $\equiv \mathrm{FeOH}^{-0.5}+\mathrm{B}(\mathrm{OH})_{3}{ }^{0}+\mathrm{H}^{+} \leftrightarrow \mathrm{FeOH}_{2}{ }^{+0.5}-\mathrm{B}(\mathrm{OH})_{3}{ }^{0}$ & 1 & 0 & 0 & $9.32^{\mathrm{e}}$ \\
\hline$\equiv(\mathrm{FeOH})_{2} \mathrm{Zn}$ & $\mathrm{S}(\mathrm{bh})$ & $2 \equiv \mathrm{FeOH}^{-0.5}+\mathrm{Zn}^{2+} \leftrightarrow(=\mathrm{FeOH})_{2}{ }^{0.17} \mathrm{Zn}^{0.83}$ & 1.17 & 0.83 & 0 & $7.97^{f}$ \\
\hline$\equiv(\mathrm{FeOH})_{2} \mathrm{ZnOH}$ & $S(b h)$ & $2 \equiv \mathrm{FeOH}^{-0.5}+\mathrm{Zn}^{2+} \leftrightarrow(\equiv \mathrm{FeOH})_{2}^{1.06} \mathrm{ZnOH}^{-0.06}+\mathrm{H}^{+}$ & 1.06 & -0.06 & 0 & $0.69^{f}$ \\
\hline$\equiv(\mathrm{FeOH}) \mathrm{ZnOH}$ & $S(a, b l)$ & $\equiv \mathrm{FeOH}^{-0.5}+\mathrm{Zn}^{2+} \leftrightarrow(\equiv \mathrm{FeOH})^{0.57} \mathrm{ZnOH}^{0.43}+\mathrm{H}^{+}$ & 0.57 & 0.43 & 0 & $-2.40^{\dagger}$ \\
\hline$\equiv(\mathrm{FeOH}) \mathrm{Zn}(\mathrm{OH})_{2} \mathrm{Zn}_{2}(\mathrm{OH})_{2}$ & $S(b l)$ & $=\mathrm{FeOH}^{-0.5}+2 \mathrm{Zn}^{2+} \leftrightarrow \equiv \mathrm{FeOH}^{0.52} \mathrm{Zn}(\mathrm{OH})_{2}^{-0.52} \mathrm{Zn}(\mathrm{OH})_{2}+4 \mathrm{H}^{+}$ & 0.52 & -0.52 & 0 & $-21.02^{f}$ \\
\hline$\equiv(\mathrm{FeOH})_{2} \mathrm{Cu}$ & $\mathrm{S}(\mathrm{a})$ & $2=\mathrm{FeOH}^{-0.5}+\mathrm{Cu}^{2+} \leftrightarrow(=\mathrm{FeOH})_{2}{ }^{1.03} \mathrm{Cu}^{0.97}$ & 1.03 & 0.97 & 0 & $7.60^{\dagger}$ \\
\hline$\equiv(\mathrm{FeOH})_{2} \mathrm{CuOH}$ & $S(a)$ & $2 \equiv \mathrm{FeOH}^{-0.5}+\mathrm{Cu}^{2+} \leftrightarrow(\equiv \mathrm{FeOH})_{2}^{1} \mathrm{CuOH}^{0}+\mathrm{H}^{+}$ & 1 & 0 & 0 & $1.83^{\dagger}$ \\
\hline$\equiv(\mathrm{FeOH}) \mathrm{CuOH}$ & $\mathrm{S}(\mathrm{a}, \mathrm{bl})$ & $\equiv \mathrm{FeOH}^{-0.5}+\mathrm{Cu}^{2+} \leftrightarrow(\equiv \mathrm{FeOH})^{0.58} \mathrm{CuOH}^{0.42}+\mathrm{H}^{+}$ & 0.58 & 0.42 & 0 & $0.23^{f}$ \\
\hline \\
\hline \multicolumn{6}{|c|}{$\begin{array}{l}{ }^{a} \text { From Hiemstra and Zhao }{ }^{58} \\
{ }^{b} \text { From Mendez and Hiemstra }\end{array}$} & \\
\hline \multicolumn{7}{|c|}{${ }^{\mathrm{c} F r o m ~ M e n d e z ~ a n d ~ H i e m s t r a ~}{ }^{87}$} \\
\hline
\end{tabular}




\section{Chapter 7}

Table S5: Formation of surface species and corresponding adsorption parameters for the GTLM model for Mn oxides. Formation reactions are with two different surface groups: $\mathrm{XOH}\left(1.08 . \mathrm{nm}^{-2}\right)$ and $\mathrm{YOH}(0.61$ $\mathrm{nm}^{-2}$ ). The SSA was taken as $746 \mathrm{~m}^{2} \mathrm{~g}^{-1}$. All structural and adsorption parameters are taken from Tonkin et al..$^{65}$

\begin{tabular}{l|l|l|l} 
Species & $\begin{array}{l}\text { Surface } \\
\text { group }\end{array}$ & Adsorption reaction & log $K$ \\
\hline$\equiv \mathrm{XO}$ & $\mathrm{X}$ & $\equiv \mathrm{XOH} \leftrightarrow \equiv \mathrm{XO}+\mathrm{H}^{+}$ & -2.35 \\
\hline$\equiv \mathrm{YO}$ & $\mathrm{Y}$ & $\equiv \mathrm{YOH} \leftrightarrow \equiv \mathrm{YO}+\mathrm{H}^{+}$ & -6.06 \\
\hline$\equiv \mathrm{XOZn}$ & $\mathrm{X}$ & $\equiv \mathrm{XOH}+\mathrm{Zn}^{2+} \leftrightarrow \equiv \mathrm{XOZn}+\mathrm{H}^{+}$ & -0.01 \\
$\equiv \mathrm{XOZnOH}$ & $\mathrm{X}$ & $\equiv \mathrm{XOH}+\mathrm{Zn}^{2+} \leftrightarrow \equiv \mathrm{XOZnOH}+2 \mathrm{H}^{+}$ & -4.4 \\
$\equiv \mathrm{YOZnOH}$ & $\mathrm{Y}$ & $\equiv \mathrm{YOH}+\mathrm{Zn}^{2+} \leftrightarrow \equiv \mathrm{YOZnOH}+2 \mathrm{H}^{+}$ & -7.6 \\
\hline$\equiv \mathrm{XOCu}$ & $\mathrm{X}$ & $\equiv \mathrm{XOH}+\mathrm{Cu}^{2+} \leftrightarrow \equiv \mathrm{XOCu}+\mathrm{H}^{+}$ & 0.85 \\
$\equiv \mathrm{XOCuOH}$ & $\mathrm{X}$ & $\equiv \mathrm{XOH}+\mathrm{Cu}^{2+} \leftrightarrow \equiv \mathrm{XOCu}+2 \mathrm{H}^{+}$ & -2.8 \\
$\equiv \mathrm{YOCu}$ & $\mathrm{Y}$ & $\equiv \mathrm{YOH}+\mathrm{Cu}^{2++} \leftrightarrow \equiv \mathrm{YOCu}+\mathrm{H}^{+}$ & 0.86 \\
$\equiv \mathrm{YOCuOH}$ & $\mathrm{Y}$ & $\equiv \mathrm{YOH}+\mathrm{Cu}^{2+} \leftrightarrow \equiv \mathrm{YOCuOH}+2 \mathrm{H}^{+}$ & -5.7 \\
\hline$\equiv \mathrm{XOCa}$ & $\mathrm{X}$ & $\equiv \mathrm{XOH}+\mathrm{Ca}^{2+} \leftrightarrow \equiv \mathrm{XOCa}+\mathrm{H}^{+}$ & -1.5
\end{tabular}




\section{E. The fraction reactive organic matter}

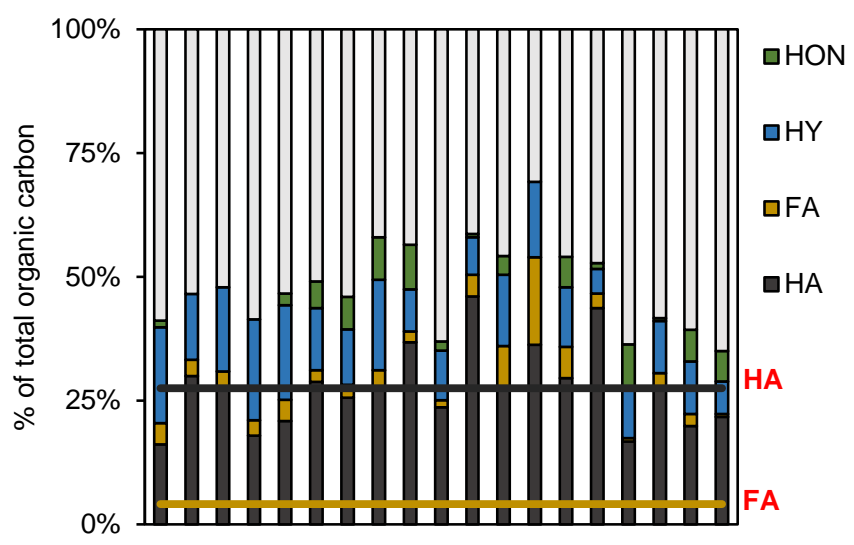

Figure S3: The solid organic carbon fractions for 19 soil samples that were selected from the different datasets: 7 from Burundi, 5 from Rwanda and 4 from Kenya. The different fractions were measured by the rapid batch procedure from Van Zomeren and Comans ${ }^{46}$ : humic acids (HA), fulvic acids (FA), hydrophilics (HY) and hydrophobic neutrals (HON). The recovery of the fractionation procedure was on average $86 \pm 8 \%$. These measured concentrations are expressed as the $\%$ of total organic carbon that was measured according to the Kurmies wet oxidation method ${ }^{45}$. The light grey bars represent the additional carbon that was measured with the Kurmies method, but not by the fractionation procedure. The HA fraction ranged between 16 and $46 \%$ of total organic carbon, the FA between 1 and $18 \%$. The grey line represents the average HA percentage of total organic carbon $(28 \%)$ and the yellow line represents the average FA fraction $(4 \%)$. These average percentages were used in the multi-surface modelling to calculate reactive $\mathrm{FA}$ and $\mathrm{HA}$ for all soil samples relative to the total organic carbon that was measured.

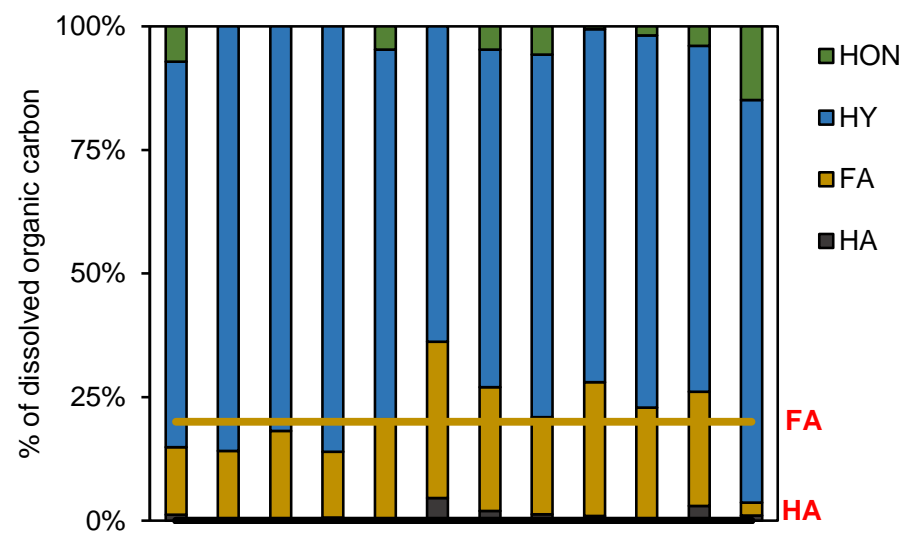

Figure S4: The dissolved organic carbon fractions that were measured on a selection of 12 soil samples from Burundi $(n=7)$ and Rwanda $(n=5)$. The different fractions were measured by the rapid batch procedure from Van Zomeren and Comans (2007): humic acids (HA), fulvic acids (FA), hydrophilics (HY) and hydrophobic neutrals (HON). The HA fraction ranged between 0.03 and $5 \%$ of total dissolved organic carbon, the FA between 3 and $32 \%$. The grey line represents the average HA percentage of total organic carbon $(1 \%)$ and the yellow line represents the average FA fraction $(20 \%)$. These average percentages were used in the multi-surface modelling to calculate reactive FA and $\mathrm{HA}$ for all soil samples relative to the total dissolved organic carbon that was measured in the $0.01 \mathrm{M} \mathrm{CaCl}_{2}$ extraction. The recovery of the fractionation procedure was on average $102 \pm 8 \%$. 

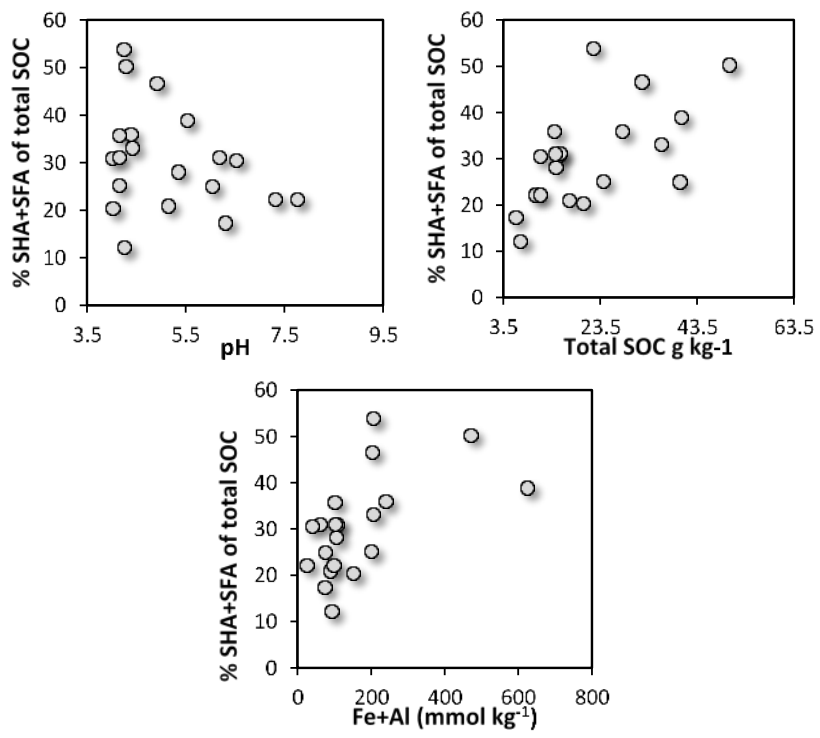

Figure S5: The \% of humic acids (HA) and fulvic acids (FA) in the solid phase of the total SOC as a function of $\mathrm{pH}$, total $\mathrm{SOC}$ and the $\mathrm{Fe}$ and $\mathrm{Al}$ content measured in the ammonium oxalate extraction. The $\mathrm{HA}$ and FA were separated according to the fractionation procedure from Van Zomeren and Comans ${ }^{46}$ for soil samples from Burundi ( $n=10)$, Rwanda $(n=5)$ and Kenya $(n=4)$.
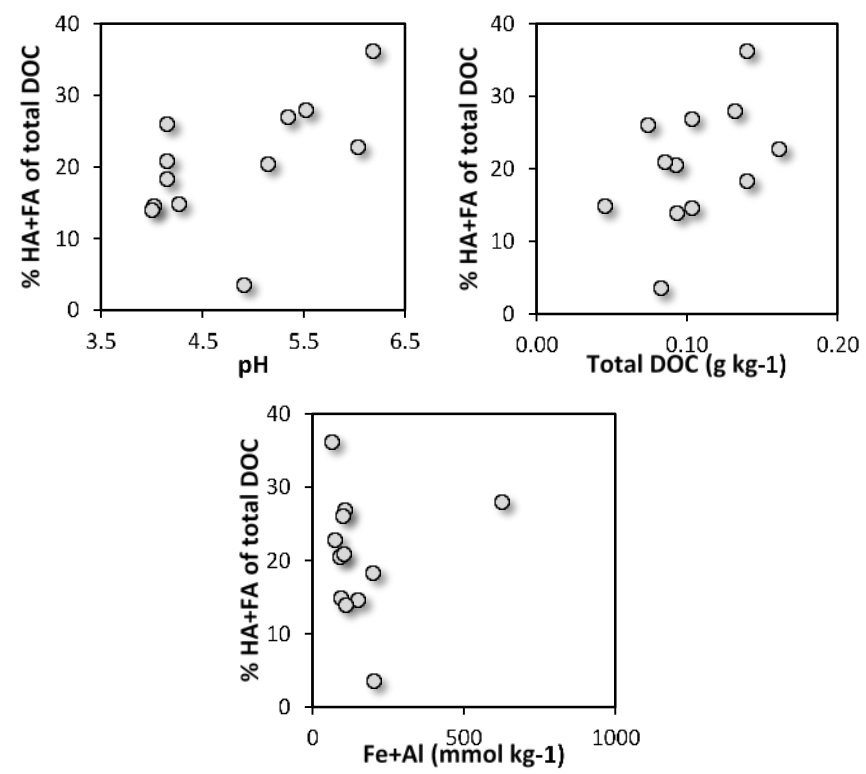

Figure S6: The \% of humic acids (HA) and fulvic acids $(\mathrm{FA})$ in the solution phase of the total DOC as a function of $\mathrm{pH}$, total $\mathrm{DOC}$ and the $\mathrm{Fe}$ and $\mathrm{Al}$ content measured in the ammonium oxalate extraction. The $\mathrm{HA}$ and FA were separated according to the fractionation procedure Van Zomeren and Comans ${ }^{46}$ for soil samples from Burundi $(n=7)$ and Rwanda $(n=5)$. 
F. Relations between $K_{D}$ and soil properties for boron
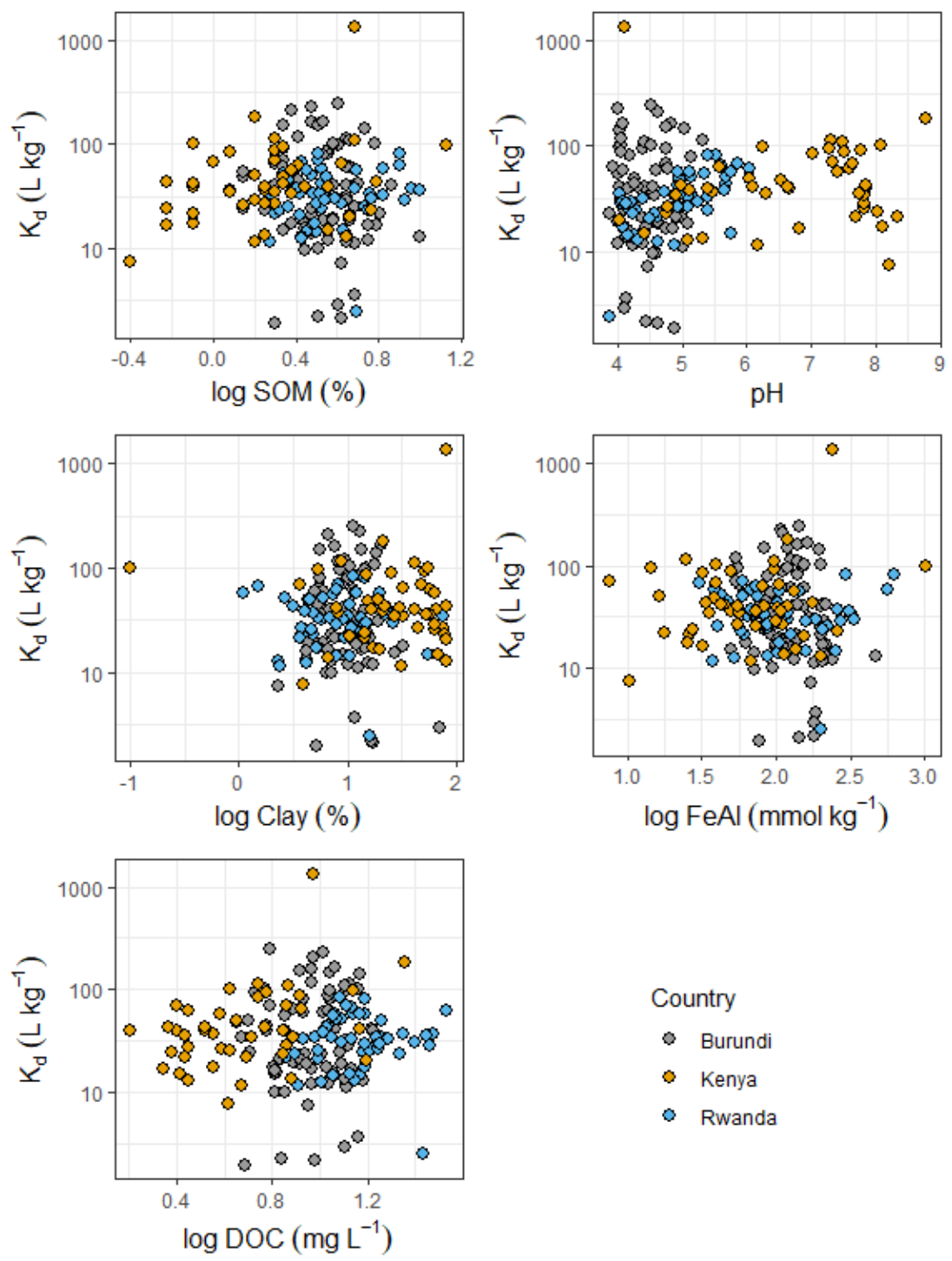

Country

- Burundi

- Kenya

- Rwanda

Figure S7: Relation between the $\mathrm{K}_{d}$ value for $\mathrm{B}\left(\mathrm{L} \mathrm{kg}^{-1}\right)$ and the soil properties that were used for derivation of the partition functions. The $K_{d}$ value was calculated based on the $B$ measured in $0.43 \mathrm{M} \mathrm{HNO}_{3}$ minus the soluble $B$ measured in $0.01 \mathrm{M} \mathrm{CaCl}_{2}\left(\mathrm{~mol} \mathrm{~kg}^{-1}\right)$ divided by the $\mathrm{B}$ measured in the $0.01 \mathrm{M} \mathrm{CaCl}_{2}(\mathrm{~mol}$ $\left.\mathrm{L}^{-1}\right)$. 


\section{G. Correlation table}

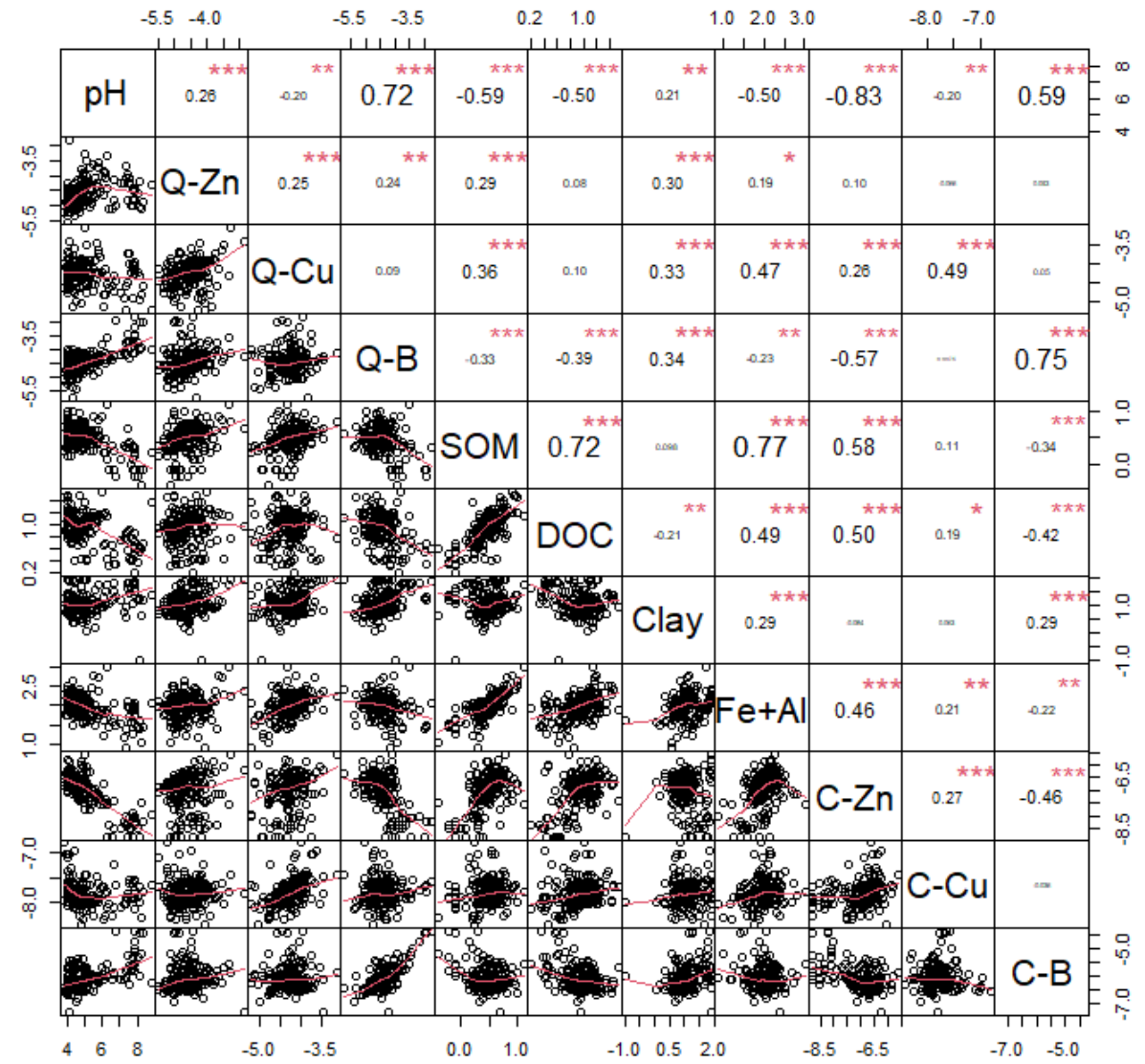

Figure S8: Correlation table. Below the diagonal, the scatter plot of the two variables is shown. Above the diagonal, the pearson correlation coefficient is given with the level of significance ${ }^{* * *}: p<0.001,{ }^{* *}$ : $\left.\mathrm{p}<0.01,{ }^{*}: \mathrm{p}<0.05\right)$. All soil variables are based on the $\log _{10}$ scale, except for $\mathrm{pH}$. The $\mathrm{Q}-\mathrm{Zn}, \mathrm{Q}-\mathrm{Cu}$ and $\mathrm{Q}-$ $B$ represent the concentrations measured in $0.43 \mathrm{M} \mathrm{HNO}_{3}\left(\operatorname{log~mol~kg}{ }^{-1}\right)$. The soil organic matter (SOM) and clay content are given as the log of the concentration in \%. SOM is assumed to consist of $50 \%$ of carbon. The dissolved organic carbon is given as the measured carbon concentration in the $0.01 \mathrm{M} \mathrm{CaCl}_{2}$ extraction, in $\mathrm{mg} \mathrm{L}^{-1}$. The AO-ox represent the sum of $\mathrm{Fe}$ and $\mathrm{Al}$ measured in the ammonium oxalate extraction and the Cryst-ox represent the sum of $\mathrm{Fe}$ and $\mathrm{Al}$ that were calculated as the difference between measured concentration in the dithionite and the ammonium oxalate extraction, both in log mmol $\mathrm{kg}^{-1}$. The $\mathrm{C}-\mathrm{Zn}, \mathrm{C}-\mathrm{Cu}$ and $\mathrm{C}-\mathrm{B}$ are the concentrations measured in the $0.01 \mathrm{M} \mathrm{CaCl}_{2}$ extraction, given as the $\log \mathrm{M}$. 


\section{H. Using $\mathrm{B}$ in $0.43 \mathrm{M} \mathrm{HNO}_{3}$ as reactive $\mathrm{B}$}

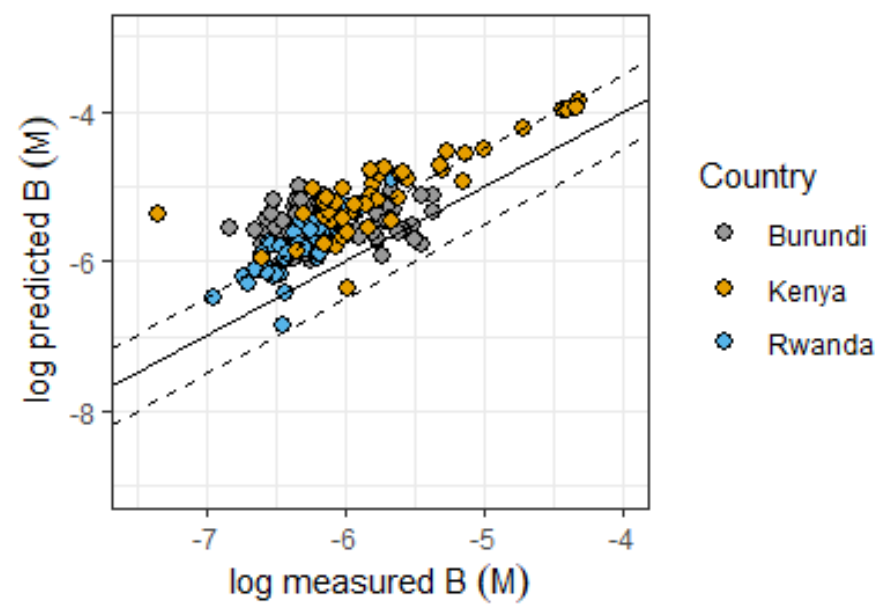

Figure S9: The calculated $B$ solubility when using the total $B$ concentration measured in $0.43 \mathrm{M} \mathrm{HNO}_{3}$ is used as input for reactive $B$ in the multi-surface model. $R M S E=0.66 ; M E=0.58$.

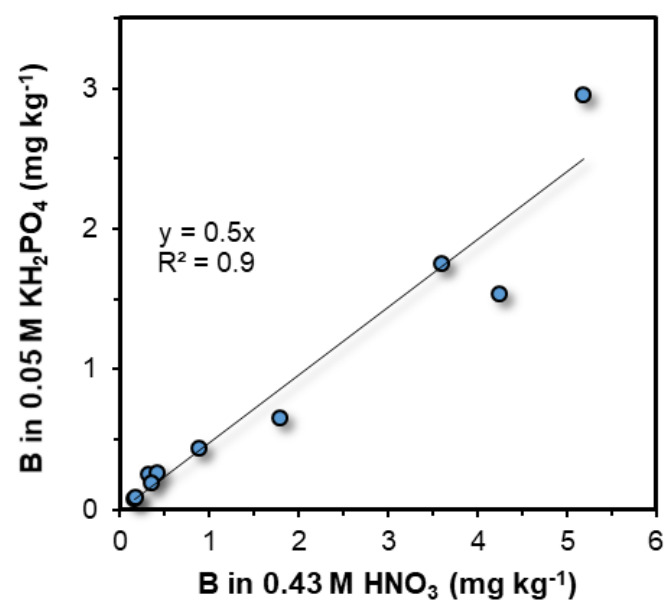

Figure S10: Relation between $\mathrm{B}$ measured in $0.43 \mathrm{M} \mathrm{HNO}_{3}$ and $\mathrm{B}$ measured in $0.05 \mathrm{M} \mathrm{KH}_{2} \mathrm{PO}_{4}$ for ten topsoil samples. Data was taken from a previous study on B speciation in Dutch and Burundi soils ${ }^{24}$. 


\section{Relation between modeling error and soil properties}
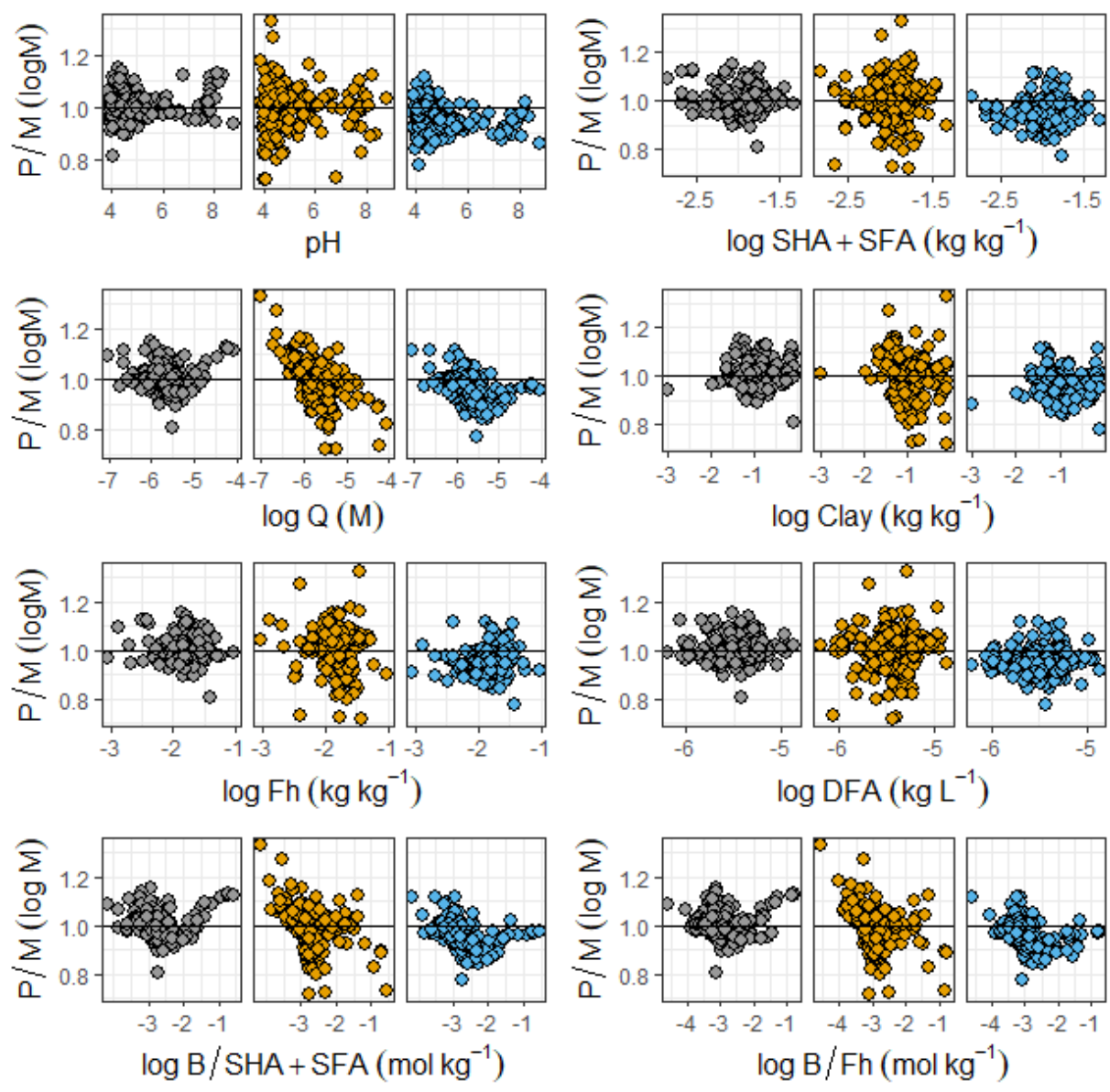

Model
- CQ Partition Relation
- Kf Partition Relation
- Multi Surface Model

Figure S11: The prediction error of the partition relations and multi-surface model based on the measured $\mathrm{B}$ in $0.01 \mathrm{M} \mathrm{CaCl}_{2}$, expressed as the ratio of predicted over measured concentration in log $\mathrm{M}$. Values below 1 are consequently over-predictions of the concentrations by the model. The prediction error is shown in relation to the input variables: $\mathrm{pH}$, solid humic acids (SHA) as a percentage of total SOC and assuming $50 \%$ of carbon, the reactive $B$ concentration measured in $0.43 \mathrm{M} \mathrm{HNO}_{3}(Q)$, the clay content, the ferrihydrite (Fh) content, which is based on the Fe and Al measured in the ammonium oxalate and dithionite extractions and the B loadings, calculated as the reactive B over the SHA and Fh content. 

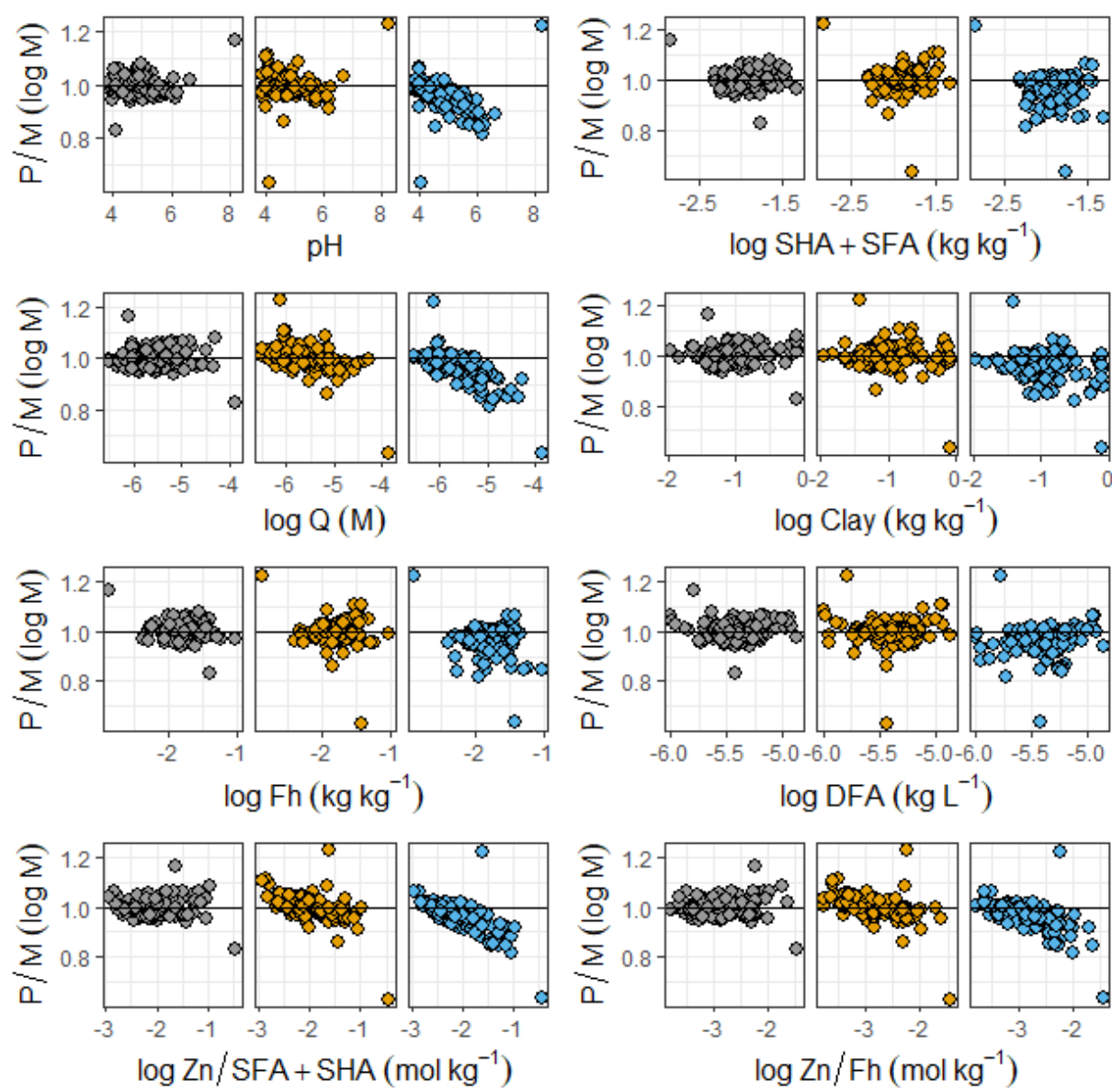

\section{Model \\ - CQ Partition Relation \\ - Kf Partition Relation \\ - Multi Surface Model}

Figure S12: The prediction error of the multi-surface model and partition relations based on the measured $\mathrm{Zn}$ in $0.01 \mathrm{M} \mathrm{CaCl}_{2}$, expressed as the ratio of predicted over measured concentration in log $\mathrm{M}$. Values below 1 are over-predictions of the $\mathrm{Zn}$ concentrations by the model. The prediction error is shown in relation to the input variables: $\mathrm{pH}$, solid humic acids $(\mathrm{SHA})$, the reactive $\mathrm{Zn}$ concentration measured in $0.43 \mathrm{M} \mathrm{HNO}_{3}(\mathrm{Q})$, the clay content, the ferrihydrite (Fh) content and the $\mathrm{Zn}$ loadings, calculated as the reactive $\mathrm{Zn}$ over the SHA and Fh content. 

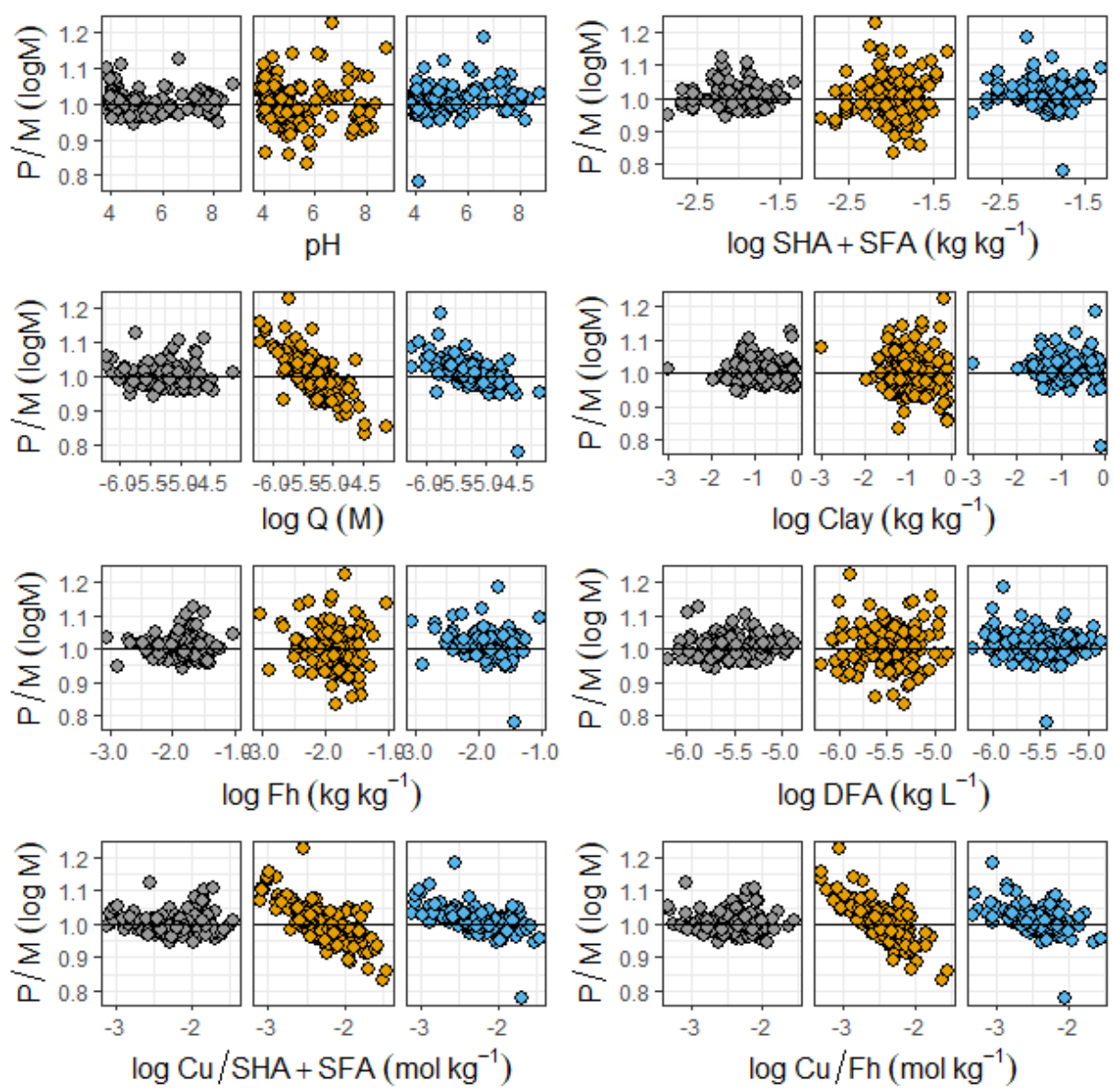

\section{Model \\ - CQ Partition Relation \\ - Kf Partition Relation \\ - Multi Surface Model}

Figure S13: The prediction error of the multi-surface model and partition relations based on the measured $\mathrm{Cu}$ in $0.01 \mathrm{M} \mathrm{CaCl}_{2}$, expressed as the ratio of predicted over measured concentration in log $\mathrm{M}$. Values below 1 are over-predictions of the $\mathrm{Cu}$ concentrations by the model. The prediction error is shown in relation to the input variables: $\mathrm{pH}$, solid humic acids (SHA) as a percentage of total SOC and assuming $50 \%$ of carbon, the reactive $\mathrm{Cu}$ concentration measured in $0.43 \mathrm{M} \mathrm{HNO}_{3}(\mathrm{Q})$, the clay content, the ferrihydrite (Fh) content, which is based on the $\mathrm{Fe}$ and $\mathrm{Al}$ measured in the ammonium oxalate and dithionite extractions and the $\mathrm{Cu}$ loadings, calculated as the reactive $\mathrm{Cu}$ over the SHA and Fh content. 


\section{J. Importance of Mn oxides for $\mathrm{Zn}$ and $\mathrm{Cu}$ adsorption}
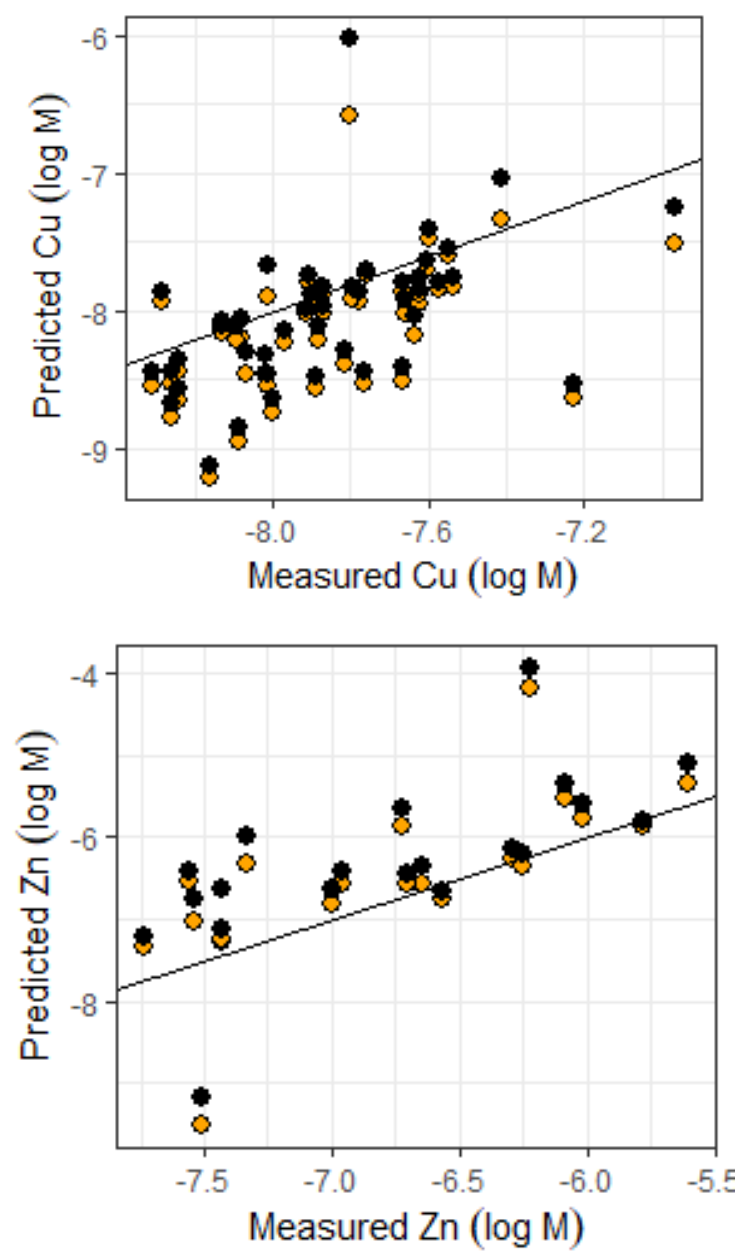

Figure S14: Comparison between the measured and predicted $\mathrm{Cu}$ and $\mathrm{Zn}$ concentrations in the $0.01 \mathrm{M}$ $\mathrm{CaCl}_{2}$ soil extract when including no manganese $(\mathrm{Mn})$ oxides (black markers) and when including $\mathrm{Mn}$ oxides for $\mathrm{Cu}$ and $\mathrm{Zn}$ adsorption (yellow markers). The dashed line represent the 1:1 line. The modeling set-up without $\mathrm{Mn}$ oxides is the same as the one evaluated in the main text. The adsorption to Mn oxides was modeled using the parameters from Tonkin et al. ${ }^{65}$ 


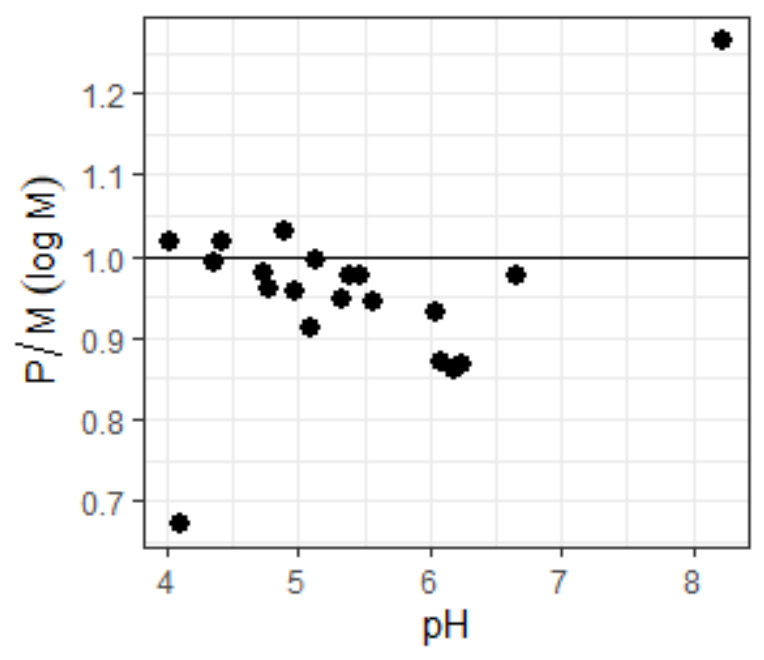

Figure S15: The prediction error of the multi-surface model (expressed as the ratio of predicted over measured concentration in $\log \mathrm{M}$ ) based on the measured $\mathrm{Zn}$ in $0.01 \mathrm{M} \mathrm{CaCl}_{2}$, in relation to the soil $\mathrm{pH}\left(\mathrm{CaCl}_{2}\right)$. The multi-surface modeling calculations include $\mathrm{Zn}, \mathrm{Cu}$ and $\mathrm{Ca}$ adsorption to $\mathrm{Mn}$ oxides, based on the parameters from Tonkin et al. ${ }^{65}$. Values below 1 represent soils for which the $\mathrm{Zn}$ solubility is overestimated by the multi-surface model.

\section{REFERENCES}

1. Kihara, J. et al. Application of secondary nutrients and micronutrients increases crop yields in sub-Saharan Africa. Agron. Sustain. Dev. (2017) doi:10.1007/s13593-017-0431-0.

2. Kihara, J., Bolo, P., Kinyua, M., Rurinda, J. \& Piikki, K. Micronutrient deficiencies in African soils and the human nutritional nexus: opportunities with staple crops. Environ. Geochem. Health 42 , 3015-3033 (2020).

3. Sillanpää, M. Micronutrient assessment at the country level: an international study. FAO Soils Bulletin (Food and Agriculture Organization of the United Nations in cooperation with the Finnish International Development Agency, 1990).

4. Gregory, P. J. et al. Approaches to reduce zinc and iron deficits in food systems. Glob. Food Sec. 15, 1-10 (2017).

5. Alloway, B. J. Soil factors associated with zinc deficiency in crops and humans. Environ. Geochem. Health 31, 537-48 (2009).

6. Black, R. E. et al. Maternal and child undernutrition and overweight in low-income and middleincome countries. The Lancet vol. 382 427-451 (2013).

7. de Vries, W., Curlík, J., Murányi, A., Alloway, B. \& Groenenberg, B. J. Assessment of relationships between total and reactive concentrations of cadmium, copper, lead and zinc in Hungarian and Slovakian soils. Ekológia (Bratislava) 24, 152-169 (2005).

8. Groenenberg, J. E., Römkens, P. F. A. M., Zomeren, A. Van, Rodrigues, S. M. \& Comans, R. N. J. Evaluation of the Single Dilute $(0.43 \mathrm{M})$ Nitric Acid Extraction to Determine Geochemically Reactive Elements in Soil. Environ. Sci. Technol. 51, 2246-2253 (2017).

9. Bonten, L. T. C. \& Groenenberg, J. E. Uitspoeling van zware metalen uit bodems in het landelijk gebied: modelberekeningen ten behoeve van Emissieregistratie 2008. Alterra-rapport;1695 http://library.wur.nl/way/bestanden/clc/1879645.pdf (2008).

10. Dijkstra, J. J., Meeussen, J. C. L. \& Comans, R. N. J. Leaching of Heavy Metals from Contaminated Soils: An Experimental and Modeling Study. Environ. Sci. Technol. 38, 43904395 (2004).

11. Degryse, F., Smolders, E. \& Parker, D. R. Partitioning of metals (Cd, $\mathrm{Co}, \mathrm{Cu}, \mathrm{Ni}, \mathrm{Pb}, \mathrm{Zn}$ ) in soils: concepts, methodologies, prediction and applications - a review. Eur. J. Soil Sci. 60, 590-612 (2009). 
12. Catlett, K. M., Heil, D. M., Lindsay, W. L. \& Ebinger, M. H. Soil Chemical Properties Controlling Zinc Activity in 18 Colorado Soils. Soil Sci. Soc. Am. J. 66, 1182 (2002).

13. Nawara, S. et al. A comparison of soil tests for available phosphorus in long-term field experiments in Europe. Eur. J. Soil Sci. 68, 873-885 (2017).

14. Groenenberg, J. E. \& Lofts, S. The use of assemblage models to describe trace element partitioning, speciation, and fate: a review. Environ. Toxicol. Chem. 33, 2181-96 (2014).

15. Gustafsson, J. P. \& Tiberg, C. Molybdenum binding to soil constituents in acid soils: An XAS and modelling study. Chem. Geol. 417, 279-288 (2015).

16. Dijkstra, J. J., Meeussen, J. C. L. \& Comans, R. N. J. Evaluation of a Generic Multisurface Sorption Model for Inorganic Soil Contaminants. Environ. Sci. Technol. 43, 6196-6201 (2009).

17. Groenenberg, J. E., Dijkstra, J. J., Bonten, L. T. C., De Vries, W. \& Comans, R. N. J. Evaluation of the performance and limitations of empirical partition-relations and process based multisurface models to predict trace element solubility in soils. Environ. Pollut. 166, 98-107 (2012).

18. Weng, L., Temminghoff, E. J. M. \& Van Riemsdijk, W. H. Contribution of Individual Sorbents to the Control of Heavy Metal Activity in Sandy Soil. Environ. Sci. Technol. 35, 4436-4443 (2001).

19. Tiberg, C., Sjöstedt, C. \& Gustafsson, J. P. Metal sorption to Spodosol Bs horizons: Organic matter complexes predominate. Chemosphere 196, 556-565 (2018).

20. Gustafsson, J. P., Pechova, P. \& Berggren, D. Modeling metal binding to soils: The role of natural organic matter. Environ. Sci. Technol. 37, 2767-2774 (2003).

21. Duffner, A., Weng, L., Hoffland, E. \& van der Zee, S. E. A. T. M. Multi-surface modeling to predict free zinc ion concentrations in low-zinc soils. Environ. Sci. Technol. 48, 5700-5708 (2014).

22. Goldberg, S. Reanalysis of boron adsorption on soils and soil minerals using the constant capacitance model. Soil Sci. Soc. Am. J. 63, 823-829 (1999).

23. Goldberg, S. Modeling Boron Adsorption Isotherms and Envelopes Using the Constant Capacitance Model. Vadose Zo. J. 3, 676-680 (2004).

24. Van Eynde, E., Weng, L. \& Comans, R. N. J. Boron speciation and extractability in temperate and tropical soils: a multi-surface modeling approach. Appl. Geochemistry 104797 (2020) doi:10.1016/j.apgeochem.2020.104797.

25. Mao, L. C. C., Young, S. D. D., Tye, A. M. M. \& Bailey, E. H. H. Predicting trace metal solubility and fractionation in Urban soils from isotopic exchangeability. Environ. Pollut. 231, 1529-1542 (2017).

26. Klinkert, S. \& Comans, R. N. J. Geochemical Multisurface Modeling of Reactive Zinc Speciation in Compost as Influenced by Extraction Conditions. Environ. Sci. Technol. 54, 2467-2475 (2020).

27. $\mathrm{Xu}$, L. et al. Latitudinal patterns and influencing factors of soil humic carbon fractions from tropical to temperate forests. J. Geogr. Sci. 28, 15-30 (2018).

28. Wei, Y., Wu, X., Zeng, R., Cai, C. \& Guo, Z. Spatial variations of aggregate-associated humic substance in heavy-textured soils along a climatic gradient. Soil Tillage Res. 197, 104497 (2020).

29. van der Perk, M., Stergiadi, M., de Nijs, T. C. M., Comans, R. N. J. \& Bierkens, M. F. P. The response of metal leaching from soils to climate change and land management in a temperate lowland catchment. Catena 171, 426-439 (2018).

30. Hengl, T. et al. Soil nutrient maps of Sub-Saharan Africa: assessment of soil nutrient content at $250 \mathrm{~m}$ spatial resolution using machine learning. Nutr. Cycl. Agroecosystems 109, 77-102 (2017).

31. Hengl, T. et al. Mapping Soil Properties of Africa at $250 \mathrm{~m}$ Resolution: Random Forests Significantly Improve Current Predictions. PLoS One 10, e0125814 (2015).

32. De Groot, A. C., Peijnenburg, W. J. G. M., van den Hoop, M. A. G. T., Ritsema, R. \& van Veen, R. P. . Heavy metals in Dutch field soils: an experimental and theoretical study on equilibrium partitioning. http://rivm.openrepository.com/rivm/handle/10029/10141 (1998).

33. Degryse, F., Broos, K., Smolders, E. \& Merckx, R. Soil solution concentration of $\mathrm{Cd}$ and $\mathrm{Zn}$ canbe predicted with a $\mathrm{CaCl} 2$ soil extract. Eur. J. Soil Sci. 54, 149-158 (2003).

34. Nolan, A. L., Zhang, H. \& McLaughlin, M. J. Prediction of zinc, cadmium, lead, and copper availability to wheat in contaminated soils using chemical speciation, diffusive gradients in thin films, extraction, and isotopic dilution techniques. J. Environ. Qual. 34, 496-507 (2005).

35. Tipping, E. et al. The solid-solution partitioning of heavy metals $(\mathrm{Cu}, \mathrm{Zn}, \mathrm{Cd}, \mathrm{Pb})$ in upland soils of England and Wales. Environ. Pollut. 125, 213-225 (2003).

36. Impellitteri, C. A., Saxe, J. K., Cochran, M., Janssen, G. M. C. M. \& Allen, H. E. Predicting the bioavailability of copper and zinc in soils: modeling the partitioning of potentially bioavailable copper and zinc from soil solid to soil solution. Environ. Toxicol. Chem. 22, 1380-6 (2003).

37. Groenenberg, B. et al. Transfer functions for solid-solution partitioning of cadmium, copper, nickel, lead and zinc in soils: derivation of relationships for free metal ion activities and validation 
with independent data. Eur. J. Soil Sci. 61, 58-73 (2010).

38. Houba, V. J. G., Temminghoff, E. J. M., Gaikhorst, G. A. \& van Vark, W. Soil analysis procedures using $0.01 \mathrm{M}$ calcium chloride as extraction reagent. Commun. Soil Sci. Plant Anal. 31, 1299$1396(2000)$.

39. Van Eynde, E., Mendez, J. C., Hiemstra, T. \& Comans, R. N. J. Boron adsorption to ferrihydrite with implications for surface speciation in soils: Experiments and modeling. ACS Earth Sp. Chem. acsearthspacechem.0c00078 (2020) doi:10.1021/acsearthspacechem.0c00078.

40. Keskinen, R. et al. Readily available concentrations of selected micronutrients and harmful metals in soils of Sub-Saharan Africa. Geoderma 347, 203-209 (2019).

41. ISO. Soil quality 17586:2016 -- Extraction of trace elements using dilute nitric acid. https://www.iso.org/standard/60060.html (2016).

42. Konert, M. \& Vandenberghe, J. Comparison of laser grain size analysis with pipette and sieve analysis: a solution for the underestimation of the clay fraction. Sedimentology vol. 44 (1997).

43. ISO. ISO 12782-3:2012 Soil quality -- Parameters for geochemical modelling of leaching and speciation of constituents in soils and materials -- Part 3: Extraction of aluminium oxides and hydroxides with ammonium oxalate/oxalic acid. (2012).

44. ISO. ISO 12782-2:2012 - Soil quality -- Parameters for geochemical modelling of leaching and speciation of constituents in soils and materials -- Part 2: Extraction of crystalline iron oxides and hydroxides with dithionite. https://www.iso.org/standard/51698.html (2012).

45. Nelson, D. W. \& Sommers, L. E. Total Carbon, Organic Carbon, and Organic Matter. in Methods of soil analysis Part 3-Chemical Methoods 961-1010 (1996). doi:10.2136/sssabookser5.3.c34.

46. van Zomeren, A. \& Comans, R. N. J. Measurement of Humic and Fulvic Acid Concentrations and Dissolution Properties by a Rapid Batch Procedure. Environ. Sci. Technol. 41, 6755-6761 (2007).

47. Keizer, M. G. \& Van Riemsdijk, W. H. ECOSAT, a Computer Program for the Calculation of Chemical Speciation and Transport in Soil-Water Systems. (1995).

48. Mendez, J. C., Hiemstra, T. \& Koopmans, G. F. Assessing the Reactive Surface Area of Soils and the Association of Soil Organic Carbon with Natural Oxide Nanoparticles Using Ferrihydrite as Proxy. Environ. Sci. Technol. 54, 11990-12000 (2020).

49. Murphy, J. \& Riley, J. P. A modified single solution method for the determination of phosphate in natural waters. Anal. Chim. Acta 27, 31-36 (1962).

50. Lindsay, W. L. Chemical equilibria in soils. (Wiley, 1979).

51. Parks, J. L. \& Edwards, M. Boron in the Environment. Crit. Rev. Environ. Sci. Technol. 35, 81114 (2005).

52. Kinniburgh, D. G. et al. Metal ion binding by humic acid: Application of the NICA-Donnan model. Environ. Sci. Technol. 30, 1687-1698 (1996).

53. Goli, E., Hiemstra, T. \& Rahnemaie, R. Interaction of boron with humic acid and natural organic matter: Experiments and modeling. Chem. Geol. 515, 1-8 (2019).

54. Milne, C. J. C. J., Kinniburgh, D. G. D. G. D. G. \& Tipping, E. Generic NICA-Donnan Model Parameters for Proton Binding by Humic Substances. Environ. Sci. Technol. 35, 2049-2059 (2001).

55. Milne, C. J., Kinniburgh, D. G., van Riemsdijk, W. H. \& Tipping, E. Generic NICA-Donnan Model Parameters for Metal-Ion Binding by Humic Substances. Environ. Sci. Technol. 37, 958-971 (2003).

56. Hiemstra, T. \& van Riemsdijk, W. H. Biogeochemical speciation of $\mathrm{Fe}$ in ocean water. Mar. Chem. 102, 181-197 (2006).

57. Hiemstra, T. \& Van Riemsdijk, W. H. A Surface Structural Approach to Ion Adsorption: The Charge Distribution (CD) Model. J. Colloid Interface Sci. 179, 488-508 (1996).

58. Hiemstra, T. \& Zhao, W. Reactivity of ferrihydrite and ferritin in relation to surface structure, size, and nanoparticle formation studied for phosphate and arsenate. Environ. Sci. Nano 3, 12651279 (2016).

59. Hiemstra, T. \& Van Riemsdijk, W. H. A surface structural model for ferrihydrite I: Sites related to primary charge, molar mass, and mass density. Geochim. Cosmochim. Acta 73, 4423-4436 (2009).

60. Hiemstra, T., Antelo, J., Rahnemaie, R. \& van Riemsdijk, W. H. Nanoparticles in natural systems I: The effective reactive surface area of the natural oxide fraction in field samples. Geochim. Cosmochim. Acta 74, 41-58 (2010).

61. Mendez, J. C. Ion complexation modelling of ferrihydrite: From fundamentals of metal (hydr)oxide nanoparticles to applications in soils systems. (Wageningen University, 2020). doi:10.18174/525623. 
62. Mossa, A. et al. Zinc lability and solubility in soils of Ethiopia - an isotopic dilution study. SOIL Discuss. 1-22 (2021) doi:10.5194/soil-2020-81.

63. Ren, Z.-L. et al. Speciation and reactivity of lead and zinc in heavily and poorly contaminated soils: Stable isotope dilution, chemical extraction and model views *. (2017) doi:10.1016/j.envpol.2017.03.051.

64. Cancès, B., Ponthieu, M., Castrec-Rouelle, M., Aubry, E. \& Benedetti, M. . F. Metal ions speciation in a soil and its solution: experimental data and model results. Geoderma 113, 341355 (2003).

65. Tonkin, J. W., Balistrieri, L. S. \& Murray, J. W. Modeling sorption of divalent metal cations on hydrous manganese oxide using the diffuse double layer model. Appl. Geochemistry 19, 29-53 (2004).

66. Sauvé, S., Hendershot, W. \& Allen, H. E. Solid-Solution Partitioning of Metals in Contaminated Soils: Dependence on pH, Total Metal Burden, and Organic Matter. Environ. Sci. Technol. 34, 1125-1131 (2000).

67. Nolan, A. L., McLaughlin, M. J. \& Mason, S. D. Chemical speciation of $\mathrm{Zn}, \mathrm{Cd}, \mathrm{Cu}$, and $\mathrm{Pb}$ in pore waters of agricultural and contaminated soils using donnan dialysis. Environ. Sci. Technol. 37, 90-98 (2003).

68. Elzinga, E. J., Van Grinsven, J. J. M. \& Swartjes, F. A. General purpose Freundlich isotherms for cadmium, copper and zinc in soils. Eur. J. Soil Sci. 50, 139-149 (1999).

69. Tye, A. M. et al. Predicting the activity of $\mathrm{Cd} 2+$ and $\mathrm{Zn} 2+$ in soil pore water from the radio-labile metal fraction. Geochim. Cosmochim. Acta 67, 375-385 (2003).

70. Webster, R. \& McBratney, A. B. On the Akaike Information Criterion for choosing models for variograms of soil properties. J. Soil Sci. 40, 493-496 (1989).

71. R Core Team \& R Development Core Team. A language and environment for statistical computing. (2020).

72. Duffner, A., Hoffland, E., Weng, L. \& van der Zee, S. E. A. T. M. Predicting zinc bioavailability to wheat improves by integrating $\mathrm{pH}$ dependent nonlinear root surface adsorption. Plant Soil 373, 919-930 (2013).

73. Novozamsky, I., Barrera, L. L., Houba, V. J. G., van der Lee, J. J. \& van Eck, R. Comparison of a hot water and cold $0.01 \mathrm{M} \mathrm{Cacl}_{2}$ extraction procedures for the determination of boron in soil. Commun. Soil Sci. Plant Anal. 21, 2189-2195 (1990).

74. Kabata-Pendias, A. \& Pendias, H. Trace Elements in Soils and Plants, Third Edition. www.crcpress.com (2001).

75. Supriatin, S., Terrones, C. A., Bussink, W. \& Weng, L. Drying effects on selenium and copper in $0.01 \mathrm{M}$ calcium chloride soil extractions. Geoderma 255, 104-114 (2015).

76. Groenenberg, J. E., Koopmans, G. F. \& Comans, R. N. J. Uncertainty analysis of the nonideal competitive adsorption - Donnan model: Effects of dissolved organic matter variability on predicted metal speciation in soil solution. Environ. Sci. Technol. 44, 1340-1346 (2010).

77. Fest, E. P. M. J. M. J., Temminghoff, E. J. M. J. M., Comans, R. N. J. N. J. \& van Riemsdijk, W. H. H. Partitioning of organic matter and heavy metals in a sandy soil: Effects of extracting solution, solid to liquid ratio and pH. Geoderma 146, 66-74 (2008).

78. Weng, L. P., Van Riemsdijk, W. H. \& Hiemstra, T. Adsorption of humic acids onto goethite: Effects of molar mass, $\mathrm{pH}$ and ionic strength. J. Colloid Interface Sci. 314, 107-118 (2007).

79. Meima, J. A. \& Comans, R. N. J. Application of surface complexation/precipitation modeling to contaminant leaching from weathered municipal solid waste incinerator bottom ash. Environ. Sci. Technol. 32, 688-693 (1998).

80. Marzouk, E. R., Chenery, S. R. \& Young, S. D. Measuring reactive metal in soil: A comparison of multi-element isotopic dilution and chemical extraction. Eur. J. Soil Sci. 64, 526-536 (2013).

81. Degryse, F., Voegelin, A., Jacquat, O., Kretzschmar, R. \& Smolders, E. Characterization of zinc in contaminated soils: complementary insights from isotopic exchange, batch extractions and XAFS spectroscopy. Eur. J. Soil Sci. 62, 318-330 (2011).

82. Bonten, L. T. C. C., Groenenberg, J. E., Weng, L. \& van Riemsdijk, W. H. Use of speciation and complexation models to estimate heavy metal sorption in soils. Geoderma 146, 303-310 (2008).

83. Weng, L., Van Riemsdijk, W. H. \& Hiemstra, T. Cu2+ and Ca2+adsorption to goethite in the presence of fulvic acids. Geochim. Cosmochim. Acta 72, 5857-5870 (2008).

84. Benedetti, M. ., van Riemsdijk, W. H. \& Koopal, L. K. Humic Substances Considered as a Heterogeneous Donnan Gel Phase. Environ. Sci. Technol 30, 1905-1913 (1996).

85. Town, R. M., Van Leeuwen, H. P. \& Duval, J. F. L. Rigorous Physicochemical Framework for Metal Ion Binding by Aqueous Nanoparticulate Humic Substances: Implications for Speciation Modeling by the NICA-Donnan and WHAM Codes. Environ. Sci. Technol. 53, 8516-8532 (2019).

86. ISRIC - World Soil Information. SoilGrids: an automated system for global soil mapping. (2013). 


\section{Chapter 7}

87. Mendez, J. C. \& Hiemstra, T. High and low affinity sites of ferrihydrite for metal ion adsorption: Data and modeling of the alkaline-earth ions $\mathrm{Be}, \mathrm{Mg}, \mathrm{Ca}, \mathrm{Sr}, \mathrm{Ba}$, and $\mathrm{Ra}$. Geochim. Cosmochim. Acta (2020) doi:10.1016/j.gca.2020.07.032.

88. Mendez, J. C. \& Hiemstra, T. Ternary complex formation of phosphate with $\mathrm{Ca}$ and $\mathrm{Mg}$ ions binding to ferrihydrite: Experiments and mechanisms. ACS Earth Sp. Chem. 4.4 acsearthspacechem.9b00320 (2020).

89. Mendez, J. C. \& Hiemstra, T. Surface area of ferrihydrite consistently related to primary surface charge, ion pair formation, and specific ion adsorption. Chem. Geol. 532, (2020).

90. Van Zomeren, A. \& Comans, R. N. J. Measurement of humic and fulvic acid concentrations and dissolution properties by a rapid ratch procedure. Environ. Sci. Technol. (2007) doi:10.1021/es0709223. 
Chapter 8

General Discussion

Elise Van Eynde 


\section{MAIN FINDINGS}

The main objective of this thesis research was to enhance the understanding of the soil chemical processes that control the environmental availability of zinc (Zn), copper $(\mathrm{Cu})$ and boron $(\mathrm{B})$ in soils from Sub-Saharan Africa. Environmental availability, or the so-called reactive pool, consists of the fraction in the solid phase that is available for interaction with the dissolved phase at short time scales (up to a few days) through processes such as sorption/desorption and precipitation/ dissolution reactions ${ }^{1}$, and the actual dissolved concentration ${ }^{2}$. The distribution of the reactive pool over the solid and solution phase depends on the soil solution chemistry such as $\mathrm{pH}$, ionic strength and the concentrations of co-occurring ions, and the amount and properties of the mineral and organic surfaces. The first objective of this thesis was therefore to assess the content, the composition and reactivity of the most relevant reactive surfaces (i.e. metal (hydr)oxides and soil organic matter) for controlling the solid-solution partitioning of micronutrients in soils from SSA. The second objective was to understand and describe the interaction of micronutrients with ferrihydrite (Fh) nanoparticles. We hypothesized that this model oxide can be used as a proxy for the reactive metal (hydr)oxide fractions in soils from SSA, which was first validated in Chapter 2 under the first objective. Finally, using the results found under the first two objectives, the environmental availability and solid-solution partitioning of $\mathrm{Zn}, \mathrm{Cu}$ and $\mathrm{B}$ was studied for a large set of SSA soils.

\subsection{Surface reactivity of tropical soils}

The distribution of the reactive or labile micronutrient content over the solid and solution phase, is often controlled by adsorption processes to reactive surfaces. In Chapter 2 of this thesis, we studied the amount, composition and reactivity of the metal (hydr)oxides in tropical soils. In Chapter 3, we looked into the role of the metal (hydr)oxides on the soil organic carbon content, with the latter being another major important reactive surface for micronutrient speciation in soils.

\subsubsection{Metal (hydr)oxides}

In Chapter 2 of this thesis, we have shown that irrespective of weathering stage and metal (hydr)oxide composition, $\mathrm{Fh}$ is a good representation of the reactive natural metal (hydr)oxides for $\mathrm{PO}_{4}$ adsorption in soils. The reactive surface area (RSA, m² $\mathrm{g}^{-1}$ soil) of a subset of typical weathered tropical topsoils from Burundi and Kenya has been consistently determined based on an interpretation of phosphate $\left(\mathrm{PO}_{4}\right)$ measurements in $0.5 \mathrm{M} \mathrm{NaHCO}_{3}$ soil extractions at different solution-to-solid ratios, using the charge distribution (CD) model. This probe-ion method was originally developed for Dutch agricultural topsoils ${ }^{3,4}$ but our results have shown that it could be successfully applied without modifications to tropical topsoils with around 5 times lower levels of reactive $\mathrm{PO}_{4}$. Despite the dominant presence of crystalline metal (hydr)oxides in these weathered soils ( $75 \%$ of the total Fe and Al (hydr)oxide content), Fh was found to be a better representation of the reactive natural metal 
(hydr)oxide fraction than goethite, which is in agreement with previous results for a series of representative Dutch agricultural topsoils 4 . The observed good relationship between the RSA and the $\mathrm{Fe}$ and $\mathrm{Al}$ measured in the ammonium oxalate (AO) extraction, suggests that despite the large mass fraction of crystalline $\mathrm{Fe}$ and $\mathrm{Al}$ (hydr)oxides, the nanocrystalline metal (hydr)oxides determine the surface reactivity in soils. Interestingly, these tropical soils from Chapter 2 were characterized by a dominant presence of $\mathrm{Al}(\sim 70 \%)$ in the nanocrystalline metal (hydr)oxide fraction. This means that although the metal (hydr)oxide reactivity can be described using Fh as proxy, the actual metal (hydr)oxides that determine the reactivity are a mixture of Fe and Al metal (hydr)oxides.

\subsubsection{Soil organic matter}

Soil organic matter (SOM) is the most important reactive surface for the adsorption of $\mathrm{Zn}$ and $\mathrm{Cu}$, and with increasing $\mathrm{pH}$ it becomes increasingly important for $\mathrm{B}$ retention in the solid phase (see Chapters 6 and 7 of this thesis). In Chapter 3 of this thesis, we have shown that SOM is predominantly stored in primary organo-oxide aggregates, in which the metal (hydr)oxide particle is surrounded by a layer of SOM with a mean thickness that is linearly related to the size of the oxide nanoparticles involved.

For all the soils analyzed in Chapter 3, a very good correlation was found between the content of $\mathrm{Fe}$ and $\mathrm{Al}$ extractable with ammonium oxalate $(\mathrm{AO})$ and $\mathrm{SOC}$, irrespective of climate region and soil chemical properties. These results confirm the important role of the soil metal (hydr)oxide nanoparticles for controlling soil organic carbon content ${ }^{5-7}$. This relationship suggests that SOM binds to the surfaces of the metal (hydr)oxides. However, the relationship between SOC and the RSA was much less pronounced, and it was found that the soil organic carbon (SOC) loading per unit of $\mathrm{m}^{2}$ is consistently related to the particle diameter. We concluded that in order to understand the role of metal (hydr)oxides in controlling the SOC storage, the mean particle size of the reactive metal (hydr)oxide fraction is a key property.

With regard to micronutrient retention and speciation in soils, not only the bulk SOC content is important, but also the solid-solution partitioning of the SOC and the reactivity for ion adsorption. The solid-solution partitioning of SOC determines how much dissolved organic carbon (DOC) will be present, which may have a positive effect on nutrient solubility. The results from Chapter 7 have shown that DOC is especially important to understand Cu speciation and solubility, in line with previous findings ${ }^{8,9}$. Figure 1 shows that the distribution coefficient of SOM is rather constant among soils, and I could not explain the observed variability by soil $\mathrm{pH}$ or the content of $\mathrm{Fe}$ and $\mathrm{Al}$ oxides. 


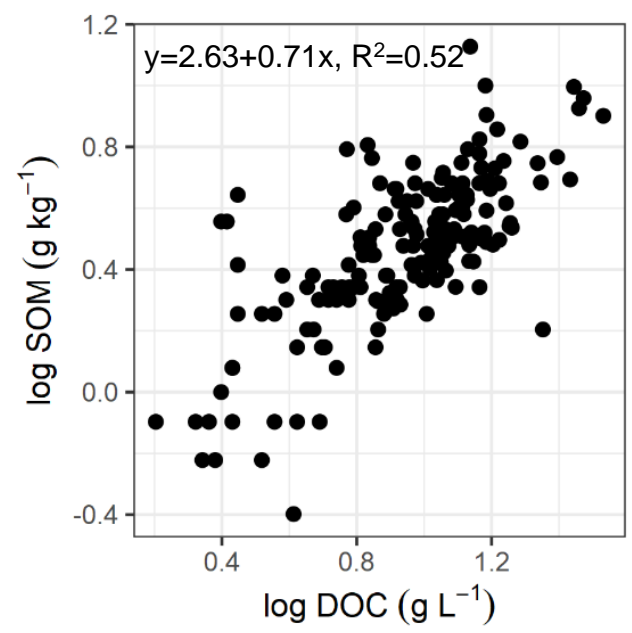

Figure 1: The relationship between bulk soil organic carbon (SOC) and dissolved organic carbon (DOC). The data is shown for tropical soils from Kenya, Burundi and Rwanda $(n=172)$ that have been described in Chapter 7 of this thesis.

Next to content and solubility, the reactivity of the SOC and DOC is important for understanding the solid-solution partitioning of nutrients in soils. The reactivity of $\mathrm{SOC}$ for ion adsorption is interpreted as the fraction of humic and fulvic acids, as these compounds have been used for the parameterization of the ion adsorption models to organic matter ${ }^{10-12}$. We have measured the fraction of humic and fulvic acids in the dissolved and solid organic matter for a limited number of tropical soils (Chapter 7). These measurements suggests a lower fraction of reactive organic carbon ( 32\%), especially in the dissolved phase ( 21\%), compared to previous measurements $\left(\sim 50 \%\right.$ in solid phase ${ }^{13}, \sim 30 \%$ in solution phase $\left.{ }^{14}\right)$ or modelled fractions $\left(65-100 \%\right.$ in solution phase $\left.{ }^{15}\right)$ in temperate soils.

\subsection{Parameterization of the CD model for $\mathrm{Zn}, \mathrm{Cu}$ and $\mathrm{B}$ adsorption to $\mathrm{Fh}$}

Multi-surface modeling is a powerful tool to study how the interplay between various soil properties control the solid-solution partitioning of nutrients and pollutants in soils. As such, these models have been applied to study the environmental availability of micronutrients in this thesis research. The application of multi-surface models to soil systems, requires well parameterized ion adsorption models for each of the relevant reactive surfaces. Most multi-surface modeling applications have used the Generalized Two Layer Model (GTLM) for describing ion adsorption to the microcrystalline metal (hydr)oxides $1,16,17$ because of its comprehensive and internally consistent set of adsorption parameters for metal cations and oxyanions ${ }^{18}$. However, the GTLM applies a simplified representation of the mineral solid-solution interface, in contrast to the charge distribution (CD) model ${ }^{19}$. As a result, the CD model provides a better description of the electrostatic interactions that are involved in the 
ion adsorption processes, and as discussed later, these interactions are key for a correct interpretation of multi-component interactions. Major progress has been made in recent years in parameterization of the $C D$ model ${ }^{19}$ in combination with the Multi Site lon Complexation (MUSIC) model for $\mathrm{Fh}^{20}$ for describing ion adsorption to freshly prepared Fh nanoparticles. Together with the results from this thesis, the CD model has now been consistently parameterized for the adsorption to Fh of major monovalent electrolyte ions $\left(\mathrm{K}^{+}, \mathrm{Na}^{+}, \mathrm{Li}^{+}, \mathrm{Cl}^{-}, \mathrm{NO}_{3}{ }^{-}, \mathrm{ClO}_{4}\right)^{-20,21}$, as well as the alkaline earth metal cations $\left(\mathrm{Mg}^{2+}, \mathrm{Ca}^{2+}, \mathrm{Sr}^{2+}, \mathrm{Ba}^{2+}, \mathrm{Ra}^{2+}\right)^{22}$, oxyanions such as $\mathrm{PO}_{4}^{3-}, 20$ $\mathrm{H}_{4} \mathrm{SiO}_{4},{ }^{23} \mathrm{CO}_{3}{ }^{2-},{ }^{24} \mathrm{~B}(\mathrm{OH})_{3}$ and the metal cations $\mathrm{Zn}^{2+}$ and $\mathrm{Cu}^{2+}$ (this thesis).

The B adsorption data that was collected in Chapter 4, was modelled with two bidentate inner-sphere and one outer-sphere surface species, based on the interpretation of spectroscopic analysis of B adsorption to metal (hydr)oxides ${ }^{25-27}$. Remarkably, we found that high affinity sites for bidentate double corner-sharing complexes were necessary in order to describe the non-linear adsorption behavior observed in the experimental data. The site density of the high affinity sites that was fitted based on the B adsorption data, was $0.25 \pm 0.08$ sites $\mathrm{nm}^{-2}$. This value is similar to the value that has been found for the adsorption of alkaline earth metal ions that also form double corner bidentate complexes with singly coordinated groups, being in the order of $0.29 \pm 0.02$ sites $\mathrm{nm}^{-2}{ }^{22}$ For bidentate surface species, this corresponds to a maximal $B$ loading of $0.2 \mu \mathrm{mol} \mathrm{m}^{-2}$. The maximal $B$ surface loading for our experiments was only $0.1 \mu \mathrm{mol} \mathrm{m}{ }^{-2}$, so too low to saturate the high affinity sites to a sufficient level at which additional adsorption to low affinity sites starts to play a role. Hence, the latter could not be revealed by modeling. The maximal measured $\mathrm{B}$ adsorption was only $23 \%$ of the added $\mathrm{B}$, pointing towards the low affinity of $\mathrm{B}$ for adsorption to Fh compared to other oxyanions, as we have clearly illustrated in Chapter 4 when introducing $\mathrm{PO}_{4}$ to the $\mathrm{B}$-Fh systems.

Zinc and $\mathrm{Cu}$ adsorption to Fh nanoparticles was studied in Chapter 5. Our CD modeling calculations for $\mathrm{Zn}$ showed that inclusion of both single and double cornersharing surface species, resulted in a good description of the experimental adsorption data, and predicts well the decrease in the $\mathrm{Zn}-\mathrm{Fe}$ coordination number with increasing loadings as observed by X-ray absorption spectroscopy (XAS) ${ }^{28}$. For $\mathrm{Cu}$, the adsorption data could be modeled with a bidentate edge-sharing complex with the option of being hydrolyzed.

In natural systems such as soils, adsorption processes are governed by the presence of co-occurring ions such as $\mathrm{PO}_{4}$, which is omnipresent and exhibits a high affinity for the adsorption to metal (hydr)oxides such as Fh. In the CD model, competitive and synergistic adsorption interactions to Fh by electrostatics mainly occur via charge attribution to the Stern plane $\left(\Delta z_{1}\right)$. As a result, the choice of surface species and corresponding $C D$ values affect the modeled adsorption in multi- 
component systems. This has been illustrated in Chapter 4 for B-Fh systems with the addition of $\mathrm{PO}_{4}$. With increasing $\mathrm{PO}_{4}$ loadings, the $\mathrm{B}$ surface speciation becomes dominated by the outer-sphere complex, which does not introduce any charge in the 1-plane.

Similarly, the choice of surface species greatly affect cooperative interactions of cations in the presence of anions such as $\mathrm{PO}_{4}$. Based on our $\mathrm{CD}$ modeling, we found that $\mathrm{Zn}$ adsorption in the presence of $\mathrm{PO}_{4}$ is mainly enhanced because of an increased adsorption of the non-hydrolyzed surface species that introduces a positive charge to the 1-plane. Interestingly, we did not find evidence for the formation of ternary $\mathrm{Zn}-\mathrm{PO}_{4}$ complexes on Fh. Only at extreme $\mathrm{Zn}$ and $\mathrm{P}$ loadings, an additional surface species was necessary for explaining the multi-component adsorption data, and the modeling suggested a Zn-bridged ternary complex. For comparison, we re-interpreted the $\mathrm{Cu}$ adsorption data from Tiberg et al. ${ }^{29}$ to $\mathrm{Fh}$ in the absence and presence of $\mathrm{PO}_{4}$, using similar loadings as our experiments for $\mathrm{Zn}$. With our CD modeling, we could not reveal the presence of a ternary $\mathrm{Cu}-\mathrm{PO}_{4}$ surface species, in line with what we have found for $\mathrm{Zn}$ : the enhanced $\mathrm{Cu}$ adsorption in the presence of $\mathrm{PO}_{4}$ was mainly a result of electrostatic interactions. Based on our results for $\mathrm{Zn}$ and $\mathrm{Cu}$, we conclude that electrostatic interactions explain metal adsorption at background levels in multi-component systems, and that ternary complexes may form only at rather extreme metal or $\mathrm{PO}_{4}$ loading conditions. The analyses in Chapter 5 have illustrated that conclusions with regard to ternary complex formation in modeling multi-component systems, depend largely on the electrostatic model, the surface species and $C D$ values. If the electrostatic part of the ion adsorption model is weakly described, for example when the GTLM is used, careful conclusions should be drawn with regard to ternary complex formation in multi-component systems ${ }^{30}$.

\subsection{Solid-solution partitioning of micronutrients in tropical soils}

In Chapter 7 of this thesis, the solid-solution partitioning of $\mathrm{Zn}, \mathrm{Cu}$ and $\mathrm{B}$ was measured experimentally for 172 agricultural soil samples from Rwanda, Burundi and Kenya, and modeled using a geochemical multi-surface model that included ion adsorption to soil organic matter, metal (hydr)oxides and clay minerals. The measured solid-solution partitioning, expressed as the distribution coefficient or $K_{d}$ value, showed that the distribution coefficient for $B$ is generally lower than for $\mathrm{Cu}$, and for $\mathrm{Zn}$ to some extent.

The affinity of soils for B adsorption is in generally low. This was shown by the adsorption experiments in Chapter 4 , in which only a maximum of $23 \%$ of added $B$ was adsorbed to $\mathrm{Fh}$ nanoparticles. In addition, on average $60 \%$ of reactive $\mathrm{B}$ (estimated by the concentration measured in $0.43 \mathrm{M} \mathrm{HNO}_{3}$ divided by two) is 
measured in the $0.01 \mathrm{M} \mathrm{CaCl}_{2}$ extract for the soils analyzed in Chapter 7, which is much higher compared to $\mathrm{Zn}(10 \%)$ and $\mathrm{Cu}(0.40 \%)$. Multi-surface modeling calculations have been done for B in Chapter 4 for a set of Dutch soils, in Chapter 6 for a limited set of tropical and temperate soils, and in Chapter 7 for the 172 soil samples from Burundi, Rwanda and Kenya. These modeling predictions confirm the low affinity of soils for $B$ adsorption, as most of reactive $B$ is predicted to be in the in the solution phase. In addition, the C-Q partition relations derived in chapter 7 showed that the variability of soluble $\mathrm{B}$ measured in a $0.01 \mathrm{M} \mathrm{CaCl}_{2}$ soil extract is mainly controlled by the reactive concentration which may be explained by a low interaction with the reactive surfaces. In terms of speciation, the multi-surface model predicts that the metal (hydr)oxides are most important for $\mathrm{B}$ adsorption at low $\mathrm{pH}$, while soil organic matter becomes more important with increasing $\mathrm{pH}$. This was also confirmed by the positive relationship between the soil organic carbon content and the experimentally observed decrease in boron concentration with increasing $\mathrm{pH}$ for the 10 soils studied in Chapter 6 . Based on this relationship, one may expect for a soil with $30 \mathrm{~g} \mathrm{~kg}^{-1}$ soil organic carbon, that the B solubility will decrease with about $0.46 \mu \mathrm{mol} \mathrm{L}^{-1}$ per unit of $\mathrm{pH}$ increase. In line with these results, we found in Chapter 7 that $\mathrm{pH}$ and SOM positively contribute to the solid-solution partitioning of $\mathrm{B}$ in the $K_{f}$ partition relations.

Generally, the retention of $\mathrm{Cu}$ in the solid phase is the highest for all three micronutrients studied. This is reflected by the highest experimental $K_{d}$ values, and predicted by the multi-surface modeling calculations from Chapter 7 for the soils from Burundi, Rwanda and Kenya. In line with previous findings, the soil organic matter content, both in the solid and solution phase, is most important for controlling $\mathrm{Cu}$ speciation and solid-solution partitioning in soils. Only for soils with $\mathrm{pH}>7$, the $\mathrm{Fe}$ and $\mathrm{Al}$ (hydr)oxides start playing an important role for $\mathrm{Cu}$ adsorption in the solid phase according to the multi-surface model. The $K_{d}$ of $\mathrm{Cu}$ was positively related to the SOC content, and negatively related to the DOC concentrations. The relation between $\mathrm{Cu}-K_{d}$ and SOC solubility, expressed as the DOC/SOC ratio, was the strongest, showing that both organic carbon fractions control simultaneously the $\mathrm{Cu}$ solid-solution partitioning. Similarly as for $\mathrm{B}$, based on the partition relations it was found that the variability in $\mathrm{Cu}$ solubility is mainly explained by the reactive concentration. For B, it was suggested that weak interaction with the soil reactive surfaces explains this result. For $\mathrm{Cu}$, this explanation is not valid, since the majority of reactive $\mathrm{Cu}$ is present in the solid phase. Instead, these results for $\mathrm{Cu}$ may be explained by the limited variability observed in $K_{d}$, which in turn may be related to the limited variability observed in the solid-solution partitioning of SOC as shown by Figure 1.

The opposite was found for $\mathrm{Zn}$ : there is a large variability in $K_{d}$ values among the soils that have been analyzed in Chapter 7 , and this variability is almost completely 
explained by $\mathrm{pH}$. The multi-surface modeling calculations showed that $\mathrm{Zn}$ speciation is dominated by the adsorption to the soil organic matter and with increasing $\mathrm{pH}$, also to metal (hydr)oxides, which is the opposite trend as what was found for $\mathrm{B}$. The dissolved organic carbon did not play an important role for $\mathrm{Zn}$ speciation, similarly as for $\mathrm{B}$.

In Chapter 7, we showed the important soil chemical processes that control the solidsolution partitioning of the three micronutrients in soils from the tropics with a multisurface model, and used this knowledge as benchmark to develop partition relations that require less input data and are more accessible tools for predicting micronutrient availability based on existing soil data. In Section 3.2 of this chapter, I will illustrate how these relations can be used for making spatial predictions of micronutrient solubility, based on currently available soil data.

\section{LIMITATIONS OF THIS STUDY AND FUTURE RESEARCH}

\subsection{Multi-surface model for micronutrients}

Multi-surface modeling was used as a tool in Chapters 6 and 7 to study the soil chemical processes that control the environmental micronutrient availability in soils. The results from the foregoing chapters were used for defining the modeling set-up and for the inclusion of ion-specific modeling parameters. However, the modeling predictions in Chapter 7 showed systematic deviations, especially for $\mathrm{Zn}$ and B, which limits a correct interpretation of the calculated micronutrient speciation by the model. For $\mathrm{Zn}$, we found an increased overestimation of the soluble concentration, with increasing $\mathrm{pH}$, increasing reactive $\mathrm{Zn}$ and increasing $\mathrm{Zn}$ loadings per mass of soil organic matter or metal (hydr)oxides. For most soil samples, the $B$ in solution was overestimated by the multi-surface model without a clear relation between the modeling error and soil variables.

As George E.P Box stated in 197631: "Since all models are wrong, we cannot obtain a "correct" one by excessive elaboration. On the contrary, we should seek an economical description of natural phenomena and be alert to what is importantly wrong." So if we want to improve geochemical multi-surface model calculations for complex soil systems, we need to identify those aspects that lead to major improvements in the modeling calculations when modified. An uncertainty analysis can be a valuable tool for doing $\mathrm{So}^{15}$ based on a thorough analysis of the uncertainties and simplifications present in geochemical modeling applications.

A geochemical multi-surface model is characterized by assumptions with regard to the input side, the modeling set-up and the adsorption parameters. The validity of these assumptions can be questioned, and may have implications on the final model calculations. 


\subsubsection{Errors associated with input variables}

The input of a geochemical multi-surface model consists of (1) the reactive concentration of the ion of interest (2) the solution composition like $\mathrm{pH}$, ionic strength and co-occurring ions that are expected to be important for competitive or cooperative adsorption effects (3) the type, amount and reactivity of the adsorption surfaces.

In Chapter 6, we have discussed the use of a $0.43 \mathrm{M} \mathrm{HNO}_{4}$ soil extraction for quantifying reactive $\mathrm{B}$ in soils. The use of $\mathrm{HNO}_{3}$-extractable $\mathrm{B}$ concentration as reactive input results in an overestimation of soluble $B$ by the multi-surface model. This was also suggested by the modeling calculations for a larger set of soil samples in Chapter 7. In Chapter 6, total B was measured for 10 selected Dutch and Burundi soil samples. These results showed that the fraction of reactive $B$ was only between 2 and $5 \%$ of total $\mathrm{B}$. This percentage is much lower than the average fraction of total $\mathrm{Zn}$ and $\mathrm{Cu}$ that is extracted by $0.43 \mathrm{M} \mathrm{HNO}_{3}$, which has been previously found to be around $40-50 \%$ for a set of Dutch soils ${ }^{1}$. So when most of total B is occluded in minerals ${ }^{32}$, progressive dissolution of these minerals that takes place in a $0.43 \mathrm{M}$ $\mathrm{HNO}_{3}$ soil extraction ${ }^{1}$ can have a relatively large impact on the measured $\mathrm{B}$ concentration. In Chapter 6, we have shown that weaker extraction methods using for example $\mathrm{PO}_{4}$ as competing anion, can be a better alternative for estimating reactive $B$ in soils.

Next to the input of the reactive concentrations, the amount of surfaces for adsorption, together with a quantification of their reactivity, is needed. Under the $1^{\text {st }}$ objective, we have found in Chapter 2 that Fh nanoparticles are a good representation of the reactive natural metal (hydr)oxide fraction in tropical topsoils based on the adsorption of natural $\mathrm{PO}_{4}$. It was rather remarkable that despite the high presence of Al metal (hydr)oxide nanoparticles, the surface reactivity of these soils for $\mathrm{PO}_{4}$ adsorption can still be adequately described using $\mathrm{Fh}$ as single proxy for the diverse and Al-dominated natural metal (hydr)oxides. Previous studies have shown that the nanocrystalline $\mathrm{Al}$ (hydr)oxides adsorb more $\mathrm{PO}_{4}$ than $\mathrm{Fh}$ per mol metal ion ${ }^{33,34}$. Interestingly, the adsorption isotherms of $\mathrm{PO}_{4}$ for both metal (hydr)oxide nanoparticles presented in these previous studies ${ }^{33,34}$ had a similar shape. It is unknown whether the adsorption isotherms remain similar in shape in the presence of $0.5 \mathrm{M} \mathrm{NaHCO}_{3}$, and how the presence of Al finally affects the outcome of the probe-ion method. The good agreement between inorganic $\mathrm{PO}_{4}$ measured in an ammonium oxalate extraction, and the reversibly bound $\mathrm{PO}_{4}$ calculated when using Fh as model oxide, led to the conclusion that Fh is a good representation of the natural reactive metal (hydr)oxides in the tropical weathered soils. These results suggests that $\mathrm{Al}$ and $\mathrm{Fe}$ metal (hydr)oxide nanoparticles have a similar affinity for $\mathrm{PO}_{4}$ adsorption. A comparison of the adsorption isotherms of $\mathrm{PO}_{4}$ 
to both $\mathrm{Fe}$ and $\mathrm{Al}$ metal (hydr)oxide nanoparticles in the presence of $0.5 \mathrm{M} \mathrm{NaHCO}_{3}$, would still be interesting to assess how the presence of Al metal (hydr)oxides affects the RSA derived with the probe-ion method using Fh as model oxide.

The probe-ion method is based on the metal (hydr)oxide reactivity for $\mathrm{PO}_{4}$. It can be questioned whether the results from the probe-ion method can be directly used for modeling the solid-solution partitioning of other elements in soils. Based on the results from Chapter 2, we have used $\mathrm{Fh}$ as model oxide for the natural metal (hydr)oxide fraction for the soils analyzed in Chapter 7. However, if $\mathrm{Zn}, \mathrm{Cu}$ or B do exhibit a distinct adsorption behavior to $\mathrm{Fe}$ and $\mathrm{Al}$ (hydr)oxide nanoparticles in contrast to $\mathrm{PO}_{4}$, the use of only $\mathrm{Fh}$ as model oxide might be problematic. In addition, other metal (hydr)oxides that are not important for $\mathrm{PO}_{4}$ adsorption, may affect the solid-solution partitioning of these micronutrients. Preliminary modeling results in Chapter 7 using GTLM, have shown that manganese oxides may play a role for $\mathrm{Zn}$ speciation. It may still be necessary to assess how the presence of $\mathrm{Al}$ and $\mathrm{Mn}$ oxide materials affects the application of the probe-ion method in soil systems for other ions than $\mathrm{PO}_{4}$.

For the multi-surface modeling calculations in Chapters 6 and 7, we did not use the RSA derived by the probe-ion method, but instead we estimated the RSA based on the amount of $\mathrm{Fe}$ and $\mathrm{Al}$ measured by $\mathrm{AO}$ and dithionite extractions, with a set of "standard" molar mass and specific surface area for the crystalline and nanocrystalline metal (hydr)oxide fraction as done previously 1,16,35,36. However, in Chapter 2 we have shown that the SSA and corresponding molar mass of the natural metal (hydr)oxides varies greatly between soils, and that this simplification is thus incorrect. For most of the soils analyzed in Chapter 2, the calculated RSA based on "standard values", was below the RSA derived by the probe-ion method. Using this fixed SSA may therefore lead to underestimation of the adsorption to metal (hydr)oxides for B, and for $\mathrm{Zn}$ in non-acidic soils.

Similarly, we have used a fixed percentage of humic and fulvic acids of the solid and dissolved organic matter, based on average values found by fractionation experiments for a limited set of soils. However, the results from the latter have shown that the fraction of reactive organic matter can vary with more than $20 \%$. This simplification is therefore invalid, and may have serious implications on the calculated speciation for all three micronutrients. Based on the limited number of samples that were used for measuring the reactive organic matter in Chapter 7 , we found that the fraction of reactive compounds increases with higher levels of SOC and DOC, and that there was an effect of $\mathrm{pH}$ and the amount of metal (hydr)oxides. Further research may improve these relations by using more datapoints, which in turn may be used in modeling applications for estimating the soil-specific fraction of reactive SOM without the need for fractionation experiments. 


\subsubsection{Errors associated with modelling set-up}

With regard to the modeling set-up, we have used a component additivity approach, which assumes that the different reactive surfaces in the multi-surface model do not mutually interact in such a way that it affects ion adsorption processes ${ }^{37}$. One may question this assumption, based on the close interaction between the metal (hydr)oxides and the soil organic matter that we have shown in Chapter 3 of this thesis. For oxyanions with a strong affinity for metal (hydr)oxides such as $\mathrm{PO}_{4}$, previous studies have clearly demonstrated that the competitive effect of the negatively charged soil organic matter at the oxide surfaces needs to be taken into account for a correct description of the $\mathrm{PO}_{4}$ solid-solution partitioning ${ }^{38-40}$. For cations, such as $\mathrm{Ca}$ and $\mathrm{Cu}$, batch experiments have shown that the component additivity approach does not work for describing metal cation adsorption processes in systems with both metal (hydr)oxides and organic matter ${ }^{41,42}$. Due to the presence of SOM, the surface of the metal (hydr)oxides becomes more negatively charged, thereby enhancing the adsorption of metal cations. In addition, the formation of ternary complexes has also been proposed ${ }^{41}$. This may lead to more $\mathrm{Zn}$ adsorption to metal (hydr)oxides with increasing $\mathrm{pH}$ than expected based on a linear additivity approach ${ }^{41}$, and could explain the overestimation of soluble $\mathrm{Zn}$ with increasing $\mathrm{pH}$ by the multi-surface model in Chapter 7 .

\subsubsection{Errors associated with modelling parameters}

The systematic multi-surface modeling deviation for $\mathrm{Zn}$ with increasing $\mathrm{pH}$ and surface loadings, has been previously attributed to uncertainties in modelling parameters of the NICA-Donnan model for $\mathrm{Zn}$ adsorption to SOM. Klinkert and Comans $^{9}$ found an overestimation of soluble $\mathrm{Zn}$ with increasing $\mathrm{Zn}$ loadings (per mass of reactive SOM), when using multi-surface modelling calculations for the assessment of $\mathrm{Zn}$ speciation in 4 composts. We have found the same modeling deviation for the 172 tropical soil samples analyzed in Chapter 7 . The relation between $\mathrm{Zn}$ loadings and modelling deviations for the compost samples was partly attributed to limitations of the electrostatic part of the NICA-Donnan model for FA that is used for modelling ion adsorption to soil organic matter ${ }^{9}$. The electrostatic part of the NICA-Donnan model is based on an ionic-strength dependent Donnan volume, in which charge neutralization of the humic substance takes place ${ }^{43,44}$. It has been suggested that the calculated Donnan volume is unrealistically large, especially for the relatively small fulvic acids ${ }^{45,46}$. Klinkert and Comans ${ }^{9}$ argued that this large Donnan volume results in an overestimation of the electrostatic binding component, and as a result, an overestimation of the competitive effect of $\mathrm{Ca}$ for $\mathrm{Zn}$ adsorption ${ }^{9}$. I have therefore tested the effect of imposing a smaller Donnan volume for the particulate humic (HA) and fulvic acids (FA), on the calculated soluble concentration of $\mathrm{Zn}$ in the soils from Chapter 7. To do so, a constant Donnan volume of $1 \mathrm{~L} \mathrm{~kg}^{-1}$ for the solid FA and HA was used, as it was proposed by Gustafsson and $\mathrm{Kleja}^{47}$ based 
on the experimentally observed smaller effect of salt level on the Donnan volume of particulate SOM compared to DOM. This value of $1 \mathrm{~L} \mathrm{~kg}^{-1}$ is smaller than the Donnan volume for $\mathrm{HA}$ and $\mathrm{FA}$ in the $0.01 \mathrm{M} \mathrm{CaCl}_{2}$ extraction that is calculated based on the salt level (i.e. $\sim 1.7$ and $\sim 2.7 \mathrm{~L} \mathrm{~kg}^{-1}$ respectively). As a result, $\mathrm{Zn}$ adsorption increases and $\mathrm{Zn}$ solubility decreases, but the effect is small (i.e. $-0.05 \pm 0.03 \log \mathrm{M} \mathrm{Zn}$ in solution), rather constant and thereby does not remove the systematic trend between prediction error and $\mathrm{pH}$ or $\mathrm{Zn}$ loading as observed in Chapter 7 when using the saltdependent Donnan volume.

According to the required charge neutrality in the Donnan phase, a smaller volume results in a more negative Donnan potential ${ }^{44}$. A lower Donnan potential enhances the adsorption of divalent cations (i.e. $\mathrm{Ca}^{2+}$ and $\mathrm{Zn}^{2+}$ ) over protons, resulting in lower proton competition and more $\mathrm{Zn}$ and $\mathrm{Ca}$ adsorption. But the competitive effect of $\mathrm{Ca}$ on $\mathrm{Zn}$ adsorption does not change with a different Donnan volume or potential. This is also shown in Figure 2 for pure systems with HA or FA, where a lower Donnan volume results in higher $\mathrm{Zn}$ adsorption due to an enhanced specific adsorption to carboxylic and phenolic groups of the humic and fulvic acids.

Figure 2 shows the $\mathrm{Zn}$ surface speciation on FA and $\mathrm{HA}$ in terms of $\mathrm{pH}$, in the absence and presence of $\mathrm{Ca}$, using the generic parameters from Milne et al. ${ }^{10}$. The main part of $\mathrm{Zn}$ adsorbed to FA is predicted to be electrostatically bound in the Donnan phase. With increasing $\mathrm{pH}, \mathrm{Zn}$ adsorption to HA and FA increases (Figure 2). However, in the presence of $\mathrm{Ca}$, the $\mathrm{Zn}$ adsorption to $\mathrm{FA}$ increases until pH $~ 5.5$, and decreases when $\mathrm{pH}$ is higher than 6.5 due to competition of $\mathrm{Ca}$ on $\mathrm{Zn}$ adsorption to the carboxylic functional groups and in the Donnan phase. For HA, $\mathrm{Zn}$ is mainly adsorbed to carboxylic groups, but due to specific competition of $\mathrm{Ca}$ on these functional groups, the increase in $\mathrm{pH}$ dependent $\mathrm{Zn}$ adsorption reduces above $\mathrm{pH}$ $\sim 5.5$. The $\mathrm{pH}$ region in which the competitive effect of $\mathrm{Ca}$ on $\mathrm{Zn}$ adsorption becomes important in pure systems of $\mathrm{HA}$ and $\mathrm{FA}$, coincides with the $\mathrm{pH}$ region in which the multi-surface model underestimates $\mathrm{Zn}$ adsorption for the soils in Chapter 7. Based on these results, it can be hypothesized that the use of the generic parameters ${ }^{10}$ results in a overestimation of the competitive effect of $\mathrm{Ca}$ on, mainly specific, $\mathrm{Zn}$ adsorption to $\mathrm{HA}$ and FA with increasing $\mathrm{pH}$. Although the NICA-Donnan parameters predict that the largest part of the $\mathrm{Ca}$ adsorbed to HA and FA is electrostatically bound $^{9}$, the competitive effect of $\mathrm{Ca}$ on $\mathrm{Zn}$ adsorption is mainly a result of the (smaller part of) specifically adsorbed $\mathrm{Ca}$ to the carboxylic groups (Figure 2). Milne et al. ${ }^{10}$ used a relatively limited set of datapoints to derive NICA parameters for $\mathrm{Zn}$ adsorption to $\mathrm{HA}(\mathrm{n}=35)$ and $\mathrm{FA}(\mathrm{n}=51)$, with only one competition experiment with $\mathrm{Ca}$ on $\mathrm{HA}^{48}$. The inclusion of additional competition experiments are necessary to test the accuracy of the current generic parameters for modelling multi-element systems with relatively high Ca background concentrations. Recent criticism on the NICA-Donnan model goes even further in which the existence of specific adsorption 
of $\mathrm{Ca}$ to the functional groups of humic substances is questioned ${ }^{46,49}$, which may also be a possible cause for an overestimation of the $\mathrm{Ca}$ competition on $\mathrm{Zn}$ binding.

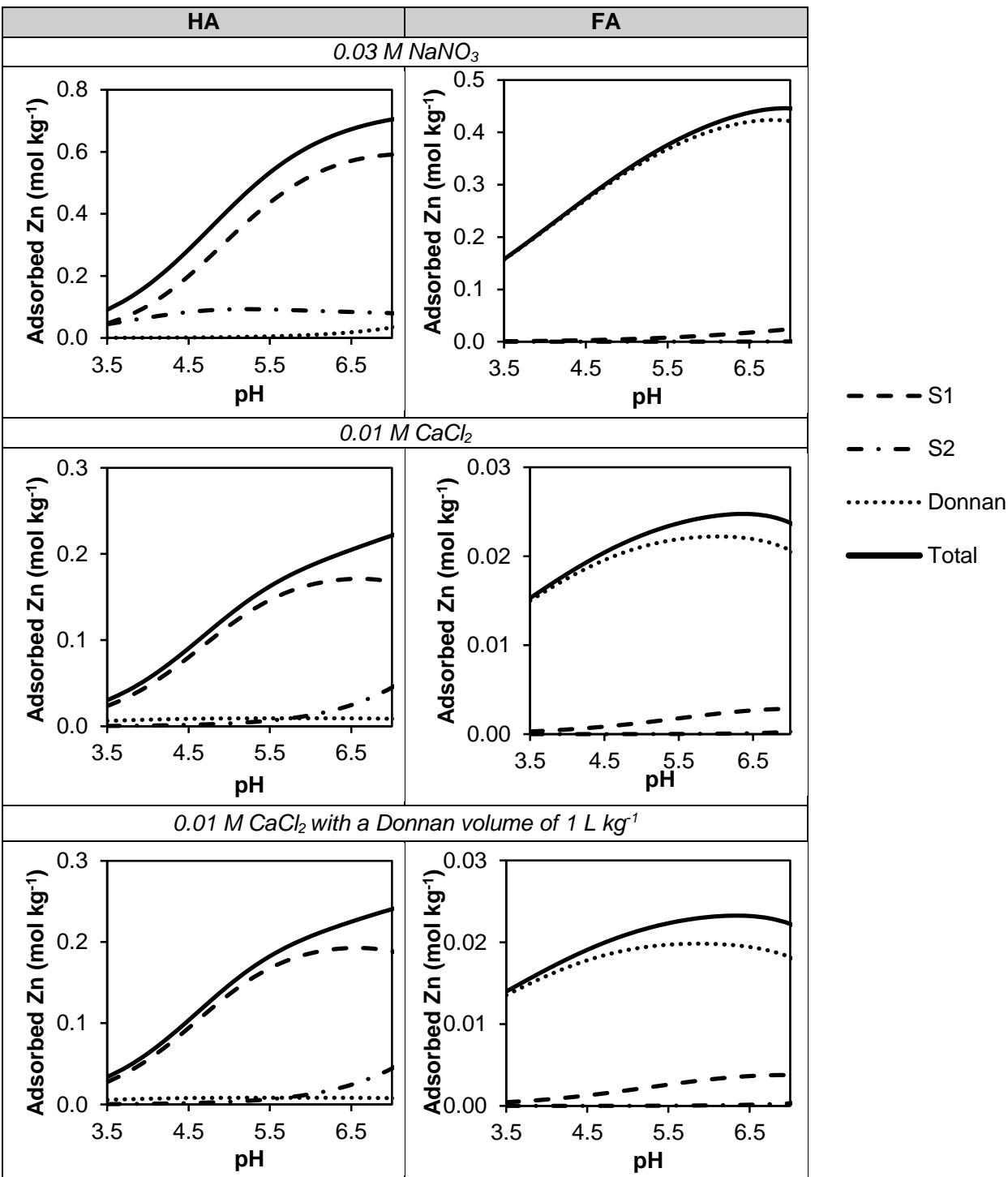

Figure 2: $\mathrm{Zn}$ adsorption to humic acids (HA) and fulvic acids (FA) in a $0.03 \mathrm{M} \mathrm{NaNO}_{3}$ and $0.01 \mathrm{M} \mathrm{CaCl}_{2}$ background electrolyte with total $\mathrm{Zn}$ of $0.5 \mathrm{~mol} \mathrm{~kg}^{-1}$. In the lower panel, $\mathrm{Zn}$ adsorption is calculated in a $0.01 \mathrm{M} \mathrm{CaCl}_{2}$ but with a constant Donnan volume of $1 \mathrm{~L} \mathrm{~kg}^{-1}$. Zn is adsorbed to the carboxylic groups (S1) or phenolic groups (S2) of the HA and FA, or it is electrostatically bound in the Donnan phase. The modeling calculations were done with the adsorption parameters from Milne et al. ${ }^{10}$, using a total concentration of $0.1 \mathrm{mM} \mathrm{Zn}$ and $0.1 \mathrm{~g} \mathrm{~L}^{-1} \mathrm{HA}$ or FA. 


\section{2 $\quad$ A $0.01 \mathrm{M} \mathrm{CaCl}_{2}$ soil extraction as proxy for the soil solution}

The availability of nutrients for plant uptake depends on their chemical speciation in soils: plants take up nutrients directly from the soil solution, which is replenished through desorption or dissolution from the solid phase. In Chapters 6 and 7 of this thesis, a $0.01 \mathrm{M} \mathrm{CaCl}_{2}$ soil extraction was used as proxy for the concentration of $\mathrm{Zn}$, $\mathrm{Cu}$ and $\mathrm{B}$ in the soil solution. In the following section, this assumption is challenged by comparison with actual soil pore water measurements.

Since plants take up nutrients from the soil solution, weak salt extracts that mimic the soil solution composition have since long been used as indicators of soil nutrient availability for plants. Houba et al. ${ }^{50}$ introduced a commonly used method in which soils are extracted with $0.01 \mathrm{M} \mathrm{CaCl}_{2}$, to approximate the ionic strength and $\mathrm{Ca}$ concentration of the actual soil solution. A number of studies have shown that the nutrient concentration measured in this $0.01 \mathrm{M} \mathrm{CaCl}_{2}$ relates to plant uptake of, for example, $\mathrm{Zn}$ and $\mathrm{Cu}^{51-53}$.

However, actual pore water analyses have resulted in Ca concentrations below 0.01 $\mathrm{M}$, suggesting the use of lower $\mathrm{Ca}$ concentrations in the extractant ${ }^{54,55}$. The choice of the molar concentration of $\mathrm{CaCl}_{2}$ extraction might affect the nutrient solubility in three ways: (1) it determines the ionic strength, which can affect ion binding to surfaces (2) The $\mathrm{Ca}$ acts as a competitor for adsorption sites and can thereby enhance solubility ${ }^{56,57}$ (3) The presence of $\mathrm{Ca}$ can lead to coagulation of DOC ${ }^{58}$, which may consequently result in lower nutrient concentrations in the extraction solution. The effect of $\mathrm{Ca}$ concentration in the soil extract on the measured $\mathrm{Zn}$ and $\mathrm{Cu}$ concentrations for 15 acidic topsoil samples from Burundi $\left(\mathrm{pH}\left(\mathrm{CaCl}_{2}\right)\right.$ between 4.1 and 4.9), is shown in Figure 3. In general, higher Ca levels in the extraction result in higher $\mathrm{Zn}$ concentrations due to competition effects, while the opposite is true for $\mathrm{Cu}$. The effect of $\mathrm{Ca}$ on Cu solubility can be related to the decrease in DOC concentrations due to the coagulation effect of increased Ca levels, as also shown in Figure 3. These results confirm the higher importance of DOC for Cu compared to Zn speciation from Chapter 7 of this thesis.

Also shown in Figure 3 is that the competitive effect of $\mathrm{Ca}$ on $\mathrm{Zn}$ is larger than the effect of $\mathrm{Ca}$ on DOC concentrations, and consequently on the measured $\mathrm{Cu}$ concentrations for these tropical topsoils. Interestingly, Weng et al. ${ }^{58}$ showed that an increase in Ca concentration from 0.001 to $0.01 \mathrm{M}$, led to a sharp decrease in humic acid concentrations due to coagulation effects, while the effect for total DOC is rather small in these Burundi soils. One explanation can be that the DOC is not dominated by humic acids but by fulvic acids (see results Chapter 7 ), which are more soluble and less susceptible for coagulation in the presence of cations ${ }^{58}$. In addition, the concentrations of DOC and the reactive fractions for these tropical topsoils, are several factors lower than the concentrations applied in the coagulation experiments 
by Weng et al. ${ }^{58}$ The lower concentrations imply a lower collision frequency ${ }^{59}$, thereby resulting in less aggregation due to increased $\mathrm{Ca}$ levels. As a result, the difference between the $\mathrm{DOC}$ and $\mathrm{Cu}$ concentrations measured 0.01 and $0.001 \mathrm{M}$ $\mathrm{CaCl}_{2}$ soil extracts, is relatively small.
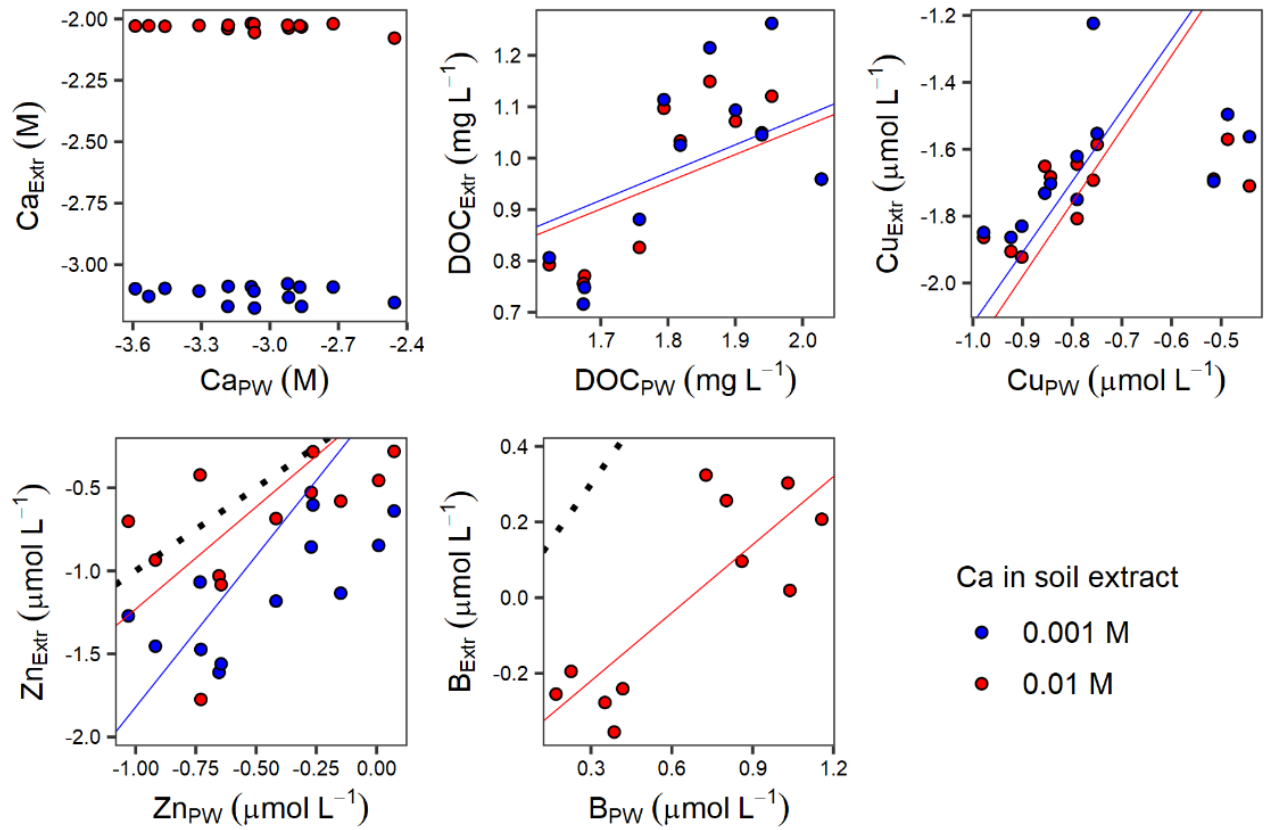

Ca in soil extract

- $0.001 \mathrm{M}$

- $0.01 \mathrm{M}$

Figure 3: Relationships between the concentrations of $\mathrm{Ca}$, dissolved organic carbon (DOC), $\mathrm{Zn}, \mathrm{Cu}$ and $B$ measured in the pore water extracts (x-axis, PW) and the soil extractions ( $y$-axis, Extr) with $0.001 \mathrm{M}$ (blue markers) or $0.01 \mathrm{M}$ (red markers) $\mathrm{CaCl}_{2}$ concentrations. Note that the concentrations are shown on the $\log 10$ scale. The black dotted line represents the 1:1 line, and is shown in figures with the appropriate $x$ and $y$ range. The blue and red lines are the linear models between the pore water extracts and the 0.01 $\mathrm{M}$ and $0.001 \mathrm{M} \mathrm{CaCl}_{2}$ extractions respectively.

From the same 15 topsoil samples from Burundi, pore water was collected. The soil samples were brought to field capacity (on average $0.25 \mathrm{~L} \mathrm{~kg}^{-1}$ ), and left for multiple days. Afterwards, the samples were used for ultracentrifugation and subsequent collection of pore water. After filtration, the pore water was analyzed for $\mathrm{Ca}, \mathrm{Zn}, \mathrm{Cu}$, $\mathrm{B}$ and DOC. As shown by Figure 3, there is for all three micronutrients a relationship between the concentrations measured in the $\mathrm{CaCl}_{2}$ extractions, and the actual pore water concentrations. In line with previous findings ${ }^{54}$, the $\mathrm{Ca}$ concentration in the pore water samples varied around $0.001 \mathrm{M}$ which is lower than the standard $\mathrm{CaCl}_{2}$ extraction procedure ${ }^{50}$. As such, a $0.01 \mathrm{M} \mathrm{CaCl}_{2}$ extraction induces a higher $\mathrm{Ca}$ concentration compared to actual pore water samples. In addition, the high solutionto-solid ratio of $10 \mathrm{~L} \mathrm{~kg}^{-1}$ that is employed in the $\mathrm{CaCl}_{2}$ extraction protocoloo, leads to sample dilution compared to pore water extraction, but meanwhile promotes desorption of elements from the solid phase, thereby changing the solid-solution partitioning. The combination of aforementioned effects does not lead to the same 
results for $\mathrm{Zn}, \mathrm{Cu}$ and $\mathrm{B}$ as shown by Figure 3. For $\mathrm{Cu}$, on average 10 times higher concentrations are measured in pore water samples compared to both $\mathrm{CaCl}_{2}$ extractions, which is similar for DOC. The difference between pore water samples and $\mathrm{CaCl}_{2}$ extraction becomes smaller for $\mathrm{B}$ ( $\sim 5$ times) and for $\mathrm{Zn}$ ( $\sim 2.5$ and 5 times for 0.01 and $0.001 \mathrm{M} \mathrm{CaCl}_{2}$ respectively). For these two nutrients, desorption and/or Ca competition because of the higher Ca concentrations in the soil extract, seems to be more important.

I have used the same multi-surface modelling approach as in Chapter 7 but now with a solution-to-solid ratio of $0.25 \mathrm{~L} \mathrm{~kg}^{-1}$ instead of $10 \mathrm{~L} \mathrm{~kg}^{-1}$ to assess the micronutrient speciation at field capacity. In these modelling calculations, the concentration of $\mathrm{Ca}$ and other competing cations was kept the same as measured in the $0.01 \mathrm{M} \mathrm{CaCl}_{2}$ extraction. Moving from field capacity to a soil extraction with a solution-to-solid ratio of 10 , a dilution factor of 40 is imposed. This results in lower concentrations $\left(\mathrm{L}^{-1}\right)$ and the dilution effect is most pronounced for $\mathrm{Cu}$, followed by $\mathrm{B}$ and $\mathrm{Zn}$ (See Figure 4 for a selection of soils from Chapter 7). It must be noted that additional DOC release in the $\mathrm{CaCl}_{2}$ extraction due to the higher solution-to-solid ratio was not taken into account, which might play a role on the nutrient solubility, especially for $\mathrm{Cu}$. Upon dilution, a significant proportion of reactive $\mathrm{Zn}$ and $\mathrm{B}$ is desorbed from the solid phase, thereby reducing the dilution effect, which is less the case for $\mathrm{Cu}$ (Figure 4). For $\mathrm{Zn}$, the desorption from the solid phase is most pronounced with increasing $\mathrm{Zn}$ loadings per reactive surface (i.e. SOM and metal (hydr)oxides). However, when expressed relative to the total reactive content, upon dilution, there is a large increase in the contribution of soluble $\mathrm{Zn}$ in soils with low $\mathrm{pH}$, as shown by Figure 4 . For $\mathrm{B}$, there is less a relation with $\mathrm{pH}$ but it can be seen in Figure 4 that upon dilution, a large fraction of reactive $B$ ends up in the solution phase. Related to the lower binding strength, $\mathrm{Zn}$ and $\mathrm{B}$ are more easily desorbed from the solid phase when the solution-to-solid ratio is increased compared to $\mathrm{Cu}$, which explains why relatively higher $\mathrm{Zn}$ and $\mathrm{B}$ than $\mathrm{Cu}$ concentrations are found in the $\mathrm{CaCl}_{2}$ extractions compared to pore water as I have shown in Figure 3. 

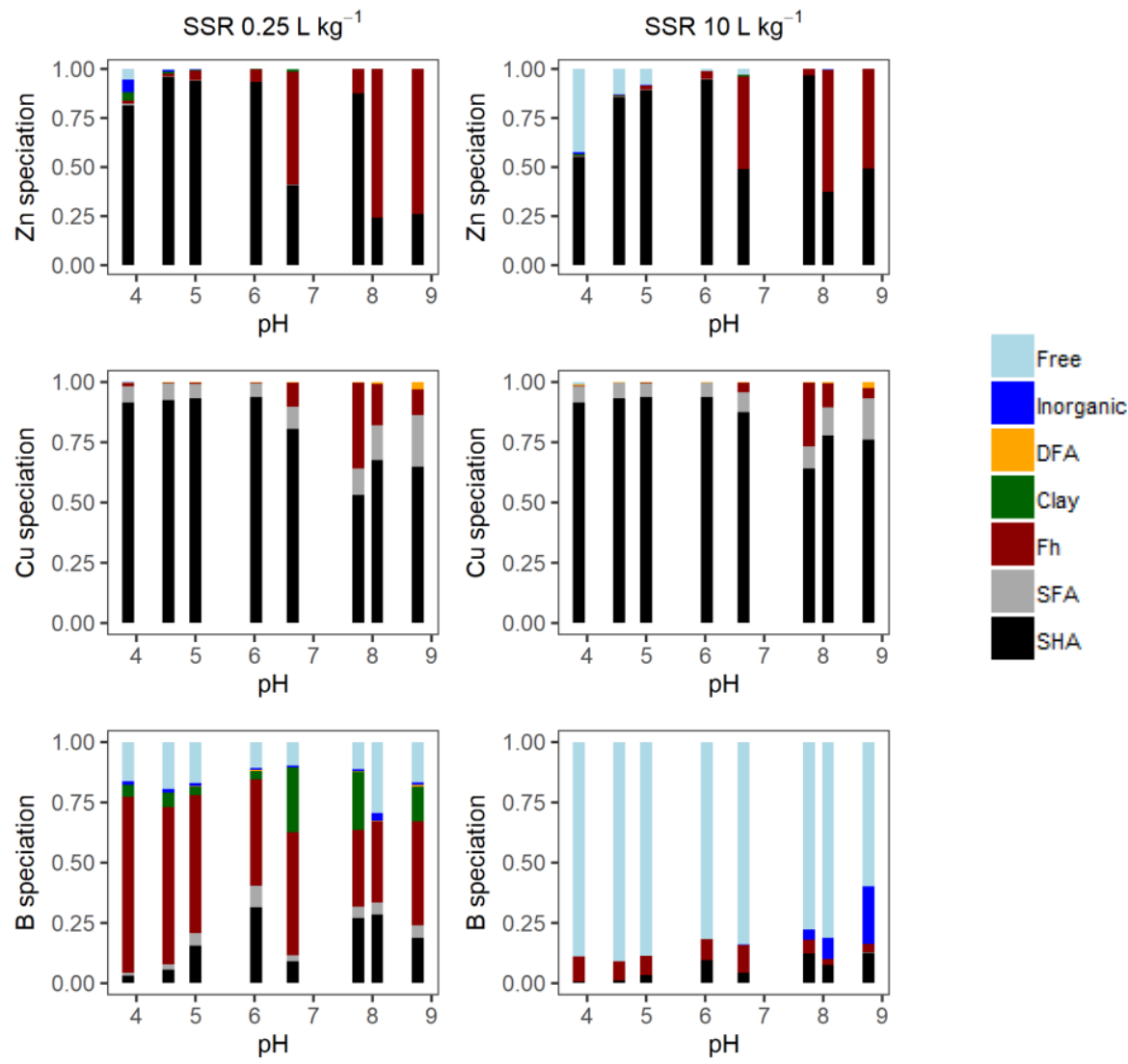

Figure 4: The speciation of $\mathrm{Zn}, \mathrm{Cu}$ and $\mathrm{B}$ calculated by a multi-surface model for a $0.01 \mathrm{M} \mathrm{CaCl}_{2}$ extraction at different solution-to-solid ratios (SSR), namely $0.25 \mathrm{~L} \mathrm{~kg}^{-1}$ and $10 \mathrm{~L} \mathrm{~kg}^{-1}$. The modeling calculations were done for a subset of soil samples from Chapter 7 to cover a wide $\mathrm{pH}$ range. The micronutrients are present in the solution phase as free ion, inorganic complex or complexeswith dissolved organic carbon (as fulvic acids DFA). In the solid phase, the micronutrients can be adsorbed to clay, metal (hydr)oxides (Fh), or solid organic matter in the form of fulvic acids (SFA) and humic acids (SHA).

\section{PRACTICAL IMPLICATIONS}

\subsection{Future modeling applications}

From a geochemical modeling perspective, this thesis research delivered novel and useful insights for future modeling applications. On the one hand, the CD parameters derived in Chapters 4 and 5 have expanded the existing set of parameters ${ }^{20,22-24,61}$ for modeling ion adsorption to Fh, that can be used consistently in future modeling applications. This widens the applicability of using the CD model for ion adsorption processes to metal (hydr)oxides in multi-surface calculations, instead of using the GTLM that is still mostly used ${ }^{1,9}$. On the other hand, the assessment of the content, composition and reactivity of the metal (hydr)oxides and the soil organic matter in soils from the tropics, may contribute to future modeling applications for these types 
of soils. We conclude that currently, using $\mathrm{Fh}$ as model oxide for describing the reactivity of the natural metal (hydr)oxide fraction, is the best choice based on what we have found in Chapter 2 with regard to the reactivity towards $\mathrm{PO}_{4}$. In addition, our results suggest that caution should be made when taking "standard" estimations of reactive soil organic matter based on previous measurements for soils from temperate regions, since we have generally found lower fractions of reactive SOM and DOM for the set of tropical soils that was studied in this thesis.

\subsection{Spatial predictions of micronutrient solubility}

A major advantage of partition relations is that that they require mainly basic soil input data, making them suitable for large-scale applications and when limited data is available 62,63 . In 2008, the Africa Soil Information Service (Afsis) project was established to increase available soil data in Africa. This resulted in soil information data of more than 28 thousand sampling locations, that have been used in combination with covariates to make spatial predictions of informative soil properties for agricultural management, including soil organic carbon ${ }^{64}, \mathrm{pH}\left(\mathrm{H}_{2} \mathrm{O}\right)^{64}$, and nutrient concentrations based on Mehlich-3 extractions ${ }^{65}$. Although relevant for nutrient availability52, no soil information exists for the soluble concentrations of micronutrients in SSA so far, but these could be derived from the reactive concentrations approximated by the Mehlich-3 extraction, if the solid-solution partitioning is known. With help from Bas Kempen (ISRIC) and Mirjam Breure (SBL, WUR), I have used the partition relations from Chapter 7 , to employ existing soil data for making spatial predictions of the $\mathrm{Zn}, \mathrm{Cu}$ and $\mathrm{B}$ concentrations in a $0.01 \mathrm{M} \mathrm{CaCl}_{2}$ soil extract for Rwanda and Burundi. These countries were chosen because of the high availability of national soil data for these two countries ${ }^{66}$ : The Rwanda dataset contained 949 georeferenced datapoints (i.e. 999 minus the 50 points used for validation as explained later), the Burundi dataset $1007^{66}$.

The methodological approach followed different steps (Figure S1). Since no information exists about the dissolved organic carbon concentrations, a first step was to re-calibrate partition relations without DOC, based on the soil samples from Burundi, Rwanda and Kenya from Chapter 7. The results are shown in Table S1. As expected, excluding DOC as input variable reduces the explained variation in the soluble $\mathrm{Cu}$ concentrations by the model.

Secondly, the available soil data were converted into appropriate units and measurements that can be used as input for the partition relations. Therefore, we established relations between the $\mathrm{Zn}, \mathrm{Cu}$ and $\mathrm{B}$ concentrations measured in $\mathrm{HNO}_{3}$ and Mehlich-3, and between the $\mathrm{Fe}$ and $\mathrm{Al}$ measured in the ammonium oxalate extraction and in Mehlich-3. In addition, the $\mathrm{pH}\left(\mathrm{H}_{2} \mathrm{O}\right)$ that was available for the Rwanda and Burundi soil datasets, was converted into corresponding $\mathrm{pH}\left(\mathrm{CaCl}_{2}\right)$ measurements. The results for these transfer functions are shown in Figure S2. The 
explained variance by the transfer functions was always above 0.70 . In general, the $\mathrm{Zn}, \mathrm{Cu}$ and $\mathrm{B}$ measured in Mehlich-3 extractions are lower than the concentrations measured in the $0.43 \mathrm{M} \mathrm{HNO}_{3}$ extraction. For $\mathrm{Cu}$, higher $\mathrm{SOC}$ levels additionally reduce the extraction efficiency of a Mehlich-3 soil extract. For Al, and especially for $\mathrm{Fe}$, the Mehlich-3 extraction also results in lower concentrations compared to an ammonium oxalate extract.

Next, based on these transformed soil data, spatial predictions of the particular soil input variables were made based on random forest (ranger package in R) modeling. The total set of soil data was used in combination with 134 covariates as described in the report by Kempen et al66. The quality of these spatial predictions of the input variables are shown in Table $S 2$. The $\mathrm{R}^{2}$ values show that the spatial models explain $20-60 \%$ of the data, with the largest variation explained for SOM and the Fe and Al (hydr)oxides. These $R^{2}$ values seem rather low, but are similar to the $R^{2}$ found by Kempen et al. ${ }^{66}$ for the same and other soil properties for Burundi and Rwanda. Based on the spatial analysis of the model residuals, they found that despite low $\mathrm{R}^{2}$ values, the models explained most of the spatial variation in the data, and that the unexplained variation is random noise.

Finally, these generated maps were used to feed the partition relations, and to predict the $\mathrm{Zn}, \mathrm{Cu}$ and $\mathrm{B}$ concentrations in $0.01 \mathrm{M} \mathrm{CaCl}_{2}$ extractions for Burundi and Rwanda. The $\mathrm{CaCl}_{2}$ measurements of Chapter 7 for the topsoils from the Burundi and Rwanda soil series, were used to validate the resulting maps.

The spatial predictions of the $\mathrm{Zn}, \mathrm{Cu}$ and $\mathrm{B}$ concentrations are shown in Figure 5. Figure 5 also shows the validation plots with the comparison between measured and predicted concentrations, based on using either the actual soil measurements or the maps as input in the partition relations. It is important to note that the spatial distribution of relatively low and high concentrations is not the same for all three micronutrients. For example, the Eastern side of Rwanda has relatively low $\mathrm{Zn}$ concentrations, while the Western side has relatively low B concentrations. These results underline the potential usefulness of spatial predictions for identifying specific regions with low levels of particular micronutrients. In addition, using critical $\mathrm{Zn}$ and $\mathrm{B}$ concentrations in a $\mathrm{CaCl}_{2}$ extraction of 1 and $7 \mu \mathrm{mol} \mathrm{kg}^{-1}$ respectively $52,67,68$, these maps suggest that a large part of Burundi and Rwanda may be at risk for $\mathrm{Zn}$ and $\mathrm{B}$ deficiencies for crop production.

The validation plots in Figure 5 next to the corresponding soil maps show that there is generally a small difference in the predicted concentrations when using either the actual soil measurements or soil maps as input data for the partition relations. It can also be seen that the quality of the prediction differs among countries. For Burundi, the experimental variation in the measured $B$ and $Z n$ concentrations is less captured 
by the model predictions when using soil maps as input. This may be partly explained by the lower number of validation points, that in turn are more spatially clustered. A larger validation set with higher coverage of the country, may help to further assess whether modeling predictions for $\mathrm{Zn}$ and $\mathrm{B}$ based on soil maps differ between Burundi and Rwanda, and to explore underlying reasons that explain these differences.

For $\mathrm{Cu}$, the variation in in the measured $\mathrm{CaCl}_{2}$-extractable concentrations is explained the least among all micronutrients. However, this is similar when using either measured soil data or soil maps as input, and therefore can be attributed to the partition relation. As shown in Chapter 7 and in Table S1, the partition relations for $\mathrm{Cu}$ have the lowest $\mathrm{R}^{2}$, which we attributed in Chapter 7 to a rather constant solidsolution partitioning that is not much related to soil properties.

Future research should look further into the error propagation originating from the use of transfer functions, input maps and the actual prediction model, when maps are created based on partition relations. The knowledge from this thesis, and the partition relations and transfer functions derived here, may form a starting point for future progress for making spatial predictions of micronutrient environmental availability that in turn can be used for regional micronutrient fertilization schemes.

\subsection{Micronutrient fertilizer recommendations}

Low micronutrient availability in SSA soils limits crop production, crop quality and affects human health. The use of micronutrient fertilizers is currently low due to little information on the soil micronutrient status and the lacking of a reliable fertilizer recommendation system that includes micronutrients. This thesis delivered valuable information on the micronutrient soil status in SSA, and knowledge on the processes that control soil micronutrient availability.

The knowledge on the solid-solution partitioning, may help to assess fertilizer use efficiency, or to identify which of the both nutrient pools (i.e. soluble or reactive) may be most relevant for identifying micronutrient deficiencies ${ }^{69}$.

Moreover, this thesis provides a low-cost and reliable method based on partition and transfer relations, for testing soils for $\mathrm{Zn}, \mathrm{Cu}$ and $\mathrm{B}$ availability and for making regional and national soil maps showing micronutrient availability, that includes both the reactive and soluble micronutrient concentrations. The soil micronutrient information obtained by these methods, can be used in future work for establishing micronutrient fertilizer recommendation systems. 


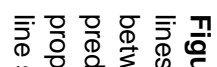

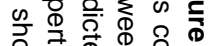

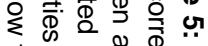

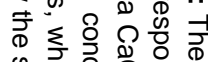

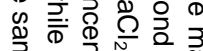

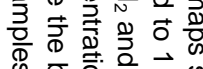

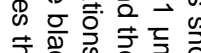

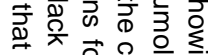

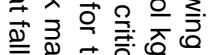

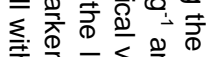

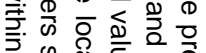
ก 䓃定

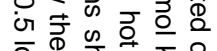

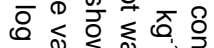

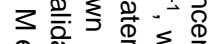

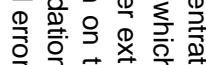

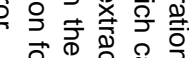

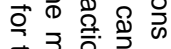

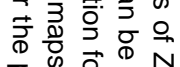

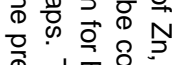
응 검 س용 응

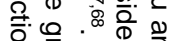
क 雨음

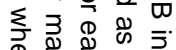

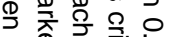
ธ. ๑ัต

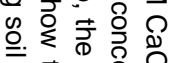

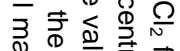

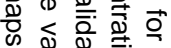

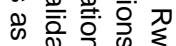

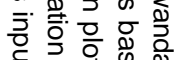
웅 क क

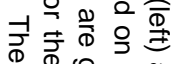
का 응 ฏ 을 응 호응 음

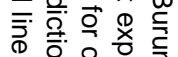
๘. 을 윽 을. 흐.

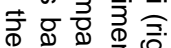

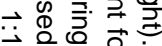

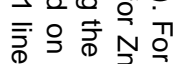
宗㤀

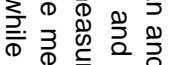

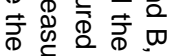

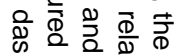

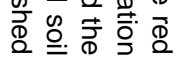
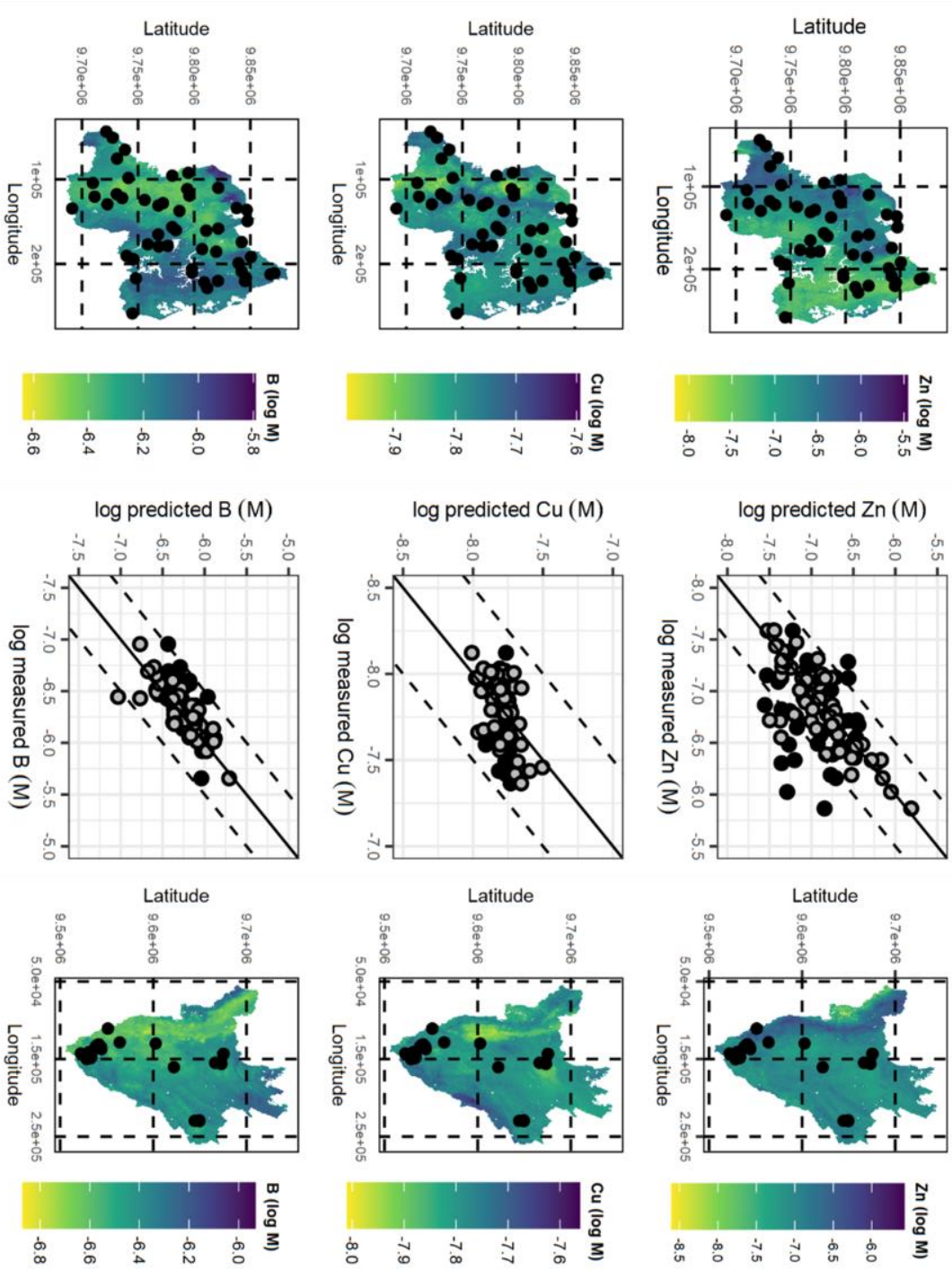

log predicted B (M)
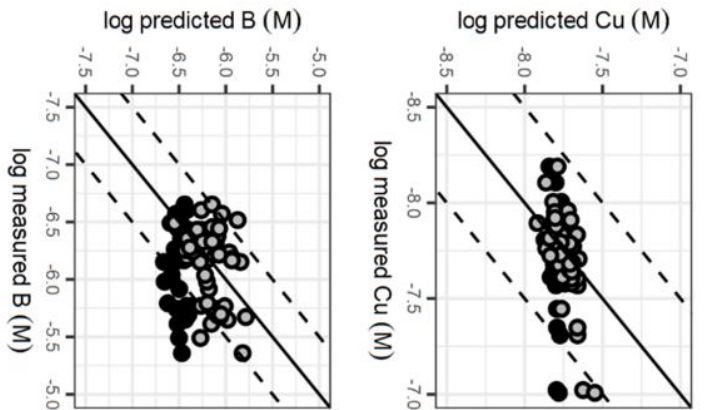

log predicted $\mathrm{Zn}(\mathrm{M})$
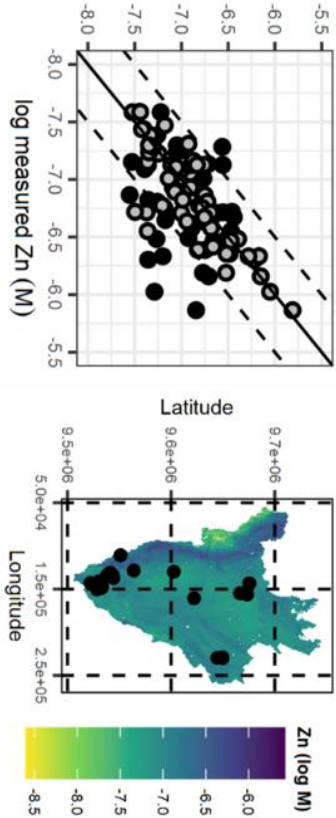

log predicted Zn (M)

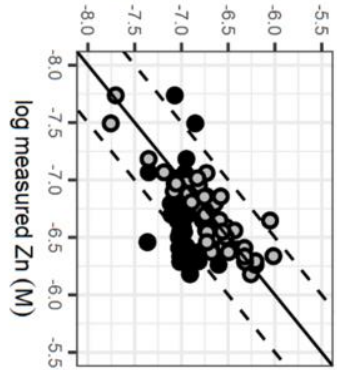




\section{SUPPORTING INFORMATION}

\section{A. Methodological approach for making spatial predictions}

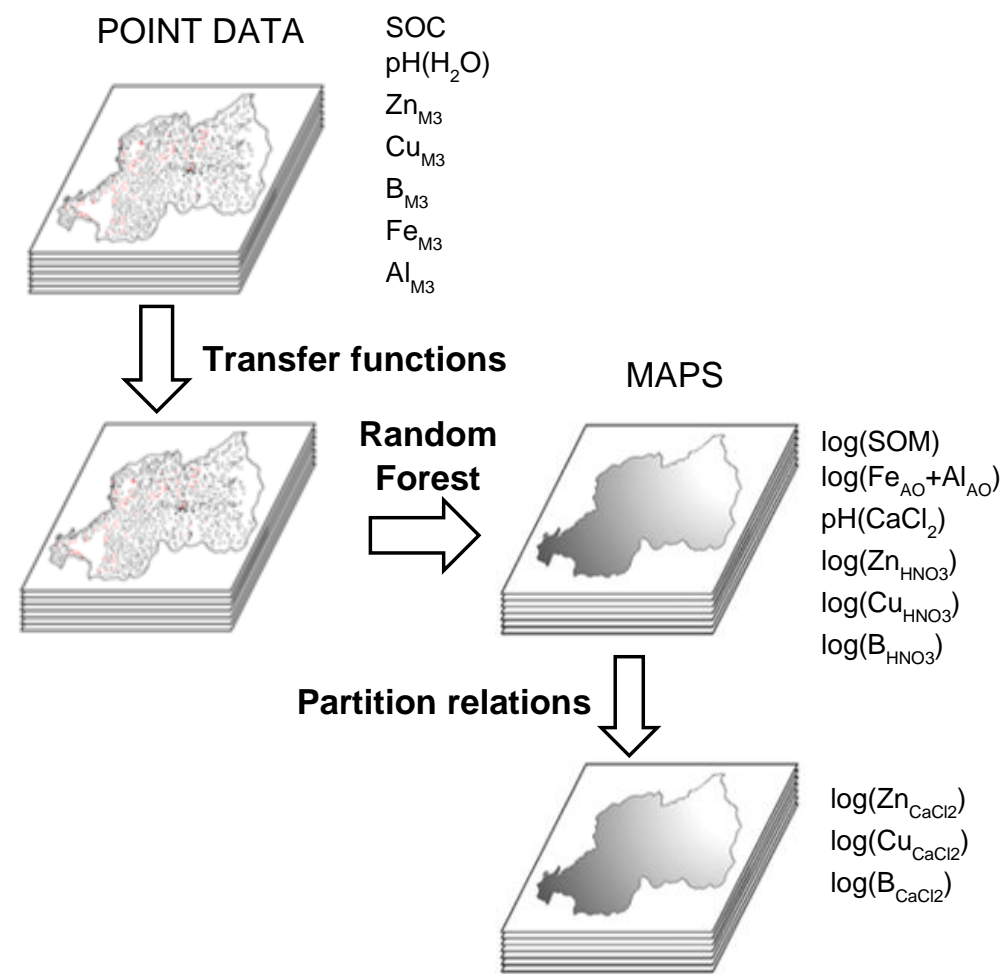

Figure S1: Overview of the different steps for making the maps of Burundi and Rwanda showing the predicted concentrations of $\mathrm{Zn}, \mathrm{Cu}$ and $\mathrm{B}$, measured in a $0.01 \mathrm{M} \mathrm{CaCl}_{2}$ based on existing soil data and the partition relations. Transfer functions were used to transform the available soil data into the units and measurements needed as input for the partition relations.

\section{B. Partition relations}

Table S1: The partition relations derived on the soil samples from Chapter 7), but without the inclusion of $\mathrm{DOC}$ no spatial soil information exist for this soil property. For $\mathrm{Cu}$ and $\mathrm{B}, \mathrm{C}-\mathrm{Q}$ relations were derived, while for $\mathrm{Zn}$ a K $\mathrm{f}_{\mathrm{f}}$ relation was calibrated. For more info on the method for derivation of these partition relations and the soil samples, we refer to Chapter 7 of this thesis.

\begin{tabular}{|c|c|c|c|c|c|c|}
\hline & $\alpha_{0}$ & pH & $\log Q$ & $\log S O M$ & $\log F e A I$ & $\mathbf{R}^{2}$ \\
\hline $\log C(\mathrm{Cu})$ & $\begin{array}{l}-5.75 \\
(0.28)\end{array}$ & $\begin{array}{c}-0.05 \\
(0.02,13)\end{array}$ & $\begin{array}{c}0.39 \\
(0.06,82)\end{array}$ & $\begin{array}{c}-0.23(0.10 \\
5)\end{array}$ & - & 0.26 \\
\hline $\log C(B)$ & $\begin{array}{l}-2.82 \\
(0.23)\end{array}$ & - & $\begin{array}{c}0.72 \\
(0.05,89)\end{array}$ & $\begin{array}{c}-0.19 \\
(0.10,11)\end{array}$ & - & 0.57 \\
\hline & $\mathrm{n}$ & $\alpha_{0}$ & $\mathrm{pH}$ & logSOM & $\log F e A I$ & $\mathbf{R}^{2}{ }_{a}$ \\
\hline $\log K_{f}(Z n)$ & 0.66 & $\begin{array}{l}-4.15 \\
(0.26)\end{array}$ & $\begin{array}{c}0.62(0.03 \\
85)\end{array}$ & $\begin{array}{c}0.69 \\
(0.15,10)\end{array}$ & $\begin{array}{c}0.35 \\
(0.11,6)\end{array}$ & 0.78 \\
\hline
\end{tabular}




\section{Transfer functions}
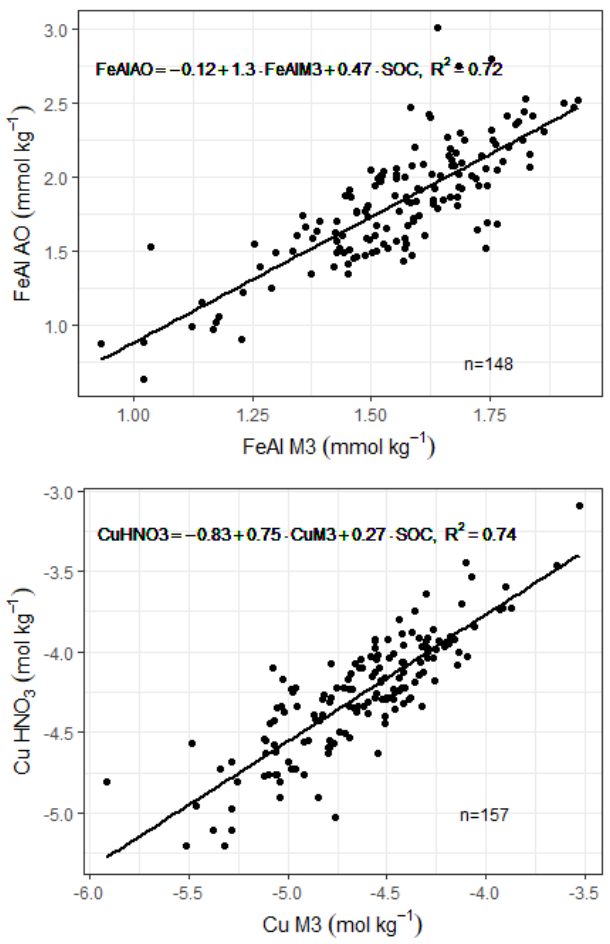
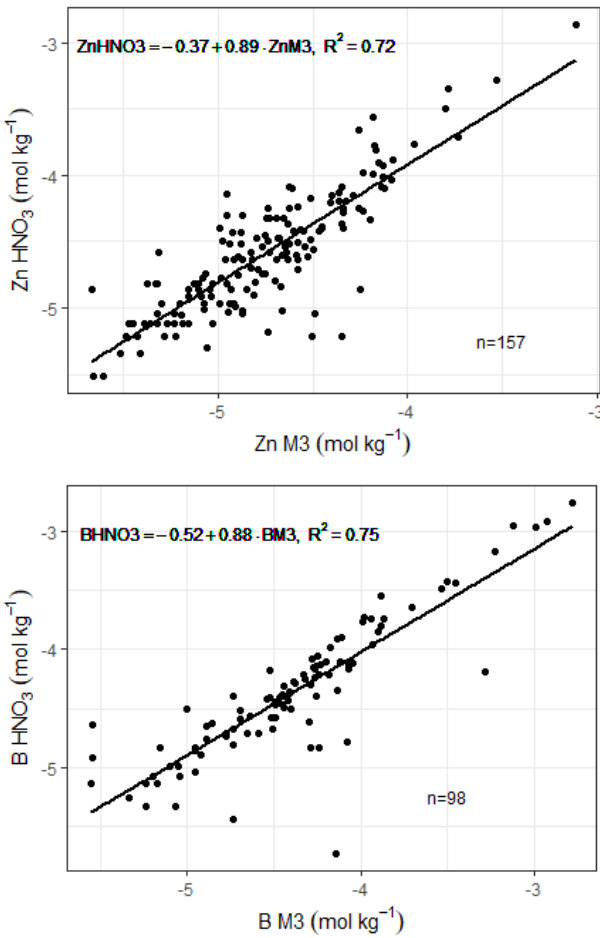

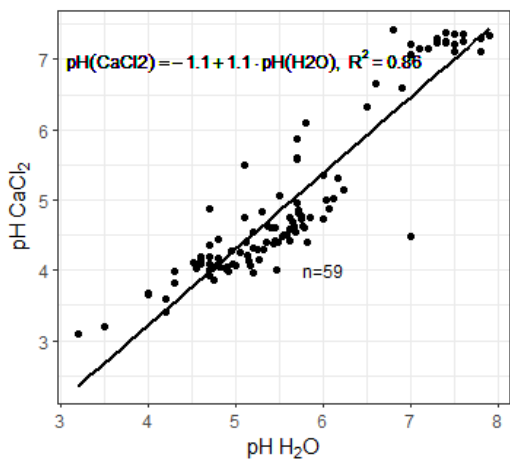

Figure S2: Soil maps exist of $\mathrm{Fe}, \mathrm{Al}, \mathrm{Zn}, \mathrm{Cu}$ and $\mathrm{B}$ based on Mehlich-3 extractions. In addition, the map showing the soil $\mathrm{pH}$ is based on $\mathrm{pH}$ measured in $\mathrm{H}_{2} \mathrm{O}$ extractions. Since the partition relations have been calibrated based on ammonium oxalate extractions ( $\mathrm{Fe}$ and $\mathrm{Al}$ ), $\mathrm{HNO}_{3}$ extractions ( $\mathrm{Zn}$, $\mathrm{Cu}$ and $\mathrm{B})$ and $\mathrm{pH}\left(\mathrm{CaCl}_{2}\right)$ measurements for predicting the soluble concentration in $0.01 \mathrm{M} \mathrm{CaCl}_{2}$, transfer functions were derived and applied on existing soil maps to use them as input in partition relations. These figures show the data and transfer functions used for converting the $\mathrm{Fe}+\mathrm{Al}$ measured in Mehlich-3 extraction to the ammonium oxalate extraction, for converting the $\mathrm{Zn}, \mathrm{Cu}$ and $\mathrm{B}$ measured by Mehlich-3 to the concentrations measured in $0.43 \mathrm{M} \mathrm{HNO}_{3}$ extraction, and lastly for converting the $\mathrm{pH}$ measured in $\mathrm{H}_{2} \mathrm{O}$ to $\mathrm{pH}$ measured in $0.01 \mathrm{M} \mathrm{CaCl}_{2}$ extract. The $\mathrm{SOC}$ content $(\log 10$, in \%) was found to be significant in the transfer functions for $\mathrm{Cu}$, and for $\mathrm{Fe}$ and $\mathrm{Al}$. 


\section{Quality of the input maps}

Table S2: The mean squared error (MSE), the explained variance $\left(R^{2}\right)$ and the root mean squared error (RMSE) of the spatial predictions of the input variables for the partition relations. The spatial predictions were based on random forest models (ranger function in R), using the point and covariate data from Kempen et al. ${ }^{66}$

\begin{tabular}{|c|c|c|c|c|c|c|}
\hline & \multicolumn{3}{|c|}{ Rwanda } & \multicolumn{3}{|c|}{ Burundi } \\
\hline & MSE & $\mathbf{R}^{2}$ & RMSE & MSE & $\mathbf{R}^{2}$ & RMSE \\
\hline $\mathrm{pH}\left(\mathrm{CaCl}_{2}\right)$ & 0.58 & 0.38 & 0.76 & 0.28 & 0.30 & 0.53 \\
\hline $\log \left(\mathrm{Fe}_{\mathrm{AO}}+\mathrm{Al}_{\mathrm{AO}}\right)\left(\mathrm{mmol} \mathrm{kg}^{-1}\right)$ & 0.03 & 0.40 & 0.16 & 0.02 & 0.56 & 0.14 \\
\hline $\operatorname{logSOM}(\%)$ & 0.01 & 0.36 & 0.11 & 0.01 & 0.43 & 0.09 \\
\hline $\left.\log \mathrm{Zn}_{\mathrm{HNO} 3}(\mathrm{~mol} \mathrm{~kg})^{-1}\right)$ & 0.10 & 0.22 & 0.32 & 0.05 & 0.18 & 0.23 \\
\hline $\log \mathrm{Cu}_{\mathrm{HNO} 3}\left(\mathrm{~mol} \mathrm{~kg}^{-1}\right)$ & 0.03 & 0.36 & 0.19 & 0.04 & 0.34 & 0.20 \\
\hline $\log B_{\mathrm{HNO} 3}\left(\mathrm{~mol} \mathrm{~kg}^{-1}\right)$ & 0.08 & 0.37 & 0.28 & 0.06 & 0.37 & 0.25 \\
\hline
\end{tabular}

\section{REFERENCES}

1. Groenenberg, J. E., Römkens, P. F. A. M., Zomeren, A. Van, Rodrigues, S. M. \& Comans, R. N. J. Evaluation of the Single Dilute ( $0.43 \mathrm{M})$ Nitric Acid Extraction to Determine Geochemically Reactive Elements in Soil. Environ. Sci. Technol. 51, 2246-2253 (2017).

2. ISO 17402. Soil quality -- Requirements and guidance for the selection and application of methods for the assessment of bioavailability of contaminants in soil and soil materials. https://www.iso.org/standard/38349.html (2008).

3. Hiemstra, T., Antelo, J., Rahnemaie, R. \& van Riemsdijk, W. H. Nanoparticles in natural systems I: The effective reactive surface area of the natural oxide fraction in field samples. Geochim. Cosmochim. Acta 74, 41-58 (2010).

4. Mendez, J. C., Hiemstra, T. \& Koopmans, G. F. Assessing the Reactive Surface Area of Soils and the Association of Soil Organic Carbon with Natural Oxide Nanoparticles Using Ferrihydrite as Proxy. Environ. Sci. Technol. 54, 11990-12000 (2020).

5. Kleber, M., Mikutta, R., Torn, M. S. \& Jahn, R. Poorly crystalline mineral phases protect organic matter in acid subsoil horizons. Eur. J. Soil Sci. 56, 717-725 (2005).

6. Rasmussen, C. et al. Beyond clay: towards an improved set of variables for predicting soil organic matter content. Biogeochemistry 137, 297-306 (2018).

7. Kaiser, K. \& Guggenberger, G. The role of DOM sorption to mineral surfaces in the preservation of organic matter in soils. in Organic Geochemistry vol. 31 711-725 (Pergamon, 2000).

8. Groenenberg, J. E., Dijkstra, J. J., Bonten, L. T. C., De Vries, W. \& Comans, R. N. J. Evaluation of the performance and limitations of empirical partition-relations and process based multisurface models to predict trace element solubility in soils. Environ. Pollut. 166, 98-107 (2012).

9. Klinkert, S. \& Comans, R. N. J. Geochemical Multisurface Modeling of Reactive Zinc Speciation in Compost as Influenced by Extraction Conditions. Environ. Sci. Technol. 54, 2467-2475 (2020).

10. Milne, C. J., Kinniburgh, D. G., van Riemsdijk, W. H. \& Tipping, E. Generic NICA-Donnan Model Parameters for Metal-Ion Binding by Humic Substances. Environ. Sci. Technol. 37, 958-971 (2003).

11. Koopal, L. K., Saito, T., Pinheiro, J. P. \& Riemsdijk, W. H. van. Ion binding to natural organic 
matter: General considerations and the NICA-Donnan model. Colloids Surfaces $A$ Physicochem. Eng. Asp. 265, 40-54 (2005).

12. Gustafsson, J. P. Modeling the acid-base properties and metal complexation of humic substances with the Stockholm Humic Model. J. Colloid Interface Sci. 244, 102-112 (2001).

13. Dijkstra, J. J., Meeussen, J. C. L. \& Comans, R. N. J. Leaching of Heavy Metals from Contaminated Soils: An Experimental and Modeling Study. Environ. Sci. Technol. 38, 43904395 (2004).

14. Fest, E. P. M. J. M. J., Temminghoff, E. J. M. J. M., Comans, R. N. J. N. J. \& van Riemsdijk, W. H. H. Partitioning of organic matter and heavy metals in a sandy soil: Effects of extracting solution, solid to liquid ratio and pH. Geoderma 146, 66-74 (2008).

15. Groenenberg, J. E., Koopmans, G. F. \& Comans, R. N. J. Uncertainty analysis of the nonideal competitive adsorption - Donnan model: Effects of dissolved organic matter variability on predicted metal speciation in soil solution. Environ. Sci. Technol. 44, 1340-1346 (2010).

16. Dijkstra, J. J., Meeussen, J. C. L. \& Comans, R. N. J. Evaluation of a Generic Multisurface Sorption Model for Inorganic Soil Contaminants. Environ. Sci. Technol. 43, 6196-6201 (2009).

17. Groenenberg, J. E. \& Lofts, S. The use of assemblage models to describe trace element partitioning, speciation, and fate: a review. Environ. Toxicol. Chem. 33, 2181-96 (2014).

18. Dzombak, D. A. \& Morel, F. F. M. M. Surface Complexation Modeling: Hydrous Ferric Oxide. (John Wiley \& Sons, 1990).

19. Hiemstra, T. \& Van Riemsdijk, W. H. A Surface Structural Approach to Ion Adsorption: The Charge Distribution (CD) Model. J. Colloid Interface Sci. 179, 488-508 (1996).

20. Hiemstra, T. \& Zhao, W. Reactivity of ferrihydrite and ferritin in relation to surface structure, size, and nanoparticle formation studied for phosphate and arsenate. Environ. Sci. Nano 3, 12651279 (2016).

21. Mendez, J. C. \& Hiemstra, T. Surface area of ferrihydrite consistently related to primary surface charge, ion pair formation, and specific ion adsorption. Chem. Geol. 532, (2020).

22. Mendez, J. C. \& Hiemstra, T. High and low affinity sites of ferrihydrite for metal ion adsorption: Data and modeling of the alkaline-earth ions $\mathrm{Be}, \mathrm{Mg}, \mathrm{Ca}, \mathrm{Sr}, \mathrm{Ba}$, and $\mathrm{Ra}$. Geochim. Cosmochim. Acta (2020) doi:10.1016/j.gca.2020.07.032.

23. Hiemstra, T. Ferrihydrite interaction with silicate and competing oxyanions: Geometry and Hydrogen bonding of surface species. Geochim. Cosmochim. Acta 238, 453-476 (2018).

24. Mendez, J. C. \& Hiemstra, T. Carbonate Adsorption to Ferrihydrite: Competitive Interaction with Phosphate for Use in Soil Systems. ACS Earth Sp. Chem. 3.1, 129-141 (2018).

25. Su, C. \& Suarez, D. L. Coordination of Adsorbed Boron: A FTIR Spectroscopic Study. Environ. Sci. Technol. 29, 302-311 (1995).

26. Xu, D. \& Peak, D. Adsorption of Boric Acid on Pure and Humic Acid Coated am-Al(OH)3: A Boron K-Edge XANES Study. Environ. Sci. Technol. 41, 903-908 (2007).

27. Lemarchand, E., Schott, J. \& Gaillardet, J. How surface complexes impact boron isotope fractionation: Evidence from $\mathrm{Fe}$ and $\mathrm{Mn}$ oxides sorption experiments. Earth Planet. Sci. Lett. 260, 277-296 (2007).

28. Waychunas, G. A., Fuller, C. C. \& Davis, J. A. Surface complexation and precipitate geometry for aqueous $\mathrm{Zn}$ (II) sorption on ferrihydrite I: X-ray absorption extended fine structure spectroscopy analysis. Geochim. Cosmochim. Acta 66, 1119-1137 (2002).

29. Tiberg, C., Sjöstedt, C., Persson, I. \& Gustafsson, J. P. Phosphate effects on copper(II) and lead(II) sorption to ferrihydrite. Geochim. Cosmochim. Acta 120, 140-157 (2013).

30. Swedlund, P. . \& Webster, J. . Cu and Zn ternary surface complex formation with SO4 on ferrihydrite and schwertmannite. Appl. Geochemistry 16, 503-511 (2001).

31. Box, G. E. P. Science and Statistics. Journal of the American Statistical Association vol. 71 (1976).

32. Hou, J., Evans, L. J. \& Spiers, G. A. Boron fractionation in soils. Commun. Soil Sci. Plant Anal. 25, 1841-1853 (1994).

33. Tiberg, C., Sjöstedt, C., Eriksson, A. K., Klysubun, W. \& Gustafsson, J. P. Phosphate competition with arsenate on poorly crystalline iron and aluminum (hydr)oxide mixtures. Chemosphere 255, 1-8 (2020).

34. Liu, Y. T. \& Hesterberg, D. Phosphate bonding on noncrystalline Al/Fe-hydroxide coprecipitates. 
Environ. Sci. Technol. 45, 6283-6289 (2011).

35. Tiberg, C., Sjöstedt, C. \& Gustafsson, J. P. Metal sorption to Spodosol Bs horizons: Organic matter complexes predominate. Chemosphere 196, 556-565 (2018).

36. Weng, L., Temminghoff, E. J. M. \& Van Riemsdijk, W. H. Contribution of Individual Sorbents to the Control of Heavy Metal Activity in Sandy Soil. Environ. Sci. Technol. 35, 4436-4443 (2001).

37. Di Bonito, M., Lofts, S. \& Groenenberg, J. E. Models of Geochemical Speciation: Structure and Applications. Environ. Geochemistry 237-305 (2018) doi:10.1016/B978-0-444-63763-5.000124.

38. Warrinnier, R. et al. Investigation on the control of phosphate leaching by sorption and colloidal transport: Column studies and multi-surface complexation modelling. Appl. Geochemistry 100, 371-379 (2019).

39. Hiemstra, T., Antelo, J., van Rotterdam, A. M. D. M. D. (Debby. \& van Riemsdijk, W. H. Nanoparticles in natural systems II: The natural oxide fraction at interaction with natural organic matter and phosphate. Geochim. Cosmochim. Acta 74, 59-69 (2010).

40. Verbeeck, M., Hiemstra, T., Thiry, Y. \& Smolders, E. Soil organic matter reduces the sorption of arsenate and phosphate: a soil profile study and geochemical modelling. Eur. J. Soil Sci. 68, 678-688 (2017).

41. Weng, L., Van Riemsdijk, W. H. \& Hiemstra, T. Cu2+ and Ca2+adsorption to goethite in the presence of fulvic acids. Geochim. Cosmochim. Acta 72, 5857-5870 (2008).

42. Du, H., Peacock, C. L., Chen, W. \& Huang, Q. Binding of Cd by ferrihydrite organo-mineral composites: Implications for $\mathrm{Cd}$ mobility and fate in natural and contaminated environments. Chemosphere 207, 404-412 (2018).

43. Kinniburgh, D. G. et al. Metal ion binding by humic acid: Application of the NICA-Donnan model. Environ. Sci. Technol. 30, 1687-1698 (1996).

44. Benedetti, M. ., van Riemsdijk, W. H. \& Koopal, L. K. Humic Substances Considered as a Heterogeneous Donnan Gel Phase. Environ. Sci. Technol 30, 1905-1913 (1996).

45. Hiemstra, T. \& van Riemsdijk, W. H. Biogeochemical speciation of $\mathrm{Fe}$ in ocean water. Mar. Chem. 102, 181-197 (2006).

46. Town, R. M., Van Leeuwen, H. P. \& Duval, J. F. L. Rigorous Physicochemical Framework for Metal Ion Binding by Aqueous Nanoparticulate Humic Substances: Implications for Speciation Modeling by the NICA-Donnan and WHAM Codes. Environ. Sci. Technol. 53, 8516-8532 (2019).

47. Gustafsson, J. P. \& Berggren Kleja, D. Modeling Salt-Dependent Proton Binding by Organic Soils with the NICA-Donnan and Stockholm Humic Models. Environ. Sci. Technol. 39, 53725377 (2005).

48. Oste, L. A., Temminghoff, E. J. M., Lexmond, T. M. \& Van Riemsdijk, W. H. Measuring and modeling zinc and cadmium binding by humic acid. Anal. Chem. 74, 856-862 (2002).

49. Town, R. M. \& Van Leeuwen, H. P. Metalion-humic acid nanoparticle interactions: Role of both complexation and condensation mechanisms. Phys. Chem. Chem. Phys. 18, 18024-18032 (2016).

50. Houba, V. J. G. G., Novozamsky, I., Lexmond, T. M., van der Lee, J. J. \& Jvnn Der, L. Applicability Of $0.01 \mathrm{M}$ Cacl2 As a Single Extraction Solution for the Assessment of the Nutrient Status of Soils and Other Diagnostic Purposes. Commun. Soil Sci. Plant Anal. 21, 2281-2290 (1990).

51. Menzies, N. W., Donn, M. J. \& Kopittke, P. M. Evaluation of extractants for estimation of the phytoavailable trace metals in soils. Environ. Pollut. 145, 121-130 (2007).

52. Duffner, A., Hoffland, E., Weng, L. \& van der Zee, S. E. A. T. M. Predicting zinc bioavailability to wheat improves by integrating $\mathrm{pH}$ dependent nonlinear root surface adsorption. Plant Soil 373, 919-930 (2013).

53. Meers, E. et al. Phytoavailability assessment of heavy metals in soils by single extractions and accumulation by Phaseolus vulgaris. Environ. Exp. Bot. 60, 385-396 (2007).

54. Schröder, T. J., Hiemstra, T., Vink, J. P. M. \& Van Der Zee, S. E. A. T. M. Modeling of the solidsolution partitioning of heavy metals and arsenic in embanked flood plain soils of the rivers rhine and meuse. Environ. Sci. Technol. 39, 7176-7184 (2005).

55. ISO - ISO 21268-2:2019 - Soil quality - Leaching procedures for subsequent chemical and ecotoxicological testing of soil and soil-like materials - Part 2: Batch test using a liquid to solid 
ratio of $10 \mathrm{l} / \mathrm{kg}$ dry matter. https://www.iso.org/standard/68251.html.

56. Iglesias, A., López, R., Fiol, S., Antelo, J. M. \& Arce, F. Analysis of copper and calcium-fulvic acid complexation and competition effects. Water Res. 37, 3749-3755 (2003).

57. Goli, E., Hiemstra, T. \& Rahnemaie, R. Interaction of boron with humic acid and natural organic matter: Experiments and modeling. Chem. Geol. 515, 1-8 (2019).

58. Weng, L., Temminghoff, E. J. M. \& Van Riemsdijk, W. H. Interpretation of humic acid coagulation and soluble soil organic matter using a calculated electrostatic potential. Eur. J. Soil Sci. 53, 575-587 (2002).

59. Van Zomeren, A. \& Comans, R. N. J. Measurement of humic and fulvic acid concentrations and dissolution properties by a rapid ratch procedure. Environ. Sci. Technol. (2007) doi:10.1021/es0709223.

60. Houba, V. J. G., Temminghoff, E. J. M., Gaikhorst, G. A. \& van Vark, W. Soil analysis procedures using $0.01 \mathrm{M}$ calcium chloride as extraction reagent. Commun. Soil Sci. Plant Anal. 31, 12991396 (2000).

61. Mendez, J. C. \& Hiemstra, T. Ternary complex formation of phosphate with $\mathrm{Ca}$ and $\mathrm{Mg}$ ions binding to ferrihydrite: Experiments and mechanisms. ACS Earth Sp. Chem. 4.4, acsearthspacechem.9b00320 (2020).

62. van der Perk, M., Stergiadi, M., de Nijs, T. C. M., Comans, R. N. J. \& Bierkens, M. F. P. The response of metal leaching from soils to climate change and land management in a temperate lowland catchment. Catena 171, 426-439 (2018).

63. de Vries, W., Curlík, J., Murányi, A., Alloway, B. \& Groenenberg, B. J. Assessment of relationships between total and reactive concentrations of cadmium, copper, lead and zinc in Hungarian and Slovakian soils. Ekológia (Bratislava) 24, 152-169 (2005).

64. Hengl, T. et al. Mapping Soil Properties of Africa at $250 \mathrm{~m}$ Resolution: Random Forests Significantly Improve Current Predictions. PLoS One 10, e0125814 (2015).

65. Hengl, T. et al. Soil nutrient maps of Sub-Saharan Africa: assessment of soil nutrient content at $250 \mathrm{~m}$ spatial resolution using machine learning. Nutr. Cycl. Agroecosystems 109, 77-102 (2017).

66. Kempen, B. et al. Preliminary evaluation of the feasibility of using geospatial information to refine soil fertility recommendations. (2015).

67. Kabata-Pendias, A. \& Pendias, H. Trace Elements in Soils and Plants, Third Edition. www.crcpress.com (2001).

68. Novozamsky, I., Barrera, L. L., Houba, V. J. G., van der Lee, J. J. \& van Eck, R. Comparison of a hot water and cold $0.01 \mathrm{M} \mathrm{Cacl}_{2}$ extraction procedures for the determination of boron in soil. Commun. Soil Sci. Plant Anal. 21, 2189-2195 (1990).

69. Nawara, S. et al. A comparison of soil tests for available phosphorus in long-term field experiments in Europe. Eur. J. Soil Sci. 68, 873-885 (2017). 
Chapter 8

264 


\section{SUMMARY}

Trace elements such as zinc $(\mathrm{Zn})$, copper $(\mathrm{Cu})$ and boron $(\mathrm{B})$, are important micronutrients for crop production. Their bioavailability is essential to crops yield quantity and quality in tropical soils from Sub-Saharan Africa (SSA). Blanket fertilizer recommendations including only macronutrients are most common practice in SSA. They have been developed for large areas or agroecological zones based on general soil and climate information and do not take into account the spatial heterogeneity in soil and management factors. Alternatively, science-based approaches have been developed for formulating site-specific fertilizer recommendations. Such sciencebased approaches could be extended to account for micronutrients, based on the knowledge about the processes that affect their availability for plant uptake. Studying the soil micronutrient status, is therefore an important first step for future development of fertilizer recommendation schemes that include micronutrients, and forms the starting point for this thesis research.

Micronutrient bioavailability depends partly on the soil micronutrient status. Soils usually contain substantial total amounts of micronutrients. A large part that is present in the solid phase is however inert and not readily available for crop uptake, because it is occluded in the matrix of soil constituents such as (hydr)oxides and clay minerals. This fraction is assumed to be released from the soil matrix only by very slow weathering processes. More relevant for plant uptake is the labile, reactive or potentially available pool that is distributed over the solid and solution phase, through sorption/desorption and precipitation/dissolution. A fraction of this labile pool is present in the soil solution and is therefore directly available for plant uptake. Together, the soluble and potential available pool represent the soil micronutrient environmental availability.

The distribution of the reactive or labile micronutrient content over the solid and solution phase, is often controlled by adsorption processes to reactive surfaces (i.e. clay minerals, organic matter, metal (hydr)oxides). The adsorption of micronutrients to the reactive surfaces in soils is a complex process influenced by the amount and surface properties of the mineral and organic phases and the chemistry of the soil solution (e.g. pH, ionic strength, type and concentration of co-existing ions). In this context, geochemical multi-surface models are envisioned as promising tools for analyzing how these multiple factors interact and affect the overall environmental availability of micronutrients in soil systems.

Geochemical multi-surface models combine surface-specific models with thermodynamic constants for inorganic speciation and mineral equilibria, to calculate nutrient speciation in soils. Multi-surface models have often been applied for studying the solid-solution partitioning of trace elements mainly in temperate and 
often contaminated soils, but these models have rarely been used in soils from the tropics with low levels of micronutrients.

From a practical perspective, geochemical multi-surface models are not easy-to-use tools for predicting soluble or reactive concentrations of $\mathrm{Zn}, \mathrm{Cu}$ and $\mathrm{B}$ in soils. More convenient for this purpose are partition relations, in which the complex processes described by the geochemical multi-surface model are lumped into one empirical regression equation that consists of input variables such as $\mathrm{pH}$ and the content of reactive surfaces for adsorption. These prediction models are convenient for largescale applications and when limited data are available. For example, based on partition relations, predictions could be made about the soluble concentrations of $\mathrm{Zn}$, $\mathrm{Cu}$ and $\mathrm{B}$ based on the spatial soil information data that currently exist for SSA.

The aim of this thesis was therefore to gain insight in the soil chemical processes that control the solid-solution partitioning of $\mathrm{Zn}, \mathrm{Cu}$, and $\mathrm{B}$, particularly in tropical soils from SSA, and to use this knowledge to develop accessible tools for predicting the soluble concentrations of these micronutrients. This was done using geochemical multi-surface models and partition relations.

Geochemical multi-surface models require input data about the amount of reactive surfaces and their corresponding reactivity for ion adsorption. The metal (hydr)oxides and soil organic matter have been previously identified as the most important reactive surfaces for micronutrient adsorption in soils. The first objective of this thesis was therefore to better understand the reactivity of metal (hydr)oxides and soil organic matter in tropical soils from SSA.

Soils from the humid tropics are often intensively weathered, resulting in a high abundance of iron (Fe) and aluminum (Al) (hydr)oxides. These metal (hydr)oxides are mainly present as well-crystallized metal (hydr)oxides while the contribution of oxide nanoparticles (i.e. ferrihydrite-like materials) may be relatively small on a mass basis. Nevertheless, it is still possible that these nano-sized materials greatly contribute to the overall reactivity of soils, even at low concentrations, because the specific surface area of these nanoparticles is substantially higher than the SSA of the crystalline metal (hydr)oxides. In Chapter 2 of this thesis, we used a novel probeion methodology combined with state-of-the-art surface complexation modeling to derive the reactive surface area (RSA) of the metal (hydr)oxides for a set of highly weathered tropical topsoils from SSA. The results showed that even though wellcrystallized materials dominate the mass fraction of metal (hydr)oxide of these soils, nanocrystalline ferrihydrite $(\mathrm{Fh})$ is a better proxy than well-crystallized goethite for describing the reactivity of the metal (hydr)oxides, in line with what has been found before for a set of Dutch topsoils. Using Fh as a proxy, the RSA of these SubSaharan topsoils ranged from $\sim 2$ to $40 \mathrm{~m}^{2} \mathrm{~g}^{-1}$ soil. Nanoparticles with a mean 
diameter of $\sim 1.5-5.0 \mathrm{~nm}$ dominate the reactive fraction of metal (hydr)oxides in these topsoils. We conclude that irrespective of weathering stage, $\mathrm{Fh}$ is a good model oxide to describe the surface reactivity of the natural metal (hydr)oxides in soils.

Next to the metal (hydr)oxides, soil organic matter (SOM) is another important reactive surface for ion adsorption in soils, especially for metal cations such as $\mathrm{Zn}$ and $\mathrm{Cu}$. Previous studies have found a positive relationship between soil organic carbon (SOC) and the nanocrystalline Fe- and Al-(hydr)oxide content, suggesting the importance of these minerals for SOM stabilization. In Chapter 3, we did find indeed a positive relationship between $\mathrm{SOC}$ and the fraction of nanocrystalline oxides for a set of tropical topsoils, which suggests that SOC binds to its surfaces. However, the scaling of SOC to the RSA measured for these soils showed a much less pronounced relationship. Our results showed that in soils, the mean particle size of the natural oxide fraction explains the SOC content found in soils, irrespective of origin, land use and soil depth. According to these results, soil organic carbon is predominantly stored in primary organo-oxide aggregates that are additionally organized by association with larger mineral particles.

In addition to the amount and reactivity of the adsorption surfaces, geochemical multi-surface models also require well-parameterized models to describe the ion adsorption processes to these surfaces. The second objective of this thesis was to use a consistent modeling approach for describing the adsorption of $\mathrm{Zn}, \mathrm{Cu}$ and $\mathrm{B}$ to Fh with the Charge Distribution (CD) model in combination with a Multi Site Ion Complexation (MUSIC) model. The resulting parameters can then be used later in multi-surface modeling applications for the final objective of this thesis.

In Chapter 4, the $\mathrm{pH}$-dependent $\mathrm{B}$ adsorption to $\mathrm{Fh}$ was studied in single-ion systems, and in the presence of phosphate $\left(\mathrm{PO}_{4}\right)$ as competing anion. The $\mathrm{pH}$ dependent $B$ adsorption envelope of ferrihydrite was found to be bell-shaped with a maximum around $\mathrm{pH}$ 8-9. In agreement with spectroscopy, modeling suggested the formation of a trigonal bidentate complex and an additional outer-sphere complex at low to neutral $\mathrm{pH}$ values. At high $\mathrm{pH}$, it was found that a tetrahedral bidentate surface species becomes important. In the presence of phosphate, B adsorption decreased strongly and only formation of the outer-sphere surface complex became relevant. Interestingly, we found that the data could be best described when using a set of singly coordinated groups that act as high-affinity sites for B adsorption to $\mathbf{F h}$, in line with what has been found previously for the adsorption of cations. The adsorption densities observed in our experimental window were below the saturation level of these high-affinity sites, and as a result, no binding to surface sites with lower affinity was detected. 
In Chapter 5, $\mathrm{Zn}$ adsorption to $\mathrm{Fh}$ was studied in the absence and presence of $\mathrm{PO}_{4}$. In agreement with spectroscopy, it was found that $\mathrm{Zn}$ is bound at low loading as a double-corner bidentate complex. At higher loading, the modeling results showed that the formation of a monodentate complex becomes important, which is in line with a decrease in the number of ions in the second shell of $\mathrm{Zn}$ as observed by spectroscopy. According to the modeling calculations, Zn polymerization only occurs at a very high molar $\mathrm{Zn} / \mathrm{Fe}$ ratio $(>0.1)$, which was sucessfully described using a neutral, hydrolyzed $\mathrm{Zn}$-dimer species. The presence of $\mathrm{PO}_{4}$ enhanced $\mathrm{Zn}$ adsorption to $\mathrm{Fh}$, especially in the $\mathrm{pH}$ range 5-6. At the $\mathrm{Zn}$ and $\mathrm{PO}_{4}$ levels studied, the modeling could not reveal the formation of ternary $\mathrm{Zn}-\mathrm{P}$ surface complexes. For comparison, $\mathrm{Cu}$ adsorption data were re-interpreted with a consistent modeling approach as applied for $\mathrm{Zn}$. Based on these calculations, we also did not find the formation ternary complexes for describing the interaction of $\mathrm{Cu}$ and $\mathrm{PO}_{4}$ with $\mathrm{Fh}$. We conclude that ternary complexes may form but only at rather extreme metal or $\mathrm{PO}_{4}$ loading conditions, as found in our experiments for $\mathrm{Zn}$.

Using the knowledge and parameters gained under the first and second objective, the third objective of this thesis was to apply a multi-surface model for calculating the solid-solution partitioning of $\mathrm{Zn}, \mathrm{Cu}$, and $\mathrm{B}$ in tropical soils, and to translate these results in easily accessible prediction tools in the form of partition relations.

Previous work on the speciation of $B$ in soils has been done with a generalized composite modeling approach, in which the binding to the different reactive surfaces in soils is approximated by one single composite surface plane. With this modeling approach, the adsorption parameters are not generically transferable to other soils, and little information is gained about the individual soil chemical processes that affect the distribution of natural reactive B over the solid and solution phase. In Chapter 6, the chemical speciation of $B$ in soils was studied with a geochemical multi-surface model that included $B$ adsorption to dissolved and solid organic matter, ferrihydrite, and clay mineral edges. This was done for five temperate and five tropical. In addition, the performance of previously proposed extraction methods for measuring reactive $B$ were evaluated, since this information is needed as input variable for multi-surface modeling calculations. Based on modeling calculations, the reactive $\mathrm{B}$ in soils corresponded best to the $B$ measured in a $0.05 \mathrm{M} \mathrm{KH}_{2} \mathrm{PO}_{4}(\mathrm{pH} 4.5)$ extraction. In general, the multi-surface modeling showed that $68 \%$ or more of reactive boron was present in the solution phase for the soils in this study and that the adsorption was dominated by oxides in the tropical soils, while solid organic matter was the main adsorbent in the temperate soils. When changing the soil $\mathrm{pH}\left(\mathrm{CaCl}_{2}\right)$, B concentration was found to decrease with increasing $\mathrm{pH}$, and both experimental data and modelling suggested that this effect is mainly due to increased binding of $B$ to organic matter. 
In Chapter 7, the solid-solution partitioning of Zn, Cu and B was studied for 172 soils from Burundi, Rwanda and Kenya, using extensive soil characterization in combination with multi-surface modelling. In addition, two types of Freundlich-like partition relations were derived, in which either the soluble concentration or the actual solid-solution partitioning were optimized for $\mathrm{Zn}, \mathrm{Cu}$ and $\mathrm{B}$ in these soils. The results showed that the generic multi-surface model applied to these tropical soils performs similarly for $\mathrm{Zn}$ and $\mathrm{Cu}$ as in previous studies on temperate and contaminated soils. The $\mathrm{Zn}$ and $\mathrm{Cu}$ speciation was dominated by adsorption to soil organic matter, with an increased importance of metal (hydr)oxides with increasing $\mathrm{pH}$. Given its generally low concentrations in these soils, dissolved organic matter was found to be important only for the solution speciation of $\mathrm{Cu}$. The multi-surface model overestimated B solubility for most soil samples, which was attributed to an inaccurate estimation of reactive B. Interestingly, the observed and modeled solid-solution partitioning of $\mathrm{Cu}$ and $\mathrm{B}$ was found to be rather constant among soils, and the soluble concentration was consistently mainly controlled by the reactive concentration. The solid-solution partitioning of $\mathrm{Zn}$ was strongly related to the soil $\mathbf{p H}$. Generally, the partition relations resulted in a smaller prediction error compared to the multi-surface models. The partition relations in which the soluble concentration was optimized, resulted in an average overestimation for the lowest observed concentrations, and an underestimation for highest concentrations of all three elements. Partition relations in which the $\mathrm{Zn}$ solidsolution partitioning was optimized, resulted in more robust predictions since the prediction error was not related to the actual measured concentration.

Finally, Chapter 8 addressed the main findings of this thesis research. In addition, major limitations were discussed. With regard to practical implications, it was shown how partition relations derived in Chapter 7 can be used for large-scale applications when limited data is available. Spatial soil information for Rwanda and Burundi was used as input into the partition relations, for making spatial predictions of soluble $\mathrm{Zn}$, $\mathrm{Cu}$ and $\mathrm{B}$ concentrations for these countries.

Overall, this thesis delivered valuable information on the micronutrient soil status in SSA, and knowledge on the processes that control soil micronutrient availability. Moreover, based on this knowledge, a low-cost and reliable method was developed for predicting soluble micronutrient concentrations, based on partition and transfer relations. This method can be used for testing soils for $\mathrm{Zn}, \mathrm{Cu}$ and $\mathrm{B}$ availability and for making regional and national soil maps showing micronutrient availability. The soil micronutrient information obtained by these methods, can be used in future work for establishing micronutrient fertilizer recommendation systems. 


\section{ACKNOWLEDGEMENTS}

Deze thesis kon enkel tot stand komen dankzij de vele boeiende, constructieve en gezellige mensen die mij de laatste 4 jaar hebben omringd.

Allereerst wil ik heel graag mijn begeleiders bedanken.

Rob, dankjewel voor het perfecte evenwicht van begeleiding en vrijheid die je me hebt gegeven als promotor. Dankjewel voor de boeiende discussies, je motivatie, je interesse, je empathie. Het is dankzij jou dat ik me heb kunnen ontwikkelen tot een zelfstandige en kritische wetenschapper.

Ellis, bedankt dat je me continu hebt uitgedaagd om na te denken wat al die bodemchemische processen nu betekenen in de praktijk. Ik apprecieer je directheid en je gezelligheid, en ik bewonder je efficiëntie. Ik heb ontzettend veel bijgeleerd van onze meetings en het samen begeleiden van MSc studenten.

In addition to my supervisors, I would like to thank the co-authors Tjisse Hiemstra, Liping Weng, Bert-Jan Groenenberg and Juan Carlos Mendez. Dankjewel Tjisse, om heel veel bij te dragen aan de inhoud van deze thesis. Het was een ontzettend leerrijke ervaring om met je samen te werken. Je interesse en passie voor wetenschap is ongelooflijk inspirerend. Dankjewel Bert-Jan, voor de belangrijke adviserende rol die je hebt gespeeld in dit thesis werk, voor de hulp bij het modelleren, en voor je kritische vragen.

A very special thanks to Juan Carlos. It has been a great pleasure to work together. Thanks for the inspiring discussions, from which I learned a lot. I really hope we meet again, whether it has to do something with ferrihydrite, or just for a beer!

I want to thank the whole consortium of the 'Micronutrients for better yields' project for the helpful meetings. Bas Kempen, thank you for teaching me so much on geospatial modeling and mapping, both during my MSc thesis and this PhD project. I really appreciated your input, and the fact that I could always pass by for questions. Peter van Erp, thank you for your interest in this thesis work, I enjoyed our discussions and our joint supervision of MSc students.

Een bijzondere dankjewel aan Mirjam Breure. Ik vond het een geweldig leerrijke ervaring om samen met jou in het micronutriënten project te mogen werken. Ik bewonder je openheid en je kritische blik.

Dit onderzoek zou onmogelijk zijn geweest zonder het CBLB lab, en al zijn geweldige mensen. Ik wil heel graag Gerlinde Vink, Miranda Vlag, Peter Nobels, Johan Uijtenbroek, Andre van Leeuwen, Wobbe Schuurmans, Wim Pape en Erna Voskuilen bedanken voor hun onmisbare hulp bij de lab analyses, en voor het leuke 
gezelschap. Ik moest initieel even wennen aan jullie Nederlandse directheid, maar ik weet heel zeker dat ik ze nu ga missen!

I want to thank all the staff members, fellow PhDs, interns, and post-doc researchers at the former Soil Quality Department. Thanks for the coffees, lunch breaks and group activities. It was a great experience to be able to interact with so many people coming from different places, doing interesting research and bringing fascinating stories. Marnella, Esther en Priskila, dank jullie wel voor alle goeie zorgen!

Thanks to the Soil Chemistry PhD's for the weekly meetings during the past months, which really helped during the corona-isolation.

A very special thanks to Yilina and Hui for introducing me in the rich taste of Chinese dinners. Yilina, thank you for being the most unpredictable but also most enjoyable office mate!

Thank you Rima for your never-ending enthusiasm for beers after work, and for adventure and cycling. A great combination!

I would like to thank all the MSc students who have contributed to this project: Frank, Koen, Juan, Stef, Sjoerd-Jan and Xinyang. I learned a lot from all of you, and I enjoyed working together.

Thanks to all the great people I have met outside campus during my time in Wageningen. A special thanks to the Droevendaal 65 family, and to Maddy! Dankjewel Maddy, om mij een warme thuis te geven in Wageningen. De tijd met jou in Wageningen waarin we samen in ons $\mathrm{PhD}$ en in ons leven zijn gegroeid, betekent heel veel voor mij.

Verder dank ik de vrienden uit België voor de weekends vol goeie babbels en zotte momenten, ver weg van Wageningen en van bodemchemische processen!

Mijn grootste dank gaat naar mijn ouders, die mij hebben geleerd om altijd de balans te zoeken tussen enerzijds hard werken en anderzijds intens van het leven te genieten. Het is dankzij jullie dat ik dit werk heb kunnen voltooien, en er terwijl heel hard van heb genoten. Sarah en Pirre, dankuwel om mijn geweldige voorbeelden te zijn, en om te zorgen voor prachtige schoonfamilie en kids!

Als laatste, maar zeker niet de minste, wil ik Pieterjan bedanken. Met jou als tochtgenoot, is het leven zoveel boeiender. Bedankt dat je in mij blijft geloven, me blijft uitdagen en mijn leven zoveel rijker maakt. 


\section{ABOUT THE AUTHOR}

Elise Van Eynde was born on the $6^{\text {th }}$ of May, 1992, in Leuven, Belgium. At the end of secondary school, she was determined to make the world a better place for all people. She postponed her dreams to travel around the world, and instead decided to study bioscience engineering at the KU Leuven (Belgium), and not anthropology as first thought. During the soil science courses during the bachelor, she was very much inspired and realized the importance of soils for sustainable food production and land use. This made her choose the major in soil and water management during her masters. During her master thesis, she learned a lot from her field work in Uganda, where she looked at soil properties of landslide affected areas. This work resulted in a first publication. Driven by her eagerness to always learn more and new things, she decided to obtain a second master's degree at Wageningen University (the Netherlands) with a specialization in soil chemistry. Here, she really enjoyed her master thesis on modeling bioavailable zinc in soils from Burundi, which formed the starting point for her PhD work afterwards. During her PhD, she discovered how much she enjoys data-analysis and modeling, especially when the work is done in collaboration with many inspiring people. 


\section{SENSE}

Netherlands Research School for the

Socio-Economic and Natural Sciences of the Environment

\section{I P L O M A \\ for specialised PhD training}

The Netherlands research school for the Socio-Economic and Natural Sciences of the Environment (SENSE) declares that

\section{Elise Van Eynde}

born on $6^{\text {th }}$ May 1992 in Leuven, Belgium

has successfully fulfilled all requirements of the educational PhD programme of SENSE.

Wageningen, 27 August 2021

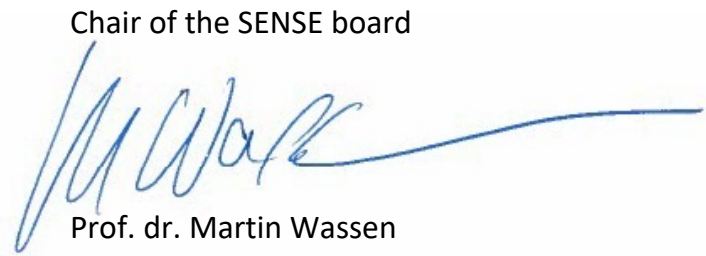

The SENSE Director

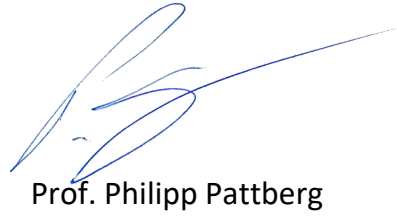

The SENSE Research School has been accredited by the Royal Netherlands Academy of Arts and Sciences (KNAW)

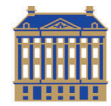

$\begin{array}{llllllllllllllllllllll}K & O & N & I & N & K & L & I & J & K & E & N & E & D & E & R & L & A & N & D & S & E\end{array}$

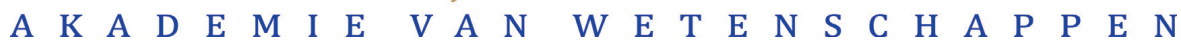




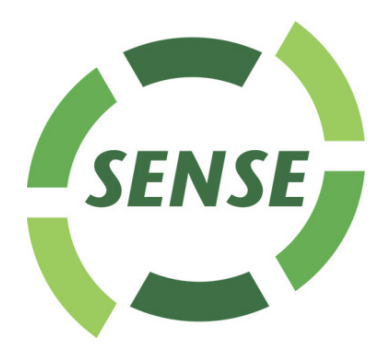

The SENSE Research School declares that Elise Van Eynde has successfully fulfilled all requirements of the educational PhD programme of SENSE with a work load of $37.9 \mathrm{EC}$, including the following activities:

\section{SENSE PhD Courses}

o Environmental research in context (2017)

- Speciation and Bioavailability of Metals, Organics and Nanoparticles, University of Antwerp \& SENSE (2017)

- Research in context activity: 'Exploring the possible acquisition of a pre-automated dilution and pre-concentration instrument at the CBLB lab' (2021)

\section{Other PhD and Advanced MSc Courses}

- Statistical Uncertainty Analysis of Dynamic Models, Wageningen University (2017)

- Workshop: Interpretation of Mid Infrared (MIR) Spectra of Soils, Pedometrics conference (2017)

- Workshop: Infrared spectroscopy as a fast and reliable method of soil testing in practice, Pedometrics conference (2017)

- Multivariate Analysis, Wageningen University (2019)

o Career orientation (2020)

\section{Selection of Management and Didactic Skills Training}

o Supervising 5 MSc students with thesis (2017-2021)

- Supervising 1 MSc student during internship (2018-2019)

o Assisting and teaching in the BSc course 'Soil-Plant relations' (2017-2019)

o Assisting and teaching in the BSc course 'Soil Quality' (2017-2020)

- Assisting and teaching in the MSc course 'Interdisciplinary Topics of Earth and Environment' (2017-2020)

- Assisting and teaching in the BSc course 'Chemical processes in soil, water, atmosphere' (2020)

- Coordinating and teaching the practicals of the BSc course 'Principles of Soil Processes' (2020)

o Teaching in the MSc course 'Applications in soil and water chemistry' (2021)

\section{Oral Presentations}

- Modelling Zn bioavailability in tropical low Zn soils: Geochemical multi-surface models and partition functions. The International Conference on the Biogeochemistry of Trace Elements, 16-21 July 2017, Zürich, Switzerland

SENSE coordinator PhD education

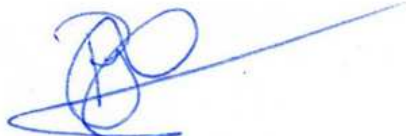

Dr. ir. Peter Vermeulen 
The research described in this thesis was financially supported by a grant from the Netherlands Organization for Scientific Research (NWO, grant number 14688).

Financial support from Wageningen University for printing this thesis is gratefully acknowledged.

Cover design by Stefanie van den Herik, herikmedia

Printed by Proefschriftmaken 